TESIS DOCTORAL

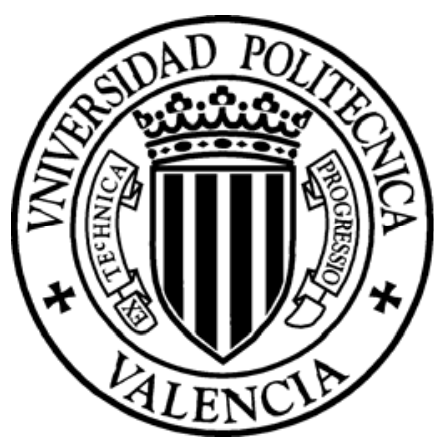

\title{
CONTRIBUCIÓN AL CONOCIMIENTO DEL COMPORTAMIENTO TÉRMICO Y LA GESTIÓN TÉRMICA DE LOS MOTORES DE COMBUSTIÓN INTERNA ALTERNATIVOS
}

\author{
Presentada por \\ CARLOS ALBERTO ROMERO PIEDRAHITA \\ En el \\ DEPARTAMENTO DE MÁQUINAS Y MOTORES TÉRMICOS de la \\ UNIVERSIDAD POLITÉCNICA DE VALENCIA \\ Para la obtención del grado de \\ DOCTOR
}

Valencia, mayo de 2009 



\section{TESIS DOCTORAL}

\section{CONTRIBUCIÓN AL CONOCIMIENTO DEL COMPORTAMIENTO TÉRMICO Y LA GESTIÓN TÉRMICA DE LOS MOTORES DE COMBUSTIÓN INTERNA ALTERNATIVOS}
Realizada por:
D. Carlos Alberto Romero Piedrahita
Dirigida por:
Dr. D. Antonio José Torregrosa Huguet
Dr. D. Pablo César Olmeda González

\section{TRIBUNAL CALIFICADOR}

Presidente: $\quad$ Dr. D. Vicente Macián Martínez

Secretario: $\quad$ Dr. D. Jaime Alberto Broatch Jacobi

Vocales: $\quad$ Dr. D. Pedro Acisclo Rodríguez Aumente

Dr. D. Juan José Hernández Adrover

Dr. D. Octavio Armas Vergel

Vocales Suplentes:

Dr. D. Andrés Melgar Bachiller

Dr. D. Manuel Valdés Del Fresno 



\section{Resumen}

El trabajo desarrollado en la presente tesis está encaminado a estudiar los fenómenos de la transmisión de calor y balance térmico en los motores de combustión interna alternativos, tanto en el cilindro como en el sistema de refrigeración. El principal objetivo de la tesis ha sido la utilización y extensión de un programa computacional de predicción de temperaturas de paredes del motor y de dos programas comerciales de modelado de sistemas termohidráulicos para estudiar los flujos de calor dentro y fuera de la estructura del motor, para evaluar el balance térmico de los motores en estado estacionario y en condiciones transitorias y para modelar, simular y validar diseños nuevos de sistemas de refrigeración de motores para locomotoras. Para lograr este objetivo se han realizado varias tareas que se explican a lo largo de este documento, y que se sintetizan en una revisión de los modelos de transferencia de calor utilizados en los cálculos de flujos de calor en las diferentes condiciones de contorno presentes en los motores de combustión; la expansión de las capacidades de un modelo nodal para obtener de él los flujos de calor discriminados entre las partes metálicas del motor y los calores intercambiados entre los fluidos del sistema y las partes metálicas; el estudio experimental de la evolución de las temperaturas del motor durante el calentamiento bajo un ciclo de conducción con el fin de cuantificar el balance térmico en este modo de operación y proponer alternativas de bajo costo para mejorar ese tiempo de calentamiento; el modelado, simulación, validación y evaluación del impacto sobre el calentamiento, el consumo y las emisiones del motor, de alternativas propuestas en el diseño de los sistemas de refrigeración; y finalmente, la elaboración y aplicación de una metodología de prediseño, modelado y simulación de sistemas de refrigeración para aplicaciones especiales.

\section{Resum}

El treball desenvolupat en la present tesi està encaminat a estudiar els fenòmens de transmissió de calor i el balanç tèrmic en els motors de combustió interna alternatius, tant en el cilindre com en el sistema de refrigeració. El principal objectiu de la tesi ha sigut la utilització i extensió d'un programa computacional de predicció de temperatures de les parets del motor i de dos programes comercials de modelat de sistemes termohidràulics per estudiar els fluxos de calor dins i fora de l'estructura del motor, per 
avaluar el balanç tèrmic dels motors en estat estacionari i en condicions transitòries i per modelar, simular i validar nous dissenys de sistemes de refrigeració de motors per a locomotores. Per assolir aquest objectiu s'han realitzat diverses tasques que s'expliquen al llarg d'aquest document, i que es sintetitzen en una revisió dels models de transferència de calor utilitzats en els càlculs de fluxos de calor en les diferents condicions de contorn presents en els motors de combustió; l'expansió de les capacitats d'un model nodal per obtindre d'ell els fluxos de calor discriminats entre les parts metàl-liques del motor i les calors intercambiats entre els fluids del sistema i les parts metàl-liques; l'estudi experimental de l'evolució de les temperatures del motor durant el calfament baix un cicle de conducció amb el fi de quantificar el balanç tèrmic en aquest mode d'operació i proposar alternatives de baix cost per a millorar eixe temps de calfament; el modelat, simulació, validació i avaluació de l'impacte sobre el calfament i les emissions del motor, d'alternatives proposades en el disseny dels sistemes de refrigeració; i finalment, l'elaboració i aplicació d'una metodologia de predisseny, modelat i simulació de sistemes de refrigeració per a aplicacions especials.

\begin{abstract}
In the thesis at hand the study of heat tranfer phenomena and thermal balance inside the engine structure, as well as in the cooling system is addressed. It has been the main goal of this thesis the use and extension of a model for the prediction of combustion chamber wall temperatures, along with the application of two commercial computational thermohydraulic programs, to the study of heat flows inside and outside the engine structure in order to assess the engine energy balance under steady and transitory operation, and also to design or redesign new cooling systems for locomotive engines. To accomplish the aforementioned goal some steps explained along this document, were followed. Those tasks consisted on: a thorough review of the heat transfer models used to calculate the heat flows in all the internal combustion engine boundaries; the enhacement of a predictive wall temperature model to detail all the heat flows between metallic parts and boundary media; the experimental study of the engine warm-up under a driving cycle to study its energy balance, and to propose low cost modifications to the original cooling system intended to accelerate the engine warm-up; the modelling and assessment of the proposed modifications in terms of their impact on the engine warm-up time, as well as on the fuel
\end{abstract}


consumption and emissions; and, finally, the development and implementation of a methodology flor the initial design, modelling and simulation of engine cooling systems for special applications. 
A mis padres

A Valentina y Carlos Alberto 


\section{Agradecimientos}

Esta tesis ha sido realizada durante mi trabajo como docente de la Escuela de Tecnología Mecánica de la Universidad Tecnológica de Pereira, a ella debo todo el apoyo económico, y de ello estoy profundamente agradecido.

Ante todo, quiero agradecer a mis directores Antonio José Torregrosa y a Pablo César Olmeda por la asesoría brindada y por vincularme a su equipo de trabajo. El Dr. Torregrosa me presentó en la primera charla que tuvimos, de una manera insuperablemente clara, sintética, precisa y completa, el problema de la transferencia de calor entre los gases y las paredes de la cámara de combustión en los motores, y este ha sido el inicio del trabajo cuyos resultados presento, pero que no culmina ahí, porque aunque la charla fue breve, proyectó todas las tareas sobre las que quisiera seguir trabajando y para las que confío contar con su profundo conocimiento y su sabia visión. Agradezco una vez más al Dr. Torregrosa su excelente disposición para conmigo, su interés en mi trabajo, sus acertadas recomendaciones y también por compartir sus ideas y pensamiento. A Pablo, incansable trabajador, le agradezco grandemente el acompañamiento continuo, las siempre oportunas observaciones y el rigor puesto en la lectura y corrección de esta tesis.

Agradezco a los responsables del Departamento de Máquinas y Motores Térmicos, quienes me han facilitado los medios necesarios para llevar a cabo los trabajos de investigación que se presentan en la tesis, así como al personal de secretaría por ayudarme en todos los trámites administrativos.

Muy especialmente me gustaría dar las gracias a Juanma por la ayuda prestada en todos los temas informáticos relacionados con la programación en $\mathrm{C}++$. Por la grata compañía durante estos años en el departamento quiero dar las gracias a la gente de la línea de Transferencia de Calor y, particularmente, a Jaime Martin y Bart Degraeuwe. No puedo menos que agradecer la ayuda de Benjamín Pla, José Manuel Lujan, Salvador Torró en la facilitación de los trabajos de medición de temperaturas. Los trabajos realizados sobre los sistemas de refrigeración de las locomotoras no se habrían podido llevar a cabo sin la colaboración de la empresa Vossloh y en particular de Toni y Jordi. Gracias a los estudiantes Edouard, Juliene, Etienne por sus contribuciones en este trabajo. 
viii

Agradezco a todos mis amigos que no menciono porque son muchos, que me han apoyado en esta aventura de venirme a hacer lo que me gusta. Es muy importante para mí reconocer el apoyo, aún en la distancia, de mis compañeros de trabajo en la UTP, pero sobre todo de Yamid, Héctor Fabio y Gabriel. Gracias a mis hermanos por querer que emprendiera esta etapa y sobre todo gracias a John Jairo y a Rosa.

Finalmente, lo más importante, de la manera más profunda, agradezco a mi compañera Luz Adriana y a mis hijos por haberme aguantado durante estos años y por aceptar mi falta de atención en ciertos momentos. 


\section{Índice General}

Lista de símbolos................................................... XV

Capítulo 1. Introducción............................................. 1

1.1. Motivación............................................................. 3

1.2. Objetivos...................................................... 8

1.3. Antecedentes................................................. 9

1.4. Metodología y planteamiento.................................. 13

1.5. Bibliografía.................................................... 15

\section{Capítulo 2. Estado del arte de la transferencia de calor en los}

M.C.I.A............................................ 19

2.1. Introducción..................................................... 21

2.2. La transferencia de calor en el motor............................ 22

2.3. Transferencia de calor entre los gases del cilindro y las paredes de la cámara de combustión..................................................... 23

2.3.1. Modelos globales.......................................... 26

2.3.2. Modelos zonales y locales................................. 32

2.3.3. Modelos multidimensionales............................... 46

2.3.4. Transferencia de calor radiativa.................................. 51

2.4. Transferencia de calor en las pipas de admisión y escape............. 62

2.5. Transferencia de calor del lado del refrigerante.................... 67

2.5.1. Modelos empíricos.......................................... 69

2.5.2. Modelado CFD ........................................ 72

2.6. Transferencia de calor entre el aceite y el motor..................... 73

2.6.1. Transferencia de calor entre el pistón y el aceite................ 73

2.6.2. Transferencia de calor entre el aceite y la estructura (bloque y culata)..................................................... 77

2.7. Transferencia de calor entre el motor y el ambiente................ 78

2.8. Conclusiones................................................. 81

2.9. Bibliografía................................................ 83 
Capítulo 3. Caracterización térmica de los M.C.I.A................ 95

3.1. Introducción................................................ 97

3.2. Proceso de combustión y flujo de calor a través de las paredes de la cámara de combustión de un motor alternativo................... 99

3.2.1. El análisis de Fourier.................................. 100

3.2.2. Transformada de Laplace................................ 102

3.3. Balance térmico del moto.................................... 103

3.3.1. Balance energético del motor en estado estacionario a carga constante............................................... 105

3.3.2. Balance energético durante el calentamiento del motor....... 107

3.3.2.1. Consideraciones teóricas y experimentales.............. 107

3.3.3. Balance energético experimental........................ 117

3.3.3.1. Balance térmico del motor durante su calentamiento bajo una carga y régimen constantes, representativos del ciclo de conducción.

3.3.3.2. Balance térmico del motor durante su calentamiento en su proceso de calentamiento en el ciclo de conducción NEDC... 123

3.3.3.3. Energía recuperada en el vehículo durante el ciclo de conducción.............................................. 130

3.3.4. Observaciones.......................................... 134

3.4. Modelado de la temperatura y los flujos de calor con un modelo predictivo................................................ 135

3.4.1. Breve descripción del modelo térmico...................... 136

3.4.1.1. Fundamentos del modelo nodal...................... 136

3.4.1.2. Interacciones y condiciones de contorno............... 138

3.4.2. Programa de cálculo..................................... 141

3.4.3. Predicción de las temperaturas del primer motor ante cambios en escalón de las condiciones de operación............... 143

3.4.3.1. Predicción de los flujos de calor entre los nodos del modelo.

3.4.3.2. Predicción de las temperaturas y los flujos de calor en estado transitorio.

3.5. Predicción de las temperaturas y los flujos de calor del segundo 
motor....................................................... 151

3.5.1. Predicción de los flujos de calor al refrigerante............... 151

3.5.2. Predicción del comportamiento térmico del motor bajo condiciones de calentamiento................................ 154

3.5.3. Observaciones.......................................... 158

3.6. Conclusiones............................................ 159

3.7. Bibliografía.................................................... 161

Capítulo 4. Consideraciones y herramientas de diseño y modelado de los sistemas de refrigeración de los motores.................... 165

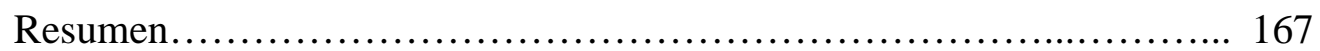

4.1. Introducción................................................... 167

4.2. Prediseño de los sistemas de refrigeración de los motores........... 169

4.2.1. Cálculo preliminar de los componentes del sistema de refrigeración.................................................... 170

4.2.2. Metodología de diseño de los sistemas de refrigeración.......... 184

4.2.3. Cálculo de los radiadores de los sistemas de refrigeración....... 187

4.3. Modelado de los sistemas de refrigeración........................ 196

4.3.1. Modelos simplificados de la temperatura del motor a nivel de sistema..................................................... 197

4.3.2. Modelos a nivel de circuito del sistema de refrigeración del

4.4. Herramientas de modelado unidimensional....................... 208

4.5. Conclusiones.................................................. 212

4.6. Bibliografía................................................ 213

Capítulo 5. Modelado, simulación y validación del sistema de refrigeración de un motor HSDI para reducir su tiempo de calentamiento.................................................. 217

5.1. Introducción.................................................. 219

5.2. Modelado térmico del motor................................... 221

5.2.1. Descripción y caracterización del sistema de refrigeración...... 221

5.2.2. Modelo 1D del sistema de refrigeración original del motor...... 226

5.3. Modelado de las modificaciones al sistema de refrigeración del 
motor para reducir el tiempo de calentamiento

5.4. Validación experimental de las modificaciones propuestas y valoración de su influencia sobre las emisiones y el consumo de combustible....

5.4.1. Flujos de refrigerante experimentales de todas las configuraciones............................................ 237

5.4.2. Respuesta de temperatura del refrigerante................... 238

5.4.3. Emisiones y consumo de combustible........................ 241

5.5. Conclusiones.................................................. 243

5.6. Bibliografía............................................... 245

Capítulo 6. Diseño, modelado y simulación del sistema de refrigeración del motor de una locomotora........................ 249

Resumen......................................................... 251

6.1. Introducción............................................... 251

6.2. Definición de las especificaciones del motor y diseño preliminar del sistema de refrigeración.......................................... 254

6.2.1. Especificaciones de diseño del sistema de refrigeración (tarea técnica).................................................. 254

6.2.2. Cálculo preliminar de los componentes del sistema de refrigeración.............................................. 258

6.2.3. Disposición de los componentes del sistema hidráulico y del sistema de ventilación.......................................... 266

6.3. Modelado y simulación del sistema de refrigeración................ 269

6.3.1. Modelado y caracterización del sistema de ventilación........... 269

6.3.2. Caracterización de los componentes de los circuitos del refrigerante............................................. 275

6.3.3. Modelado en FlowMaster del sistema de refrigeración del motor de la locomotora.................................... 280

6.3.4. Alcance de resultados de los modelos........................... 287

6.4. Validación experimental del sistema de refrigeración del motor de la locomotora.......................................................... 289

6.4.1. Instrumentación del sistema de refrigeración................... 290

6.4.2. Resultados de las mediciones en el sistema de refrigeración 
operando el motor a plena carga.............................. 294

6.4.3. Cálculos de los flujos de calor en el sistema de refrigeración operando el motor a plena carga

6.4.4. Comparación y análisis de las mediciones en el sistema de refrigeración operando el motor a plena carga con los resultados de la simulación 1D.

6.5. Conclusiones................................................ 303

6.6. Bibliografía................................................ 304

Capítulo 7. Conclusiones y trabajos futuros........................ 309

7.1. Conclusiones................................................ 311

7.2. Trabajos futuros......................................... 318

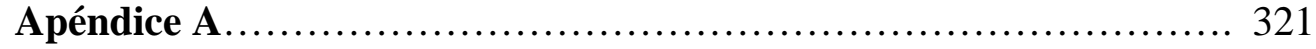

A.1. Acerca de AMESim............................................ 321

A.2. Acerca de FlowMaster y PowerFlow........................... 327

A.3. Bibliografía.............................................. 330 


\title{
LISTA DE SÍMBOLOS
}

\author{
Latinos \\ $A \quad$ Área \\ $C_{p} \quad$ Calor específico a presión constante de un gas \\ $c_{m} \quad$ Velocidad lineal media del pistón. \\ $c_{u} \quad$ Componente tangencial del gas en la cámara de combustión \\ $D, d \quad$ Diámetro \\ $f \quad$ Factor de fricción \\ $F \quad$ Área \\ $h \quad$ Coeficiente de transmisión de calor \\ $h \quad$ Entalpía \\ $i \quad$ Número de cilindros del motor \\ $k \quad$ Conductividad térmica \\ $K \quad$ Conductancia térmica, constante \\ $K \quad$ Coeficiente de momento, coeficiente de pérdidas de presión \\ $L \quad$ Longitud característica \\ $M \quad$ Par \\ $m \quad$ Masa \\ $\dot{m} \quad$ Flujo másico \\ $n \quad$ Velocidad de motor \\ $N \quad$ Potencia \\ $p \quad$ Presión \\ pme Presión media efectiva del motor. \\ $Q \quad$ Calor \\ $\dot{Q} \quad$ Flujo de calor por unidad de tiempo \\ $S \quad$ Carrera del pistón \\ $T$ Temperatura \\ $t$ Tiempo \\ $u \quad$ Velocidad del fluido
}


U Coeficiente global de transferencia de calor, energía interna del sistema

V Volumen, velocidad

$\dot{V} \quad$ Flujo volumétrico

W Trabajo mecánico

\section{Griegos}

$\alpha \quad$ Coeficiente de difusividad

$\varepsilon \quad$ Emisividad, eficiencia del intercambiador.

$\eta \quad$ Eficiencia

$\mu \quad$ Viscosidad dinámica

$v \quad$ Viscosidad cinemática

$\rho \quad$ Densidad

$\sigma \quad$ Constante de Stefan-Boltzmann

$\omega \quad$ Velocidad angular

$\Delta \quad$ Incremento

\section{Subíndices}

$A C \quad$ Relativo al enfriador de aire

$a d m$ Relativo a la admisión

aire Relativo al aire

bl Relativo al bloque del motor

comb Relativo al combustible

$e \quad$ Relativo a las condiciones efectivas de motor

en Relativo a las condiciones de entrada

esc Relativo al escape del motor

equiv Relativo a valores equivalentes

fr Frontal

$g \quad$ Relativo a las condiciones del gas

$i \quad$ Relativo a las condiciones en el interior del cilindro

rad Relativo al radiador, relativo a la radiación 
refr Relativo al refrigerante

sal Relativo a las condiciones de salida

$w \quad$ Relativo a las condiciones en la pared

$\infty \quad$ Relativo a condiciones basadas en corriente libre de un fluido

\section{Superíndices}

en Relativo a las condiciones de entrada

sal Relativo a las condiciones de salida

\section{Grupos adimensionales}

$B i \quad$ Número de Biot

Fo Número de Fourier

j $\quad$ Número de Colbourn

$\mathrm{Nu} \quad$ Número de Nusselt

$\mathrm{Pr} \quad$ Número de Prandtl

$R a \quad$ Número de Rayleigh

Re Número de Reynolds

St Número de Stanton

\section{Siglas}

$C F D \quad$ Computational Fluid Dynamics.

$E D C+E U D C$ European Driving Cycle + Extra Urban Driving Cycle.

EGR Exhaust Gas Recirculation.

EVC Cierre de la válvula de escape

EVO Apertura de la válvula de escape

HSDI High Speed Direct Injection.

ITD Diferencia de temperaturas de entrada

IVC Cierre de la vávula de admisión

IVO Apertura de la válvula de admisión

M.C.I.A. Motor de combustión interna alternativo.

NEDC Nuevo ciclo de conducción europeo.

NTU Número de unidades de transferencia de calor. 
xviii

UPV Universidad Politécnica de Valencia.

WAM Wave Action Model (Modelo de acción de ondas)

$A C T \quad$ Apparent combustion time 


\section{Capítulo 1}

\section{Introducción}

1.1. Motivación ………………………………………………………….... 3

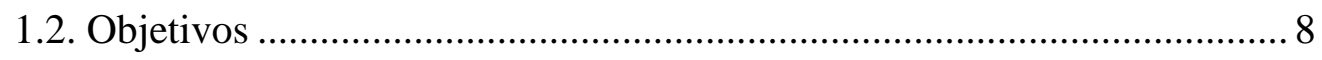

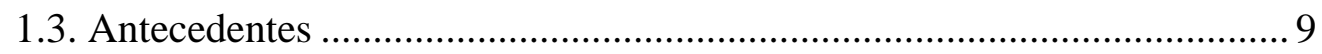

1.4. Metodología y planteamiento................................................................ 13

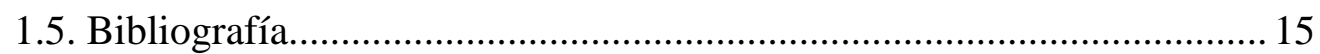




\subsection{Motivación}

La mayor parte de los esfuerzos por mejorar los sistemas de potencia de los medios de transporte se ha enfocado a mejorar sus características dinámicas, reducir las emisiones y reducir también el consumo de combustible mediante desarrollos aplicados sobre todo a los sistemas de combustible, los sistemas de intercambio de gases, los mecanismos de distribución de gases, los sistemas de inyección y los sistemas de control. Los incrementos en ganancias logradas con los diferentes sistemas se han ido haciendo más pequeños y sólo en los últimos años se ha vuelto la mirada hacia los sistemas de refrigeración, el eslabón olvidado en los sistemas de control de los sistemas de potencia, como foco potencial de perfeccionamiento del motor y ganancias traducibles en ahorros energéticos y reducción de emisiones.

Con los avances tecnológicos aplicados a los sistemas de transporte, ya no es suficientemente preciso hablar de sistemas de refrigeración del motor, sino de sistemas de gestión térmica por la complejidad de los fluidos que participan de los intercambios de calor y la variedad de los componentes, algunos de ellos no pertenecientes al grupo funcional del motor. Es por esto que a lo largo de esta tesis se entenderá por sistema de gestión térmica al conjunto de sistemas dedicado al intercambio de calor en los sistemas de potencia de los vehículos, acoplados directa o indirectamente con el sistema básico de refrigeración del motor.

Las exigencias de reducción de emisiones han conducido a la implementación de sistemas de recirculación de gases refrigerados; el aumento de la potencia específica de los motores ha hecho obligatorio en casi todos los motores modernos el montaje de refrigeradores del aceite del motor y, desde hace poco también, de la transmisión. La búsqueda de una mayor potencia ha conducido a la aplicación de tecnologías de turboalimentación y sobrealimentación con al menos una etapa de refrigeración del aire fresco comprimido antes de entrar a los cilindros. Se cuentan así al menos tres intercambiadores de calor que antes no existían y que se integran en los sistemas convencionales de refrigeración, haciendo necesaria la implementación de sistemas de control de flujos y temperaturas (de refrigerantes, gases, aire de admisión y aceite), que se complementen con los sistemas de control del motor, de las emisiones y de la transmisión. 
En mayor o menor grado y respetando las escalas y las particularidades, la actualización de los sistemas de gestión térmica representa una reconversión tecnológica que tiene lugar en los sistemas de potencia de los vehículos de pasajeros, los vehículos de transporte pesado, la maquinaria industrial y que hoy empieza a investigarse en las locomotoras [1.21].

El papel desempeñado por los sistemas de gestión térmica es complejo. Originalmente destinados a garantizar los niveles de temperatura seguros desde el punto de vista del mantenimiento de las tensiones térmicas dentro de los niveles permitidos por los materiales de la estructura y los fluidos, los sistemas de gestión térmica hoy se han convertido también en objeto de ingeniería térmica con identidad propia, con restricciones y necesidades de control adicionales a las mínimas tradicionalmente impuestas por su papel de sistema complementario. Los sistemas de gestión térmica actuales, además de su función preventiva de temperaturas máximas de los materiales y de los fluidos, cumplen con funciones de control de temperatura de las partes metálicas en los puntos de interés de la estructura del motor y de los fluidos involucrados (refrigerantes, gases de escape, aire de admisión y aceite) en diferentes puntos de los circuitos. Para ilustrar con un ejemplo las nuevas tareas asumidas por los sistemas de gestión térmica, puede citarse el control del flujo de refrigerante a su paso por las superficies entre las válvulas de escape, recomendado como estrategia de control de la temperatura media de la cara de la culata expuesta a los gases del cilindro.

Complementando lo anterior, en la búsqueda por optimizar el proceso de combustión de los motores y mejorar su respuesta transitoria, tanto durante el tiempo de calentamiento como en los cambios de punto de operación dentro del mapa de posibles condiciones de funcionamiento, se le está dedicando mucha atención al modelado de la transferencia de calor entre el gas de trabajo dentro de los cilindros del motor y las paredes [1.1]-[1.3]. Una razón adicional para ahondar en las investigaciones relacionadas con la transferencia de calor entre las paredes de los cilindros y los gases de trabajo es la necesidad de disponer de modelos detallados de las temperaturas de las partes metálicas del motor y la identificación de las rutas que siguen los flujos de calor dentro del mismo.

La gestión térmica en los sistemas de automoción modernos se ha convertido en una tecnología transversal cuyo futuro tiende a trascender los límites del 
sistema de potencia y a convertirse, nombrándolos con un término más amplio, en sistemas de gestión energética. El concepto de gestión energética es más integral en lo que se refiere al análisis, modelado y simulación de la distribución de la energía del combustible consumido por un vehículo o medio de transporte. Adicionalmente, el concepto de sistemas de gestión energética cubre, en su definición más amplia, las tecnologías híbridas de tracción, en las cuales se emplean simultáneamente motores eléctricos y motores de combustión para la propulsión del vehículo. Buena parte de la energía de los motores eléctricos de los vehículos híbridos puede obtenerse no sólo por la recuperación de la energía no utilizada por el motor de combustión, sino también por la utilización de la energía de regeneración de otros sistemas del automóvil como los sistemas de frenado y suspensión. En el esquema de la Figura 1.1 se sintetiza la utilización de la energía del combustible suministrado al motor del vehículo. Sólo cerca de un $40 \%$ de la energía del combustible se realiza en forma de energía mecánica efectiva. El tratamiento holístico de la distribución de la energía en los medios de transporte hace parte hoy de las directrices de los departamentos gubernamentales de energía en lo que se refiere a las políticas energéticas en los sistemas de transporte (un ejemplo de esto es el programa PNGV, patrocinado por el gobierno de los E.U., al igual que otros programas similares [1.4]).

Es en el marco de la gestión energética del vehículo donde se inscriben las últimas investigaciones y desarrollos de los sistemas de gestión térmica. En particular, para el modelado y simulación de los sistemas térmicos en los medios de transporte, actualmente en las investigaciones se estudia (bien sea a nivel predictivo o de diagnóstico) la operación del grupo motor-transmisión del vehículo sometido a unas condiciones de operación establecidas según su utilización. Para dicho estudio se integran modelos a nivel de componentes y modelos a nivel de sistema. Así, el vehículo con su modelo dinámico conforma un sistema que interactúa funcionalmente con el modelo del grupo de potencia, demandando de éste la operación en unas condiciones de par y velocidad dados, según el régimen de conducción establecido. A nivel de componente el motor requiere de modelos de intercambio de gases (renovación de la carga), modelos de combustión y modelos de transferencia de calor. A nivel de sistema, la gestión térmica del vehículo acopla los modelos de flujos de calor en los diferentes componentes del vehículo de manera que se pueda tener un control de dichos flujos en favor del 
desempeño integral del vehículo, valorado por el consumo de combustible y las emisiones.

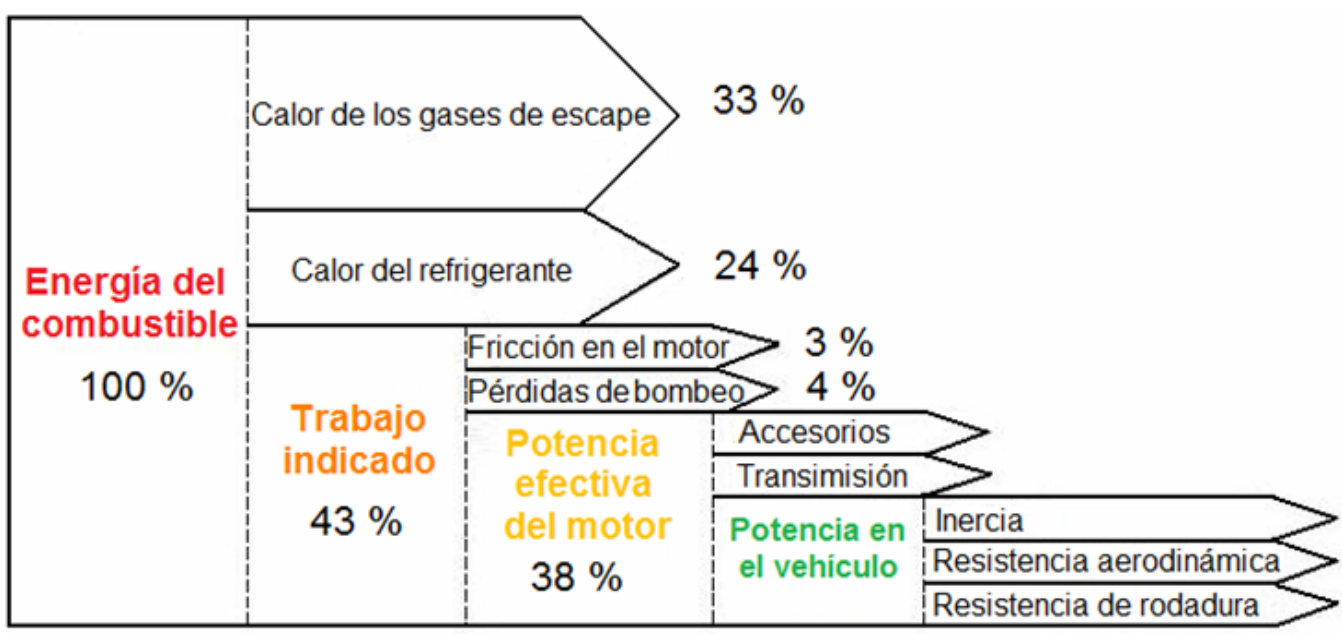

Figura 1.1. Esquema genérico de la distribución de la energía del combustible en un vehículo equipado con motor de combustión (las proporciones de las componentes variarán dependiendo del tipo de vehículo y de las condiciones de operación).

Tras esta breve presentación del contexto actual en el que se hilvanan los diferentes esfuerzos de investigación en el campo de los sistemas energéticos relacionados con los sistemas propulsivos de los medios de transporte, y atendiendo la finalidad particular de la presente tesis, puede decirse que, en general, las investigaciones de modelado de los sistemas de gestión térmica se conducen según un esquema cercano al de la Figura 1.2, en el que: a nivel de componente, el motor es estudiado bien sea con un modelo de diagnóstico o con un modelo predictivo, que permite determinar o predecir, entre muchas otras características, el calor liberado por el combustible, la potencia indicada y el calor disipado por el motor, y puede acoplarse a un modelo de predicción de temperaturas y flujos de calor dentro del motor y a un modelo de simulación de tracción del automóvil (como CRUISE de AVL [1.5]) que predice la dinámica del vehículo a partir del régimen de operación suministrado (en la figura el régimen ilustrado corresponde al nuevo ciclo de conducción europeo - NEDC). La utilización de programas de modelado de sistemas termohidráulicos 1D (como Amesim ${ }^{\circledR}$ o FlowMaster $^{\circledR}$ ) y de 
dinámica de fluidos computacional - 3D-CFD (como Fluent ${ }^{\circledR}$, Star-CD ${ }^{\circledR}$ o PowerFlow $^{\circledR}$ ) permite acoplar los modelos de los sistemas de refrigeración y ventilación del motor, así como otros sistemas de intercambio de calor, a los tres grandes bloques de modelos descritos, y también al modelo integral de gestión térmica del vehículo.

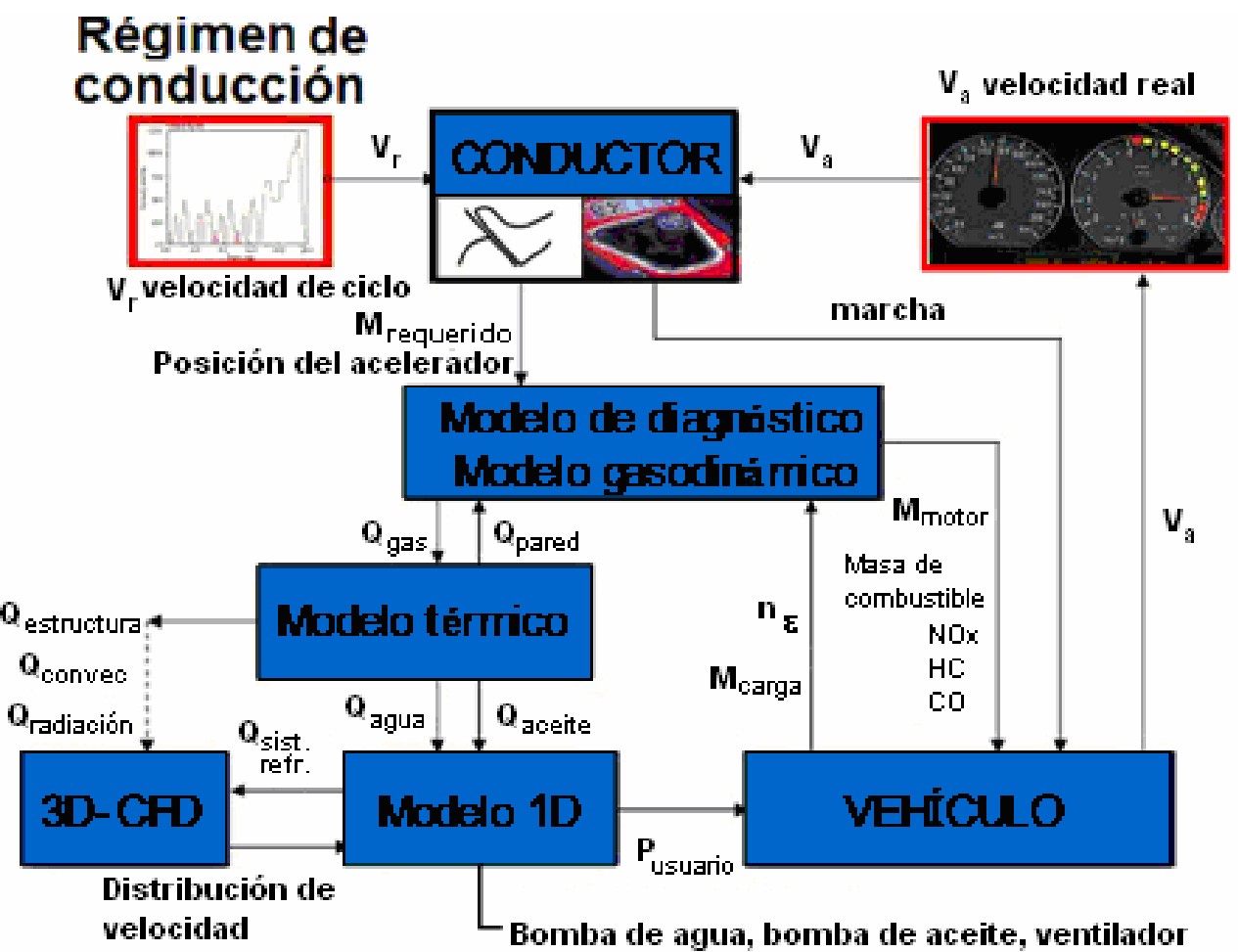

Figura 1.2. Diagrama de bloques del modelado dinámico del vehículo con énfasis en el modelado de la gestión térmica.

El tema de la presente tesis se inscribe en el estudio de la gestión térmica de los sistemas de transporte y, más específicamente, en el estudio del balance energético experimental del motor durante su calentamiento, y en el estudio teórico-experimental de los flujos de calor en estado estacionario y transitorio dentro del motor con ayuda de un modelo térmico. Del estudio del balance energético del motor durante su calentamiento se ha concluido la necesidad de proponer estrategias de refrigeración que, sin afectar mucho la topología del sistema de refrigeración, produzcan un calentamiento más rápido del 
motor y permitan mejorar las prestaciones de emisiones y consumo. Aparte de esto, parte de la tesis se dedica a la exposición de una metodología para el diseño de los sistemas de refrigeración de motores, con aplicación a las locomotoras. Para lograr estos objetivos se han utilizado las instalaciones experimentales disponibles en el CMT, las herramientas computacionales Amesim $^{\circledR}$ y FlowMaster ${ }^{\circledR}$ y se ha extendido la funcionalidad de un modelo de predicción de temperaturas de paredes del motor.

\subsection{Objetivos}

Esta tesis ha sido realizada con el fin de estudiar los fenómenos de transmisión de calor y el balance térmico en motores de combustión interna alternativos, tanto en el cilindro como en sus sistemas de refrigeración, en condiciones estacionarias y transitorias, mediante la utilización y adaptación de un programa computacional de predicción de temperaturas de paredes del motor y de dos programas comerciales de modelado de sistemas termohidráulicos, también utilizados para modelar, simular y validar diseños nuevos de sistemas de refrigeración de motores para locomotoras. Para lograr este objetivo general se han considerado los siguientes objetivos específicos:

- Revisar los modelos de transferencia de calor utilizados en los cálculos de flujos de calor con las condiciones de contorno presentes en motores modernos.

- Expandir las capacidades del modelo nodal desarrollado, para obtener de él los flujos de calor discriminados entre las partes metálicas del motor y los calores intercambiados entre los fluidos del sistema y las partes metálicas.

- Estudiar experimentalmente la evolución de la temperatura del motor y su balance térmico durante el calentamiento bajo una carga y régimen constantes, y bajo un ciclo de conducción europeo (NEDC), para caracterizar su comportamiento hidráulico y térmico, así como para cuantificar el balance térmico en esas condiciones de operación y proponer alternativas de bajo costo para mejorar el tiempo de calentamiento del motor.

- Modelar, simular, validar y evaluar el impacto sobre el calentamiento y las emisiones del motor, de alternativas de bajo costo en el diseño de los sistemas de refrigeración. 
- Elaborar una metodología de prediseño, modelado y simulación de sistemas de refrigeración para aplicaciones especiales.

\subsection{Antecedentes}

El modelado térmico del motor, visto este como fuente principal de disipación de calor en los medios de transporte, comprende el tratamiento de seis subsistemas: las pipas de admisión y escape, la cámara de combustión o el cilindro, las camisas de enfriamiento en el bloque y la culata, el aceite de enfriamiento (aprovechado para enfriar la parte inferior del pistón y que desempeña un papel importante en la fricción entre la camisa y los anillos, fricción que genera un flujo de calor adicional entre el pistón y la camisa) y, el sexto y último subsistema, las partes sólidas que conforman el motor y sirven para separar los otros cinco subsistemas. Estas partes se someten a transferencia de calor inestable tridimensional. La inestabilidad es de dos formas: las fluctuaciones rápidas en las superficies cilindro-gas y los transitorios algo menos rápidos causados por cambios en la carga y la velocidad. El constante almacenamiento y entrega de energía a ambos niveles de frecuencia causa una pérdida potencialmente grande de la energía disponible, porque la energía es absorbida a elevadas temperaturas y liberada luego a bajas temperaturas. Otros subsistemas importantes que entran en juego en la transferencia de calor son el turbocompresor, los interenfriadores y el sistema de tratamiento de emisiones.

Muchos de los trabajos relevantes relativos a la transferencia de calor y la gestión térmica en los motores de combustión interna han sido divulgados en las investigaciones de Borman y Nishiwaki [1.17], Finol y Robinson [1.20], y Pang y Brace [1.1]. Algunos de los trabajos teóricos y experimentales relacionados con el estudio y mejora de los sistemas de refrigeración son los realizados por Luptowski et al. [1.22], Cortona [1.23], Chalgren y Traczyk [1.3], Allen [1.19], Mohan et al. [1.24], entre otros.

Los modelos para valorar las temperaturas del motor pueden ser de un solo nodo (globales), de tres nodos (tres nodos representados por la camisa, el pistón y la culata), de nodos múltiples, y modelos de elementos finitos. Los tres primeros se cuentan entre los modelos de capacitancias concentradas. El método de capacitancias concentradas es uno de los métodos más utilizados 
para evaluar las temperaturas de las partes metálicas de los motores de combustión y ha sido aplicado particularmente por Kaplan y Heywood [1.6], Shayler [1.7], Veshagh y Chen [1.8]. El trabajo de Bohac, Baker y Assanis [1.9] da una descripción detallada de dicho método.

Como en otros centros motorísticos de investigación, en CMT-Motores Térmicos, tratando de desarrollar propuestas de ahorro de combustible, de reducción de emisiones, de mejoramiento de la dinámica del motor y del vehículo, se ha buscado continuamente la implementación de códigos detallados de cálculo del ciclo de trabajo destinados a cuantificar y correlacionar la transferencia de calor. Actualmente los programas propios del centro tienen submodelos que predicen la transferencia de calor basándose en las relaciones termodinámicas disponibles como la temperatura de la carga de trabajo, la velocidad del pistón, etc. Durante el proceso de modelado con el programa WAM, todos los subsistemas del motor enumerados anteriormente son modelados incluyendo los términos de flujo de calor. Esos submodelos han sido publicados en los trabajos de Santos [1.10], Reyes [1.12] y Dolz [1.13].

El modelo con el que se trabajará en la presente tesis es el modelo nodal desarrollado recientemente por Degraeuwe [1.14], que utiliza el método de las capacitancias concentradas. El modelo construido para predecir los flujos de calor a través de las paredes de la cámara de combustión del motor emplea un tratamiento de analogía eléctrica, de manera similar a como lo hicieron los autores anteriormente mencionados. El motor es tratado como una red térmica en la cual el calor se puede transferir entre masas concentradas a través de resistencias eléctricas. El pistón, la culata y el cilindro del motor son divididos en un número razonable de partes (nodos) que se interconectan por resistencias térmicas y condiciones de contorno, representadas éstas por el gas del cilindro, el refrigerante, el aceite lubricante y el aire y los gases de escape en las pipas de admisión y escape. La envolvente externa del bloque y la culata es considerada adiabática.

El modelo nodal ha sido validado con la información experimental de un motor (denominado en esta tesis "primer motor") y puede emplearse con fiabilidad en motores de la misma clase, siempre que se provea al modelo de la geometría detallada requerida. El modelo tiene en cuenta la transferencia 
de calor en los seis subsistemas mencionados en la parte inicial de este parágrafo. En la Figura 1.3. se ilustra el diagrama de flujo que sirve para describir la operación del programa.
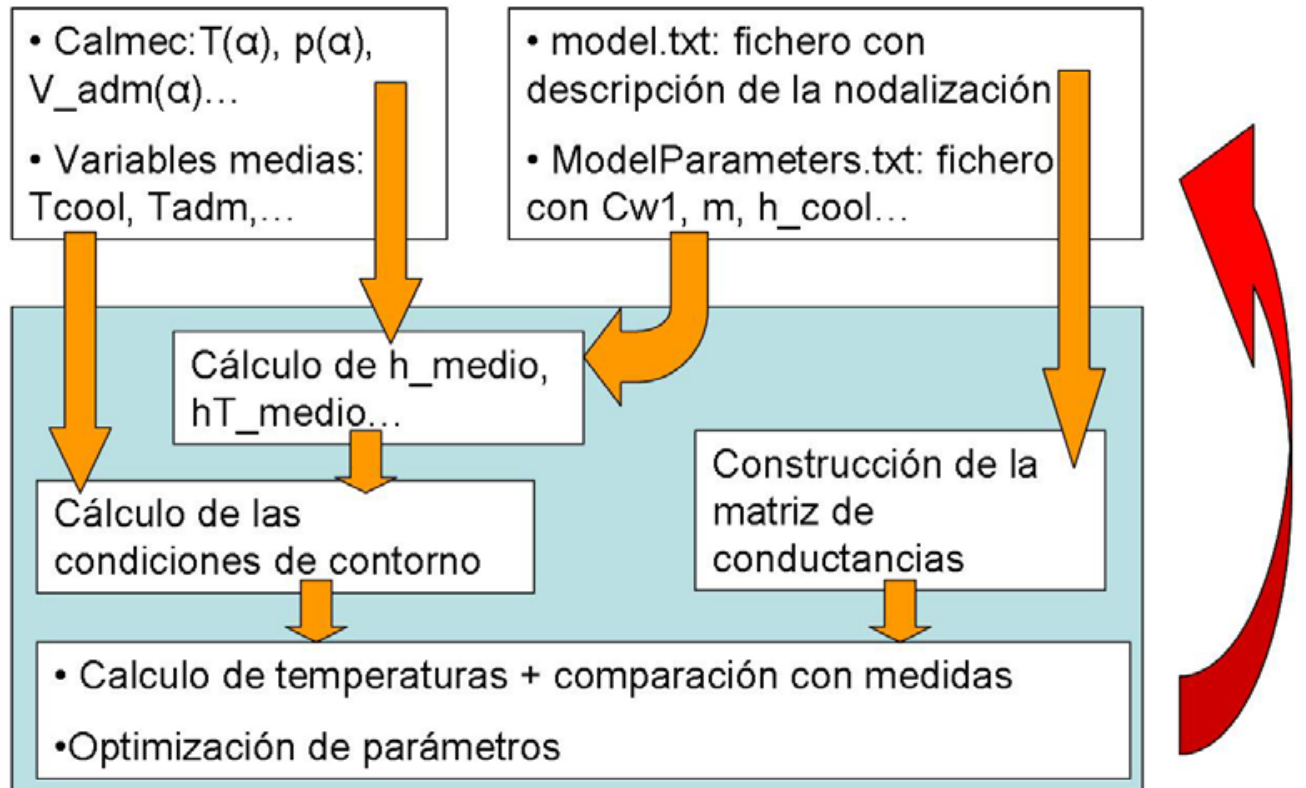

Figura 1.3. Estructura actualizada del programa computacional desarrollado por Degraeuwe [1.14] para predecir las temperaturas de pared. El esquema ha sido actualizado en su último campo, relativo a la predicción y presentación al usuario de los flujos de calor entre nodos.

El programa inicia con la lectura de la geometría del motor, la obtención de las variables medias de operación, presión, temperatura y volumen instantáneos, y luego pasa a la determinación de las otras variables instantáneas conducentes a la definición de las condiciones de contorno del problema térmico a resolver. Los parámetros del modelo pueden ser cambiados en un fichero accedido por el programa. El modelo basa sus rutinas de transferencia de calor en las expresiones conocidas de Woschni [1.17] para la interfase gases-paredes de cilindro, y Nu-Re (con las adaptaciones pertinentes) para las otras fronteras en las pipas de admisión y escape, para el intercambio de calor en las camisas de enfriamiento del bloque y la culata, y para el sistema aceite-pistón. Degraeuwe utilizó su modelo para evaluar la influencia de la refrigeración separada (separación de 
circuitos de refrigeración para el bloque, para la parte inferior de la culata en contacto con los gases del cilindro, y para la parte superior de la culata en contacto con los gases de escape) y de las temperaturas del refrigerante en las emisiones del motor. En el capítulo cuarto de esta tesis se presentarán mayores detalles del modelo.

Todos los submodelos de transferencia de calor pueden cambiarse en el código del programa, permitiendo valorar la influencia de cambiar, por ejemplo, las diferentes correlaciones de transferencia de calor entre los gases del cilindro y las paredes de éste, comentados en el reporte de Borman y Nishiwaki [1.18] (Annand, Woschni, Hohenberg, etc.) y otros modelos de intercambio de calor en las otras fronteras del motor, descritos en el capítulo segundo de esta tesis. Así mismo, la estructura del programa permite acceder a los parámetros de los submodelos que pueden cambiarse para buscar mejores ajustes según la disponibilidad de la información experimental del motor que se esté trabajando. En esta tesis se realizará, como se mencionó en los objetivos, una ampliación de las capacidades de este programa, que lo habilitan para ser empleado en la predicción de los flujos de calor y temperaturas del metal también en estado transitorio, y que lo hacen utilizable en la predicción de la evolución de las temperaturas del motor y los flujos de calor a medida que el motor se somete a una secuencia de trabajo por diferentes puntos de operación, incluyendo un ciclo de conducción estándar como el NEDC (New European Driving Cycle).

En lo que tiene que ver con el modelado de los sistemas de gestión térmica (sistemas de refrigeración extendidos), los desarrolladores de programas como GT-Power ${ }^{\circledR}$, Amesim ${ }^{\circledR}$ y FlowMaster $^{\circledR}$ proveen librerías de componentes para ser integrados en sistemas de refrigeración según el diseño especificado. La utilización de estos programas permite modelar y simular circuitos termohidráulicos una vez que sus componentes han sido debidamente caracterizados. El número de publicaciones relacionadas con el modelado de sistemas de refrigeración con programas como los mencionados ha ido en aumento y, como se mencionó en el inicio de esta introducción, el modelado termohidráulico se ha constituido como parte sustantiva del modelado integral del vehículo con fines de optimización.

En lo que respecta particularmente a los trabajos de que trata la presente tesis debe comentarse que: a) tras el trabajo de revisión de la transferencia de calor 
en los motores de combustión de Borman y Nishiwaki [1.18], no se ha intentado una actualización de esa revisión; b) aunque son varios los estudios de cálculo de temperaturas y flujos de calor realizados en condiciones de calentamiento (Veshagh y Chen [1.8] [1.21], Jarrier et al. [1.16], Shayler et al. [1.7], Chalgren [1.2]) la adquisición de la información referida a las condiciones de operación del motor se realiza para puntos estabilizados y no se conoce (en lo que el autor ha podido comprobar) una exploración de factibilidad actual de muestrear las variables requeridas para alimentar los modelos de predicción de temperaturas y flujos de calor durante un proceso transitorio de motor; c) en los trabajos de control de los sistemas de refrigeración para reducir el tiempo de calentamiento de los motores es recurrente el empleo de sistemas con algún grado de sofisticación electrónico, sin que se haya evaluado la posibilidad de controlar el calentamiento del motor con dispositivos de bajo costo; d) en las aplicaciones no automotrices no se dispone de metodologías claras que permitan diseñar o rediseñar los sistemas de refrigeración sin incurrir en los riesgos y mayores costos demandados por la experimentación.

Dentro de este marco, en la presente tesis se aborda la actualización y la extensión de la revisión del estado del arte de la transferencia de calor, se realiza la valoración experimental del balance energético de un motor operado en frío bajo condiciones de calentamiento en un ciclo de conducción homologado, se valora la posibilidad de predecir las temperaturas y los flujos de calor utilizando la información muestreada bajo estas condiciones, se estudia la influencia de algunas modificaciones realizadas al sistema de refrigeración base de un motor sobre su tiempo de calentamiento y sobre el consumo y las emisiones y, finalmente, se propone una metodología para el diseño de sistemas de refrigeración acoplando tres programas de modelado termohidráulico, de diseño de radiadores y de modelado CFD en el recorrido del aire de refrigeración, según la tendencia actual antes comentada.

\subsection{Metodología y planteamiento}

La metodología seguida para alcanzar los objetivos en esta tesis se ha basado primeramente en realizar un estudio de los modelos utilizados para determinar la transmisión de calor en las diferentes interfases (superficies) del motor, con atención especial a los modelos para predecir los flujos de calor 
entre los gases de trabajo y las paredes de la cámara de combustión, con la intención de determinar los posibles modelos que podrían adaptarse al código de predicción de temperaturas desarrollado por Degraeuwe [1.14], y también para ubicar los alcances de los resultados esperados con la utilización de las herramientas descritas y perfilar posibles trabajos futuros en lo que respecta a la experimentación y al modelado térmico de los motores. La revisión del estado del arte será presentada en el capítulo dos de este documento.

En segundo lugar se realiza el calentamiento de un motor operando bajo carga constante y también bajo un ciclo de conducción estándar (el motor sobre el que se realizan las medidas en este trabajo y que es objeto de modelado termohidráulico se denomina en esta tesis "segundo motor"), con el fin de analizar su respuesta térmica y analizar las variables que inciden en ella a la luz del balance térmico experimental realizado, y también de proponer estrategias para mejorar esa respuesta y reducir las emisiones. Después de describir brevemente el modelo térmico validado por Degraeuwe y mostrar su aplicación en la determinación de los flujos internos de calor en el motor, el modelo es utilizado para predecir las temperaturas y los flujos de calor en el segundo motor, así como el calor disipado por las paredes de sus cilindros. Los ensayos, el balance térmico durante el calentamiento, y la aplicación del modelo térmico de predicción de temperaturas y flujos de calor son explicados en el capítulo tres de esta tesis.

Como un objetivo de la tesis es sugerir una metodología de diseño de sistemas de refrigeración para aplicaciones especiales, en el cuarto capítulo se propone dicha metodología y se recomienda un proceso de prediseño de los sistemas de refrigeración, el cual comprende la determinación de los parámetros básicos para iniciar la selección o el diseño detallado del radiador, la bomba y el ventilador de un sistema de refrigeración. Allí mismo se explican algunos elementos teóricos de cálculo de radiadores y de modelos de sistemas de refrigeración, sobre los que, de manera aproximada, se fundamentan los códigos de los programas de cálculo comerciales utilizados en esta tesis. Complementa el capítulo cuarto la descripción de las características de un programa diseñado para el cálculo, selección y análisis de intercambiadores.

El estudio de las posibles estrategias de mejora del calentamiento del segundo motor estudiado en el capítulo tercero se realiza en el capítulo quinto. A partir 
de las características hidráulicas y térmicas de los componentes del sistema de refrigeración original del motor, se realiza el modelado termohidráulico (modelado 1D) de éste y de sus posibles circuitos alternativos y se evalúan las respuestas térmicas. Una vez establecida la variante que mejor comportamiento tiene, se realiza la validación experimental de la misma y se comparan las características de calentamiento del refrigerante del sistema alternativo y del original, y se sacan conclusiones sobre las ventajas adicionales del nuevo sistema de refrigeración en términos de consumo de combustible y de emisiones contaminantes.

La metodología de diseño propuesta en el capítulo cuarto se aplica al diseño del sistema de refrigeración del motor de una locomotora, a partir de la potencia del motor y de unas condiciones de espacio y dimensiones definidas, como se expone en el capítulo sexto. Se explica aquí también el proceso de modelado del sistema hidráulico y del sistema de ventilación, describiendo la caracterización y el modelado de los componentes. Realizado el modelado y la simulación, y considerados cumplidos los niveles de temperatura requeridos por la tarea técnica, se prueba el motor a plena carga, se miden los flujos de refrigerante, de aire y las temperaturas, y se verifica el cumplimiento de los balances energéticos en las fuentes y los intercambiadores del sistema. Finalmente se comparan los resultados experimentales con los del modelo y se sacan conclusiones sobre las mediciones, los calores calculados, las limitaciones del modelo y las incertidumbres de medición que deben tenerse en cuenta al ponderar los resultados de los modelos.

Para cerrar la tesis, en el capítulo 7 se extraen las conclusiones obtenidas del trabajo realizado y se proponen los posibles temas de desarrollo futuro.

\subsection{Bibliografía}

[1.1] Pang, H.H., Brace, C. J. Review of engine cooling technologies for modern engines. Proc. Instn. Mech. Engrs., part D: J Automobile Engineering, vol. 218, pp. 1209-1215, 2004.

[1.2] Chalgren, R. D. Thermal comfort and engine warm-up optimization of a low-flow advanced thermal management system. SAE paper 2004-01-0047.

[1.3] Chalgren, R. D., Traczyk, T. Advanced secondary cooling systems 
for light trucks. SAE paper 2005-01-1380.

[1.4] Polverini, M. International PNGV-Equivalent Programs: Where does the United States stand? Disponible en http://www.wiseintern.org/journal/1998/Polverini.pdf.

[1.5] Regner, G., Loibner E., Krammer J. Analysis of transient drive cycles using CRUISE-BOOST co-simulation techniques. SAE paper 2002-01-0627.

[1.6] Kaplan, J. A., Heywood, J. B. Modeling the spark ignition engine warm-up process to predict component temperatures and hydrocarbon emissions. SAE paper 910302.

[1.7] Shayler, P. J., Christian, S. J. A model for the investigation of temperature, heat flow and friction characteristics during engine warm-up. SAE paper 931153.

[1.8] Veshagh, A., Chen, C. A computer model for thermofluid analysis of engine warm-up process. SAE paper 931157.

[1.9] Bohac, S.V., Baker, D.M., Assanis, D.N. A global model for steadystate and transient S.I. engine heat transfer studies. SAE paper 960073.

[1.10] Santos, R. Estudio del aprovechamiento de la energía de los gases de escape en motores Diesel. Tesis doctoral, Universidad Politécnica de Valencia. 1999.

[1.11] Reyes M. Desarrollo de un modelo para el análisis térmico de MCIA. Seminario en el CMT, Universidad Politécnica de Valencia. España. Septiembre, 2003.

[1.12] Reyes M. Modelo de transferencia de calor para colectores de escape de motores alternativos. Tesis doctoral, Universidad Politécnica de Valencia. España. 1993.

[1.13] Dolz, V. R. Contribucion al modelado de la transmisión de calor en los MCIA y su aplicacion en el aprovechamiento energético de los gases de escape durante los transitorios de carga. Tesis doctoral, Universidad Politécnica de Valencia. España. 2006.

[1.14] Degraeuwe, B. M. A. Contribución al modelado de la transmisión de calor en los MCIA. Tesis doctoral, Universidad Politécnica de Valencia. España. 2006.

[1.15] Torregrosa, A.J., Olmeda, P., Degraeuwe, B., Reyes, M. A concise wall temperature model for DI diesel engines. Appl. Therm. Eng., 
vol. 26, pp.1320-13277.

[1.16] Jarrier, L., Gentile, D. Simulation du comportement thermique transitoire d'un motear à combustión interne et à allumage commandé. Rev. Gén Therm, vol. 36, nº 7, pp. 520-533, 1997.

[1.17] Woschni, G. A Universally applicable equation for the instantaneous heat transfer coefficient in the I.C. Engine. SAE paper 670931.

[1.18] Borman, G., Nishiwaki, K. Internal combustion engine heat transfer. Progress in Energy Combustion Sciences, vol. 13, pp. 1-46, 1987.

[1.19] Allen, D. J. Lasecki, M. P. Thermal management evolution and controlled coolant flow. SAE paper 2001-01-1732.

[1.20] Finol, C. A., Robinson, K. Thermal modelling of modern engines: a review of empirical correlations to estimate the in-cylinder heat transfer coefficient. JAUTO202. Proc. Instn. Mech. Engrs., part D: J. Automobile Engineering, vol. 220, pp. 1765-1781, 2006.

[1.21] Hill, N., Kollamthodi, S., Cross, S. Rail Diesel study, WP2 final report: Technical and operational measures to improve the emissions performance of Diesel rail, diciembre de 2005.

[1.22] Luptowski, B., Parker, G., Arici, O. Johnson, J. Development of the enhanced vehicle and engine cooling system simulation and application to active cooling control. SAE paper 2005-01-0697.

[1.23] Cortona, E., Onder, C. H. Engine thermal management with electronic cooling pump. SAE paper 2000-01-0965.

[1.24] Mohan, K. V, Arici, O., Yang, S., Johnson, J. H. A computer simulation of the turbocharged diesel engine as an enhancement of the vehicle engine cooling system simulation. SAE paper 971804.

[1.25] Lapuerta, M., Armas, O., Hernández, J.J. Diagnosis of DI Diesel combustion from in-cylinder pressure signal by estimation of mean thermodynamic properties of the gas. Appl. Therm. Eng., vol. 19 pp. 513-529, 1999. 


\section{Capítulo 2}

\section{Estado del Arte de la Transferencia de Calor en los M.C.I.A.}

2.1. Introducción 21

2.2. La transferencia de calor en el motor 22

2.3. Transferencia de calor entre los gases del cilindro y las paredes de la cámara de combustión 23

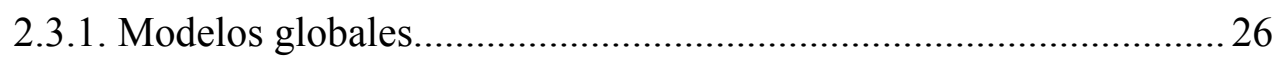

2.3.2. Modelos zonales y locales.......................................................... 32

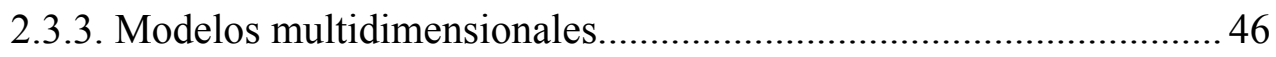

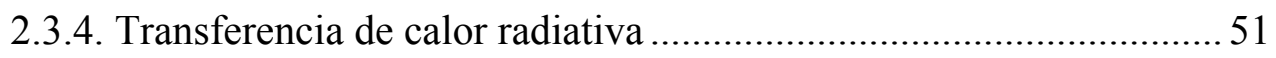

2.4. Transferencia de calor en las pipas de admisión y escape ......................62 62

2.5. Transferencia de calor del lado del refrigerante......................................6 67

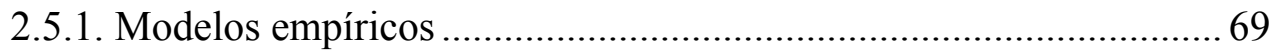

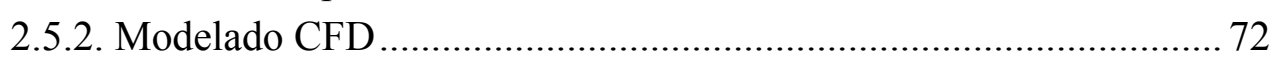

2.6. Transferencia de calor entre el aceite y el motor ................................... 73

2.6.1. Transferencia de calor entre el pistón y el aceite ............................ 73

2.6.2. Transferencia de calor entre el aceite y la estructura (bloque y

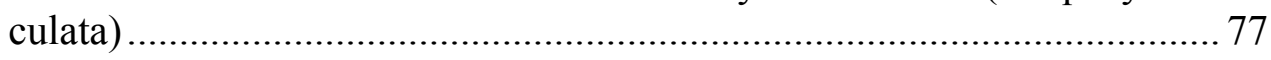

2.7. Transferencia de calor entre el motor y el ambiente ............................. 78

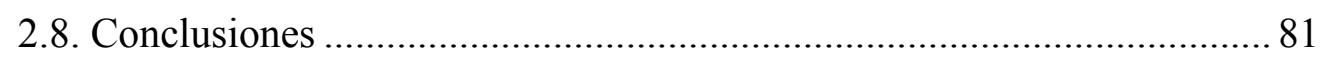

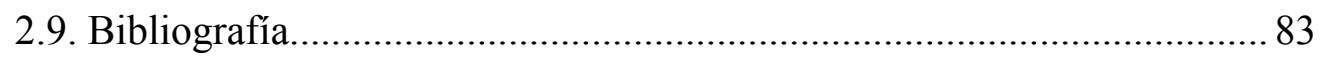




\subsection{Introducción}

La transferencia de calor es un problema de gran interés para la comunidad involucrada en el estudio de los motores de combustión interna (M.C.I.) por las implicaciones que tienen las pérdidas de calor hacia las paredes sobre el rendimiento termodinámico del proceso de conversión de energía, por la relación que tiene sobre la elevación de la temperatura de la superficie y con ello la influencia sobre el comportamiento tribológico y la durabilidad de los componentes de la cámara de combustión y en particular del grupo cilindropistón. Además de esto, la cinética de las reacciones químicas que controlan la formación de emisiones, la formación del hollín y la aparición del golpeteo dependen en gran medida de la temperatura de los productos en la cámara, la cual generalmente varía ampliamente dentro del espacio y al pasar el tiempo dentro de la cámara de combustión. No es reciente ya la controversia entorno al desempeño de los motores adiabáticos y son muy actuales los desarrollos en la gestión térmica de los vehículos. Todo esto motiva a repasar el estado en el que se encuentra la temática de la transferencia de calor en los motores modernos.

En 1987 Borman y Nishiwaki [2.1] publicaron una vasta revisión del estado de la transferencia de calor en los motores de combustión interna. Finol y Robinson [2.84] presentaron también una revisión, pero ésta se limitó a enumerar las principales correlaciones encontradas en la literatura del coeficiente global de transferencia de calor en el cilindro del motor. Puede adelantarse que las conclusiones del trabajo de Borman y Nishiwaki no han variado mucho y lo que ha venido marcando la diferencia es el incremento de trabajos basados en los métodos computacionales. Se ha trabajado más sobre los modelos detallados del coeficiente de película local, el procesado de mediciones de temperaturas instantáneas de pared y el refinamiento de algoritmos matemáticos para el procesamiento de esta información.

El estudio del estado del arte de la transferencia de calor en los M.C.I. en esta tesis se ha abordado con el objetivo de explorar los modelos y trabajos experimentales actuales empleados en el diagnóstico y la predicción de las temperaturas de las paredes del motor y los flujos de calor en su interior, así como la interrelación térmica con los sistemas térmicos periféricos relacionados con él, incluyendo, claro está, al más convencional de ellos, al 
sistema de refrigeración. La revisión se ha realizado según el esquema de la Figura 2.1.

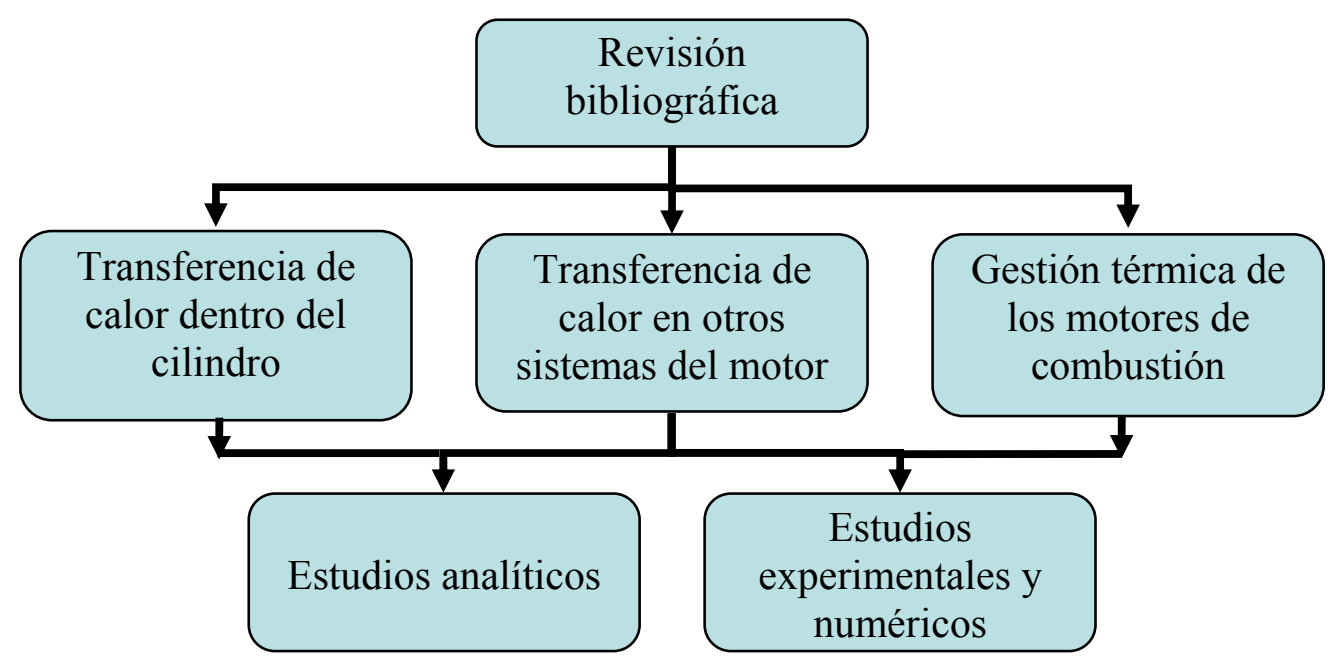

Figura 2.1. Esquema de la revisión del estado del arte de la transferencia de calor en los motores de combustión alternativos.

Por razones de espacio se ha decidido consignar en este capítulo sólo la parte de la revisión relativa a los modelos de la transferencia de calor en las diferentes fronteras del motor como estructura. La parte del esquema relacionada con la gestión térmica de los motores de combustión no se trata en este capítulo, como tampoco la revisión de las técnicas experimentales.

\subsection{La transferencia de calor en el motor}

El motor, como máquina térmica provista de una estructura con superficies limitadas por al menos seis fronteras fluidicas diferentes (gases de cilindros, gases de admisión, gases de escape, refrigerante, lubricante y aire ambiente) y una frontera de fricción pistón-cilindro, es una máquina muy compleja para ser modelada desde el punto de vista térmico, sobre todo porque todas esas fronteras son de naturaleza transitoria y dependientes del punto de operación del motor. 
Las temperaturas de la estructura y de los fluidos son los factores que limitan el incremento de la potencia específica del motor (potencia por unidad de volumen de trabajo del motor) y es por esto que es importante, no sólo continuar garantizando los niveles seguros de temperaturas según las limitaciones de tensiones térmicas de los materiales estructurales, las tolerancias en los pares cinemáticos, las exigencias tribológicas y los límites impuestos por las propiedades de los fluidos, sino también avanzar en la identificación térmica de los motores, esto es, en la obtención de modelos que permitan predecir y controlar las temperaturas de los fluidos de las fronteras y de las superficies de interés. Es importante poder predecirlas y controlarlas para poder optimizar la combustión y reducir con ello el consumo y las emisiones de contaminantes en todos los regímenes de operación del motor, en primer lugar, y luego para potenciar la recuperación de la energía evacuada con los gases de escape y con el refrigerante.

Para identificar térmicamente el motor se debe disponer de modelos que permitan validar los intercambios de calor entre los gases de admisión y escape y las pipas correspondientes, entre los gases del cilindro y las paredes de la cámara de combustión, y así mismo entre el refrigerante, el aceite lubricante y el aire circundante y las correspondientes superficies del motor. En las partes siguientes de este apartado se comentarán los tratamientos de modelado más usados para estas interfases.

\subsection{Transferencia de calor entre los gases del cilindro y las paredes de la cámara de combustión}

La transferencia de calor en el motor se manifiesta como el flujo de energía térmica debido por los gradientes de temperatura en las diferentes interfases del motor. Particularmente para el cilindro, básicamente la transferencia de calor se puede describir como el flujo de energía térmica, $q_{p}$, desde los gases del cilindro (generalmente a una mayor temperatura promedio) hacia el refrigerante (generalmente a una menor temperatura promedio), a través de las paredes metálicas, como se esquematiza en la Figura 2.2.

En la figura se destaca la variación de la temperatura de los gases durante el ciclo, como motor principal del proceso de intercambio de calor, y la 
variación del coeficiente de transferencia de calor del lado del gas, correspondiente a un modelo simplificado. Realmente los mecanismos de transferencia de calor a ambos lados de la pared del cilindro son más complejos que lo mostrado en el esquema, como se hará evidente a lo largo de este capítulo.

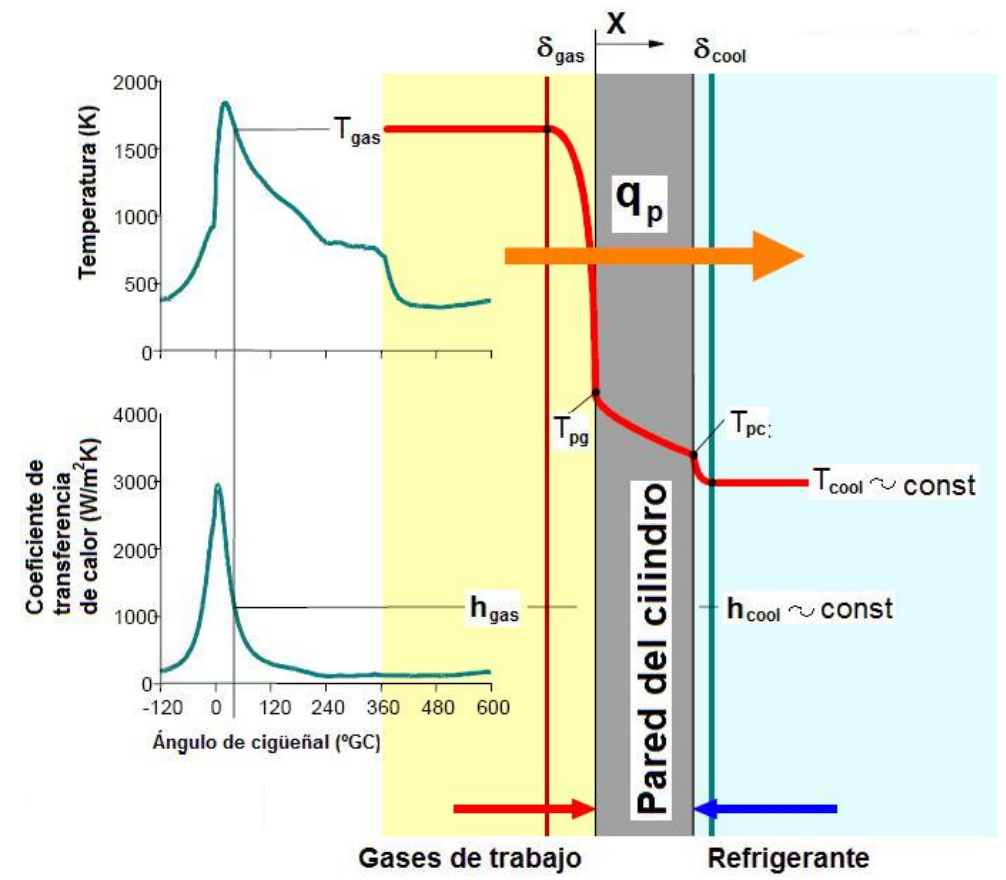

Figura 2.2. Esquema reducido de la transferencia de calor en el motor.

La transferencia de calor dentro del cilindro es uno de los problemas más complicados de transferencia de calor en el motor, asociado a cambios rápidos de la temperatura, la presión y la velocidad de la masa de trabajo, y con grandes variaciones en la distribución de los campos de temperatura y velocidad, aparte de la participación de dos mecanismos muy diferentes como la convección y la radiación. El flujo de calor de los gases hacia las paredes del cilindro varía durante el ciclo desde valores muy pequeños, negativos en los momentos que siguen a la apertura de las válvulas de escape, hasta valores positivos del orden de los $\mathrm{MW} / \mathrm{m}^{2}$, como en los resultados mostrados en la Figura 2.3, obtenida por Nijeweme et al. [2.12] en su estudio de la transferencia de calor en un motor de encendido provocado (la gráfica de la izquierda ilustra el comportamiento negativo del flujo de calor durante parte 
del tiempo de renovación de la carga). Se aprecian muchas variaciones en los flujos de calor locales y entre diferentes regiones de la cámara de combustión a lo largo del ciclo. La onda de flujo de calor hacia el interior de las paredes sigue con cierto retraso y atenuación hacia el interior la onda del frente de llama a medida que éste se aproxima y pasa por las diferentes superficies de la cámara de combustión. No es, pues, simple modelar la transferencia de calor entre el cilindro y las paredes, como sí puede serlo entre un fluido con flujo establecido unidireccional e isóbaro y las paredes de un recipiente o una tubería.
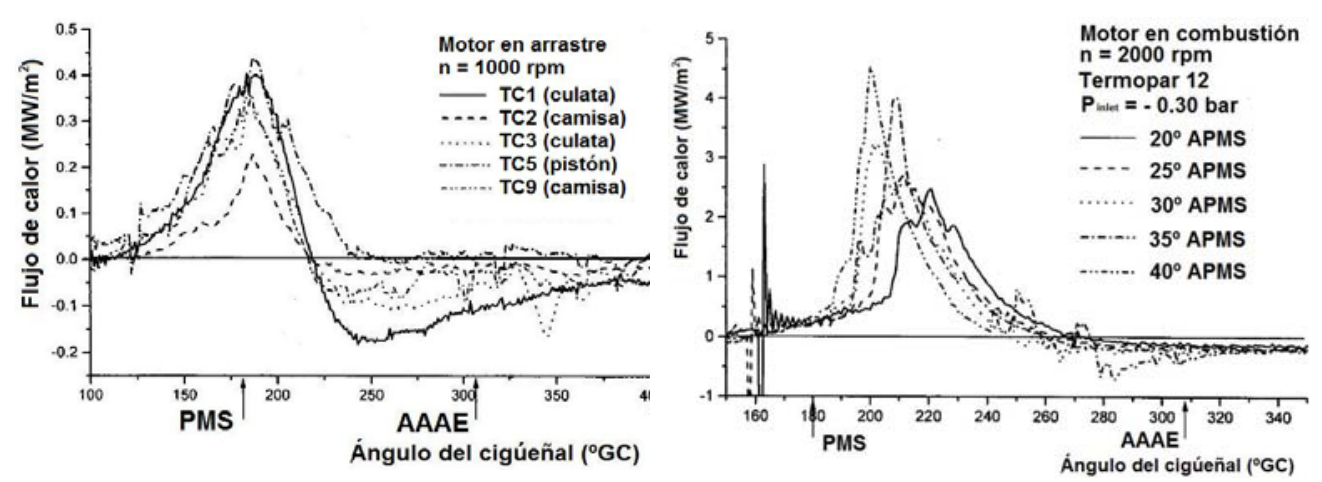

Figura 2.3. Coeficiente de transferencia de calor entre los gases del cilindro y las paredes de la cámara de combustión. Las pruebas fueron realizadas en un motor a gasolina Rover K16 [2.12].

Para cualquier zona de la cámara de combustión, lo correcto al determinar el flujo de calor a través de cualquier superficie es resolver la ecuación de la energía que, en su forma adimensional simplificada y con los supuestos de que la conductividad y el calor específico de los gases son constantes y de que la presión es espacialmente uniforme, puede presentarse constituida por sus términos de almacenamiento, conducción, convección, trabajo de presión y fuente de calor (por convección y radiación), como se describe en la ecuación (2.1):

$$
\frac{\partial T^{*}}{\partial \tau}=\frac{1}{\operatorname{Re} \operatorname{Pr}} \nabla^{2} T^{*}-v^{*} \nabla T^{*}+\left(\frac{\gamma-1}{\gamma}\right) \frac{T^{*}}{p^{*}} \frac{d p^{*}}{d \tau}+\dot{q}^{*},
$$

donde $\mathrm{p}^{*}=p / p_{0}, T^{*}=T / T_{0}$ y $v^{*}=v / v_{0}$ son la presión, la temperatura y las velocidades adimensionales, escaladas por los valores de referencia $p_{0}, T_{0} \mathrm{y}$ 
$v_{0}$, respectivamente; $\tau=t v_{0} / D$ es el tiempo adimensional, $R e=\rho v_{0} D / \mu$, es el número de Reynolds con $D$ como longitud característica, $\operatorname{Pr}=c_{p} \mu / k$ es el número de Prandtl del gas y $\gamma=c_{p} / c_{v}$ es el exponente adiabático.

Aunque desde la segunda década del siglo pasado, cuando se iniciaron los primeros trabajos de modelado de la transferencia de calor, ha habido grandes avances en los análisis teóricos y experimentales, aún no se ha desarrollado un modelo predictivo satisfactorio de cálculo del calor transferido entre las paredes de la cámara de combustión y los gases. Así lo confirma también la revisión realizada por Finol y Robinson [2.84].

\subsubsection{Modelos globales}

Ante las dificultades de "acoplar" los términos de la ecuación (2.1), la insuficiencia de información necesaria para resolverla, y dada la necesidad de estimar las pérdidas de calor dentro del cilindro, son muchos los modelos que se han propuesto para valorar esas pérdidas. La solución más sencilla ha sido su valoración a través de coeficientes globales de transferencia de calor promediados a lo largo del ciclo, generalmente asumidos como representativos para todas las superficies de transferencia de calor (cilindro, pistón, culata) y obtenidos mediante diferentes correlaciones, unas empíricas y otras con mayor grado de elaboración teórica. Como base para el modelado se toma la asunción de estado cuasi-estacionario, el cual puede describirse con la expresión de Newton:

$$
q^{\prime \prime}=h\left(T-T_{w}\right)
$$

donde $q^{\prime \prime}$ es el flujo de calor, $h$ es el coeficiente de transferencia de calor (o coeficiente de película), $T$ y $T_{w}$ corresponden a la temperatura media del gas (promediada en masa) y de la superficie de la pared, respectivamente [2.25]. La componente radiativa de la transferencia de calor puede estar integrada en una sola expresión o detallada como un término independiente del modelo. Los modelos globales de transferencia de calor en el cilindro fueron bien recogidos en el trabajo de Borman y Nishiwaki [2.1].

En la tabla 2.1 se resumen las expresiones más usadas propuestas para el modelado del coeficiente global de transferencia de calor dentro del cilindro. 
Tabla 2.1. Expresiones utilizadas para modelar la transferencia de calor en la cámara de combustión.

\begin{tabular}{|c|c|c|}
\hline Autor & Método & Expresión y comentarios \\
\hline \multicolumn{3}{|r|}{ Análisis experimentales. } \\
\hline $\begin{array}{l}\text { Nusselt en } \\
\text { (1923) }\end{array}$ & $\begin{array}{l}\text { Recipiente de alta } \\
\text { presión y experimentos } \\
\text { en un motor real. } \\
\text { Originalmente obtenida } \\
\text { para predecir el flujo de } \\
\text { calor promediado en el } \\
\text { tiempo o estacionario. }\end{array}$ & $\begin{array}{l}h=h_{c}+h_{r}= \\
=5,41 \cdot 10^{-3}\left(1+1,24 c_{m}\right) \sqrt[3]{p^{2} T}+, \quad \mathrm{kW} / \mathrm{m}^{2} \mathrm{~K} \\
+\frac{4,21 \cdot 10^{-4}}{\left(1 / \varepsilon_{g}+1 / \varepsilon_{w}-1\right)} \frac{(T / 100)^{4}-\left(T_{w} / 100\right)^{4}}{T-T_{w}}\end{array}$ \\
\hline $\begin{array}{l}\text { Briling } \\
(1935)\end{array}$ & $\begin{array}{l}\text { Mejora de la ecuación de } \\
\text { Nusselt }\end{array}$ & $h=5,41 \cdot 10^{-3}\left(3,5+0,185 c_{m}\right) \sqrt[3]{p^{2} T}$ \\
\hline $\begin{array}{l}\text { Van Tyen } \\
(1962)\end{array}$ & $\begin{array}{l}\text { Mejora de la ecuación de } \\
\text { Nusselt }\end{array}$ & $h=\left(3,19+0,885 c_{m}\right) \sqrt[3]{p^{2} T}$ \\
\hline $\begin{array}{l}\text { Eichelberg, } \\
(1939)\end{array}$ & $\begin{array}{l}\text { Consideró la radiación. } \\
\text { Primera medición del } \\
\text { flujo instantáneo de } \\
\text { calor. }\end{array}$ & $h=2,1 \sqrt[3]{c_{m}} \sqrt{p T}$ \\
\hline $\begin{array}{l}\text { Pflaum } \\
(1961)\end{array}$ & $\begin{array}{l}\text { Basándose en la ecuación } \\
\text { de Eichelberg, incorpora } \\
\text { el efecto del } \\
\text { supercargador, diferentes } \\
\text { empleando para la } \\
\text { coeficientes para el } \\
\text { culata, el pistón y el } \\
\text { cilindro. }\end{array}$ & $\begin{array}{l}h=K_{D, K, B}^{\prime} \sqrt{p T} f\left(c_{m}\right), \\
K_{D, K}^{\prime}=1,10+0,366\left(p_{L}-p_{0}\right) / p_{0} \text { para la culata y el pistón; } \\
K_{B}^{\prime}=0,36+0,12\left(p_{L}-p_{0}\right) / p_{0} \text { para el cilindro; } p_{L} \text { es la presión } \\
\text { del supercargador; } \quad \mathrm{p}_{0} \quad \text { es la presión atmosférica; } \\
f\left(c_{m}\right)=3 \pm 2,57\left\{1-\exp \left[ \pm\left(1,50-0,416 c_{m}\right)\right]\right\} ; \text { si } c_{m}>3,6 \text { se } \\
\text { emplea el signo "más", si } c_{m}<3,6 \text { se emplea el signo "menos". }\end{array}$ \\
\hline & Análisis & e flujo turbulento estacionario \\
\hline $\begin{array}{l}\text { Annand } \\
(1963)\end{array}$ & $\begin{array}{l}\text { Basado en la } \\
\text { transferencia de calor por } \\
\text { convección turbulenta } \\
\text { estacionaria. Término } \\
\text { independiente para la } \\
\text { radiación. }\end{array}$ & $\begin{array}{l}q=a \frac{k}{D}(\mathrm{Re})^{0,7}\left(T-T_{w}\right)+b\left(T^{4}-T_{w}^{4}\right), \quad a=0,35-0,8 \\
\text { dependiendo de la intensidad del movimiento de la carga; el segundo } \\
\text { término representa el flujo de calor radiativo, asumiendo radiación de } \\
\text { cuerpo gris; } b=3,3 \cdot 10^{-11} \text { para motores Diesel y } b=4,3 \cdot 10^{-12} \mathrm{~kW} / \mathrm{m}^{2} \mathrm{~K}^{4} \\
\text { para motores a gasolina. }\end{array}$ \\
\hline $\begin{array}{l}\text { LeFeuvre } \\
(1969)\end{array}$ & $\begin{array}{l}\text { Incorpora en la expresión } \\
\text { para el flujo instantáneo } \\
\text { de calor el efecto del } \\
\text { swirl. Utiliza un número } \\
\text { de Reynolds local (por lo } \\
\text { que estrictamente } \\
\text { hablando no es un } \\
\text { modelo global). }\end{array}$ & $\begin{array}{l}q=a \frac{k(t)}{r} \operatorname{Re}(t)^{0,8} \operatorname{Pr}(t)^{0,33}\{T(t)-T(t)\}, r \text { es un radio en el } \\
\text { punto de medición en la culata, Re el número de Reynolds, } \\
\operatorname{Re}=\frac{r^{2} \omega}{v}, \omega \text { la velocidad angular de los gases en el cilindro } \\
\text { ("swirl"); } a=0,047 .\end{array}$ \\
\hline $\begin{array}{l}\text { Annand y } \\
\text { Ma (1970) }\end{array}$ & $\begin{array}{l}\text { Extienden la fórmula } \\
\text { inicial de Annand, } \\
\text { introduciendo una } \\
\text { corrección para la } \\
\text { transitoriedad. Emplea } \\
\text { los resultados de un } \\
\text { motor de inyección } \\
\text { directa con swirl en la } \\
\text { admisión. Adoptó la }\end{array}$ & $\begin{array}{l}q=a \frac{k}{D}(\operatorname{Re})^{0,7}\left\{a\left(T-T_{w}\right)+\frac{a^{\prime}}{\omega} \frac{d T}{d t}\right\}+b \sigma\left(T^{4}-T_{w}^{4}\right), \omega \text { es la } \\
\text { velocidad angular del cigüeñal, } \sigma \text { es la constante de Stefan-Boltzman }\end{array}$ \\
\hline
\end{tabular}




\begin{tabular}{|c|c|c|}
\hline & $\begin{array}{l}\text { velocidad media } \\
\text { propuesta por Knight. }\end{array}$ & \\
\hline $\begin{array}{l}\text { Dent } \quad y \\
\text { Suliaman } \\
(1977)\end{array}$ & $\begin{array}{l}\text { Mejora a la expresión de } \\
\text { LeFeuvre. Aplicaron un } \\
\text { análisis de transferencia } \\
\text { de calor convectiva } \\
\text { turbulenta para flujo a lo } \\
\text { largo de una placa. Se } \\
\text { utiliza un número de } \\
\text { Reynolds local. }\end{array}$ & $q=0,023 \frac{k}{r}\left(\frac{r^{2} \omega}{v}\right)^{0,8}\left(T-T_{w}\right)$ \\
\hline $\begin{array}{l}\text { Woschni } \\
(1967)\end{array}$ & $\begin{array}{l}\text { Trabajó sobre el balance } \\
\text { de energía , para } \\
\text { determinar el calor total } \\
\text { transferido a las paredes } \\
\text { de la cámara en cada } \\
\text { proceso del ciclo } \\
\text { completo de trabajo del } \\
\text { motor }\end{array}$ & $\begin{array}{l}h=0,820 D^{-0,2} p^{0,8} W^{0,8} T^{-0,53} \\
W=\left[C_{1} C_{m}+C_{2} \frac{V_{s} T_{1}}{p_{1} V_{1}} p-p_{0}\right]\end{array}$ \\
\hline $\begin{array}{l}\text { Sitkei } \\
(1962)\end{array}$ & $\begin{array}{l}\text { Adoptó la relación para } \\
\text { la transferencia de calor } \\
\text { convectiva turbulenta de } \\
\text { Annand. }\end{array}$ & $h=2,36 \cdot 10^{-4}(1+b) \frac{p^{0,7} C_{m}^{0,7}}{T^{0,2} d_{e}^{0,3}} \mathrm{~kW} / \mathrm{m}^{2} \mathrm{~K}$, donde $d_{e}=4 V / A$ \\
\hline $\begin{array}{l}\text { Zapf } \\
(1966)\end{array}$ & $\begin{array}{l}\text { Aportó un efecto del } \\
\text { swirl durante el proceso } \\
\text { de intercambio de gases }\end{array}$ & $\begin{array}{c}N_{u}=N_{u_{0}}\left(1+0,062 C_{u} / C_{m}\right), \text { siendo } N_{u_{0}} \text { el número de Nusselt } \\
\text { sin contar el swirl. }\end{array}$ \\
\hline $\begin{array}{l}\text { Nishiwaki } \\
(1979)\end{array}$ & $\begin{array}{l}\text { Número de Nusselt en las } \\
\text { carreras de admisión y } \\
\text { escape }\end{array}$ & $N u_{i}=0,168 \mathrm{Re}^{0,867} N u_{e}=1,69 \mathrm{Re}^{0,578}$ \\
\hline $\begin{array}{l}\text { Hohenberg } \\
(1979)\end{array}$ & $\begin{array}{l}\text { Modificó la ecuación de } \\
\text { Woschni, para ajustar } \\
\text { mejor las predicciones a } \\
\text { los flujos instantáneos de } \\
\text { calor medidos. }\end{array}$ & $h=a V_{c y l}^{-0.06} p^{0,8} W^{0,8} T^{-0,4}\left(C_{m}+1.4\right)^{0,8} \mathrm{~kW} / \mathrm{m}^{2} \mathrm{~K}, p$ en bar. \\
\hline \multicolumn{3}{|c|}{ Análisis de la energía de la capa límite térmica no estacionaria. } \\
\hline $\begin{array}{l}\text { Elser } \\
(1954)\end{array}$ & $\begin{array}{l}\text { Aplicó las leyes de } \\
\text { semejanza (números } \\
\text { adimensionales) }\end{array}$ & $N u=6,5\left(1+0,5 \frac{\Delta S}{c_{p}}\right)(\operatorname{Re} \operatorname{Pr})^{0,5}$ \\
\hline $\begin{array}{l}\text { Oguri } \\
(1960)\end{array}$ & $\begin{array}{l}\text { Mejora a la expresión } \\
\text { de Elser }\end{array}$ & $N u=1,75\left(1+0,5 \frac{\Delta S}{c_{p}}\right)(\operatorname{Re} \operatorname{Pr})^{0,5}\left[2+\cos \left(\theta-20^{\circ}\right)\right]$ \\
\hline \multicolumn{3}{|c|}{$\begin{array}{l}\text { * En las expresiones de la tabla } h \text { es el coeficiente de transferencia de calor }\left(\mathrm{kW} / \mathrm{m}^{2} \mathrm{~K}\right) ; \varepsilon_{g}, \varepsilon_{w} \text { son las emisividades } \\
\text { del gas y de la pared, respectivamente; } c_{m} \text { es la velocidad del pistón, en } \mathrm{m} / \mathrm{s} \text { y } p \text { es la presión del gas en MPa; } T \text { y } T_{w} \\
\text { son las temperaturas del gas y de la pared, respectivamente; siendo } k \text { la conductividad térmica del gas; } D \text { el diámetro } \\
\text { del cilindro; en la fórmula de Annand y Ma } a \text { a } a^{\prime} \text { y } b \text { son constantes obtenidas para el motor en cuestión y dependen } \\
\text { de las revoluciones del motor y de la relación aire combustible. La derivada en el tiempo de la temperatura media } \\
\text { global se agrega en la fórmula para compensar la naturaleza no estacionaria del flujo. El número de Reynolds se } \\
\text { calcula con la velocidad obtenida a partir del valor medio de la energía cinética del gas. } \\
\text { En la ecuación de Woschni [2.16] } W \text { es la velocidad de referencia, la cual representa la velocidad media del gas que } \\
\text { afecta la transferencia de calor y está dada para cada proceso. } C_{m} \text { es la velocidad media del pistón. Para el proceso de } \\
\text { intercambio de gases } C_{l}=6,18, C_{2}=0 \text {. Para el proceso de compresión } C_{l}=2,28, C_{2}=0 \text {. Para los procesos de } \\
\text { combustión y expansión } C_{l}=2,28, C_{2}=3,24 \cdot 10^{-3} \text {. } p_{0} \text { es la presión en MPa obtenida en arrastre, } V_{s} \text { es el volumen } \\
\text { barrido por el pistón, en } \mathrm{m}^{3} . T_{l}, V_{l} \text { y } p_{l} \text { son determinados para un tiempo determinado en el que se conocen la } \\
\text { presión y la temperatura, por ejemplo, el cierre de la válvula de admisión o el inicio del proceso de combustión. } \\
\text { Aunque fue escrita en la forma de transferencia de calor convectivo turbulento estacionario, la fórmula de Woschni }\end{array}$} \\
\hline
\end{tabular}


incluye de manera concentrada todos los efectos de la convección y la radiación. Posteriormente Woschni agregó un término de "swirl" a su ecuación, cambiando la forma del coeficiente $C_{l}$ [2.115]. Para el proceso de intercambio de gases $C_{1}=6,18+0,417 C_{u} / C_{m}$; para el resto del ciclo $C_{1}=2,28+0,308 / C_{m} ; C_{u}=\pi D n_{D}, y n_{D}$ es la velocidad de rotación del rodete usado en el banco de pruebas de swirl.

En la expresión de Sitkei $V$ es el volumen del cilindro, $A$ es el área que absorbe el calor, la constante adimensional $b$ se agregó en consideración a velocidades de turbulencia adicionales y está entre 0 y 0,35 , dependiendo del tipo de cámara de combustión

En general, los modelos globales no se emplean para describir los efectos transitorios de la transferencia de calor, ya que se han propuesto para valorar la transferencia de calor con consideraciones de cuasi-estacionaridad de los procesos dentro del cilindro, y son por tanto apropiados para simular el ciclo del motor y predecir las prestaciones globales del motor como la potencia efectiva, el consumo de combustible, las pérdidas totales de calor, la carga térmica del motor o la temperatura de los gases de escape.

La correlación de Woschni [2.16], basada en las leyes de semejanza de la transferencia de calor para flujos estacionarios turbulentos a través de tubos, descrita con la ecuación semiempírica $N u=C \operatorname{Re}^{0,8} \operatorname{Pr}^{0,4}$ es la más empleada en la literatura. Hohenberg [2.17] modificó la expresión de Woschni, para mejorar la predicción de los flujos de calor obtenidos con sensores de flujos de calor. Las modificaciones incluyeron el empleo del volumen instantáneo del cilindro en lugar del diámetro del pistón como dimensión característica y cambios en la velocidad efectiva, $W$, y en el exponente del término de temperatura.

El uso de las relaciones de Annand y Eichelberg también es frecuente en los trabajos de modelado. Cuando el modelo de transferencia de calor no se requiere para el cálculo del calor transferido al refrigerante o para determinar la temperatura de los componentes, la elección del modelo de cálculo puede no ser tan trascendente. Stone [2.21] afirma que, de manera típica, un error de un $10 \%$ en la predicción de la transferencia de calor en el cilindro conduce a un error del orden del $1 \%$ en las prestaciones del motor. La Figura 2.4 reproduce el comportamiento del coeficiente de transferencia de calor valorado con diferentes expresiones globales, para las mismas condiciones de operación de un motor analizado por Hiroyasu [2.18]. Las diferencias en los resultados calculados con las diferentes expresiones se deben a los diferentes supuestos incluidos en ellas y las diferentes condiciones de operación, afirma el autor. 


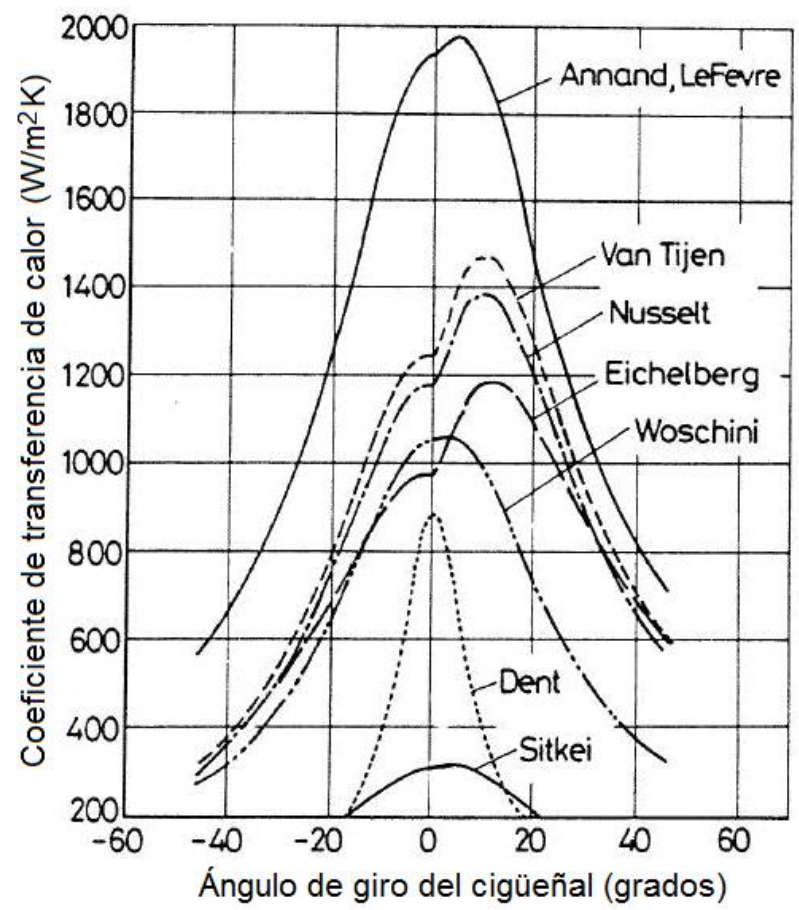

Figura 2.4. Comparación de los coeficientes de transferencia de calor calculados por Hiroyasu empleando diferentes correlaciones [2.18].

Visto el desarrollo que tienen los actuales sistemas de control de los motores, hoy es posible utilizar algoritmos de control menos dependientes de tablas de caracterización del motor experimentales y aprovechar la capacidad computacional de los microcontroladores empleados en ellos para implementar algoritmos basados en funciones. Para estos nuevos sistemas de control es ya, entonces necesaria la utilización de modelos que todavía pueden corresponder a modelos globales para todos los procesos que ocurren en el interior del cilindro. Un ejemplo de este tipo de aplicación es el tratamiento propuesto por Allmendinger et al. [2.23], quienes desarrollaron un modelo predictivo para ser implementado en un programa para el control en lazo cerrado, independiente de mapas, de un motor Diesel de inyección de colector común. Basándose en la relación lineal aparente entre el coeficiente de transferencia de calor y la temperatura de los gases, los autores concibieron una expresión con coeficientes gausianos de la forma:

$$
h(\varphi, T)=a(\varphi)+b(\varphi) T ; a, b(\varphi)=\frac{1}{\sqrt{2 \pi \sigma^{2}}} e^{\frac{-\left(\varphi-\varphi_{E}\right)^{2}}{2 \sigma^{2}}}
$$


Con este tratamiento el coeficiente de transmisión de calor para una carga dada, varía según el régimen como aparece en la Figura 2.5.

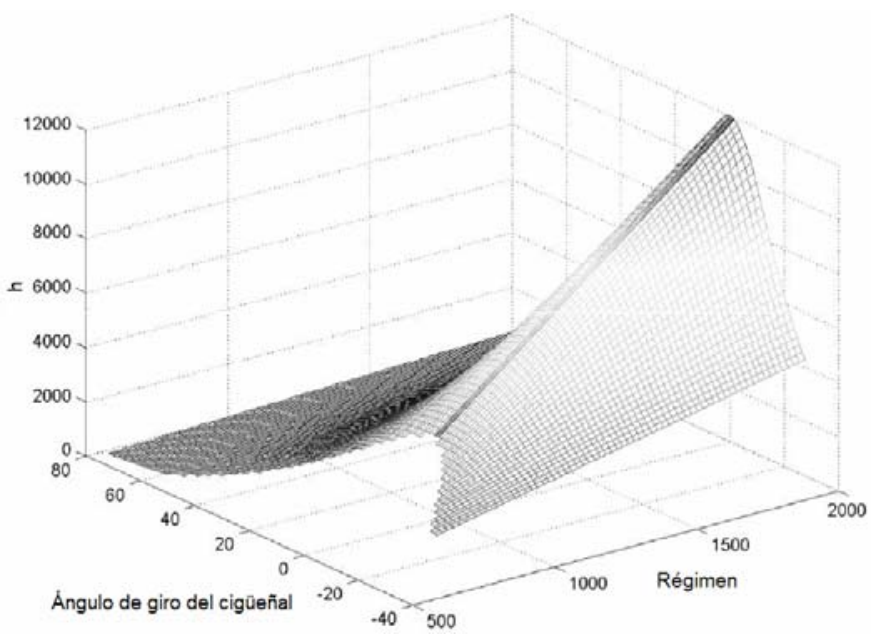

Figura 2.5. Mapa del coeficiente de transferencia de calor instantáneo para un estado de carga según el régimen del motor [2.23].

Recientemente Kavtaratze et al. [2.24] emplearon una hipótesis de aditividad de la transferencia de calor convectiva y radiativa para determinar un coeficiente de transferencia de calor integral local, tras emplear información experimental del factor de emisividad de los productos de la combustión. Las investigaciones se realizaron en la cámara de combustión de un motor Diesel de altas revoluciones. El comportamiento del coeficiente integral se ha reproducido en la Figura 2.6.

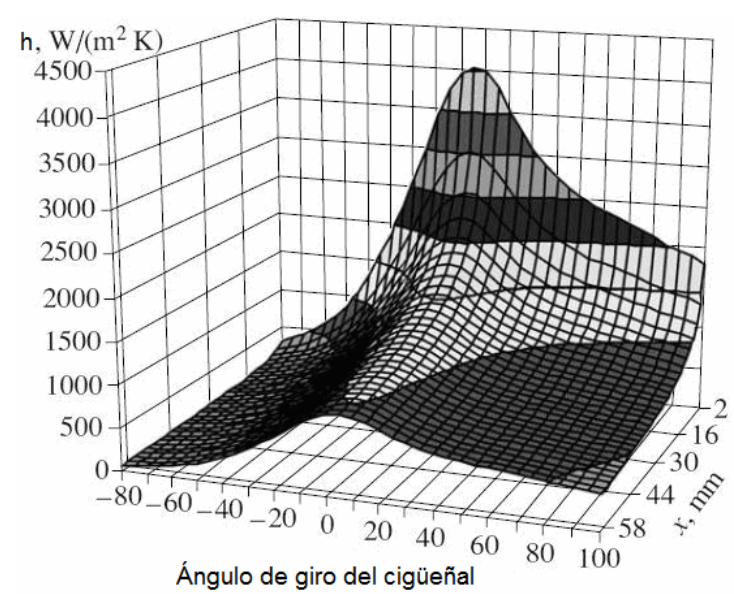

Figura 2.6. Variación del coeficiente de transferencia de calor integral (convección más radiación) sobre la superficie de la culata en función de la distancia radial (1600 rpm, $191,6 \mathrm{~kW})[2.24]$. 
La transferencia de calor en el cilindro se da a través de las capas límite térmicas de la cámara de combustión y las superficies metálicas, encontrándose el mayor gradiente de temperatura en la pared (Figura 2.2). La transferencia de calor depende del gradiente de temperatura, afectado por las velocidades de turbulencia de los gases en la zona externa de la capa límite, y de la conductividad térmica de los gases en dicha capa límite. La presencia de las paredes, donde se hace cero la velocidad de los gases, también tiene una profunda influencia en la distribución del momento. La transferencia de calor en cualquier posición es, pues, una función tanto de la capa límite térmica como de la capa límite cinemática (viscosa). Estos detalles no son descritos por los modelos globales y por ello no se puede lograr un conocimiento, ni siquiera aproximado, del mapa de distribución de los coeficientes de la transferencia de calor a lo largo de la geometría de la cámara. Woschni [2.16] procuró tener en cuenta los efectos de la turbulencia alterando la velocidad en la que se basa su número de Reynolds en los diferentes procesos para acercarse a las velocidades reales del gas. La mayoría de los trabajos que siguen la ecuación de Woschni han incorporado correcciones para tener en cuenta los factores anteriormente mencionados. En la búsqueda de una mayor exactitud, algunos autores [2.6], [2.28], [2.27], [2.25] también han incorporado efectos de la turbulencia en sus modelos, teniendo como principal elemento distintivo el tratamiento de la velocidad del gas.

Los modelos globales carecen de la resolución para tener en cuenta y analizar procesos como la radiación en los motores de encendido por compresión o la detonación en motores de encendido provocado. Los modelos globales sólo permiten el cálculo de los valores medios de los flujos de calor radiativos hacia las paredes y no permiten discriminar qué partes de las paredes están más o menos radiadas. Se requiere entonces el empleo de modelos de al menos dos zonas para considerar los dos mecanismos de convección y radiación de manera separada.

\subsubsection{Modelos zonales y locales}

Las limitaciones descritas de los modelos globales son de alguna manera superadas, al menos para la aplicación en modelos predictivos y de diagnóstico, en los modelos zonales desarrollados a partir de los trabajos de Morel [2.6], Poulos y Heywood [2.28] y Borgnakke [2.31]. 
En la gráfica de la Figura 2.7 se ilustra la correlación de los mecanismos de transmisión de calor por radiación y por convección durante el ciclo del motor. En esta figura se ha dibujado el coeficiente de transferencia de calor "equivalente", resultado de la combinación de la convección y la radiación, en el trabajo de Boulouchos [2.58], quien realizó estudios en un motor de dos tiempos de carrera larga y consideró la influencia de elevar de manera separada la temperatura del gas (mediante el empobrecimiento de la mezcla) y la temperatura de la pared (empleando camisas de titanio para los cilindros).

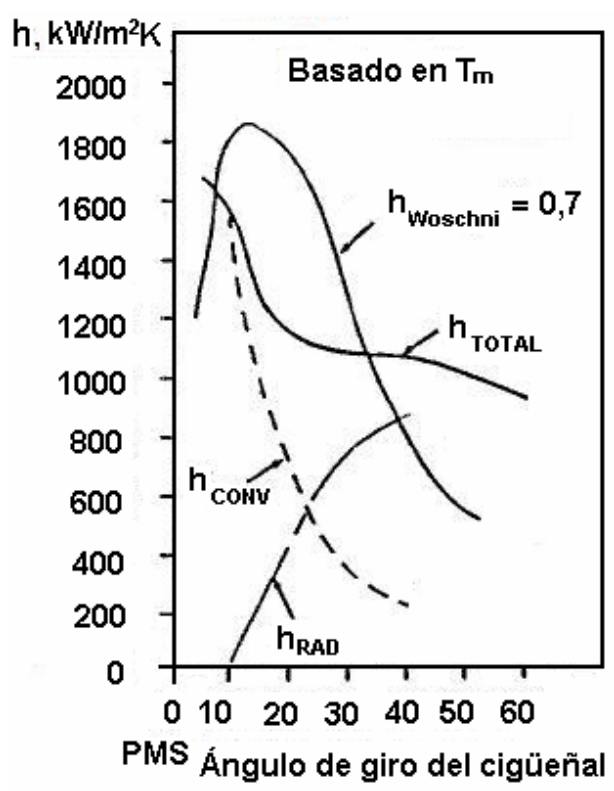

Figura 2.7. Coeficientes de transferencia de calor en un motor con camisas de acero y de fundición, calculados en función de la temperatura termodinámica media del proceso. El coeficiente de Woschni se ha multiplicado por 0,7 para obtener el coeficiente de convección predicho excluyendo la parte radiativa [2.58].

De la figura se ve claro que, aunque es similar en magnitud al coeficiente de Woschni, el coeficiente de transferencia de calor equivalente exhibe un comportamiento instantáneo diferente, principalmente debido al elevado flujo radiativo durante la combustión.

En los motores de encendido provocado, en el momento de la detonación los mayores flujos de calor locales tienen lugar en aquellas zonas donde la llama se está extinguiendo. Estas razones y otras más han demandado la necesidad de desarrollar modelos zonales y multidimensionales para estudiar la combustión y la transferencia de calor, modelos que son más exigentes en lo 
que respecta al equipo experimental para las validaciones. El desarrollo de modelos zonales es necesario también para valorar desde el punto de vista fenomenológico las interacciones llama-pared y chorro-pared. Con el avance de los equipos de computación se han podido programar modelos más refinados, en los que se hace uso de expresiones basadas en la ecuación de la energía y los conceptos de la capa límite térmica en toda la geometría de la cámara de combustión y que dan cuenta de las inestabilidades de los flujos dentro y fuera de la capa térmica límite, el comportamiento complejo del campo de velocidades, la compresibilidad de la capa límite, las variaciones de la composición de los gases y los depósitos en las paredes. En la tabla $2.2 \mathrm{se}$ resumen las expresiones propuestas para modelar la transferencia de calor entre el gas y las paredes con resolución zonal o dimensional.

Tabla 2.2. Correlaciones basadas en modelos zonales.

\begin{tabular}{|c|c|c|}
\hline Autor & Método & Expresión y comentarios \\
\hline \multicolumn{3}{|c|}{ Análisis energético de la capa límite térmica } \\
\hline $\begin{array}{l}\text { Morel y } \\
\text { Keribar } \\
(1985)\end{array}$ & $\begin{array}{l}\text { Basaron su trabajo en la } \\
\text { analogía de Colburn, } \\
\text { acoplada a un modelo de } \\
\text { dos zonas. }\end{array}$ & $\begin{array}{l}h=\frac{1}{2} C_{f} \rho U_{e f f} C_{p} P^{-2 / 3} \quad \text { (23). } C_{f} \text { y } C_{p} \text { son coeficientes de } \\
\text { fricción superficial y de presión, respectivamente, , } U_{e f f} \text { es la } \\
\text { velocidad efectiva del gas, } \\
U_{\text {eff }}=\left(U_{z}^{2}+U_{y}^{2}+2 k\right)^{1 / 2} ; U_{z} \text { y } U_{y} \text { se obtienen del modelo } \\
\text { de flujo promediado, que tiene en cuenta la velocidad del pistón, los } \\
\text { movimientos de swirl y squish. La energía cinética turbulenta k se } \\
\text { calcula aplicando un modelo turbulento k- } \varepsilon \text {. La temperatura del gas, } \\
\text { Tg, se determina a partir de la cantidad y ubicación de los gases } \\
\text { quemados. }\end{array}$ \\
\hline $\begin{array}{l}\text { Poulos y } \\
\text { Heywood } \\
(1983)\end{array}$ & $\begin{array}{l}\text { Incorporaron los efectos de } \\
\text { turbulencia, como lo hizo } \\
\text { Morel, en el ciclo de } \\
\text { simulación de un motor a } \\
\text { gasolina. }\end{array}$ & $\begin{array}{l}\text { Calcularon la velocidad efectiva como } \\
U_{\text {eff }}=\left[2 K+2 k+\frac{V_{p}^{2}(t)}{4}\right]^{1 / 2} \text {, donde } K \text { y } k \text { corresponden a la } \\
\text { energía cinética del flujo promediado y de la turbulencia, } \\
\text { respectivamente. La longitud característica se emplea como macro- } \\
\text { escala de la turbulencia. }\end{array}$ \\
\hline $\begin{array}{l}\text { Bargende } \\
(1990)\end{array}$ & $\begin{array}{l}\text { Inicialmente utilizó los } \\
\text { postulados de Woschni, al } \\
\text { describir el número de } \\
\text { Nusselt } \\
\left(\mathrm{Nu}=\mathrm{CRe}^{0,78} \mathrm{Pr}^{0,33}\right)\end{array}$ & $h=C \underbrace{D^{-0,22}}_{\text {Char.length }} \underbrace{k\left(\frac{\rho}{\mu}\right)}_{\text {Materialproperties }} \underbrace{w_{\text {Combustion }}^{0,78}}_{\text {Gasvelocity }} \underbrace{\Delta}$ \\
\hline
\end{tabular}




\begin{tabular}{|c|c|c|}
\hline & $\begin{array}{l}\text { Las constantes en la } \\
\text { ecuación } \\
\text { determinadas para motoren } \\
\text { a gasolina de inyección } \\
\text { directa. }\end{array}$ & $\begin{array}{l}\text { La longitud característica se expresa en función del diámetro de la } \\
\text { esfera de volumen igual al volumen instantáneo del cilindro, } \\
D^{-0,22} \equiv 1,11 V^{-0,073} ; w=\frac{\sqrt{\frac{8 k}{3}+C_{p}^{2}}}{2} \text {. La velocidad del gas se } \\
\text { basa en la energía turbulenta y en la velocidad del pistón. } \\
\frac{d k}{d t}=\left[-\frac{2}{3} \frac{k}{V} \frac{d V}{d t}-\varepsilon \frac{k}{L}+\left(\varepsilon_{s} \frac{k_{S}^{1,5}}{L}\right)_{\varphi>T D C}\right]_{I V C \leq \varphi \leq E V O} \\
\varepsilon=\varepsilon_{S}=2,184, y \text { la longitud característica del swirl se halla como } \\
L=\sqrt[3]{6 /(\pi V)} ; k_{s} \text { es la energía cinética del squish. Al describir las } \\
\text { condiciones iniciales en la capa límite se utiliza un promedio de } \\
\text { temperatura entre la temperatura del gas y la temperatura de la pared. }\end{array}$ \\
\hline \multicolumn{3}{|c|}{ Modelos basados en el concepto de la capa límite. } \\
\hline $\begin{array}{l}\text { Borgnakke, } \\
\text { Arpaci y } \\
\text { Tabaczyns- } \\
\text { ki (1980) }\end{array}$ & $\begin{array}{l}\text { Un parámetro alternativo al } \\
\text { coeficiente de transferencia } \\
\text { de calor es la constante de } \\
\text { conductividad equivalente } \\
\text { de la capa límite de espesor } \\
\delta_{T}\end{array}$ & $\begin{array}{l}\text { El flujo de calor no se determina por la expresión de Newton, sino por } \\
\text { la expresión de Fourier, en la forma } q_{w}=k_{\text {eff }} \frac{T_{g}-T_{w}}{\delta_{T}}, k_{\text {eff }} \text { es la } \\
\text { conductividad térmica efectiva del gas en la capa límite, determinada } \\
\text { a partir de la teoría de turbulencia. }\end{array}$ \\
\hline $\begin{array}{l}\text { Chen y } \\
\text { Veshagh } \\
\text { (1989) }\end{array}$ & $\begin{array}{l}\text { Basada en la analogía de } \\
\text { Colburn. Emplean una ley } \\
\text { de potencia para definir el } \\
\text { perfil de velocidad: } \\
u^{+}=C\left(y^{+}\right)^{n} \text {, igual que } \\
\text { para definir la distribución } \\
\text { de temperatura en la capa } \\
\text { límite interna: } \\
\theta=\frac{T_{\infty}-T}{T_{\infty}-T_{w}}=D\left(\frac{y}{\Delta}\right)^{n}\end{array}$ & 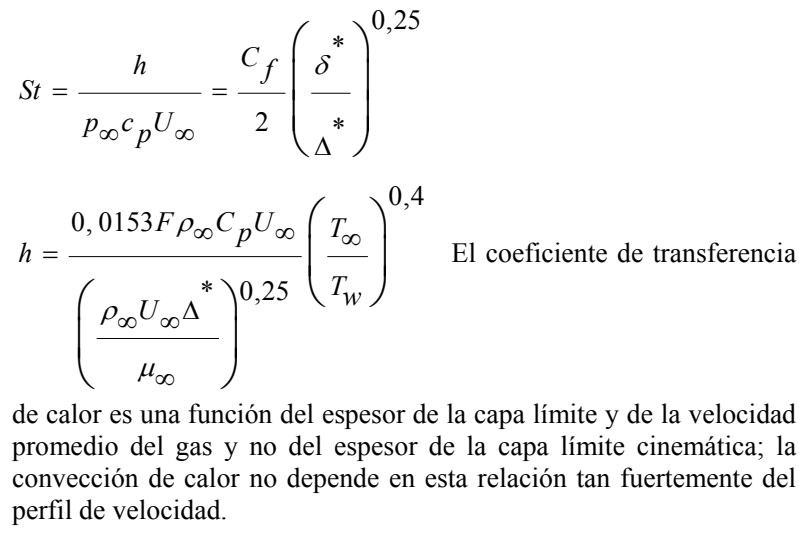 \\
\hline $\begin{array}{l}\text { Fiveland y } \\
\text { Assanis } \\
\text { (2002) }\end{array}$ & $\begin{array}{l}\text { Aplicaron su modelo a un } \\
\text { motor HCCI. El modelo } \\
\text { tiene en cuenta la zona } \\
\text { gruesa (central) del gas o } \\
\text { zona de núcleo gaseoso } \\
\text { adiabático, las regiones de } \\
\text { capa límite y las regiones } \\
\text { intersticiales del grupo de } \\
\text { anillos. }\end{array}$ & $h=\frac{1}{\int_{0}^{\delta_{t}} \frac{1}{\rho \cdot C_{p} \frac{v_{\text {eff }}}{\operatorname{Pr}_{\text {eff }}}}}$ \\
\hline
\end{tabular}




\begin{tabular}{|c|c|c|}
\hline \multicolumn{3}{|c|}{$\begin{array}{c}\text { Modelos de transferencia de calor locales. Modelos de capa límite con empleo de leyes } \\
\text { de pared. }\end{array}$} \\
\hline $\begin{array}{l}\text { Pinchon } \\
{[2.101],} \\
\text { Kleeman } \\
{[2.51],} \\
\text { referencian } \\
\text { a Launder } \\
\text { y Spalding } \\
(1974) .\end{array}$ & $\begin{array}{l}\text { Ajustaron una función de } \\
\text { pared sólo para flujo } \\
\text { unidimensional estable no } \\
\text { compresible en tuberías } \\
\text { lisas y con números de } \\
\text { Reynolds moderados. Se } \\
\text { ignoran las variaciones de } \\
\text { las propiedades } \\
\text { termofísicas del fluido } \\
\text { (densidad, viscosidad, } \\
\text { conductividad térmica) } \\
\text { dentro de la capa límite. }\end{array}$ & 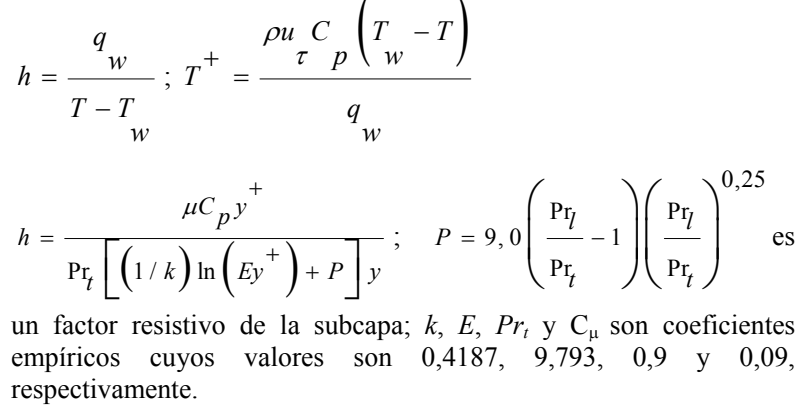 \\
\hline $\begin{array}{l}\text { Han y } \\
\text { Reitz } \\
\text { (1997) }\end{array}$ & $\begin{array}{l}\text { Modificaron la ley de la } \\
\text { pared. La turbulencia en las } \\
\text { regiones de números de } \\
\text { Reynolds elevados la } \\
\text { modelan empleando un } \\
\text { modelo RNG k-e. Se } \\
\text { consideran las variaciones } \\
\text { de las propiedades } \\
\text { termofísicas del fluido } \\
\text { dentro de la capa límite. }\end{array}$ & $\begin{array}{l}\text { El flujo de calor a la pared, } q_{w} \text {, con sus componentes de estado estable } \\
\left(q_{s s}\right) \text { y transitorio }\left(q_{u s}\right) \text { se expresa como: } q=q s s+q u s: \\
q_{w}=\frac{\rho C_{p} u^{*} T \ln \left(T / T_{w}\right)-\left(2,1 y^{+}+33,4\right) G v / u^{*}}{2,1 \ln \left(y^{+}\right)+2.5}-k T_{0}\left(\frac{p}{p_{0}}\right)^{\lambda-1 / \lambda} * \\
* \frac{k \rho_{0} u^{*}}{\mu_{w}}\left\{\int_{0}^{\tau}\left[\frac{1}{\sqrt{\pi(\tau-\theta)}}+0,082\left(1-\exp \left(-\frac{\sqrt{(\tau-\theta)}}{3}\right)\right)\right] \frac{d \phi_{u s}(0 . \theta)}{d \theta}\right. \\
u^{*}=\sqrt{k C_{\mu}^{1 / 2}} \text {. El flujo de calor es proporcional al logaritmo de la } \\
\text { relación de temperaturas del gas y la pared, en lugar de la diferencia } \\
\text { aritmética de temperaturas, empleada en flujos incompresibles. }\end{array}$ \\
\hline
\end{tabular}

Capa límite térmica. En las condiciones en las que se desarrollan los procesos dentro del cilindro del motor, la turbulencia es el principal mecanismo promotor no sólo de la combustión, sino también de la transferencia de calor entre el gas y las paredes. La analogía de Reynolds es la manera más sencilla para acoplar los fenómenos de momento y de transferencia de calor que tienen lugar entre el grueso del gas y las paredes en modelos de flujos estacionarios a través de tuberías lisas, y también ha sido adaptada por diferentes autores para determinar las variaciones de temperatura en las inmediaciones de la capa límite dentro del cilindro del motor. Dicha analogía introduce suposiciones que imponen algunas condiciones a la capa límite, como son que el flujo en ella es isotérmico, no se produce liberación de calor ni se realiza trabajo en ella, el gradiente de temperatura a lo largo de la pared es nulo y el número de Prandtl turbulento dentro de la capa es constante. El estudio de la capa límite térmica permite no 
sólo acercarse a la determinación de los flujos de calor, sino también a la determinación del espesor de la capa de extinción de la llama.

Borgnakke, Arpaci y Tabaczynski [2.31] determinaron el espesor de la capa límite térmica, $\delta_{T}$, a partir de la ecuación de energía formulada en el entorno de su volumen de control. Heywood y Lyford-Pike [2.5] midieron el espesor de las capas límite térmicas en el cilindro, durante todo el ciclo de operación de un motor de encendido provocado a diferentes condiciones de velocidad y de carga, empleando fotografías Schlieren. Las mediciones permitieron valorar en cerca de $2 \mathrm{~mm}$ el espesor máximo de la capa límite. La reducción de la velocidad produce una reducción del espesor de la capa límite, mientras que la variación de la carga no genera variaciones significativas del mismo. Heywood y Lyford-Pike concluyeron que el espesor de la capa límite térmica depende de la difusividad térmica $(\alpha)$ y el tiempo $(t)$ disponible por la capa para desarrollarse. Ellos relacionaron estas variables y el número de Reynolds mediante una expresión de la forma $\delta_{T} \sqrt{\alpha t}=a \cdot \operatorname{Re}^{0,2}$, donde $a$ es una constante de proporcionalidad.

Chen y Veshagh [2.32] evaluaron el coeficiente de transferencia de calor como función del espesor de la capa límite térmica, utilizando la analogía de Colburn $\left(S t \cdot \operatorname{Pr}^{2 / 3}=C_{f} / 2\right.$ para $\left.0,5<P r<100\right)$ y tomando en cuenta la variación de las propiedades del gas a través del espesor de las capas límite tanto hidrodinámica como térmica, es decir, considerando las distorsiones causadas en los perfiles de velocidad y de temperatura. En el modelo unidimensional de Chen y Veshagh, al tiempo que se tienen en cuenta las variaciones globales, espaciales y temporales, de las capas límite, el comportamiento de los perfiles de velocidad y temperatura dentro de las mismas capas se rige por una ley potencial estacionaria. El modelo referido es una alternativa a los modelos de ley de pared que también puede integrarse tanto a los modelos de llenado-vaciado, como a los métodos de dinámica de fluidos computacional.

En su investigación sobre motores HCCI, Fiveland et al. [2.13] presentaron un modelo de combustión de dos zonas en el que acoplaron la zona núcleo adiabática con una zona de capa límite a través de la cual se realizaba la transferencia de calor hacia las paredes (Figura 2.8 y 2.9). El modelo permitió predecir muy bien el coeficiente de transferencia de calor, en comparación con un modelo en el que se empleaba un coeficiente de transferencia de calor 
global, determinando el espesor de la capa límite térmica y la masa atrapada, dos magnitudes necesarias para predecir la formación de hidrocarburos en las regiones cercanas a la pared. Los autores validaron su modelo de dos zonas con información experimental y también con los resultados obtenidos al aplicar un modelo de transferencia de calor empírico. El tratamiento de la capa límite se basó en los trabajos propuestos por primera vez por Borgnakke et al. [2.31] y Puzinauskas y Borgnakke [2.29] en la parte cerrada del ciclo.

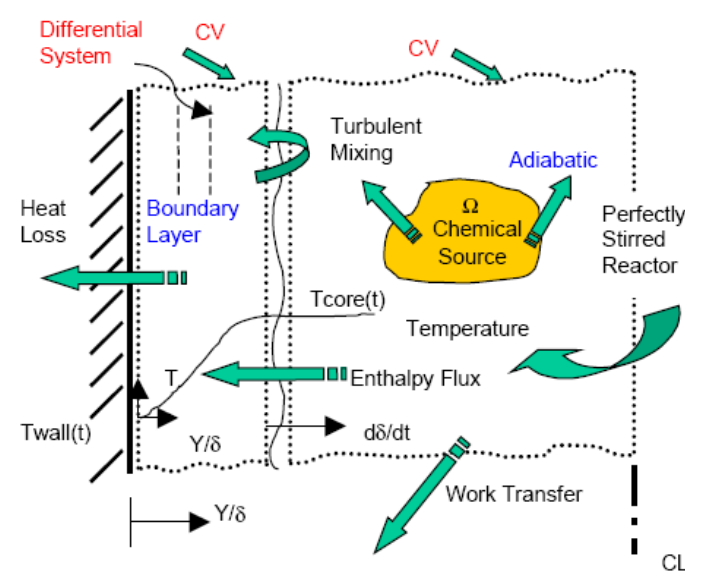

Figura 2.8. Interacción del núcleo adiabático con la capa límite [2.13].

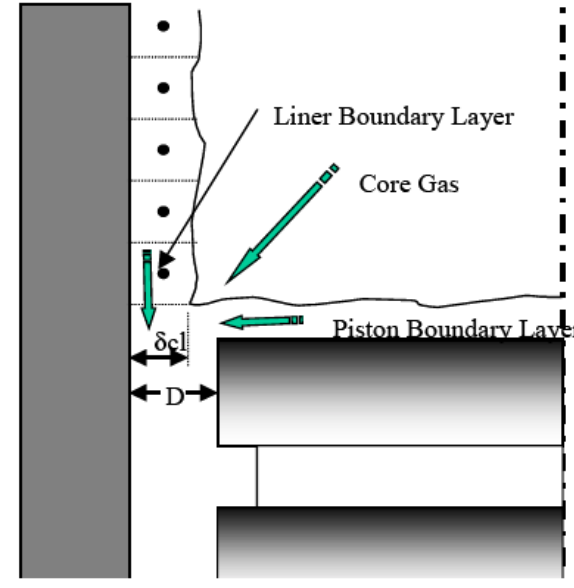

Figura 2.9. Representación esquemática de los flujos provenientes de las masas del núcleo y de la capa límite hacia los intersticios de los anillos [2.13].

El modelo de Fiveland et al. contempla un espesor característico de capa límite, asumiendo un flujo de calor unidimensional cuasi-estacionario, con lo que la ecuación de Fourier para la conducción de calor pudo integrarse para finalmente obtener un coeficiente de transferencia de calor en la forma en la que aparece en la tabla 2.2.

El modelo de capa límite fue implementado en zonas locales de la cámara de combustión y los espesores de la capa límite a lo largo del ciclo fueron calculados como ilustra la Figura 2.10. El máximo espesor de la capa límite se produce durante la carrera de escape y es aproximadamente de $3 \mathrm{~mm}$, 
mientras que su espesor mínimo desciende a cerca de $0,3 \mathrm{~mm}$ cerca del punto muerto superior. La variación de la densidad en el espesor de la capa límite hace que la masa atrapada en la capa límite en este punto corresponda a aproximadamente el $30-35 \%$ de la masa total del cilindro.

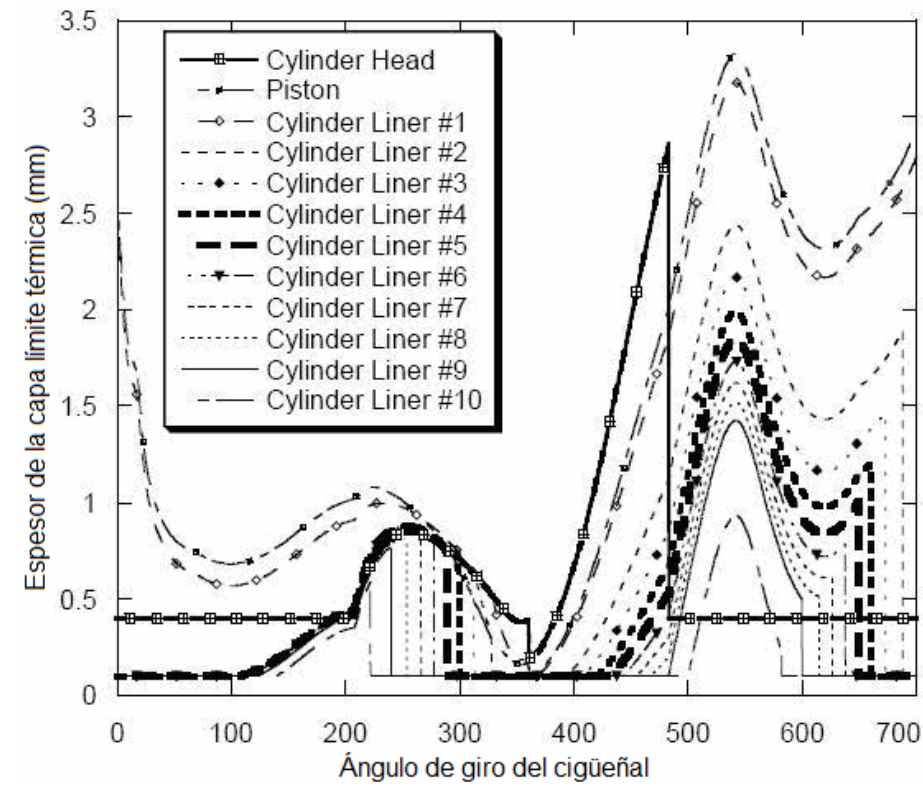

Figura 2.10.

Variación del espesor de la capa límite térmica a lo largo del ciclo de un motor HCCI sobre las superficies de algunos nodos seleccionados en la camisa, el pistón y la culata [2.13].

Tratamiento de la capa límite en los códigos de dinámica de fluidos computacional mediante la ley de la pared. El tratamiento general en los contornos de pared busca evitar la capa molecular y las zonas de transición contiguas a ésta y alcanzar la solución en la primera celda con las propiedades de la pared. Esto se logra con las conocidas "funciones de pared", un conjunto de funciones semiempíricas que han sido desarrolladas a partir de información experimental y leyes de afinidad.

El flujo dentro del cilindro del motor es turbulento y el espesor de la capa límite es muy pequeño, comparado con la resolución de la malla que, en la práctica, puede realizarse con los programas computacionales. Por esta razón, para determinar el esfuerzo cortante y la transferencia de calor en las vecindades de la pared, se recurre al empleo de funciones de pared para los cálculos de la velocidad y de la temperatura (Figura 2.11). 
En los modelos de turbulencia generalmente empleados, los efectos directos de la viscosidad molecular sobre las componentes representativas de la energía del movimiento fluctuante (escalas de mayor contenido energético), al igual que los del flujo medio sobre las correspondientes escalas disipativas (escala de turbulencia menor), se asume que son despreciables. Estas asunciones, en general, son válidas allí donde el flujo es totalmente turbulento, pero no lo son en las regiones contiguas a la pared, donde se reducen las diferencias entre las escalas grandes y pequeñas del movimiento turbulento.

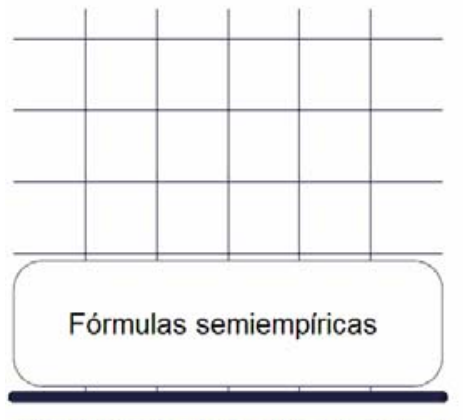

Tratamiento de función de pared

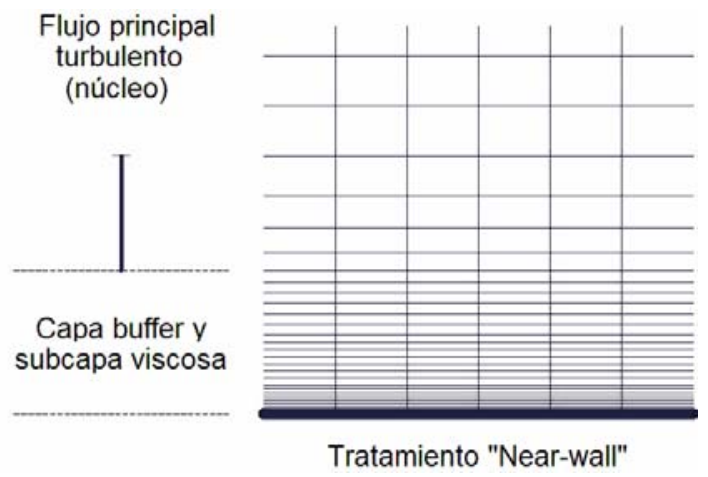

Tratamiento "Near-wall"

Figura 2.11. El mallado grueso no puede resolver la capa interna, por lo que se requiere una relación auxiliar que sirva de condición de contorno, la función de pared, para acoplar los flujos interno y externo.

El método más sencillo para modelar el flujo y los fenómenos asociados que ocurren cerca de la pared ha sido el empleo de la ley de la pared (tratamiento de función de pared), mediante la cual se suele expresar la velocidad media paralela a la pared y las propiedades de turbulencia fuera de la subcapa viscosa, en términos de la distancia a la pared y de las condiciones de la pared como son el esfuerzo cortante, el gradiente de presión, y la transferencia de calor a la pared. Así, las funciones de pared pueden usarse para proveer las condiciones de contorno en las inmediaciones de la pared para las ecuaciones de transporte de momento y turbulencia, en lugar de las condiciones en la pared misma, de manera que no hay necesidad de resolver la subcapa viscosa y se supera así la exigencia de un mallado muy fino. Este método fue propuesto por Launder y Spalding [2.91], quienes obtuvieron los perfiles de velocidad y temperatura para las vecindades de la pared a partir de un análisis de flujo de Couette, asumiendo un flujo unidimensional estacionario e 
ignorando las variaciones de las propiedades termofísicas (como la densidad, la viscosidad, la conductividad térmica) dentro de la capa. En la Figura 2.12 se puede ver la manera como con la ley de la pared de Spalding se logra el acuerdo en la región de mezclado para el perfil de velocidad. De manera similar la ley de la pared negocia la zona correspondiente para el perfil de temperatura.

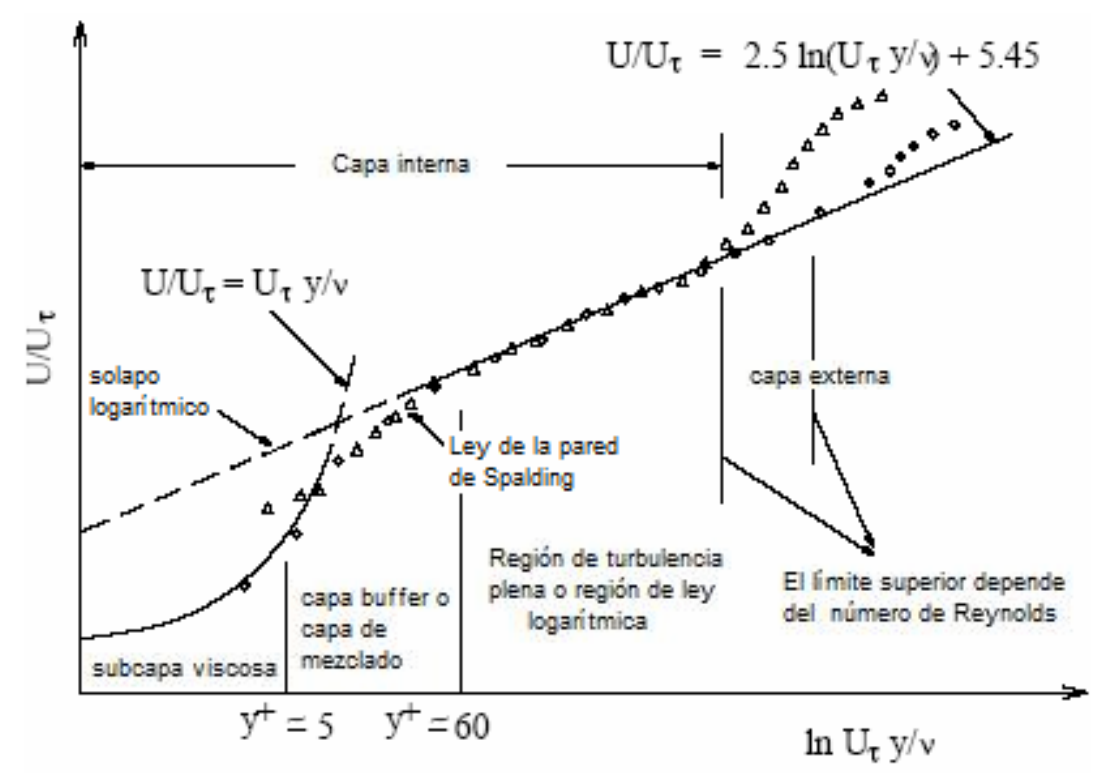

Figura 2.12. Comparación de la expresión de Spalding para superficies internas de tubos, con información experimental obtenida [2.49].

La mayoría de los códigos de mecánica de fluidos computacional (CFD) utilizan el método de función de la pared. La capa límite hidrodinámica se descompone en una subcapa laminar viscosa y una capa buffer para adaptar la parte laminar de la capa límite al núcleo turbulento. Las principales asunciones tomadas en cuenta al usar la ley de la pared son: a) el flujo es estacionario, incompresible (es decir, no afectado por la presión), esencialmente unidimensional (lo que quiere decir que los gradientes de velocidad y las propiedades escalares son todas normales a la pared); b) los efectos de los gradientes de presión son pequeños; c) la turbulencia corresponde a un estado de equilibrio local; d) el tamaño de la escala de turbulencia es función lineal de la distancia a la pared. Adicionalmente a estas restricciones, las ondulaciones más pequeñas de la superficie pueden alterar 
los resultados de los modelos implementados con la ley de la pared. Para la ecuación de momento medio y energía, las funciones de pared se basan en la distribución logarítimica de la velocidad y la temperatura, escaladas con la velocidad de fricción $u_{\tau}=\sqrt{\tau_{w} / \rho}$ y la viscosidad $\mu$ para la velocidad media y por el flujo de calor a la pared y la difusividad térmica para la temperatura media.

Con las suposiciones hechas, la velocidad tangencial y la temperatura en el nodo más próximo a la pared, presumiblemente localizado dentro de la capa límite turbulenta, pueden determinarse por las expresiones:

En la subcapa viscosa:

En la subcapa inercial:

$$
\begin{aligned}
& u^{+}=y^{+} \\
& T^{+}=\operatorname{Pr} \cdot u^{+}
\end{aligned}, \text {para } y^{+} \leq 11,63
$$

$$
\begin{aligned}
& u^{+}=\frac{1}{k} \ln \left(E y^{+}\right) \\
& T^{+}=\operatorname{Pr}_{t} \cdot\left[u^{+}+P\left(\frac{\operatorname{Pr}}{\operatorname{Pr}_{t}}\right)\right], \text { para } y^{+}>11,63
\end{aligned}
$$

donde $u^{+}, T^{+}$y $y^{+}$son valores locales adimensionales ("coordenadas de pared") de la velocidad tangencial $u$, la temperatura $T$ y la distancia normal a la pared $y$, definidas por:

$$
\begin{aligned}
& u^{+}=\frac{u^{*}}{u_{\tau}} \\
& T^{+}=\frac{\rho u_{\tau} C_{p}\left(T-T_{w}\right)}{q_{w}} \\
& y^{+}=\frac{y u_{\tau}}{v}=\frac{C_{\mu}^{0,25} k^{0,5} y}{v}
\end{aligned}
$$

En las expresiones descritas $T_{w}$ y $q_{w}$ son los valores locales de la temperatura de pared y del flujo de calor, respectivamente, $u_{\tau}=\sqrt{\tau_{w} / \rho}$ es la velocidad de fricción definida en términos del esfuerzo cortante local $\tau_{w}$ y $P\left(P r / P r_{t}\right)$ es un 
factor de resistencia térmica de la subcapa viscosa, dependiente de los números de Prandtl laminar y turbulento; $v$ es la viscosidad cinemática y $c_{p}$ es el calor específico; $k, E, P r_{t}$ y $C_{\mu}$ son coeficientes empíricos cuyos valores son $0,4187,9,793,0,9$ y 0,09 , respectivamente.

Como el modelado de la capa límite empleando funciones de pared se basa en una serie de suposiciones acerca del flujo, la exactitud de la solución es, en consecuencia, dependiente de qué tan válidas sean esas asunciones en cada caso en particular.

LeFeuvre [2.33], Nijeweme [2.12] y Reitz [2.11] han contribuido al estudio de la influencia de la inestabilidad y la compresibilidad de la capa límite en la transferencia de calor del motor. Han y Reitz [2.11], basándose en la ecuación de conservación de la energía unidimensional desarrollaron una función de pared apropiada para el estudio de los flujos turbulentos de densidad variable en los motores de combustión interna. La función de pared se implementó y se probó con un motor Diesel Caterpillar, en el que se instalaron tres sensores de flujo de calor, HT-1 cerca al inyector, HT-2 en la cavidad del pistón cerca al borde y HT-3 sobre el área de squish cerca de la camisa, a unas distancias del eje de 15, 45, y $60 \mathrm{~mm}$, respectivamente. Los flujos de calor obtenidos y los medidos se han reproducido en la Figura 2.13, donde se aprecia un par de picos (claros para el pistón y la culata, sobre todo) correspondientes a la combustión inicial premezclada después de la ignición y al paso de la llama. La rapidez de desprendimiento de calor también aparece graficada.

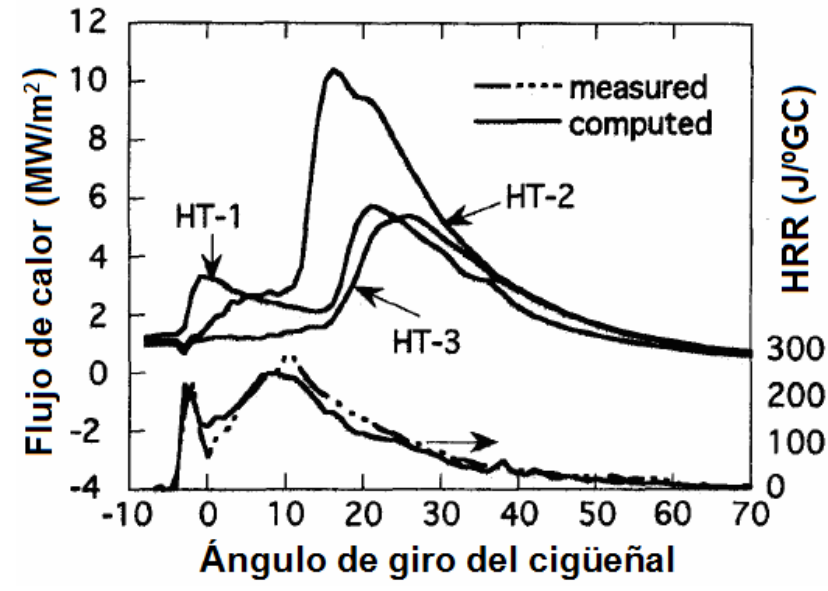

Figura 2.13. Flujos de calor calculados en la cámara de combustión de un motor Caterpillar [2.11]. 
Nijeweme et al. [2.12], tras demostrar las inconsistencias debidas al empleo de la ley de la pared, modelaron el fenómeno de la transferencia de calor dentro del cilindro de un motor de encendido provocado aplicando la ecuación de la energía, encontrando una solución en la forma:

donde $\alpha_{0}=k_{0} / \rho C_{p}$.

$$
\frac{\partial U}{\partial t}=\underbrace{\alpha_{0} \frac{p}{p_{0}} \frac{\partial^{2} U}{\partial z^{2}}}_{\text {la } \min a r}+\underbrace{\alpha_{0} \frac{p}{p_{0}} \frac{\partial}{\partial z}\left(\frac{\operatorname{Pr}}{\operatorname{Pr}_{t}} \frac{\mu_{t}}{\mu} \frac{\partial U}{\partial z}\right)}_{\text {turbulento }}
$$

Nijeweme [2.12] aplicó la información experimental para determinar la conductividad térmica turbulenta $k_{t}$, el número de Prandtl turbulento $P r_{t}$, y la viscosidad turbulenta $\mu_{t}$, a través de la capa límite. Buscó un ajuste de la conductividad térmica turbulenta hasta que obtuvo un campo de temperaturas (distribución de temperaturas) entorno a la pared, que se ajustaba a los flujos de calor medidos en esas posiciones del cilindro. Luego calculó las intensidades turbulentas con un programa de cálculo, y así fue ajustando la expresión para la conductividad térmica por posiciones. La solución de Nijeweme permitió observar el flujo de calor negativo durante la carrera de expansión del pistón y también sirvió para ilustrar las contribuciones relativas de los términos de convección y de trabajo en la ecuación de energía. La Figura 2.14 ilustra el flujo total de calor considerando todos los componentes de la ecuación de energía, comparado con los flujos obtenidos al excluir el término convectivo, o el término de influencia de la presión, o ambos.

El término más importante es el de convección, como muestra la figura. El término de variación de la presión es muy importante para la ubicación o fase de la transferencia de calor. Con variaciones de presión de 5 bar, el flujo de calor se adelanta en 10 grados de giro del cigüeñal, en comparación con el flujo de calor sin el término de trabajo de presión.

Son muchos los autores que han reportado modificaciones y mejoras a la ley de la pared y de ellos, lo más notable recientemente ha sido el trabajo del grupo de Nuutinen [2.49]. Ellos han desarrollado un método para integrar las ecuaciones de momento y energía en su forma compresible a formas de funciones de pared modificadas sensibles a la variación de la densidad (definen una nueva densidad variable adimensional referida a la densidad en el punto más próximo a la pared) y también tienen en cuenta la variación del 
número de Prandtl turbulento. En todos los casos de funciones de pared y sus modificaciones, el objetivo final es reemplazar las ecuaciones que se resuelven dentro de un mallado muy fino en la subcapa laminar por una formulación no muy pesada en términos de cómputo y que dé buena cuenta de la resistencia total de la región al transporte de momento y de calor [2.92].

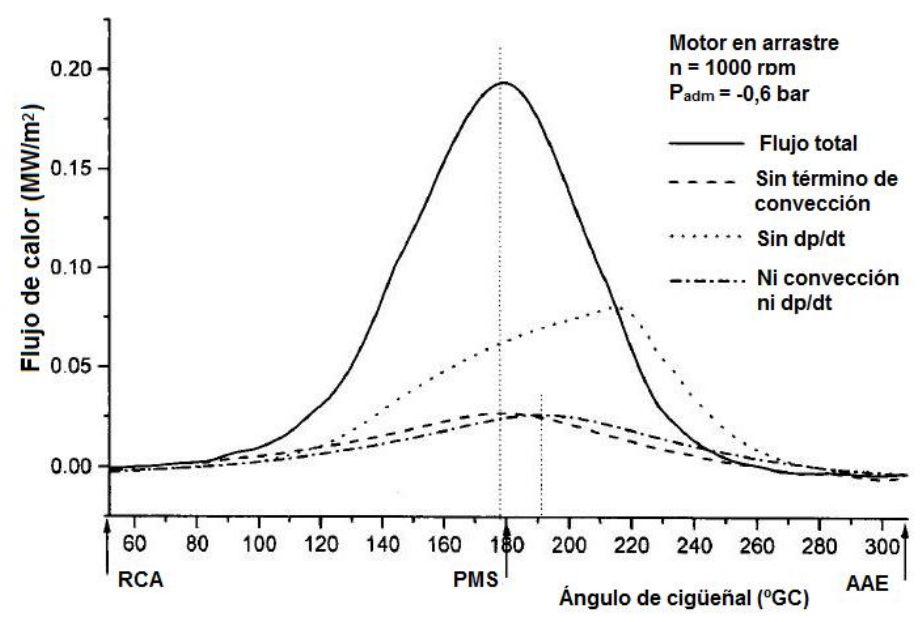

Figura 2.14. Importancia relativa de los componentes individuales de la ecuación de energía [2.12]

Recientemente se han propuesto algunos métodos complementarios o sustitutivos de los modelos $k$ - $\varepsilon$ (energía cinética turbulenta - velocidad de disipación) que están en la base de los modelos de turbulencia con los que se tratan los flujos complejos dentro del cilindro del motor. Entre esos métodos se cuentan:

- El método $k$-w (energía cinética turbulenta - velocidad de disipación específica), el cual combina la ley de la pared y el tratamiento para números de Reynolds bajos mediante una función de continuidad de acople (o función de amortiguación) [2.107].

- El método de relajación elíptica, conocido también como método $\bar{\vartheta}^{2}-f$, definido por Durbin [2.109], en el cual se toma el tensor de esfuerzos de Reynolds $\overline{v v}$ (denotado $\bar{\vartheta}^{2}$ ) como la escala de velocidad en lugar de la energía cinética turbulenta $k$, y donde el término de acople presión-velocidad en la ecuación de transporte de momento 
junto con la diferencia entre las partes no homogénea y homogénea de la velocidad de disipación $\varepsilon$ se evalúan de manera integrada como el producto de $k \mathrm{y}$ una función de relajación $f ; \mathrm{y}$

- El método $\varsigma-f$ de Hanjalic [2.50], el cual es una modificación del método de Durbin y en el cual la ecuación de transporte se resuelve para la relación de escalas de velocidades $\varsigma$, en lugar del tensor de esfuerzos de Reynolds $\bar{\vartheta}^{2}$. Este método se acopla a códigos de simulación numérica directa (DNS).

\subsubsection{Modelos multidimensionales}

Son los modelos más detallados en el estudio de los motores de combustión alternativos. Ellos tienen en cuenta el comportamiento del flujo de la masa de trabajo tanto en lo temporal como en lo espacial, e incluyen submodelos para los procesos físicos como el movimiento periódico del pistón, los flujos en las válvulas, los procesos de inyección, atomización, vaporización y distribución del combustible, la impregnación de las paredes con combustible, la química, la combustión, las capas límite y la transferencia de calor. Los modelos multidimensionales resuelven numéricamente las ecuaciones generales de conservación de los flujos de fluidos reactivos (ecuaciones de Navier Stokes), es decir, las ecuaciones de conservación de la masa, de momento, de energía, de especies y algunas variables de turbulencia, empleando criterios para el promediado de esas variables y sus fluctuaciones turbulentas.

Desde los trabajos de Jennings y Morel [2.34] los códigos numéricos computacionales (CFD) se han constituido en la herramienta analítica más importante para el modelado de los motores de combustión. Todos los programas computacionales comerciales $\left(\mathrm{KIVA}^{\circledR}[2.45][2.88]\right.$, Fluent ${ }^{\circledR}$ [2.85], Star- $\mathrm{CD}^{\circledR}$ [2.87], FIRE $^{\circledR}$ [2.15]) tienen librerías de modelos para el análisis de la transferencia de calor.

Los principales componentes de estos programas, en general, son: los modelos matemáticos (ecuaciones) usados para describir los procesos turbulentos; los procedimientos de discretización usados para transformar las ecuaciones diferenciales del modelo matemático en relaciones algebraicas entre los valores discretos de la velocidad, la presión y la temperatura en puntos de la malla conforme con la geometría de la cámara de combustión, 
con sus partes móviles (pistón y válvulas); y los algoritmos de solución, cuya función es resolver las ecuaciones algebraicas.

La mayoría de los programas CFD comerciales emplean o una función de pared o una modificación de ésta para describir el flujo en las cercanías de las paredes en los cálculos de transferencia de calor. Las ecuaciones promediadas de Navier-Stokes para flujo compresible tridimensional se resuelven en un mallado móvil, mientras que los flujos turbulentos son modelados bajo un concepto de viscosidad turbulenta, empleando el modelo $\kappa-\varepsilon$.

Los programas CFD comerciales pueden acoplarse de manera iterativa a programas de cálculo de temperaturas de pared basados en elementos finitos, FE (Fluent ${ }^{\circledR}$ se puede acoplar con ANSYS $^{\circledR}$, por ejemplo) y permitir el cálculo también de esfuerzos y deformaciones térmicas. En la primera parte de la metodología, el ciclo del motor se calcula con temperaturas constantes asignadas para las diferentes partes de la cámara de combustión. Luego, en la ejecución del segundo ciclo, el programa CFD utiliza las temperaturas calculadas por el programa FE, que ya son más cercanas a las que se asignaron en el primer ciclo [2.47].

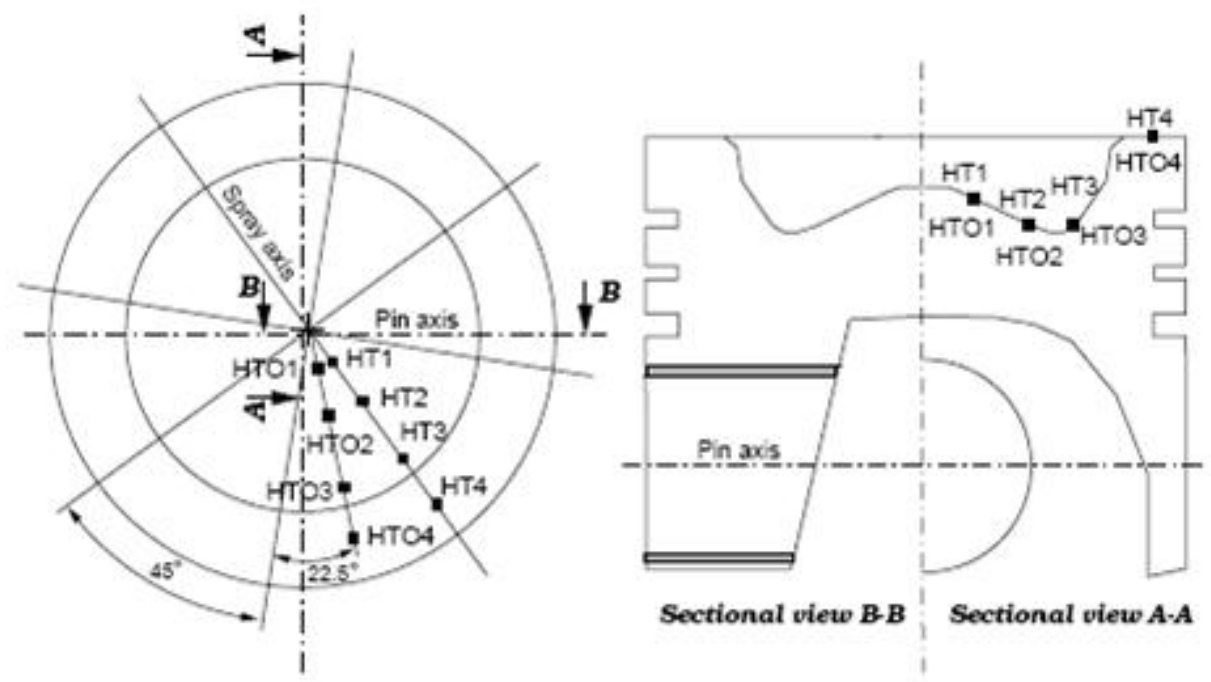

Figura 2.15. Ubicación de los sensores de flujo de calor en el pistón del motor HPP [2.51]. 
Kleemann et al. [2.51] usaron el programa STAR-CD ${ }^{\circledR}$, junto con medidas experimentales, para determinar la magnitud y distribución espacial y temporal de los flujos de calor en la superficie del pistón de un motor Diesel de pulso de alta presión de inyección, HPP. Ellos compararon las medidas con los resultados de la simulación empleando tanto una función de pared estándar, como una función de pared mejorada que consideraba la variación de las propiedades termofísicas del gas, propuesta por ellos. Para los puntos del pistón donde se realizaron las mediciones, observados en la Figura 2.15, se calcularon los flujos de calor.

Los resultados de los flujos de calor en cuatro momentos del ciclo, empezando justo antes de la llegada del frente de llama a la superficie se reproducen en la Figura 2.16.

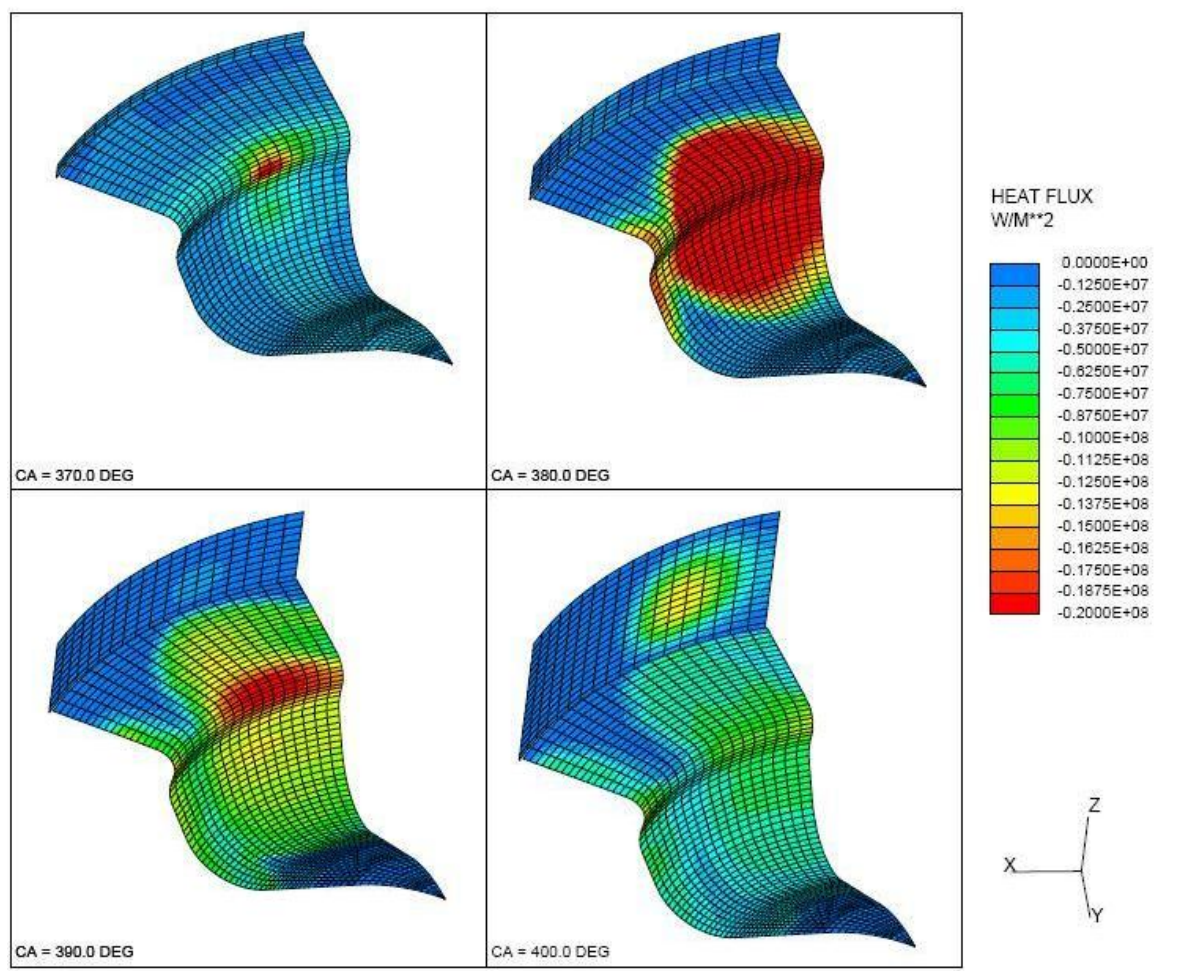

Figura 2.16. Flujos de calor predichos en el pistón del motor HPP con el modelo CFD, empleando una función de pared modificada [2.51]. 
El flujo de calor en la zona pico es unas 10 veces mayor que el valor promedio fuera de la zona pico. La zona de flujo de calor pico se desplaza a medida que se desplaza la llama. La llegada de la llama produce un aumento grande de la temperatura del gas haciendo que la cantidad de movimiento aumente y que se aumente también el coeficiente de transferencia de calor debido a la influencia sobre las variaciones de las propiedades termofísicas de la capa límite.

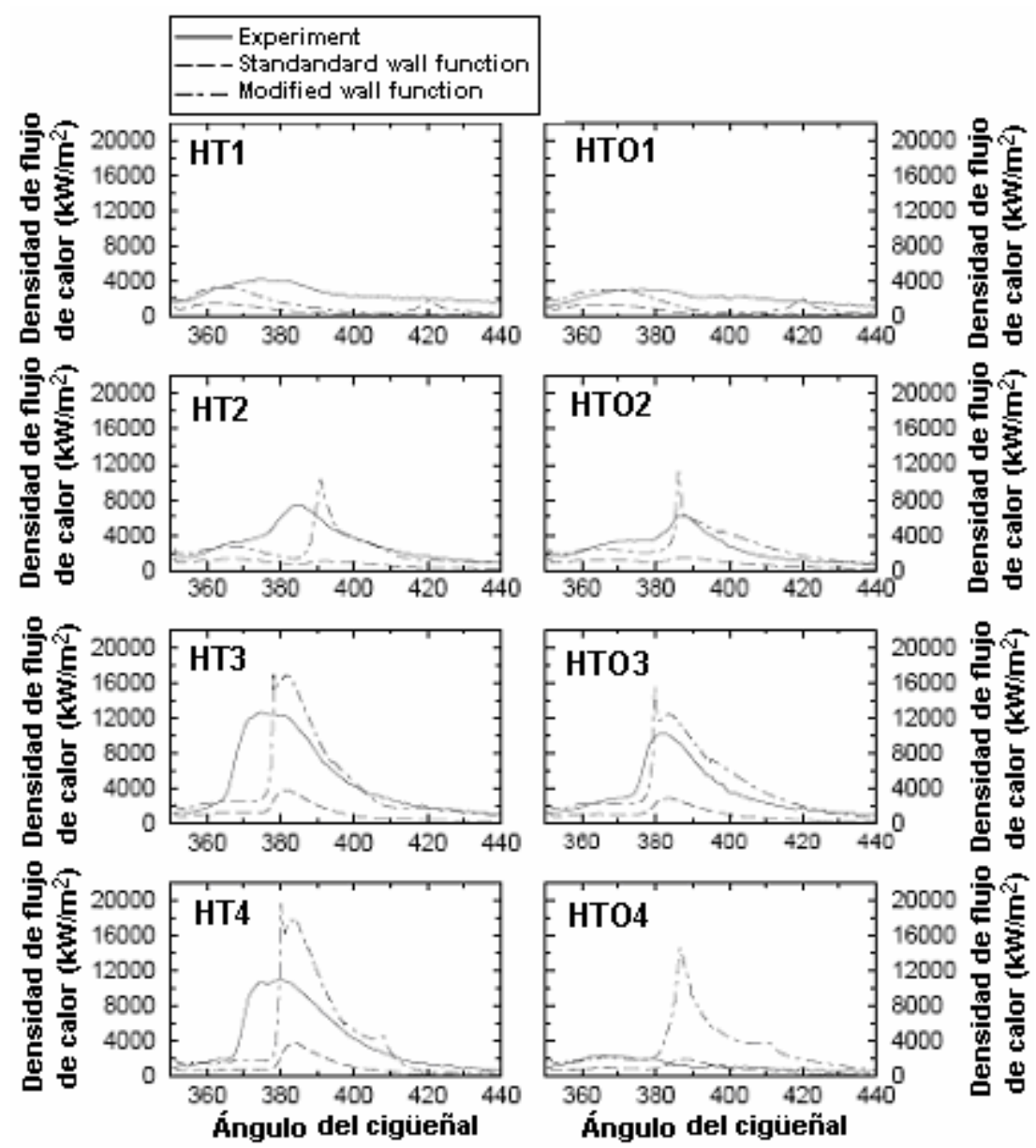

Figura 2.17. Comparación de los flujos de calor experimentales con los modelados con la ley de pared estándar y una ley de pared modificada, en ocho posiciones de la superficie del pistón [2.51]. 
En la Figura 2.17 se reproduce la comparación de los resultados de la simulación de los flujos de calor a través de las superficies del pistón en las 8 localidades del pistón de la Figura 2.15, empleando un modelo con función de pared estándar, con respecto a un modelo con función de pared modificada, y con los resultados experimentales. En la Figura 2.17 las gráficas de la izquierda corresponden a las ubicaciones bajo el eje de uno de los chorros de combustible, mientras que las de la derecha corresponden a espacios intermedios entre dos chorros. Con la función de pared estándar los flujos de calor obtenidos son muy bajos.

La mejora de la función de pared en el modelo de Kleeman et al. [2.51] permitió concluir que, además de no proveer resolución espacial, las correlaciones empíricas para la transferencia de calor tienden a subestimar substancialmente la transferencia de calor durante la fase de combustión (Figura 2.18). También, independientemente del tipo de función de pared, las simulaciones predicen bien el comportamiento de la presión en el cilindro.

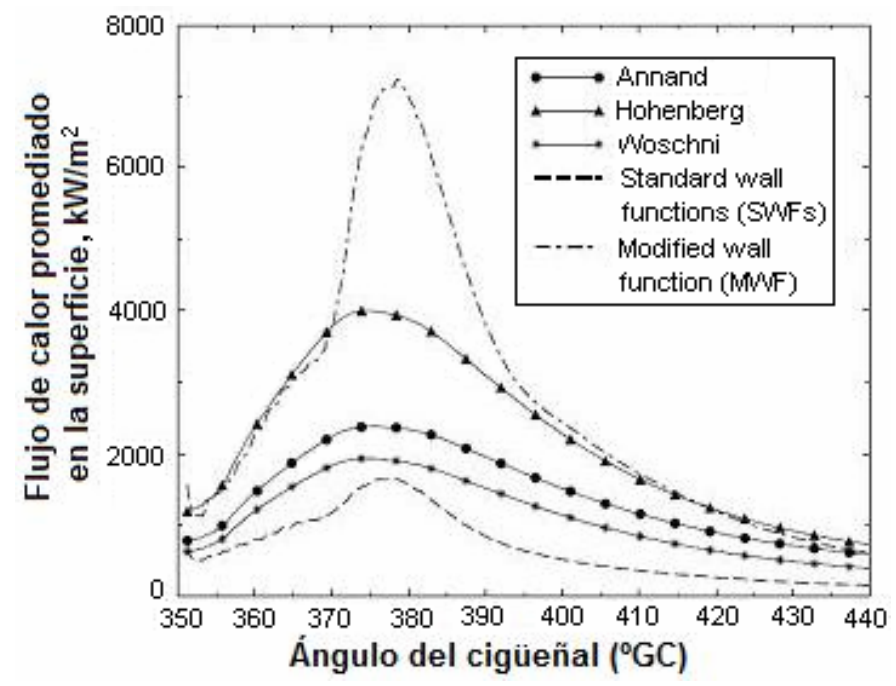

Figura 2.18. Comparación del promediado espacial de los flujos de calor predichos por los modelos CFD y por las relaciones empiricas [2.51].

Más recientemente Popovac et al. [2.50] desarrollaron una propuesta generalizada de las condiciones de contorno para el cálculo computacional de los flujos turbulentos y la transferencia de calor empleando RANS. El método conjuga la integración hasta la pared (ItW) con las funciones generalizadas de pared (GWF), que tienen en cuenta los efectos de compresibilidad y variación de las propiedades de los fluidos y también brindan buena 
estabilidad en las soluciones. Las condiciones de contorno pueden definirse sin tener que considerar si el punto de la malla más cercano a la pared se encuentra dentro de la subcapa viscosa, en la zona de transición o en la región totalmente turbulenta.

Como anota Hanjalic [2.110], a pesar de los grandes avances de las tres últimas décadas, todavía son varios los aspectos cuyo modelado aún no se acepta como satisfactorio entre la comunidad investigadora de la turbulencia: el seguimiento de las superficies curvas, los esfuerzos de deformación, la inestabilidad, la proximidad a la pared, la tridimensionalidad, la separación y el regreso, el "swirl" y la rotación son los ejemplos más destacables. El modelado de estos fenómenos presenta dificultad no sólo por el insuficiente entendimiento de su física, sino también por la necesidad aparente de recurrir a formulaciones matemáticas y esquemas numéricos de gran complejidad comparados con los actualmente utilizados. El trabajo de Hanjalic [2.110] presenta una revisión de algunos de los principales modelos de turbulencia para entornos cerrados disponibles en la literatura.

\subsubsection{Transferencia de calor radiativa}

La transferencia de calor radiativa en el motor proviene de dos fuentes: la radiación del gas y la radiación de las nubes de partículas. En los motores de combustión homogénea la cantidad de hollín producido es pequeña y por ello la radiación de gas es la que más se considera. En los motores Diesel la radiación del gas es una pequeña fracción del calor transferido, pero la radiación de las partículas es importante.

El modelado de la transferencia de calor por radiación está íntimamente relacionado con el modelado de la combustión. Para correlaciones de una sola zona la radiación puede incluirse en la fórmula convectiva, como en la fórmula de Woschni, o separada como en la fórmula de Annand. En las fórmulas en las que la convección y la radiación van separadas el problema es usar la emisividad y la temperatura radiativa adecuadas, porque un error de sólo 50 grados en la temperatura radiativa se traduce en un error del flujo de calor del 10\% (por estar la temperatura elevada a la cuarta potencia); Si como temperatura radiativa se toma la temperatura del gas, promediada por mol, el error es de casi $200 \%$ [2.1]. 
Morel [2.7] formuló un modelo de longitud de haz promedio simple para ser usado en un programa de análisis de ciclo. El modelo desprecia la radiación del gas, la radiación pared-pared y la dispersión. La radiación de material particulado es tomada como gris. El flujo hacia una superficie con emisividad $\varepsilon_{i}$ y temperatura $T_{i}$ es entonces:

$$
q_{i}^{\prime \prime}=\sigma_{i} \varepsilon_{i} \varepsilon_{s i}\left(T_{r}^{4}-T_{i}^{4}\right),
$$

donde $T_{r}$ es la temperatura de radiación del hollín, $\varepsilon_{s i}=1-\exp [-$ $\left.1575\left(f_{m} / \rho_{s}\right) \rho T_{r} L_{i}\right]$ (2.10) y $f_{m}$ es la fracción de la masa de hollín, $\rho_{s} / \rho$ es la relación entre las densidades del hollín y el gas, $L_{i}$ es el recorrido radiativo medio para la iésima superficie. El recorrido radiativo medio se toma de alguna manera arbitraria, relacionada estrictamente con la geometría de la cámara, por ejemplo, para una superficie del cilindro se puede tomar el diámetro de éste. La temperatura de radiación fue definida arbitrariamente en términos de la temperatura de la "zona quemada". La correlación para $f_{m}$ se obtuvo de los resultados empíricos de Sitkei [2.22]. Borman y Nishiwaki [2.1] estiman que es un modelo de trabajo razonable si se cuenta con información para ajustar las fórmulas de $T_{r} \mathrm{y} f_{m}$.

El modelo matemático que describe el campo de radiación en el cilindro del motor es la ecuación de transferencia radiativa (RTE) [2.98],[2.93]:

$$
(\Omega \cdot \nabla) I(r, \Omega)=-\left(a_{n e t}+\sigma_{s}\right) I(r, \Omega)+k I_{b}(r)+\frac{\sigma_{s}}{4 \pi} S(r, \Omega)
$$

donde $a_{\text {net }}$ representa la absortividad combinada del gas y el hollín, $\sigma_{s}$ es el coeficiente de dispersión y $k=a_{n e t}+\sigma_{s}$ es el coeficiente de extinción. $I(r, \Omega)$ es la intensidad de la radiación a una distancia $r$ en la dirección $\Omega$. $I_{b}(r)$ es la intensidad de radiación de un cuerpo negro a la distancia $\boldsymbol{r}$ y es independiente de la dirección. $S(r, \Omega)$ es un término fuente que tiene en cuenta el incremento de la intensidad debido a la dispersión y definido como:

$$
S(r, \Omega)=\int_{\Omega^{\prime}=4 \pi} I\left(r, \Omega^{\prime}\right) \Phi(\beta) d \Omega^{\prime}
$$

donde $\Phi(\beta)=\Phi\left(\Omega^{\prime} \rightarrow \Omega\right)$ es la función de fase de la transferencia de energía de la dirección incidente $\Omega^{\prime}$ a la dirección saliente $\Omega$. 
La intensidad radiativa espectral es función de la ubicación, la longitud de onda y el ángulo sólido, lo que hace que sea muy difícil de resolver en el entorno de la geometría del motor de combustión.

Para la solución de la ecuación de transferencia radiativa se emplean varias técnicas numéricas, entre las cuales se cuenta el método zonal de Hottel, métodos basados en la técnica de Monte Carlo, el método de flujos, el método de transferencia discreta de Lockwood y Shah y el método de ordenadas discretas [2.95], [2.96], [2.102]. El método de transferencia discreta combina las mejores características de los métodos zonales, Monte Carlo y el método de flujos. El método de transferencia discreta es particularmente útil en el modelado de volúmenes de geometría complicada, ya que las condiciones de contorno son fáciles de acomodar y es económico desde el punto de vista computacional. Dicho método emplea volúmenes de control para sus cálculos de flujo en diferencias finitas; el procedimiento incluye el trazado de rayos en el dominio de cálculo.

El método de Monte Carlo. Con la utilización de este método, como modelo probabilístico, se asume que la energía emitida por los gases (hollín mayormente), se compone de un número finito de "historias" de fotones (paquetes de energía) que pueden reproducirse mediante un generador de números aleatorios. Para cada paquete de energía se generan y utilizan números aleatorios que muestrean distribuciones probabilísticas apropiadas de los ángulos de dispersión y distancias de recorrido entre colisiones. Cada historia de fotón se empieza asignándole a éste un conjunto de parámetros, que incluyen su energía, posición y dirección iniciales. Luego de esto se determina, de manera estocástica, el número de recorridos libres medios por los que se propaga el fotón. Después se muestrea la información de la sección transversal (los coeficientes de absorción y dispersión) y se determina si el fotón que colisiona es absorbido o dispersado por las moléculas del gas o las partículas del medio. Si es absorbido la historia del fotón termina ahí. Si es dispersado, se muestrea la distribución de ángulos de dispersión y se asigna una nueva dirección al fotón. En caso de dispersión elástica se determina una nueva energía observando las leyes de conservación de energía y momento, repitiendo el procedimiento de colisiones sucesivas con el nuevo conjunto de energía, posición y dirección asignados hasta que el fotón es absorbido o escapa del sistema. Con una rutina de lanzamiento de cada uno de los paquetes de energía hasta que todos ellos son absorbidos, se obtiene el 
número de éstos que han sido absorbidos por cada uno de los elementos discretizados de la superficie. El flujo de calor radiativo $Q_{A j}$ para la superficie elemental $A_{j}$ de la cámara de combustión puede definirse como:

$$
\begin{aligned}
Q_{A j}= & \int_{A j} \int_{V} \frac{k_{a i}}{\pi r^{3}} \exp \left(-\int_{0}^{r} k_{a i} r d r\right) \sigma T_{g i}^{4} d V_{i} d A_{j}+ \\
& +\int_{A j V} \frac{\cos \eta_{i} \cos \eta_{j}}{\pi r^{3}} \exp \left(-\int_{0}^{r} k_{a i} r d r\right) \varepsilon_{s i} T_{s i}^{4} d A_{i} d A_{j}
\end{aligned}
$$

En la ecuación (2.11) el primer término es la transferencia de calor radiativa entre todos los volúmenes elementales $V$ y las superficies elementales $A_{j}$, en tanto el segundo término es la transferencia de calor radiativa entre todas las superficies elementales y la superficie elemental $A_{j} ; k_{a i}$ es el coeficiente de absorción de un volumen elemental; $\eta$ es el ángulo entre la normal a la superficie y la dirección de la radiación; $\varepsilon_{s i}$ es el coeficiente de absorción de la superficie elemental, $r$ es la distancia desde la fuente de radiación hasta la superficie; $\sigma$ es la constante de Stefan-Boltzman y $T$ es la temperatura del elemento; los subíndices $i$ e $j$ se refieren a las zonas, $g$ se refiere al volumen elemental y $s$ a la superficie elemental.

Si el volumen elemental $d V_{i}$ emite $n$ paquetes de energía, de los cuales $n_{i}$ son absorbidos por $d A_{j}$, entonces la fracción de paquetes de energía emitida por $d V$ y absorbida por $d A_{j}$, es $P_{g i j}=n_{i} / n$. De manera análoga, $P_{s i j}$ representa la fracción de paquetes de energía emitida por todas las superficies elementales absorbida por $d A_{j}$, con lo que la ecuación (2.11) puede reescribirse como:

$$
Q_{A j}=\sum_{i=1}^{M_{g}} P_{g i j} Q_{g i}+\sum_{i=1}^{M_{s}} P_{s i j} Q_{s i}
$$

donde $M_{g}$ y $M_{s}$ son, respectivamente, la cantidad de volúmenes y superficies elementales, mientras que $Q_{g i}$ y $Q_{s i}$ son, la energía emitida por el volumen y la superficie elemental.

Los pasos para evaluar el flujo de calor radiativo hacia las paredes de la cámara de combustión y el flujo de calor global son: 
- Creación de la geometría, para cada posición angular del cigüeñal durante la etapa de combustión difusiva, la cámara de combustión se parcela en $M_{g}$ volúmenes y $M_{s}$ superficies elementales.

- Se determina el coeficiente de extinción, la temperatura de radiación, los coeficientes de emisión y absorción de los volúmenes y superficies elementales, a partir del modelo de radiación que se tenga.

- La energía radiativa emitida por cada elemento se parte en $\mathrm{N}$ paquetes de energía y se aplica el método de Monte-Carlo para simular la dirección y la trayectoria (incluyendo la parte que se refleja) de cada uno de estos paquetes y determinar la posición en la que es absorbido.

- Se suma el número de paquetes de energía absorbidos por cada elemento de la superficie. A partir de la ecuación (2.12) puede determinarse la distribución del flujo de calor radiativo entre las superficies y el flujo radiativo global.

Yan et al. [2.100] emplearon el método de Monte-Carlo para calcular la transferencia de calor radiativa; repartieron el volumen total del cilindro en 8 volúmenes elementales y la superficie de la cámara de combustión la discretizaron en 7 superficies independientes (una correspondiente a la superficie de la culata, otra correspondiente al cilindro y las cuatro restantes a la superficie del pistón). La Figura 2.19 ilustra esta partición.

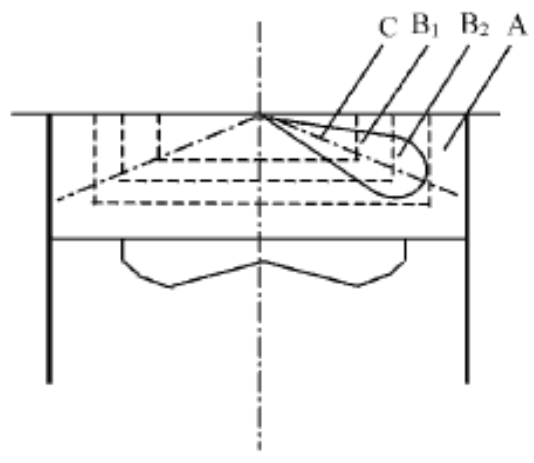

Figura 2.19. Partición de las zonas de combustión.

La Figura 2.20 muestra los flujos de calor radiativos en la parte superior del pistón y en centro de la culata en un motor Diesel de inyección directa, junto con los valores promedio en todas las superficies de la cámara de combustión predichos con el método multidimensional de Chen et al. [2.59], para cuya parte radiativa se utilizó el método de Monte Carlo. 
El modelo desarrollado por Chen et al. [2.59] incluye submodelos de la ley de liberación de calor, descripción geométrica, temperatura de radiación, formación y oxidación del hollín coeficiente de absorción y el método de Monte-Carlo para todas las áreas de intercambio de calor radiativo. En este código el cilindro fue dividido en 10 zonas de superficie y 4 zonas de gas.

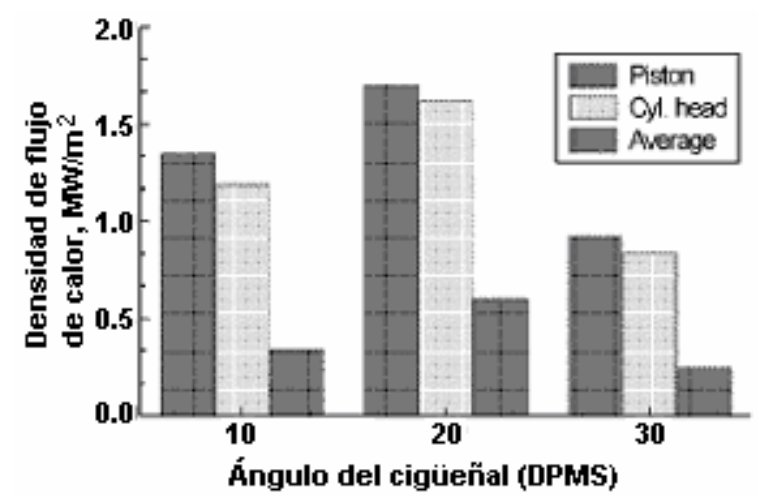

Figura 2.20. Flujos radiativos pico en la parte superior del pistón y en la culata y valores medios de la transferencia radiativa considerando todas las superficies de la cámara [2.59].

Modelo fenomenológico de la transferencia de calor. Merker y Eiglmeier [2.27] presentan un modelo fenomenológico multizonal para la transferencia de calor en motores Diesel, en el cual se tiene en cuenta el efecto radiativo del hollín aparte del efecto convectivo, y con resolución espacial. La ecuación de transporte radiativo entre la nube de hollín y la capa de hollín depositada sobre la superficie fue resuelta mediante el método de Hottel and Sarofim.

El trabajo de Merker y Eiglmeier sirvió de base para una implementación en CFD. El esquema de trabajo del modelo mencionado corresponde al de la Figura 2.21, en la cual se esquematiza la interacción entre los diferentes modos de transferencia de calor en el motor y las variables involucradas. Los autores subdividieron la cámara de combustión en seis sub-superficies isotérmicas: las partes de la culata sobre la corona y el bowl del pistón, la corona del pistón, la parte cilíndrica y el fondo del bowl del pistón y el cilindro. Esta subdivisión da lugar aproximadamente a superficies isotérmicas. La deposición del hollín, la conducción transitoria del calor de la pared, la radiación de temperatura y la transferencia de calor convectiva se calculan de manera separada para cada una de estas sub-superficies. Las paredes del motor reciben calor por convección y por radiación de los gases 
resultado de la combustión y del hollín formado. En el modelo de Eiglemeier se observa la variación del componente convectivo con el movimiento turbulento del fluido, mientras que el componente radiativo depende de las temperaturas del gas y de la temperatura de llama adiabática. La mayor influencia sobre el componente radiativo la tiene la radiación del hollín. Las temperaturas de las superficies de los componentes dependerán de las propiedades físicas de los materiales de los que estos están hechos y de las condiciones de contorno (refrigerante y aceite). Los flujos de calor experimentales, obtenidos con medidores de temperatura instantánea en varios puntos de la cámara de combustión, y los modelados se reproducen en la Figura 2.22.

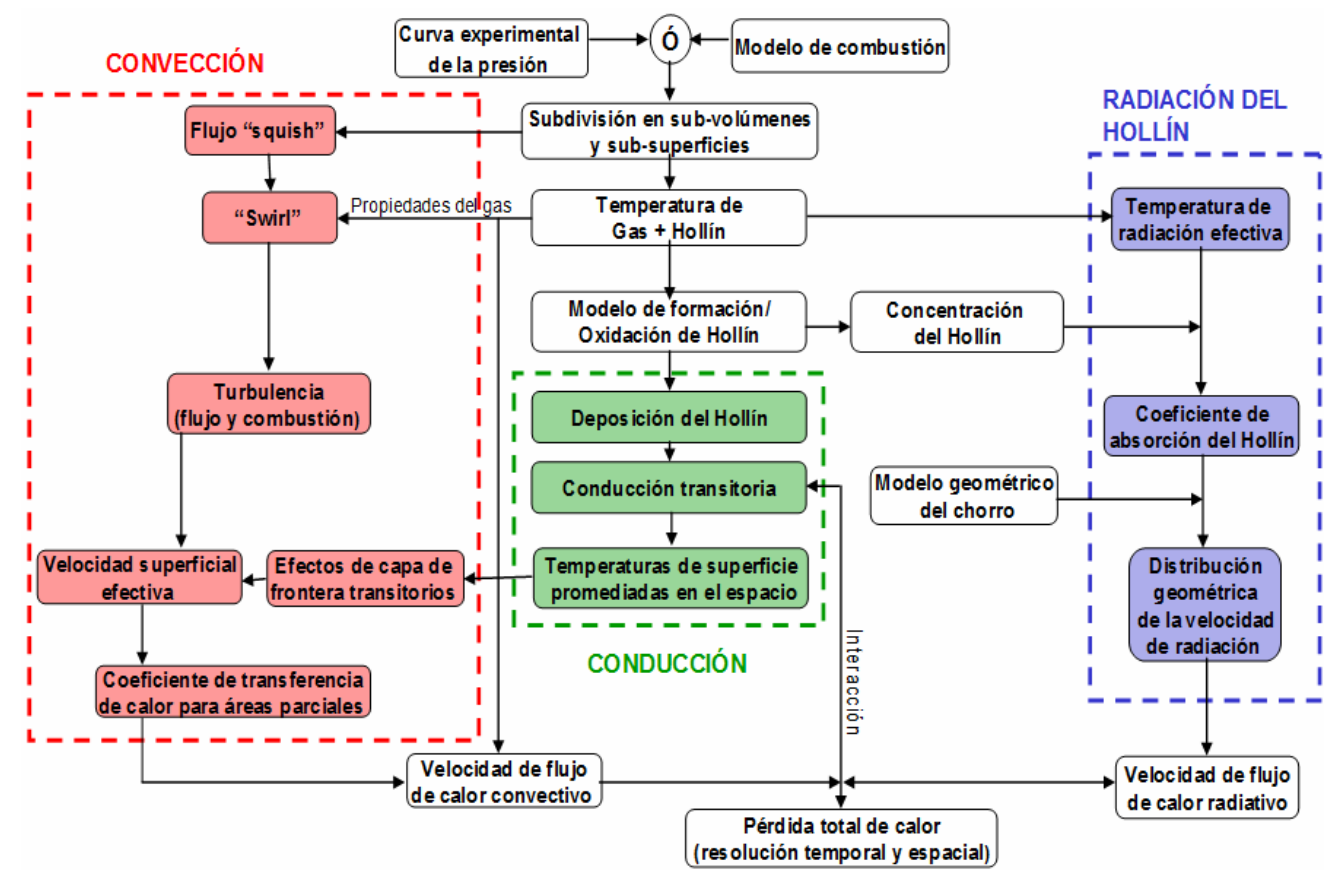

Figura 2.21. Esquema para un modelo fenomenológico detallado de la transferencia de calor en los motores Diesel [2.27].

Por lo que se aprecia en las figuras del trabajo reportado, el modelo describe bien los mecanismos que causan la transferencia de calor en los puntos medidos, con una ligera desviación del flujo predicho en la camisa del 
cilindro durante la carrera de expansión. Los autores atribuyen esta desviación a factores de medición, dado que los termopares se ubicaron muy arriba en la camisa para no ser estropeados por el anillo, con lo que, probablemente, los termopares no son tan sensibles a los cambios de flujo de calor producidos a lo largo de la superficie de la camisa a medida que desciende el pistón, durante la carrera de expansión.
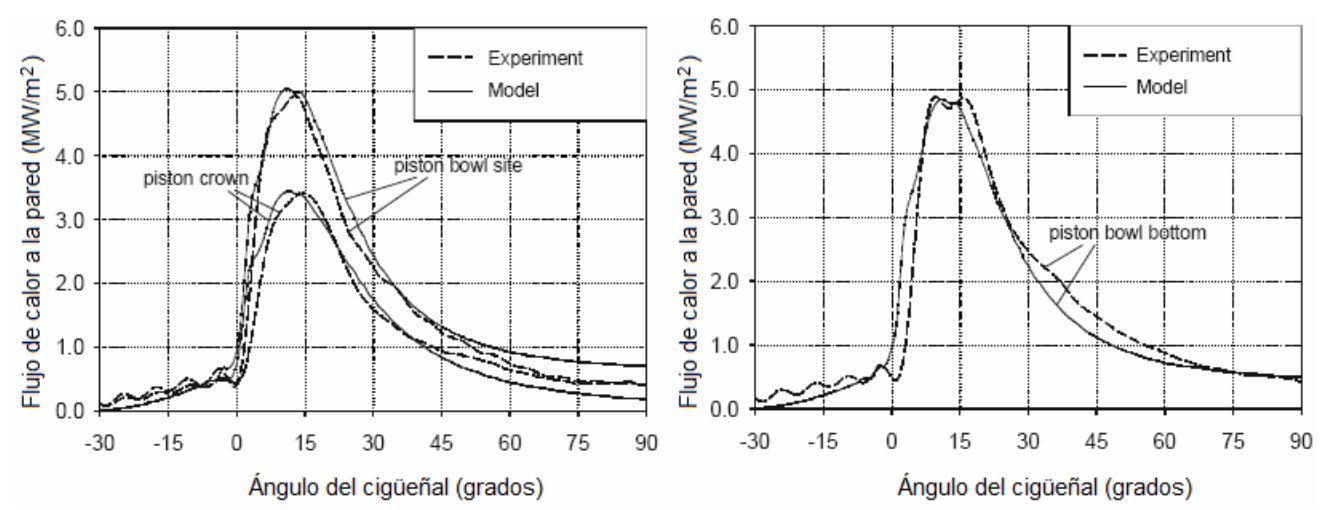

Figura 2.22. Resultados obtenidos por Eiglemeier con su modelo fenomenológico [2.27].

El método de ordenadas discretas (DOM). Wiedenhoefer y Reitz [2.48] idearon un modelo de radiación basado en este método integrado en la versión ERC ${ }^{\circledR}$ del programa de CFD KIVA-3V ${ }^{\circledR}$ [2.88] para estudiar los efectos de la radiación en las emisiones y en las temperaturas de los componentes en un motor Diesel Cummins N14. El método DOM resuelve la ecuación de transferencia radiativa (RTE) en una serie de direcciones $u$ ordenadas, cada una de ellas ponderada por el ángulo sólido subtendido por esa ordenada.

En su trabajo Wiedenhoefer y Reitz [2.48] no consideraron la dispersión por considerar que el coeficiente de absorción era mucho mayor que ésta. La condición de contorno para la ecuación RTE, viene dada por:

$$
I_{w}(r, \Omega)=\varepsilon I_{b}(r)+\frac{\rho}{\pi} \int_{n \cdot \Omega^{\prime}<0}\left|n \cdot \Omega^{\prime}\right| I\left(n \cdot \Omega^{\prime}\right) d \Omega^{\prime}
$$


donde $I_{w}$ es la intensidad de la radiación en la pared y $\varepsilon$ e $I_{b}(r)$ son la emisividad y la intensidad de cuerpo negro de la pared, respectivamente. $\rho=1-\varepsilon$ es la reflectividad de la superficie. La intensidad del cuerpo negro viene dada por $I_{b}(r)=\sigma T^{4} / \pi . \sigma=5,67051 \times 10^{-8} \mathrm{~W}\left(\mathrm{~m}^{2} \cdot \mathrm{K}^{4}\right)$ es la constante de Stefan-Boltzmann. $I(r, \Omega)$ es la intensidad de la radiación a una distancia $\boldsymbol{r}$ en la dirección $\Omega$. $\Omega^{\prime}$ es el ángulo que define la dirección de la radiación incidente.

Wiedenhoefer y Reitz validaron su modelo radiativo comparando sus resultados con una solución analítica y con datos de literatura publicada. También se compararon resultados con la información medida con un sensor instalado en un punto de la culata del motor. La información de la distribución de las temperaturas en la superficie necesaria para correr el programa KIVA ${ }^{\circledR}$ se obtuvo con un código numérico de elementos finitos. Otros submodelos empleados fueron: un submodelo exponencial de banda ancha para el coeficiente de absorción, dependiente de la presión, la temperatura y la concentración de especies; un submodelo de absorción de hollín, basado en un modelo empírico para la emisividad del hollín y dependiente de la concentración y la temperatura; un submodelo de deposición por termoforesis del hollín para la radiación en la zona cercana a la pared y dependiente a su vez de la temperatura del gas en esta zona y del gradiente de temperatura normal a la pared.

Entre las conclusiones a las cuales Wiedenhoefer y Reitz llegaron se cuentan: la deposición de hollín por termoforesis es responsable de cerca del 1-3\% del total del hollín en el escape; la combinación de la temperatura no uniforme de la superficie junto con los efectos de radiación tomados en consideración tiene un impacto profundo en los niveles predichos de óxidos de nitrógeno; los niveles de hollín expulsados se incrementan hasta en un $13 \%$ en comparación con la situación cuando no se consideraba la radiación y se consideraba una distribución de temperatura uniforme, los niveles predichos de NOx descienden hasta un 46\%; un incremento del $30 \%$ en pérdidas de calor cuando se tiene en cuenta la radiación junto con una distribución no uniforme de temperatura. En las condiciones base, las pérdidas de calor por convección a través de la cámara de combustión representaron aproximadamente el $70 \%$ por un $30 \%$ para las pérdidas por radiación. Para los casos analizados, aproximadamente el $32.7 \%$ del total de las pérdidas de 
calor se produjo a través de la culata, mientras que el $19.1 \%$ fue a través de la camisa y el $48.2 \%$ restante por las superficies del pistón. La radiación aumentó la magnitud total de las pérdidas de calor en un $40 \%$ en comparación con el caso en que se despreció la radiación. La temperatura de los componentes metálicos aumentó cuando se aplicó la radiación. En la tabla 2.3 se reproduce el resumen de las pérdidas de calor por el pistón, la camisa y la culata para diferentes relaciones de swirl publicados por Wiedenhoefer y Reitz. En la Figura 2.23 se reproducen las gráficas del flujo de calor radiativo y del hollín y se aprecia la manera como el flujo de calor sigue la forma del hollín. Los efectos del swirl en la radiación predichos con el modelo se ilustran en la Figura 2.24. Los resultados de Wiedenhoefer y Reitz se han presentado aquí por ser muy ilustrativos de la influencia del swirl en la transferencia de calor radiativa y convectiva.

Tabla 2.3. Pérdidas de calor para diferentes relaciones de swirl, (J) [2.48].

\begin{tabular}{|c|c|c|c|c|c|c|}
\hline & \multicolumn{3}{|c|}{ Relación de swirl } & \multicolumn{2}{|c|}{ \% de variación } \\
\hline & & 0,0 & 1,4 & 2,2 & 0,0 a 1,4 & 1,4 a 2,2 \\
\hline \multirow{3}{*}{ 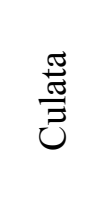 } & Convectiva & 22,722 & 24,796 & 26,227 & $-3,60$ & 5,77 \\
\hline & Radiativa & 7,221 & 7,147 & 10,119 & $-1,02$ & 41,58 \\
\hline & Total & 32,942 & 31,943 & 36,346 & $-3,03$ & 13,78 \\
\hline \multirow{3}{*}{ 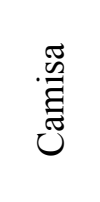 } & Convectiva & 21,970 & 10,660 & 8,443 & $-51,48$ & $-20,80$ \\
\hline & Radiativa & 13,570 & 9,714 & 7,532 & $-28,42$ & $-22,46$ \\
\hline & Total & 35,540 & 20,374 & 15,975 & $-42,67$ & $-21,59$ \\
\hline \multirow{3}{*}{ 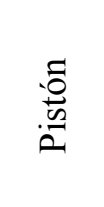 } & Convectiva & 38,751 & 34,477 & 29,685 & $-11,35$ & $-13,90$ \\
\hline & Radiativa & 11,89 & 10,490 & 6,543 & $-11,77$ & $-37,626$ \\
\hline & Total & 50,650 & 44,967 & 36,229 & $-11,22$ & $-19,43$ \\
\hline \multirow{3}{*}{ 葛 } & Convectiva & 86,443 & 69,933 & 64,356 & $-19,10$ & $-7,97$ \\
\hline & Radiativa & 32,689 & 27,351 & 24,194 & $-16,33$ & $-11,54$ \\
\hline & Total & 119,132 & 97,284 & 88,550 & $-18,34$ & $-8,98$ \\
\hline
\end{tabular}


La influencia de tener en cuenta la transferencia de calor en el modelo se ilustra en la gráfica de la Figura 2.25.

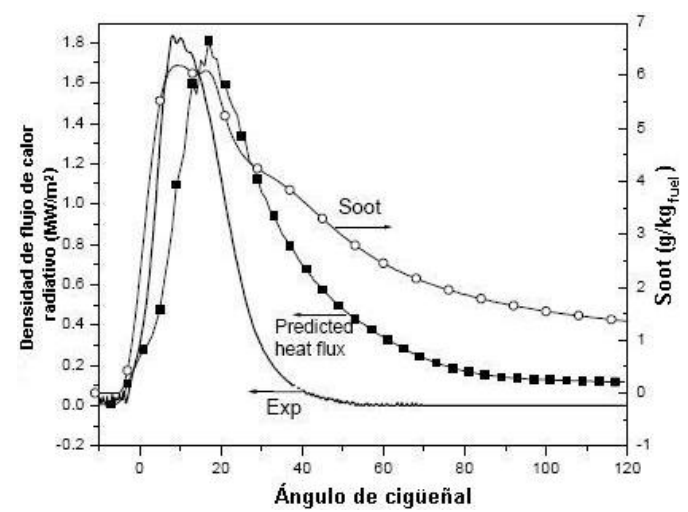

Figura 2.23. Comparación del flujo de calor radiativo en el sensor, en el motor probado a plena carga y $1500 \mathrm{rpm}$ [2.48].

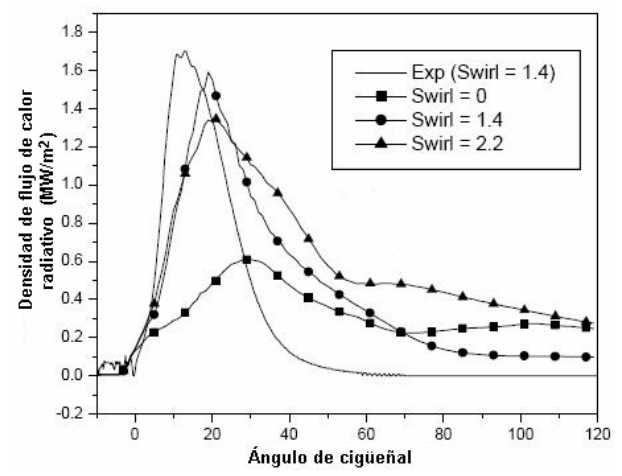

Figura 2.24. Flujo de calor radiativo predicho en la ubicación del sensor para diferentes relaciones de swirl [2.48].

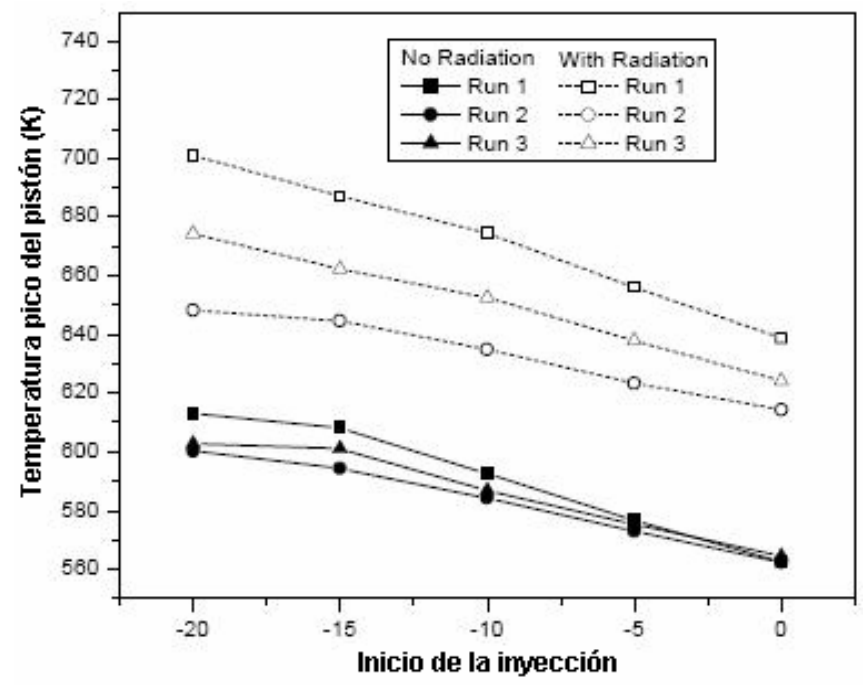

Figura 2.25.

Temperatura pico del pistón en función de la fase de dispersión $\Phi(\beta)$ [2.48].

Abraham y Magi [2.63] reportaron relaciones de pérdidas por radiación sobre pérdidas por convección de $12 \%$ a relaciones de equivalencia de 0,3 y 0,4 y 
velocidades del motor de 1500 y $1900 \mathrm{rpm}$.. A relaciones de equivalencia de 0,5 , la relación fue cercana a $15,5 \%$.

\subsection{Transferencia de calor en las pipas de admisión y escape}

La disponibilidad de herramientas para valorar la transferencia de calor en el sistema de escape es importante para definir las condiciones de operación del turbogrupo y de los dispositivos de tratamiento de emisiones. La transferencia de calor en la pipa de escape es mucho mayor que en la pipa de admisión debido a las mayores temperaturas y velocidades del gas; también debido a la elevada velocidad de transferencia de calor, la temperatura de los gases de escape desciende rápidamente a medida que viajan por el sistema de escape.

Al igual que sucede con el cilindro, son muchos los trabajos que tratan los procesos de transmisión de calor entre los gases de admisión y escape y sus pipas (y sistemas en general). Recientemente Dolz [2.104] realizó una revisión extensa de los modelos de transferencia de calor en los sistemas de escape, en la cual comparó diferentes correlaciones de coeficientes de transmisión de calor para el interior de tubos, tanto las de carácter general, desarrolladas por Dittus-Boelter, Colburn, Sieder-Tate, Petukhov, Gnielinski y Hubber, como las correlaciones más específicas fundamentales desarrolladas para MCIA.

La transferencia de calor desde la pipa y el colector de admisión influyen más sobre el funcionamiento del motor que la transferencia asociada a sus válvulas, principalmente porque sus áreas de superficie son mayores. Zapf [2.60] obtuvo, para pruebas de flujo estacionarias, correlaciones de transferencia de calor para la pipa de admisión, $N u=0,214 R e^{0,68}(1-$ $\left.0,765 \delta / D_{v}\right)$ y para la pipa de escape, $N u=2,58 R e^{0,5}\left(1-0,797 \delta / D_{v}\right)$, tomando como longitud de referencia para los números de Nusselt y Reynolds el diámetro hidráulico de las pipas, $\delta$ es el levantamiento de la válvula y $D_{v}$ es el diámetro del asiento de la válvula. Bauer [2.61] realizó experimentos en flujos estacionarios y pulsantes de aire a números de Reynolds medios de 3000-35,000 en ductos seleccionados para sintonizar colectores de admisión en motores de encendido provocado comerciales. 
Para el cálculo de la transferencia de calor entre las pipas de escape y de admisión, Bohac [2.3] empleó una correlación para flujo totalmente turbulento a través de tubos circulares rectos con modificación para la curvatura y los efectos de entrada y salida, la correlación de Sleicher y Rouse:

$$
N u_{D}=5+0,015 \operatorname{Re}_{D, f}^{a} \operatorname{Pr}_{w}^{b}
$$

donde $a$ y $b$ son funciones del número de Prandtl (con valores típicos de $a=$ $0,83$ y $b=0,66) ; \operatorname{Re}_{D, f}$ es el número de Reynolds basado en el diámetro modificado de la pipa (considerando su curvatura y geometría de entrada). La transferencia de calor hacia la válvula se modela como un cilindro expuesto a flujo cruzado.

Debido a que no se ha dispuesto de una expresión clásica de transferencia de calor transitoria para calcular el transporte de energía en los sistemas de admisión y escape, se han usado mucho relaciones estacionarias de la forma $N u=a \operatorname{Re}^{b}$ o $N u=a \operatorname{Re}^{b} \operatorname{Pr}^{n}$ para calcular el coeficiente de película en los colectores de admisión y escape de los motores [2.64]-[2.69]. Algunos ejemplos de estas correlaciones son las siguientes:

Dittus y Boelter:

$$
N u=0,023 \operatorname{Re}^{0,8} \operatorname{Pr}^{n}
$$

Bauer et al.:

$$
\begin{aligned}
& N u=0,062 \overline{\operatorname{Re}}^{0,73} \text { para tubos de admisión rectos [2.62] } \\
& N u=0,140 \overline{\operatorname{Re}}^{0,66} \text { para tubos de admisión curvos [2.62] }
\end{aligned}
$$

Shayler et al.:

Meisner y Sorenson:

$$
N u=0,135 \overline{\mathrm{Re}}^{0,713}[2.64]
$$

$$
N u=0,0774 \overline{\operatorname{Re}}^{0,769} \text { para tubos de escape [2.65] }
$$

Wendland:

$$
N u=0,027 \overline{\operatorname{Re}}^{0,8} \operatorname{Pr}^{0,33}\left(\frac{\mu}{\mu_{s}}\right)^{0,14} C_{\text {entr }} \text { para tubos de escape [2.66] }
$$


En esta última, el termino $C_{\text {entr }}$ tiene en cuenta los efectos de separación del fluido de las paredes al entrar al puerto. $\mu$ y $\mu_{s}$ corresponden a la viscosidad evaluada a las temperatura media del flujo y de la superficie, respectivamente.

El número de Nusselt en el cálculo del coeficiente de película en las pipas de admisión y escape emplea velocidades instantáneas en lugar de velocidades medias, para tener en cuenta las características pulsantes del fluido, particularmente en el estudio del escape. Huber [2.107], basándose en medidas de velocidad y temperatura realizadas en conductos de escape, observó desfases temporales entre la señal de velocidad y temperatura, por lo que propuso correlacionar el número de Nusselt con una velocidad promedio mediante la ecuación $N u=1,6 \mathrm{Re}^{0,4}$, en la que se pondera la historia de velocidades a su paso por el ducto. Reyes [2.105] modificó la expresión de Huber para dar cuenta la dependencia que tiene el coeficiente de película en el colector de escape no sólo de la velocidad instantánea del fluido en el instante considerado, sino también de la historia de esa velocidad en instantes anteriores. En el modelo de Reyes, la velocidad característica se calcula como la suma de la velocidad presente y las velocidades previas multiplicadas por sus respectivos coeficientes de disipación:

$$
\bar{V}(t)=\frac{\sum_{k=0}^{\infty} c^{k} V(t-k \Delta t)}{\sum_{k=0}^{\infty} c^{k}}
$$

La velocidad promedio se calcula con la velocidad instantánea $V(t)$ y la velocidad promedio anterior $\bar{V}(t-\Delta t)$ por la formula:

$$
\bar{V}(t)=c \bar{V}(t-\Delta t)+(1-c) V(t)
$$

Reyes ajustó la constante de disipación $c$ de manera que sus ensayos se aproximaran a los del modelo de Huber. La velocidad instantánea fue obtenida a partir de los análisis de un programa de combustión.

Tras analizar varios métodos de cálculo de temperaturas de pared para ser utilizados en un modelo de acción de ondas unidimensional, Dolz [2.104] argumentó la utilización de un modelo que combina el coeficiente de película 
de Caton [2.106], durante el período de válvula de escape cerrada, y el de Reyes [2.105], durante el período de válvula abierta.

Bauer, Balun y Heywood [2.62] realizaron mediciones instantáneas de temperaturas y flujos de calor en la pipa de admisión de un motor de encendido provocado trabajando con combustibles gaseosos y líquidos. En la pared de la pipa los flujos de calor tuvieron un pico de cerca de $50 \mathrm{~kW} / \mathrm{m}^{2}$, y en la parte posterior de la válvula los valores fueron del orden de $200 \mathrm{~kW} / \mathrm{m}^{2}$, valores que sirven de base para determinar la influencia de la transferencia de calor en el rendimiento del motor y para fijar magnitudes de cálculo de las tensiones térmicas en estos elementos.

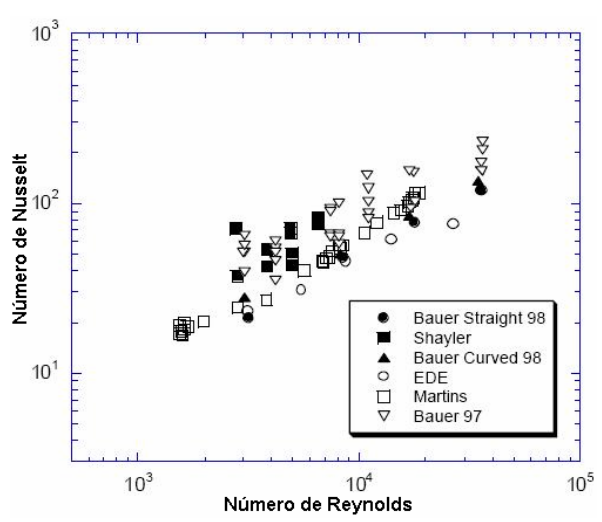

Figura 2.26. Información experimental en la pipa de admisión de un motor de combustión interna detallando la relación entre los números de Nusselt y Reynolds. La información usada correspondió a condiciones de estado estable [2.67].

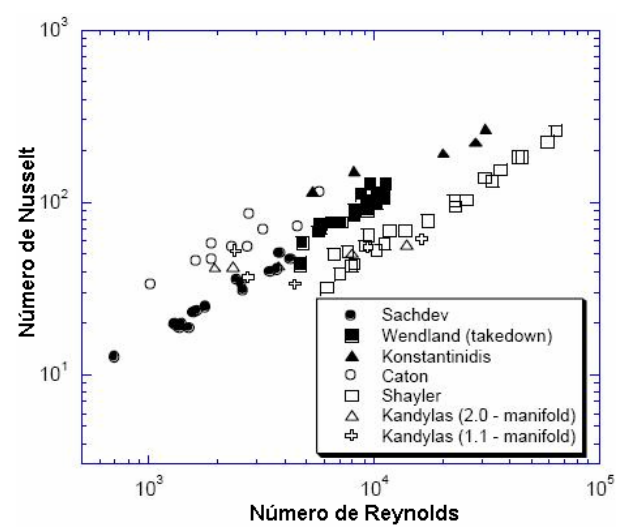

Figura 2.27. Información experimental en la pipa de escape de un motor de combustión interna detallando la relación entre los números de Nusselt y Reynolds. La información usada correspondió a condiciones de estado estable [2.67].

Depcik y Assanis [2.67] graficaron la información experimental de la relación entre el número de Nusselt (transferencia de calor) y el número de Reynolds (cantidad de movimiento) en los puertos de admisión y escape, disponibles en la literatura. Estos gráficos se han reproducido en la Figura 2.26 para las 
pipas de admisión y, en la Figura 2.27 para las pipas de escape. Basándose en los resultados de esta revisión $\mathrm{y}$ en los fundamentos físicos de las microescalas de turbulencia, propusieron una relación universal cuasiestacionaria que puede usarse para determinar la transferencia de calor en las pipas de admisión y escape de los motores de combustión interna:

$$
N u=0,070 \overline{\operatorname{Re}}^{3 / 4} \quad \text { [2.67] }
$$

Transferencia de calor transitoria en los sistemas de admisión y escape. Recientemente Zeng y Assanis [2.68] obtuvieron, basándose en la expresión de Ditus y Boelter, una correlación de dos etapas para la transferencia de calor transitoria en la que el coeficiente de película no es sólo una función de los números de Reynolds y Prandtl, sino que incorpora también la rapidez de cambio de la velocidad, mediante la velocidad caracterísitica, $U$ (definida como el valor RMS de la velocidad instantánea):

$$
\begin{aligned}
& N u=0,023 \operatorname{Re}^{0,8} \operatorname{Pr}^{0,4}\left(1-0,75 \frac{D}{U^{2}} \frac{d U}{d t}\right)^{0,8} \text { para la primera etapa, } \\
& N u=\underbrace{0,018 \operatorname{Re}^{0,8} \operatorname{Pr}^{0,4}}_{\begin{array}{c}
\text { solución } \\
\text { estable }
\end{array}} \underbrace{\left(1-0,75 \frac{D}{U^{2}} \frac{d U}{d t}\right)^{0,8}}_{\begin{array}{c}
\text { corrección } \\
\text { no_estable }
\end{array}} \text { para la segunda etapa, }
\end{aligned}
$$

En el límite de la primera etapa, la corrección de estado no estable, se define el criterio para el establecimiento del proceso de transferencia de calor turbulento decreciente. La transición, según los resultados experimentales de Zeng y Assanis se da según el parámetro denotado por ellos como $\Psi_{\text {criitico }}$ :

$$
\Psi_{\text {criitico }}=\left(\frac{D}{U^{2}} \frac{d U}{d t}\right)_{\text {critical }}=-0,7 \ldots-0,8
$$

donde $D$ es el diámetro del tubo de admisión.

Los modelos de transferencia de calor no estacionarios fueron aplicados a los datos experimentales tomados por Bauer et al. [2.62]. En la Figura 2.28 se han copiado las gráficas que comparan la información experimental con las 
predicciones basadas en los modelos de transferencia para los estados transitorio y estacionario. La Figura 2.28, $a$ se relaciona con el proceso de admisión dentro de los tiempos de apertura y cierre de las válvulas de admisión; la figura cubre la transferencia de calor para todo el ciclo de trabajo del motor.

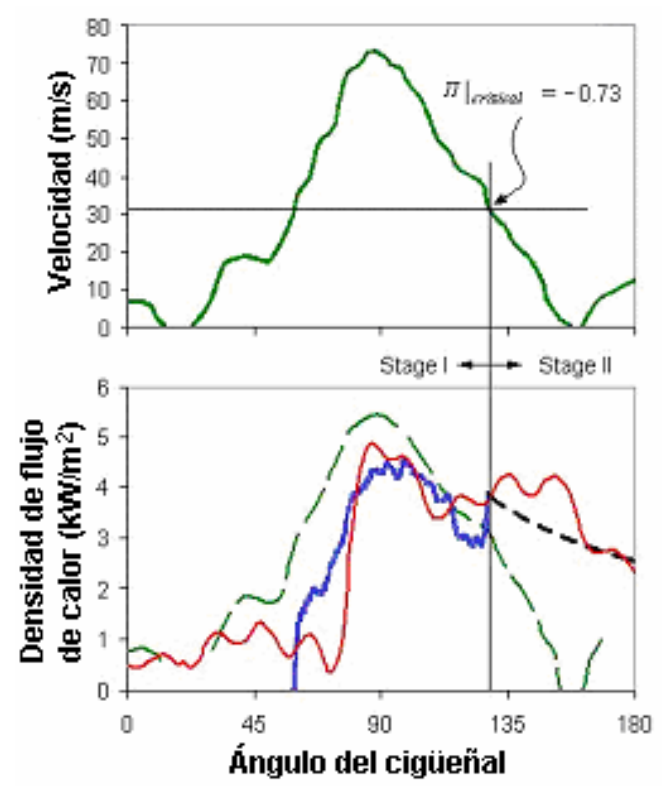

a) Proceso de admisión ( 0 a 180 grados)

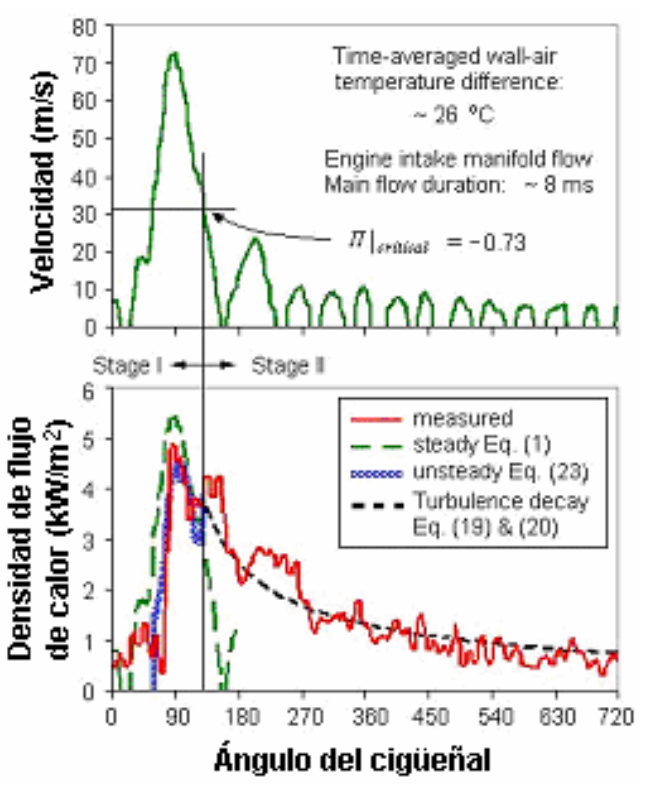

b) Ciclo de trabajo completo.

Figura 2.28. Velocidad del aire y mediciones de flujo de calor de Bauer y otros, comparados con las predicciones del modelo de Zeng y Assanis [2.68].

\subsection{Transferencia de calor del lado del refrigerante}

En los motores con sistemas de refrigeración convencional el refrigerante se hace llegar a las camisas de los cilindros y después el refrigerante fluye a través de perforaciones en la parte superior del bloque hacia la culata (los orificios de transferencia en la culata son calibrados y tienen diferentes diámetros según el control y la distribución del caudal que fluye hacia la culata). Dependiendo de la topología en la culata los flujos de refrigerante siguen hacia los entornos de las válvulas y del inyector, a la salida de los cuales se dirigen hacia la parte superior de la culata. De manera que, en lo 
que respecta a la camisa de enfriamiento, el motor se comporta como un intercambiador de calor con flujos complicados que transportan calor, gases de combustión en la parte interna (con direcciones de movimiento complejas) y fluido refrigerante en la parte externa del entorno de combustión. La Figura 2.29 ilustra algunos esquemas de circulación del refrigerante en motores en línea.

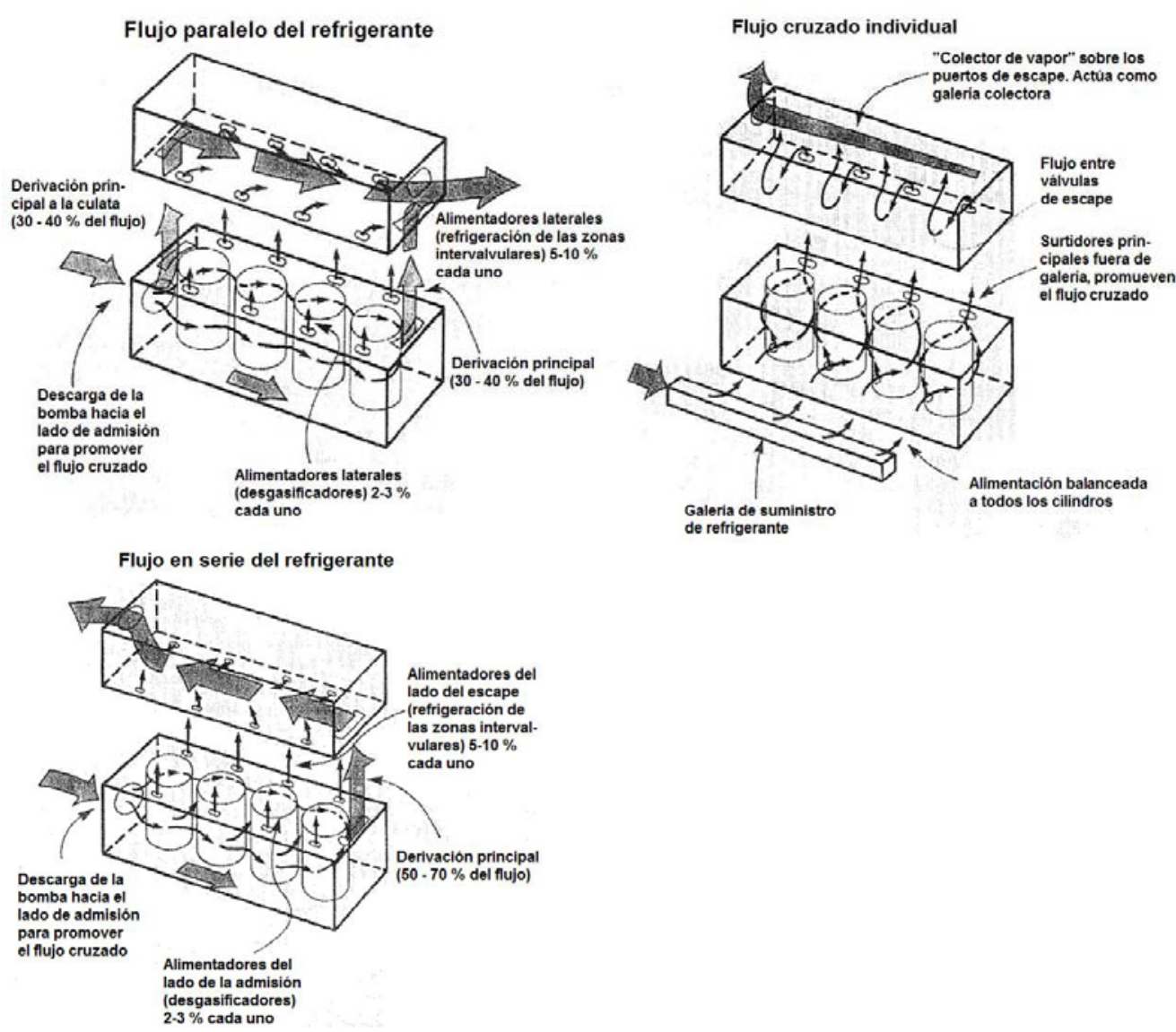

Figura 2.29. Formas de organización del flujo de refrigerante por la camisa de enfriamiento del motor.

En los motores de combustión la transferencia de calor está muy influenciada por el campo del fluido en los pasajes de refrigeración, el cual es turbulento bajo todas las condiciones de operación. En estos motores la transferencia de 
calor en la camisa depende de las propiedades termofísicas del refrigerante, del patrón del flujo y de la presencia de ebullición y cavitación.

Los modelos para predecir la transferencia de calor hasta hace poco eran sólo empíricos, pero con las investigaciones de optimización de la refrigeración y la búsqueda de control de las zonas térmicamente peligrosas del motor, los modelos han ido teniendo un tratamiento más analítico, aunque siempre con muchas correcciones experimentales. También, los métodos analíticos y los algoritmos usados desarrollados para flujos turbulentos han posibilitado el modelado CFD.

\subsubsection{Modelos empíricos}

La mayoría de los modelos para las camisas de enfriamiento de los motores son exclusivamente empíricos debido a las dificultades teóricas causadas por el flujo turbulento y también a dificultades experimentales de visualización e instrumentación. Los recientes trabajos de Norris [2.69] y Robinson [2.70] han permitido un modelado más ajustado de la transferencia de calor entre el refrigerante y las paredes. Trabajos como el de Bohac et al. [2.3], hacen uso de una expresión $\mathrm{Nu}$-Re-Pr para calcular la transferencia de calor entre las superficies mojadas del motor y el refrigerante. Así, Bohac modela la transferencia de calor entre refrigerante y las camisas en el bloque y la culata empleando la correlación de Grimson-Nusselt, para un flujo que atraviesa un banco de cilindros, modificada para ser usada con fluidos diferentes al aire:

$$
N u_{D}=1,13 C_{1} C_{2} \operatorname{Re}_{D}^{m} \operatorname{Pr}^{1 / 3}
$$

donde $C_{1}$ y $m$ dependen de la distribución de los cilindros y $C_{2}$ depende del número de cilindros. En caso de presentarse temperaturas del metal superiores a la temperatura de saturación del refrigerante, el modelo de Bohac hace uso de la correlación de Chen [2.71] para ajustar el valor del coeficiente de transferencia de calor por ebullición nucleada:

$$
\begin{gathered}
h_{\text {nuc,boiling }}=0,00122 \frac{k_{f}^{0,79} c_{p, f}^{0,45} \rho_{f}^{0,49}}{\sigma^{0,5} \mu_{f}^{0,29}\left(\Delta h_{f g} \rho_{g}\right)^{0,24}} \cdot F \cdot S \\
F=\left(T_{\text {wall }}-T_{\text {sat }}\right)^{0,24}\left(P_{\text {sat,wall }}-P_{\text {cool }}\right)^{0,75}
\end{gathered}
$$




$$
S=3,4-0,6 \log (\mathrm{Re})
$$

La primera parte de la correlación contiene las propiedades relevantes del fluido: $k_{f}$ (conductividad), $c_{p, f}$ (calor específico), $\rho_{f}$ (densidad), $\sigma$ (tensión superficieal), $\mu$ (viscosidad) y $\Delta h_{f g}$ (calor de vaporización). $P_{\text {sat,wall }}$ y $P_{\text {cool }}$ son la presión de saturación a la temperatura de pared y del fluido, respectivamente. $\mathrm{S}$ es un factor de supresión de ebullición nucleada que interpreta que en los flujos más turbulentos la formación de burbujas es contrarrestada por el mezclado con el fluido más frío. La ebullición nucleada en el circuito de refrigeración sólo tiene lugar en algunas zonas térmicamente muy cargadas como, la parte superior de la camisa y cerca de las válvulas de escape en a elevadas cargas del motor. Diferentes autores han empleado la expresión de Dittus Boelter para el cálculo de la transferencia de calor en la camisa de refrigeración de los motores [2.37]:

$$
N u=0,023 \operatorname{Re}_{D}^{0,8} \operatorname{Pr}^{0,4}
$$

válida para temperaturas de pared $\left(T_{\text {wall }}\right)$ superiores a la temperatura media del refrigerante $\left(T_{\text {bulk }}\right), T_{\text {bulk }}<T_{\text {wall }}$. El rango de validez de esta expresión se fija por el número de Reynolds, $R e>10000$ (flujo totalmente turbulento), 0,7 $<\operatorname{Pr}<160$, tuberías lisas, $x / D_{\mathrm{h}}>60$ (flujo totalmente desarrollado) y $T_{\text {bulk }}-T_{\text {wall }}<5,6^{\circ} \mathrm{C}$. Todas las propiedades del fluido se evalúan a su temperatura media. Cuando la diferencia entre las temperaturas de la pared y el refrigerante es mayor de $6{ }^{\circ} \mathrm{C}$ se introduce la corrección propuesta por Sieder y Tate:

$$
N u=0,027 \operatorname{Re}_{D}^{0,8} \operatorname{Pr}^{1 / 3}\left(\frac{\mu_{\text {bulk }}}{\mu_{\text {wall }}}\right)^{0,14}
$$

siendo $\mu_{\text {bulk }} \mathrm{y} \mu_{\text {wall }}$ la viscosidad del fluido a las temperaturas media del flujo y de pared, respectivamente. Otros autores usan correlaciones similares con correcciones específicas según el motor.

Finlay et al. [2.72] demostraron que tanto la ebullición nucleada como la ebullición de película se presentan con frecuencia en las camisas de refrigeración de los motores. Norris et al. [2.69] realizaron mediciones de temperaturas y un análisis de transferencia con elementos finitos para encontrar las regiones de convección pura, ebullición nucleada y ebullición 
de película en la culata de un motor. El motor se operó en un amplio rango de velocidades y de carga y dividieron la cara de la culata y la camisa de refrigeración en ocho zonas para determinar los coeficientes de transferencia de calor necesarios para ajustarse a las temperaturas obtenidas durante la prueba. Los autores constataron la presencia de ebullición en la mayoría de las áreas críticas. Sin embargo, la gran disparidad en los resultados obtenidos para los diferentes cilindros hizo difícil definir con seguridad los modos de transferencia de calor, y específicamente la presencia de transferencia de calor por ebullición. También encontraron que varios de los coeficientes encontrados eran menores que los coeficientes esperados de convección pura, con lo que asumieron que en esas posiciones se presentaba ebullición de película.

Robinson et al. [2.70] realizaron experimentos en una maqueta de enfriamiento especialmente acondicionada para simular la dinámica fluidica real del refrigerante a lo largo de una galería de refrigeración real de un motor de combustión, con el objetivo validar un modelo compuesto que tiene en cuenta los efectos de la dinámica de entrada del fluido, la longitud inicial no caliente, la rugosidad de la superficie y la sensibilidad de las propiedades físicas del fluido a las variaciones de la temperatura. Los resultados los compararon con los derivados de la correlación de Dittus-Boelter. Esta última resultó que subvaloraba el coeficiente de transferencia de calor en aproximadamente 3,5 veces. En el mismo trabajo propusieron encontrar un modelo basado en la correlación de Dittus-Boelter, pero modificado por un factor de entrada, un factor de longitud fría inicial, un factor de rugosidad y un factor de viscosidad. Así obtuvieron la relación:

$$
\begin{aligned}
N u=\left(0,023 \operatorname{Re}^{0,8} \operatorname{Pr}^{0,4}\right) \frac{1+23,99 \operatorname{Re}^{-0,23}\left(x / D_{h}\right)^{n}}{\left[1-\left(x_{0} / x\right)^{9 / 10}\right]^{1 / 9}} * \\
*\left[0,091\left(\frac{\varepsilon}{D_{h}}\right)^{-0,125} \operatorname{Re}^{0,363\left(\varepsilon / D_{h}\right)^{0,10}}\right]\left(\frac{\mu_{\text {bulk }}}{\mu_{\text {wall }}}\right)^{0,14}
\end{aligned}
$$

donde $\varepsilon$ es la altura de rugosidad de la superficie $(\mathrm{m}) ; x$ es la distancia relativa a la distancia de flujo totalmente desarrollado donde se evalúa el número de Nusselt de transferencia de calor. 


\subsubsection{Modelado CFD}

La capacidad de los programas CFD para realizar cálculos de flujos turbulentos se ha estado incorporando en el modelado de las camisas de refrigeración, con lo que se logra la evaluación espacial de los campos de presión, de velocidades y de transferencia de calor. El análisis de los flujos de refrigerante actualmente es isotérmico, es decir, aún no se modela directamente la transferencia de calor y los coeficientes de transferencia de calor son inferidos a partir de los perfiles de velocidad y los niveles de turbulencia cerca de de la pared. Sin embargo, según afirman Robinson et al. [2.70], los niveles de turbulencia y los coeficientes reales de transferencia de calor son usualmente subestimados. Popovac et al. [2.50] aplicaron un modelo alternativo a la ley de la pared, denominado híbrido $\zeta-f / \mathrm{CWT}$ ( $\zeta-f$ con tratamiento integrado de pared) para valorar el campo de flujo y la distribución de los coeficientes de transferencia de calor en una camisa de refrigeración de un motor. Los resultados permitieron optimizar los pasajes de refrigeración de la culata, como lo ilustra la Figura 2.30.
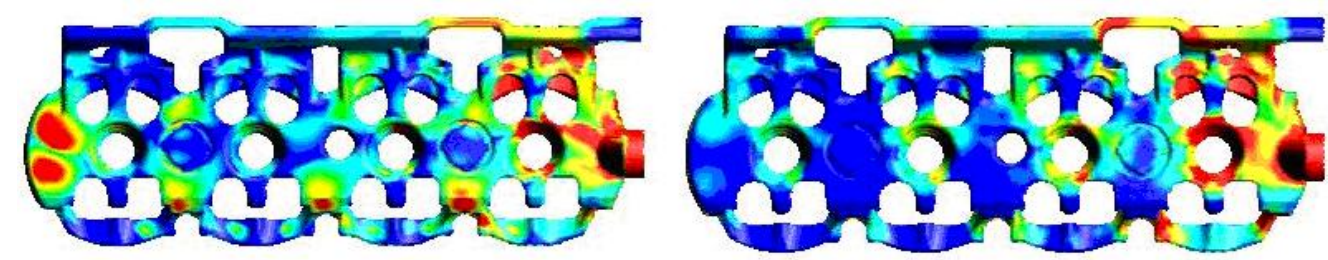

Figura 2.30. Flujo de refrigerante y distribución local del coeficiente de transferencia de calor. A la izquierda, el modelo $k-\varepsilon$; a la izquierda el modelo híbrido $\varsigma-f / C W T$ [2.50].

Los resultados de las temperaturas de la superficie determinados con el acople de programas CFD/FEM se ilustran en la Figura 2.31. Cualitativamente los resultados son similares, pero los valores absolutos de las temperaturas difieren en las áreas críticas como los puentes entre válvulas o entre los cilindros. Los autores afirman que las temperaturas del modelo en los lugares de medición de la culata, el bloque y el pistón son muy similares a los valores obtenidos experimentalmente. 

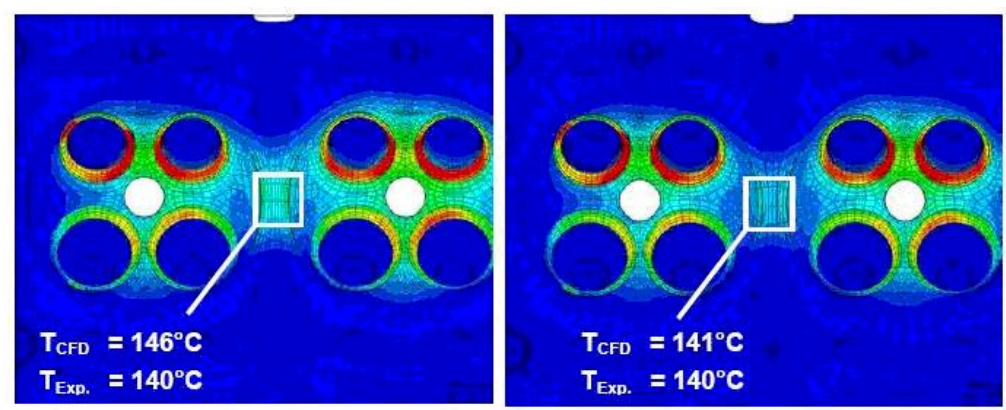

Figura 2.31. Temperatura en las superficies de la culata obtenidas con el método acoplado CFD/FEM: a la izquierda, el modelo $k-\varepsilon$; a la izquierda el modelo híbrido $s-f / C W T$ [2.50].

\subsection{Transferencia de calor entre el aceite y el motor}

El aceite es, como se mencionó, una de las condiciones de contorno. Usualmente, para el modelado del motor se evalúan los intercambios entre el aceite y el pistón y el aceite y la camisa. Para el caso del pistón, el aceite desempeña un papel importante en su refrigeración y sobre todo en los motores de elevada potencia específica, en los cuales se hace uso de toberas para inyectar aceite a canales anulares practicados en la cabeza del pistón.

\subsubsection{Transferencia de calor entre el pistón y el aceite}

La refrigeración de la parte inferior del pistón con el aceite depende del diseño del motor y puede ser por el barboteo generado por el cigüeñal, inyectado desde el bulón del pistón o inyectado por una tobera especial. Algunos de los esquemas de enfriamiento del pistón empleados en los motores se ilustran en la Figura 2.32.

En su modelo Bohac [2.3], asume valores para los coeficientes de convección asociados al barboteo, el chorro desde el bulón y el chorro desde la tobera:

Refrigeración por salpique

$1000 \mathrm{~W} / \mathrm{m}^{2} \mathrm{~K}$ para la transferencia en la corona; $240 \mathrm{~W} / \mathrm{m}^{2} \mathrm{~K}$ para la transferencia en la parte interna de la falda 
Refrigeración forzada desde el bulón

Refrigeración por chorro

$$
\begin{aligned}
& 2100-2800 \mathrm{~W} / \mathrm{m}^{2} \mathrm{~K} \\
& 2050-3230 \mathrm{~W} / \mathrm{m}^{2} \mathrm{~K}
\end{aligned}
$$

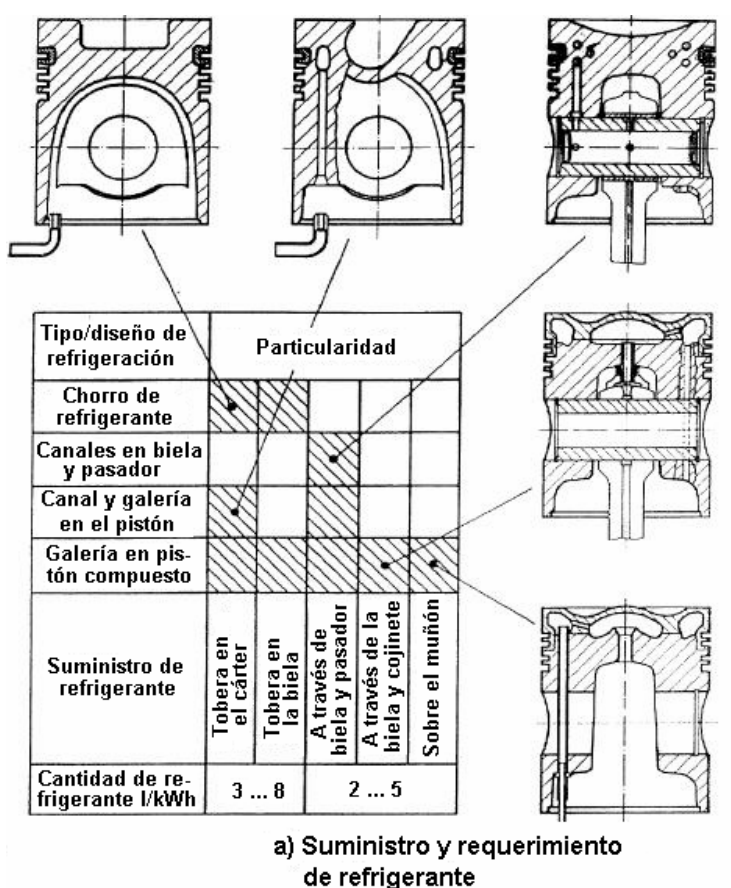

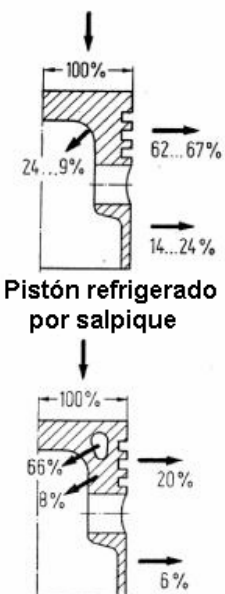

Por galería de refrigeración en el pistón b) Reparto de los flujos de calor
a traves del pistón

Figura 2.32. Influencia del tipo de refrigeración del pistón sobre las direcciones del flujo de calor hacia la camisa y el aceite [2.112].

En dicho trabajo, se estimó necesario introducir una corrección por velocidad del motor:

$$
h_{a c t}=h_{r e f}\left(\frac{n}{n_{r e f}}\right)^{b}
$$

donde $h_{\text {ref }}$ es el coeficiente de transferencia de calor a las revoluciones de referencia $n_{r e f}$. El exponente $b$ se obtiene por ajuste experimental para acercarse a las temperaturas experimentales que se hayan obtenido. 
Según Mohan et al. [2.73] y French [2.74], en sus experimentos sobre refrigeración del pistón por medio de aceite, el coeficiente de transferencia de calor es sensible a la velocidad del motor:

$$
h_{p c}=A_{p c} \operatorname{Re}^{0,8} \operatorname{Pr}^{0,33} D_{i}^{0,33}\left(\frac{k_{0}}{D}\right)
$$

donde $b$ es la longitud de la cavidad, en $\mathrm{m}, t$ es el período de movimiento del pistón, en $s, D_{i}$ es el diámetro de la cavidad del pistón, en m, $D$ es el diámetro del pistón, $k_{0}$ es la conductividad térmica del aceite de refrigeración del pistón, en $\mathrm{W} /(\mathrm{mK}), A_{p c}$ es una constante de correlación.

Liu y Reitz [2.4] en sus resultados experimentales encontraron una mayor intensidad en la transferencia de calor del aceite hacia las superficies internas de la corona del pistón, que hacia la falda de éste, lo que se explica en parte por la manera como los chorros de aceite impactan e inciden sobre estas dos superficies. Las expresiones utilizadas para los dos casos son:

Para la corona

$$
\begin{aligned}
& h_{u c 1}=900\left(\frac{N}{4600}\right)^{0,35} \\
& h_{u c 2}=240\left(\frac{N}{4600}\right)^{0,35}
\end{aligned}
$$

Agarwal y Varghese [2.75] desarrollaron un modelo numérico para predecir el coeficiente de transferencia de calor entre el aceite y la superficie del pistón empleando elementos finitos para estudiar el enfriamiento por chorro de aceite de los pistones. El modelo fue validado mediante ensayos de impacto de chorros de aceite sobre una superficie plana. Los autores partieron de una correlación empleada en el cálculo de transferencia de calor de chorros líquidos que impactan uniformemente sobre superficies planas, para obtener una correlación que permite valorar la influencia del tipo de aceite, la velocidad del chorro, el diámetro y la distancia de la tobera a la superficie del pistón, sobre el coeficiente de película. La expresión tiene la forma:

$$
\frac{N u}{N u_{0}}=\left[1+\left(a \cdot e^{b(r / d)}\right)^{-9}\right]^{-1 / 9}, N u_{0}=2,67 \operatorname{Re}^{0,567} \operatorname{Pr}^{0.4}\left(\frac{z_{0}}{d}\right)^{-0,336}\left(\frac{v}{d}\right)^{-0,237}
$$


donde los valores de $a$ y $b$ son función del diámetro de la tobera de aceite, $d$, $\mathrm{y} z_{0}$ es la distancia desde la salida de la tobera hasta la superficie interior del pistón. La función es recomendada para valores de Reynolds comprendidos en el rango 4000-52000.

En el trabajo de Rakopoulos et al. [2.76] el cálculo del coeficiente de transferencia de calor entre el chorro de aceite y la parte inferior del pistón, considerando la velocidad angular del motor, $w$, se determina por:

$$
h_{\text {oil }}=68,17\left[(r w) \frac{D_{n}}{v_{b}}\right]^{1 / 2}
$$

donde $r$ es el radio de la manivela del cigüeñal, $D_{n}$ es el diámetro de la tobera, $v_{b}$ es la viscosidad cinemática del aceite a la temperatura de operación.

En el modelo de predicción de temperaturas de pared de un motor, con enfriamiento del pistón mediante chorro inyectado a la galería de refrigeración por aceite en la corona del pistón, recientemente desarrollado por Torregrosa et al. [2.37], el coeficiente de película en el interior de la galería se obtiene a partir de la correlación $N u_{g a l}=a \operatorname{Re}^{m} \operatorname{Pr}^{n}$. Dado que en las mediciones experimentales no se incluyeron ensayos con los que se pudiera determinar el exponente $n$ del número de Prandtl, el factor $\operatorname{Pr}^{n}$, se consideró agrupado en un factor $C_{g a l}=a \operatorname{Pr}^{n}$. Los autores emplearon como longitud característica el diámetro de la sección toroidal de la galería, $d_{g a l}$, como velocidad característica la velocidad media del pistón, $C_{m}, \mathrm{y}$ considerando las propiedades termofísicas del aceite (conductividad, $k_{o i l} \mathrm{y}$ viscosidad cinemática, $v_{\text {oil }}$ ), la expresión que representa el modelo tiene la forma:

$$
h_{p i s 2 o i l}=C_{g a l} \frac{k_{o i l}}{d_{g a l}}\left(\frac{C_{m} d_{g a l}}{v_{o i l}}\right)^{m}
$$

Buscando excluir la dependencia en la expresión de las propiedades termofísicas del aceite, durante el proceso de validación, se buscó un coeficiente de la forma $C_{g a l}^{\prime}=C_{g a l} \cdot k_{o i l} / v_{o i l}^{m}$ Con estas consideraciones los valores encontrados fueron $C_{\text {gal }}^{\prime}=228,5$ y $m=0,73$. Los autores compararon 
el coeficiente de película obtenido con los valores obtenidos mediante modelado CFD por Kajiwara, encontrando que los valores reportados por éste son mucho menores. Aunque los autores no explican las grandes discrepancias obtenidas en la comparación, la explicación puede estar en que la naturaleza de las pruebas experimentales no permite la comparación con el modelo CFD de Kajiwara.

\subsubsection{Transferencia de calor entre el aceite y la estructura (bloque $y$ culata)}

Para las partes de la culata en contacto con el aceite, Jarrier y Gentile [2.54] utilizan una expresión $\mathrm{Nu}$-Re para superficies planas en la forma:

$$
N u=0,664 \operatorname{Re}^{0,5} \operatorname{Pr}^{0,33}
$$

Para el aceite que se escurre por las paredes del cilindro hacia el bloque, Jarrier y Gentile utilizan una expresión establecida por Hausen:

$$
N u=3,66 \frac{0,00668 \frac{D_{h y d}}{2 L} \operatorname{Re} \operatorname{Pr}}{1+0,04 \frac{D_{h y d}}{2 L} \operatorname{Re} \operatorname{Pr}}
$$

siendo $L$ la longitud de la parte de la camisa en contacto con el aceite.

Según Jarrier y Gentile, el coeficiente de transferencia de calor por barboteo entre el aceite y la parte inferior del bloque puede calcularse por una expresión, con corrección de temperatura para la viscosidad, de la forma:

$$
h_{\text {oil_lowerblock }}=h_{R E F}\left(\frac{N}{2000}\right)\left(\frac{v_{90^{\circ}}}{v_{T}}\right)^{0.5}
$$

En la literatura consultada, aparte de la correlación de (2.41), no se han encontrado otras que modelen el coeficiente de transferencia de calor entre el aceite y la parte inferior del bloque. Autores como Bohac [2.3], entre otros utilizan un valor constante para este coeficiente, a partir de las recomendaciones generales de los textos de transferencia de calor. 
Transferencia de calor entre el pistón y la camisa. Las condiciones del contorno del pistón son la interfaz entre la falda y los segmentos con la camisa, la parte inferior del pistón con el aceite, la interfaz con el bulón en la biela y los gases de combustión.

Para efectos de modelado térmico del motor, la interacción térmica entre el pistón y la camisa suele ser vista como una conducción a través de varias conductancias en paralelo, representativas de los contactos mediados por el aceite, entre la cabeza, la falda y los segmentos del pistón de un lado, y la camisa del cilindro del otro lado. Las resistencias empleadas son las promediadas en el tiempo (derivadas de ciclos termodinámicos promediados), por ser ésto más sencillo que tener en cuenta el movimiento del pistón. Los segmentos del pistón conducen el calor a la zona central de la camisa a través de una película lubricante. En el motor real el espesor de esta película lubricante varía a lo largo del ciclo, siendo el espesor máximo de 5-12 $\mu \mathrm{m}$ a mitad de la carrera cuando la velocidad del pistón es máxima, y el mínimo de cerca de $1 \mu \mathrm{m}$ en los puntos superior e inferior, cuando el pistón se detiene momentáneamente. Bohac [2.3] asume un espesor medio por defecto de la película lubricante, de $4 \mu \mathrm{m}$ y, al calcular el flujo de calor entre la falda del pistón y el cilindro, asume un espesor de $24 \mu \mathrm{m}$, basándose en las tolerancias funcionales del par cilindro pistón y las menores las dilataciones térmicas en esta zona.

Mohan et al. [2.73], en el modelo térmico reducido de un motor, desarrollado para acoplarse a un modelo de sistema de refrigeración, calcularon el flujo de calor del pistón a la camisa como el flujo de calor a través de dos masas entre las cuales se interpone una resistencia térmica de $248 \mathrm{~K} / \mathrm{kW}$. En el trabajo los autores tomaron como un elemento la camisa del cilindro y el bloque y la caracterizaron con una única temperatura.

\subsection{Transferencia de calor entre el motor y el ambiente}

Como lo afirman Jarrier et al. [2.26] y Bohac [2.3], no se dispone de modelos para el calculo de la transferencia de calor entre el motor y su ambiente y usualmente se hace uso de las expresiones de convección natural aplicadas a 
las superficies externas del motor. Dependiendo de la aplicación, la transferencia de calor entre el motor en su conjunto y su alrededor es compleja. Tratándose de aplicaciones automotrices, actualmente se emplean códigos CFD para el modelado del flujo de aire en los alrededores del motor y en su compartimento. Los modelos obtenidos permiten tener en cuenta las implicaciones aerodinámicas y de reparto de flujo de aire producido por los ventiladores del sistema de gestión térmica y el efecto "RAM" (incremento de presión a la entrada de aire al sistema de ventilación producido por el aire de encuentro durante el movimiento del vehículo). Una idea de las temperaturas en la parte externa del motor la da la Figura 2.33. La transferencia de calor entre el motor y su alrededor es convectiva y radiativa.

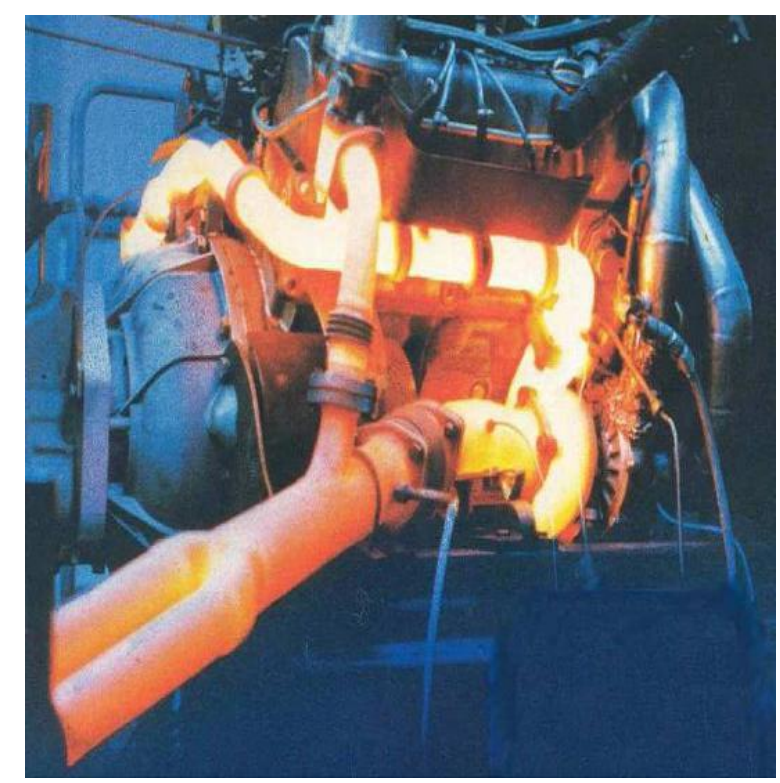

Figura 2.33. Vista de la medición con cámara termográfica de las temperaturas externas de un motor.

Aproximando el motor como un cilindro de altura y diámetro iguales, Mohan et al. [2.73] determinan la transferencia de calor convectiva y radiativa entre el motor y el ambiente (compartimento del motor) por las expresiones:

$$
\begin{gathered}
\dot{Q}_{a c}=h_{a m b} A_{b}\left(T_{b}-T_{a}\right) \\
\dot{Q}_{a r}=\varepsilon_{a m b} \sigma A_{b}\left(T_{b}^{4}-T_{a}^{4}\right)
\end{gathered}
$$


El coeficiente de transferencia de calor por convección lo expresan los autores como una función del número de Reynolds, la conductividad del aire y la longitud del motor:

$$
h_{a m b}=0,32 \operatorname{Re}^{0,675} \operatorname{Pr}^{0,4}\left(\frac{k}{l_{e}}\right)
$$

La emisividad radiativa de la superficie del bloque se aproxima a la del material del bloque, para el hierro fundido, $\varepsilon_{a m b}=0,44$. Por su parte, $T_{b}$ y $T_{a}$ son la temperatura del bloque y del compartimento del bloque del motor, respectivamente; $A_{b}$ es el área del bloque en contacto con el aire externo y $l_{e}$ es la longitud efectiva del motor.

Para los motores refrigerados por aire, Rakopoulos et al. [2.76] emplearon ecuaciones basadas en el número de Nusselt, dependiendo del estado del flujo, para calcular la transferencia de calor entre las aletas de los cilindros (las cuales forman un número de pasos paralelos cerrados por el enfocador) y el aire:

Para flujo laminar:

$$
\begin{aligned}
& N u=1,86\left[\operatorname{Re} \operatorname{Pr} \frac{D 1}{L}\right]^{1 / 3}\left(\frac{\mu_{b}}{\mu_{s}}\right)^{0,14} \\
& \operatorname{Re}<2100 \text {, } \\
& \text { basada en Sieder y } \\
& \text { Tate } \\
& N u=0,116\left[\operatorname{Re}^{2 / 3}-125\right] \operatorname{Pr}^{1 / 3}\left(\frac{\mu_{b}}{\mu_{s}}\right)^{0,14}\left[1+\left(\frac{D 1}{L}\right)^{2 / 3}\right]^{1 / 3} \begin{array}{l}
2100<\operatorname{Re}<10000 \\
\text { basada en Hausen }
\end{array}
\end{aligned}
$$

Para flujo turbulento:

$$
N u=0,023 \operatorname{Re}^{0,8} \operatorname{Pr}^{1 / 3}\left(\frac{\mu_{b}}{\mu_{s}}\right)^{0,14} \quad \begin{aligned}
& \operatorname{Re}>10000 \\
& \text { basada en Sieder } \mathrm{y} \\
& \text { Tate. }
\end{aligned}
$$

En estas ecuaciones las propiedades del aire se evalúan a la temperatura " $T_{b}$ " global, que es la media aritmética de las temperaturas de entrada y salida, mientras que " $S$ " se refiere a la temperatura de la superficie; $L$ es la longitud del recorrido del flujo, $D 1=4 A / P$ es el diámetro hidráulico equivalente, $A$ es el área de flujo superficial en cada pasaje y $P$ es el perímetro interno de refrigeración. 
En la Figura 2.34 se reproduce una pantalla de resultados de la simulación de las temperaturas del aire en el entorno del motor de un vehículo de pasajeros, empleando un código CFD.

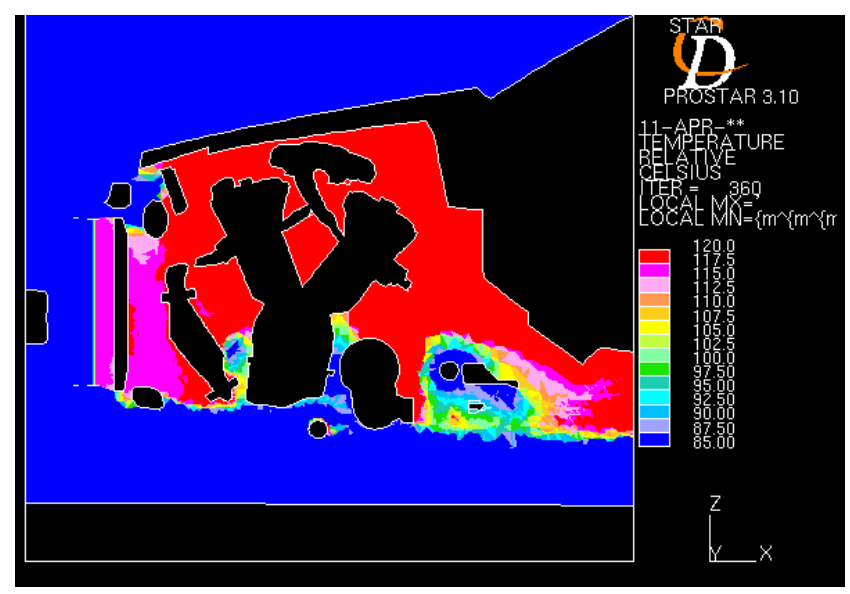

Figura 2.34.

Temperatura en el compartimento del motor en el plano de simetría (configuración condensador-radiadorventilador, $R F M)$ [2.114].

El código comercial CFD Star-CD fue el empleado en el análisis, considerando los tres mecanismos de transferencia de calor, convección, conducción y radiación. El modelo, basado en volúmenes finitos, fue representado con un mallado no estructurado y probaron tanto el modelo estándar $\mathrm{k}-\varepsilon$, como el modelo $\mathrm{k}-\varepsilon$ integrado con las funciones de la pared para representar las condiciones de contorno turbulentas en las inmediaciones de ésta. Ambos modelos dieron resultados comparables en la región cercana al motor. El núcleo de los intercambiadores fue modelado como un medio poroso. El calor disipado del núcleo se modeló usando un modelo basado en un macro de intercambiador de calor de Star-CD, el cual usa el NTU del intercambiador de calor para predecir el calor transferido al aire.

\subsection{Conclusiones}

En el presente capítulo se ha realizado un análisis del estado del arte en el modelado de la transferencia de calor en los motores de combustión interna con especial atención a las expresiones empleadas para calcular los flujos térmicos a través de las principales interfases entre los elementos. 
Se ha seguido el orden del trabajo realizado por Borman y Nishiwaki [2.1], repasando sus comentarios y complementándolos con la información extraída de los trabajos de investigación recientes relacionados. En la primera parte se ha abordado el modelado de la transferencia de calor entre los gases del cilindro y las paredes de la cámara de combustión, concluyendo que los modelos que se siguen empleando para la predicción y el diagnóstico de la combustión siguen haciendo uso de la expresión de Woschni [2.16], aunque siempre con modificaciones encaminadas a ajustar los resultados de los modelos a las medidas experimentales particulares. A pesar que la expresión de Woschni, como las de Annand, Eihelberg, Hohenberg y otras, subestima el calor transferido por los gases a las paredes, las estimaciones alcanzadas con ellas son suficientes para el estudio de las características de funcionamiento del motor con exactitud aceptable. En esta primera parte también se han resumido algunos modelos zonales, basados en trabajos como los de Morel [2.6], Boulouchos [2.58], Borgnakke [2.31] y otros. Se ha dejado en evidencia la ayuda prestada por los modelos CFD en el esclarecimiento de las limitaciones de los modelos globales en lo que respecta a las magnitudes de calor predichas y las particularidades del fenómeno de la transferencia de calor no cubiertas por éstos. Relativo a los modelos CFD, queda claro que sus predicciones han mejorado mucho gracias a la mayor disponibilidad de medidas experimentales y a la evolución de la capacidad de las máquinas de cálculo que están posibilitando mejores resoluciones y mejores tratamientos analíticos de la capa límite térmica, superando las deficiencias debidas a la utilización de la función de pared estándar. Los actuales modelos multidimensionales prácticamente dan cuenta de todos los componentes de la ecuación de energía en la interfaz gases-pared. En particular son notables los trabajos de Reitz [2.11][2.48], Kleeman [2.51], Popovac [2.50], Nijeweme [2.12] y Buttsworth [2.14].

Los modelos para la transferencia de calor radiativa también han sido revisados, aunque con menos amplitud que la convección, no por ser menos importante, sino por ser menor la cantidad de trabajos encontrados en la literatura. El desarrollo de las técnicas experimentales ópticas y de las técnicas de computación ha hecho posible el modelado cada vez mejor de este mecanismo de transferencia de calor. Una mención especial debe hacerse de los trabajos del grupo de Morel [2.7], Eiglmeier [2.27] y Reitz [2.48] quienes han hecho aportes metodológicos apreciables para el cálculo de la radiación en la cámara de combustión. 
En una segunda parte del capítulo, se repasaron los modelos usados para calcular el calor transmitido entre los gases de admisión y escape y sus respetivas pipas. La mayoría de las correlaciones encontradas valoran la transferencia de calor a partir de la relación $\mathrm{Nu}-\mathrm{Re}-\mathrm{Pr}$, con ajuste para la viscosidad del gas. Se destaca el trabajo de Depcik y Assanis [2.67], quienes presentaron una correlación universal y luego también presentaron una propuesta para evaluar la transferencia de calor transitoria.

En una tercera parte de este capítulo se ha explicado la complejidad del proceso de transferencia de calor hacia el refrigerante y se han descrito algunos de los modelos encontrados en la literatura, la mayoría de ellos basados en la ecuación de Dittus-Boelter. Los trabajos más importantes son los trabajos de Norris [2.69] y de Robinson [2.70]. Los avances en los modelos computacionales se están aplicando a los flujos en la camisa de refrigeración y se espera que así como los modelos globales y zonales dentro del cilindro han sido mejorados con los resultados de los modelos CFD, así mismo sean mejorados los modelos para la interfaz paredes motor refrigerante.

En el resto del capítulo, y de una manera menos detallada se ha comentado algunos de los tratamientos dados al cálculo de los flujos de calor en las otras interfases del motor, como entre el aceite y la estructura, entre el pistón y el cilindro y entre la estructura del motor y el aire ambiente.

Los modelos de transferencia de calor se han estudiado dentro de la presente tesis porque constituyen la base del modelado de las temperaturas y los flujos de calor del motor. Se entiende así, que la adecuada integración de las expresiones para el cálculo de los flujos de calor entre los componentes del motor, en su parte interna y desde su superficie es la base de un modelo de balance energético del motor (modelo interno) que puede acoplarse al modelo de gestión térmica del motor (modelo externo).

\subsection{Bibliografía}

[2.1] Borman, G., Nishiwaki, K. Internal combustión engine heat transfer. Progress in Energy Combustion Sciences, 1987. Vol 13, páginas 1-46.

[2.2] Rakopoulos, C. D., Mavropoulos, G. C. Experimental instantaneous 
heat fluxes in the cylinder head and exhaust manifold of an air-cooled diesel engine. Energy Conversion and Management, vol. 41, issue 12, pp. 1265-1281, 2000.

[2.3] Bohac, S.V., Baker, D.M., Assanis D.N. A global model for steadystate and transient S.I. engine heat transfer studies. SAE paper 960073.

[2.4] Liu, Y., Reitz, R. D. Modeling of heat conduction within chamber walls for multidimensional internal combustion engine simulations. Int. J. Heat Mass Transfer, vol 41, no 6-7, pp. 859 - 869, 1998.

[2.5] Lyford-Pike, E.J., Heywood, J.B. Thermal boundary layer thickness in the cylinder of a spark-ignition engine. Int. J. Heat Mass Transfer, vol. 27, no 10, pp. 1873, 1984.

[2.6] Morel, T., Keribar, R. A model for predicting spatially and timeresolved convective heat transfer in bowl-in-piston combustion chambers. SAE paper 850204.

[2.7] Morel, T., Keribar, R. Heat radiation in D. I. Diesel engines. SAE paper 860445 .

[2.8] Boulouchos, K, Isch, R. Modeling heat transfer during combustion: a quasi-dimensional approach with emphasis on large low-speed Diesel engines. International Symposium, COMODIA 90, pp. 321-328, 1990.

[2.9] Borman, G. L. In-Cylinder Heat Transfer Research at the U. W. Engine Research Center. International Symposium, COMODIA 90, pp. 1-10, 1990.

[2.10] Nishiwaki, K. Modeling engine heat transfer and flame-wall interaction. The Fourth International Symposium on Diagnostics and Modeling of Combustion in Internal Combustion Engines, COMODIA 98, pp. 35 - 44, 1998.

[2.11] Han, Z., Reitz, R. D. Temperature wall function formulation for variable-density turbulent flows with application to engine convective heat transfer modelling. Int. J. Heat Mass Transfer, vol. 40, no 3, pp. 613-25, 1997.

[2.12] Nijeweme, D. J., Kok, J. B. W., Stone, C. R., Wyszynski, L. Unsteady in-cylinder heat transfer in a spark ignition engine: experiments and modelling. Proc. Instn. Mech. Engrs., vol. 215, part D, part D. pp. 747-760, 2001

[2.13] Fiveland S. B., Assanis D. N. Development of a two-zone HCCI combustion model accounting for boundary layer effects. SAE paper 
2001-01-1028.

[2.14] Buttsworth, D. R. Transient response of an erodable heat flux gauge using finite element analysis. Proc. Instn. Mech. Engrs, part D, Journal of Automobile engineering, vol. 216, pp. 701-706, 2002.

[2.15] R. Tatschl, B. Basara, J. Schneider, K. Hanjalic, M. Popovac, A. Brohmer, J. Mehring. Advanced turbulent heat transfer modeling for IC-engine applications using AVL FIRE. International Multidimensional Engine Modeling User's Group Meeting. April 2, 2006, Detroit, MI.

[2.16] Woschni, G. A Universally Applicable Equation for the Instantaneous Heat Transfer Coefficient in the I.C. Engine. SAE paper 670931.

[2.17] Hohenberg, G. F. Advanced approaches for heat transfer calculations, Diesel engine thermal loading. SAE SP-449, pp. 61-79, 1979.

[2.18] Hiroyasu, H. Diesel engine combustion and its modeling. International Symposium, COMODIA 85, pp. 53 -76, 1985.

[2.19] Wilson, T.S., Bryanston-Cross, P. J., Chana, K. S. Dunkley, P., Jones, T. V., Hannah, P. High bandwidth heat transfer and optical measurements in an instrumented spark ignition internal combustion engine. SAE paper 2002-01-0747.

[2.20] Heywood, J.B. Internal combustion engine fundamentals. McGrawHill, 1988.

[2.21] Stone, R. Introduction to internal combustion engines. MacMillan publishers, 1999.

[2.22] Sitkei, G. Heat transfer and thermal loading in internal combustion engines. Akademiai Kiado, 1973.

[2.23] Allmendinger, K. Guzzella, L., Seiler, A., Loffeld, O. A method to reduce the calculation time for an internal combustion engine model. SAE paper 2001-01-0574.

[2.24] Kavtaradze, A. I., Gaivoronskii, V. A. Fedorov, D. D. Onishchenko, A. V. Shibanov. Calculation of radiative-convective heat transfer in the combustor of Diesel engine. High Temperature, vol. 45, $\mathrm{n}^{\mathrm{o}} 5$, pp. 673-680, 2007.

[2.25] Schubert, C., Wimmer, A., Chmela, F. Advanced heat transfer model 
for CI engines. SAE paper 2005-01-0696.

[2.26] Jarrier, L. Champoussin, J. C., Yu, R., Gentile, D. Warm-up of a D. I. Diesel engine: experiment and modeling. SAE paper 2000-01-0299.

[2.27] Eiglmeier, C., Stiesch, G., and Merker, G. P. A detailed phenomenological model for wall heat transfer prediction in Diesel engines. SAE paper 2001-01-3265.

[2.28] Poulos, S.G., Heywood, J.B. The effect of chamber geometry on spark ignition engine combustion. SAE paper 830334.

[2.29] Puzinauskas, P., Borgnakke, C. Evaluation and improvement of an unsteady heat transfer model for spark ignition engines. SAE paper 910298.

[2.30] Bargende, M. Ein Gleichungsansatz zur Berechnung der Instationaeren Wandwaermeverluste im Hochdruckteil von Ottomotoren. PhD dissertation, Technische Hochschule Darmstadt, Darmstadt, Germany, 1991.

[2.31] Borgnakke, C., Arpaci, V.S., Tabaczynski, R.J. A model for the instantaneous heat transfer and turbulence in a spark ignited engine. SAE paper 800287.

[2.32] Chen, C., Veshagh, A. A one-dimensional model for in-cylinder heat convection based on the boundary layer theory. SAE paper 921733.

[2.33] LeFeuvre, T. Instantaneous Metal Temperature And Heat Fluxes in a Diesel engine. PhD dissertation, University of Wisconsin-Madison, 1968.

[2.34] Chapman K. S., Johnson, J. H., Chiang, E. The enhancement and validation of a vehicle engine cooling system simulation for a heavy duty Diesel truck. SAE paper 880601.

[2.35] Jennings, M. J., Morel, T. A computational study of wall temperature effects on engine heat transfer. SAE paper 910459.

[2.36] Torregrosa, A., Olmeda, P., Martín, J., Degrauwe, B. Experiments on the influence of inlet charge and coolant temperature on performance and emissions of a DI Diesel engine. Exp. Therm. Fluid Sci., vol. 30, issue 7, pp. 633-641, 2006.

[2.37] Torregrosa, A. Olmeda, P., Broatch, A., Degrauwe, B., Reyes, M. A concise wall temperature model for DI Diesel engines. Appl. Therm. Eng., vol. 26, issue 11-12, pp. 1320-1327, 2006. 
[2.38] Meingast, U., Reichelt, L., Renz. U. Measuring transient wall heat flux under Diesel engine conditions. Int. J. Eng. Research, vol. 5, $\mathrm{n}^{\mathrm{o}}$ 5, pp. 443-452, 2004.

[2.39] Wang, X., Price, P., Stone, C. R., Richardson, D. Heat release and heat flux in a spray-guided direct-injection gasoline engine. Proc. Instn. Mech. Engrs., vol. 221, $\mathrm{n}^{\mathrm{o}}$ 11, part D: J. Automobile Engineering, pp. 1441-1452, 2007.

[2.40] Descombes, G. Maroteaux, F., Feidt, M. Study of the interaction between mechanical energy and heat exchanges applied to IC engines. Appl. Therm. Eng., vol. 23, issue 16, pp. 2061-20078, 2003.

[2.41] Lee, K. S., Assanis, D. N., Lee, J., Chun, K. M. Measurements and predictions of steady-state and transient stress distributions in a Diesel engine cylinder head. SAE paper 1999-01-0973.

[2.42] Dec, J. E. HCCI and stratified-charge compression-ignition engine combustion research. Advanced Combustion Engine R\&D FY 2004 Progress Report.

[2.43] Kuboyama, T., Kosaka, H., Aizawa, T., Matsui., Y. A Study on heat loss in DI Diesel engines. J-EAST, vol. 2001, $\mathrm{n}^{\mathrm{o}}$ 2, pp. 517-518, 2001 .

[2.44] Jenkins, R. J., James, E. H., Malalasekera, W. M. Modelling the effects of combustion and turbulence on near-wall temperature gradients in the cylinders of spark ignition engines. Proc. Instn. Mech. Engrs., vol 212, part D, pp. 533-546, 1998.

[2.45] Amsden, A.A., O'Rourke, P.J., Butler, T.D. KIVA: A computer program for two - and three - dimensional fluid flows with chemical reactions and fuel sprays. Los Alamos Report $n^{\circ}$ LA-11560-MS, 1989.

[2.46] Stanton, D., Rutland, C. Modeling fuel film formation and wall interaction in Diesel engines, SAE paper 960628.

[2.47] Wieserand, K. J., Ennemoser, A. O. 3D-CFD Diesel combustion and accurate heat transfer modeling, THIESEL 2002 Conference on Thermo - and Fluid-Dynamic Processes in Diesel Engines.

[2.48] Wiedenhoefer J. F., Reitz R. D. A multidimensional radiation model for diesel engine simulation with comparison to experiment. Numerical Heat Transfer, part A: Applications, vol. 44, nº 7, pp. 665$682,2003$. 
[2.49] Nuutine, M.. Improved near wall treatment for ci engine CFD simulations. Post Graduate Seminar on Heat and Mass Transfer. Helsinki, april 28, 2008.

[2.50] Popovac, M., Hanjalic, K. Compound wall treatment for RANS computation of complex turbulent flows and heat transfer. Applied Scientific Research, vol. 78, n² 2, pp. 177-202, 2007.

[2.51] Kleemann, A. P., Gosman, A. D., Binder, K. B. Heat transfer in Diesel engines: a CFD evaluation study, Proceeding of the Fifth International Symposium on Diagnostic and Modeling of Combustion in Internal Combustion Engines, COMODIA, pp. 123$131,2001$.

[2.52] Lucht, R. P., Walter, T., Dreier, T., Dunn-Rankin, D., Bopp, S. C. Heat transfer in engines: comparison of CARS thermal boundary layer measurements and heat flux measurements. SAE paper 910722, 1991.

[2.53] Reichelt, L., Meingast, U., Renz, U. Calculating transient wall heat flux from measurements of surface temperature. Int. J. Heat Mass Transfer, vol. 45, pp. 579-584, 2002.

[2.54] Jarrier, L., Gentile, D. Simulation du comportement thermique transitoire d'un motear à combustión interne et à allumage commandé. Rev. Gén Therm, vol. 36, nº 7, pp. 520-533, 1997.

[2.55] Sanders, S. T., Kim, T., Ghandhi, J. B. Gas temperature measurements during ignition in an HCCI engine SAE paper 200301-0744.

[2.56] Kranendonk, L. A., Caswell, A. W., A. M. Myers, S. T. Sanders Wavelength-agile laser sensors for measuring gas properties in engines. SAE paper 2003-01-1116.

[2.57] Chang, J., Güralp, O., Filipi, Z., Assanis, D., Kuo, T., Najt, P., Rask, R. New heat transfer correlation for an hcci engine derived from measurements of instantaneous surface heat flux. SAE paper 200401-2996.

[2.58] Boulouchos, K, Eberle, M. K., Ineichen, B., Klukowski, C. New insights into the mechanisms of in-cylinder heat transfer in Diesel engines. SAE paper 890573.

[2.59] Chen S., Chan T. L., Leung C. W., Liu M. A., Pan K. Y., Zhou L. B. Multidimensional numerical simulation of heat radiation in direct 
injection diesel engines. Proc. Instn. Mech. Engrs., part D: J. Automobile Engineering, vol. 214, ${ }^{\circ} 4$, pp. 453-466, 2000.

[2.60] Zaph, H. Beitrag zur Unterzuchung des Warmeubergangs wahrend des Ladungsvechsels im Viertaktdieselmotor, MTZ vol. 30, $\mathrm{n}^{\mathrm{0}} 12$, pp. 461-465, 1969.

[2.61] Bauer, W., Balun, P., Heywood, J. B. Heat transfer and mixture preparation in intake port of spark-ignition engine. SAE paper 972983.

[2.62] Bauer, W.D., Wenisch, J., Heywood, J.B. Averaged and timeresolved heat transfer of steady and pulsating entry flow in intake manifold of a spark-ignition engine. Int. J. Heat and Fluid Flow, vol. 19, pp. 1-9, 1998.

[2.63] Abraham, J. V. Magi. Modeling Radiant heat loss characteristics in a Diesel engine. SAE paper 970888.

[2.64] Shayler, Harb, P.J., C.M., Ma, T. Time-dependent behaviour of heat transfer coefficients for exhaust systems. IMechE paper C496/046/95, VTMS 2 Conf. Proc., 1995.

[2.65] Meisner, S., Sorenson, S.C. Computer simulation of intake and exhaust manifold flow and heat transfer. SAE paper 860242.

[2.66] Wendland, D. W. The effect of periodic pressure and temperature fluctuation on unsteady heat transfer in a closed system. $\mathrm{PhD}$ dissertation, 1968.

[2.67] Depcik, C., Assanis, D. A universal heat transfer correlation for intake and exhaust flows in a spark-ignition internal combustion engine. SAE paper 2002-01-0372.

[2.68] Zeng, P., Assanis, D. N. Unsteady convective heat transfer modeling and application to engine intake manifolds. Proceedings of IMECE2004, IMECE2004-60068.

[2.69] Norris, P.M., Wepfer, W., Hoag, K. L., Courtine-White, D. Experimental and analytical studies of cylinder head. SAE paper 931122.

[2.70] Robinson, K., Hawley, J. G., Hammond, G. P., Owen, N. J. Convective coolant heat transfer in internal combustion engines. Proc. Instn Mech. Engrs., part D: J. Automobile Engineering, vol. 217 pp. 136-146, 2003. 
[2.71] Chen, J. C. Correlation for boiling heat transfer to saturated fluids in convective flow. I \& E C Process Design and Development, vol. 5, $\mathrm{n}^{\mathrm{o}}$ 3, pp. 322-329, 1966.

[2.72] Finlay, I. C., Boyle, R. J., Pirault, J. P., Biddulph, T. Nucleate and film boiling of engine coolants flowing in a uniformly heated duct of small cross section. SAE paper 870032.

[2.73] Mohan, K. V, Arici, O., Yang, S., Johnson, J. H. A computer simulation of the turbocharged diesel engine as an enhancement of the vehicle engine cooling system simulation. SAE paper 971804.

[2.74] French C.C.J. Piston cooling. SAE Paper 720024.

[2.75] Agarwal, A. K., Varghese, M. B. Numerical investigations of piston cooling using oil jet in heavy duty Diesel engines, Int. J. Eng. Research, vol. 7, pp. 411-421.

[2.76] Rakopoulos, C. D. , Mavropoulos G. C., Hountalas, D. T. Modeling the structural thermal response of an air-cooled Diesel engine under transient operation including a detailed thermodynamic description of boundary conditions. SAE paper 981024 .

[2.77] Ikegami, M., Hida, M., Nishiwaki, K. Computational fluid-dynamic predictions of heat transfer in the combustion-chamber walls of Diesel engines. Trans. JSME, vol. 57, $\mathrm{n}^{\mathrm{o}}$ 534, ser. B, pp-744-749, 1991.

[2.78] Woschni, G., Huber, K. The influence of soot deposits on combustion chamber walls on heat losses in diesel engines. SAE paper 910297.

[2.79] Annand, W. J. D., Ma, T. H. Instantaneous heat transfer to the cylinder head surface of a small compression engine. Proc. Instn. Mech. Engrs. 185, 72/71, pp. 976-987, 1970.

[2.80] Uchimi, T., Taya, K., Hagihara, Y., Kimura, S., Enomoto, Y. Heat loss to combustion chamber wall in a D.I. diesel engine -First report: Tendency of heat loss to piston surface. JSAE Review 21, pp. 133$141,2000$.

[2.81] Hottel, H. C., Broughton, F. P. Determination of the true temperature and total radiation from luminous gas flames. Industrial and engineering chemistry, vol. 4, $\mathrm{n}^{\mathrm{o}}$ 2, pp. 166-175, 1932.

[2.82] Kubicki, M., Watson, H. C., Williams, J., Stryker, P. C. Spatial and temporal temperature distributions in a spark ignition engine piston at 
WOT. SAE paper 2007-01-1436.

[2.83] Morel, T., Blumberg, P.N., Fort, E.F., Keribar, R. Methods for heat analysis and temperature field analysis of the insulated Diesel. NASA CR-174783, 1984.

[2.84] Finol, C. A., Robinson, K. Thermal modelling of modern engines: a review of empirical correlations to estimate the in-cylinder heat transfer coefficient.. Proc. IMechE, vol. 220, part D: J. Automobile Engineering, vol. 202, pp. 1765 - 1781, 2006.

[2.85] Fluent Inc, FLUENT 6.2--User's Guide, vol. 2, 2001.

[2.86] Gosman, A.D. State of the art of multi-dimensional modeling of engine reacting flows. Oil \& Gas Science Technology, vol. 54, 1999.

[2.87] STAR-CD V3.15 PROSTAR \& es-ice are Trademarks of CD-adapco Group.

[2.88] Amsden, A. A. KIVA-3V: A block-structured KIVA program for engines with vertical or canted valves. Los Alamos National Laboratory Report LA-13313-MS, 1997.

[2.89] Dec, J.E. A Conceptual model of DI Diesel combustion based on laser-sheet imaging, Society of Automotive Engineers report SAE970873, 1997.

[2.90] Yi, Y., Investigating Engine Systems with CFD Tools. Fluent Inc. http://www.erc.wisc.edu/Symosium_and_meetings/2005_Symposiu m.

[2.91] Launder, B., Spalding, D. The numerical computation of turbulent flows. Comp Methods in App. Mech and Eng., vol. 3, , pp. 269-289, 1974.

[2.92] Craft, T. J., Gant, S. E., Gerasimov, A. V., Iacovides, H., Launder, B.E. Development and application of wall-function treatments for turbulent forced and mixed convection flows. Fluid Dynamics Research, vol. 38, issues 2-3, pp. 127-144, 2006,

[2.93] Mengüç, M.P., Viskanta, R., Ferguson, C.R., Multidimensional modeling of radiative heat transfer in Diesel engines, SAE paper 850503 .

[2.94] Viskanta, R., Mengüç, M. P. Radiation Heat Transfer In Combustion Systems. Prog. Energy Combust. Sci., vol. 13, pp. 97-160, 1987.

[2.95] Viscanta, R. Computation of radiative transfer in combustion 
systems. International Journal of Numerical Methods for Heat and Fluid Flow, vol. 18, pp. 415-442, 2008.

[2.96] Jaluria, Y., Torrance, K. E. Computational heat transfer. Taylor \& Francis, 2003.

[2.97] Lockwood, F. C., Shah, N. G. A new radiation solution method for incorporation in general combustion prediction procedures. 18 International Symposium on Combustion, pp. 1405-1414, 1981.

[2.98] Chai, J.C., Patankar, S.V. Finite-volume method for radiation heat transfer. Advances in Numerical Heat Transfer, vol. 2, pp. 109-138, 2000.

[2.99] Furmanski, P., Banaszek J., Wisniewski, T. S. Radiation heat transfer in a combustion chamber of Diesel engine with partially transparent burnt gas zone. SAE paper 980504.

[2.100] Yan, Z., Liu, Z., Rui, Y., Chen, J., Yu, X. Simulation of in-cylinder radiative heat transfer of diesel engine with Monte-Carlo Method. Journal of Zhejiang University SCIENCE, vol. 1, $\mathrm{n}^{\mathrm{o}} 3$, pp. 306$310,2000$.

[2.101] Pinchon, P. Modelling of fluid dynamics and combustion in piston engines. International Symposium, COMODIA 90, pp. 31 -47, 1990.

[2.102] Blunsdon, C.A., Malalasekera, W.M.G., Dent, J.C. Application of the Discrete Transfer Model of Thermal Radiation in a CFD simulation of Diesel engine combustion and heat transfer. SAE paper 922305.

[2.103] Huber, E.W., Koller, T., Pipe friction and heat transfer in the exhaust pipe of a firing combustion engine, CIMAC, pp. 2075-2105, 1977.

[2.104] Dolz, V. R. Contribucion al Modelado de la transmisión de calor en los MCIA y su aplicacion en el aprovechamiento energético de los gases de escape durante los transitorios de carga. Tesis doctoral, Universidad Politécnica de Valencia. España. 2006.

[2.105] Reyes M. Modelo de transferencia de calor para colectores de escape de motores alternativos. Tesis doctoral, Universidad Politécnica de Valencia. España. 1993.

[2.106] Caton, J.A. Comparisons of thermocouple, time-averaged and massaveraged exhaust gas temperatures for a spark-ignited engine. SAE 820050 . 
[2.107] Ladommatos, N., Xiao, Z., Zhao, H. The effect of piston bowl temperature on diesel exhaust emissions. Proc. Instn. Mech. Engrs., part D: J. Automobile Engineering, vol. 219, pp. 371-388, 2005.

[2.108] Bredberg, J., Peng _S-H., Davidson, L. On the wall boundary condition for computing turbulent heat transfer with $k-w$ models. Proceedings of the ASME Heat Transfer Division, vol. 366-5, pp. 243-250, 2000.

[2.109] Durbin, P.A. Near-wall turbulence closure modelling without "damping functions", Theoret. Comput. Fluid Dynamics 3, pp. 1-13, 1991.

[2.110] Hanjalic, K. Closure models for incompressible turbulent flows. Department of Applied Physics, Delft University of Technology.

[2.111] Arcoumanis, C, Cutter, P., Whitelaw, D.S. Heat transfer processes in Diesel engines. Trans. IChemE, vol. 76, parte A, pp. 124-132, 1998.

[2.112] Bach, E. Triebwerk der Verbrennungsmotoren, Lehrbrief V5 Kolben- Hrsg. Hochscule für Verkehrswesen "Friedrich List" section Fahrzeugtechnik, LG Kolbenmaschinen, Dresden, 2006.

[2.113] Kajiwara, H., Fujioka, Y., Suzuki, T. Negishi, H. An analytical approach for predicition of piston temperature distribution in Diesel engines, JSAE Review, vol. 23, pp. 429-434, 2002

[2.114] Yang, Z., Bozeman, J. Shen, F., Acre, J. CFRM concept at vehicle idle conditions. SAE paper 2003-01-0613.

[2.115] Woschni, G. A Die Berechnung der Wanderluste und der Thermischen Belastung der Bauteile von Diesel-motoren, MTZ, 30, 12, pp. 491-499, 1970. 


\section{Capítulo 3}

\section{Caracterización térmica de los M.C.I.A.}

3.1. Introducción

3.2. Proceso de combustión y flujo de calor a través de las paredes de la cámara de combustión de un motor alternativo

3.2.1. El análisis de Fourier.................................................................. 100

3.2.2. Transformada de Laplace .............................................................. 102

3.3. Balance térmico del motor ................................................................ 103

3.3.1. Balance energético del motor en estado estacionario a carga constante

3.3.2. Balance energético durante el calentamiento del motor.................. 107

3.3.2.1. Consideraciones teóricas y experimentales......................... 107

3.3.3. Balance energético experimental

3.3.3.1. Balance térmico del motor durante su calentamiento bajo una carga y régimen constantes, representativos del ciclo de conducción

3.3.3.2. Balance térmico del motor durante su calentamiento en su proceso de calentamiento en el ciclo de conducción NEDC

3.3.3.3. Energía recuperada en el vehículo durante el ciclo de conducción 
3.3.4. Observaciones

3.4. Modelado de la temperatura y los flujos de calor con un modelo predictivo. 135

3.4.1. Breve descripción del modelo térmico.......................................... 136

3.4.1.1. Fundamentos del modelo nodal.......................................... 136

3.4.1.2. Interacciones y condiciones de contorno ............................. 138

3.4.2. El programa de cálculo................................................................ 141

3.4.3. Predicción de las temperaturas del primer motor ante cambios en escalón de las condiciones de operación

3.4.3.1. Predicción de los flujos de calor entre los nodos del modelo

3.4.3.2. Predicción de las temperaturas y los flujos de calor en estado transitorio.

3.5. Predicción de las temperaturas y los flujos de calor del segundo motor

3.5.1. Predicción de los flujos de calor al refrigerante.

3.5.2. Predicción del comportamiento térmico del motor bajo condiciones de calentamiento.

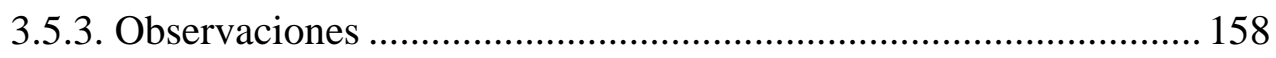

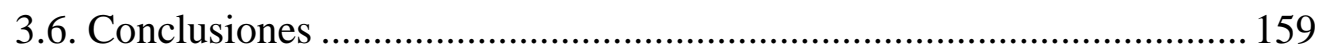

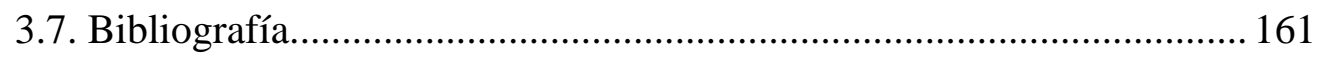




\subsection{Introducción}

El objetivo de este capítulo es estudiar a la caracterización térmica de los motores, entendiendo por caracterización la determinación de las temperaturas del motor, sus flujos de calor internos y externos y la inercia térmica de sus paredes internas. Esta caracterización es particularmente necesaria para alimentar programas de modelado de la combustión, de operación de sistemas de refrigeración y también para derivar conclusiones relativas a la utilización de la energía suministrada al motor. El trabajo presentado en este capítulo es el resultado de la combinación de las evaluaciones experimentales realizadas en un motor Diesel y la aplicación y extensión de un modelo térmico a la predicción de temperaturas y flujos de calor de este mismo motor.

Para lograr el objetivo mencionado se ha dispuesto la realización de ensayos y adquisición de información en el motor en las siguientes fases:

- Ensayos del motor en caliente bajo diferentes combinaciones constantes y estabilizadas de carga y revoluciones. Las variables medias de operación del motor y las variables instantáneas medidas sirven para alimentar el modelo de predicción de temperaturas y flujos de calor y obtener el mapa de disipación de calor al refrigerante.

- Ensayos del motor durante su arranque y proceso de calentamiento bajo una condición de carga y revoluciones bajas, representativa de la operación en vehículo circulando en ciudad. Se miden continuamente las variables medias de operación del motor y los flujos y temperaturas de refrigerante en diferentes puntos del sistema de refrigeración y de igual manera se miden las variables instantáneas dentro del cilindro ( 25 ciclos consecutivos de motor) en 14 instantes del tiempo de calentamiento. Estas mediciones alimentan el modelo de predicción de temperaturas y flujos de calor y también se utilizan para calcular y analizar el balance térmico del motor. El comportamiento de las temperaturas del motor durante el calentamiento es usado para estimar la inercia térmica global de sus paredes internas.

- Ensayos del motor durante su arranque y proceso de calentamiento en condiciones simuladas de operación propulsando un vehículo de 
pasajeros bajo un ciclo de conducción europeo, NEDC. Se hacen las mismas mediciones que en la fase anterior con los mismos propósitos, las variables instantáneas se registran en 23 puntos escogidos del ciclo de conducción y se concluye cualitativamente sobre la factibilidad de usar la información adquirida durante el ciclo (información registrada en puntos no estabilizados $y$ en condiciones transitorias de calentamiento del motor) para la predicción de las temperaturas y flujos de calor. Se sacan conclusiones sobre el tiempo de calentamiento del motor y sobre el comportamiento del balance térmico en los diferentes tiempos de operación del ciclo NEDC.

Se ha usado un programa de diagnóstico de combustión, denominado CALMEC [3.49], para calcular, aparte de los parámetros importantes de la liberación de calor del combustible, la temperatura de los gases en el cilindro y los gastos másicos de aire de admisión y gases de escape, así como las áreas efectivas de paso de estos flujos.

En el primer apartado de este capítulo se describe el proceso de transferencia de calor entre el gas y las paredes de la cámara de combustión, puntualizando sobre la variación transitoria de la temperatura de la pared. En el segundo apartado, tras hacer unos comentarios breves sobre el balance energético de los motores Diesel, se describe la distribución energética experimental del motor, con lo que se ven las implicaciones del régimen de funcionamiento del motor, del diseño de su sistema de refrigeración y de las masas del motor sobre el proceso de calentamiento. En el tercer apartado se describe brevemente el modelo de cálculo de temperaturas de paredes utilizado para la predicción de las temperaturas y los flujos de calor en el motor y construir el mapa de calor disipado al refrigerante. Con los tres apartados descritos, se logra conocer el carácter de variación de las temperaturas instantáneas y medias de las paredes y del refrigerante y, para efectos de modelado del sistema de refrigeración del motor, se calcula el mapa de calores disipados al sistema de refrigeración y se estima la masa térmicamente sensible. Se concluye finalmente discutiendo las necesidades del sistema de refrigeración en los motores de combustión interna. 


\subsection{Proceso de combustión y flujo de calor a través de las paredes de la cámara de combustión de un motor alternativo}

La localización de los focos calientes en las partes metálicas del motor es un punto de partida en el diseño de mejores sistemas de refrigeración. Para comprender mejor los efectos de la combustión y sus fenómenos asociados en la disipación de calor se requiere estudiar los fenómenos físicos que ocurren dentro del cilindro, incluyendo el flujo instantáneo de calor entre los gases de trabajo y las paredes. Un esquema bastante simplificado de la transferencia de calor en las paredes de la cámara de combustión es el representado en la Figura 3.1.

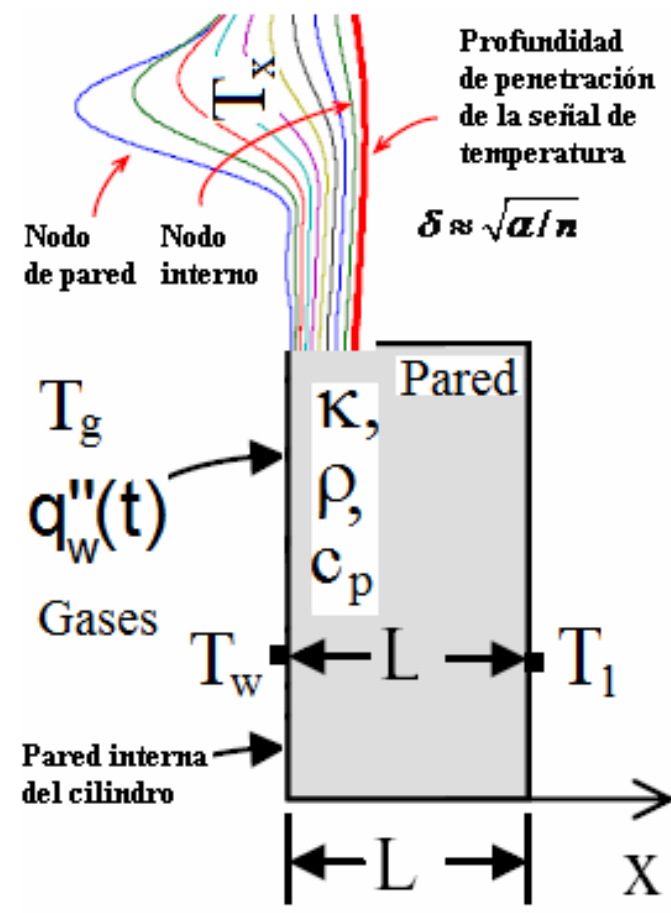

Figura 3.1. Ilustración de la propagación de la onda de temperatura al interior de las paredes de la cámara de combustión.

Aunque el flujo de calor a través de las paredes del cilindro es esencialmente tridimensional, en la práctica se asume la unidimensionalidad para facilitar la solución del problema y por las dificultades prácticas de instalar sensores rápidos de temperatura en espacios reducidos. La aproximación se basa en el 
hecho de que la parte transitoria de los correspondientes transitorios de flujo de calor penetran sólo hasta una escasa profundidad de la pared (Whitehouse [84]). Con esta aproximación la expresión del flujo transitorio de calor empleada tiene la forma [3.36]:

$$
\frac{\partial T}{\partial t}=\frac{1}{\rho c} \frac{\partial}{\partial x}\left(k \frac{\partial T}{\partial x}\right)
$$

El flujo de calor dado por la ecuación (3.1) puede solucionarse analíticamente utilizando el procedimiento de Fourier, empleando la transformación de Laplace o método de respuesta a una señal de impulso y también empleando métodos numéricos.

\subsubsection{El análisis de Fourier}

La solución por series de Fourier se puede aplicar cuando la temperatura en los sólidos es de variación periódicamente estable y las propiedades térmicas del material son constantes. La temperatura periódica de la superficie $T_{w}(t)$ de cualquier parte de la cámara de combustión del motor se compone de una parte constante, $T_{m}$, y de una parte transitoria que es función de la frecuencia angular $\omega(\mathrm{rad} / \mathrm{s})$ [3.36]:

$$
T_{w}=T_{m}+\sum_{n=1}^{N}\left[A_{n} \cos (n \omega t)+B_{n} \sin (n \omega t)\right]
$$

$A_{n}$ y $B_{n}$ son los coeficientes de Fourier y $n$ es el número del armónico.

Las condiciones de contorno son:

$T=T_{w}(t)$ en $x=0$ y $T=T_{l}=$ constante en $x=l$

La solución de la ecuación (3.1) con estas condiciones de contorno está dada por la expresión:

$$
T(x, t)=T_{m}-\left(T_{m}-T_{l}\right) \frac{x}{l}+\sum_{n=1}^{N} \exp \left(-\phi_{n} x\right) F_{n}(x, t)
$$


Donde $F_{n}=A_{n} \cos \left(n \omega t-\phi_{n} x\right)+B_{n} \sin \left(n \omega t-\phi_{n} x\right), \phi_{n}=\sqrt{n \omega / 2 \alpha}$, siendo $\alpha=$ $k / \rho c$ la difusividad térmica del material de la pared.

Combinando la ecuación (3.3) y la ley de Fourier, $q_{w}=-k \partial T / \partial x$, se obtiene la expresión para determinar el flujo de calor a través de la pared:

$$
q_{w}^{\prime \prime}(t)=\frac{k}{l}\left(T_{m}-T_{l}\right)+k \sum_{n=1}^{N} \phi_{n}\left[\left(A_{n}+B_{n}\right) \cos n \omega t-\left(A_{n}-B_{n}\right) \sin n \omega t\right]
$$

Generalmente se aplica la Transformada Rápida de Fourier (FFT) a las mediciones de la temperatura de superficie para determinar los coeficientes $A_{n}$ y $B_{n}$. Al realizar la transformada rápida de Fourier para determinar $A_{n}$ y $B_{n}$, es importante definir el número apropiado de armónicos, $n$. Si éste se elige muy bajo, el perfil de la temperatura reconstruido no se ajustará tan exactamente a los datos de temperatura medidos; si $n$ es muy grande, se aumenta la sensibilidad del cálculo a los ruidos en la señal medida y puede reproducir fluctuaciones (inestabilidades) no presentadas en el perfil simulado. Chang et al. [3.37] encontraron que un valor de $n=40$ era apropiado para mediciones con resoluciones angulares elevadas $(0,5$ grados de giro del cigüeñal). Una vez que $A_{n}$ y $B_{n}$ se han determinado, se aplica la ley de Fourier a la ecuación (3.4) para obtener el flujo de calor.
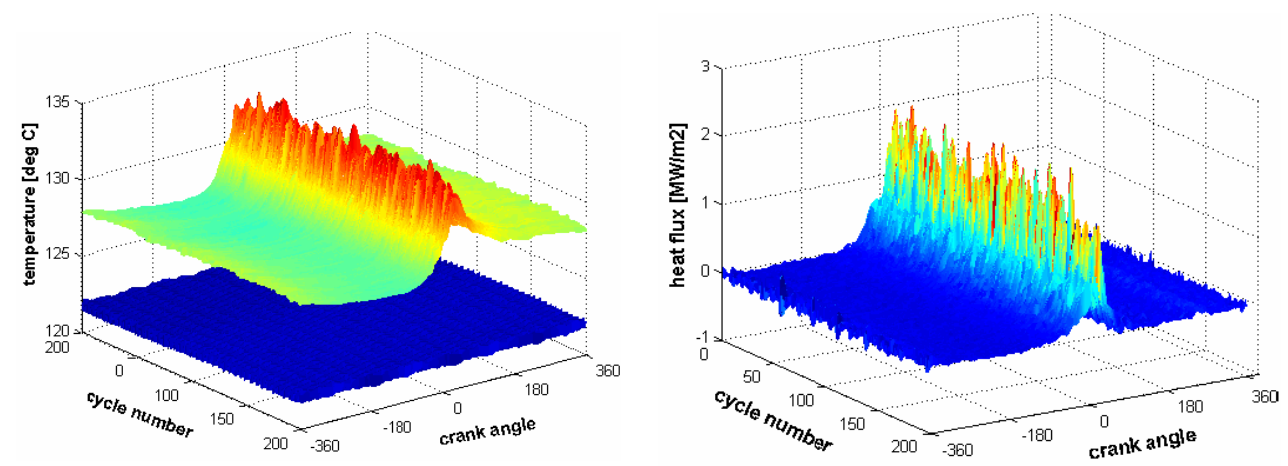

Figura 3.2. Representación de la variabilidad cíclica de las temperaturas instantánea en la superficie y temperatura en la profundidad del material (a) y del flujo de calor calculado durante 200 ciclos consecutivos (b) en un punto de la culata de un motor [3.37]. 
Como anotan Chang et al. [3.37], la temperatura superficial medida varía considerablemente de ciclo a ciclo aún bajo condiciones estables de operación del motor, incluso cuándo la variación cíclica de la presión es pequeña. La Figura 3.2 es un ejemplo para ilustrar esa variación de la temperatura de pared. Las razones para estas fluctuaciones son múltiples y pueden ser debidas a la aleatoriedad del flujo y del proceso de combustión. El conocimiento de este problema de variabilidad hace necesario promediar un número grande de grupos de muestras de temperaturas de ciclo, con el riesgo de que se escondan o pierdan aquellas componentes que no son aleatorias, sino que son reales (por ejemplo las componentes de frecuencias inferiores a la frecuencia del ciclo del motor, que están relacionadas con inestabilidades de funcionamiento).

El perfil de flujos de calor promediado en la superficie permite determinar experimentalmente los flujos de calor y las pérdidas de calor acumuladas. Una vez que se ha calculado el coeficiente de transferencia de calor experimental $h_{\text {exp }}$ a partir del flujo de calor promediado en la superficie, $\bar{q}_{w}$, puede también calcularse el flujo de calor a través de todas las superficies:

$$
\left(\frac{d Q_{h t}}{d t}\right)_{\exp }=h_{\exp } \sum_{i}\left(T-T_{w, i}\right) A_{i},
$$

donde $h_{\exp }=\frac{\bar{q}_{w}}{T-T_{w}}$

$T$ es la temperatura global del gas. $T_{w}$ es la temperatura promediada de la superficie medida con el sensor de flujo. El subíndice $i$ denota cada una de las áreas de transfencia consideradas en las mediciones.

\subsubsection{Transformada de Laplace}

Reichelt et al. [3.38] aplicaron la transformada de Laplace para calcular el flujo de calor transitorio entre el chorro de combustible y la pared bajo condiciones de operación de un motor Diesel. En el cálculo asumieron que el flujo de calor era unidimensional y utilizaron las lecturas temporales de los termopares rápidos ubicados en tres sitios de la zona inyectada. La solución de la ecuación la obtuvieron en la forma: 


$$
\bar{\theta}(\xi, s)=\bar{\theta}_{s}(s) \frac{e^{-p \xi}-e^{-p(\xi-2)}}{1-e^{-2 p}}
$$

donde $p^{2}=s$ es la variable independiente en el dominio de la frecuencia, $\xi$ es la distancia adimensional, $\bar{\theta}$ es la temperatura transformada. El flujo de calor a la pared se determina como:

$$
\dot{q}^{\prime \prime}(\tau)=\frac{k}{\sqrt{\pi} \cdot x} \int_{0}^{\tau} \frac{T_{s}}{d \tau^{*}}\left(\tau^{*}\right) \frac{1}{\sqrt{\tau-\tau^{*}}}\left(1+2 \sum e^{-n^{2} /\left(\tau-\tau^{*}\right)}\right) d \tau^{*}
$$

\subsection{Balance térmico del motor}

Usualmente los balances de energía en los motores se realizan bajo condiciones estacionarias estabilizadas, con lo que se garantizan condiciones estables y la repetibilidad de los resultados. Sin embargo, los motores de automóvil siempre están funcionando en condiciones variables de operación, lo que resulta ser diferente de una secuencia de puntos de operación estabilizados. En tanto esto, la realización de un balance térmico del motor a partir de pruebas puntuales, aún representativas de condiciones de operación bajo ciclo de conducción, resulta ser no muy exacta y no da cuenta de las fluctuaciones causadas por los procesos transitorios. Paralelamente, los ciclos normalizados son representativos de la utilización media que se les da a los vehículos por parte de los usuarios. Las mediciones acumuladas durante el ciclo pueden permitir profundizar más en los caminos tomados por la energía, en comparación con las valoraciones hechas mediante las mediciones realizadas en puntos de operación discretos y estabilizados. Puede agregarse además que la valoración de los flujos de energía por ciclo de conducción arrancando el motor en frío puede arrojar una información muy representativa y útil desde el punto de vista normativo, para fijar estrategias de ahorro energético basadas en el ciclo de conducción.

La motivación para incluir el balance energético en esta tesis es la ausencia, en la literatura revisada, de información experimental relativa a la valoración energética de motores durante su calentamiento. Muchos investigadores han realizado evaluaciones térmicas de motores bajo condiciones de conducción para validar modelos de predicción de temperaturas. Morel [3.17] expuso un 
modelo detallado para calcular los transitorios térmicos del motor, pero como puede inferirse de su trabajo, el modelo fue validado empleando un proceso transitorio térmico controlado (la temperatura del motor se varió manualmente entre $300 \mathrm{~K}$ y $380 \mathrm{~K}$, realizando las mediciones cuando la temperatura se estabilizaba). El trabajo de Morel no estaba dirigido a obtener un balance energético. Jarrier et al. [3.18] encerraron un motor en un calorímetro para realizar el balance térmico instantáneo para diferentes condiciones de operación, identificando las rutas principales seguidas por los flujos de energía. Aunque en el artículo se sugiere que la parte experimental se ha realizado para simular ciclos de conducción urbanos (ECE), no se presentan en el trabajo resultados de balance energético bajo el ciclo NEDC completo. Jarrier et al. asumieron que durante el calentamiento del motor las presiones instantáneas de los gases en el cilindro eran idénticas a las del estado estacionario, no considerando el hecho de que durante el calentamiento la presión media indicada es afectada por la presión media efectiva de fricción, la cual depende de la viscosidad del aceite (esto es, de la temperatura). Shayler et al. [3.19] desarrollaron un modelo computacional para predecir las características de calentamiento de un motor utilizando un modelo de masas globales junto con correlaciones empíricas para el cálculo disipado al refrigerante por el motor (correlación de Taylor y Toong) y las pérdidas de fricción (correlación de Patton). Los objetivos de otros trabajos relacionados (Taymaz [3.20], Perez-Blanco [3.21], Lehner [3.22]) son diferentes a la realización de un balance energético. Además en los trabajos encontrados se hace uso de información obtenida bajo condiciones estabilizadas.

En este apartado se hace mención del balance energético del motor de manera genérica, a partir de la información disponible en la literatura, y luego, para el motor estudiado, se calcula el balance energético bajo condiciones de calentamiento a carga y revoluciones constantes y también bajo el ciclo de operación NEDC. También se evalúa la energía que realmente se recupera en los dispositivos suplementarios del sistema de refrigeración del motor, representados por el enfriador de gases de escape recirculados, EGR, y el calentador del habitáculo. La información relativa a los gradientes de temperatura y flujos de calor en condiciones de operación transitorias puede usarse para prever las tecnologías de recuperación de energía que mejor se ajustan a esos niveles de gradientes de temperaturas y flujos de calor. 


\subsubsection{Balance energético del motor en estado estacionario a carga constante}

En la literatura, de forma general, se reporta que en los motores alternativos la energía se descompone casi por partes iguales entre el trabajo útil, el calor evacuado con el refrigerante y el calor fugado con los gases de escape, con proporciones mayores de pérdidas a bajas cargas [3.23]. Las proporciones varían mucho en función de la aplicación del motor, de su tecnología y también de la potencia del mismo. A mayores potencias se suele tener menos pérdidas relativas de calor disipado al refrigerante.

En consideración a las tendencias de control de la combustión y de la renovación de la carga del motor Diesel, y a la gestión térmica en general del motor, la descomposición porcentual de la energía suministrada con el combustible en condiciones nominales de operación para el nivel de perfeccionamiento actual de los motores Diesel, se acerca a la descrita por la Figura 3.3. La figura incorpora las disipaciones de calor al refrigerante en los enfriadores de los gases de escape recirculados al cilindro y en el enfriador del aire (mezcla de gases) de admisión. El calor disipado al ambiente no es fácil de evaluar, como se hizo evidente al explorar la información disponible y como se anotó en el parágrafo 2.6.

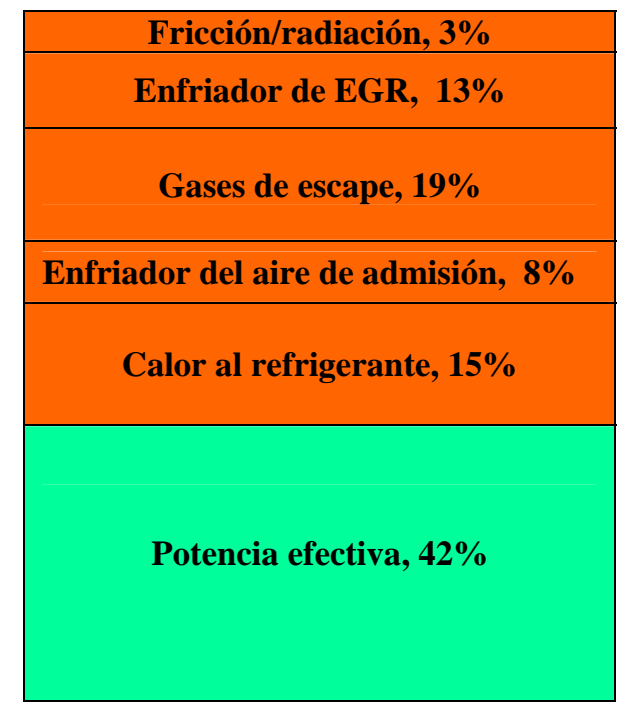

Figura 3.3. Aproximación al balance térmico de un motor Diesel moderno. 
En un plano menos prospectivo, en la gráfica de la Figura 3.4 se ha particularizado el calor disipado al refrigerante de algunos motores comerciales, discriminando los calores disipados directamente al sistema de refrigeración del motor y al enfriador del aire de admisión. La aplicación de enfriamiento de la carga fresca ha sido, por razones de costo, más empleada en los motores de potencias mayores, pero gradualmente se empieza a aplicar también en los motores de vehículos de pasajeros.

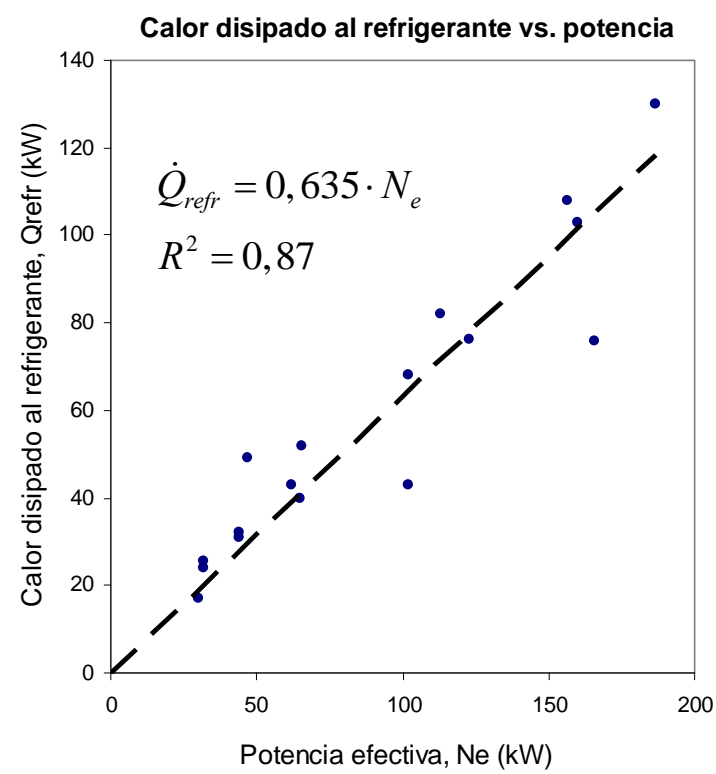

Figura 3.4. Calor disipado al refrigerante que pasa por el motor. Los motores analizados incluyen por igual motores con enfriador de aire $y$ sin él.

Ha existido la idea de que si las superficies internas de la cámara de combustión se mantienen a mayor temperatura, las pérdidas de calor pueden ser menores (Morel [3.39], [3.48]), lo que ha dado lugar a la experimentación con aislamientos de las superficies empleando materiales cerámicos (Taymaz [3.20] realizó una revisión bastante extensa de los trabajos más relevantes realizados en este tema). En las gráficas de la Figura 3.5 se ilustran los balances energéticos de un motor con aislamiento y sin aislamiento, reproducidas del trabajo de Descombes [3.40], quien recientemente presentó los resultados de un estudio experimental de un motor Diesel sobrealimentado. Este motor tenía un aislamiento en la precámara de combustión y un recubrimiento cerámico en la cara superior del pistón. Los resultados de las pruebas mostraron que la eficiencia energética del motor permanece casi constante y que la reducción de las pérdidas de calor a las paredes redunda en un incremento simultáneo de la energía contenida en los 
gases de escape. La fracción acumulada de los flujos de calor hacia el refrigerante, de un lado, y hacia el escape, por otro lado, permanece casi constante cuando el motor opera bajo carga. Una análisis bastante fundamental de las implicaciones termodinámicas del empleo de aislamientos y los posibles alcances, apartir de la Segunda Ley de la Termodinámica, fue realizado por Caton [3.28], quien, entre otras conclusiones, afirma que, promoviendo que los procesos de combustión se realicen a mayores temperaturas (como resultado, por ejemplo, de la utilización de aislamientos cerámicos en la cámara de combustión), se minimiza la destrucción de la disponibilidad de la energía del combustible resultado de los procesos de combustión, aumentándose las temperaturas de los gases de escape, e incrementándose los niveles de disponibilidad en ellos.

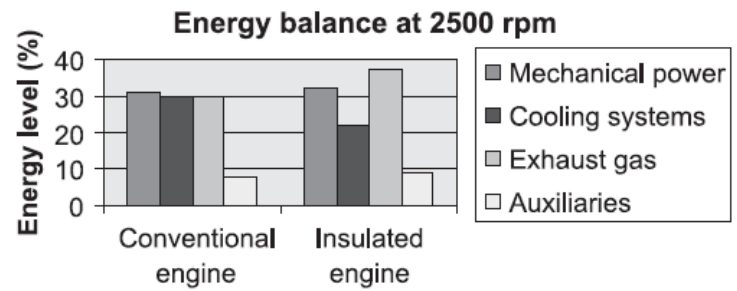

Figura 3.5. Comparación entre los balances

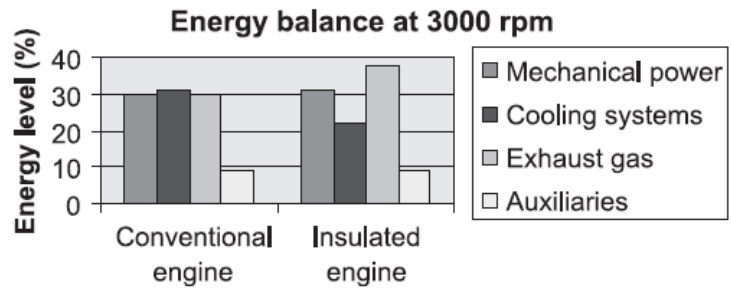
energéticos de un motor con aislamiento y sin aislamiento [3.40].

\subsubsection{Balance energético durante el calentamiento del motor}

\subsubsection{Consideraciones teóricas y experimentales}

Un motor térmico convierte la energía térmica de un fluido en trabajo mecánico, de acuerdo a una secuencia de procesos termodinámicos que se suceden en su interior. Esto incluye la transferencia de calor y la variación del volumen del fluido. El tipo de motor más sencillo opera recibiendo calor de un foco térmico que está a una temperatura superior, tras lo cual realiza un trabajo mecánico y libera parte del calor recibido a un segundo foco térmico de menor temperatura, como se esquematiza en la Figura 3.6 (esta forma de presentar el motor se corresponde con el enunciado de la Segunda Ley de la 
Termodinámica). Un motor de combustión es un motor térmico en el que la energía térmica es producida por un proceso de combustión. Un motor de combustión interna es un motor de combustión en el que los productos de la reacción de un oxidante (usualmente aire) actúan como fluido de trabajo. Esto necesariamente incluye el flujo de masas frescas de oxidante y combustible hacia el motor y el flujo de sustancias quemadas fuera del motor. Un motor de combustión interna alternativo utiliza un mecanismo biela-manivela para la aspiración y expulsión de las sustancias de trabajo de su interior y para la transformación de la fuerza expansiva de los gases, en movimiento lineal reciprocante de un pistón y movimiento circular de una manivela solidaria con el árbol de salida de la potencia mecánica.

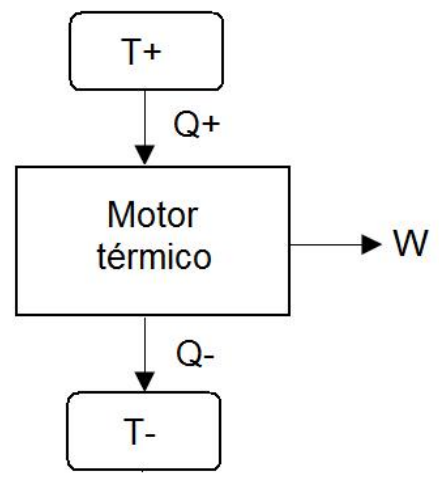

Figura 3.6. Representación esquemática de un motor térmico. El motor recibe $Q+$ Joulios de calor del foco térmico a temperatura $T+K y$ libera $Q$ - Joulios de calor al foco térmico que se encuentra a $T$ - $K$ de temperatura. El motor entrega $W=$ $Q^{+}$- Q- Nm de trabajo mecánico.

\section{El motor entendido como máquina de trabajo}

Al analizar la termodinámica de la combustión en un motor alternativo, se entiende que el sistema está comprendido por el gas atrapado en la cámara de combustión durante la compresión, la combustión y la expansión, como se ilustra en la Figura 3.7. Este gas es una mezcla de combustible, aire, gases residuales y EGR. Durante la combustión los reactantes se convertirán en productos de combustión $\left(\mathrm{CO}_{2}, \mathrm{H}_{2} 0\right.$, $\mathrm{CO}$, etc.) y la energía química se convertirá en energía térmica.

Esquematizando la cámara de combustión como se hace en la Figura 3.7, puede decirse que ésta es el volumen encerrado por las paredes del cilindro, la superficie interna de la culata, la superficie superior del pistón y las válvulas. Las paredes de la cámara de combustión constituyen las fronteras del sistema. Todo el espacio de combustión puede verse como un volumen lleno por una masa de aire fresco, gases quemados, gases que pasan desde el cárter del 
motor y combustible inyectado. Aplicando la Primera Ley de la Termodinámica se obtiene la descomposición de la energía del sistema, como se plantea en la ecuación (3.8) y que literalmente equivale a decir que la variación de la energía del sistema $\left(E_{s i s}\right)$, asumiendo que no hay pérdidas de energía en deformaciones del volumen de control, se reduce a la variación de la energía interna del sistema $(U)$.

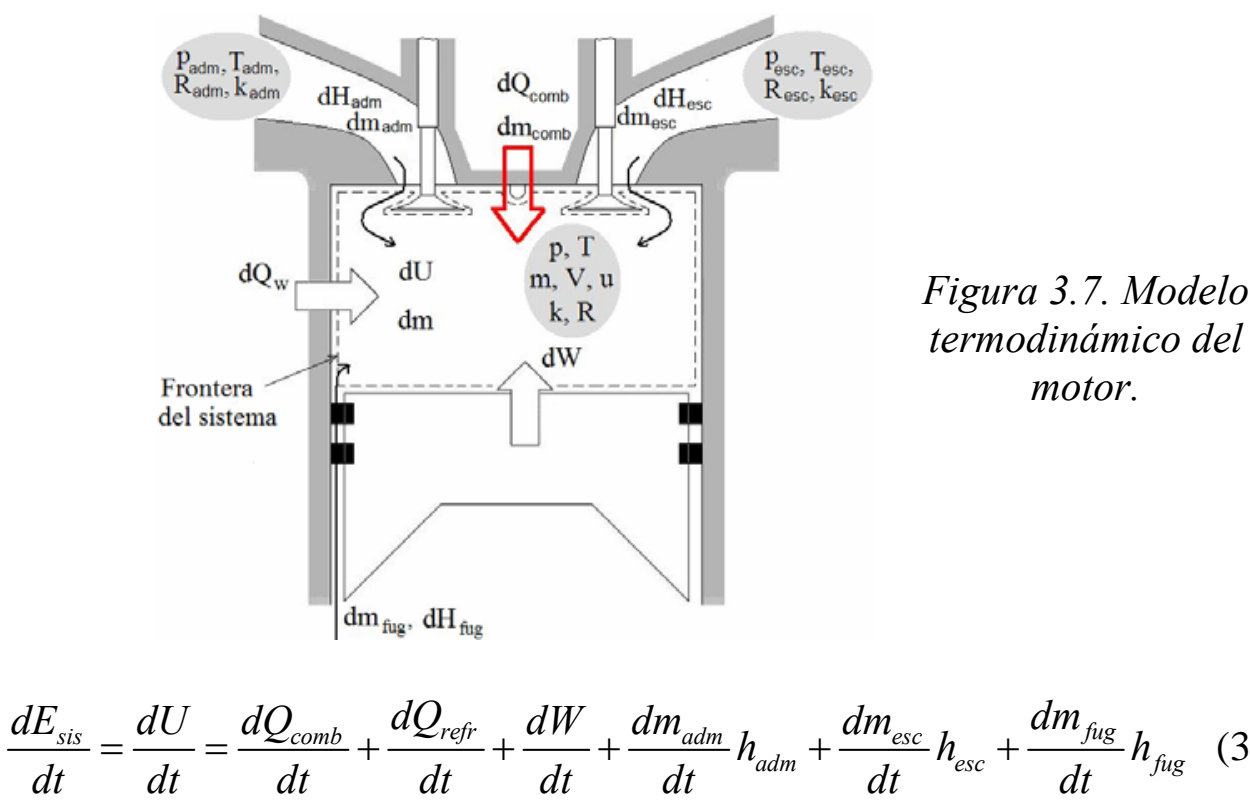

La variación de la energía interna del sistema en el motor de combustión interna es la resultante de la variación por unidad de tiempo de la energía suministrada al sistema con el combustible $\left(Q_{c o m b}\right)$ y la carga de admisión $\left(m_{a d m} h_{a d m}\right)$, el calor realizado en forma de trabajo mecánico incluyendo las pérdidas mecánicas $(W)$, el calor disipado a través de las paredes al refrigerante $\left(Q_{\text {refr }}\right)$, el calor equivalente de los gases de escape referidos a las condiciones de admisión $\left(m_{\text {esc }} h_{\text {esc }}\right)$ y el calor equivalente de los gases de la cámara fugados a través de los intersticios en los segmentos $\left(m_{f u g} h_{f u g}\right)$.

Entendido el motor como máquina de realización de trabajo, la Figura 3.7 da una cabal representación del sistema para ser modelado desde el punto de vista termodinámico. No obstante esto, el motor no puede funcionar con su solo volumen de trabajo y debe interactuar con el medio externo para renovar 
la fuente de energía (inyección de combustible y admisión de aire fresco), evacuar los productos de combustión, asegurar mediante un sistema adecuado (sistema de refrigeración) los límites de temperatura seguros para la operación de sus componentes y garantizar el mínimo de pérdidas mecánicas y desgaste de los componentes (necesidad de un sistema de lubricación). La integración de los subsistemas le da funcionalidad e integralidad al motor, pero también se corresponde con el esquema de repartición de las pérdidas del motor como máquina.

\section{El motor visto como sistema de gestión térmica}

Físicamente, el motor como parte integral del sistema propulsivo de un vehículo se compone de la máquina propiamente hablando, fundamentada en el mecanismo manivela-deslizador para la realización del trabajo mecánico y el conjunto de subsistemas que posibilitan que este mecanismo pueda funcionar continuamente y que la combustión sea eficiente y ambientalmente aceptable. Para esto, como se explicó anteriormente en la introducción, la estructura del motor debe complementarse con los subsistemas de lubricación, refrigeración, escape, admisión e inyección de combustible. Los subsistemas de refrigeración y escape pueden integrar funciones adicionales demandadas por las estrategias de renovación de la carga y combustión (turboalimentación, refrigeración de la carga de admisión y de los gases recirculados) y demandas de sistemas externos al motor como el sistema de calentamiento del habitáculo del conductor o de refrigeración del aceite de la transmisión, entre otros. Al momento de analizar la manera en que se descompone la energía proveniente del combustible es útil mirar todos los subsistemas descritos como volúmenes de control, como de manera sintética y respondiendo a la práctica experimental se representa en el esquema de la Figura 3.8.

La Figura 3.8 representa esencialmente el equivalente mecánico en diagrama de bloques del modelo termodinámico representado en la Figura 3.7, con la diferencia de que en ella se ha introducido el término de pérdidas misceláneas que dan cuenta no sólo de las pérdidas por fugas de gases, sino también de otras pérdidas propias del motor real como las disipadas al ambiente a través de su superficie externa y la energía almacenada en la masa estructural del motor y en las masas de los fluidos. También debe agregarse que en el modelo termodinámico no se tienen en cuenta las pérdidas mecánicas, 
mientras que en el esquema de la Figura 3.8 las pérdidas por fricción sí están presentes e incluidas en la energía del refrigerante.

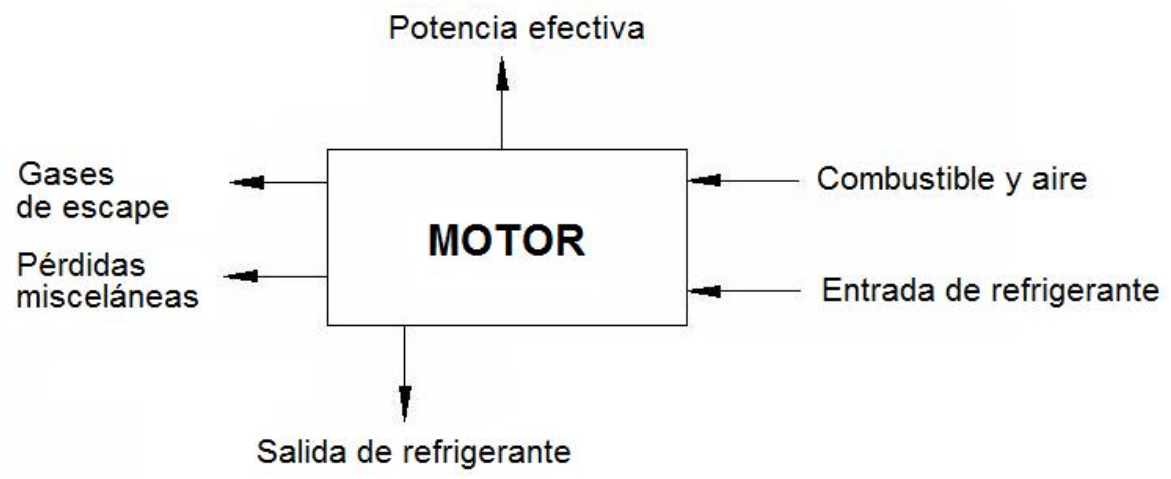

Figura 3.8. Esquema para la descripción del balance energético del motor.

Las sustancias de entrada y salida son transportadoras de energía.

Basándonos en el esquema de la Figura 3.8, el balance energético del motor se realiza en este trabajo, de manera práctica, midiendo los flujos másicos de aire y combustible, el refrigerante, los gases de escape y también midiendo las revoluciones y el momento torsor del motor, con los que se calcula la potencia efectiva.

Para efectos prácticos de cálculo del balance energético del motor, la ecuación (3.8) puede reescribirse como:

$$
\dot{m}_{\text {comb }} H_{i_{-} \text {comb }} \eta_{\text {comb }}+\dot{m}_{\text {air }} h_{\text {air }}=\dot{Q}_{\text {equiv }}+\dot{m}_{\text {air }} h_{\text {air }}=N_{e}+\dot{Q}_{\text {refr }}+\dot{Q}_{\text {misc }}+\dot{Q}_{\text {esc }}
$$

donde, $\dot{m}_{\text {comb }} \mathrm{y} \dot{m}_{\text {air }}$ corresponden a los flujos másicos de combustible y aire, respectivamente, $h$ denota la entalpía, $\dot{Q}_{\text {equiv }}$ es la energía desprendida por la combustión del combustible de poder calorífico $H_{i_{-} \text {comb }}, \eta_{\text {comb }}$ representa el rendimiento de la combustión, $N_{e}$ es la potencia media efectiva, $\dot{Q}_{\text {refr }}$ es el calor disipado al refrigerante (calor transferido a través de las paredes de la cámara de combustión, más el calor proveniente del intercambio con el aceite del motor, los gases de admisión y escape), y $\dot{Q}_{\text {misc }}$ es el término que cierra el 
balance e integra el calor evacuado al aceite refrigerante (en caso de que el aceite tenga refrigeración independiente del sistema de refrigeración del motor), más el calor disipado por convección y por radiación al ambiente que rodea el motor y también la energía almacenada en las masas del motor durante los procesos transitorios. Durante el calentamiento del motor parte de (si no todas) las pérdidas misceláneas se utilizan en el calentamiento de las partes estructurales y de la masa de los fluidos, mientras no se opera el termostato.

La potencia efectiva del motor se obtiene conociendo las revoluciones y el par del motor:

$$
N_{e}=M_{e} \frac{\pi n}{30}
$$

Siendo $n$ las revoluciones (en rpm) y el par efectivo del motor, respectivamente. La energía térmica disipada en el motor por convección al refrigerante puede calcularse por la expresión:

$$
\dot{Q}_{\text {refr }}=\dot{m}_{r e f r} c_{p_{-} r e f r}\left(T_{r e f r}^{s a l}-T_{r e f r}^{e n t}\right)
$$

Siendo $\dot{m}_{\text {refr }} \mathrm{y} \quad c_{p_{-} \text {refr }}$ el flujo másico y el calor específico del refrigerante, $\mathrm{y}$ $T_{\text {refi }}^{\text {ent }}$ y $T_{\text {refr }}^{\text {sal }}$ las temperaturas del refrigerante a la entrada y la salida del motor, respectivamente. La energía que se evacúa con los gases de escape referenciada a la temperatura de admisión se determina midiendo los gastos másicos de combustible y de aire $\left(\dot{m}_{\text {comb }}, \dot{m}_{\text {air }}\right)$, y las temperaturas del aire de admisión y de los gases de escape $\left(T_{a d m}, T_{e s c}\right)$, tras determinar las entalpías de la carga de trabajo, $h_{a d m}$ y de los gases de escape, $h_{e s c}$, y asumiendo que la temperatura del combustible ( $T_{\text {comb }}$ ) es igual a la temperatura de admisión, empleando la expresión:

$$
\dot{Q}_{e s c}=\left(\dot{m}_{c o m b}+\dot{m}_{a i r}\right)\left[h_{e s c}\left(T_{e s c}\right)-h_{a d m}\left(T_{a d m}\right)\right]
$$

Durante el calentamiento del motor, despreciando la disipación de calor a través de las superficies externas del motor, parte de la energía suministrada con el combustible se almacena en forma de calor en las masas de la 
estructura, mientras que otra parte se transporta con los fluidos, refrigerante y aceite, progresivamente según la expresión:

$$
\frac{d Q_{d i s}}{d t}=m_{b l} c_{p b l} \frac{d T_{b l}}{d t}+\dot{m}_{r e f r} c_{p_{-} r e f r} d T_{r e f r}+\dot{m}_{a c} c_{p_{-} a c} d T_{a c}
$$

donde $Q_{d i s}$ representa el calor total disipado por el motor a través de las paredes; $T_{r e f r}$ y $T_{a c}$ son las temperaturas del refrigerante y del aceite, $m_{b l}$ y $c_{p b l}$ representan la masa térmicamente sensible del bloque y el calor específico del material, $\dot{m}_{r e f r}$ y $\dot{m}_{a c}$ son los flujos másicos de refrigerante y aceite a través de la camisa de refrigeración y las galerías de lubricación, respectivamente (el flujo del aceite es más complejo y la inclusión del término en la expresión es una aproximación grosera que no contempla el volumen de aceite en el cárter), y $c_{p_{-} a c}$ representa el calor específico del aceite.

\section{Descripción de la instalación experimental}

La instalación está integrada por siete sistemas constituidos por el motor equipado totalmente respondiendo a su aplicación vehicular, el sistema de control del motor basado en un dinamómetro, el sistema de control de temperatura del agua, el sistema de instrumentación, el sistema de medida del gasto de combustible, el sistema de medida del gasto de aire y el sistema de adquisición de información. El motor utilizado es un motor Diesel rápido turboalimentado de inyección directa (HSDI), cuyas características principales se describen en la tabla 3.1.

Tabla 3.1. Características del motor

\begin{tabular}{ll}
\hline Tipo & 4 cilindros 2.0 l HSDI \\
\hline Relación de Compresión & 18 \\
Potencia máxima & $100 \mathrm{~kW}$ a $4000 \mathrm{rpm}$ \\
Revoluciones máximas & $4750 \mathrm{rpm}$ \\
\hline
\end{tabular}

El dinamómetro es un motor AC, controlado por un actuador, que opera a frecuencia variable, con características especiales para controlar el par de 
frenado y arrastrar el motor en prueba con rapidez. El dinamómetro está dotado de un programa computacional que permite simular transitorios de carga y condiciones de operación de vehículo en carretera, configurando previamente las características del sistema motor-vehículo-carretera. Para reproducir el funcionamiento del motor instalado en un vehículo marchando bajo un ciclo de conducción, el dinamómetro debe configurarse con las características dinámicas básicas del sistema de tracción, las características aerodinámicas del vehiculo y las características resistivas del terreno por el cual "circulará" el vehículo. En la tabla 3.2 se presenta un resumen de los parámetros del sistema vehículo-carretera.

Tabla 3.2. Configuración de los parámetros vehículo-carretera

\begin{tabular}{ll}
\hline Vehículo & ------ \\
Parámetro & Valor \\
Tipo de embrague & Disco seco \\
Marchas de la caja de velocidades & Relación de transmisión \\
$1^{\mathrm{a}}$ & 3,417 \\
$2^{\mathrm{a}}$ & 1,841 \\
$3^{\mathrm{a}}$ & 1,167 \\
$4^{\mathrm{a}}$ & 0,8238 \\
$5^{\mathrm{a}}$ & 0,6730 \\
Transmisión principal & 3,690 \\
Masa del vehículo & $1700 \mathrm{~kg}$ \\
Batalla & $2,795 \mathrm{~m}$ \\
Inercia caja de velocidades & 0,0140 \\
Inercia cardán & $0,410 \mathrm{kgm}$ \\
Inercia rueda delantera & $1,033 \mathrm{kgm}{ }^{2}$ \\
Inercia rueda trasera & $1,033 \mathrm{kgm}^{2}$ \\
Eficiencia de la transmisión & 0,89 \\
Radio ruedas & $0,321 \mathrm{~m}$ \\
Coeficiente de resistencia aerodinámico & 0,300 \\
Constante de rozamiento & $185 \mathrm{~N}$ \\
Superficie frontal & $2,223 \mathrm{~m}^{2}$ \\
Densidad del aire & $1,202 \mathrm{~kg}^{3}$ \\
\hline
\end{tabular}

El refrigerante del motor instalado en el vehículo se enfría cediendo calor al aire que atraviesa el radiador a una velocidad determinada por la velocidad 
del vehículo y la operación del ventilador. En el banco de ensayos, el radiador del motor en el vehículo es sustituido por un intercambiador agua-agua de características equivalentes al radiador original. La temperatura del refrigerante se controla a la salida del motor con un controlador PID.

Los parámetros que se registran en el banco dinamométrico son el par del motor, el régimen de giro, el consumo de combustible, el gasto de aire, la temperatura del refrigerante a la entrada y la salida del motor, la temperatura de los gases de escape, las condiciones ambientales de la prueba $y$, eventualmente, las emisiones contaminantes de los gases de escape. Las variables instantáneas (presión en el cilindro, presión de admisión, presión en el escape) se miden mediante un sistema de adquisición con velocidad de muestreo de hasta $100 \mathrm{kHz}$ por canal. Para sincronizar de manera precisa la información de las variables instantáneas con la posición correspondiente del cigüeñal, se emplea un codificador angular óptico de $0,1^{\circ}$ de giro de cigüeñal de resolución angular, como señal de muestreo. La resolución angular seleccionada para la adquisición de la información es de 0,5 grados de giro del cigüeñal, es decir, 1440 puntos por cada ciclo termodinámico del motor, de manera que la frecuencia de muestreo queda comprendida entre $15 \mathrm{kHz}$ y $27 \mathrm{kHz}$, dependiendo de las revoluciones del motor.

La variación de la presión en los flujos de admisión y escape se mide mediante sensores piezoresistivos. La presión dentro del cilindro se mide con un sensor piezoeléctrico no refrigerado. La presión de referencia se fija asumiendo que la presión en el cilindro correspondiente al punto muerto inferior posterior a la carrera de admisión es igual a la presión media de admisión.

El flujo de combustible se mide mediante dos procedimientos independientes: empleando una balanza gravimétrica, instalada en la línea de combustible que alimenta la bomba de inyección, y que realiza mediciones a una frecuencia de $10 \mathrm{~Hz}$, y empleando la información de la unidad electrónica de control, la cual provee una estimación del flujo volumétrico instantáneo de combustible a una frecuencia de $200 \mathrm{~Hz}$. Para obtener una medida precisa del flujo de combustible se combinan las mediciones de estas dos señales.

La medición del par se logra mediante la señal entregada por una célula de carga instalada en uno de los extremos de la carcasa basculante del 
dinamómetro. La señal del codificador angular sirve de base para la medición del régimen de giro.

El flujo másico de aire se evalúa mediante la señal de un medidor de hilo caliente. Los flujos de refrigerante en los tramos del sistema de refrigeración se miden con medidores de flujo electromagnéticos. Las temperaturas de la carga de admisión, del refrigerante, del aceite y de los gases de escape se realizan con termopares tipo $\mathrm{K}$, con aislamiento de radiación para la medición de la temperatura de los últimos. Se posicionaron dos termopares en la parte posterior de la culata del motor para monitorizar la temperatura de la superficie exterior del motor, en contacto con el ambiente. Estas temperaturas son usadas como referencia durante los estudios de calentamiento del motor.

Todos los parámetros medidos durante los ensayos son registrados, acondicionados y enviados a un ordenador para su almacenamiento y tratamiento posterior.

\section{El sistema de refrigeración del motor}

El sistema de refrigeración del motor integra, además de los elementos de un sistema de refrigeración convencional, dos intercambiadores de calor para enfriar los gases de escape recirculados y para calentar el habitáculo de pasajeros. La Figura 3.9 ilustra la composición de la parte hidráulica del sistema de refrigeración del motor. La otra parte del sistema, relacionada con el flujo de aire para la disipación del calor en el radiador, no fue objeto de mediciones y no aparece representada en el esquema, pues para efectos del estudio interesa solamente el proceso de calentamiento, hasta que el termostato entra en funcionamiento. Como se explicó en el apartado anterior el radiador original es reemplazado en el banco de ensayos por un radiador agua-agua de flujos y temperaturas controladas.

El calentador del habitáculo usado en los ensayos corresponde al original y se instala con el ventilador apagado, con lo que el calor transferido es el correspondiente a la convección natural impuesta por la temperatura de la sala de ensayos, mantenida a un valor constante de $23-24{ }^{\circ} \mathrm{C}$.

Como se desprende de la Figura 3.9, el sistema de refrigeración del motor de prueba es un sistema convencional con la introducción de un ramal adicional 
a la salida de la caja de agua del motor, conducente al enfriador de los gases de escape recirculados al motor (EGR), en serie con el calentador del habitáculo de pasajeros. En la instalación se posicionaron medidores de temperatura y caudal a la entrada y salida de los intercambiadores de calor del circuito.

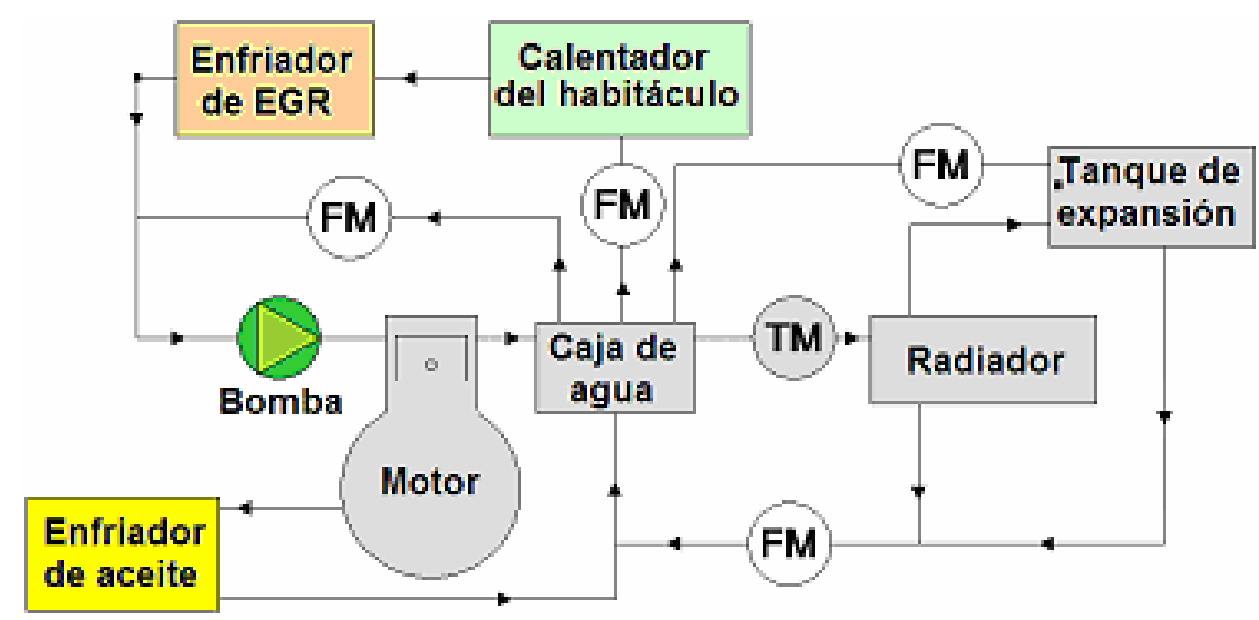

Figura 3.9. Representación esquemática del sistema de refrigeración del motor utilizado para la prueba de calentamiento durante el ciclo de conducción (FM-medidores de flujo de refrigerante).

\subsubsection{Balance energético experimental}

Previo al análisis energético del motor es pertinente ubicar los puntos característicos del ciclo NEDC en el mapa de consumo del motor. En la Figura 3.10(a) se ilustra el mapa de consumo de combustible en función de la presión media efectiva y de las revoluciones del eje de salida del motor. En la misma gráfica se han dibujado los puntos de consumo correspondientes a las revoluciones y presión media efectiva desarrollados por el motor cuando el vehículo en el que éste se instala marcha a las velocidades características del ciclo NEDC, luego de que el motor se ha calentado. Los puntos de operación son correspondientes a los del ciclo pero se han tomado en condiciones estacionarias.

Como se ve en la figura el ciclo de conducción europeo es modesto en la demanda de potencia del motor y ocupa la zona baja del mapa, donde los 
consumos de combustible y también los rendimientos del motor son muy bajos. Sin embargo el ciclo es representativo de la utilización que se le da al vehículo durante su vida útil y la identificación térmica del motor en esas condiciones de operación, en consecuencia, es de mucha importancia para las estrategias asociadas con la optimización de las prestaciones y del consumo de combustible y también de la reducción de las emisiones contaminantes.

En la Figura 3.10(b) se ilustran los resultados experimentales del balance térmico, referido a los valores de energía suministrada con el combustible, del motor bajo cuatro puntos de operación estabilizados de bajas cargas estando el motor caliente. Se destaca la reducción de las pérdidas específicas por refrigeración del motor a medida que aumenta la carga, constituyendo cerca del 19 \% para el más cargado de los ensayos (35 \% de carga).

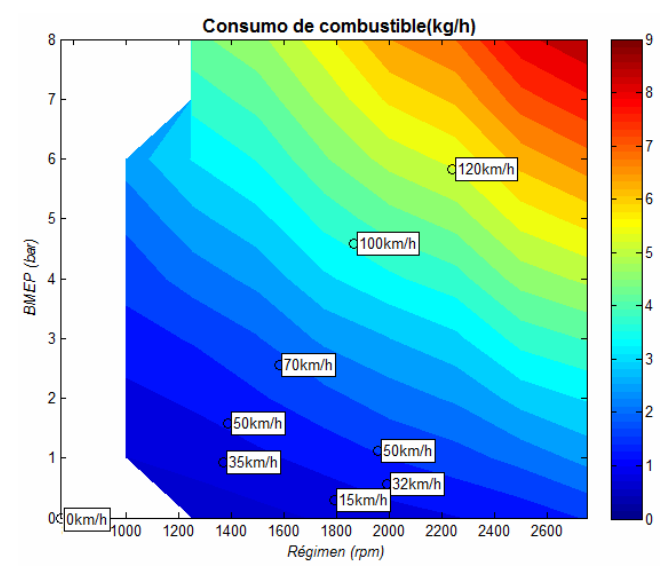

a)

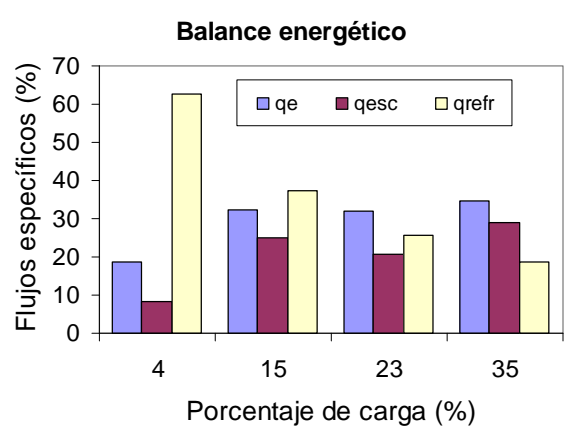

b)

Figura 3.10, a) Mapa de consumo de combustible del motor y representación de los puntos de operación a las condiciones impuestas por el ciclo de conducción NEDC. b) Balance térmico del motor operando en varios puntos de operación a cargas parciales bajas.

\subsubsection{Balance térmico del motor durante su calentamiento bajo una carga y régimen constantes, representativos del ciclo de conducción}

Para analizar la variación del balance energético del motor durante su calentamiento bajo carga constante, se ha elegido el punto de operación correspondiente a $1500 \mathrm{rpm}$ y $50 \mathrm{Nm}$, frecuente en la conducción del 
vehículo en ciudad. Las temperaturas del motor (temperatura de salida del refrigerante, $T_{\text {refr }}$, temperatura de los gases de escape, $T_{\text {esc }}$, temperatura del aceite, $T_{a c}$,y la temperatura de la parte externa del bloque, $T_{b l}$ ), registradas en 14 instantes a lo largo de los 400 segundos de duración de las mediciones, se ilustran en la Figura 3.11. El número de mediciones durante el ciclo es limitado por las características de muestreo y registro de información del sistema de adquisición.

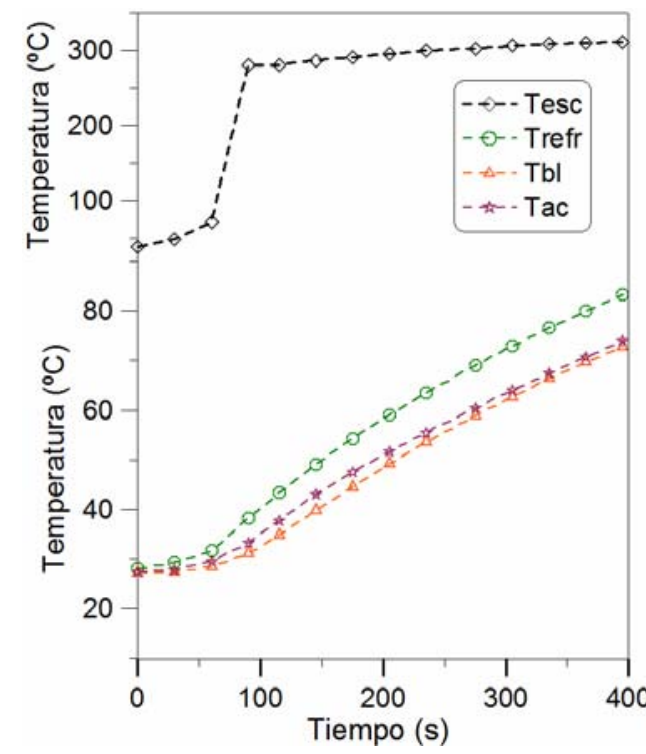

Figura 3.11. Temperaturas del motor durante su calentamiento bajo carga constante.

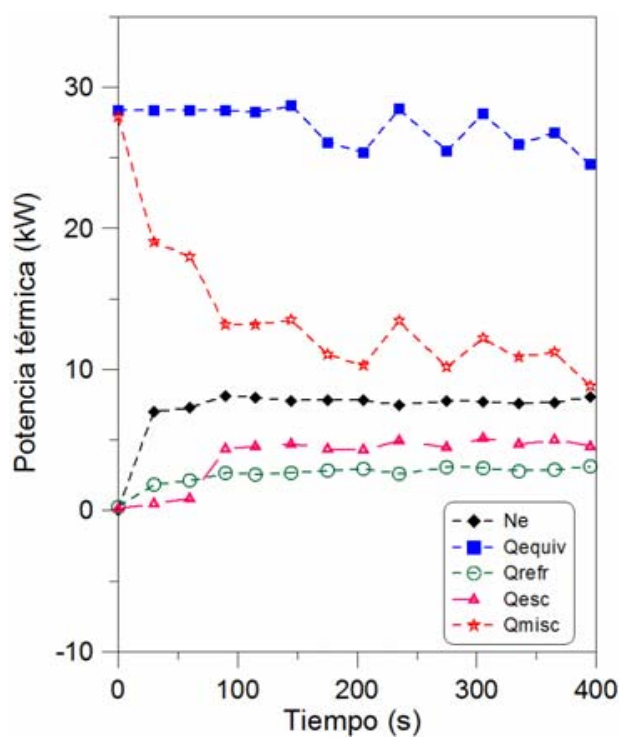

Figura 3.12. Distribución de la energía del motor durante su calentamiento bajo carga constante.

La duración de la prueba se limitó al tiempo requerido para que el refrigerante alcanzara los $80{ }^{\circ} \mathrm{C}$, temperatura de consigna, a pesar de que aún no se había abierto el termostato y las temperaturas del aceite, el bloque, el refrigerante y el aceite no se habían estabilizado. El refrigerante alcanzó los $80{ }^{\circ} \mathrm{C}$ a los 395 segundos después de iniciada la prueba. La temperatura podría alcanzarse más rápidamente si fuera menor el flujo de refrigerante por la camisa de refrigeración y fueran también menores las masas térmicamente sensibles del refrigerante y del entorno de la cámara de combustión (la masa 
del bloque de la parte externa a la camisa de refrigeración también influye, pero no es tan determinante como la primera). La temperatura de los gases de escape aumenta bastante rápido, como es de esperar, mientras que las otras temperaturas tardan más en estabilizarse. La temperatura medida en la superficie externa de la estructura del motor sigue ligeramente por debajo el crecimiento de la temperatura del aceite.

Los procesos de transferencia de calor en el motor son muy complejos, puesto que tienen que ver con todas las condiciones de contorno estudiadas en el capítulo dos, y no es sencillo realizar una identificación del sistema. Asumiendo que, alcanzada la temperatura de consigna del refrigerante también se ha alcanzado la temperatura "estabilizada" de las paredes internas del motor a los 395 segundos, y atreviéndonos a considerar esta parte interna del motor como un sistema de primer orden con constante de tiempo $\tau=m_{t} C_{t} / \overline{h_{g}} A_{t}$, como se esquematiza en la Figura 3.13, su temperatura se puede describir por la expresión:

$$
T_{m_{t}}(t)=T_{0}+\left(T_{e s t}-T_{0}\right)\left(1-\exp \left(-\frac{t \cdot \bar{h}_{g} A_{t}}{m_{t} c_{t}}\right)\right)
$$

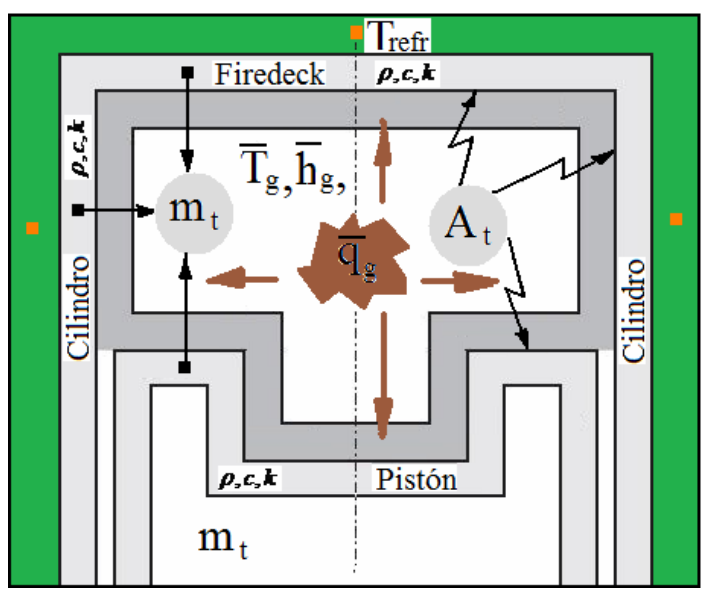

Figura 3.13. Esquema de reducción del calentamiento del motor a un proceso de primer orden.

En el modelo representado por la expresión (3.14), la inercia térmica está dada por la masa interna del motor (masa de las paredes de la cámara de combustión), $m_{t}$, y el calor específico de material $c_{t}=900 \mathrm{~J} / \mathrm{kgK}$ (aluminio). 
La masa se calienta por la transferencia de calor por convección de los gases de la cámara de combustión, con un coeficiente de transferencia de calor $\bar{h}_{g}=$ $320 \mathrm{~W} / \mathrm{m}^{2} \mathrm{~K}$ (calculado para el punto de operación estudiado), a través de un área equivalente, $A_{t}=0,112 \mathrm{~m}^{2}$ (área mayor de la cámara de combustión considerando los pistones en el $P M I$ ). Tomando la constante de tiempo igual a la cuarta parte del tiempo requerido para alcanzar el punto de consigna, $\tau=$ $99 \mathrm{~s}$, llegamos a estimar la masa térmica equivalente como $m_{t}=3,96 \mathrm{~kg}$ (masa sensible entre los cilindros y las condiciones de contorno, incluye parte de las masas del pistón, los cilindros y la culata).

Tras alcanzarse los $80^{\circ} \mathrm{C}$ el termostato está pronto a abrirse y la temperatura del refrigerante empezará a mantenerse en torno a este valor, en función de la característica del sistema y del termostato. No obstante, las temperaturas del aceite y el bloque continuarán creciendo ligeramente, i. e. aún hay inercia térmica por superar en el sistema. La masa térmica esbozada corresponde a una masa sensible del motor, la cual responde más rápidamente al calentamiento, pero en la parte metálica externa de la camisa de refrigeración (con una mayor masa) la temperatura continuará aumentando.

Si consideráramos estabilizada la temperatura del refrigerante y de la estructura del motor al mismo tiempo, asumiendo un coeficiente de convección entre el refrigerante y las paredes de la camisa de refrigeración de $10200 \mathrm{~W} / \mathrm{m}^{2} \mathrm{~K}$, un calor específico combinado de las masas metálicas y del refrigerante de $1360 \mathrm{~J} / \mathrm{kgK}$ y un área interna en contacto con el refrigerante de $0,13 \mathrm{~m}^{2}$, la masa térmica del motor para la dinámica del sistema de refrigeración constituiría cerca de $97 \mathrm{~kg}$.

La energía total liberada, denominada en este trabajo la energía equivalente suministrada con el combustible, ( $\dot{Q}_{\text {equiv }}$ ) se obtiene, asumiendo que no hay pérdidas de combustión, como el producto del flujo de combustible $\dot{m}_{\text {comb }}$ por el poder calorífico del combustible $H_{i}\left(H_{i}=42500 \mathrm{~kJ} / \mathrm{kg}\right)$ y aparece representada durante el calentamiento en la Figura 3.12. La fluctuación dibujada de la energía equivalente del combustible es debida a las fluctuaciones en las mediciones instantáneas experimentales del consumo de combustible. Realmente el consumo debe caer ligeramente de manera 
monótona a medida que el motor se calienta bajo la carga y el régimen constantes. La energía suministrada con el aire no se tiene en cuenta porque será considerada en la energía de los gases de escape (dado que el cálculo de la energía térmica de los gases de escape se referencia a la temperatura de admisión).

Las expresiones (3.9 - 3.12) permiten determinar los flujos de potencia efectiva, $N_{e}$, disipada al refrigerante, $\dot{Q}_{\text {refr }}$, evacuada con los gases de escape, $\dot{Q}_{e s c}$ y las pérdidas misceláneas, $\dot{Q}_{\text {misc }}$. Se observa la reducción continua de la energía correspondiente a las pérdidas misceláneas, a favor del aumento del calor disipado al refrigerante y de la reducción del consumo de combustible.

La Figura 3.12 es la representación gráfica del balance térmico descrito por la ecuación (3.9), a medida que el motor se calienta bajo la carga constante, mientras que la Figura 3.14 muestra el balance térmico al final del calentamiento del motor. Se destaca la influencia del término de "pérdidas misceláneas", una componente del balance en la que, como se dijo anteriormente, se incluyen los flujos de energía no valorados explícitamente en la expresión y que cierra el balance energético. Este término es difícil de descomponer, pero ya se ha explicado que incluye el calor almacenado en las masas del motor y el calor disipado a través de las superficies externas del mismo. Para el punto de operación analizado el calor disipado a través de la superficie externa es bastante elevado cuando se ha llegado a los 395 segundos, que es el tiempo al cual se ha disparado el termostato $\left(T_{\text {refr }}=80\right.$ ${ }^{\circ} \mathrm{C}$ ), y la temperatura externa del bloque es cercana a los $73{ }^{\circ} \mathrm{C}$. Realmente este término continuará descendiendo a medida que toda la estructura del motor y el aceite lleguen a sus temperaturas de estabilización. Un estimativo del flujo de energía hacia el ambiente teniendo en cuenta esa temperatura media del bloque de $73{ }^{\circ} \mathrm{C}$ y una temperatura media del ambiente de $23{ }^{\circ} \mathrm{C}$, con un coeficiente de convección medio entre el cuerpo del motor y el aire circundante de $35 \mathrm{~W} /\left(\mathrm{m}^{2} \mathrm{~K}\right)$ y un área de transferencia de calor externa de 1 metro cuadrado (subestimada) da como resultado un valor de $1,7 \mathrm{~kW}$ de potencia térmica disipada, que viene a corresponder al $7 \%$ del flujo energético suministrado para este instante con el combustible, y a un $20 \%$ de las pérdidas misceláneas. El otro $80 \%$ de las pérdidas misceláneas está comprometido con el calentamiento que todavía están sufriendo las masas del motor, incluyendo el aceite. 


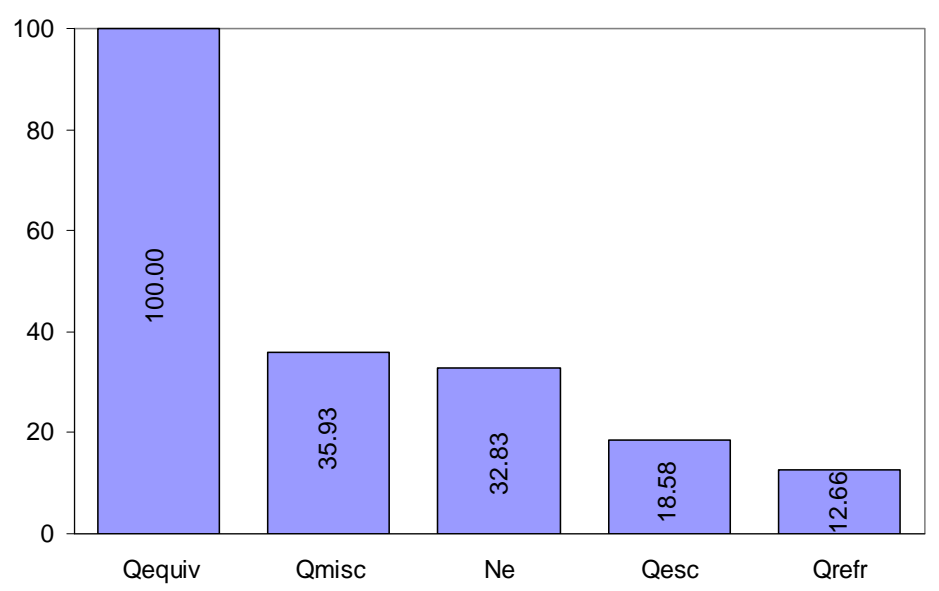

Figura 3.14. Distribución global de la energía en el motor a los 395 segundos de su periodo de calentamiento bajo carga y régimen constante: momento torsor de $50 \mathrm{Nm}$ y $1500 \mathrm{rpm}$.

Si asumimos que el término misceláneo se usa enteramente para aumentar la temperatura del bloque, conociendo la temperatura media de éste (la temperatura medida de la superficie externa), puede estimarse la capacitancia térmica del motor, es decir, la masa térmica equivalente referida al material del bloque. Luego de aproximar el motor a un sistema de primer orden sometido a una entrada de calor en escalón, se obtiene una masa de $97 \mathrm{~kg}$ (esta masa comprendería toda la estructura interna y externa del motor y sus líquidos). De manera inversa, si quisiéramos una reducción de las pérdidas misceláneas o del tiempo de calentamiento, prescribiendo una temperatura de estabilización durante el calentamiento, puede hallarse la reducción equivalente requerida de la masa del motor para lograr ese objetivo. De esta manera se ilustra, aunque de una manera muy burda, el significado de la reducción de la masa en la reducción del tiempo de calentamiento.

\subsubsection{Balance térmico del motor durante su calentamiento en su proceso de calentamiento en el ciclo de conducción NEDC}

En la Figura 3.15 se muestra el comportamiento del flujo másico de combustible y las revoluciones del cigüeñal del motor bajo el ciclo de conducción del vehículo. El consumo de combustible sigue el 
comportamiento del ciclo de conducción, consistente en varias cargas parciales bajas. Asumiendo que todo el combustible suministrado se convierte en energía térmica, la gráfica de la energía suministrada es una versión a escala del combustible consumido. El flujo de energía equivalente se ilustra en la Figura 3.16.

Mediante la expresión 3.10 se ha calculado la variación de la potencia a lo largo del ciclo, aunque no aparece graficada. La máxima potencia demandada en los tramos urbanos del ciclo es de $20 \mathrm{~kW}$. La potencia efectiva promedio a lo largo del ciclo es de $4,75 \mathrm{~kW}$, mientras que la máxima entrega de potencia se aproxima a $40 \mathrm{~kW}$ (en la parte extraurbana del ciclo).

En la Figura 3.16 se representa el aumento de las temperaturas del refrigerante a la entrada y la salida del motor y también de la temperatura externa del bloque (promedio de dos puntos) a medida que transcurre el ciclo. El conocimiento de las temperaturas del refrigerante y el flujo a través de las camisas permiten calcular, con la expresión (3.11), el calor transferido al refrigerante, que se representa también en la Figura 3.16. El valor promediado del flujo de energía al refrigerante durante las cuatro partes ECE del ciclo NEDC es de 2,18 kW.

Como se observa de la gráfica de temperatura de salida del refrigerante, el tiempo en que se alcanzan los $80{ }^{\circ} \mathrm{C}$ (temperatura de apertura del termostato) es cercano a los 720 segundos. Son doce minutos durante los cuales el motor tiene mayor tendencia a producir emisiones de hidrocarburos por encima de los niveles permitidos [3.33].

La evolución de las temperaturas del refrigerante y el bloque dan una idea de la inercia térmica del motor descrita de manera simplificada por la expresión (3.13). Aún viendo el motor como una masa concentrada, es evidente que un tratamiento simplificado para determinar su respuesta a una entrada de calor como la representada en la Figura 3.16, no es una tarea trivial. A pesar de ésto, algunos autores han validado modelos observadores de temperatura de pared a partir de mapas de temperaturas obtenidos en estacionario [3.1], [3.24], mientras que otros han elaborado sistemas de control de la temperatura del motor basándose en funciones de regresión de respuestas del motor a cargas constantes o a cargas de ciclo [3.22]. 
El interés, en la presente tesis, en el estudio de la temperatura del motor y particularmente del refrigerante y el bloque es cualitativo, pues interesa saber qué tiempo tarda el motor que se analiza en alcanzar la temperatura objetivo de $80{ }^{\circ} \mathrm{C}$ y sugerir medidas para reducir ese tiempo de calentamiento. Esto se hará en el capítulo quinto.

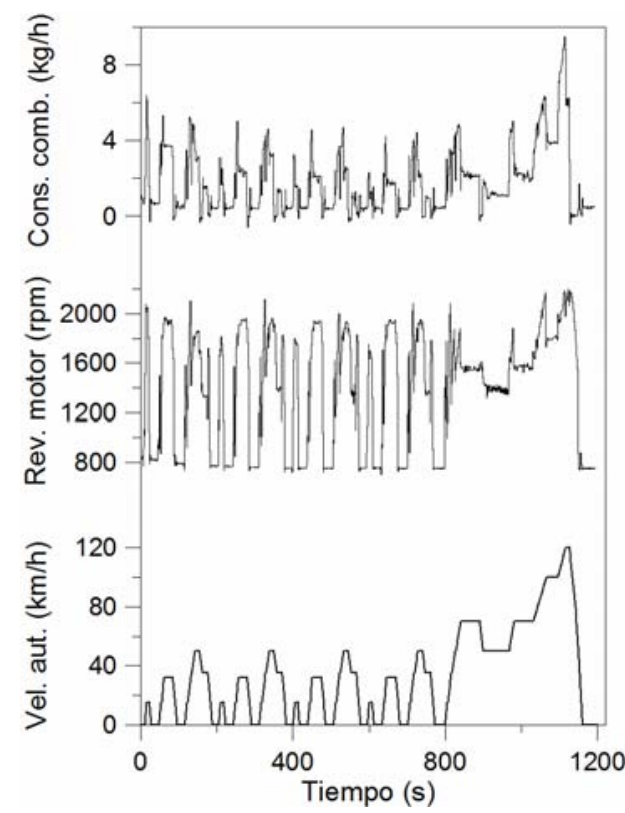

Figura 3.15. Consumo de combustible medido como función de las revoluciones del motor y del perfil del ciclo de conducción NEDC del vehículo.

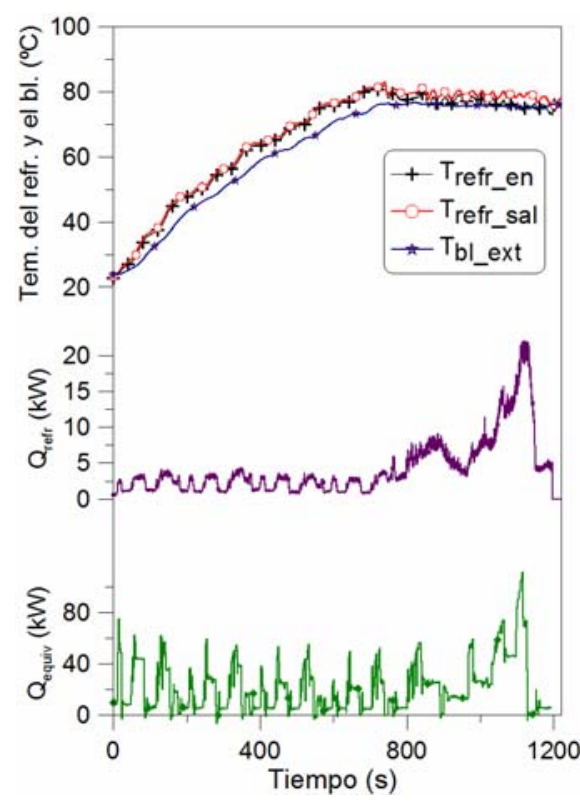

Figura 3.16. Calor disipado al refrigerante, energía equivalente del combustible y temperaturas del refrigerante y de la superficie externa del motor.

Las mediciones de los flujos de aire y combustible y de las temperaturas de admisión y escape, graficadas en la Figura 3.17, sirven como se describe con la expresión (3.12) para calcular la energía transportada con los gases de escape. El valor promedio de la temperatura de los gases de escape es inferior a $200{ }^{\circ} \mathrm{C}$ durante las partes urbanas del ciclo (780 segundos) y después de este tiempo la temperatura aumenta, llegando a alcanzar valores hasta de $500^{\circ} \mathrm{C}$. Las temperaturas son relativamente bajas pensando en las tecnologías de recuperación de calor basadas en celdas termoeléctricas [3.16], 
y aunque sí están dentro de los umbrales en los que se aplican tecnologías de recuperación de energía en sistemas de capacidades grandes, la implementación de éstas está también condicionada por el espacio disponible, el aumento de la masa y las limitaciones de espacio. El valor promedio del flujo de energía transportado con los gases de escape durante las cuatro partes ECE del ciclo NEDC es de 2,84 kW.

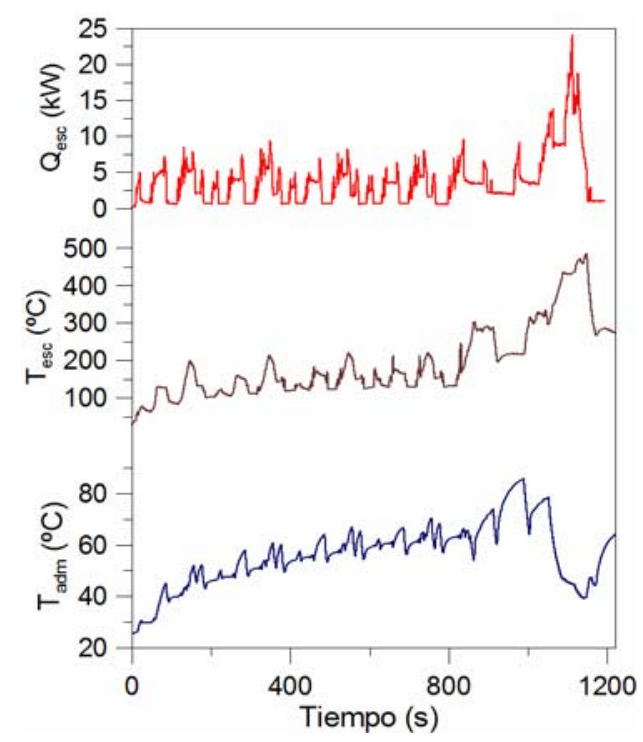

Figura 3.17. Flujo de calor transportado con los gases de escape. La temperatura del aire de admisión también crece con el paso del ciclo.

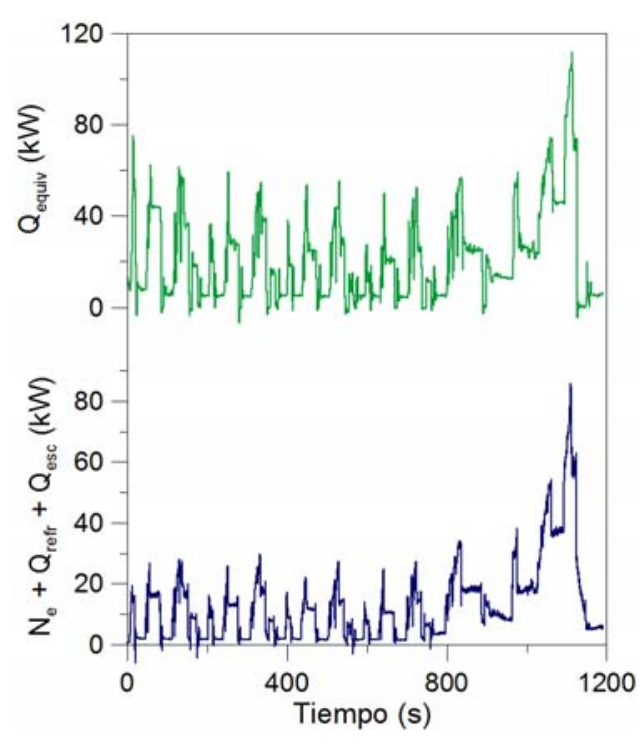

Figura 3.18. Flujo de energía equivalente suministrada al motor y flujo resultante de la potencia efectiva sumado a las pérdidas de calor a través de los gases de escape y el refrigerante.

Como se mencionó anteriormente, el remanente de la diferencia entre la energía suministrada y la suma de las energías convertidas en trabajo útil, calor de refrigerante y gases de escape corresponde al término misceláneo de la ecuación (3.9). En la Figura 3.18 se representa la variación de los términos de esta diferencia durante el calentamiento del motor bajo la operación en ciclo NEDC. La gráfica de la Figura 3.19 muestra el comportamiento de las pérdidas misceláneas, cuya mayor parte se emplea en el calentamiento de las masas del motor. 


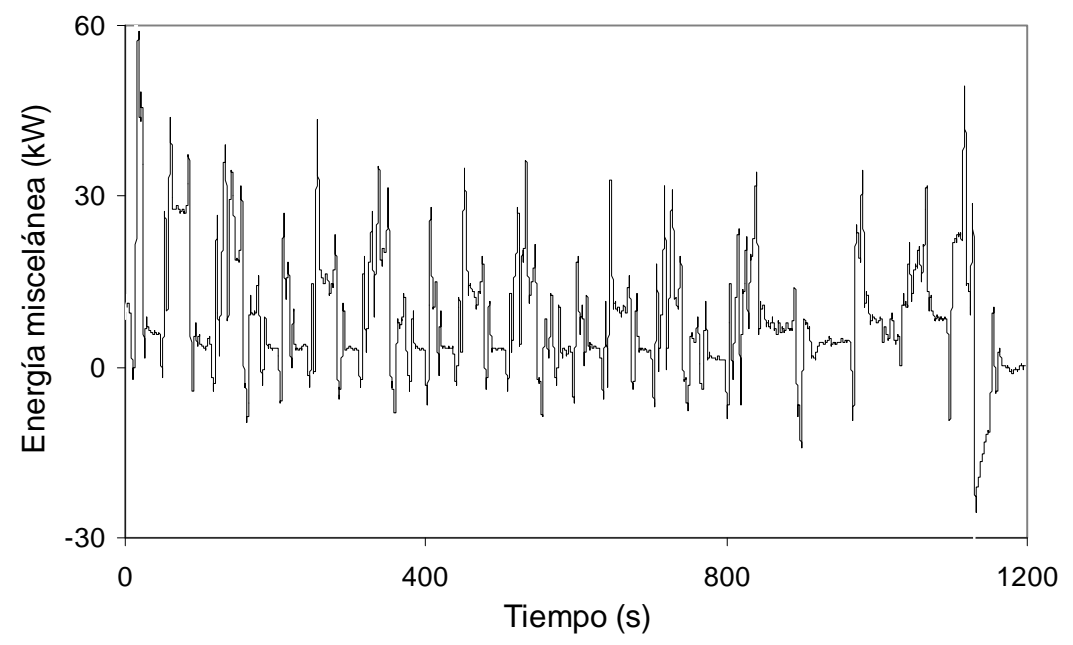

Figura 3.19. Perfil del flujo de energías misceláneas durante el calentamiento del motor.

El carácter picudo de las gráficas es resultado del cálculo con las señales reales, sobre todo las señales de temperatura de los gases de escape. Las gráficas de variación de la temperatura del refrigerante y el bloque del motor son más suaves, por la mayor inercia térmica asociada.

Los valores promedio de los flujos de energía transportados por los gases de escape y el refrigerante a lo largo de todo el ciclo de conducción NEDC son 3,75 y 4,31 kW, respectivamente. Las pérdidas de calor constituyen más del $30 \%$ de la energía suministrada con el combustible, mientras que la mayor parte de la energía suministrada se dedica al calentamiento de las partes del motor (durante el calentamiento es muy poco el calor evacuado a través de la superficie externa del motor al ambiente [3.18]).

En el diagrama de barras de la Figura 3.20 se resumen los porcentajes promedio de los flujos de energía componentes del balance descrito por la expresión (3.9) referidos al flujo de energía suministrado al motor durante el ciclo. Desafortunadamente las pérdidas misceláneas no las podemos detallar (es decir, el flujo neto de energía hacia las diferentes capacitancias del motor, el calor evacuado con el aceite no transferido al refrigerante, el calor disipado al ambiente), pero los resultados globales están de acuerdo con los resultados 
de Jarrier [3.18], quien afirma que durante el primer minuto de calentamiento del motor cerca del $65 \%$ de la energía proveniente de la combustión se emplea para el calentamiento de las masas, siendo despreciable el calor disipado al ambiente. A medida que el ciclo transcurre la parte de energía que se transfiere al ambiente va creciendo, llegando a constituir un valor importante. Una estimación del flujo de energía hacia el ambiente al finalizar la última parte urbana (último ECE) del ciclo arroja un valor de 1,8 kW, calculado teniendo en cuenta la temperatura experimental del bloque, una temperatura ambiente promedio de $24{ }^{\circ} \mathrm{C}$ y un coeficiente de convección entre el cuerpo del motor y el aire circundante de $35 \mathrm{~W} /\left(\mathrm{m}^{2} \mathrm{~K}\right)$. Este flujo de calor corresponde al $12 \%$ de la energía suministrada al motor, dando una idea de su nivel de importancia.

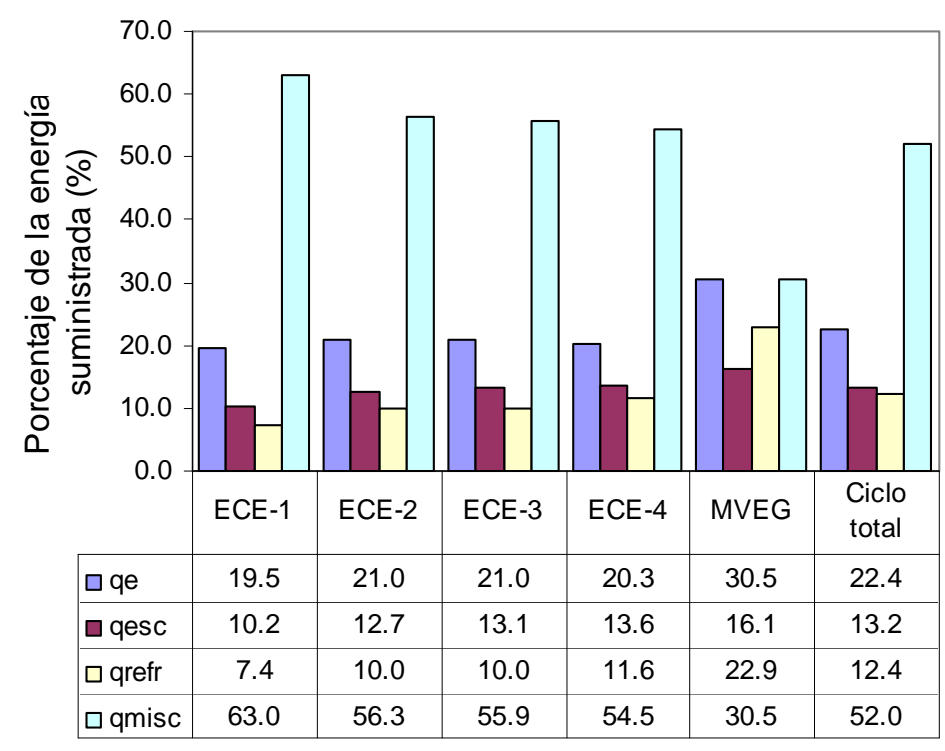

Figura 3.20. Reparto de los flujos energéticos en las diferentes etapas del ciclo.

Los porcentajes de las pérdidas de calor hacia los gases de escape y el refrigerante aumentan a medida que el vehículo se calienta y la demanda de almacenamiento de calor en la estructura del motor y los fluidos disminuye, tendiendo a estabilizarse el promedio una vez que se ha alcanzado la temperatura de operación regulada por el termostato. 


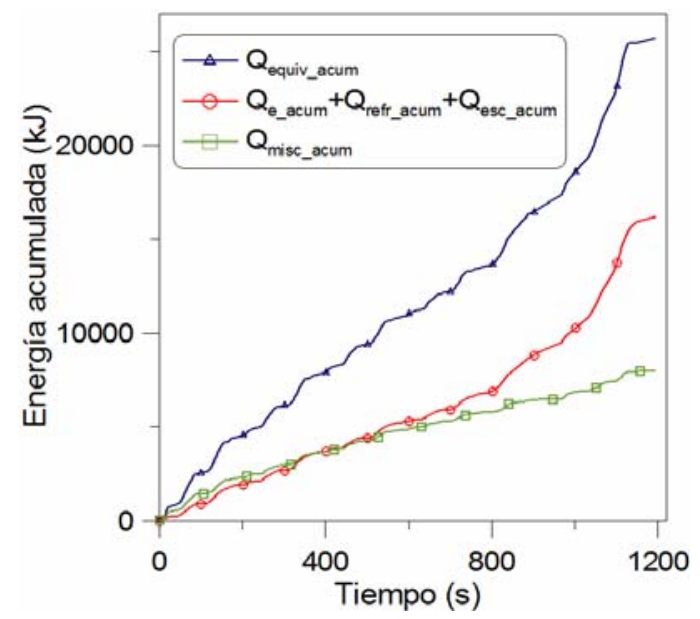

Figura 3.21. Importancia relativa de las pérdidas misceláneas en relación con la energía acumulada suministrada con el combustible y la energía acumulada resultante del trabajo útil y las pérdidas de calor con los gases de escape y el refrigerante.

Otra manera de ver la importancia relativa de la componente miscelánea en relación con las otras pérdidas energéticas durante el calentamiento del motor es el cálculo del acumulado de las energías del balance. En la Figura 3.21 se ilustran los valores acumulados, a lo largo de la prueba, de la energía suministrada con el combustible, $Q_{\text {equiv_acum }}$, la suma de las energías correspondientes al trabajo efectivo y a las energías evacuadas con el refrigerante y los gases de escape, $Q_{e_{-} a c u m}+Q_{\text {refr_acum }}+Q_{\text {esc_acum }}$, y la energía acumulada de las pérdidas misceláneas, $Q_{\text {misc_acum }}$. Se aprecia la ralentización en el crecimiento de la energía acumulada de las pérdidas misceláneas. Si el motor estuviera aislado del exterior y la carga se mantuviera constante después del calentamiento, la energía miscelánea se estabilizaría en el valor equivalente a la energía almacenada.

La inercia térmica influencia el calentamiento del motor hasta que se logra el equilibrio térmico, pero se requieren medidas adicionales para aumentar el calor disponible para alcanzar la temperatura de trabajo óptima del motor en menos tiempo. Estas medidas adicionales pueden ser la reducción del volumen del refrigerante con consideración del vaciado de la camisa de refrigeración durante el tiempo que las propiedades de los materiales lo permitan [41], el mejoramiento del encapsulamiento o aislamiento del motor, el empleo de sistemas de calentamiento adicionales, o el empleo de la unidad electrónica de control para manipular la termodinámica de los gases de 
escape, conmutando el control del motor a un modo de arranque en frío. Algunas de estas ideas se han estado experimentando [3.11], [3.14]-[3.16] y forman parte del concepto de gestión térmica del motor. Basándose en la información disponible en los sensores que actualmente se montan en los motores modernos, pueden manipularse las temperaturas del refrigerante y los gases de escape empleando mapas de observación y control prediseñados. El control de estas temperaturas estará mediado por el control de las múltiples interrelaciones de los flujos de refrigerante (de los posibles circuitos), aire de admisión refrigerado, gases de escape y aceite lubricante.

El aumento del calor disponible durante el calentamiento redunda en unas mejores características del sistema de calentamiento del habitáculo de pasajeros. En los automóviles modernos se busca un compromiso entre el logro de la temperatura de trabajo del motor en el menor tiempo posible y el logro de los niveles exigidos de confort, que está relacionado con el calor transferido por el refrigerante al habitáculo del vehículo.

\subsubsection{Energía recuperada en el vehículo durante el ciclo de conducción}

En el parágrafo anterior se comentó la variación de los calores evacuados con los gases de escape y el refrigerante. Como se ha representado en la Figura 3.9, el sistema de refrigeración del motor incluye tres intercambiadores: el radiador principal del motor, el enfriador de los gases de escape que han de recircularse a los cilindros del motor y el calentador del habitáculo de pasajeros. El radiador opera sólo bajo el umbral de temperatura previsto por el termostato, si se omite el pequeño flujo de refrigerante que pueda pasar por él a través del ramal de desaireación desde el tanque de expansión. Dependiendo de la característica del termostato, habrá un momento en que por el radiador empiece a fluir refrigerante para ceder calor allí al aire inducido por el movimiento del vehículo y también por el trabajo del ventilador. Este calor actualmente se disipa sin compensación alguna, aunque se trabaja en medidas tecnológicas para utilizar esta energía.

La ecuación (3.11) sirve para hallar el calor recibido por el refrigerante en el enfriador de los gases de escape recirculados y también el cedido al aire del habitáculo de pasajeros. Estos son dos intercambiadores que de manera separada recuperan energía de los gases de escape y del refrigerante. El calor 
recuperado en estos dos dispositivos durante el ciclo de operación del vehículo tiene el comportamiento ilustrado en la Figura 3.22.
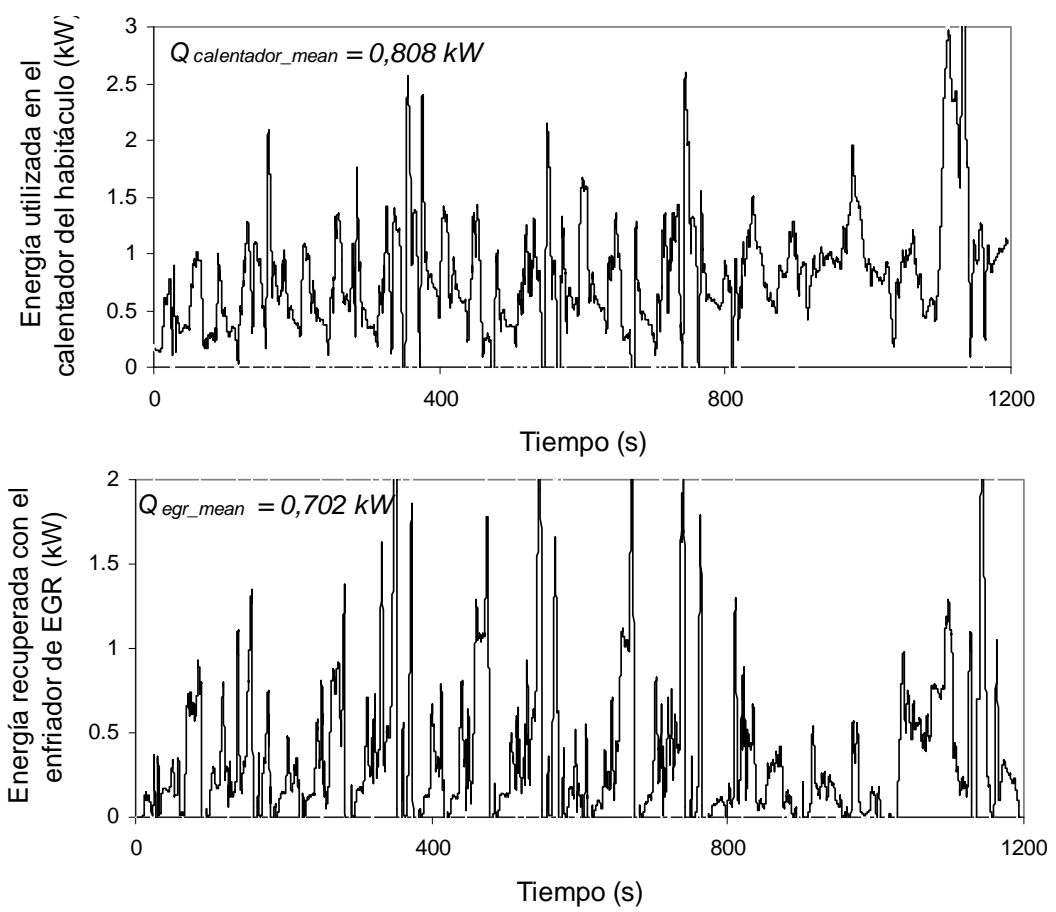

Figura 3.22. Calores recuperados en el enfriador de EGR y en el calentador del habitáculo del vehículo.

En el circuito examinado el enfriador de EGR sirve a los efectos de controlar las emisiones de óxidos de nitrógeno, extrayendo calor de los gases de escape y pasándolo al refrigerante; estrictamente hablando no hay una recuperación de energía allí. El calor disipado por el motor que realmente se está recuperando es el empleado para calentar el habitáculo de pasajeros.

Se ve que durante el calentamiento los flujos de calor en el enfriador de EGR y en el calentador del habitáculo son de magnitud comparable (para las condiciones de la prueba), por lo que el enfriador de EGR podría proveer directamente el calor necesario para el confort de los pasajeros del vehículo. Sin embargo, en términos prácticos el calor que recibe en este momento el habitáculo de pasajeros es pequeño porque las pruebas se han realizado bajo unas condiciones climáticas controladas, con temperatura en la sala cercana a los 23-24 ${ }^{\circ} \mathrm{C}$. Cuando se presenta demanda de calor en condiciones de 
invierno, la carga térmica del calentador del habitáculo para un vehículo sedán de cuatro pasajeros puede ser de un promedio de $3 \mathrm{~kW}$ en modo de recirculación, como se ilustra en la característica de la Figura 3.23 [3.42], con lo que el enfriador de EGR se queda corto para abastecer esta demanda de energía. El motor normalmente, detectada la demanda de calor del habitáculo de pasajeros, responde aumentando el consumo de combustible y aumentando el régimen, pero lo que sí podría buscarse es que el calor de confort requerido en el vehículo provenga de los gases de escape y del refrigerante.

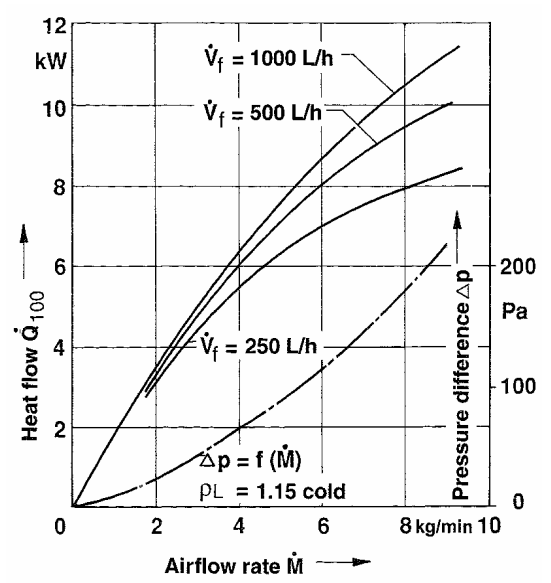

Figura 3.23. Característica de un calentador para habitáculo de pasajeros [3.42].

Si los circuitos de refrigerante del intercambiador de EGR y del calentador del habitáculo se montaran en circuitos independientes, la suma de los flujos térmicos a través del enfriador de EGR y del calentador del habitáculo podría verse como la potencia térmica recuperada durante el calentamiento del motor. En la Figura 3.24 se ilustra la variación de esta potencia térmica "recuperada". El valor promedio de la energía "recuperada" durante la operación del motor bajo regímenes urbanos es de aproximadamente $1 \mathrm{~kW}$, o sea, un $20 \%$ de la potencia evacuada con los gases de escape y el refrigerante. Cabe anotar que en los vehículos modernos es cada vez mayor el número de motores equipados con enfriadores de EGR y de aire de admisión con circuitos de refrigeración independientes, de refrigerante líquido para los primeros y de aire para los segundos. Esto genera posibilidades de recuperación de calor adicionales [3.8], [3.9]. 


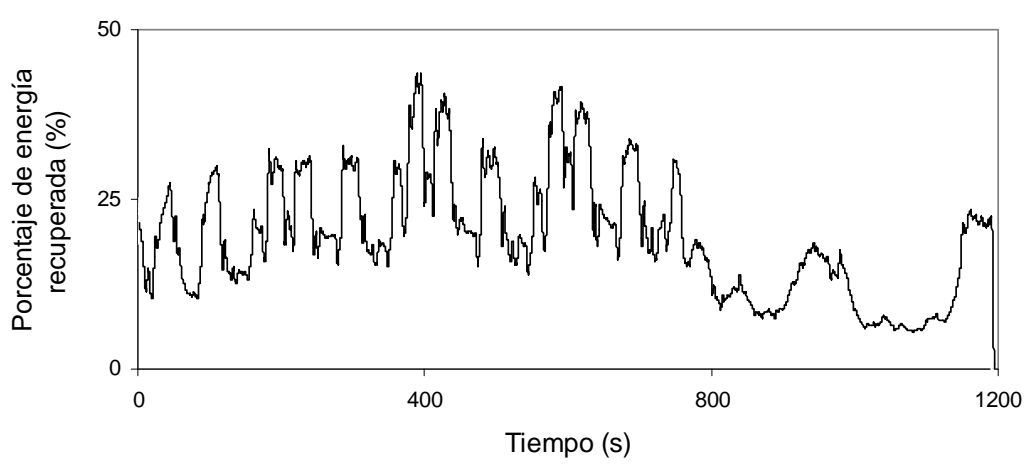

Figura 3.24. Porcentaje de la "energía recuperada" en relación al total de las pérdidas de calor con los gases de escape y el refrigerante.

El análisis de la información obtenida en el banco de ensayos ha permitido realizar un balance térmico del motor, pudiéndose analizar las proporciones relativas de las pérdidas de calor y los factores que influyen sobre el tiempo de calentamiento del motor. Un tratamiento integral de la gestión de los flujos de calor tiene el potencial no sólo de mejorar la respuesta térmica del motor, sino también de reducir las pérdidas parásitas (bombeo de refrigerante en volúmenes innecesarios), optimizar el consumo de combustible y mejorar el calentamiento del habitáculo de pasajeros. Desacoplando los componentes del sistema de refrigeración (bomba y ventiladores) del cigüeñal y accionándolos con motores eléctricos y reemplazando los termostatos de acción mecánica por válvulas controladas electrónicamente es posible mejorar el comportamiento del motor y reducir las pérdidas de potencia en auxiliares no sólo durante el calentamiento, sino también durante la operación estacionaria y transitoria en caliente del motor.

Algunos de los trabajos teóricos y experimentales relacionados con el estudio y la mejora del calentamiento del motor y de los systemas de refrigeración son los realizados por Luptowski et al. [3.1], Cortona [3.2], Hnatczuk et al. [3.5], Eberth et al. [3.6], Chanfreau [3.7], Cho et al. [3.10], Chalgren y Traczik [3.8], Chalgren y Allen [3.9], Chastain y Wagner [3.3], Pang y Brace [3.11], Robinson et al. [3.29], Norris et al. [3.12], Brace y Burham [3.14].

La introducción de nuevos modos de control de la combustión de bajas temperaturas y sistemas HCCI requieren de mayor recirculación de gases de escape, con lo que el calor disipado allí llega a tener valores muy importantes. 
Igual sucede con los sistemas modernos de enfriamiento del aire de admisión, que llegan a disipar potencias térmicas del mismo orden que los radiadores principales [3.8].

\subsubsection{Observaciones}

Esta sección del capítulo tenía dos motivaciones. La primera consistente en presentar un tratamiento del balance energético del motor durante su calentamiento bajo carga constante y en ciclo de conducción, y analizar los factores que en él influyen. La segunda motivación era la valoración de la energía recuperada y recuperable en el motor bajo el ciclo de conducción del vehículo. A diferencia de otros balances energéticos publicados en la literatura, el balance energético aquí presentado fue registrado durante la operación real del motor bajo el ciclo de conducción NEDC, con la idea de desvelar la dinámica del reparto de la energía y también de dar una estimación de las pérdidas de calor promediadas a lo largo del ciclo.

Los valores promedio de los flujos térmicos evacuados con los gases de escape y el refrigerante son 3,75 y 4,31 kW, respectivamente. De estos flujos, aproximadamente el $20 \%$ es recuperado por el enfriador de EGR y el calentador del habitáculo. El flujo térmico disponible es del orden de $4 \mathrm{~kW}$. El acumulado de las pérdidas de energía correspondientes disipadas con los gases de escape y el refrigerante al final del ciclo de conducción es próximo a los $9500 \mathrm{~kJ}$. El acumulado de las denominadas pérdidas misceláneas es de cerca de 8000 kJ. Estos números hablan del potencial de energía recuperable que se tiene. Sí debe anotarse que los niveles de las temperaturas de los gases de escape, así como el carácter fluctuante de los flujos de calor son condicionantes para las propuestas de tecnologías de recuperación de calor como es el caso de las pilas termoeléctricas.

El balance energético efectuado para el motor durante su calentamiento permite ver que el término de pérdidas misceláneas es el mayor componente de las pérdidas de energía del motor durante su calentamiento bajo su operación en ciclo de conducción y en carga constante, como se observó en la primera parte. En el término de pérdidas misceláneas, la capacitancia térmica del material del motor es lo que más influye en el control del tiempo de calentamiento, hasta que las temperaturas del motor se estabilizan. Así, entonces, una reducción de la masa del motor se traduce en una reducción del tiempo de calentamiento, lo que otros autores han apuntado que se traduce en 
una reducción de consumo de combustible y de las emisiones contaminantes [3.17], [3.18].

\subsection{Modelado de la temperatura y los flujos de calor con un modelo predictivo}

En esta parte del capítulo se presenta y resume un modelo de predicción de temperaturas para luego comentar su empleo en el cálculo de las temperaturas y los flujos de calor en dos motores. El modelo fue validado inicialmente en el trabajo de Degraeuwe [3.13]. Dicho modelo utiliza los valores instantáneos de la presión y la temperatura del gas obtenidos con el modelo de diagnóstico de combustión CALMEC [3.49] y también los valores instantáneos de los flujos másicos y las áreas de paso, también calculadas por CALMEC, de los gases de admisión y escape para calcular las condiciones de contorno en el cilindro y las pipas. Las condiciones de contorno restantes del lado del refrigerante, el aceite y la interfaz pistón-segmentos-cilindro se obtienen por un proceso de optimización descrito en [3.13]. Basándose en la geometría del motor (ingresada mediante un fichero) y las condiciones de contorno, el programa construye un modelo térmico de resistencias y capacitancias. Las medidas experimentales en la culata, la camisa y el pistón se emplean para ajustar el modelo.

El modelo se optimizó a partir de la información aportada por 23 termopares ubicados en el cilindro, 16 en la culata y dos en el pistón. El código del programa es abierto y permite su modificación para que, entre otras posibilidades, se puedan probar diferentes submodelos de transferencia de calor en todas las condiciones de contorno (refrigerante, aceite, gases en los puertos, etc.), como se presentaron en el estado del arte del capítulo 2.

En este parágrafo se analizarán las predicciones de temperaturas del modelo en estado transitorio, al pasar de un punto de operación a otro, una parte del programa original no explotada. El trabajo aquí presentado contiene una breve descripción del modelo discretizado de motor, una descripción de las condiciones de contorno y una discusión sobre los flujos de calor entre los volúmenes finitos del modelo y entre éstos y las condiciones de contorno bajo condiciones de operación estacionaria y transitoria del motor. Luego de esto se aplica el modelo térmico a la determinación de las temperaturas del motor 
estudiado en el parágrafo anterior operando bajo carga constante durante el calentamiento y bajo condiciones de operación de ciclo NEDC, empleando las variables medias e instantáneas determinadas durante las pruebas experimentales. Al final se obtienen conclusiones cualitativas sobre la conveniencia de la adquisición de información para predicción de temperaturas durante los propios ciclos de conducción, en contraste con la práctica habitual de adquirir información del motor bajo condiciones estacionarias.

\subsubsection{Breve descripción del modelo térmico}

El modelo construido por Degraeuwe [3.13] para predecir los flujos de calor a través de las paredes de la cámara de combustión y las temperaturas de los componentes se basa en la analogía existente entre los sistemas de flujo eléctricos y de transferencia de calor, como lo hicieron otros autores como Bohac [3.45], Veshagh [3.46], o Gentile [3.18]. El motor es tratado como una malla térmica, donde el calor puede transportarse entre capacitancias concentradas a través de conductancias concentradas (Reyes [3.44] las llama globales). Para hacer corresponder el sistema real a su análogo eléctrico, el pistón, la culata y el cilindro del motor se descomponen en trozos elementales (nodos) de acuerdo al número de sensores de temperatura instalados en el motor para validar el modelo y cuidando de observar el número de Biot para asegurar el criterio de uniformidad de la temperatura en cada nodo. Estos nodos se interconectan por resistencias térmicas de conducción o convección, estas últimas asociadas a las condiciones de contorno (convección y contacto variable) de los nodos. Las condiciones de contorno, se reitera, están representadas por la temperatura y coeficientes de convección de los gases del cilindro, los gases de admisión y escape, el refrigerante y el aceite. Además cabe la condición de contorno especial en el grupo pistónsegmentos-cilindro. La fundamentación experimental, teórica y la validación del modelo fueron ampliamente explicados por Degraeuwe [3.13] en su trabajo de tesis doctoral.

\subsubsection{Fundamentos del modelo nodal}

Los modelos de la culata y el pistón son creados empleando un programa computacional de diseño (CAD). La camisa del cilindro se divide a lo largo de las coordenadas cilíndricas y se tiene en cuenta que, generalmente, no toda la longitud de la camisa del cilindro es refrigerada. El número de nodos de la 
camisa puede variarse automáticamente, mientras que los nodos del pistón y la culata son difíciles de automatizar por lo complejo de la geometría de ambas partes, como se ve en los modelos dibujados en las Figura 3.25. En el modelo geométrico el usuario es libre de discretizar estos elementos como más le convenga.
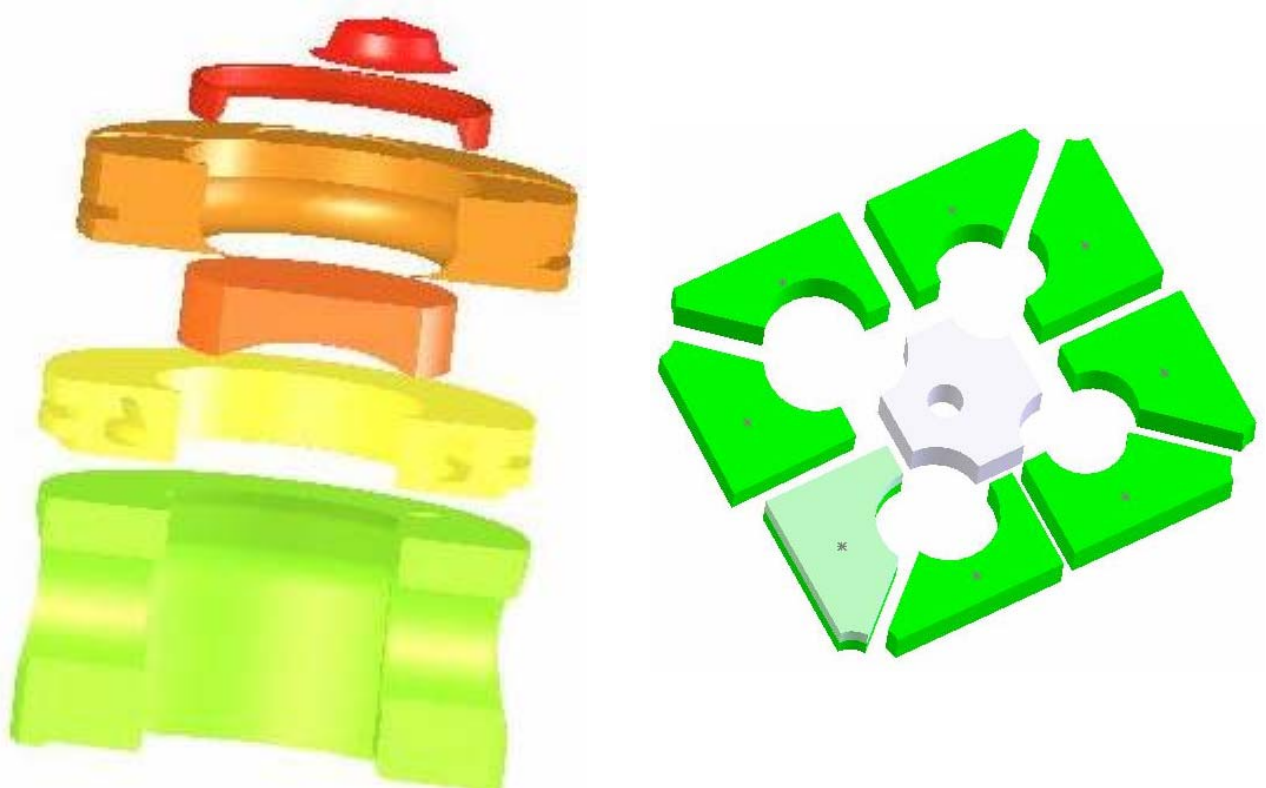

Figura 3.25. Nodalización del pistón y de la cara de la culata en contacto con los gases.

Para cada componente (nodo) las condiciones de contorno quedan definidas por la temperatura del fluido y el coeficiente de transferencia de calor, ó directamente por la temperatura de la superficie.

En el modelo cada nodo está conectado a otros nodos y condiciones de contorno. Estas conexiones se caracterizan por su conductancia térmica. Son tres los posibles tipos de conductancias: por conducción, convección o radiación.

Para el modelo geométrico suministrado definido por el usuario, el programa resuelve la ecuación de conservación de la energía en estado transitorio expresada en forma matricial como: 


$$
\left([K]+\frac{[C]}{\Delta t}\right)\left[T_{t+\Delta t}\right]=[Q]+\frac{[C]}{\Delta t}\left[T_{t}\right]+[H]
$$

donde $\left[T_{t}\right]$ y $\left[T_{t+\Delta t}\right]$ son vectores columna de $n$ elementos formados por las temperaturas precedentes y actuales de los nodos. $[Q]$ es un vector columna con la suma de los flujos de calor hacia el nodo $i$ en la $i$-ésima fila. Este puede ser, por ejemplo, un flujo de calor generado por fricción. $[H]$ es un vector columna en el que la i-ésima fila es la suma de los productos $T_{l} h_{l i} A_{l i}$ de las condiciones de contorno del nodo $i$. $[K] \mathrm{y}[C]$ son, respectivamente, las matrices de conductancias y capacitancias, de dimensiones $n \times n$.

El elemento i-ésimo de la diagonal principal de la matriz de conductancias es la suma de todas las conductancias conductivas y convectivas relacionadas con el nodo $i$.

Cuando todas las conductancias son conocidas, las temperaturas de la pared pueden ser calculadas con este sistema de ecuaciones. Para las condiciones estacionarias los términos inerciales de la ecuación (3.14) desaparecen porque $T_{t+\Delta t}^{i}=T_{t}^{i}$, con lo que la ecuación matricial de conservación de la energía se reduce a:

$$
[K] \cdot[T]=[Q]+[H]
$$

El programa realiza automáticamente el ensamble de la ecuación (3.14) en la rutina de preprocesamiento, cuando integra el modelo geométrico con las condiciones de contorno calculadas con las variables medias y las variables instantáneas (condiciones dentro del cilindro). Luego el programa halla por el método implícito el vector de temperaturas $[T]$, empleando un procedimiento de eliminación Gausiana.

\subsubsection{Interacciones y condiciones de contorno}

Los cálculos térmicos incluyen la determinación de los flujos de calor entre los gases del cilindro y las paredes de la cámara de combustión, en las partes metálicas entre sí, entre los gases de admisión y escape y las superficies de 
las válvulas y pipas de admisión y escape, y también entre las partes metálicas y el lubricante y el refrigerante. También para las superficies en contacto con los gases de admisión y escape, en el modelo se utiliza un valor del coeficiente de transferencia de calor promediado en el ciclo, pero además adaptado para tener en cuenta la historia de los procesos de propagación de ondas en los sistemas. Las interacciones de contacto entre las válvulas y sus asientos se describen por los flujos de calor $q_{A B}$ de la superficie sólida A a B, a través de la diferencia de sus temperaturas de superficie $T_{A}, T_{B}$ por $\dot{q}_{A B}=k\left(T_{B}-T_{A}\right)$, donde $k$ es la conductancia de contacto.

Todas las expresiones analíticas para el cálculo de las interacciones térmicas en las fronteras han sido ampliamente detalladas en el trabajo de Degraeuwe [3.25]. En general, aparte de las conductancias definidas para los nodos de las paredes de la cámara de combustión, todas las demás conductancias del modelo que dependen de una variable de operación están definidas por una correlación validada de la forma:

$$
K_{i 2 j}=\left(K_{\text {const }}^{-1}+\left(C_{i 2 j} \cdot(x)^{E x p_{-} i 2 j}\right)^{-1}\right)^{-1}
$$

donde $x$ puede ser el flujo del refrigerante o la velocidad de pistón, $C_{i 2 j}$, Exp_i2j y $K_{\text {const }}$ son parámetros de ajuste, sujetos a optimización.

Aparte de la conductancia entre el gas y los nodos de la cámara de combustión en contacto con ellos, las conductancias finales empleadas en el modelo actual tienen las expresiones descritas en la tabla 3.3.

Tabla 3.3. Expresiones para las conductancias del modelo

\begin{tabular}{|l|l|}
\hline & $\begin{array}{c}\frac{1}{720} \int_{0}^{720} T_{\text {gas }}(\alpha) h(\alpha) \cdot d \alpha \\
\bar{h}\end{array}$ \\
$\begin{array}{l}\text { Entre el gas y el } \\
\text { pistón o la culata: }\end{array}$ & $\begin{array}{l}K_{\text {gas } 2 \text { pis }}=A_{\text {pis }} \cdot \frac{1}{720} \int_{0}^{720} h(\alpha) d \alpha ; \\
K_{\text {gas } 2 \text { head }, d}=A_{\text {head }} \cdot \frac{1}{720} \int_{0}^{720} h(\alpha) d \alpha\end{array}$ \\
\hline
\end{tabular}




\begin{tabular}{|c|c|}
\hline $\begin{array}{l}\text { Entre el gas y el plato } \\
\text { de las válvulas }\end{array}$ & $K_{\text {gas } 2 \text { valv }}=A_{\text {valv }} \cdot \frac{1}{720} \int_{0}^{720} h(\alpha) d \alpha$ \\
\hline $\begin{array}{l}\text { Entre el plato de } \\
\text { válvula y su asiento }\end{array}$ & $K_{\text {valves } 2 \text { head }}=f \cdot K_{\text {seat }} \cdot A_{\text {seats }}$ \\
\hline $\begin{array}{l}\text { Entre el gas y algún } \\
\text { nodo } i \text { del cilindro en } \\
\text { contacto con él a una } \\
\text { distancia axial } z \text { de la } \\
\text { superficie de la culata }\end{array}$ & 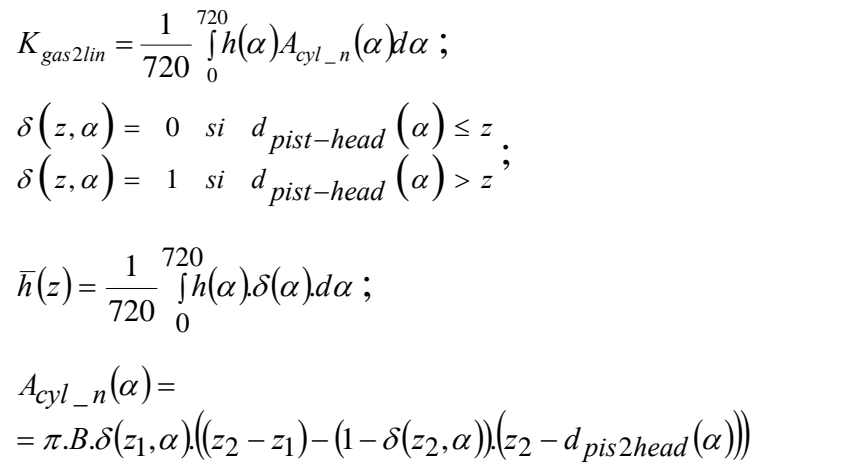 \\
\hline $\begin{array}{l}\text { Entre el pistón y el } \\
\text { cilindro }\end{array}$ & $K_{p i s 2 c y l}=\left(K_{\text {seg }}^{-1}+\left(C_{\text {pis } 2 c y l}\left(S_{p}\right) E x p_{-} \text {pis } 2 o i l\right)^{-1}\right)^{-1}$ \\
\hline $\begin{array}{l}\text { Entre el cilindro } \\
\text { (culata) y el } \\
\text { refrigerante, } \\
\text { basados en una } \\
\text { relación Nu-Re-Pr, } \\
N u=0,023 \operatorname{Re}_{D}^{0,8} \operatorname{Pr}^{0,4} \text {. }\end{array}$ & $\begin{array}{l}K_{c y l 2 c o o l}=\left(K_{c y l}^{-1}+\left(C_{c y l 2 c o o l} \cdot\left(Q_{c o o l}\right)^{E x p \_c y l 2 c o o l}\right)^{-1}\right)^{-1} ; \\
K_{\text {head } 2 \text { cool }}=\left(\left(K_{\text {head }}\right)^{-1}+\left(C_{c y l 2 c o o l} \cdot\left(Q_{\text {cool }}\right)^{E x p \_c y l 2 c o o l}\right)^{-1}\right)^{-}\end{array}$ \\
\hline Ente cilindro y aceite: & $K_{\text {lin-oil }}=A_{i j} \cdot h_{\text {lin-oil }}$ \\
\hline $\begin{array}{l}\text { Entre aceite y el canal } \\
\text { en el pistón, } \\
\text { basados en una } \\
\text { relación } N u-\operatorname{Re}-\mathrm{Pr} \\
N u_{g a l}=C_{g a l} \operatorname{Re}_{\text {gal }}^{m}:\end{array}$ & $\begin{array}{l}N u_{g a l}=\frac{h_{g a l} \cdot d_{g a l}}{k_{\text {oil }}} ; \mathrm{Re}_{\text {gal }}=\frac{S_{p} \cdot d_{\text {gal }}}{v_{\text {oil }}} ; \\
K_{\text {pis-oil }}=C_{\text {pis-oil }} \cdot\left(S_{p}\right) \operatorname{Exp}_{-} \text {pis-oil } ; \\
K_{\text {pis-oil }}=A_{\text {gal }} \cdot C_{\text {gal }} \cdot \frac{k_{\text {oil }}}{d_{\text {gal }}} \cdot\left(\frac{\bar{S}_{p} \cdot d_{\text {gal }}}{v_{\text {oil }}}\right)^{m}\end{array}$ \\
\hline
\end{tabular}




\begin{tabular}{|l|l|}
\hline $\begin{array}{l}\text { Entre superficies de } \\
\text { los puertos y los } \\
\text { gases: }\end{array}$ & $K_{\text {esc,adm }}=\bar{h}_{\text {esc }, a d m} A_{\text {esc }, a d m}$ \\
\hline $\begin{array}{l}\text { Entre el pistón y el } \\
\text { cilindro, con } K_{\text {seg }} \\
\text { conductancia de los } \\
\text { anillos por unidad de } \\
\text { longitud: }\end{array}$ & $K_{P i s_{-} i-\text { Lin }_{-} j}=\frac{t_{c o n}}{T_{\text {cycle }}} K_{\text {seg }} \alpha_{L_{\text {Lin }} j} j \frac{D}{2} ;$ \\
\hline
\end{tabular}

\subsubsection{El programa de cálculo}

El programa original ha sido modificado para ser usado de manera simultánea por otros programas de diagnóstico y predicción del motor como son los de renovación de la carga y la combustión. Así, el programa puede utilizarse como una rutina que calcula el flujo de calor desde los gases a través del pistón, la culata y el cilindro, al igual que la temperatura media de estos componentes para unas condiciones de operación dadas a partir de las variables medias suministradas. Tras recibir todos los puntos del ciclo el programa calcula los flujos de calor y las temperaturas de todos los nodos, pudiendo correrse una secuencia de puntos de operación correspondientes, por ejemplo, a un ciclo de conducción de vehículo, si se le han pasado consecutivamente todas las variables medias e instantáneas de cada instante de operación del motor.

El programa modificado para correr secuencias de condiciones de operación opera según el diagrama de flujo representado en la Figura 3.26.

Los ensayos originales realizados sobre el primer motor han sido utilizados para reajustar los parámetros del modelo, buscando ajustar el exponente en la conductancia aceite-canal de pistón a un valor más parecido al de las relaciones Nu-Re-Pr. Así, los nuevos valores hallados

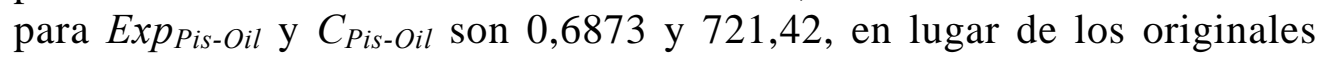
1,63 y 161,3, respectivamente. Los parámetros actualizados del modelo se presentan en la tabla 3.4. 


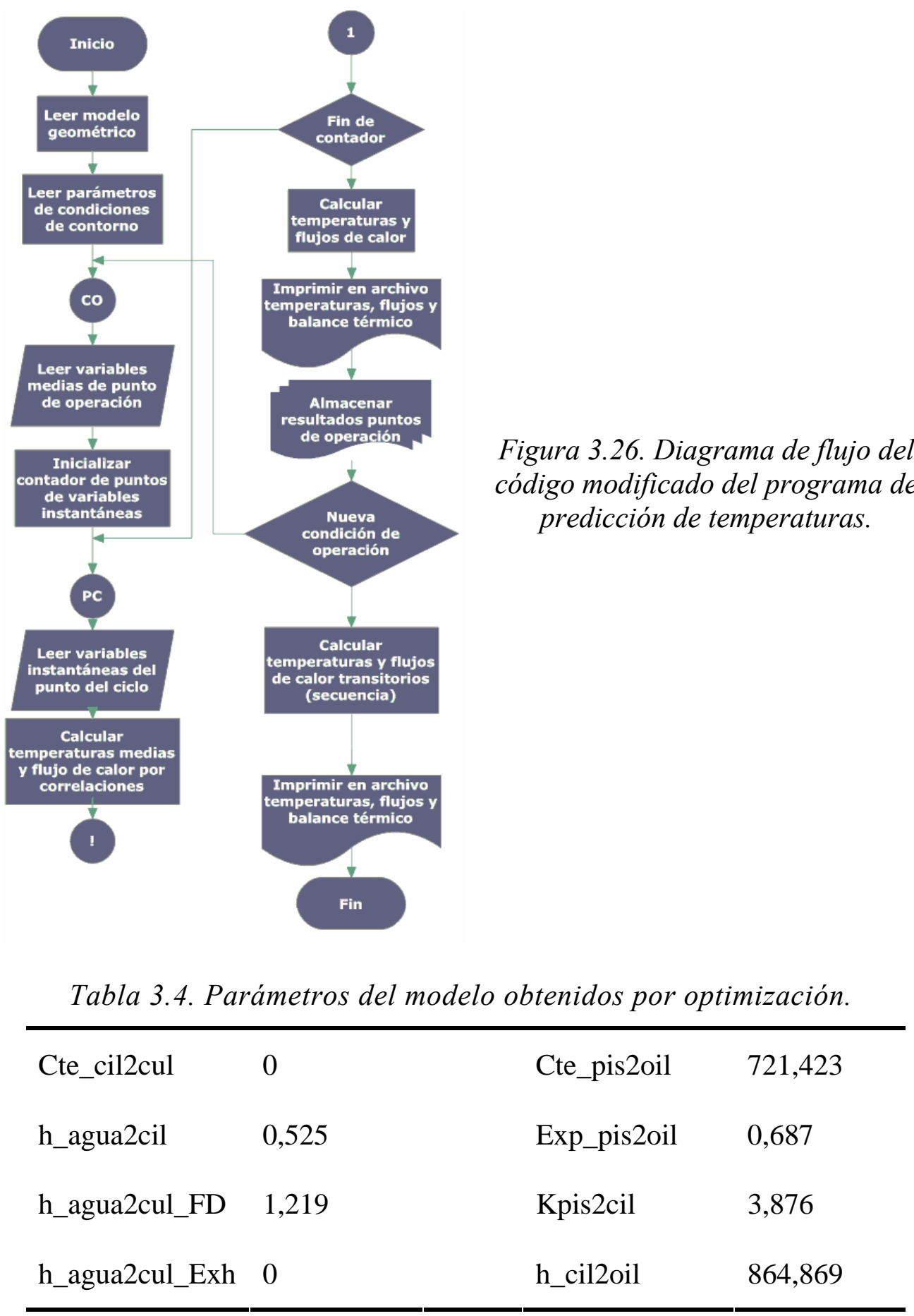


Aunque no hay un cambio apreciable en los errores de predicción de temperaturas, los nuevos valores de los parámetros sí alteran, obviamente los errores puntuales para los diferentes puntos de operación del motor, como se ilustra en la gráfica de la Figura 3.27, en la que se representan los errores en las predicciones de las temperaturas del borde y el fondo de la cavidad del pistón, en cada prueba con los parámetros originales y con los parámetros nuevos. Para el caso de los nodos del pistón, la magnitud de los errores máximos es menor.

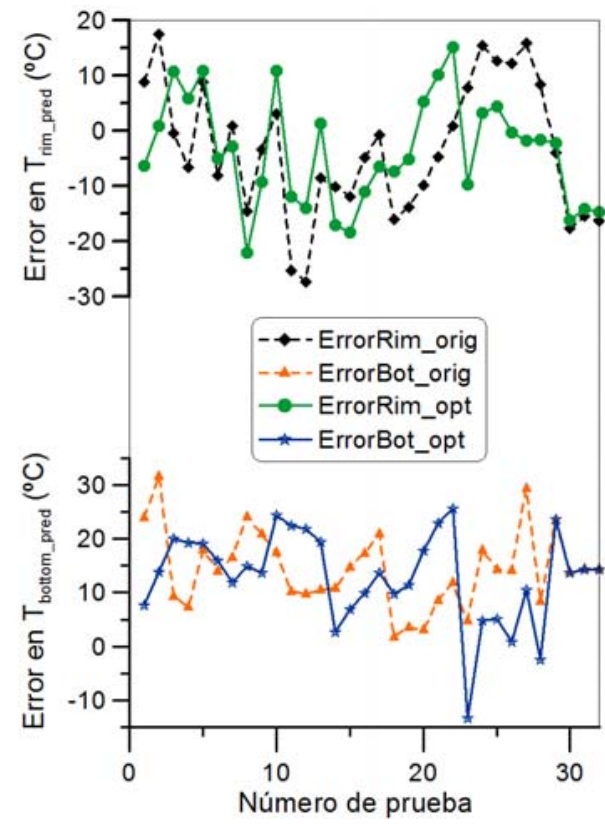

Figura 3.27. Comparación entre los errores entregados por el modelo utilizando los parámetros originales [3.13] y los parámetros nuevos, para todas las pruebas estacionarias.

\subsubsection{Predicción de las temperaturas del primer motor ante cambios en escalón de las condiciones de operación}

\subsubsection{Predicción de los flujos de calor entre los nodos del modelo}

Dado que el programa en el modo de operación básicamente predice las temperaturas de todos los nodos del modelo, en este trabajo se amplió la información del archivo de salida para que la información de los flujos de calor también fueran visibles. Más concretamente, la misma información utilizada para la validación del modelo, basada en las mediciones en el primer motor, permitió realizar el balance térmico detallado del motor para todos los 
puntos experimentales disponibles. Para ilustración, en la Figura 3.28 se resumen los flujos de calor entre los nodos del modelo para el motor operando a $1500 \mathrm{rpm}$ a una presión media efectiva de 3,93 bar. En esta condición particular, parte del calor de los gases del cilindro se transmite al pistón (42\%), a la superficie de la culata (32 \%) y a la camisa del cilindro (26 $\%)$. Hacia la camisa el calor se transmite en casi un $50 \%$ a través de la primera zona longitudinal (en el modelo el cilindro se ha dividido en 5 zonas axiales desde la superficie de la culata), debido al mayor tiempo de contacto de los gases con esta zona.

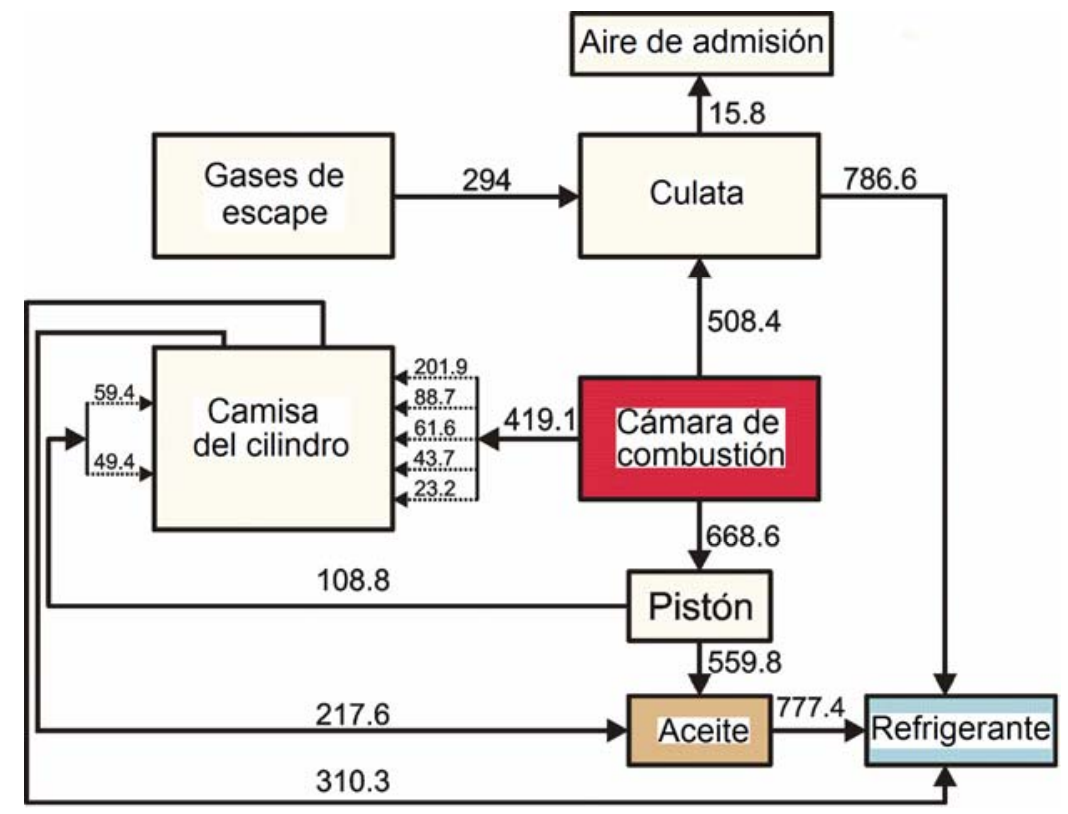

Figura 3.28. Flujos de calor (en W) entre los nodos del modelo.

El flujo de calor recibido por el pistón diverge por dos caminos: por uno de ellos el calor fluye hacia la camisa a través de los segmentos (16\%), mientras que por otro el calor fluye hacia el aceite a través de la galería de lubricación y las superficies internas inferiores (84\%). Este calor recibido por el aceite se transfiere al refrigerante y al medio circundante a través del cárter inferior (para efectos del balance térmico, todo el calor recibido por el aceite se considera transferido al refrigerante, como se comentó en la segunda sección de este capítulo). En el caso de la camisa, el calor proviene de los gases de combustión y del pistón (a través de los segmentos). Todo este calor se 
transfiere al refrigerante de manera directa (51\%) o pasando a través del aceite (49 \%).

Finalmente, la culata recibe calor de los gases de combustión (63 \%) y de los gases de escape (36 \%). Este calor recibido por la culata es, a su vez (en el modelo no se han considerado los flujos de calor entre los nodos de la culata y el aceite), transmitido parte al aire de admisión (2\%) y el resto al refrigerante (98\%). Así, el calor recibido por el refrigerante llega directamente de la culata (42\%) y de la camisa (16 \%) e indirectamente del pistón y la camisa a través del aceite (42\%).

El análisis se ha presentado bastante resumido, pero es claro que el modelo provee las temperaturas y los flujos de calor entre todos los nodos, pudiéndose detallar todas las superficies del pistón, la culata y el cilindro, para efectos de cálculos de tensiones térmicas por ejemplo, o para el estudio de la influencia de los materiales de las superficies en las temperaturas de las superficies y los flujos de calor, previendo la resolución adecuada en el fichero del modelo.

El balance ilustrado puede hacerse para todos los puntos de operación, haciendo del modelo una herramienta apropiada para determinar los mapas de pérdidas de calor al refrigerante y para ser acoplada a una herramienta de análisis de sistemas de refrigeración del motor. Una aplicación inmediata del modelo es su acoplamiento a los modelos de predicción y diagnóstico de la combustión y de renovación de la carga, en los cuales el conocimiento de las temperaturas medias de las superficies en contacto con los gases del cilindro y los gases de admisión y escape es indispensable.

\subsubsection{Predicción de las temperaturas y los flujos de calor en estado transitorio}

El modelo térmico permite calcular también las temperaturas en todos los nodos del motor en condiciones transitorias. El programa ofrece la evolución temporal de las temperaturas del motor, que se produce al pasar de un punto de funcionamiento (ensayo inicial) a otro de condiciones de contorno conocidas (ensayo final) - cambio dinámico en escalón de las condiciones de operación -. 
La ejecución del programa en modo de operación transitoria requiere que el modelo haya sido ajustado en estacionario. Una vez que este ajuste ha sido hecho, puede emprenderse el estudio de la respuesta del modelo a los cambios en escalón de los puntos de operación. Para el primer motor el modelo fue probado bajo una serie de pruebas transitorias (28) variando la carga y también variando las revoluciones. El resumen de esas pruebas es el que aparece en la tabla 3.5. El paso temporal de cálculo es un parámetro a elección, que se elige considerando la eficiencia y la exactitud del cálculo, pero dada la resolución de la adquisición de las temperaturas experimentales en dicho motor, la duración del paso se elige de un segundo. La calidad de las predicciones de las temperaturas se valora por las mediciones de 39 termopares dispuestos en la culata y la camisa del cilindro. Para el pistón no se dispuso de medidas experimentales.

Tabla 3.5. Resumen de los procesos transitorios de prueba del motor DV6.

\begin{tabular}{|c|c|c|}
\hline Parámetro & Rango de variación & Condiciones \\
\hline & Transición de carga & Revoluciones \\
\hline \multirow[t]{7}{*}{$\begin{array}{c}\text { Carga } \\
\text { (pme, bar) }\end{array}$} & $\begin{array}{l}3,29-10,66 \\
10,66-3,29\end{array}$ & 1000 \\
\hline & $\begin{array}{c}3,9-16,86 \\
16,86-3,85\end{array}$ & 1500 \\
\hline & $\begin{array}{l}6,54-18,77 \\
3,28-12,03 \\
18,77-6,48 \\
12,03-3,28\end{array}$ & 2000 \\
\hline & $\begin{array}{l}4,18-12,64 \\
12,64-4,14\end{array}$ & 2500 \\
\hline & $\begin{array}{c}4,42-12,79 \\
5,42-1,69 \\
12,79-4,21 \\
\end{array}$ & 3000 \\
\hline & $\begin{array}{l}4,22-12,22 \\
12,22-4,21\end{array}$ & 3500 \\
\hline & $\begin{array}{l}2,55-8,55 \\
8,55-2,55\end{array}$ & 4000 \\
\hline
\end{tabular}




\begin{tabular}{|c|c|c|}
\hline & $\begin{array}{l}2,41-8,81 \\
8,81-2,37\end{array}$ & 4500 \\
\hline & Transición de revoluciones & Carga \\
\hline & $1078-3013$ & \multirow{2}{*}{4,04} \\
\cline { 2 - 3 } Régimen & $3013-1078$ & \\
\cline { 2 - 3 }$(\mathrm{rpm})$ & $1092-2710$ & \multirow{2}{*}{8,08} \\
& $1042-4001$ & \\
& $2710-1091$ & \\
\hline
\end{tabular}

Los resultados de las pruebas del modelo con este motor son muy satisfactorios. A continuación se describen algunos resultados para ilustrar la validez del modelo.

El comportamiento transitorio de las temperaturas de la camisa del modelo del motor se puede observar en las gráficas de la Figura 3.29, en la que se muestra la evolución de la temperatura en dos puntos extremos a lo largo de la camisa, distanciados 8,8 y $89,1 \mathrm{~mm}$ de la superficie de la culata, bajo dos transitorios de carga: de 2,41 a 8,81 bar de presión media efectiva a un régimen constante de 4500 rpm y de 3,29 a 10,66 bar a 1000 rpm.

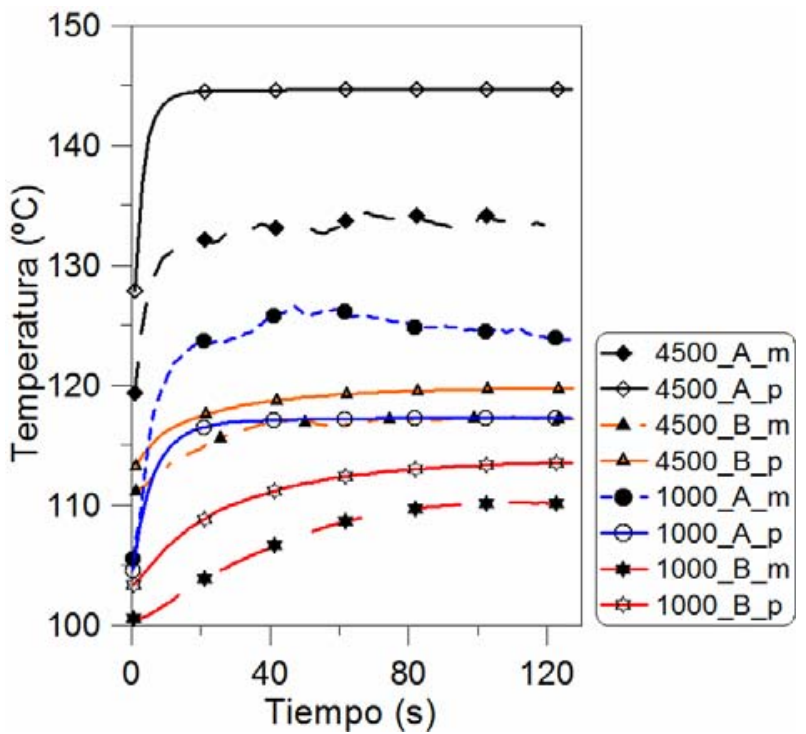

Figura 3.29.

Temperaturas transitorias para un par de nodos longitudinales, a y $B$, extremos de la camisa bajo dos procesos transitorios: a) $4500 \mathrm{rpm}$ (2 - 8 bar $), \mathrm{b}$ ) $1000 \mathrm{rpm}$ (3 - $10 \mathrm{bar})$. 
Parece que el modelo sobrevalora las temperaturas para la parte superior de la camisa, especialmente a velocidades y cargas mayores. Esto se debe de un lado al error del modelo en la predicción de las temperaturas estacionarias, cuyo valor medio total es de $7,37^{\circ} \mathrm{C}$ [3.13] y de otro lado a la finura de la discretización de la camisa, la cual determina la masa y, en consecuencia, la respuesta temporal de los nodos del modelo. La mayor fuente de error está en los errores de estado estacionario, puesto que la dimensión de los nodos en lo que respecta a la camisa puede hacerse lo bastante pequeña. Si se recuerda que un mallado más fino redunda en un mayor tiempo de cálculo, esto puede no ser deseable si el modelo ha de acoplarse a otros programas. Los resultados de las temperaturas para la parte inferior de la camisa son muy satisfactorios.

En el caso de la culata, en la Figura 3.30 y Figura 3.31 se puede ver el curso seguido por la temperatura en dos de sus nodos. La valoración cualitativa es muy buena para el asiento de las válvulas, mientras que para el nodo de la superficie de la culata situado entre las válvulas de escape, la valoración a bajas velocidades no es igual de buena. Aquí las observaciones hechas para los nodos de la camisa también son válidas, con el añadido de que el proceso de transferencia de calor del lado del refrigerante para está zona es complejo, el mecanismo de transferencia de calor no está plenamente determinado (en esta zona puede presentarse ebullición nucleada, por ejemplo), y difícilmente el modelo de convección del lado del refrigerante evaluado con un solo coeficiente de transferencia de calor para todo el motor, puede dar cuenta de lo que allí este pasando. De necesitarse una mejor resolución de la temperatura en esta zona, deberá incorporarse un modelo que considere la aparición de la ebullición nucleada y de película en la camisa de refrigeración del motor [2.71], como se comentó en el capítulo 2.

El error medio del modelo en la predicción de las temperaturas del pistón en estado estacionario es de $10,87{ }^{\circ} \mathrm{C}$. Como no se dispone de valores experimentales para valorar las predicciones de las temperaturas del motor al pasar de un punto de operación a otro, no se puede hacer una valoración sobre la calidad de las predicciones. Sí puede decirse que el error es comparable al presentado en estado estacionario. 


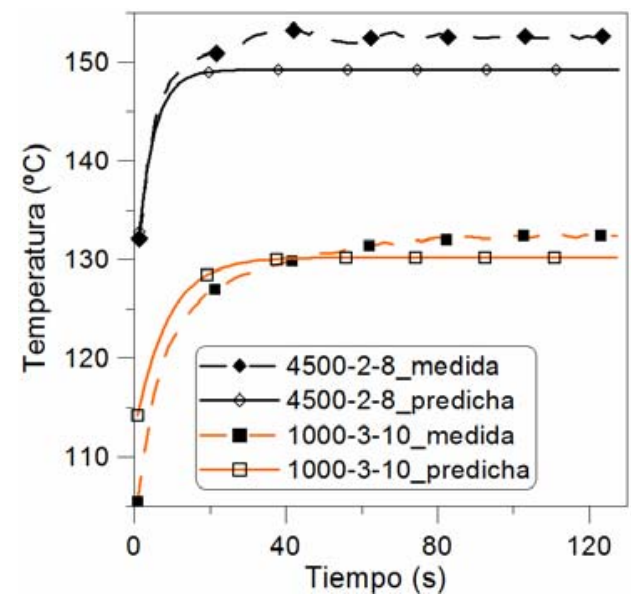

Figura 3.30. Evolución de la temperatura del asiento de la válvula bajo dos procesos transitorios de carga a bajas y a elevadas revoluciones.

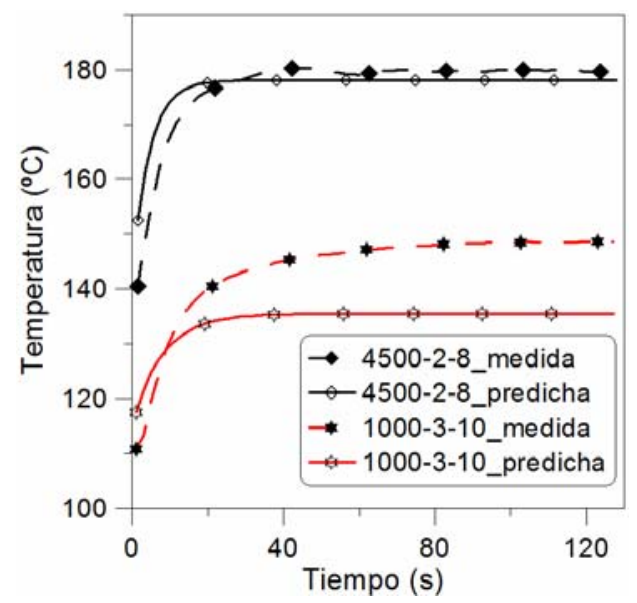

Figura 3.31. Evolución de la temperatura del nodo superficial de la culata, ubicado entre las válvulas de escape.

En la Figura 3.32 se puede apreciar la respuesta del modelo al cambio de carga del motor bajo para el borde y el fondo de la cavidad del pistón. La estabilización de la temperatura del borde es más rápida debida a su menor inercia. A bajas revoluciones el proceso de estabilización tarda más tiempo.

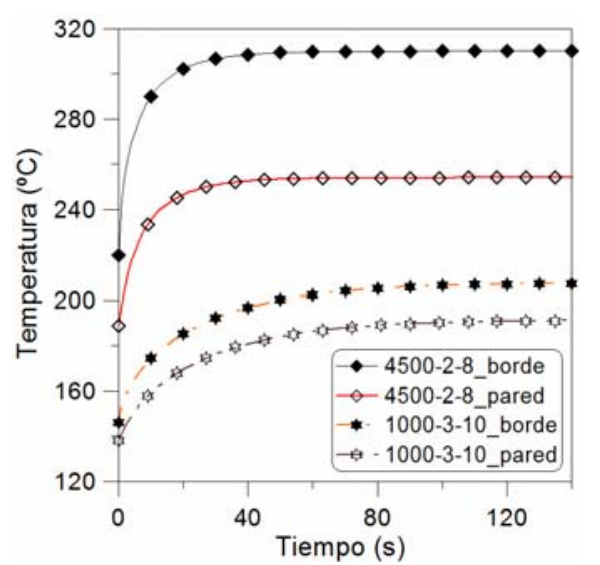

Figura 3.32. Evolución de las temperaturas predichas por el modelo para el borde y el fondo de la cavidad del pistón durante dos procesos transitorios de carga. 
Para algunos nodos del modelo las predicciones de temperaturas durante los cambios de carga presentan grandes desviaciones con relación a los valores experimentales. Estas desviaciones están asociadas tanto con las incertidumbres (por ejemplo, el modelo asume que todo el calor proviene de la combustión y no hace una discriminación de los flujos de calor generados por la fricción), como con las simplificaciones del modelo (reducido número de nodos, correlaciones simplificadas y globales para valorar las condiciones de contorno, etc.). En general, el modelo da una valoración satisfactoria de la evolución de la temperatura y los flujos de calor durante los cambios de operación transitorios.

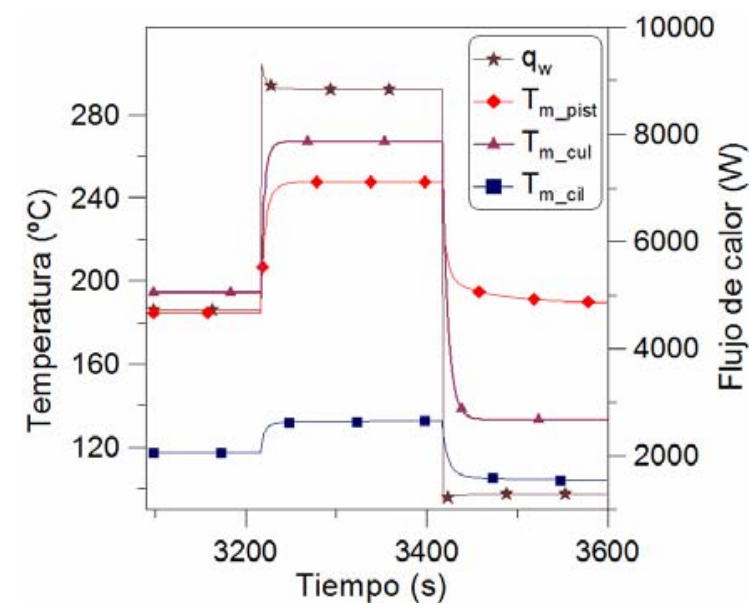

Figura 3.33. Evolución del flujo de calor hacia las superficies de la cámara de combustión y de las temperaturas medias de ésta, a medida que el motor experimenta transiciones en sus condiciones de operación.

El programa de predicción de temperaturas puede usarse para predecir la evolución de las temperaturas de los nodos del modelo de motor y sus flujos de calor para una secuencia de ciclos transitorios aleatorio, conocidas las variables medias e instantáneas en el cilindro. En la Figura 3.33 se presentan el comportamiento del flujo de calor desde los gases hacia las paredes de la cámara de combustión del motor, junto con las temperaturas medias alcanzadas por estas paredes durante la transición. La transición en la operación del motor (calentado ya) de un estado de funcionamiento a otro provoca un cambio muy rápido en la magnitud de los flujos de calor hacia las superficies (el proceso de combustión, dependiendo de los estados de carga, puede tardar entre 0,2 y 1,5 segundos en estabilizarse), mientras que, debido a la inercia térmica (más ampliamente, a las constantes de tiempo de los nodos), las temperaturas requieren en promedio de 35 a 65 segundos para estabilizarse. 
En la Figura 3.34 se representa la evolución de las temperaturas de la cámara de combustión del primer motor cuando se somete a un ciclo aleatorio de operación. La variación de los flujos de calor también se presenta. Como una información, aportada por el modelo, es el flujo de calor discriminado para todas las condiciones de contorno, conocidas las condiciones de operación del motor para puntos conocidos de un ciclo, es posible predecir el flujo de calor hacia el refrigerante, y en general predecir el comportamiento del balance térmico del motor bajo ese ciclo.

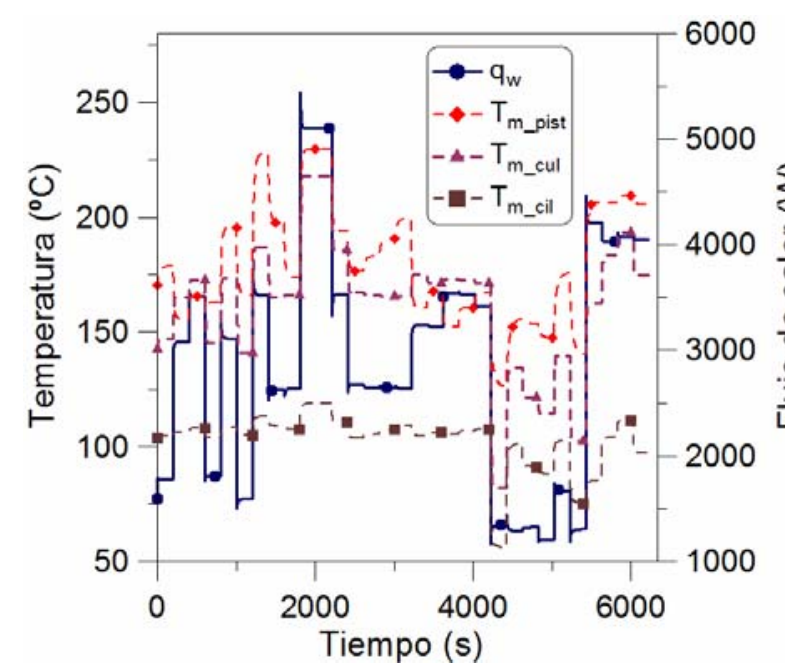

Figura 3.34. Predicción de los flujos de calor hacia las paredes de la cámara de combustión y temperaturas medias de éstas, cuando el motor se somete a un ciclo de operación aleatorio.

Una aplicación particularmente importante para el modelo es la predicción de las temperaturas de pared de la cámara de combustión durante un ciclo de conducción homologado como los ciclos NEDC o FTP.

\subsection{Predicción de las temperaturas y los flujos de calor del segundo motor}

\subsubsection{Predicción de los flujos de calor al refrigerante}

En el parágrafo 3.2 del presente capítulo se comentó que para este motor se habían registrado las variables medias e instantáneas operando bajo diferentes condiciones de operación estacionarias. Estas condiciones de operación se detallan en la tabla 3.7. 
Tabla 3.7. Condiciones de prueba estacionarias del motor.

\begin{tabular}{cccc}
\hline pme, bar & Régimen & pme, bar & Régimen \\
\hline 0 & $1750,2250,3500$ & 10 & 2500 \\
2 & $1000,1500,2500,4000$ & 12 & 2000,3000 \\
3 & 1500,3000 & 14 & 2500,3000 \\
4 & 1000,3000 & 16 & $1750,2000,2500$ \\
6 & 2500 & 18 & 1750,2000 \\
8 & 2500 & 20 & 2000,2250 \\
8,8 & 3500 & & \\
\hline
\end{tabular}

Utilizando el programa se predijeron las temperaturas del motor y los flujos de calor entre nodos bajo cada una de esas condiciones. Las temperaturas medias de las superficies del pistón, la culata y la camisa calculadas con el modelo a un régimen constante de $2500 \mathrm{rpm}$ y varios valores de la presión media efectiva se han graficado en la Figura 3.35. También se ilustra la sensibilidad de las temperaturas del motor a la variación del régimen bajo dos condiciones de carga correspondientes a 2 bar y 16 bar en la Figura 3.36.

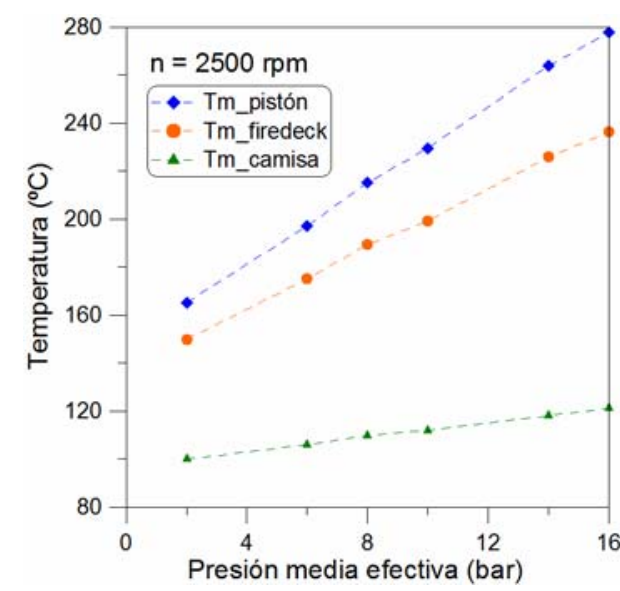

Figura 3.35. Sensibilidad de las temperaturas medias de las superficies del pistón, la culata y la camisa del motor a la variación de la carga (pme) bajo un régimen constante.

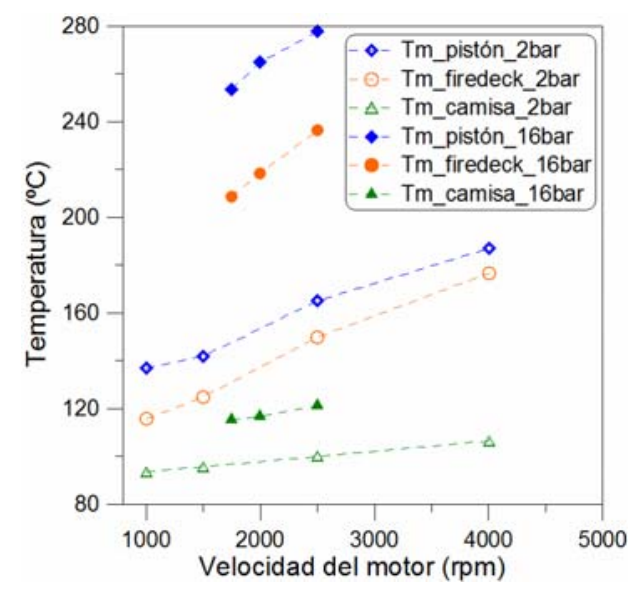

Figura 3.36. Sensibilidad de las temperaturas medias de las superficies del pistón, la culata y la camisa del motor a la variación del régimen bajo dos condiciones de carga (pme de 2 y 16 bares). 
Los resultados del modelo permitieron calcular el mapa de disipación de calor al refrigerante. La resolución del mapa, ilustrado en la Figura 3.37, está limitada por el número de pruebas disponible.

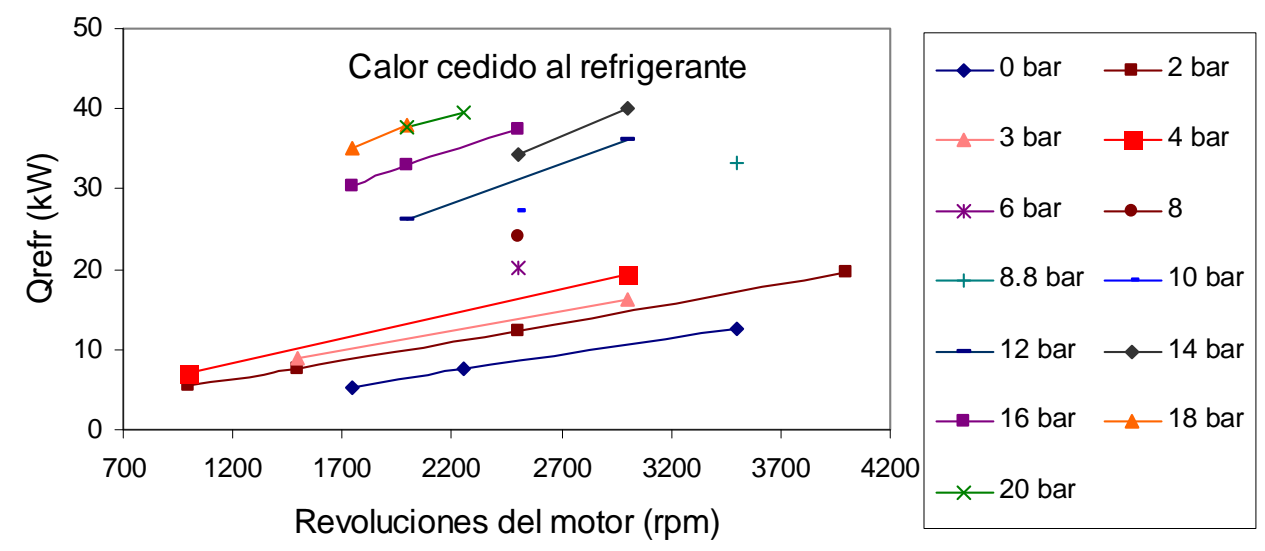

Figura 3.37. Mapa de flujos de calor al refrigerante del motor ensayado.

De manera análoga a lo realizado por Fisher [3.24], se halló una expresión para correlacionar los calores disipados al refrigerante con la temperatura media del motor, $T_{\text {refr }}\left({ }^{\circ} \mathrm{C}\right)$, la presión media efectiva, pme (bar), y las revoluciones, $n$ (rpm), en el punto de operación, con el fin de extender la predicciones del mapa de operación a condiciones diferentes a las experimentales. De esta manera se obtuvo la ecuación lineal (3.17) con un coeficiente $R^{2}$ de $88 \%$ :

$$
\dot{Q}_{\text {refr }}=14370,82+1759,05 \cdot p m e+6,07 \cdot n-225,31 \cdot T_{\text {refr }}
$$

La gráfica de superficies de los calores disipados al refrigerante, en función de la carga y el régimen del motor y para diferentes valores de la temperatura del refrigerante, obtenida con la correlación (3.17), es la representada en la Figura 3.38. La sola influencia de la temperatura del refrigerante sobre el calor disipado a éste para un punto de carga típico de 4 bar a diferentes regímenes de operación del motor se ha representado en el gráfico de la Figura 3.39. De estas gráficas se concluye la gran importancia que tiene la temperatura del refrigerante sobre las pérdidas de calor. 


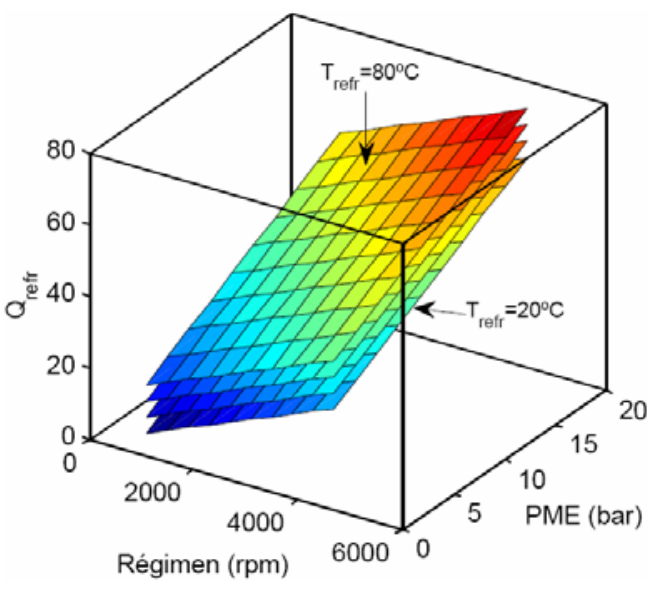

Figura 3.38. Mapa térmico del motor. En él se destacan las superficies de los calores disipados en caliente $\left(T_{\text {refr }}=80^{\circ} \mathrm{C}\right)$ y en frio $\left(T_{\text {refr }}=20^{\circ} \mathrm{C}\right)$.

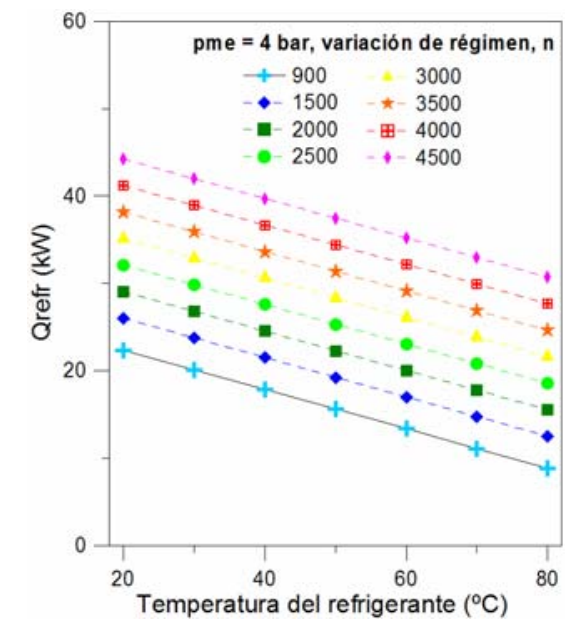

Figura 3.39. Flujo de calor disipado al refrigerante en función del régimen, para un punto de carga correspondiente a una presión media efectiva de 4 bar.

El mapa experimental de la Figura 3.37 y las predicciones obtenidas con la correlación (3.17) pueden usarse como información inicial para el diseño, el modelado y la simulación del sistema de refrigeración del motor.

\subsubsection{Predicción del comportamiento térmico del motor bajo condiciones de calentamiento}

La información experimental de las variables de operación del motor durante su calentamiento bajo carga constante y bajo condiciones de ciclo de conducción NEDC se emplea para predecir las temperaturas del motor y los flujos de calor. En la Figura 3.40 puede verse la evolución de las temperaturas del motor en calentamiento bajo condiciones de carga y régimen constantes (50 Nm - 1500 rpm). Las temperaturas predichas del cilindro están muy acordes y son razonablemente cercanas en magnitud a las temperaturas experimentales del aceite, el refrigerante y la superficie externa del bloque. El promedio predicho de la diferencia de temperaturas entre el cilindro y el refrigerante es de aproximadamente $22{ }^{\circ} \mathrm{C}$, lo que parece razonable durante el calentamiento bajo cargas bajas de ciclo. 


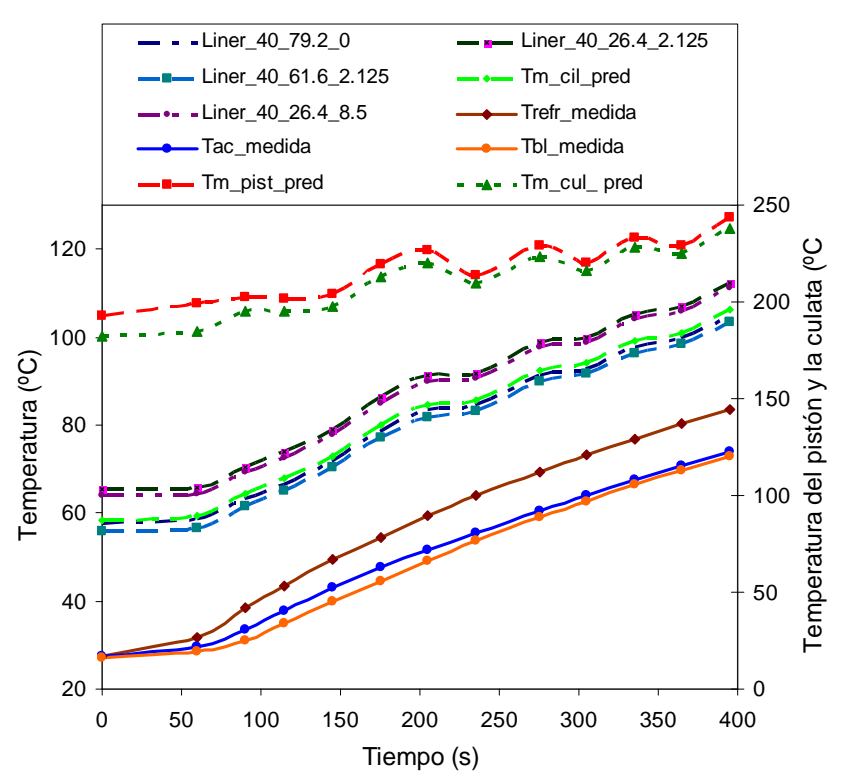

Figura 3.40. Temperaturas medidas y predichas por el modelo durante el calentamiento bajo carga constante, junto con las temperaturas del refrigerante, el aceite y la superficie externa del motor.

En la Figura 3.40, se conserva la designación original para los nodos del cilindro, provista en el programa, en la que, por ejemplo, "liner 40 61.6 2.125" referencia la ubicación longitudinal, circular y radial de un nodo de la camisa del cilindro. Las temperaturas predichas del cilindro están muy cercanas a la temperatura del refrigerante, con valores mayores a ésta para la mitad superior, y menores para la mitad inferior.

El modelo térmico, validado para el primer motor se utiliza aquí para predecir las temperaturas del segundo motor, dándole el uso para el que fue diseñado, es decir, para predecir las temperaturas y los flujos de calor de cualquier motor de características y dimensiones semejantes. Sin embargo, es oportuno observar la variación de las temperaturas de la camisa para valorar cualitativamente su tendencia en relación con las temperaturas medidas del aceite, el refrigerante y la temperatura externa.

Como se anotó en la introducción de este capítulo, un objetivo del trabajo aquí presentado ha sido la valoración de la conveniencia de adquisición de las variables medias e instantáneas en el cilindro durante el propio calentamiento del motor, particularmente durante el ciclo de conducción, sin recurrir a la 
utilización de puntos registrados durante estados estacionarios. Efectivamente, en la literatura abierta que el autor ha podido revisar, lo tradicional es encontrar predicciones de temperatura del motor a partir de información obtenida bajo puntos de operación estabilizados, más no utilizando información registrada durante el propio proceso de calentamiento. La información obtenida con puntos de operación estabilizados demanda la realización de un número de pruebas correspondientes a puntos de operación del motor durante su calentamiento con el requerido control de la temperatura que se asume tendrá el motor en ese proceso de calentamiento (actividad que demanda tiempo y recursos). La razón de este procedimiento ha sido la aparente lentitud en el muestreo de los equipos de adquisición de la información y la poca confianza en la utilización de variables medias no estabilizadas. Como se aprecia en la Figura 3.41 las predicciones de las temperaturas del motor operando bajo ciclo de conducción NEDC, a partir de la información registrada durante el mismo ciclo, son buenas, en lo que tiene que ver con los niveles de temperatura comparados con las temperaturas medidas de refrigerante, aceite y temperatura externa. Son especialmente confiables las predicciones de la camisa.

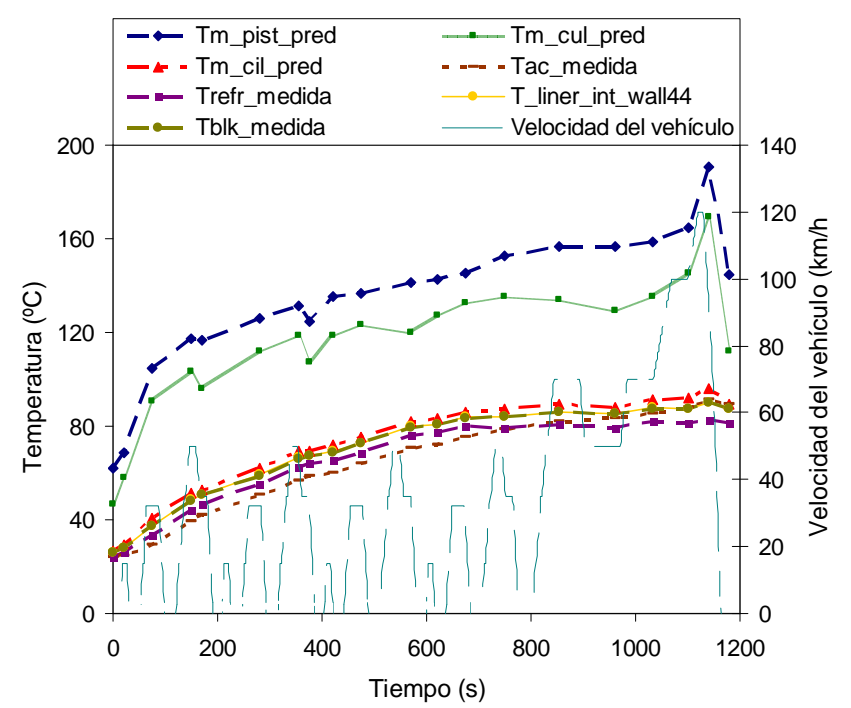

Figura 3.41. Evolución de las temperaturas medias de la superficie del pistón, la culata y la camisa y de un nodo interno de esta, comparadas con las temperaturas del refrigerante, el aceite y la temperatura externa medida, a medida que pasa el ciclo NEDC. 
Las fluctuaciones en los perfiles de las gráficas se pueden considerar una consecuencia de la adquisición de las variables durante el ciclo de conducción, dado que se utilizan valores no estabilizados de las temperaturas de los fluidos, sobre todo de los gases de admisión y escape. No obstante el rizado de las temperaturas, en general la medición de las variables durante el calentamiento del motor permite calcular temperaturas que guardan proporciones cercanas con las esperadas. Como no se tienen medidas de temperaturas internas del motor no se puede valorar el error, pero cualitativamente el resultado es bueno y tiene el beneficio de ahorrar trabajos y tiempo de ajuste y experimentación cuando se trata de predecir el comportamiento térmico del motor durante su calentamiento. Una resolución más fina en la adquisición de las variables seguro puede mejorar la calidad de las predicciones.

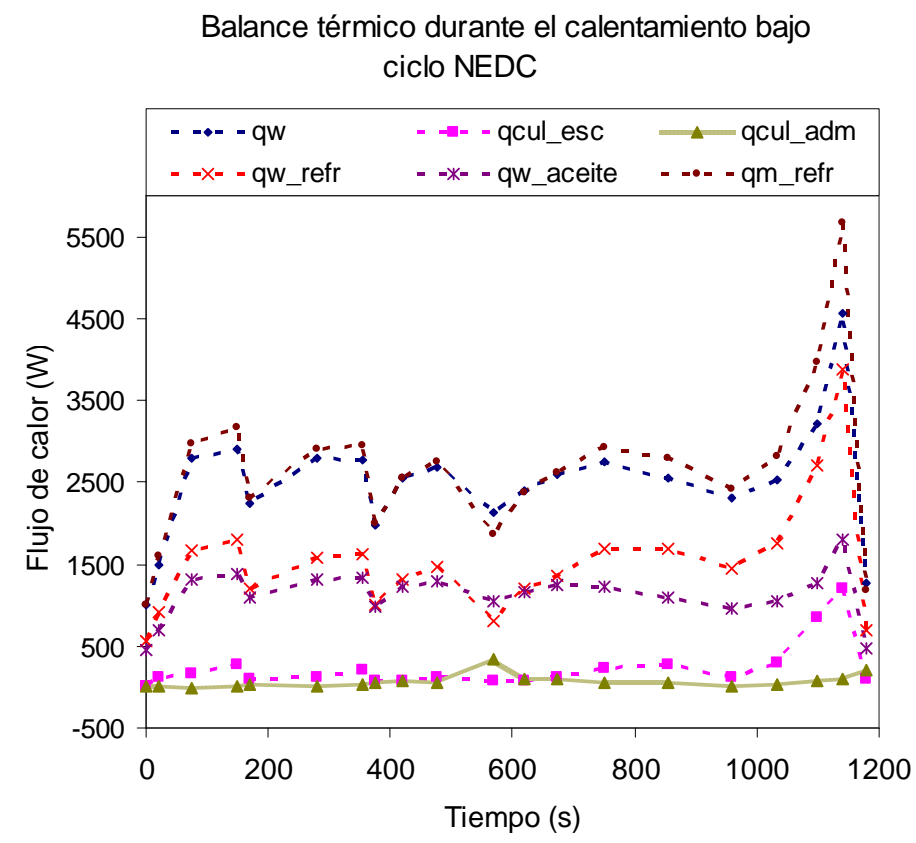

Figura 3.42. Componentes del balance energético interno predicho del motor durante el calentamiento bajo un ciclo de conducción NEDC (considerado para un cilindro).

Una comparación de los flujos de calor al refrigerante durante el calentamiento del motor a partir del modelo actualmente no es posible porque 
el modelo geométrico del motor que se ha introducido incluye sólo la parte interna; la parte externa a las galerías de refrigeración constituyen fronteras adiabáticas. Sin embargo, el programa sí permite realizar el balance de los flujos energéticos internos, como se explicó en el parágrafo 3.3.3.1, Figura 3.28. En la gráfica de la Figura 3.42 se ilustra este balance energético, en el que con $q_{w}$ se identifica el calor que fluye a través de las paredes de la cámara de combustión. El calor total recibido por el refrigerante incluye este calor más los calores provenientes del aceite y los gases de escape; la carga de admisión, por el contrario, recibe calor de las partes metálicas, en general. Este balance energético es útil para el dimensionamiento de los sistemas de refrigeración y los cálculos térmico-mecánicos y tribológicos de las partes del motor y el lubricante.

\subsubsection{Observaciones}

En esta sección del capítulo se pretendía presentar la extensión de un modelo de predicción de temperaturas a la predicción de los flujos de calor entre los nodos del modelo geométrico del motor y emplear dicho modelo en la predicción de las temperaturas en estado transitorio de los motores. Tras mostrar la validación sobre las mediciones experimentales en un primer motor, el modelo se ha utilizado para predecir las temperaturas durante el calentamiento de un segundo motor, para el cual las variables medias e instantáneas se habían registrado bajo condiciones no estabilizadas de calentamiento.

Del lado del gas en la cámara de combustión, se ha promediado en el ciclo de motor el coeficiente de transferencia de calor, calculado a partir de 25 ciclos de motor consecutivos para cada punto de operación. Se ha asumido que cuando el motor opera en una cierta condición, el coeficiente de transferencia de calor y la temperatura promedio del gas no dependen mucho de la temperatura de las paredes de la cámara de combustión. Esta aproximación ha sido justificada por Stone [3.47], quien demostró que el proceso de combustión alcanza su condición de estado “estable” en menos de 10 ciclos después de que se produce la primera combustión del motor, y las siguientes combustiones se estabilizarán más rápidamente.

Las temperaturas se predicen con buena tendencia y los flujos de calor desde las paredes son buenos, salvo algunos pocos puntos en los que probablemente 
las temperaturas se adquirieron justo durante transitorios severos de flujos y temperaturas de gases de admisión y escape. Esto sugiere que podría mejorarse la predicción realizando mediciones más continuas durante el ciclo y obteniendo medias móviles de las variables medias de operación del motor adquiridas.

\subsection{Conclusiones}

En este capítulo se ha realizado la caracterización térmica de un motor, a partir de las pruebas experimentales y de la utilización de un programa predictivo de temperaturas. Como resultado se tienen valores estimados de la inercia térmica de la "masa sensible interna" del motor y del motor integrado al sistema de refrigeración, se dispone del mapa de disipación de calor registrado para un buen número de puntos de operación y se cuenta con valores de las temperaturas y los flujos de calor hacia las paredes, que sirven como condiciones de contorno en otros modelos de gestión térmica del motor y en modelos de predicción y diagnóstico de la combustión. También se cuenta con la caracterización hidráulica. Concretamente el conocimiento de la inercia térmica del motor y los calores disipados a las paredes puede emplearse en el diseño, modelado y simulación de los sistemas de refrigeración.

La información de las predicciones también se ha usado para obtener una correlación lineal del calor disipado al refrigerante en función de la presión media efectiva, la velocidad del motor y la temperatura del refrigerante. Un resultado importante de este capítulo ha sido también la determinación experimental del balance térmico del motor durante su calentamiento bajo carga constante y bajo un ciclo de conducción estándar, tras lo cual se han sacado conclusiones referentes a los factores que influyen sobre el calentamiento del motor. Ha quedado clara la influencia de la masa como resistencia dinámica importante durante el calentamiento y se han calculado los calores transportados y las temperaturas del refrigerante y los gases de escape, como información inicial utilizable en los proyectos de recuperación de energía en los vehículos.

Las variables medias e instantáneas adquiridas durante el calentamiento del motor bajo carga constante y bajo ciclo de operación pudieron emplearse en 
el modelo térmico y las temperaturas predichas son muy razonables, mostrándose así que una alternativa a la obtención de la información en caliente del motor y en condiciones estabilizadas para los puntos de operación representativos de un ciclo de calentamiento (actividad costosa y dispendiosa) es la adquisición de las variables durante el mismo ciclo de conducción. Aunque el modelo utilizado no calcula los flujos de calor transportados por el refrigerante durante el calentamiento, sí permite predecir los flujos de calor transmitidos a las paredes de la cámara de combustión. Una actividad por realizar en el modelo es la extensión del modelo hasta la superficie externa del motor.

La próxima etapa en la investigación presente consiste en el acoplamiento de los resultados de la identificación y el mapa del motor para el modelado del sistema de refrigeración y la propuesta de estrategias de refrigeración que reduzcan el tiempo de calentamiento del motor.

Para mejorar la capacidad predictiva del modelo puede ser importante que este:

- $\quad$ incorpore el modelo de fricción de manera separada;

- valide con mayor resolución las temperaturas del pistón, puede ser empleando valores experimentales de un motor similar de dimensiones y masas conocidas;

- considere en detalle los pares térmicos pistón-película de aceitesegmentos-película de aceite-cilindro;

- extienda la geometría del modelo hasta la parte externa del motor, para poder integrar la masa externa y considerar otras interfases como la del aceite con las partes metálicas de la culata;

- discrimine la topología del recorrido del refrigerante a través de la camisa de refrigeración;

- realimente las temperaturas predichas dentro de un ciclo de modelado, de suerte que sólo se deban introducir al modelo las temperaturas iniciales del aceite y el refrigerante. Así para dentro de cada ciclo completo, el modelo podría ejecutarse para predecir las temperaturas de los nodos en el ciclo siguiente. 


\subsection{Bibliografía}

[3.1] Luptowski, B., Parker, G., Arici, O. Johnson, J. Development of the enhanced vehicle and engine cooling system simulation and application to active cooling control. SAE paper 2005-01-0697.

[3.2] Cortona, E. Engine Thermomanagement for Fuel Consumption Reduction. PhD dissertation, ETH Zürich, 2000.

[3.3] Chastain, J. H., Wagner, J. R. Advanced thermal management for internal combustion engines - valve design, component testing and block redesign. SAE paper 2006-01-1232.

[3.4] Koch, F. W., Haubner, F. G. Cooling system development and optimization for DI engines. SAE paper 2000-01-0283.

[3.5] Hnatczuk, W., Kozierowski, J. Thermal management for the 21 century - Improved control and fuel economy in an army medium tactical vehicle. SAE paper 2005-01-2068.

[3.6] Eberth, J., Wagner, J. Afshar, B., Foster, R. Modeling and validation of automotive thermal management system architectures. SAE paper 2004-01-0048.

[3.7] Chanfreau, M., Gessier, B., Farkh, A., Geels, P. Y. The need for an electrical water valve in a thermal management intelligent system. SAE paper 2003-01-0274.

[3.8] Chalgren, R. D., Traczyk, T. Advanced secondary cooling systems for Light trucks. SAE paper 2005-01-1380.

[3.9] Chalgren, R. D., Allen, D. J. Light duty Diesel advanced thermal management. SAE paper 2005-01-2020.

[3.10] Cho, H., Jung, D., Filipi, Z. S., Assanis, D. N., Vanderslice, J., Bryzik, W. Application of Controllable Electric Coolant Pump for Fuel Economy and Cooling Performance Improvement. IMECE200461056.

[3.11] Pang, H.H., Brace, C. J. Review of engine cooling technologies for modern engines. Proc. Instn. Mech. Engrs., part D: J Automobile Engineering, vol. 218, pp. 1209-1215, 2004.

[3.12] Norris, P. M., Wepfer, W., Hoag, K. L., Courtine-White, D. Experimental and analytical studies of cylinder head cooling. SAE paper 931122. 
[3.13] Degraeuwe, B.M.A. Heat transfer model of a Diesel engine. Tesis doctoral, Universidad Politécnica de Valencia. España, 2006.

[3.14] Brace, C. J., Burnham, H. Integrated cooling systems for passenger vehicles. SAE paper 2001-01-1248.

[3.15] Wambsganss, M. W. Thermal management in heavy vehicles: A review identifying issues and research requirements.

[3.16] Schock, $\mathrm{H}$, et al. Thermoelectric conversion of waste heat to electricity in an IC engine powered vehicle. Advanced Combustion Engine Technologies, FY 2005 progress report.

[3.17] Morel, T., Keribar, R. Warm-up characteristics of a spark ignition engine as a function of speed and load. SAE paper 900683.

[3.18] Jarrier, L. Champoussin, J. C., Yu, R., Gentile, D. Warm-up of a D. I. Diesel engine: experiment and modeling. SAE paper 2000-01-0299.

[3.19] Shayler, P. J., Christian S. J., Ma, T. A model for the investigation of temperature, heat flow and friction characteristics during engine warm-up. SAE paper 931153.

[3.20] Taymaz, I. An experimental study of energy balance in low heat rejection diesel engine. Energy, vol. 31, pp. 364-371, 2006.

[3.21] Perez-Blanco, H. Experimental characterization of mass, work and heat flows in an air cooled, single cylinder engine. Energy conversion and management, vol. 45, $\mathrm{n}^{\circ}$ 2, pp. 157-179, 2004.

[3.22] Lehner, C., Parker, G., Arici, O. Johnson, J. Design and development of a model based feedback controlled cooling system for heavy duty diesel truck applications using a vehicle engine cooling system simulation. SAE paper 2001-01-0336.

[3.23] Heywood, J.B., Internal combustion engine fundamentals, Mc-Graw Hill, 1988.

[3.24] Fisher, G. Expertenmodell zur Berechnung der Reiungsverluste von Ottomotores. PhD dissertation, Technische Universitaet Darmstadt, Germany, 2000.

[3.25] Torregrosa, A, Olmeda, P, Degraeuwe, B., Reyes, M. A concise wall temperature model for DI Diesel engines. Appl. Therm. Eng., vol. 26, issue 12, pp. 1320-1327, 2006.

[3.26] Allen, D. J. Lasecki, M. P. Thermal management evolution and controlled coolant flow. SAE paper 2001-01-1732. 
[3.27] Kamo R, Bryzik, W. Cummins-TRADOCOM adiabatic turbocompounded engine program. SAE Paper 810070.

[3.28] Caton, J. A. A review of investigations using the Second Law of Thermodynamics to study internal combustion engines. SAE paper 2000-01-0952.

[3.29] Robinson, K., Campbell, N.A., Hawley, J.G., Tilly, D. G. A review of precision engine cooling. SAE paper 1999-01-0578.

[3.30] Rakopoulos, C.D., Andritsakis, E.C., Huontalas, D.T. The influence of the exhaust system unsteady gas flow and insulation on the performance of a turbocharged diesel engine. Heat Recovery Systems and CHP Journal vol. $15 \mathrm{n}^{\circ}$ 1, pp. 51-72, 1995.

[3.31] Torregrosa, A, Olmeda, P, Martin, J., Degraeuwe, B. Experiments on the influence of inlet charge and coolant temperature on performance and emissions of a DI Diesel engine. Exp. Therm. Fluid Sci., vol. 30, pp. 633-641, 2006.

[3.32] Torregrosa, A.J., Broatch, A., Olmeda, P, Romero, C. Assessment of the influence of different cooling system configurations on engine warm-up, emissions and fuel consumption. IJAT, vol. 9, $\mathrm{n}^{\circ} 4$, pp. 447458, 2008.

[3.33] Broatch, A., Luján, J.M., Ruiz, S., Olmeda, P. Measurement of hydrocarbon and carbon monoxide emissions during the starting of automotive DI Diesel engines. IJAT, vol. 9, $\mathrm{n}^{\circ}$. 2, pp. 129-140.

[3.34] Payri, F., Molina, S., Martin, J., Armas, O. Influence of measurement errors and estimated parameters on combustion diagnosis. Appl. Therm. Eng., vol. 26, n 2, pp. 226-236, .

[3.35] Arregle, J., Bermúdez, V., Serrano, J. R., Fuentes, E. Procedure for engine transient cycle emissions testing in real time. Exp. Therm. Fluid Sci., vol. 30, nº 5, pp. 485-496, 2006.

[3.36] Borman, G., Nishiwaki, K. Internal Combustión Engine Heat Transfer. Progress in Energy Combustion Sciences, vol. 13, pp. 1-46, 1987.

[3.37] Chang, J., Güralp, O., Filipi, Z., Assanis, D. Kuo, T., Najt, P., Rask, R. New heat transfer correlation for an hcci engine derived from measurements of instantaneous surface heat flux. SAE paper 2004-012996. 
[3.38] Reichelt, L., Meingast, U., Renz, U. Calculating transient wall heat flux from measurements of surface temperature. Int. J. Heat Mass Transfer, vol. 45, pp. 579-584, 2002.

[3.39] Morel, T., Keribar, R. Heat radiation in D. I. Diesel engines. SAE paper 860445.

[3.40] Descombes, G., Maroteaux, F, Feidt, M. Study of the interaction between mechanical energy and heat exchanges applied to IC engines. Appl. Therm. Eng., vol. 23, issue 16, 2003, pp. 2061-20078.

[3.41] Alkidas, A. C. Performance and emissions acheivements with an uncooled heavy_duty, single_cylinder Diesel engine. SAE paper 890144.

[3.42] Wolf-Heinrich, H. Aerodynamics of road vehicles. SAE, Warrandale, 1998.

[3.43] Woschni, G. A Universally applicable equation for the instantaneous heat transfer coefficient in the I.C. engine. SAE paper 670931.

[3.44] Reyes M. Desarrollo de un modelo para el análisis térmico de MCIA. Seminario en el CMT, Universidad Politécnica de Valencia. España. Septiembre, 2003.

[3.45] Bohac, S.V., Baker, D.M., Assanis D.N. A global model for steadystate and transient S.I. engine heat transfer studies. SAE Paper 960073.

[3.46] Veshagh, A., Chen, C. A computer model for thermofluid analysis of engine warm-up process. SAE paper 931157.

[3.47] Stone, R. Introduction to Internal Combustion Engines. MacMillan publishers. 1999.

[3.48] Morel, T., Blumberg, P.N., Fort, E.F., Keribar, R. Methods for heat analysis and temperature field analysis of the insulated Diesel. NASA CR-174783, 1984.

[3.49] Lapuerta, M., Armas, O., Hernández, J.J. Diagnosis of DI Diesel combustion from in-cylinder pressure signal by estimation of mean thermodynamic properties of the gas. Appl. Therm. Eng., vol. 19 pp. 513-529, 1999. 


\section{Capítulo 4}

\section{Consideraciones y herramientas de diseño y modelado de los sistemas de refrigeración de los motores.}

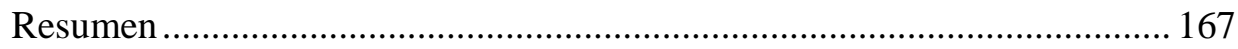

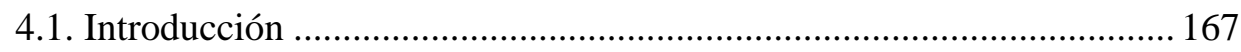

4.2. Prediseño de los sistemas de refrigeración de los motores .................... 169

4.2.1. Cálculo preliminar de los componentes del sistema de

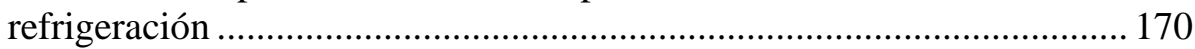

4.2.2. Metodología de diseño de los sistemas de refrigeración ................ 184

4.2.3. Cálculo de los radiadores de los sistemas de refrigeración............. 187

4.3. Modelado de los sistemas de refrigeración ............................................ 196

4.3.1. Modelos simplificados de la temperatura del motor a nivel de

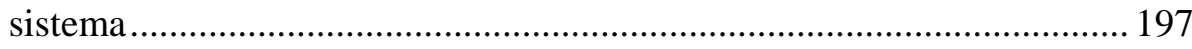

4.3.2. Modelos a nivel de circuito del sistema de refrigeración del

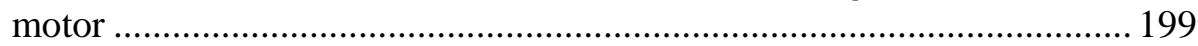

4.4. Herramientas de modelado unidimensional ........................................ 208

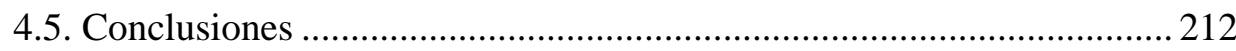

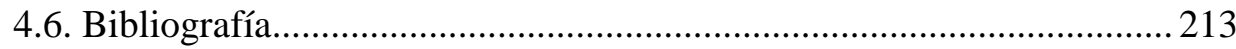




\section{Resumen}

En el capítulo anterior, aparte del estudio de los flujos de calor y las temperaturas en el interior del motor como componente, fue analizado el balance térmico de éste acoplado a su circuito de refrigeración, así como también se pudo ver el tiempo que tarda el motor en calentarse y se conocieron los factores de los que depende ese calentamiento. Pensadas estas actividades como diagnóstico de la respuesta de los sistemas de refrigeración modernos, cabe preguntarse qué trabajos pueden emprenderse para mejorar la respuesta térmica de sistemas ya diseñados o en operación y qué herramientas de computación pueden integrarse a las tareas de rediseño, modelado y simulación de nuevos sistemas de refrigeración. En los capítulos quinto y sexto de esta tesis se da respuesta a esos interrogantes, particularizando dos tareas: la modificación al sistema original del motor probado para mejorar su calentamiento, y la concepción, modelado y simulación original del sistema de refrigeración de un motor. Como preparación a esos trabajos, en el presente capítulo se describe el procedimiento de diseño de los sistemas de refrigeración, se introducen los fundamentos termohidráulicos que están en la base de los programas comerciales de modelado de estos sistemas y se describe brevemente la utilización de los mismos.

\subsection{Introducción}

El diseño funcional de los sistemas de refrigeración parte de la necesidad de proveer la suficiente capacidad de evacuación de calor demandada por el motor, y otras posibles fuentes, a intercambiadores de calor externos al motor, como radiadores, recuperadores de calor, calentadores de cabinas, etc., conservando las temperaturas de los fluidos del sistema y las partes metálicas dentro de unos valores de control propicios desde el punto de vista del consumo de combustible y las emisiones, ajustándose a un balance energético entre fuentes y sumideros como se sintetiza en el cuadro de la Figura 4.1.

Al inicio sólo se conoce la aplicación, el motor que propulsa el vehículo o medio de transporte (en general sin características térmicas), las opciones para el posible trazado y ubicación de su sistema hidráulico, el espacio y la disposición para los radiadores y los ventiladores y las condiciones ambientales de operación. Lo que se tiene que hacer es seleccionar los radiadores y ventiladores adecuados para evacuar el calor disipado por las 
fuentes al refrigerante y acoplar estos componentes al espacio disponible y observando los niveles de temperatura de los fluidos y las partes metálicas.

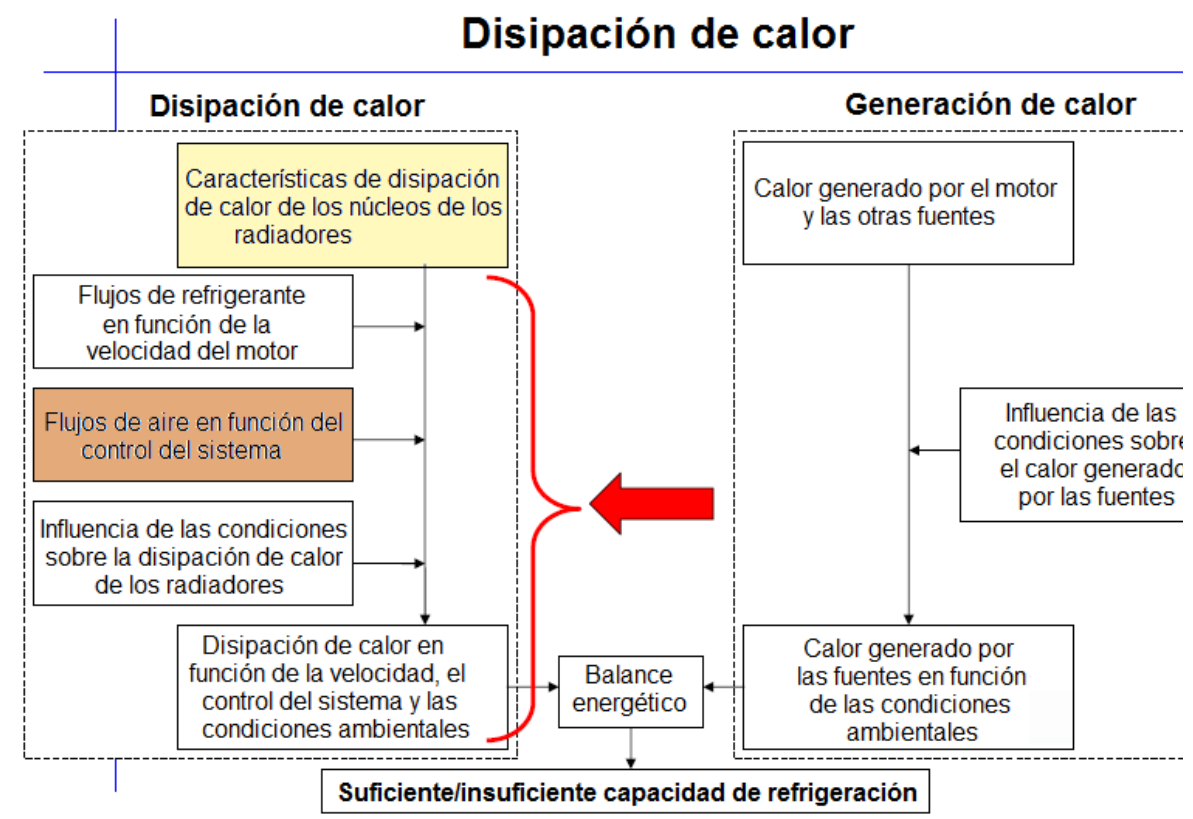

Figura 4.1. Planteamiento del problema de disipación de calor en los motores.

Aunque el problema energético resumido en la Figura 4.1 se ve sencillo y se resuelve a nivel de componentes concentrados, en la práctica su solución satisfactoria demanda de un cuidadoso trabajo de modelado termohidráulico de flujos de refrigerante líquido y de aire, en función del trazado de la red del refrigerante y del sistema de ventilación. El refinamiento adicional del diseño comprende tareas de optimización mediante diferentes tecnologías entre las cuales se encuentran el control de los flujos de refrigerante y, en sistemas más avanzados, la refrigeración localizada del motor, tareas que demandan del estudio detallado de las camisas de refrigeración del motor con cálculos CFD y del modelado del sistema de refrigeración a la luz de la teoría de los sistemas dinámicos y de control. Para efectos del presente trabajo, el diseño se trata desde la perspectiva clásica funcional, pero involucrando las 
herramientas de cálculo computacional disponibles para la selección y cálculo de radiadores y para el modelado de los circuitos del refrigerante y de ventilación del sistema. Bajo este marco de referencia, en los siguientes parágrafos de este capítulo se abordará el prediseño del sistema de refrigeración, la teoría de cálculo de los intercambiadores, la teoría básica que subyace en los códigos computacionales de modelado de sistemas termohidráulicos 1D y una descripción del tipo de información requerida por los programas utilizados en los capítulos siguientes, para el modelado de los sistemas de refrigeración del motor ensayado en el capítulo tercero y para el diseño del sistema de refrigeración del motor considerado en el capítulo sexto.

\subsection{Prediseño de los sistemas de refrigeración de los motores}

En general, el proceso de diseño de los sistemas de refrigeración comprende la definición de las variables funcionales de los principales componentes del sistema, la configuración provisional del circuito con diámetros y longitudes tentativas de las tuberías y conexiones requeridas según la topología, el diseño de detalle de los componentes o su selección, el diseño de los circuitos de refrigerante y de ventilación, y los cálculos de los flujos y las temperaturas en el circuito o el modelado y la simulación del sistema, seguidos opcionalmente por trabajos de validación. Los pasos descritos se corresponden con las rutinas tradicionales en el diseño de los sistemas de refrigeración y, aunque aún hoy son vigentes, es cierto que la disponibilidad de bases de datos de componentes y de programas computacionales para el cálculo de los mismos hace que esas rutinas tradicionales se combinen en la práctica moderna sin obedecer necesariamente a una secuencia operacional, siendo frecuente la solución simultánea iterativa del problema [4.28], [4.4]. Teniendo esto presente, en este parágrafo se comenta un procedimiento de prediseño de los parámetros básicos de la bomba, el ventilador y el radiador conducentes a un trabajo de selección de los mismos en bases de datos correspondientes (o a diseños constructivos) y luego se pasa a contextualizar la operatividad del diseño de los sistemas de refrigeración a la luz de las herramientas de cálculo y programas de modelado existentes, proponiendo una metodología de cálculo iterativa para los sistemas de refrigeración de los motores. 


\subsubsection{Cálculo preliminar de los componentes del sistema de refrigeración}

El cálculo preliminar del sistema de refrigeración convencional de un motor consiste en la determinación del área de superficie de refrigeración del radiador, la característica de la bomba de agua y la selección del ventilador. $\mathrm{Si}$ el sistema incluye otros intercambiadores, como refrigeradores de aire ("aftercooler" e "intercooler"), refrigeradores de aceite de motor y transmisión (oil coolers) y refrigeradores de gases de escape recirculados ("EGR coolers"), en circuitos independientes, puede preverse varios circuitos independientes de refrigeración, en cuyo caso posiblemente se requiera calcular múltiples radiadores, bombas y ventiladores. Puede pasar que, dependiendo de la configuración del sistema de ventilación, los circuitos compartan la parte de ventilación. Cada diseño tendrá sus particularidades.

Calor disipado al sistema de refrigeración. La cantidad total de calor a disipar por el sistema de refrigeración es un parámetro que, en general, no se conoce al inicio del diseño y que es de importancia fundamental no sólo para el dimensionamiento del sistema de refrigeración, sino también para el diseño de la toma de aire de ventilación de todo el grupo motopropulsor y para el diseño aerodinámico del vehículo en el cual se instala. Esta cantidad de calor puede venir definida por una persona experta en el diseño del motor particular, puede calcularse empleando un modelo térmico como el empleado en el capítulo tercero (véase (3.29)), puede obtenerse por alguna relación empírica o de contenido físico establecida, o puede asumirse a partir de las tendencias de los motores de la misma familia, por referencia a motores semejantes en función de la potencia del motor o en función del consumo de combustible. A continuación se resumirán las alternativas para la determinación del calor disipado al refrigerante que se han tenido presentes dentro de la presente tesis:

- La correlación empírica de Lahvic [4.2], aplicable a motores de encendido provocado y motores Diesel y obtenida tras estudiar la disipación de calor en un número de motores Ford:

$$
\dot{Q}_{r e f r}[k W]=\left(8,66 V_{d} \cdot n+108,93 \cdot M_{T}+1119,74 \cdot N_{e}-1010 \cdot V_{d}+2890\right) / 3412,2
$$


donde $V_{d}$, en l, es la cilindrada; n, en rpm, corresponde al régimen;

$M_{T}$, en Nm, es el par del motor; y $N_{e}$, en $\mathrm{kW}$ es la potencia efectiva del motor.

- La correlación con fundamento físico de Parish [4.1] desarrollada por éste para reemplazar la correlación de Lahvic, también para Ford, menos expedita en su aplicación pero más elaborada, de mayor exactitud y más representativa de los fenómenos de transferencia de calor por convección y radiación dentro del cilindro y con consideración de las pérdidas por fricción. Expresado en forma normalizada, con relación al volumen admitido por minuto $\left(V_{d} \cdot n / 2\right)$, el producto del calor específico a evacuar por el sistema de refrigeración, $q_{\text {refr }}\left(q_{\text {refr }}=\dot{Q}_{\text {refr }} / N_{e}\right)$, y la presión media efectiva (pme), tiene la forma:

$$
q_{r e f r} p m e=\frac{2,50 k_{g} \operatorname{Re}^{0,7} \operatorname{Pr}^{0,3} \pi D}{4 V_{d} n / 2}\left(\bar{T}_{g}-T_{w}\right)+\frac{0,341 \sigma D^{2}}{4 V_{d} n / 2}\left(\bar{T}_{f}^{4}-T_{w}^{4}\right)+5,98 \frac{n}{2}
$$

donde $\sigma$ es la constante de Stefan-Boltzman; $D$ es el diámetro del cilindro; $k_{g}, \operatorname{Pr}$ son la conductividad y el número de Prandtl de los gases en el cilindro, respectivamente; $R e$ es el número de Reynolds utilizado en el cálculo de la transferencia de calor entre los gases y las paredes del cilindro; $\bar{T}_{g}$ y $\bar{T}_{f}$ son las temperaturas medias de los gases y de la llama, respectivamente; y $T_{w}$ es la temperatura de la pared de la cámara de combustión.

Parish ha llegado a esta expresión partiendo de una extensión de la formulación de Annand [4.33] para el calor disipado por las paredes de la cámara de combustión - $\dot{Q}_{r e f r}=h_{g} A\left(\bar{T}_{g}-T_{w}\right)+h_{r} \sigma A\left(\bar{T}_{f}^{4}-T_{w}^{4}\right)+N_{f}-$. La información requerida es el diámetro de los cilindros, la cilindrada $\left(V_{d}\right)$, la potencia efectiva y las revoluciones del motor. Asumiendo conocidas la eficiencia neta de conversión del combustible en el motor, como $\eta_{f}=0,40$ y la presión media efectiva de fricción, fmep $=136 \mathrm{kPa}$ (determinadas por correlaciones de cuatro motores Diesel turboalimentados ensayados en diferentes puntos de calibración), se halla la presión media efectiva disponible del motor, pmed (que es función de 
la densidad del aire, el poder calorífico del combutible, $H_{i}$, la relación aire-combustible, $A / C$ y el rendimiento volumétrico, $\eta_{v}$ : pmed $\left.=\eta_{v} \rho_{a} H_{i} /(A / C)\right)$ con la que se obtiene la relación de presiones del sobrealimentador $\left(p_{r}\right)$ y el rendimiento volumétrico del motor $\left(\eta_{V}=0,8031 p_{r}=2 \dot{m}_{a} / n V_{d} \rho_{a m b}\right)$, conociendo el poder calorífico del combustible $\mathrm{Hi}$. Estas magnitudes permiten encontrar la relación aire/combustible y con ello los gastos másicos de aire y combustible. Con toda esta información y definiendo el cambio en la temperatura de los gases en el cilindro producida por el desprendimiento de calor $\left(\Delta T_{c}=H_{i} / \dot{m} C_{p}\right)$ y asumiendo un valor para la temperatura de las paredes, $T_{w}$ (Parish tomó para su trabajo $T_{w}=110^{\circ} C$ ), pueden determinarse la temperatura media del gas $\left(\bar{T}_{g}=\Delta T_{c} / 2+T_{w}\right)$ en los cilindros, la temperatura representativa de radiación $\bar{T}_{f}=\Delta T_{c}+288,16 \mathrm{y}$ las propiedades del gas (viscosidad, conductividad térmica y calor específico), necesarias para calcular los números de Prandtl y Reynolds y, finalmente determinar el calor a evacuar al sistema de refrigeración.

- En función de la potencia efectiva del motor, el calor total a disipar a través del sistema de refrigeración puede asumirse a partir de una relación o de una gráfica, como la de la Figura 4.2, en la que se resume parte de los resultados obtenidos por Parish.

- En función del consumo de combustible puede calcularse por la expresión:

$$
\dot{Q}_{\text {refr }}=q_{\text {refrC }} H_{i} \dot{m}_{\text {comb }}
$$

donde $q_{\text {refrc }}=\dot{Q}_{\text {refr }} / \dot{Q}_{\text {equiv }}$ es la entrega relativa de calor al sistema de refrigeración, en función del calor suministrado por el combustible, $\dot{Q}_{\text {equiv }}$.

Los cuatro métodos considerados para determinar el calor disipado al refrigerante sirven para obtener el calor total, pero dado que en la mayoría de los motores modernos se está implementado la sobrealimentación y la refrigeración intermedia del aire, así como la refrigeración del aceite del motor, mediante refrigeradores de aire y de aceite ubicados cercanos al radiador principal del motor, se hace necesario tener estimativos de la 
distribución del calor total entre estos componentes. Este es un problema difícil de resolver y para él las únicas opciones que quedan son: el uso de información experimental suministrada por un experto o el análisis de las tendencias de los motores de la misma familia, en función de la potencia del motor o en función del consumo de combustible.

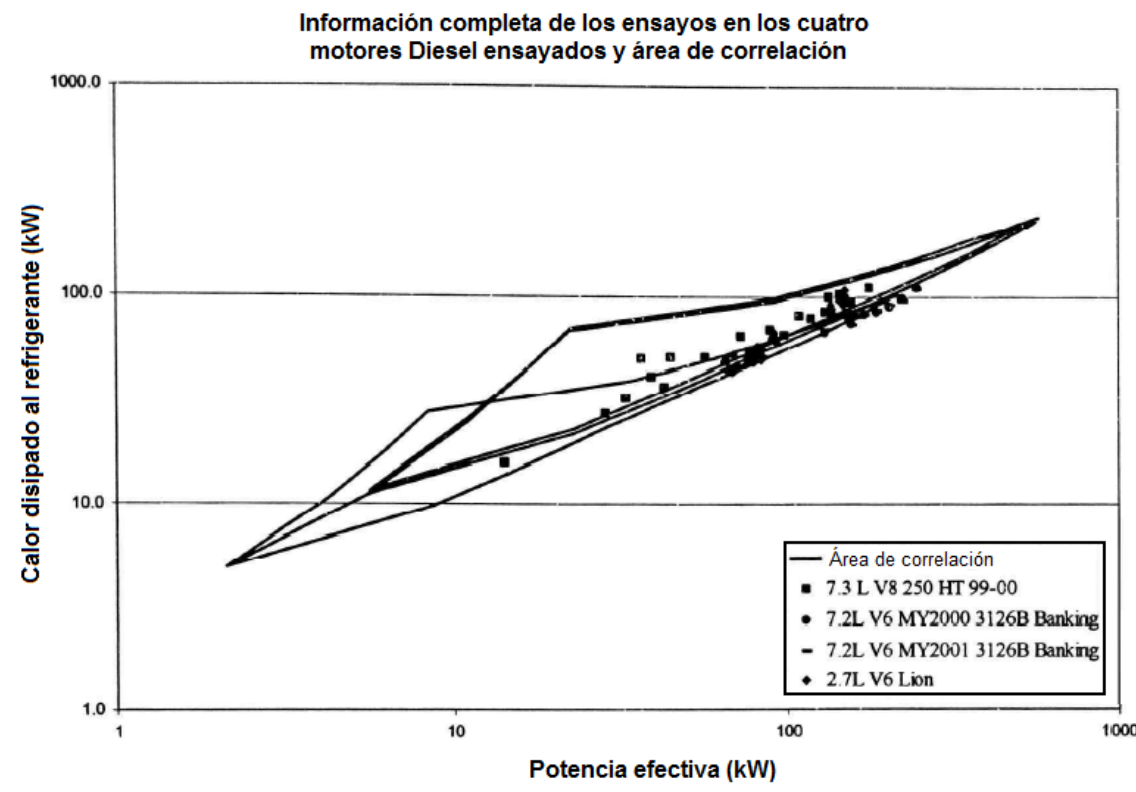

Figura 4.2. Calor disipado al refrigerante en función de la potencia efectiva del motor [4.1].

La entrega relativa de calor al sistema de refrigeración no es sencilla de estimar, pues varía mucho para los diferentes motores y tamaños, y en función de los regímenes de carga y revoluciones. En las Figuras 4.3 y 4.4 se han insertado dos gráficas que muestran la variación del calor disipado al sistema de refrigeración de motores a través del bloque del motor y a través del enfriador de aire de admisión del motor (“aftercooler"). La disipación de calor a través del refrigerador de aceite puede ir incorporada en la disipación a través del bloque del motor y otras veces puede no estar referenciada.

A medida que aumenta la carga el valor relativo de las pérdidas de calor disminuye. Un estimativo basado en un $28 \%$ de la potencia equivalente 
aportada por el combustible es un valor conservador y seguro para los cálculos de prediseño del sistema de refrigeración (selección de radiador) de motores Diesel. Los motores a gas actuales tienen una mayor disipación de calor al refrigerante (equivalente a cerca de un $66 \%$ de la potencia equivalente). Para el caso de un "aftercooler”, la capacidad de su radiador puede ser cercana a un $20 \%$ de la potencia efectiva.

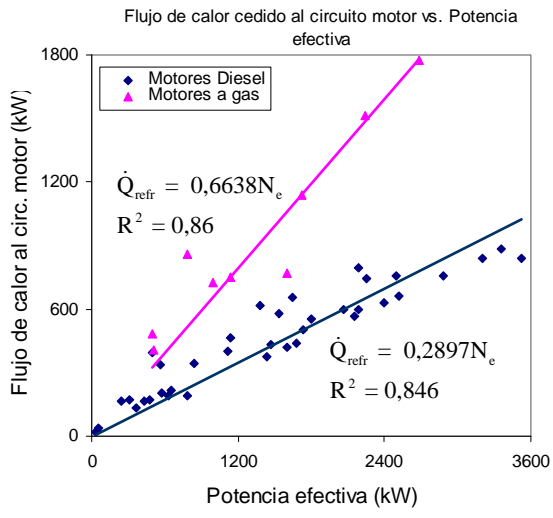

Figura 4.3. Calor disipado al refrigerante que pasa por el motor.

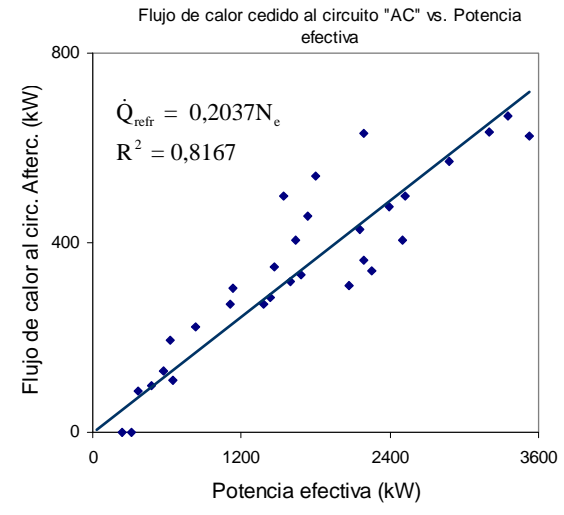

Figura 4.4. Calor disipado al refrigerante que pasa por el "aftercooler".

A medida que crece la potencia nominal de los motores, la disipación específica de calor también se reduce. Las gráficas se han obtenido para motores de rangos de potencias desde los más pequeños en grupos electrógenos, hasta los más grandes utilizados en locomotoras. Se ha utilizado información de motores Perkins, Volvo, Caterpillar, Cummins, General Motors, John Deere y Detroit Diesel.

Se reitera que debe tenerse cuidado con la estimación de la disipación específica. De tres motores de la GE analizados (GE7FDM8, GE7FDM12 y GE7FDM16), los calores a disipar en los dos circuitos de camisa del motor y del refrigerador de aire, según la información de catálogo, son de 34\% y 29\%, es decir, el calor evacuado por el circuito de alta (incluye "camisa de refrigeración del motor" y refrigerador de aceite) corresponde a aproximadamente un $34 \%$ de la potencia entregada por el motor a plena carga y el evacuado por el circuito de baja corresponde aproximadamente a 
un $29 \%$ de esa potencia máxima. El calor entregado directamente por la “camisa de refrigeración” en el circuito de alta es de un $65 \%$, con un $35 \%$ para entregarse por el refrigerador de aceite.

El radiador. En general el cálculo del radiador se puede efectuar en el siguiente orden, válido para el cálculo de otros intercambiadores en el sistema:

1. Partiendo de la cantidad de calor $\dot{Q}_{\text {refr }}(\mathrm{kJ} / \mathrm{s})$, a disipar a través del sistema de refrigeración durante el trabajo del motor en el régimen de potencia nominal, se obtiene el valor corregido a disipar, considerando el eventual ensuciamiento del radiador:

$$
\dot{Q}_{\text {refr_c }}=1,1 \dot{Q}_{\text {refr }}
$$

2. La cantidad de calor del motor evacuada por el refrigerante $\left(\dot{Q}_{r e f r_{-} c}\right)$, se toma igual a la cantidad de calor transmitida al aire circundante $\left(\dot{Q}_{\text {refr_c }}=\right.$ $\left.\dot{Q}_{\text {aire }}\right)$.

3. El caudal del aire $\left(\mathrm{m}^{3} / \mathrm{s}\right)$ que pasa a través del radiador:

$$
\dot{V}_{\text {aire }}=\dot{Q}_{\text {aire }} /\left(C_{\text {aire }} \rho_{\text {aire }} \Delta T_{\text {aire }}\right)
$$

donde $C_{\text {aire }}$ y $\rho_{\text {aire }}$ son el calor específico y la densidad del aire calculados para una temperatura media del aire de entrada ambiente de $T_{\text {aire }}^{e n}=40{ }^{\circ} \mathrm{C}$ (según Zhelezko [4.35], ésta es la temperatura promedio del aire a la entrada al radiador).

El caudal de aire (en $\mathrm{m}^{3} / \mathrm{s}$ ) para motores Diesel puede tomarse en el rango $(0,015 \ldots 0,02) N_{e}$ [4.35], siendo $N_{e}$ la potencia efectiva del motor. Según un proveedor de maquinaria pesada, para la refrigeración adecuada de un motor Diesel, se requiere un caudal mínimo de aire de $0,04 \mathrm{~m}^{3} / \mathrm{s}$ por cada kilovatio de potencia neta del motor [4.34]. Otra posibilidad es fijar el valor del $\Delta T_{\text {aire }}$ ( $\Delta T_{\text {aire }}=45-55^{\circ} C$ ).

4. El gasto volumétrico de refrigerante que pasa a través del radiador es: 


$$
\dot{V}_{r e f r}=\dot{Q}_{\text {refr_c }} /\left(C_{r e f r} \rho_{r e f r} \Delta T_{r e f r}\right)
$$

donde $C_{r e f r}, \rho_{r e f r}$ son, respectivamente, el calor específico y la densidad del refrigerante calculados para una temperatura media estimada de paso del refrigerante por la camisa del radiador; $\Delta T_{\text {refr }}$ es el salto de temperatura del refrigerante a su paso por el radiador, usualmente suele encontrarse en el rango $\Delta T_{\text {refr }}=T_{\text {refr }}^{e n}-T_{\text {refr }}^{\text {sal }}=6 \ldots 12^{\circ} \mathrm{C}$.

5. La temperatura media del refrigerante en el radiador:

$$
T_{r e f r}^{m}=T_{r e f r}^{e n}-\Delta T_{r e f r} / 2
$$

6. La temperatura media del aire que pasa a través del radiador:

$$
T_{\text {aire }}^{m}=T_{\text {aire }}^{e n}+\Delta T_{\text {aire }} / 2
$$

7. El área necesaria $\left(\mathrm{m}^{2}\right)$ de superficie de refrigeración del radiador :

$$
F_{\text {rad }}=10^{3} \dot{Q}_{\text {refr_c }} /\left[U_{\text {refr }}\left(T_{\text {refr }}^{m}-T_{\text {aire }}^{m}\right)\right]
$$

donde $U_{\text {refr }}$ es el coeficiente de transferencia de calor del refrigerante al cuerpo del radiador (coeficiente global de transferencia de calor basado en el área de la superficie húmeda, $U),\left(\mathrm{W} / \mathrm{m}^{2} \mathrm{~K}\right)$ [4.35]:

$$
U_{\text {refr }}=\left[\left(1 / h_{\text {refr }}+\delta / k\right) \psi+1 / h_{\text {aire }}\right]^{-1}
$$

$h_{\text {refr }}$ es el coeficiente de película (convección) entre el refrigerante y las paredes del radiador, $\mathrm{W} /\left(\mathrm{m}^{2} \mathrm{~K}\right) ; \delta$ es el espesor de la pared, $\mathrm{m} ; k$ es el coeficiente de conductividad térmica del material de los tubos del radiador; $h_{\text {aire }}$ es el coeficiente de película entre las paredes del radiador y el aire, $\mathrm{W} /\left(\mathrm{m}^{2} \mathrm{~K}\right)$.

El valor de $h_{\text {refr }}$ depende principalmente de la velocidad de movimiento del líquido en los tubos del radiador. Para tubos rectos lisos, a las velocidades de flujo de líquido características en los motores de automóviles, $h_{\text {refr }}=2500 \ldots$ 
$4100 \mathrm{~W} /\left(\mathrm{m}^{2} \mathrm{~K}\right)$ [4.35]. El coeficiente de aristado $\psi$, para los radiadores de paneles cruzados y de paneles en serpentín, puede tomarse entre 3,5 y 6 [4.35]. Los valores del coeficiente de entrega de calor de las paredes del radiador al aire $h_{\text {aire }}=70 \ldots 140$ [4.35]. Para los radiadores de los automóviles $U_{\text {refr }}$ tiene valores de $140 \ldots 180 \mathrm{~W} /\left(\mathrm{m}^{2} \mathrm{~K}\right.$ ) (los valores mayores son para los vehículos de pasajeros) [4.36]; para el caso de las locomotoras $U_{\text {refr }}$, tomando valores promedios, puede ser del orden de $110 \mathrm{~W} /\left(\mathrm{m}^{2} \mathrm{~K}\right)$.

8. El área de la superficie frontal del radiador, en $\mathrm{m}^{2}$ :

$$
F_{f r}=\dot{V}_{\text {aire }} / v_{\text {aire }}
$$

Donde $v_{\text {aire }}$ es la velocidad del aire en el frente del radiador $\left(v_{\text {aire }}=6 . . .18 \mathrm{~m} / \mathrm{s}\right.$ [4.4]) sin tener en cuenta la velocidad de movimiento del vehículo.

9. La profundidad del panel del radiador, en mm:

$$
l_{\text {rad }}=F_{\text {rad }} /\left(F_{f r} \varphi_{\text {rad }}\right)
$$

donde $\varphi_{\text {rad }}$ es el coeficiente de compacidad volumétrica. Para los radiadores modernos $\varphi_{\text {rad }}=0,6 \ldots 1,8 \mathrm{~mm}^{-1}$ [4.36]. La profundidad del núcleo de los radiadores de automóviles varía de 25 a $150 \mathrm{~mm}$.

En los métodos de cálculo y de caracterización de los radiadores es común referir la potencia térmica disipada por el radiador a una diferencia nominal entre las temperaturas de entrada del refrigerante y del aire, definida como $I T D=T_{\text {refr }}^{e n}-T_{\text {aire }}^{e n}$, con lo que la potencia térmica del radiador referida a la diferencia de las temperaturas de entrada se expresa por:

$$
\dot{Q}_{\text {refr_c }} / I T D=U_{r e f r} F_{r a d}
$$

Si se tienen en cuenta los gastos másicos de refrigerante y de aire, otra característica común para definir un radiador es la potencia térmica del radiador por diferencia de temperaturas de entrada referida al área frontal del paso de aire, $\dot{Q}_{\text {refr_c }} /\left(I T D \cdot F_{f r}\right)=f\left(\dot{m}_{r e f r}, \dot{m}_{\text {aire }} \cdot F_{f r}\right)$. 
Los productos $\dot{m} C_{p}$ calculados para el refrigerante y para el aire se emplean corrientemente también para caracterizar los radiadores, calculando su número de unidades de transferencia de calor, NTU.

En la Figura 4.5 pueden verse los componentes principales que participan de la evacuación del calor por parte del aire, con el radiador como elemento central.

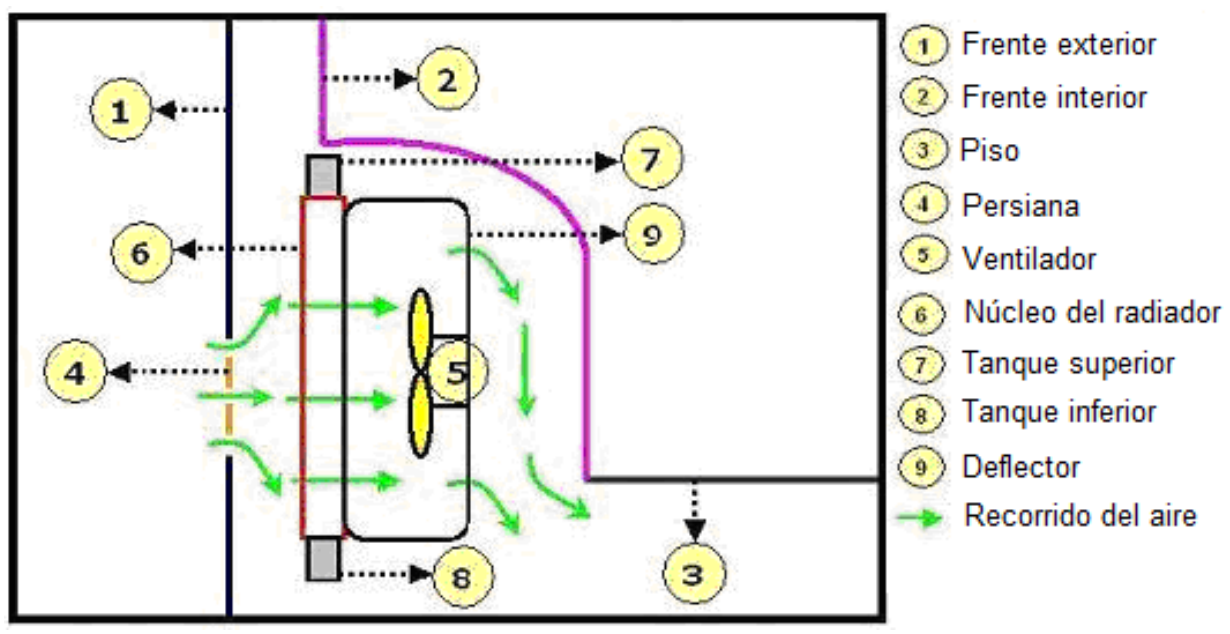

Figura 4.5. Recorrido convencional del aire del ambiente hacia el radiador de un vehículo.

En los vehículos modernos el volumen de los radiadores y el diseño de los pasajes de aire está comprometido por la configuración de los motores, la integración de nuevos componentes como los requeridos para el tratamiento de emisiones, la refrigeración de la carga fresca, la refrigeración del aceite, la ubicación del condensador del sistema de aire acondicionado del vehículo y las características estéticas y aerodinámicas del vehículo.

El mostrado es un orden para calcular los parámetros iniciales de diseño o selección del radiador. La metodología bien podría basarse estrictamente en el espacio disponible solamente y partirse de allí. Con los parámetros de diseño iniciales, que se reducen a los gastos másicos de refrigerante y de aire, 
más las dimensiones, lo que sigue es realizar el diseño del radiador, pero siempre partiendo de alguna arquitectura o diseño tipo de radiador (flujo cruzado de una o varias etapas, número de pasos, tipo de aletas, etc.), acotando también, si se puede, las pérdidas permisibles de presión, las cuales pueden tomarse por comparación con radiadores de aplicaciones análogas. El cálculo de detalle del radiador dará estos valores con exactitud.

El ventilador. Al seleccionar el ventilador deben conocerse la presión del aire creada por éste, $p_{a i}(\mathrm{~Pa})$ y el caudal de aire $\dot{V}_{\text {aire }} V_{\text {aire }}\left(\mathrm{m}^{3} / \mathrm{s}\right)$. El caudal de aire ha sido definido y la presión se debe establecer tras resolver simultáneamente el problema de circulación del aire, considerando la topología del recorrido del aire (que aún siendo simplificado puede corresponder a un esquema como el ilustrado en la Figura 4.5). Lo recomendado, si no se ha realizado un cálculo 1D ó 3D del sistema de ventilación, es prever una resistencia del sistema a partir de valores estimados de pérdidas de presión en los diferentes tramos de un esquema simplificado como el de la Figura 4.6.

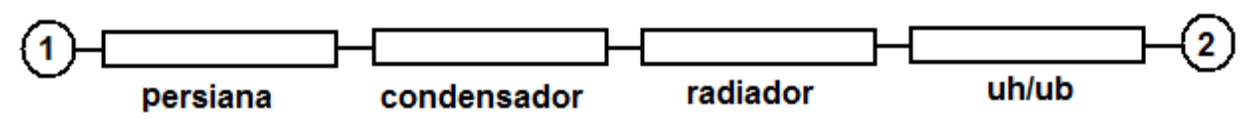

Figura 4.6. Esquema simplificado de las resistencias al paso del aire por el sistema de ventilación del motor y los radiadores.

En general, para los sistemas de ventilación de los motores, la presión de los ventiladores está en el rango de $p_{a i}=600 \ldots . .1000 \mathrm{P}_{\mathrm{a}}$.

La velocidad circular del aspa del ventilador, en $\mathrm{m} / \mathrm{s}$, en el borde externo de se determina por [4.36]:

$$
u=\psi_{a} \sqrt{p_{a i} / \rho_{a i}}
$$

donde $\psi_{a}$ es un coeficiente que depende de la forma de las aspas: $\psi_{a}=2,2 \ldots$ 2,9 para aspas curvas, $\psi_{a}=2,8 \ldots 3$ 3,5 para aspas planas. Partiendo de premisas de carácter acústico se toma $u \leq 110 \mathrm{~m} / \mathrm{s}$ [4.36]. 
El diámetro del ventilador, en metros, puede determinarse por la expresión [4.35]:

$$
D_{V}=1,3 \sqrt{\frac{\dot{V}_{\text {aire }}^{\prime}}{v_{\text {aire }}^{\prime}}}
$$

donde $\dot{V}_{\text {aire }}$ es el caudal del ventilador, determinado por la fórmula (4.5) si el sistema de refrigeración es líquido, $\mathrm{y} v^{\prime}$ aire es la velocidad de cálculo del aire en el rotor. En los conductos de aire esta velocidad depende de la relación entre el área "de sección viva" del círculo descrito por las aspas del ventilador, y la superficie frontal del radiador; esta relación se puede tomar igual a $0,45 \ldots . .0,6$ con lo que $v^{\prime}{ }_{\text {aire }}=13 \ldots 40 \mathrm{~m} / \mathrm{s}$ [4.36].

La frecuencia de rotación, en rpm, del árbol del ventilador se determina como:

$$
n_{v}=\frac{60 u}{\pi D_{v}}
$$

La potencia, en $\mathrm{kW}$, consumida por el accionamiento del ventilador se determina por:

$$
N_{V}=\frac{10^{-3} p_{a i} \dot{V}_{\text {aire }}}{\eta_{V}}
$$

donde $\eta_{V}$ es el rendimiento del ventilador: $\eta_{V}=0,6 \ldots$ 0,7 [4.35]. En la selección comercial de los ventiladores suele también hacerse uso de los coeficientes de presión, $\psi_{f d}$, y de flujo, $\varphi_{f d}$, del ventilador, definidos por:

$$
\psi_{f d}=\frac{2 \Delta p_{v e n t}}{\rho v_{p s}^{2}} ; \quad \varphi_{f d}=\frac{2 \dot{V}_{v e n t}}{\pi D_{v}^{2} v_{p s}^{2}}
$$

Los parámetros determinados constituyen los factores de entrada para el diseño de detalle o para la selección del ventilador, en general. De manera práctica el ventilador puede seleccionarse por un programa computacional de algún proveedor comercial y la información aportada por éste puede venir acompañada de características como las presentadas en la Figura 4.7. 


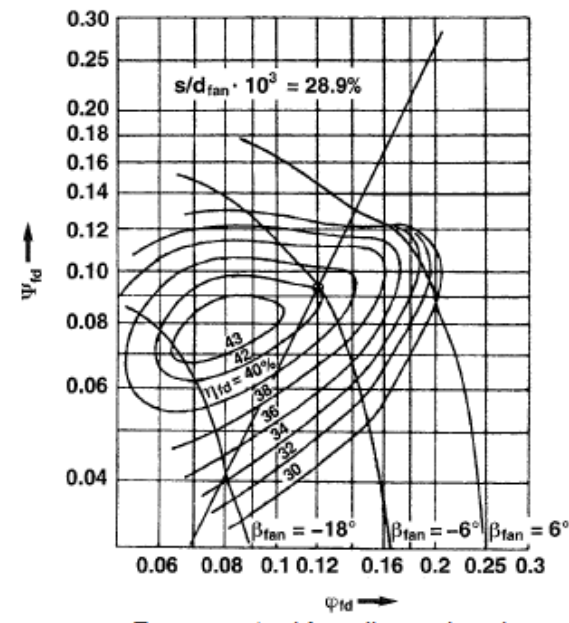

Representación adimensional

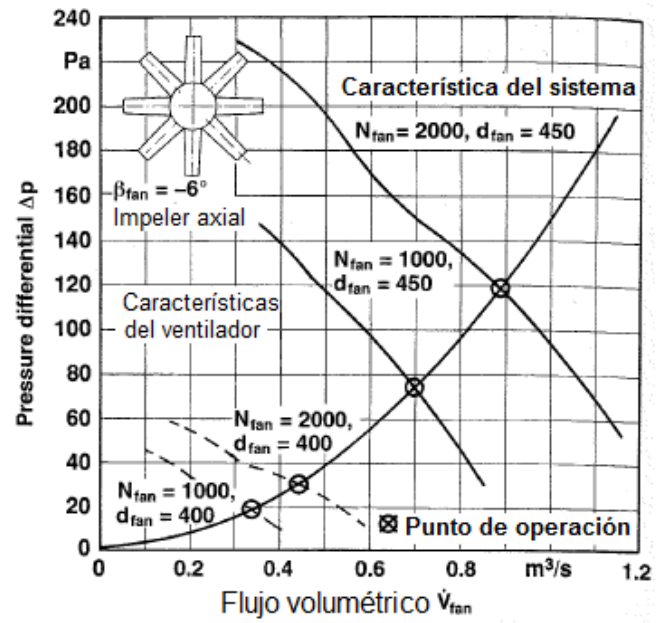

Representación adimensional

Figura 4.7. Características de un ventilador comercial [4.4].

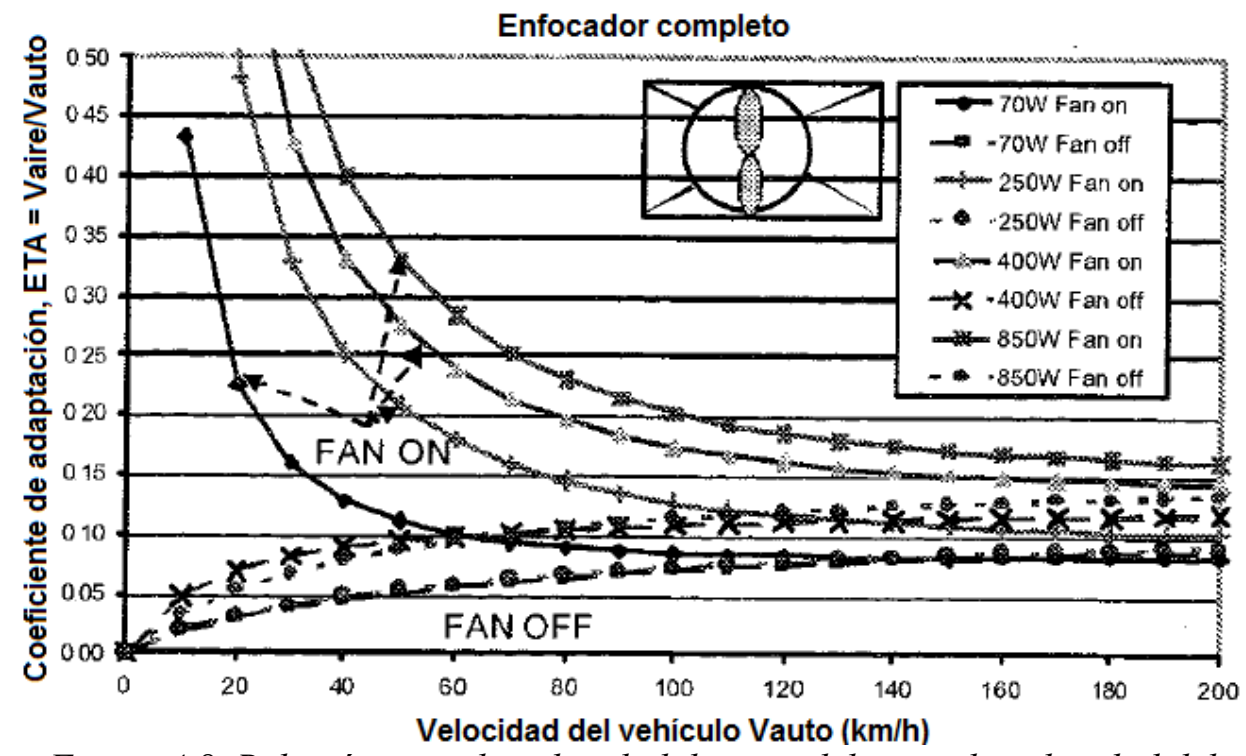

Figura 4.8. Relación entre la velocidad de paso del aire y la velocidad del vehículo para diferentes estados de encendido del ventilador en función de la velocidad del vehículo [4.3]. 
La importancia de la operación del ventilador es mayor a bajas velocidades de los vehículos y la potencia consumida es significativa mayormente para los vehículos de potencias medianas y altas. En la Figura 4.8 se reproducen los resultados experimentales de [4.3], de la que se concluye los posibles valores de la relación entre la velocidad del aire al paso por los radiadores y la velocidad del vehículo. La gráfica es representativa de los ventiladores instalados en los vehículos comerciales de cilindradas bajas y medias (los ventiladores y radiadores probados correspondieron a motores de cilindradas entre 1,6 y 2,7 litros) y puede servir de referencia durante la etapa de prediseño y estudios de selección de los componentes del sistema de ventilación.

La bomba de refrigerante. Las bombas más utilizadas son bombas centrífugas de rotor abierto. Suponiendo que haya necesidad de calcular la bomba de refrigerante, que no venga accionada por el motor, el cálculo de su caudal $(l / s)$ se realiza por la expresión:

$$
\dot{V}_{\text {refer_c }}=\frac{\dot{V}_{\text {referl }}}{\eta_{b}}
$$

donde $\eta_{b}$ es el rendimiento volumétrico de la bomba, $\eta_{b}=0,8 \ldots 0,9$.

El radio $r_{1}(\mathrm{~m})$ del conducto de succión del rotor (ver Figura 4.9) se determina de la relación $\pi\left(r_{1}^{2}-r_{0}^{2}\right)=\frac{10^{-3} \dot{V}_{r e f r_{-} c}}{c_{1}}$, cuyo cumplimiento garantiza el caudal de refrigerante a través del conducto de succión de la bomba:

$$
\begin{gathered}
\pi\left(r_{1}^{2}-r_{0}^{2}\right)=\frac{10^{-3} \dot{V}_{r e f r_{-} c}}{c_{1}}, \\
r_{1}=\sqrt{\frac{\dot{V}_{r e f e r \_c}}{\left(10^{3} \pi \cdot c_{1}\right)}+r_{0}^{2}}
\end{gathered}
$$

donde $r_{0}$ es el radio del cubo del rotor, $\mathrm{m} ; c_{1}$ es la velocidad del líquido a la entrada de la bomba: $c_{1}=1 \ldots 2,5 \mathrm{~m} / \mathrm{s}$ [4.40]. 

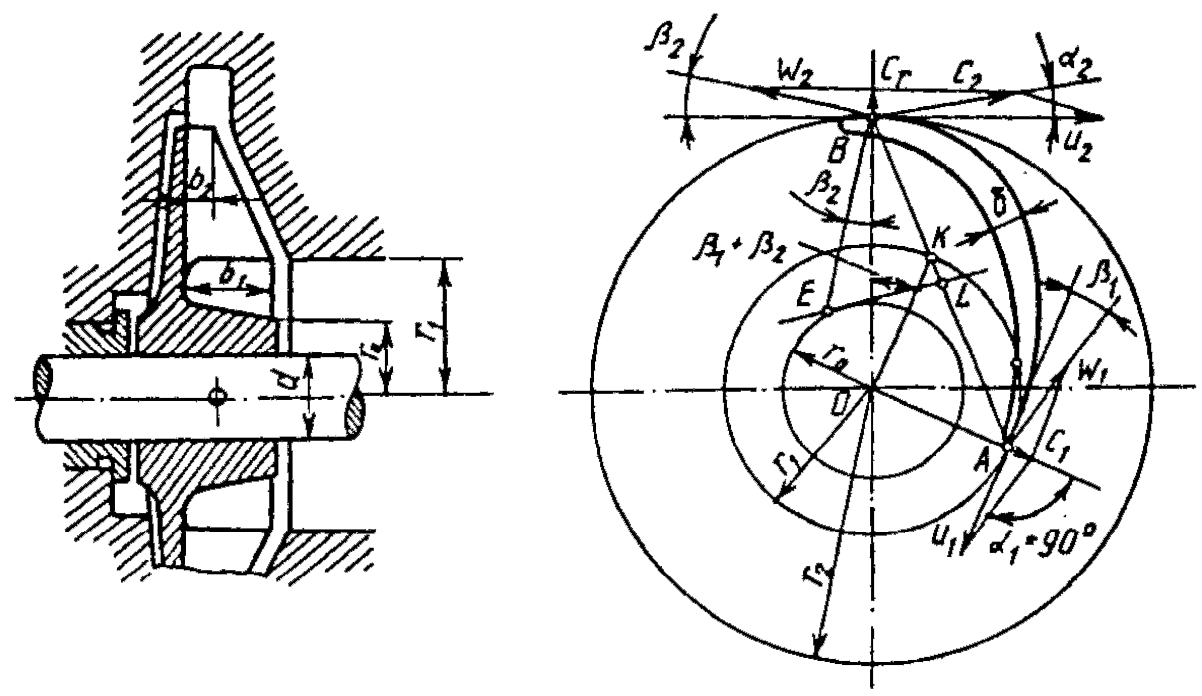

Figura 4.9. Esquema de cálculo de una bomba centrífuga.

La potencia $(\mathrm{kW})$, consumida por la bomba de agua,

$$
N_{b}=\dot{V}_{r e f r_{-} c} p_{1} / \eta_{M}
$$

donde $\eta_{M}$ es el rendimiento mecánico de la bomba de agua; $\eta_{M}=0,7 \ldots 0,9$ [4.40]. El valor de $N_{b}$ en los motores es del orden de $0,35 \ldots 1 \%$ de $N_{e}$.

Para realizar el diseño de detalle de la bomba se requiere determinar la energía cinética requerida, la cual a su vez depende de la resistencia externa al motor y de la resistencia al paso del refrigerante por el motor mismo, parámetros que pueden no ser conocidos. En la Figura 4.10, para referencia por ser representativa de las bombas instaladas en vehículos comerciales pequeños y medianos, se ilustra la característica de la bomba acoplada al sistema hidráulico del motor ensayado en el tercer capítulo (segundo motor).

La capacidad del sistema de refrigeración líquido (en litros) se puede estimar por la relación $(0,13 \ldots 0,25) N_{e}[4.35]$. 


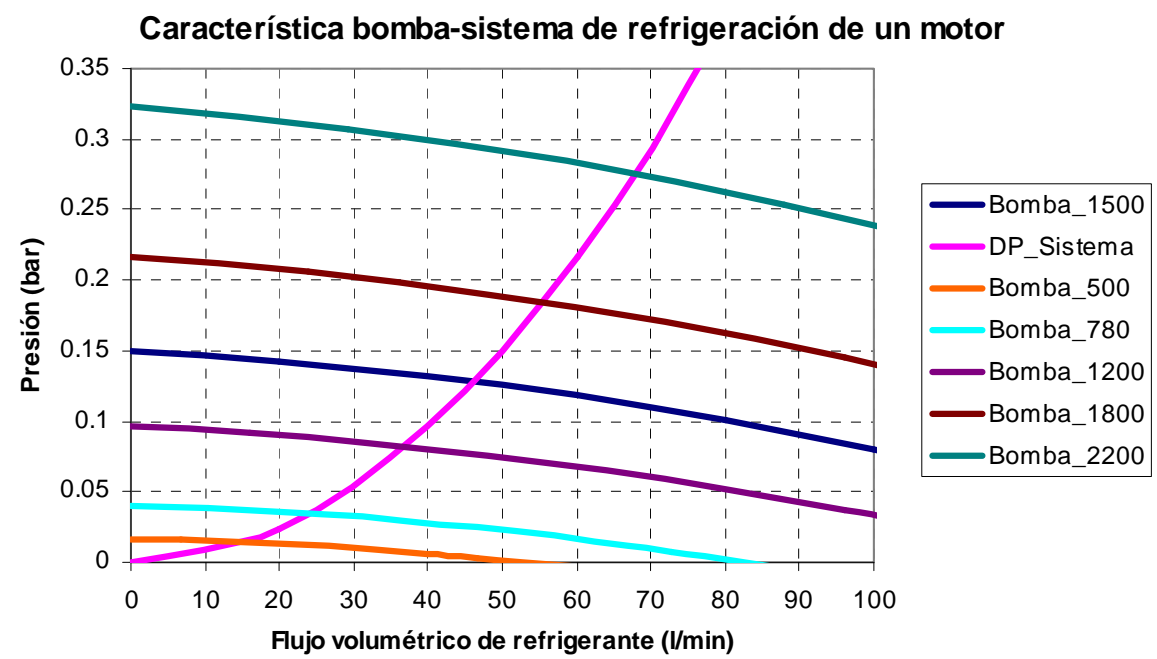

Figura 4.10. Característica de operación de una bomba acoplada al sistema de refrigeración del motor de un vehículo comercial.

Después de realizar los cálculos previos del radiador, la bomba y el ventilador, se continúa con el diseño de detalle y refinamiento de los tres sistemas funcionales: sistema hidráulico, sistema térmico motor-radiadores y sistema de ventilación. El resultado es la selección de los radiadores y el modelado y la validación del trazado de los sistemas de refrigeración y ventilación que aseguran los caudales de refrigerante y aire y las temperaturas de operación exigidas por el fabricante del motor.

\subsubsection{Metodología de diseño de los sistemas de refrigeración}

El procedimiento de prediseño pormenorizado en el apartado anterior da como resultado las características principales de la bomba, el radiador y el ventilador, dejando abierta la tarea de selección o diseño directo de estos componentes. Aunque ahora estos componentes se calculan o seleccionan de manera separada como elementos concentrados, a continuación se propone una metodología que permite ejecutar simultáneamente estas tareas, aprovechando al tiempo las ventajas de los códigos computacionales para el modelado 3D de los sistemas de ventilación y 1D de los sistemas de refrigeración. Los ventiladores y las bombas son elementos que pueden 
caracterizarse de una manera relativamente sencilla en el entorno de los programas de modelado $1 \mathrm{D}$, pero los intercambiadores de calor representan un componente complejo de modelar, con solución individual para cada aplicación y cuya geometría al detalle debe ser conocida para poder ser modelada con programas CFD.

Disponiendo de programas computacionales para el modelado termohidráulico de la parte del refrigerante, para el modelado del sistema de ventilación y para el cálculo de los intercambiadores de calor, una metodología que permite acercarse a una solución ideal de un sistema de refrigeración para una aplicación particular es la resumida en el esquema de la Figura 4.11.

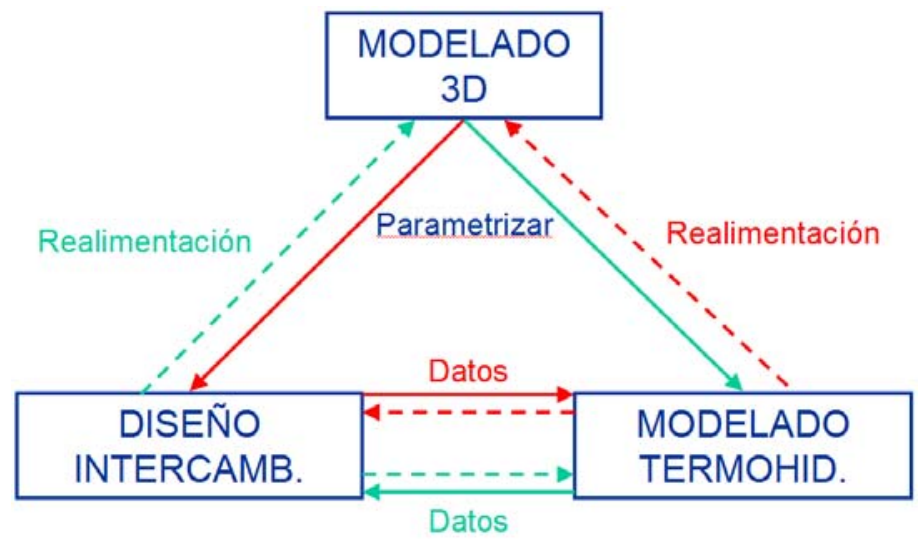

Figura 4.11. Metodología de diseño de los sistemas de refrigeración de motores de combustión.

Descripción de la metodología. Con los parámetros hallados con el cálculo preliminar se selecciona una geometría estándar para el (los) intercambiador(es) (radiador(es)) y se realiza su dimensionamiento y determinación de características térmicas e hidráulicas. Luego se elabora el modelo 3D de la parte de ventilación según la disponibilidad de espacio y ubicación de componentes, contando con el (los) ventilador(es) seleccionado(s), se simula esta parte de la ventilación y con los resultados obtenidos se caracterizan las pérdidas en los diferentes componentes y tramos del circuito de ventilación, refinando la selección del ventilador que garantice 
los caudales de aire bajo las condiciones de operación de diseño y despejando los caudales de aire "reales" que pasan por el radiador. Luego de un postprocesamiento de la información 3D se obtienen las pérdidas de presión "reales" del (de los) radiador(es) y los diferentes tramos del sistema de ventilación.

Simultáneo con el modelado de la parte de ventilación se realiza el modelado 1D de la parte hidráulica del refrigerante según también las necesidades de conexión y ubicación de los componentes, lo que permite caracterizar las pérdidas de presión en el circuito de refrigerante externo a la estructura del motor y asegurar el cumplimiento de los caudales demandados por el balance térmico del sistema.

Posteriormente, las características de pérdidas de presión obtenidas con el programa 3D para los tramos de la parte de ventilación se introducen al modelo 1D del circuito de refrigeración completo. Tras la simulación del modelo termohidráulico del sistema completo se comparan las temperaturas del refrigerante y del aire con los valores exigidos. Si las temperaturas de los fluidos no satisfacen los valores requeridos, se recalcula el radiador o los radiadores y el proceso se repite. Es así como la mayor demanda de cálculo recae sobre el diseño del radiador.

La solución iterada del sistema bajo el esquema de la Figura 4.11 puede realizarse para un número determinado de variantes de radiadores y trazados de los circuitos del refrigerante y de ventilación, ofreciendo alternativas para el usuario. En un sistema como el de refrigeración, las mayores pérdidas energéticas están en el lado del aire, con lo que la simulación de varias alternativas de radiadores y ventiladores puede dar la combinación adecuada dentro de un abanico de opciones comerciales.

La metodología de diseño que aquí se explica sólo tiene que ver con la parte termohidráulica del sistema de refrigeración, con la capacidad de disipación de calor del sistema según el esquema de la Figura 4.1, más no con el diseño del sistema de control. Es claro que el sistema de control determinará los umbrales de las temperaturas de refrigerante y aire y los tiempos de conexión y desconexión de las bombas (cuando no son accionadas por el mismo motor) y el ventilador. El sistema de control podrá integrar o no válvulas termostáticas y controles de accionamiento de los ventiladores. 


\subsubsection{Cálculo de los radiadores de los sistemas de refrigeración}

Geometría de los radiadores. Los radiadores son elementos comerciales fabricados por diferentes firmas comerciales como Valeo ${ }^{\circledR}$, Wabtech ${ }^{\circledR}$, Young Touchstone $^{\circledR}$, entre otras, las cuales tienen sistemas de nomenclatura para designar los diferentes diseños, con referencia especial del tipo, el número de pasos, la geometría del núcleo, el tipo de aletas y de tubos.

La mayoría de los radiadores utilizados actualmente para motores son de aletas apersianadas. Como se ilustra en la Figura 4.12, un radiador se compone principalmente de cuatro partes: un tanque de entrada, un tanque de salida, una tapa de control de presión y un panel. Los tanques pueden tener ubicación horizontal o vertical.

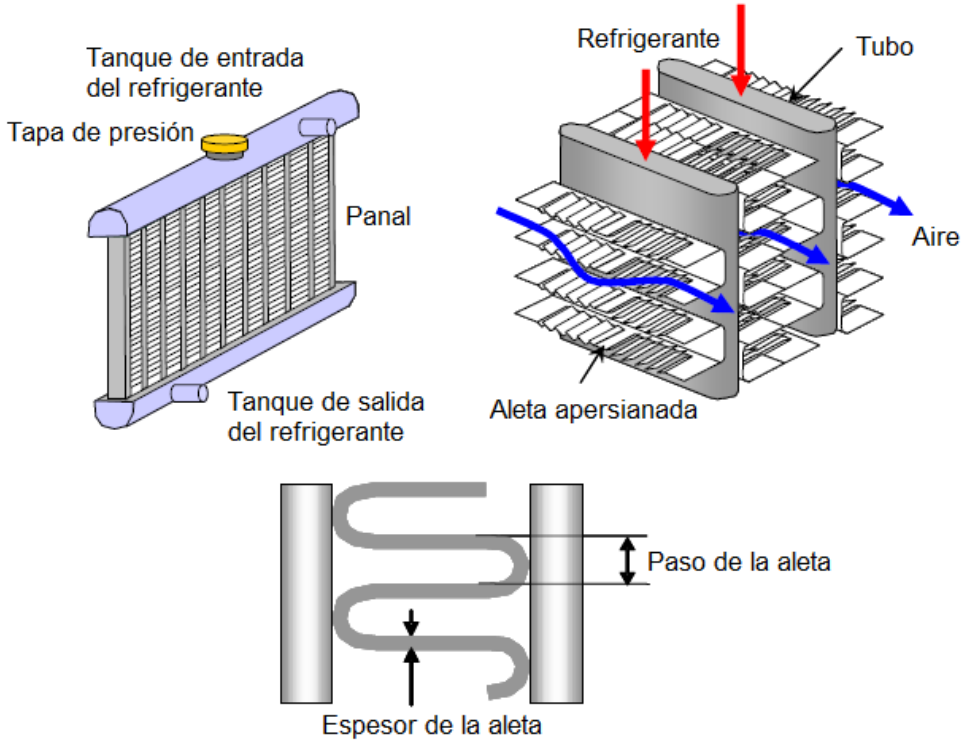

Figura 4.12. Estructura básica de un radiador

Los principales componentes del núcleo o panal son los tubos del refrigerante y las aletas. Los tubos planos son los más utilizados en la fabricación de los núcleos de los radiadores debido a su menor resistencia aerodinámica comparada con la de los tubos redondos. Las aletas pueden tener diseños muy variados, imponiéndose últimamente las aletas apersianadas, con las cuales se 
introduce un mezclado turbulento y se aumenta la transferencia de calor, aunque, como contraprestación, se aumentan las pérdidas de presión.

Entre los principales parámetros de diseño de los radiadores están las dimensiones principales del panal: altura, ancho y profundidad, las cuales dependen de la disponibilidad de espacio y cuyo cálculo preliminar fue descrito en el apartado 4.2.1. Para ajustarse a las necesidades de diseño se requiere determinar otros parámetros relacionados con el panal, de los cuales dependerán también el coeficiente global de transferencia de calor y las resistencias al paso de los fluidos. Para el cálculo de las áreas de intercambio de calor y de la resistencia al paso del refrigerante y del aire se requiere obtener, a partir de la información de la geometría del núcleo, otras medidas derivadas, como la sección transversal total (lado del refrigerante) y el perímetro interno de los tubos, el área de transferencia de calor del lado del tubo, el área total del intercambiador de calor del lado del aire, el área bloqueada por las aletas y el área de flujo libre, el perímetro del lado de la aleta y el diámetro hidráulico de su paso, el número de aletas, las áreas de transferencia de calor del lado del aire directas e indirectas por unidad de longitud, la eficiencia de la aleta y el área efectiva de la aleta. Las expresiones para el cálculo de los parámetros del panal pueden encontrarse en la referencia [4.5].

Es copiosa la literatura existente sobre geometrías de intercambiadores de calor compactos, categoría de intercambiadores dentro de la que se inscriben los radiadores para motores. La información básica que puede encontrarse en la bibliografía general sobre radiadores es la que, a manera de ejemplo, se muestra en la Tabla 4.1.

En la mayor parte de la literatura los intercambiadores de calor se calculan mediante los métodos $\varepsilon$-NTU y LMTD. En el trabajo de Kays y London [4.6] se puede seguir la metodología de cálculo de estos intercambiadores, una metodología que, aún hoy, constituye la base de cálculo de los programas computacionales existentes. Otras referencias permiten profundizar más en las particularidades de la transferencia de calor en los intercambiadores con aletas apersianadas: Webb y Trauger [4.7], Achachia y Cowell [4.8], Namai, et al. [4.9]. Tafti, et al. [4.10] resolvieron modelos computacionales tridimensionales de aletas multiapersianadas. 
Tabla 4.1. Ejemplo de las características de los núcleos de los radiadores [4.6].

\begin{tabular}{|c|c|c|}
\hline Superficie del radiador & 11.32-737-SR & Unidades SI \\
\hline Ordenamiento de los tubos & Escalonado & \\
\hline Tipo de aletas & Serpentín & \\
\hline Longitud del tubo, WET, plg & 0,737 & $18,72 \mathrm{~mm}$ \\
\hline Ancho del tubo, HET, plg & 0,1 & $2,54 \mathrm{~mm}$ \\
\hline Aletas/pulg & 11,32 & 0,446 aletas $/ \mathrm{mm}$ \\
\hline Diámetro hidráulico, 4rh,plg & 0,138 & $3,51 \mathrm{~mm}$ \\
\hline Espesor de aletas, $\delta$, plg & 0,004 & $0,102 \mathrm{~mm}$ \\
\hline $\begin{array}{l}\text { Área libre/ Área frontal del lado } \\
\text { del aire, } \sigma_{\mathrm{s}}\end{array}$ & 0,78 & 0,78 \\
\hline $\begin{array}{l}\text { Área de transferencia de } \\
\text { calor/volumen total, } \alpha_{\mathrm{a}}, \mathrm{pie}^{2} / \mathrm{pie}^{3}\end{array}$ & 270 & $0,886 \mathrm{~mm}^{2} / \mathrm{mm}^{3}$ \\
\hline Área de aletas/Área total & 0,845 & 0,845 \\
\hline Longitud de la aleta, $l$, plg & 0,225 & $5,715 \mathrm{~mm}$ \\
\hline $\begin{array}{l}\text { Espesor de la lámina del tubo, } \\
\text { DT, plg }\end{array}$ & 0,001 & $0,254 \mathrm{~mm}$ \\
\hline Paso de tubos a lo ancho, PL, plg & 0,789 & $20,066 \mathrm{~mm}$ \\
\hline Paso de tubos a lo alto, $\mathrm{PH}$, plg & 0,55 & $13,97 \mathrm{~mm}$ \\
\hline
\end{tabular}

\section{Procedimiento de Kays y London para el cálculo de los radiadores}

Para calcular la capacidad térmica de un radiador, visto como un intercambiador de flujo cruzado con ambos fluidos sin mezclar, de forma global, se suelen seguir los siguientes pasos [4.6]:

- Se determinan las propiedades geométricas a ambos lados del intercambiador. Esto incluye: área de paso mínima, área de la superficie de transferencia de calor (primaria y secundaria), longitudes de flujo, diámetros hidráulicos, densidad de área de superficie de transferencia de calor, relación de área de flujo libre mínima sobre el área frontal, longitud de las aletas y su espesor. Pueden presentarse otras especificaciones en función del diseño del radiador. Las características de los radiadores anotadas en este punto permiten identificar el núcleo o panal del radiador. 
- Se calculan la temperatura global media de los fluidos y sus propiedades termofísicas. Se asumen las temperaturas de salida. A menos que se tenga un estimativo más cercano, se puede asumir una eficiencia del $60 \%$ - $75 \%$ y con esto calcular las temperaturas probables de salida:

$$
\begin{aligned}
& T_{\text {refr }}^{\text {sal }}=T_{\text {refr }}^{e n}-\varepsilon\left(C_{\text {min }} / C_{\text {refr }}\right)\left(T_{\text {refr }}^{e n}-T_{\text {aire }}^{e n}\right) \\
& T_{\text {aire }}^{\text {sal }}=T_{\text {aire }}^{e n}+\varepsilon\left(C_{\text {min }} / C_{\text {aire }}\right)\left(T_{\text {refr }}^{e n}-T_{\text {aire }}^{e n}\right)
\end{aligned}
$$

Siendo $C=\dot{m} C_{p} ; C_{\text {min }}=\left(\dot{m} C_{p}\right)_{\text {min }} ; C_{\text {máx }}=\left(\dot{m} C_{p}\right)_{\text {máx }}$

donde $T_{\text {aire }}^{\text {sal }}$ y $T_{\text {aire }}^{\text {en }}$ son las temperaturas de salida y entrada del fluido frío, respectivamente, y $T_{\text {refr }}^{\text {sal }}$ y $T_{\text {refr }}^{\text {en }}$ son las temperaturas de salida y entrada del fluido caliente, respectivamente. Para los radiadores, la temperatura global media del refrigerante puede tomarse como el promedio de las temperaturas de entrada y salida, mientras que para el aire es más adecuado el cálculo de la Diferencia de Temperatura Media Logarítmica (DTML). Una vez que se tienen las temperaturas aproximadas en ambos lados, se pueden obtener las propiedades de los fluidos requeridas para el cálculo de la capacidad térmica: la viscosidad dinámica $\mu$, el calor específico $C_{p}$, la conductividad térmica $k$, el número de Prandtl y la densidad $\rho$. Seguidamente se puede calcular de nuevo la temperatura de salida del lado caliente $\mathrm{y}$, de esta manera, calcular de nuevo la temperatura media, refinando así la determinación de las propiedades del fluido.

- Se calculan los números de Reynolds, $\operatorname{Re}=\dot{m} D_{h} / \mu$ (siendo $D_{h}$ el diámetro hidráulico y $\dot{m}$ el gasto másico del fluido correspondiente), requeridos para determinar las características adimensionales de transferencia de calor y de fricción de las superficies del radiador. A continuación se calculan los números de Nusselt $\left(N u=h D_{h} / k\right)$ y Colbourn ( $j=S t \operatorname{Pr}^{2 / 3}$, con el número de Stanton dado por $S t=N u / \operatorname{Re} \operatorname{Pr})$. 
- A partir del número de Reynolds, se determina, por un lado, el coeficiente de fricción $(f)$ y, por otro lado, el coeficiente de película (h), definido este último mediante las expresiones, para ambas corrientes de fluidos:

$$
N u=\frac{h D_{h}}{k} \text { ó } j=\frac{h}{\rho \dot{V} C_{p}} \operatorname{Pr}^{2 / 3}
$$

El coeficiente de película del lado del aire también puede hallarse utilizando el número de Stanton. Con el número de Reynolds pueden determinarse la relación $S t \cdot \operatorname{Pr}^{2 / 3}$ y el coeficiente de fricción $f$. El número de Stanton se determina a partir de la relación $S t \cdot P^{2 / 3}$ y tras conocer el número de Prandtl para el aire:

$$
h_{\text {aire }}=S t \cdot \dot{m}_{\text {air }} \cdot C_{p}{ }_{\text {aire }}
$$

Luego se determina la eficiencia de la aleta, a partir de su longitud efectiva $l$ y del parámetro $\mathrm{m}\left(m=\sqrt{2 h_{\text {aire }} / k \delta}\right)$, mediante la expresión $\eta_{a}=\tanh (m l) / m l$. Se calcula también la conductancia térmica global del intercambiador, $U_{a} A_{a}$. El coeficiente global de transferencia de calor $U_{a}$, basado en el área del lado del aire y despreciando la resistencia metálica, está dado por:

$$
\frac{1}{U_{a}}=\frac{1}{\eta_{0} h_{\text {aire }}}+\frac{1}{\left(\alpha_{w} / \alpha_{a}\right) h_{\text {refr }}}
$$

En (4.25) $\alpha_{w}$ es la relación entre el área total de transferencia de calor del lado de agua y el volumen total del radiador, $\alpha_{a}$ es la similar relación pero para el lado de aire, $h_{\text {aire }} \mathrm{y} h_{\text {refr }}$ son los coeficientes de película para el aire y el refrigerante, respectivamente, y $\eta_{0}$ es la efectividad total de la superficie (si no se conoce la efectividad, su valor puede asumirse como $\left.\eta_{0}=0,8\right)$.

- A partir de las capacidades calóricas de los flujos de ambos lados, se puede calcular el número de unidades de transferencia de calor, NTU, por la expresión: 


$$
N T U_{\text {máx }}=\frac{A_{a} \cdot U_{a}}{\left(\dot{m} c_{p \min }\right)}
$$

- Se determina la capacidad térmica del intercambiador con la relación $C=C_{\text {min }} / C_{\text {máx }}$ y la expresión de la eficiencia $\varepsilon$, para intercambiadores de calor de flujo cruzado sin mezclar:

$$
\varepsilon=1-\exp \left[\frac{\exp \left(-N T U \cdot C \cdot N T U^{-0,22}\right)-1}{C \cdot N T U^{-0,22}}\right]
$$

De manera que la capacidad térmica del equipo es:

$$
\dot{Q}=C_{\text {min }}\left(T_{r e f r}^{e n}-T_{\text {aire }}^{e n}\right) \cdot \varepsilon
$$

$T_{\text {refr }}^{e n} \mathrm{y} T_{\text {aire }}^{e n}$ son las temperaturas de entrada del refrigerante y del aire, respectivamente.

- Conociendo el valor de la eficiencia se puede determinar ya el valor de las temperaturas de salida:

$$
\varepsilon=\frac{C_{r e f r}\left(T_{r e f r}^{e n}-T_{r e f r}^{s a l}\right)}{C_{\min }\left(T_{r e f r}^{e n}-T_{\text {aire }}^{e n}\right)}
$$

Se obtienen así $T_{\text {refr }}^{\text {sal }}$ y $T_{\text {aire }}^{\text {sal }}$. Si las temperaturas de salida son muy diferentes de las asumidas en el segundo punto, se itera el procedimiento hasta que las temperaturas asumidas y las finales converjan dentro de los límites de tolerancia aceptables.

- Se calculan los volúmenes específicos a la entrada y la salida del intercambiador y se determinan las pérdidas de presión para cada fluido. Las pérdidas relativas de presión del lado del aire se determinan por la expresión:

$$
\frac{\Delta p}{p_{1}}=\frac{\dot{m}^{2}}{2} \frac{v_{1}}{p_{1}}\left[\left(K_{c}+1-\sigma^{2}\right)+2\left(\frac{v_{2}}{v_{1}}-1\right)+f \frac{A}{A_{c}} \frac{v_{m}}{v_{1}}-\left(1-\sigma^{2}-K_{e}\right) \frac{v_{2}}{v_{1}}\right]
$$


El primer término dentro de los corchetes valora las pérdidas a la entrada, el segundo término cuantifica las pérdidas por aceleración de flujo, el tercero valora las pérdidas por fricción y el último término tiene en cuenta las pérdidas de salida; $v_{1}, v_{2} \mathrm{y} v_{m}$ son los volúmenes específicos a la entrada, a la salida y el valor medio; $K_{c}$ y $K_{e}$ son los coeficientes de pérdidas a la entrada y a la salida; $\sigma$ es la relación de área de flujo libre del núcleo sobre el área frontal; $A$ es el área de la superficie sobre la que se basa el cálculo de $U ; A_{c}$ es el área del lado frío o del lado del aire; $\frac{A}{A_{c}}=\frac{L}{r_{h}} ; L$ es la longitud de flujo del intercambiador de calor y $r_{h}$ es el radio hidráulico. Del lado del agua, las pérdidas de presión se determinan por la expresión:

$$
\Delta p=\frac{\dot{m}^{2}}{2} v f \frac{L}{r_{h}}
$$

En primera aproximación pueden despreciarse las pérdidas a la entrada y a la salida.

Los métodos $\varepsilon$-NTU y LMTD demandan que las curvas que determinan las relaciones entre $\varepsilon$ y $N T U$ se conozcan de antemano para el tipo de intercambiador a calcular [4.20].

\section{Programas computacionales para el cálculo de radiadores}

Las especificaciones mínimas del radiador descritas en 4.2.1 permiten que se estudien diferentes alternativas constructivas para el radiador o los radiadores requeridos por métodos manuales o utilizando programas de cálculo. Basados en la metodología recientemente descrita de Kays y London [4.6] y con adaptaciones propias para cada tipo de radiador o núcleo de radiador, fabricantes e investigadores han desarrollado códigos computacionales para calcular todas las características de los radiadores conducentes a obtener su característica de capacidad de disipación de calor y las pérdidas de presión por los lados de aire y refrigerante, según el diagrama de la Figura 4.13. 


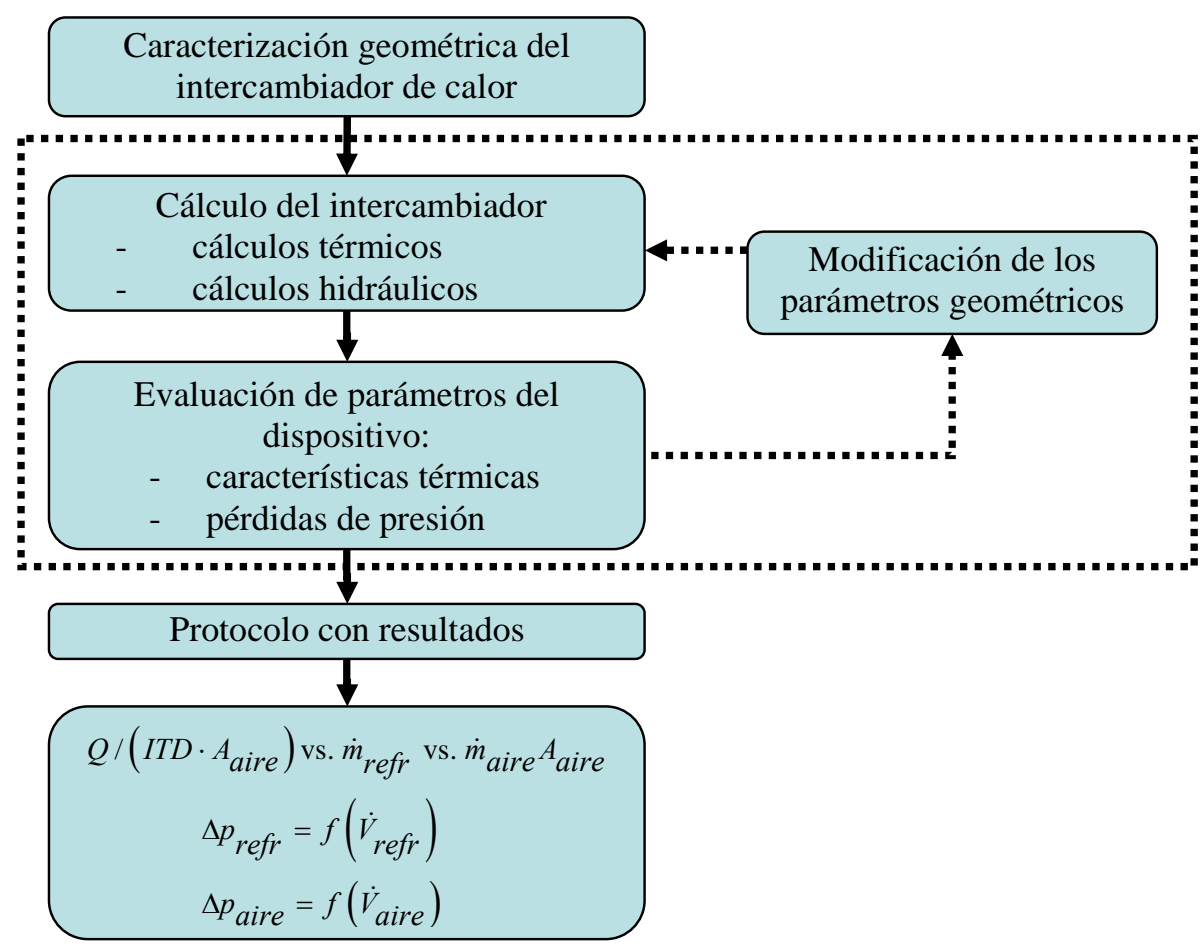

Figura 4.13. Diagrama de bloques ilustrativo de las operaciones de cálculo de los radiadores.

Los programas comerciales usualmente pueden trabajar en modo de predicción de la capacidad térmica o en modo de diseño, entregando parámetros geométricos con los que se puede realizar la solicitud técnica o compra del radiador. Entre esos programas figuran CORESEL ${ }^{\circledR}$ ó RAD $^{\circledR}$. Para efectos de esta tesis se ha sistematizado una hoja de cálculo fundamentada en las expresiones descritas y en la información de geometría de radiadores aportada por Kakac y Liu [4.11] y por Oliet et al. [4.12]. La hoja de cálculo requiere las siguientes entradas: flujos y temperaturas de entrada de los fluidos; dimensiones y materiales del radiador y tipo de núcleo, paso de las aletas, número de filas de tubos, pérdidas máximas de presión y magnitud del calor a disipar. Aparte de servir para dimensionar y predecir la capacidad de los intercambiadores, el programa puede usarse para calcular propiedades de los fluidos, realizar cálculos predictivos y de diagnóstico de intercambiadores no sólo de flujo cruzado, sino también de intercambiadores 
de carcasa y tubos. La base de datos del programa es abierta para que el usuario pueda ampliarla. Las ventanas de presentación de la hoja de cálculo de la Figura 4.14 ilustran las tareas resueltas por el programa.

\begin{tabular}{|c|c|c|c|c|c|}
\hline \multicolumn{2}{|c|}{ CÁLCULO DE RADIADORES } & $\Rightarrow$ & \multicolumn{2}{|c|}{ CONSULTA PARÁMETROS GEOMÉTRICOS } & $\Rightarrow$ \\
\hline \multicolumn{2}{|l|}{ AYUDA } & ? & & & \\
\hline \multicolumn{6}{|c|}{ HERRAMIENTAS EXTRAS } \\
\hline \multicolumn{3}{|c|}{ CÁLCULO DE PROPIEDADES } & \multicolumn{2}{|c|}{ INSERTAR } & TOTAL \\
\hline OPCIÓN & MATERIALES & FLUIDOS & MATERIALES & $\Rightarrow$ & 57 \\
\hline Rango de temperaturas & $\Rightarrow$ & $\Rightarrow$ & FLUIDOS & $\Rightarrow$ & 26 \\
\hline Comparativa (1 temperatura) & $\Rightarrow$ & $\Rightarrow$ & SUPERFICIE & $\Rightarrow$ & 53 \\
\hline \multicolumn{3}{|c|}{ EFFICIENCIA - NTU } & \multicolumn{3}{|c|}{ CARCASA Y TUBOS } \\
\hline Sin mezclar & $\Rightarrow$ & & Calcular UA & $\Rightarrow$ & \\
\hline Mezclados & $\Rightarrow 1$ & & Calcular T & $\Rightarrow 1$ & \\
\hline Cmin mezclado & $\Rightarrow$ & & & & \\
\hline Cmax mezclado & $\Rightarrow 1$ & & & & \\
\hline Contracorriente & $\Rightarrow$ & & & & \\
\hline Equicorriente & $\Rightarrow$ & & & & \\
\hline 2 pasos por tubo & $\Rightarrow$ & & & & \\
\hline
\end{tabular}

a) Vista inicial del programa.

\begin{tabular}{|c|c|c|c|}
\hline Superficie & \multicolumn{3}{|c|}{ AXB_10 } \\
\hline Tubo & \multicolumn{2}{|c|}{ Plano } & \\
\hline Aletas & \multicolumn{2}{|c|}{ Continuas } & \\
\hline Arrangement & \multicolumn{2}{|c|}{ En linea } & \\
\hline Pin pattern & \multicolumn{2}{|c|}{ Plano } & \\
\hline $\mathrm{xt}$ & \multicolumn{2}{|c|}{22.2250} & $\mathrm{~mm}$ \\
\hline $\mathrm{xl}$ & \multicolumn{2}{|c|}{15.875} & $\mathrm{~mm}$ \\
\hline фtubo. Interior / Exterior & & & $\mathrm{mm}$ \\
\hline Aletas: Espesor / фexterior & 0.0762 & & $\mathrm{~mm}$ \\
\hline Densidad & 0.3937 & & aletas $/ \mathrm{mm}$ \\
\hline Lexterior & \multicolumn{2}{|c|}{17.907} & $\mathrm{~mm}$ \\
\hline pexterior & \multicolumn{2}{|c|}{3.759} & $\mathrm{~mm}$ \\
\hline Espesor & \multicolumn{2}{|c|}{0.4572} & $\mathrm{~mm}$ \\
\hline \multicolumn{4}{|c|}{ Cálculos } \\
\hline $\mathrm{pt}$ & \multicolumn{2}{|c|}{19.3779} & $\mathrm{~mm}$ \\
\hline xd & \multicolumn{2}{|c|}{27.3124} & $\mathrm{~mm}$ \\
\hline$\sigma$ & 0.136 & 0.788 & -- \\
\hline$\alpha$ & 108.1 & 754.8 & $\mathrm{~m}^{2} / \mathrm{m}^{3}$ \\
\hline$D_{h}$ & 5.02 & 4.18 & $\mathrm{~mm}$ \\
\hline $\mathrm{A}_{\text {aletas }} / \mathrm{A}_{\text {total }}$ & & 0.852 & $\mathrm{~m}^{2} / \mathrm{m}^{2}$ \\
\hline
\end{tabular}

b) Presentación de la geometría del radiador consultado. 


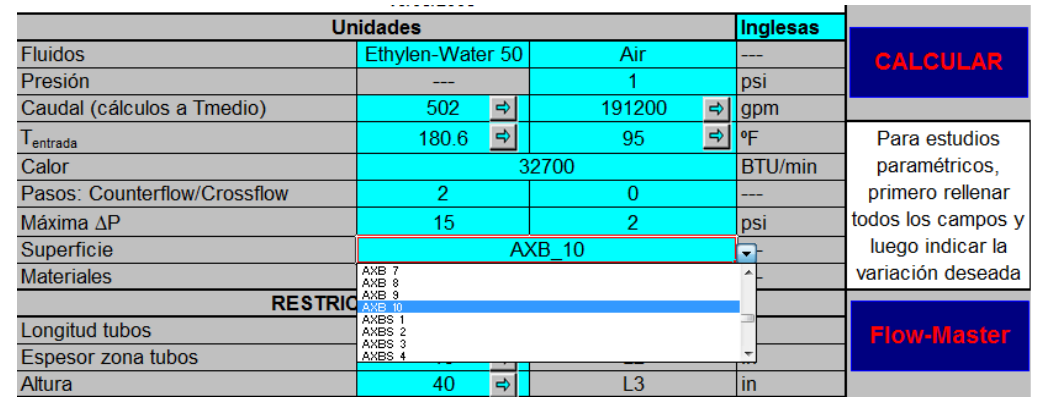

c) Pantalla de diálogo del programa de cálculo de radiadores.

\begin{tabular}{|c|c|c|c|}
\hline \multicolumn{3}{|c|}{ Unidades } & \multirow{2}{*}{\begin{tabular}{|l|} 
Inglesas \\
--- \\
\end{tabular}} \\
\hline Fluidos & Ethylen-Water 50 & Air & \\
\hline Presión & -- & 1 & psi \\
\hline Caudal (cálculos a Tmedio) & 502 & 191209 & gpm \\
\hline Tentrada & 180.6 & 95 & ${ }^{\circ} \mathrm{F}$ \\
\hline Calor & \multicolumn{2}{|c|}{32700} & BTU/min \\
\hline Pasos: Counterflow/Crossflow & 2 & 0 & --- \\
\hline Máxima $\Delta \mathrm{P}$ & 2 & 2 & psi \\
\hline Superficie & \multicolumn{2}{|c|}{ AXB_10 } & --- \\
\hline Materiales & Aluminio puro & Aluminio puro & -- \\
\hline \multicolumn{4}{|c|}{ RESTRICCIONES GEOMÉTRICAS } \\
\hline Longitud tubos & 101 & $\mathrm{~L} 1$ & in \\
\hline Espesor zona tubos & 15 & L2 & in \\
\hline Altura & 41.25 & L3 & in \\
\hline Tsalida & 172 & 175 & ${ }^{\circ} \mathrm{F}$ \\
\hline Calor & 32551 & & BTU/min \\
\hline$\Delta \mathrm{P}$ & 2.053 & 0.007 & psi \\
\hline
\end{tabular}

d) Presentación de resultados del programa de cálculo de radiadores.

Figura 4.14. Visualización de las ventanas de diálogo de la hoja de cálculo de radiadores desarrollada en el CMT para el cálculo de intercambiadores de calor.

\subsection{Modelado de los sistemas de refrigeración}

El modelado de los sistemas de refrigeración puede abordarse a nivel de conjunto de unidades funcionales que participan de la gestión térmica del vehículo, a nivel de sistema de control en interrelación con el control de potencia del motor, a nivel funcional como sistemas fluidodinámicos, y 
finalmente a nivel de componente. Para estudios que se reducen a la operación de los motores alternativos en los vehículos, los tres últimos niveles son los que se abordan en el marco del presente trabajo.

\subsubsection{Modelos simplificados de la temperatura del motor a nivel de sistema}

Para analizar el comportamiento del sistema de refrigeración desde el punto de vista dinámico y para incorporar sus características de respuesta en entornos amplios de modelado dinámico de los motores (por ejemplo el modelado de la respuesta de la salida del cigüeñal a un cambio de carga del vehículo), es suficiente el empleo de un modelo dinámico de temperatura de motor, del que se pueda obtener el gradiente de la temperatura del refrigerante (o del motor) a partir de unas entradas básicas como su temperatura media, el régimen y el par. Estos modelos se integran usualmente en bloques de control y son suficientes, por ejemplo, para considerar las estrategias de control observador en las unidades electrónicas de control de los motores. Un modelo sencillo que permite analizar la evolución de la temperatura del motor es:

$$
C \frac{d T}{d t}=\dot{Q}_{r e f r}-\dot{Q}_{r}
$$

donde $C$ es la del sistema de refrigeración, $C=C_{r e f r} m_{r e f r}+C_{m} m_{m}$, siendo $C_{r e f r}$ y $C_{m}$ los calores específicos del líquido y del metal; $m_{r e f r}$ y $m_{m}$ las masas del líquido y del metal que participan en la acumulación de calor; $\dot{Q}_{\text {refr }}$ es la cantidad de calor que recibe el sistema de refrigeración por parte del motor por unidad de tiempo, y $\dot{Q}_{r}$ es la cantidad de calor disipada por el sistema de refrigeración por unidad de tiempo, a través del radiador y los elementos recuperadores o aprovechadores de energía.

Si, tras una estabilidad térmica alcanzada por el motor, se produce alguna desviación del régimen o de la carga, esto se traducirá en una variación de la temperatura del motor, según la variación del calor evacuado por el motor y el disipado por el sistema, con lo que la ecuación (4.32) toma la forma:

$$
C \frac{d \Delta T}{d t}=\Delta \dot{Q}_{r e f r}-\Delta \dot{Q}_{r}
$$


La cantidad de calor $\Delta \dot{Q}_{\text {refr }}$, transmitida por el motor por unidad de tiempo al sistema de refrigeración depende de la temperatura $T$ del sistema de refrigeración, de la velocidad angular del cigüeñal $\omega$ y de la posición $h$ del acelerador. Por consiguiente, $\dot{Q}_{\text {refr }}=f(T, \omega, h)$, expresión que tras un proceso de linealización se traduce en:

$$
\Delta \dot{Q}_{r e f r}=\frac{\partial \dot{Q}_{r e f r}}{\partial T} \Delta T+\frac{\partial \dot{Q}_{r e f r}}{\partial \omega} \Delta \omega+\frac{\partial \dot{Q}_{r e f r}}{\partial h} \Delta h
$$

La cantidad de calor $\Delta \dot{Q}_{r}$, disipada por el sistema de refrigeración al medio exterior depende de la temperatura del refrigerante a la salida de la culata, $T_{r e f r}^{\text {sal }}$, del caudal $\dot{m}_{\text {refr }}$ a través del volumen de las galerías de refrigeración y de la temperatura de entrada, $T_{\text {refr }}^{e n}$ a la que ingresa al sistema de refrigeración, es decir, $\dot{Q}_{r}=f\left(T_{r e f r}^{\text {sal }}, \dot{m}_{\text {refr }}, T_{r e f r}^{e n}\right)$. Luego de desarrollar esta función en serie de Taylor y de linealizarla, se tiene:

$$
\Delta \dot{Q}_{r}=\frac{\partial \dot{Q}_{r}}{\partial T} \Delta T+\frac{\partial \dot{Q}_{r}}{\partial \dot{m}} \Delta \dot{m}+\frac{\partial \dot{Q}_{r}}{\partial T_{r e f r}^{e n}} \Delta T_{r e f r}^{e n}
$$

En los sistemas de refrigeración convencionales la variación del caudal $\Delta \dot{m}$ del refrigerante depende de la frecuencia de rotación del rotor de la bomba y del desplazamiento $\Delta x$ del órgano de control (la válvula de control usualmente es el termostato).

Reemplazando esta expresión en la fórmula (4.34), considerando $\Delta T_{\text {refr }}^{e n}=0 \mathrm{y}$ definiendo el factor de estabilidad del proceso de refrigeración como $F_{e}$ : $F_{e}=\frac{\partial \dot{Q}_{r}}{\partial T}-\frac{\partial \dot{Q}_{r e f r}}{\partial T}$, se obtiene una expresión para describir la variación de la temperatura del motor:

$$
C \frac{d \Delta T}{d t}+F_{e} \Delta T=-\frac{\partial \dot{Q}_{r}}{\partial x} \Delta x+\frac{\partial \dot{Q}_{r e f r}}{\partial h} \Delta h+\left(\frac{\partial \dot{Q}_{r e f r}}{\partial \omega}-\frac{\partial \dot{Q}_{r}}{\partial \omega}\right) \Delta \omega
$$


La expresión (4.36) sirve para efectos de caracterizar el sistema de refrigeración en un modelo de sistema, donde la variable que importa es la temperatura del motor en función de la condición de operación, sin detallar la influencia del diseño y la topología del sistema de refrigeración.

\subsubsection{Modelos a nivel de circuito del sistema de refrigeración del motor}

Los tratamientos a nivel de circuito consideran la variación de los flujos másicos, la temperatura y otras variables a lo largo de los diferentes ramales del sistema (modelos 1D), teniendo en cuenta con mayor o menor grado de refinamiento el efecto de las conexiones o empleando funciones de acople (modelos 0D). Cada componente es descrito por las leyes de conservación de masa, momento y energía. A continuación se presentan las expresiones base que describen los procesos físicos hidráulicos y térmicos de los componentes del sistema de refrigeración convencional, concentrados a nivel 0D, susceptibles de ser representados con programas genéricos de modelado de sistemas dinámicos para, en el siguiente apartado, describir las particularidades del modelado 1D de los sistemas termo-hidráulicos.

Con el diagrama de la Figura 4.15 se ilustran los grandes bloques en los que se pueden agrupar los componentes (y sus submodelos) de un modelo 0D de un sistema de refrigeración. El esquema también da una idea de las interrelaciones que se resuelven con un código de modelado de sistemas de refrigeración de motores.

En la parte central del diagrama se representa la estructura del motor por cuya camisa de refrigeración pasa el refrigerante que transporta calor hacia el circuito externo y transmite el calor a la envoltura externa del bloque, mediado por el coeficiente de transferencia de calor del lado del refrigerante. Se diría que ésta es la parte que ha sido objeto de estudio en el capítulo tercero. En la parte izquierda se representan aquellas variables que en los motores convencionales dependen del motor en sí, como las variables instantáneas de presión, temperatura y coeficiente de transferencia de calor de los gases a las paredes; el modelo disponible de predicción de temperaturas y flujos de calor del motor; y las posibles características de la bomba del refrigerante y las pérdidas de presión hidráulica del sistema. En la parte derecha del diagrama se esquematizan las posibles características hidráulicas y térmicas de los componentes del circuito externo y los ramales, tales como 
las características de pérdidas de presión y de potencia térmica de los intercambiadores.

El diagrama de la Figura 4.15 es, en cierta medida, la base conceptual de los programas de modelado de los sistemas de refrigeración de los motores, en los que éstos, como fuente de calor con impedancia y capacitancia hidráulicas y térmicas, se acoplan a sus circuitos termohidráulicos externos para disipar calor e interactuar dinámicamente según los regímenes de operación y estrategias de control de temperaturas.

La fuente principal de calor en el sistema es, obviamente, el motor y la fuente principal de flujo de refrigerante es la bomba accionada por el motor.

Para cada componente del sistema de refrigeración la ley de conservación de la cantidad de movimiento puede expresarse como la relación entre la caída de presión a través de él, $\Delta p$ originada tras el paso de un flujo de masa $\dot{m}$ :

$$
\begin{gathered}
\Delta p=K_{f} \cdot \dot{m}^{2} \\
\Delta p=K \cdot \dot{V}^{2}
\end{gathered}
$$

El coeficiente de momento $K_{f}$ en esta expresión es determinado a partir de medidas experimentales estacionarias para todos los componentes del sistema de refrigeración.

La característica de pérdidas de presión para una rama particular del circuito se obtiene sumando las pérdidas individuales de presión en todos los componentes en serie a condiciones iguales de flujo. La ley de conservación del momento se complementa con la ley de Kirchoff aplicada a los circuitos hidráulicos, que determina el balance de presiones en cualquier bucle cerrado de un circuito hidráulico:

$$
\sum \Delta p_{i}=0
$$

La conservación de la masa implica la igualdad de las masas que entran y salen del nodo, para todas las ramas $n$ conectadas por ese nodo:

$$
\sum_{i=1}^{n} \dot{m}=0
$$




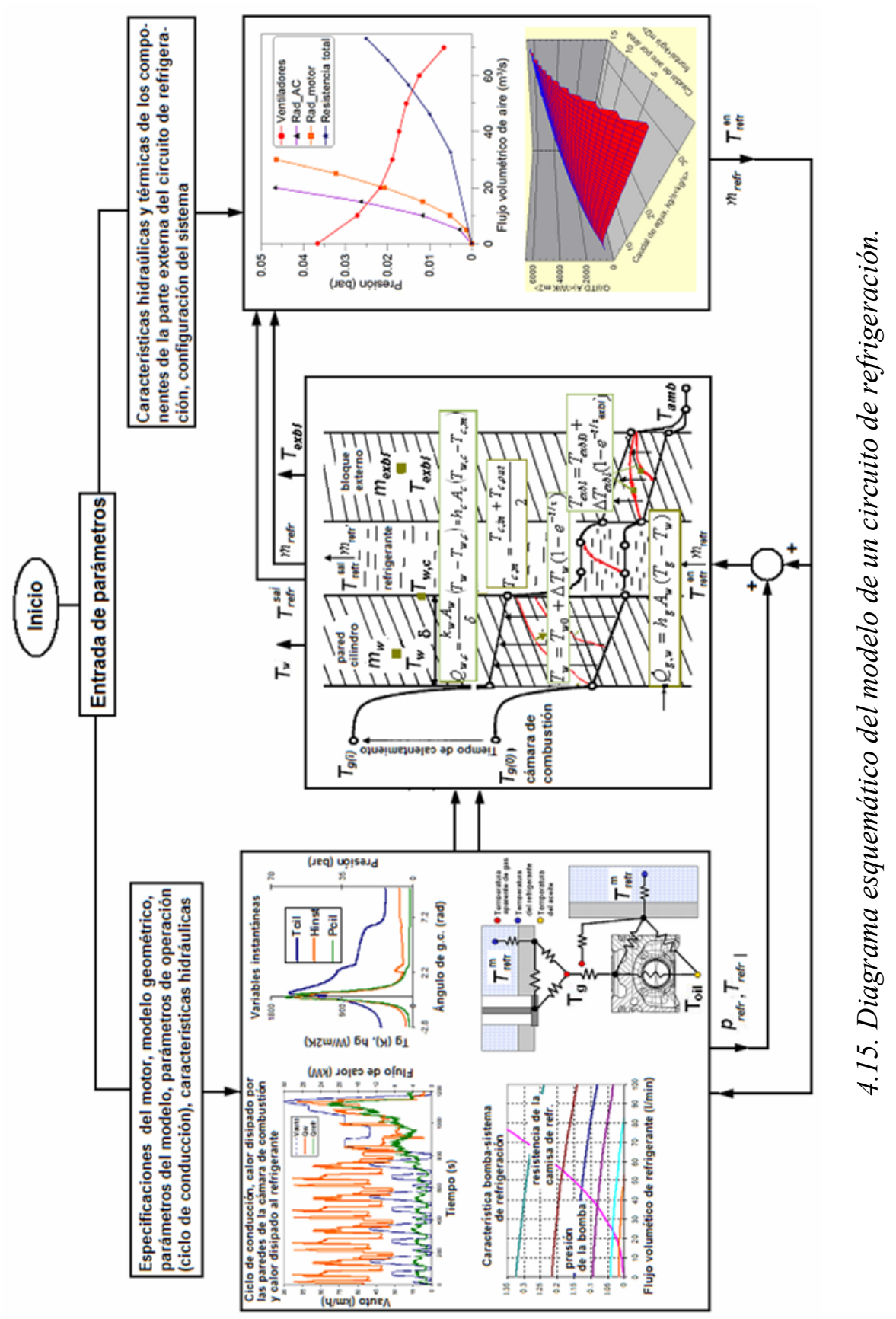


El flujo másico de refrigerante a través del circuito de refrigeración está determinado por la solución simultánea de la ecuación característica de la bomba para las condiciones de operación del motor y la característica total de pérdidas de presión del sistema (Figura 4.10).

La ecuación de conservación de la energía aplicada a cada componente, en combinación con el balance de energía en cada nodo, es la base para obtener la temperatura del refrigerante en los diferentes nodos del sistema de refrigeración. De esta manera el modelo térmico del sistema de refrigeración del motor puede dividirse en dos subconjuntos: uno para el circuito interno y otro para el circuito externo. El modelo de temperatura del circuito interno incluye la disipación de calor de las paredes del motor al refrigerante que atraviesa la camisa de refrigeración y que intercambia calor con la parte externa del bloque. El modelo de temperatura del refrigerante en la parte externa del motor incluye el intercambio de energía a lo largo de las ramas de elementos como el by-pass, el tanque de expansión, el calentador del habitáculo, y el enfriador de los gases de escape recirculados. Estos dos subsistemas interactúan a medida que evoluciona la temperatura del motor.

La temperatura del refrigerante a la salida del bloque del motor puede hallarse si se conoce el calor disipado a través de las paredes del motor al refrigerante, el caudal de éste y su temperatura de entrada al bloque. De manera análoga, si se conoce la temperatura de entrada del refrigerante a cada componente de la parte externa del circuito de refrigeración, el flujo másico total de refrigerante, las impedancias hidráulicas de las ramas externas del circuito y las características térmicas de los intercambiadores de calor, es posible determinar las temperaturas del refrigerante aguas abajo de cualquier componente en cualquier ramal de la parte externa del circuito.

\section{Circuito interno del motor}

Los fundamentos de los modelos 0D se enmarcan dentro de los aplicados por Kaplan y Heywood [4.13], Cortona et al. [4.14],Veshagh y Chen [4.15], Arici [4.16]. Con el tratamiento de modelo de parámetros concentrados, el motor es considerado como un intercambiador integrado compuesto de tres zonas como ilustra la Figura 4.15. La primera zona está representada por la cámara de combustión, la segunda zona está constituida por el refrigerante y la tercera zona representa el comportamiento térmico del bloque del motor. 
Primera zona

La primera zona se caracteriza por las dimensiones y la masa de la pared del cilindro, cuya temperatura $T_{w}$ puede obtenerse tras establecer el balance de calor presentado en la ecuación (4.40):

$$
\frac{d T_{w}}{d t}=\frac{\dot{Q}_{g, w}-\dot{Q}_{w, r e f r}}{m_{w} c_{p w}}
$$

donde, $\dot{Q}_{g, w}$ es el flujo de calor promediado en el ciclo, transmitido a través de las paredes del cilindro, incluyendo el calor transmitido por los gases y también parte del calor transferido a las paredes del cilindro a través de los anillos del pistón y del aceite; $m_{w}$ es la masa de las paredes de la cámara de combustión; $\dot{Q}_{w, r e f r}$ representa el calor transferido desde las paredes hacia el refrigerante, $\mathrm{y} c_{p w}$ es el calor específico del material de la pared.

El flujo de calor promediado $\left(\dot{Q}_{g, w}\right)$ para un punto particular de operación del motor se predice a partir de consideraciones termodinámicas y de transferencia de calor del ciclo como [4.19]:

$$
\dot{Q}_{g, w}=\bar{h}_{g} A_{w}\left(\bar{T}_{g}-T_{w}\right)
$$

donde $\bar{h}_{g}$ y $\bar{T}_{g}$ son el coeficiente de transferencia de calor promediado en el ciclo y la temperatura aparente de los gases del cilindro, y $A_{w}$ es el área de la pared en contacto con los gases del cilindro.

El calor transferido desde las paredes hacia el refrigerante por convección ( $\dot{Q}_{w, \text { refr }}$ ) depende de la magnitud de la temperatura de las paredes del cilindro del lado del refrigerante, $T_{w, r e f r}$, del coeficiente de convección, $h_{r e f r}$ y del área de convección, $A_{c}$ :

$$
\dot{Q}_{w, \text { refr }}=\frac{k_{w} A_{w}}{\delta}\left(T_{w}-T_{w, \text { refr }}\right)=h_{\text {refr }} A_{c}\left(T_{w, \text { refr }}-T_{\text {refr }}^{m}\right)
$$


Aquí, $k_{w}$ es la conductividad del material de la pared y $\delta$ es el espesor de ésta.

Conociendo las temperaturas de entrada y salida del refrigerante, puede calcularse su temperatura media:

$$
T_{r e f r}^{m}=\frac{T_{r e f r}^{s a l}+T_{r e f r}^{e n}}{2}
$$

En el caso de sistemas de refrigeración líquida, el coeficiente de convección al interior de la camisa de refrigeración puede calcularse por alguna de las expresiones (2.23 - 2.27), reducidas a la expresión de Lehner et al. [4.17]:

$$
h_{c}=c\left(\operatorname{Re}^{0,8} \operatorname{Pr}^{0,4} \frac{k}{D}\right)
$$

donde $\operatorname{Pr}$ y $k$ son el número de Prandtl y la conductividad del refrigerante, respectivamente, $c$ es una constante de correlación para ser ajustada a partir de los valores de los flujos de calor experimentales, y $R e$ el número de Reynolds calculado por la expresión [4.17]:

$$
\operatorname{Re}=\frac{4 \dot{m}_{c y l}}{\rho_{\text {refr } r} \nu_{r e f r} \pi D}
$$

donde $\dot{m}_{c y l}$ es el flujo másico de refrigerante, $D$ es el diámetro del cilindro del motor, $\rho_{\text {refr }}$ y $v_{\text {refr }}$ son la densidad y la viscosidad cinemática del refrigerante, respectivamente.

\section{Segunda zona}

La segunda zona corresponde al refrigerante en la camisa de refrigeración del motor. La temperatura en esta zona puede asumirse aproximadamente igual a la temperatura de salida del motor, con su variación descrita por la ecuación diferencial [4.14]:

$$
\frac{d T_{r e f r}^{s a l}}{d t}=\frac{\dot{Q}_{w, r e f r}-\dot{Q}_{r e f r, e x b l}-\dot{Q}_{r e f r}}{m_{r e f r} c_{p_{-} r e f r}}
$$


donde $\dot{Q}_{\text {refr,exbl }}$ representa el calor intercambiado entre el refrigerante y la parte externa del bloque, mientras que $\dot{Q}_{r e f r}=\dot{m}_{r e f r} c_{p_{-} r e f r}\left(T_{r e f r}^{s a l}-T_{r e f r}^{e n}\right)$ es el flujo térmico entre el refrigerante y las paredes del cilindro. $\dot{m}_{r e f r} \mathrm{y} c_{p_{-} r e f r}$ son el flujo másico y el calor específico del refrigerante, respectivamente.

Tercera zona.

Finalmente, asumiendo que la parte externa del bloque y el aceite están a la misma temperatura, la temperatura externa del bloque (no la de la superficie externa) se relaciona con la expresión:

$$
\frac{d T_{e x b l}}{d t}=\frac{\dot{Q}_{r e f r, e x b l}-\dot{Q}_{b l, e x}}{m_{e x b l} c_{p b l}+m_{a c} c_{p_{-} a c}}
$$

El término $\dot{Q}_{b l, e x}$ en la ecuación (4.16) representa el calor intercambiado entre la parte externa del bloque y el entorno. Este término incluye la interfaz con el colector de escape y una fracción de calor resultado del trabajo de fricción de los mecanismos del motor, no incluida en el término $\dot{Q}_{g, w}$ en la ecuación (4.40).

\section{Circuito externo de refrigeración del motor}

Para facilitar las explicaciones en este apartado, se considera el circuito de refrigeración estudiado en el capítulo anterior (Figura 3.9), por ser representativo de los encontrados en los motores convencionales modernos. El sistema se compone de cuatro ramales en paralelo: el de paso por el radiador (cerrado durante el calentamiento del motor, según la característica del termostato), el que incluye al enfriador de EGR en serie con el calentador del habitáculo, el del by-pass y el ramal de desaireación hacia el tanque de expansión. El porcentaje de refrigerante por cada ramal es función de las impedancias hidrodinámicas.

El flujo total de refrigerante que atraviesa la camisa de refrigeración del motor, regido por la característica de la bomba y la resistencia hidrodinámica total del sistema, se descompone entre los cuatro ramales anotados según esa relación de impedancias: 


$$
\dot{m}_{r e f r}=\dot{m}_{b y}+\dot{m}_{h c}+\dot{m}_{e x . t k}+\dot{m}_{r a d}
$$

donde $\dot{m}_{r a d}, \dot{m}_{b y}, \dot{m}_{h c} \mathrm{y} \dot{m}_{\text {ex.tk }}$ representan los flujos másicos de refrigerante por el radiador, el by-pass, el calentador del habitáculo y el tanque de expansión, respectivamente.

Las conexiones entre los componentes se caracterizan por sus capacitancias y por sus pérdidas de presión. Los retrasos en la respuesta de los componentes del sistema dependen de sus volúmenes, $V$, y de los flujos volumétricos, $\dot{v}$, que los atraviesan [4.14]:

$$
\tau=\frac{V}{\dot{v}}
$$

El modelo puede o no considerar los intercambios de calor a través de las tuberías y conexiones (los intercambios de calor a través de estos componentes suelen ser muy pequeños en relación con los intercambios de calor en los elementos principales). En un modelo simplificado OD las pérdidas de presión en las uniones se suelen despreciar.

Los intercambiadores de calor como el enfriador de EGR, el radiador y el calentador del habitáculo se pueden modelar como elementos concentrados con la expresión genérica:

$$
\frac{d T_{i c}}{d t}=\frac{\dot{Q}_{i c}^{e n}-\dot{Q}_{i c}^{s a l}}{m_{i c} c_{p i c}}
$$

definiendo el calor transmitido al intercambiador de calor por la expresión:

$$
\dot{Q}_{i c}^{e n}=\dot{m}_{r e f r, i c} c_{p_{-} r e f r}\left(T_{i c}^{e n}-T_{i c}^{\text {sal }}\right)
$$

Las temperaturas del refrigerante a la entrada de los intercambiadores de calor son función de la temperatura a la salida del motor (caja de agua) y de los retrasos causados por las conexiones.

La descripción del calor intercambiado en los intercambiadores, $\dot{Q}_{i c}^{\text {sal }}$, puede basarse en:

1) El método eficiencia (método NTU - $C_{r}-\varepsilon$ [4.20]), ecuación (4.26), con la eficiencia definida por expresiones como las descritas por las ecuaciones (4.27) y (4-29); 
2) la característica $Q /(I T D \cdot A)=f\left(\dot{m}_{r e f r}, \dot{m}_{\text {air }} / A_{\text {air }}\right)$, en función de los flujos másicos de refrigerante $\dot{m}_{r e f r}$ y del aire, $\dot{m}_{\text {air }}$, del área de la sección de paso del aire del intercambiador, $A_{\text {air }}$ y de la diferencia de temperaturas de entrada del refrigerante y el aire $\left(I T D=T_{r e f r}^{e n}-T_{a i r}^{e n}\right.$ ) para la cual se especifica la característica.

Durante el calentamiento del motor (cerrado el paso por el radiador), los flujos por los ramales principales, el del by-pass y del calentador del habitáculo se unen antes de entrar al motor, con lo que se puede calcular una temperatura promedio:

$$
T_{r e f r}^{e n}=\frac{T_{b y}^{s a l} \cdot \dot{m}_{b y}+T_{\text {exp.tn }}^{s a l} \cdot \dot{m}_{\text {exp.tn }}+T_{E G R}^{s a l} \cdot \dot{m}_{h c}}{\dot{m}_{\text {refr }}} \pm f(t-\tau)
$$

donde el término $f(t-\tau)$ da cuenta de los efectos de retraso en la temperatura causados por las conexiones y las uniones entre los elementos (tuberías, mangueras, uniones y accesorios) [4.14].

Finalmente, el termostato se puede modelar como una válvula de resistencia variable, con un área de paso dependiente de la temperatura (el flujo y la pérdida de presión dependen de la temperatura).

Un conjunto de ecuaciones similar al constituido por las expresiones (4.37 4.52) está detrás de los programas de modelado OD de los sistemas de refrigeración del motor. El objetivo del modelo es la predicción del comportamiento termohidrodinámico del sistema de refrigeración, evaluado principalmente por la respuesta de temperatura del refrigerante a varias condiciones de operación como el arranque en frío, el calentamiento, los puntos de carga y régimen constante y los ciclos de conducción. En general, las entradas requeridas por el modelo son las características térmicas del motor, la topología del circuito, las características térmicas e hidráulicas de los componentes de éste, las condiciones ambientales y las funciones de operación y control con las que se definen los flujos másicos por el motor y los flujos de calor en las fuentes.

Las expresiones matemáticas referidas aquí pueden estar también detrás de los programas de modelado 1D de los sistemas termo-fluido-dinámicos. La 
diferencia y ventaja adicional será la introducción de la resolución en una dimensión de las variables de los procesos en el circuito, particularmente a lo largo de las mangueras y las tuberías.

\subsection{Herramientas de modelado unidimensional}

Como se ha venido diciendo, los sistemas de refrigeración son sistemas en los que se verifican procesos tanto hidráulicos como térmicos, caracterizados por variaciones temporales y espaciales de las propiedades de los fluidos involucrados. Esas variaciones se producen a lo largo de la topología del circuito y también tienen resolución espacial dentro de cada componente. Actualmente existen programas comerciales, unos de propósito múltiple y otros especializados, que resuelven los modelos físico/matemáticos de los sistemas termohidráulicos con muy buena flexibilidad, exactitud y rapidez. Entre los programas de propósito dedicado se cuentan FlowMaster ${ }^{\circledR}$ [4.23] y Hopsan $^{\circledR}$ [4.25]. Easy5 $^{\circledR}$ [4.25], AMESim ${ }^{\circledR}$ [4.24], ITI-Sim ${ }^{\circledR}$ [4.25], Modelica ${ }^{\circledR}$ [4.25], [4.26], o GT-Power ${ }^{\circledR}$ [4.22] son programas de simulación de propósito múltiple. El modelado también se realiza con programas de propósito general como Matlab/Simulink [4.27].

Las ecuaciones que sirven de base a los programas virtuales son la ecuación de conservación de la masa, la energía y el momento y/o sus simplificaciones (expresiones (4.37) - (4.52)), que se establecen para todos los nodos del circuito que se resuelve y conforman un sistema de ecuaciones simultáneas. Estas ecuaciones se usan para calcular de manera iterativa el flujo hacia y desde los componentes como función de la presión. Cualquier componente impone una diferencia de presiones entre sus nodos de conexión, para un flujo de masa determinado. Los programas incluyen rutinas que permiten resolver estas ecuaciones simultáneas de manera iterativa hasta que se obtienen valores estables dentro de unos márgenes de tolerancias y criterios de convergencia. Al resolverse en el programa el problema de transferencia de calor, se usan rutinas de solución iterativas, dado que la solución depende del calor específico del fluido y éste a su vez es función de la temperatura.

Los programas de simulación como Amesim ${ }^{\circledR}$, GT-Cooling ${ }^{\circledR}$ y FlowMaster ${ }^{\circledR}$ se usan para analizar flujos de masa y de energía en circuitos termohidrodinámicos complejos. Con estos programas se puede diseñar, 
refinar y probar el sistema completo, incluyendo no sólo los circuitos de refrigerante y de aire, sino también la parte del control. En ellos cada componente del sistema real tiene su objeto virtual, que incluye su modelo matemático equivalente y sus respectivos atributos gráficos y paramétricos para su presentación en pantalla y el diálogo con el modelador. Los modelos virtuales de los componentes se conectan a través de los nodos para formar las redes y los circuitos; los parámetros físicos de los componentes, las propiedades de los fluidos y los tipos de los procesos se especifican mediante tablas de datos, curvas, ecuaciones y superficies. La ejecución de estos programas da como resultado las presiones, los flujos másicos y volumétricos y las temperaturas en cada parte del circuito. Los resultados que se pueden obtener en cada conexión son: la velocidad, el flujo másico, la temperatura, la densidad, la viscosidad del fluido, y la energía intercambiada, entre otros.

Actualmente en el mercado se dispone de herramientas de modelado 3D de componentes del sistema de refrigeración que se integran a las herramientas de modelado $1 \mathrm{D}$, permitiendo intercambiar información de suerte que en los componentes de interés se pueden visualizar, haciendo uso de las herramientas CFD, los gradientes hidráulicos y térmicos del refrigerante a su paso por los componentes.
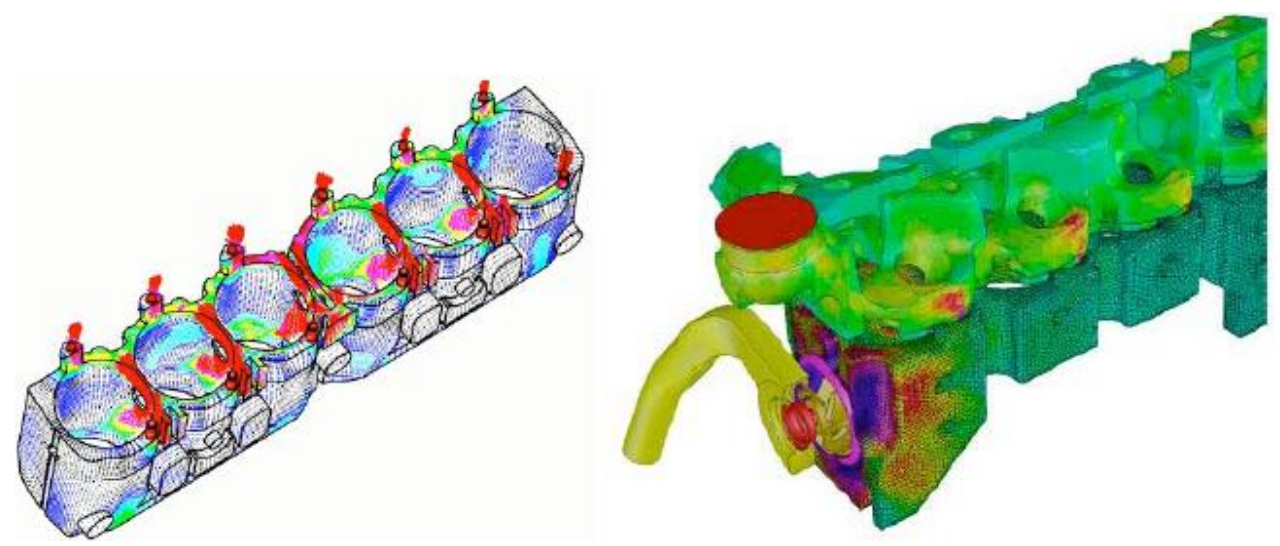

Figura 4.16. Visualización de resultados de los modelos $3 D$ de la camisa de enfriamiento del bloque y la culata de un motor [4.28]. 
La combinación de modelos fluidos 1D y 3D se logra empleando una metodología de co-simulación, con lo que el flujo interno que se simula en un componente 3D (ver Figura 4.16, a manera de ejemplo) es incorporado a la red (sistema) que contiene el resto de los componentes modelados en 1D. Esta metodología da los detalles del flujo interno a través de los componentes de interés como la camisa de enfriamiento del motor o una manguera y permite que puedan diagnosticarse o preverse problemas como la cavitación o la ebullición nucleada en partes específicas de los componentes. La metodología permite trabajar el flujo de manera cercana a la real, con toda la masa de líquido en el sistema y evitando así las incertidumbres propias de la asignación de condiciones de frontera a los modelos de los componentes en las simulaciones 3D; se reduce también el tiempo de simulación. Como ejemplo de esta forma de comunicación entre modelos 1D y 3D se conoce la combinación FlowMaster - CRADLE, descrita en el trabajo de Watanbe et al. [4.28]. La Figura 4.17 ilustra la inserción de un modelo 3D de una conexión en un circuito $1 \mathrm{D}$ de un sistema de enfriamiento.

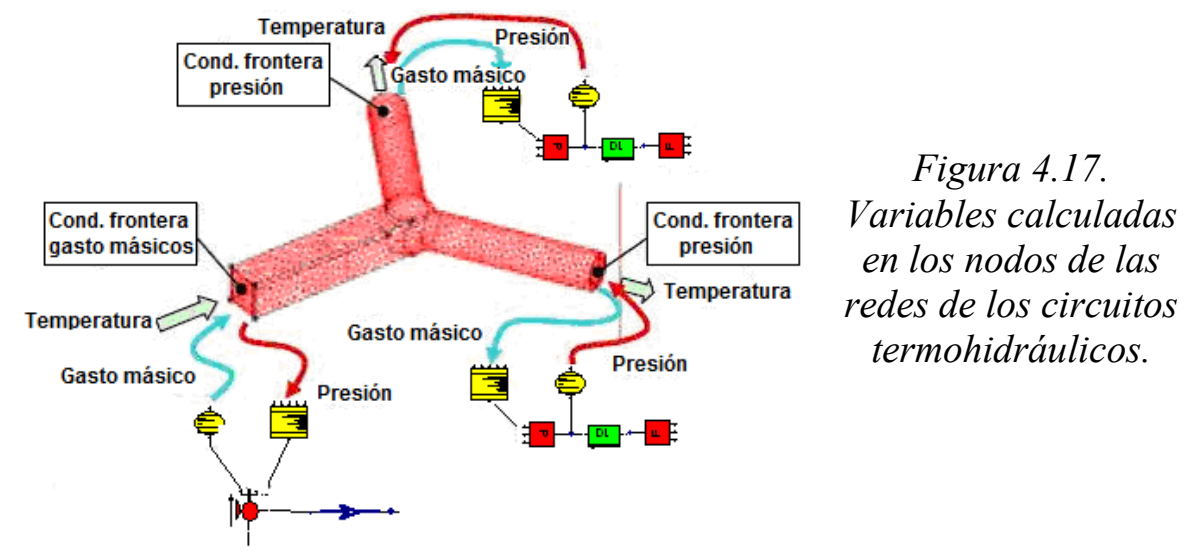

Para los trabajos de esta tesis se han utilizado los programas AMESim, que basa sus soluciones en las técnicas bond graph y multipuerto, y FlowMaster, también multipuerto, pero que hace uso del método de las características para resolver las ecuaciones. Para el modelado del sistema de ventilación del motor estudiado en el capítulo sexto se ha utilizado el programa PowerFlow. En el apéndice A se presenta un breve resumen de estos programas. 


\section{Información requerida para el modelado de componentes}

Previo al modelado de los sistemas de refrigeración, independientemente del programa computacional que se maneje, se debe reunir la información mínima que se resume a continuación:

Los bloques de motor, compresores, carcasas, enfriadores de aceite se modelan normalmente como intercambiadores de calor y dentro del submodelo o externamente a través de plantillas de controlador se les asigna la ley de entrega de calor. Para su modelado se requiere: la característica de disipación de calor en función del tiempo (para análisis dinámicos) o máxima disipación de calor (para análisis de capacidad), el área representativa de la sección de paso, la curva característica de pérdidas de presión en función del caudal del refrigerante para el rango de caudales de funcionamiento del sistema, la estimación del área interna de transferencia de calor, el material y masa del bloque y la capacidad de fluído que almacena o cabe en el componente.

Las bombas y ventiladores se modelan como bombas centrífugas y debe tenerse la siguiente información: tipo de bomba o ventilador (de flujo radial o axial), revoluciones de referencia, caudal, presión y potencia a las revoluciones de referencia, inercia de la bomba, inercia del motor que la acciona, relación de transmisión entre el motor y la bomba, par de fricción, masa, material y volumen o capacidad de fluido, curvas características (presión y par contra caudal) para diferentes revoluciones, curvas Suter de caudal y de par (este es el tipo de característica que pide FlowMaster, no muy común en la literatura).

Las tuberías demandan de la siguiente información: longitud, diámetro, rugosidad absoluta, coeficiente de fricción, tipo de material, masa y espesor de la tubería.

Los codos demandan de la siguiente información: ángulo de deflexión, relación radio/diámetro, diámetro, rugosidad absoluta, coeficiente de fricción, tipo de material, masa y espesor, curva o superficie para los factores de corrección del número de Reynolds para $\operatorname{Re}>10000$ y r/d $>2$, relación de los coeficientes de fricción laminar sobre turbulento, curva o superficie para determinar el coeficiente de pérdidas para codos de sección circular. 
Los radiadores y otros intercambiadores de calor necesitan, si el cálculo que se va a hacer es de capacidad del sistema, la capacidad de disipación de calor nominal del radiador o "Thermal Duty". Sin embargo, siempre es mejor contar con la característica completa $\mathrm{Q} /\left(\mathrm{ITD} \cdot \mathrm{A}_{\mathrm{fr}}\right)$ en función de los flujos másicos del refrigerante y de aire por unidad frontal de área. Aquí ITD es la diferencia de temperaturas de entrada de los fluidos. Aparte de esta característica se deben conocer el área de la sección de entrada del refrigerante, el diámetro hidráulico, el área de la sección de paso del aire, el material y la masa del radiador seco, la capacidad o volumen de fluido que aloja el radiador y las curvas características de pérdidas de presión tanto para el flujo del refrigerante como para el flujo de aire.

Para el termostato se necesita conocer la constante de tiempo (mCp/UA), el offset de histéresis, las curvas características de levantamiento y descenso contra temperatura, el diámetro de derivación hacia la succión de la bomba, la curva característica de pérdidas de presión en función de la posición de ascenso en el ramal hacia el by-pass o succión de la bomba, el diámetro de derivación hacia el radiador o vía principal, la curva característica de pérdidas de presión en función de la posición de ascenso en el ramal principal y las curvas características de variación de las secciones de paso en las dos salidas del termostato (necesaria para conocer la variación de la repartición de los caudales hacia la bomba y hacia los radiadores)

Control de temperatura puede hacerse controlando los flujos de refrigerante con termostatos o controlando el flujo del aire por variación de la velocidad del (de los) ventilador(es) o por la conexión y desconexión de ventiladores, o con la combinación de control de termostato y de ventiladores.

\subsection{Conclusiones}

El presente ha sido un capítulo de transición en el que, como parte sustancial de la conceptualización de los sistemas de refrigeración de los motores, se ha abordado la composición y prediseño de los componentes de estos sistemas, desde una perspectiva “convencional”. Se ha retomado la valoración del calor evacuado al sistema de refrigeración como factor clave para el prediseño del sistema resumiendo el modelo aportado por Parish [4.1] y se ha sintetizado la metodología de cálculo térmico de diseño de los radiadores fundamentada en 
el trabajo de Kays y London [4.6]. Tras realizar la presentación de una propuesta iterativa de diseño y modelado de los sistemas de refrigeración basada en herramientas computacionales y como preparación a los trabajos de modelado que se explicarán en los siguientes capítulos, se ha presentado un acercamiento a los modelos matemáticos que de alguna manera subyacen en la estructura de los programas computacionales Amesim y FlowMaster. También se ha comentado brevemente el modo de operación de éstos y se ha resumido la información mínima demandada por los submodelos de los principales componentes del sistema de refrigeración.

\subsection{Bibliografía}

[4.1] Parish, O. L. Prediction methodology for the heat rejection from turbocharged or naturally aspired automobile engines. $\mathrm{PhD}$ dissertation, Texas Tech University, 2003.

[4.2] Lahvic, T. R. Investigation of engine heat rejection, agosto 1986, Ford Motor Co. (citado por Parish [4.1])

[4.3] Ap, N., Guerrero, P., Jouanny, Ph. Influence of fan system electric power on the heat performance of engine cooling module. SAE paper 2003-01-0275.

[4.4] Wolf-Heinrich, H. Aerodynamics of road vehicles. SAE, Warrandale, 1998.

[4.5] Carranza, S. Y., Romero, P. C. Evaluación de la capacidad térmica de un radiador de calor. Scientia et Technica, vol. XIII, issue 35, pp. 225-230, 2007.

[4.6] Kays, W. M., London, A. L. Compact heat exchangers. New York, 1984.

[4.7] Webb, R. L.,Trauger, P. Flow structure in the louvered fin heat exchanger geometry. Exp. Therm. Fluid Sci., vol. 4, pp. 205-214, 1991.

[4.8] Achachia, A., Cowell, T. A. Heat transfer and pressure drop characteristics of flat tube and louvered plate fin surfaces. Exp. Therm. Fluid Sci., vol. 1, pp. 147-157, 1988.

[4.9] Namai, K., Muramoto, H., Mochizuki, S. Flow visualization in the louvered fin heat exchanger. SAE paper 980055. 
[4.10] Tafti, D.K. and Cui, J. Computations of flow and heat transfer in a three dimensional multilouvered fin geometry. Int. J. Heat and Fluid Flow, vol. 45, n 25, pp. 5007-5023, 2002.

[4.11] Kakac S., Liu H.: Heat exchangers - selection, rating and thermal design, CRC Press LLC, 2002.

[4.12] Oliet, C., Oliva, A., Castro, J. y Pérez-Segarra, C.D. Parametric studies on automotive radiators. Appl. Therm. Eng., vol. 27, pp. 2033-2043, 2007.

[4.13] Kaplan, J. A., Heywood, J. B. Modelling the spark ignition engine warm-up process to predict component temperatures and hydrocarbon emissions. SAE paper 910302.

[4.14] Cortona, E., Onder, C. H. Engine thermal management with electronic cooling pump. SAE paper 2000-01-0965.

[4.15] Chen, C., Veshagh, A. A one-dimensional model for in-cylinder heat convection based on the boundary layer theory. SAE paper 921733.

[4.16] Mohan, K. V, Arici, O., Yang, S., Johnson, J. H. A computer simulation of the turbocharged Diesel engine as an enhancement of the vehicle engine cooling system simulation. SAE paper 971804.

[4.17] Lehner, C., Parker, G., Arici, O. Johnson, J. Design and development of a model based feedback controlled cooling system for heavy duty diesel truck applications using a vehicle engine cooling system simulation. SAE paper 2001-01-0336.

[4.18] Jarrier, L. Champoussin, J. C., Yu, R., Gentile, D. Warm-up of a Diesel engine: experiment and modeling. SAE paper 2000-010299.

[4.19] Torregrosa, A. J., Olmeda, P., Degraeuwe, B., Reyes, M. A concise wall temperature model for DI diesel engines. Appl. Therm. Eng., vol. 26, 1320-1327, 2006.

[4.20] Incropera, F. P., DeWitt, D. P. Introduction to Heat Transfer. John Wiley, New York, 1996.

[4.21] Strupp, N., Lemke, N., Tegethoff, W., Köhler, J. MAC simulation using Modelica/Dymola. 7th IIR Gustav Lorentzen Conf. Natural Working Fluids, Trondheim, Norway, 2006. 
[4.22] GT-Power User's manual (GT-Cool). Versión 5.2. Diciembre 2001.

[4.23] FlowMaster user’s manual. Versión 7.2.

[4.24] AMESim user’s manual. Versión 7.1. Septiembre 2008.

[4.25] Sjöstedt, C. J. On the modular modelling for dynamical simulation with application to fluid systems. Licentiate thesis, Stockholm, 2005.

[4.26] Modelica homepage, www.modelica.org.

[4.27] The Mathworks homepage, www.themathworks.com.

[4.28] Watanabe, N., Kubo, M., Yomoda, N. An 1D-3D integrating numerical simulation for engine cooling problem. SAE paper 2006-01-1603.

[4.29] Plazas, A. T., Modelado unidimensional de inyectores common rail Diesel. Tesis doctoral, Universidad Politécnica de Valencia. España, 2005.

[4.30] PowerFlow user's manual. Version 4.1.

[4.31] Miller, D. S. Internal flow systems, segunda edición, BHR Group Limited, 1996.

[4.32] Puntigam, W., Hörmann, T., Bernasch, J., Schierl, K., Wiesler, B. y Hager J. Thermisches Management im Fahrzeug durch Kopplung unterschiedlicher Simulationsmodelle Wärmemangement des Kraftfahrzeuges IV, Essen, expert verlag, 2004 (consultado en http://www.vif.tugraz.at en diciembre de 2008).

[4.33] Annand, W. J. D., Ma, T. H. Instantaneous heat transfer to the cylinder head surface of a small compression engine. Proc. Instn. Mech. Engrs. 185, 72/71, 976-987, 1970.

[4.34] Mesa, C.A. The engine cooling system. Livonia, 1997, $4^{\text {th }}$ edition.

[4.35] Zhelezko, B. E., Adamov, V. M., Esman, R. I. Termodinámica, Transferencia de Calor y Motores de Combustión Interna. Escuela Superior. Minsk, 1985.

[4.36] Zeitnetdinov, R. A., Diakov, I. F., Iarigin, S. V. Diseño de motores para automóviles y tractores. UGTU, Ulianovsk, 2004. 
[4.37] Romero, P. C. Máquinas de combustión interna. Fundamentos de construcción y cálculo. UTP, 2003.

[4.38] Frid, A., Abom, Y., Wang, Y., Fehse, K. Cooling fans in railway vehicles- Application of noise control measures to a roof-mounted engine cooler. Fan Noise 2007, Lyon, 2007.

[4.39] Joas, K., kirschiger, C. Fans in rail vehicle cooling systems. Energy systems as a result of total systems optimisation. Voith Turbo. Disponible en http://www.voithturbo.de (consultada en enero de 2009).

[4.40] Karassik, I. Manual de bombas. Mc Graw Hill, 1976. 


\section{Capítulo 5}

\section{Modelado, simulación y validación del sistema de refrigeración de un motor HSDI para reducir su tiempo de calentamiento}

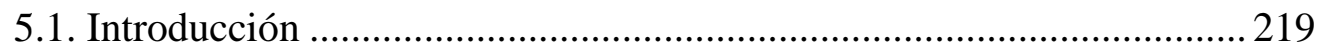

5.2. Modelado térmico del motor............................................................ 221

5.2.1. Descripción y caracterización del sistema de refrigeración........... 221

5.2.2. Modelo 1D del sistema de refrigeración original del motor. .........226

5.3. Modelado de las modificaciones al sistema de refrigeración del motor para reducir el tiempo de calentamiento ............................................ 231

5.4. Validación experimental de las modificaciones propuestas y valoración de su influencia sobre las emisiones y el consumo de combustible

5.4.1. Flujos de refrigerante experimentales de todas las

configuraciones. ............................................................................... 237

5.4.2. Respuesta de temperatura del refrigerante.................................. 238

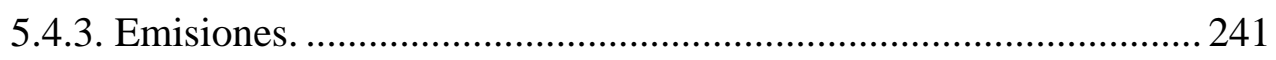

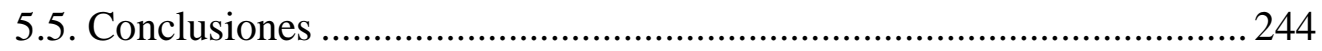

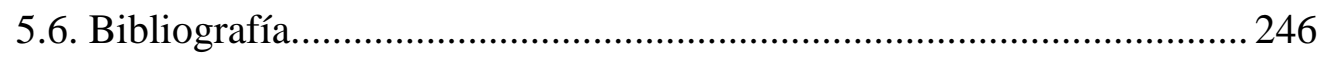




\subsection{Introducción}

El diseño de los sistemas de refrigeración parte de la necesidad de proveer la suficiente capacidad de disipación de calor a plena carga, no obstante que la mayor parte del tiempo los vehículos son conducidos a condiciones parciales de carga. Así, estando sobredimensionados, a cargas parciales de operación los sistemas de refrigeración tradicionales son responsables de pérdidas innecesarias de calor, particularmente durante el calentamiento del motor. Las medidas tecnológicas tomadas para adaptar el motor a las condiciones actuales de operación pueden dar ganancias en la eficiencia del motor (es decir, menor consumo de combustible), lo mismo que una reducción de emisiones nocivas. En trabajos publicados recientemente, como los de Campbell et al. [5.5], Allen y Lasecki [5.6], y Pang y Brace [5.7], ya se han venido demostrando los beneficios de las configuraciones de sistemas de refrigeración con control flexible. Las mejoras propuestas van desde la introducción de bombas controladas electrónicamente (Cortona y Onder [5.8], Cho et al. [5.9]), ventiladores, persianas y termostatos (Chanfreau et al. [5.10], Chalgren y Barron [5.11]), en los cuales se explota el potencial ofrecido por las tecnologías de control computarizado, hasta la implementación de diferentes técnicas como la refrigeración de precisión en Robinson et al. [5.12], la ebullición nucleada en Campbell et al. [5.2], la refrigeración evaporativa en Porot et al. [5.14], o el aislamiento térmico de las paredes, revisado por Jaichandar y Tamilporai [5.15]. Adicionalmente, la importancia de la temperatura tanto del aire de admisión como del refrigerante sobre las emisiones de un motor Diesel, bajo condiciones estables de operación en el motor caliente, han sido investigados recientemente por Torregrosa et al. [5.16].

La introducción de estrategias tendientes a reducir el tiempo de calentamiento ha adquirido especial atención también porque cuanto menos tiempo opere el motor en regímenes ineficientes y con temperaturas de sus componentes por debajo de los umbrales óptimos de operación, menor será el desgaste experimentado por el motor, como lo anotan Broatch et al. [5.1].

El desarrollo de herramientas computacionales con capacidad para modelar y analizar sistemas termohidráulicos con control integrado ha permitido en los últimos 10 años avanzar mucho en el estudio de los sistemas de refrigeración, de manera particular. Recurriendo a la simulación numérica son ostensibles 
los ahorros de tiempo y trabajo experimental en las actividades y pruebas experimentales de los motores. En efecto, los programas de modelado 0D/1D/3D se han convertido hoy en una ayuda rápida y eficiente para analizar los sistemas de gestión térmica del vehículo en sus diferentes circuitos de refrigerante, aire, aceite y carga del motor [5.18]-[5.23].

En el capítulo tres fue presentada la caracterización térmica de un motor HSDI sometido a ensayos bajo condiciones de operación estacionarias y también durante su operación en calentamiento bajo un ciclo de conducción homologado, con lo que se obtuvo el tiempo que tarda el motor en calentarse y se conocieron los factores de los que depende ese calentamiento. En el capítulo anterior se trataron los fundamentos para el modelado hidráulico y térmico de los sistemas de refrigeración, con los que podría construirse su modelo a nivel de sistemas dinámicos o a nivel de sistemas con parámetros distribuidos en presentación 0D ó 1D como otros autores lo han hecho [5.2], [5.3], y que están en la base de programas de modelado comerciales desarrollados específicamente para estos propósitos, uno de los cuales, Amesim, es empleado en el presente capítulo para modelar el sistema de refrigeración del motor ensayado. Como objetivo principal en esta parte del trabajo, se proponen modificaciones sencillas al sistema de refrigeración de ese motor, consistentes en la introducción de sólo dos válvulas de control sencillo (conservando los componentes y disposición del sistema original) fácilmente instalables en la topología actual y que redundan en la reducción del tiempo de calentamiento. Las modificaciones propuestas son simples y la fiabilidad de implementación es alta, con lo que sería posible y rápida su aplicación en los vehículos fabricados en serie. También se intenta la evaluación de los beneficios en el consumo de combustible y en las emisiones contaminantes generados con los cambios hechos en el sistema.

Para lograr este objetivo, en la primera parte de este capítulo, tras describir brevemente el sistema de refrigeración del motor ensayado, se hace uso de la información experimental (térmica e hidrodinámica) obtenida durante los ensayos descritos en el tercer capítulo, para configurar en Amesim los submodelos de correspondientes del sistema; se modela y simula el sistema completo original para obtener la respuesta del motor, expresada en términos de la temperatura de salida del motor a lo largo del tiempo, bajo condiciones constantes de operación y bajo condiciones de ciclo de conducción. En la segunda parte, habiendo comprobado la idoneidad del modelo para reproducir 
el comportamiento hidráulico y térmico del sistema de refrigeración del motor con la configuración original, se modelan tres configuraciones sugeridas del sistema de refrigeración modificadas con la introducción de dos válvulas adicionales, concluyendo sobre la influencia en la respuesta térmica del sistema. En la cuarta parte se discute la validación experimental de las modificaciones definidas con el modelo. Adicionalmente se analiza la influencia de estas modificaciones en el consumo de combustible y las emisiones contaminantes. Finalmente se sacan las conclusiones principales del trabajo realizado.

\subsection{Modelado térmico del motor}

\subsubsection{Descripción y caracterización del sistema de refrigeración}

El esquema del sistema de refrigeración del motor original es el presentado en la Figura 5.1. En el esquema, la parte del aire del sistema de refrigeración se ha omitido por no ser relevante para el modelado del calentamiento del motor. Aunque en el esquema el enfriador de aceite aparece separado, la descarga del refrigerante que pasa por él se realiza internamente en el motor.

El sistema se compone de las fuentes de calor representadas por la estructura del motor y los enfriadores de gases de escape y del aceite, los sumideros de calor, representados por el radiador y el calentador del habitáculo, la bomba, el tanque de expansión, el termostato y los elementos de conexión. El termostato es la válvula originalmente prevista en el sistema para permitir que el refrigerante fluya al radiador una vez el motor adquiera la temperatura de consigna. El refrigerante ingresa al motor impulsado por la bomba, circula por las camisas de los cilindros y luego por la culata. La parte del refrigerante desviada dentro del motor hacia el enfriador del aceite se reúne con el resto del refrigerante en la caja de agua, ubicada a la salida del motor. La caja de agua es un volumen de mezclado que recoge también el refrigerante proveniente del radiador cuando el motor ya se ha calentado. De la caja, el refrigerante se reparte por cuatro ramas paralelas: hacia el radiador, si la temperatura de consigna se ha alcanzado, hacia el calentador del habitáculo en serie con el enfriador de EGR, hacia el tanque de expansión y hacia el bypass que es el retorno más expedito de nuevo hacia la entrada del motor. Generalmente el curso por el bypass es un paso calibrado. 


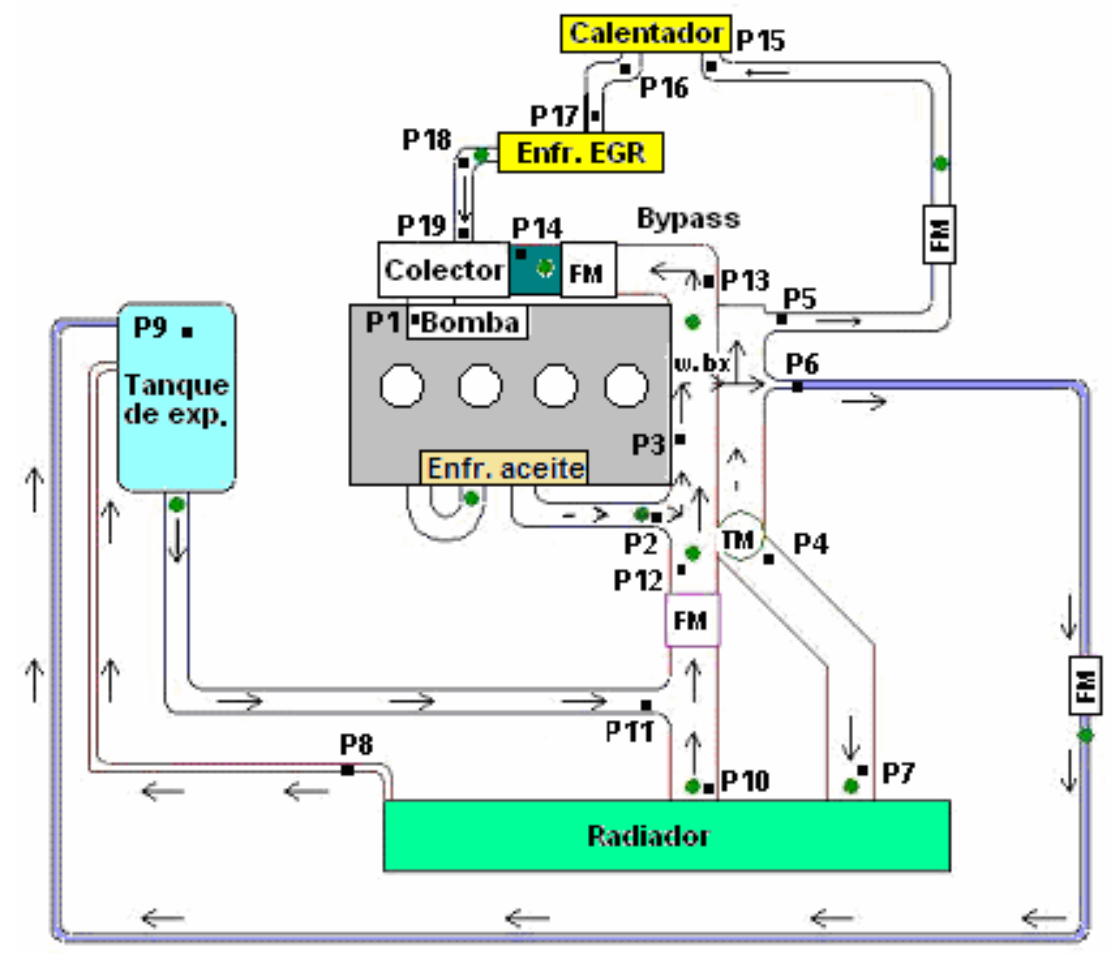

Figura 5.1. Diagrama esquemático del sistema de refrigeración del motor ensayado. FM - medidores de caudal, TM - termostato; las marcas redondas y cuadradas representan los sensores de temperatura y presión, respectivamente.

La caracterización hecha de los flujos de refrigerante, las inercias térmicas, el transporte de calor y la conducción térmica en el sistema original constituyó parte sustancial del trabajo expuesto en el capítulo tercero. Entre los mismos trabajos experimentales explicados en ese capítulo, se realizaron ensayos del motor en caliente bajo seis regímenes de operación (no explicados allí) para efectuar la caracterización hidráulica de los componentes y del sistema, es decir, para obtener las características de pérdidas de presión en los componentes principales. Adicionalmente se complementaron, bajo estas condiciones, las características térmicas de los componentes: radiador, calentador del habitáculo, enfriadores del aceite y de los gases de escape recirculados. En la Figura 5.2 se representa la característica de la bomba, mecánicamente accionada por el motor para varios valores de la velocidad de 
éste, mientras que en la Figura 5.3 se pueden observar las proporciones de los flujos de refrigerante, $\dot{V}_{\text {refr }}$, a través de los diferentes ramales del circuito en relación con el flujo promovido por la bomba.

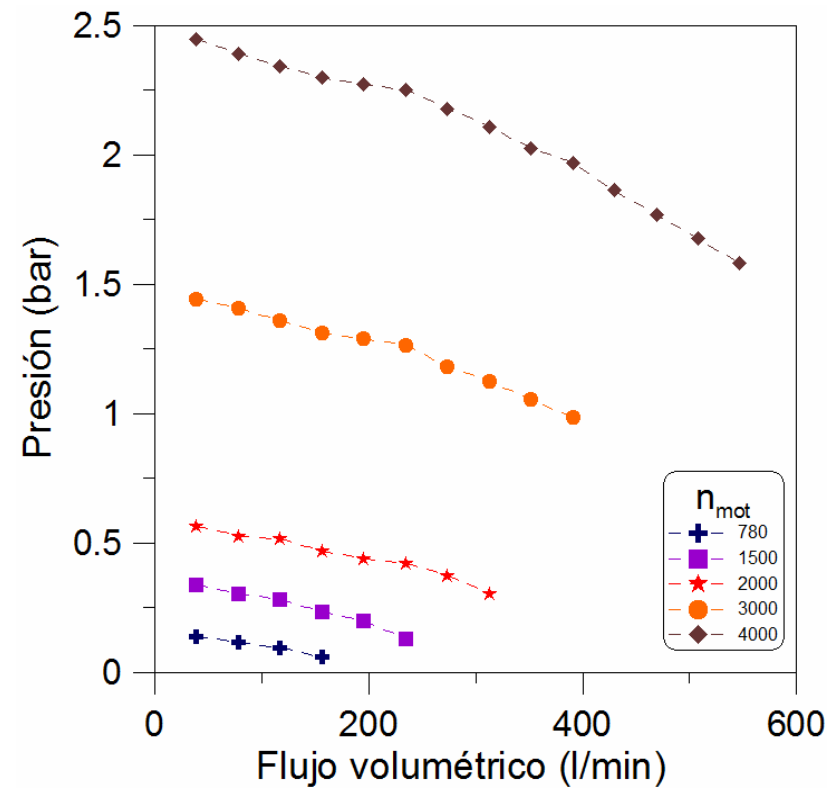

Figura 5.2.

Característica presión vs. flujo volumétrico de la bomba del motor ensayado a diferentes revoluciones.

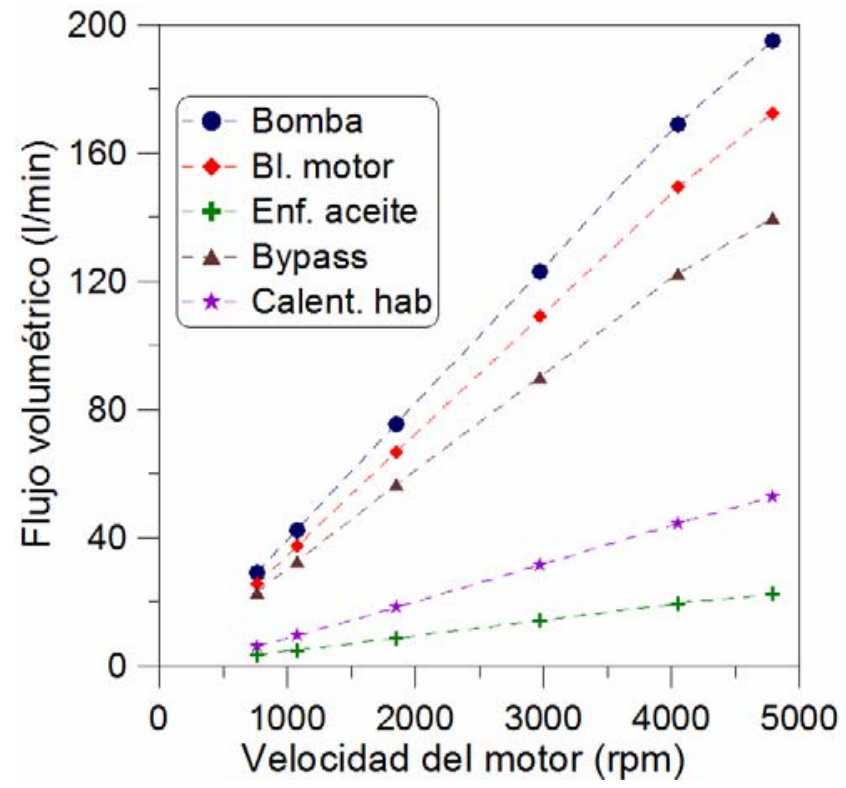

Figura 5.3. Flujos de refrigerante por las ramas del circuito de refrigeración, obtenidos para algunas revoluciones del motor. 
Con los flujos de refrigerante a través de los componentes y las mediciones de presión realizadas en sus extremos de entrada y salida, se han obtenido sus áreas equivalentes que, junto con las dimensiones de los componentes, constituye el conjunto de parámetros requerido por el programa de modelado y simulación para realizar los cálculos hidráulicos. En tabla 5.1 se resumen las áreas equivalentes de los componentes principales del sistema $\left(A_{\text {equiv }}=\dot{V}_{\text {refr }} \sqrt{\rho / 2 \Delta p}\right.$ ).

Tabla 5.1. Cálculo del área equivalente de los componentes del sistema.

\begin{tabular}{|l|ccc|}
\hline \multicolumn{1}{|c|}{ Dispositivo } & $\begin{array}{c}\text { Flujo volu- } \\
\text { métrico, } Q, \mathrm{l} / \mathrm{min}\end{array}$ & $\begin{array}{c}\text { Pérdida de } \\
\text { presión, } \Delta p, \mathrm{~Pa}\end{array}$ & $\begin{array}{c}A_{\text {equiv }}, \\
\mathrm{mm}^{2}\end{array}$ \\
\hline Calentador habitáculo & 18,3 & 12200 & 61,75 \\
Manguera motor-calentador & 18,3 & 700 & 257,77 \\
Enfriador de EGR & 18,3 & 1800 & 160,75 \\
Manguera EGR-calentador & 18,3 & 700 & 257,77 \\
Manguera EGR-motor & 18,3 & 1900 & 156,46 \\
Enfriador de aceite & 8,80 & 24200 & 87,10 \\
Motor & 75,5 & 23100 & 185,13 \\
Manguera tan. de exp.- & 50,0 & 500 & 833,38 \\
radiador & & & \\
Manguera salida radiador & 52,0 & 400 & 968,97 \\
Termostato & 56,5 & 20300 & 148 \\
Radiador & 125,0 & 30000 & 268,96 \\
\hline
\end{tabular}

La tabla 5.2 corresponde a la caracterización del calentador del habitáculo del vehículo utilizado en el modelo.

Tabla 5.2. Flujo de calor intercambiado en el calentador del habitáculo según los flujos de refrigerante y de aire, en $\mathrm{kW}$.

\begin{tabular}{|c|ccccc|}
\hline Flujo de refrigerante, l/h & \multirow{2}{*}{200} & 500 & 800 & 1200 & 1600 \\
\cline { 1 - 5 } Flujo másico de aire, kg/h & & & & & \\
\hline 150 & 3,92 & 4,02 & 4,05 & 4,1 & 4,16 \\
200 & 4,86 & 5,08 & 5,16 & 5,28 & 5,31 \\
250 & 5,43 & 5,93 & 6,16 & 6,23 & 6,32 \\
300 & 5,88 & 6,7 & 6,99 & 7,18 & 7,24 \\
\hline
\end{tabular}




\begin{tabular}{|c|ccccc|}
\hline 400 & 6,61 & 8,02 & 8,43 & 8,67 & 8,84 \\
500 & 7,15 & 9,13 & 9,67 & 9,98 & 10,26 \\
600 & 7,63 & 9,98 & 10,72 & 11,13 & 11,37 \\
\hline
\end{tabular}

La tabla 5.3 es la correspondiente a la caracterización del radiador utilizado en el modelo. Es cierto que el radiador no opera mientras no se alcance el umbral de temperatura establecido por el termostato.

Tabla 5.3. Flujo de calor intercambiado en el radiador del motor según los flujos de refrigerante y la velocidad del de aire, en $\mathrm{kW}$.

\begin{tabular}{|c|cccccc|}
\hline & 1,5 & 2,5 & 4,0 & 5,5 & 7,0 & 7,5 \\
\cline { 1 - 1 } $\begin{array}{c}\text { Vlujo volumétrico de } \\
\text { agua, l/h }\end{array}$ & & & & & & \\
\hline 3000 & 23 & 31,5 & 40 & 46 & 50 & 52,5 \\
5000 & 25 & 34 & 43 & 51,5 & 58 & 62,5 \\
8000 & 26 & 37 & 50 & 58,5 & 67 & 73 \\
10000 & 27 & 39,5 & 55 & 67 & 74 & 82,5 \\
\hline
\end{tabular}

En la tabla 5.4 se presentan los flujos de calor transferidos por los gases de escape al refrigerante en el enfriador de EGR, obtenidos a partir de las mediciones de calor transferido al refrigerante, la presión media efectiva calculada y las correspondientes revoluciones del motor, en kW.

Tabla 5.4. Flujo de calor transferido por los gases de escape al refrigerante en el enfriador de EGR, en $k W$.

\begin{tabular}{|c|c|c|c|c|}
\hline Régimen del motor, rpm & \multirow{2}{*}{1635} & \multirow{2}{*}{1728} & \multirow{2}{*}{1890} & \multirow{2}{*}{2040} \\
\hline pme, bar & & & & \\
\hline 2,2 & 0,98 & 0,7 & 0,667 & 0,485 \\
\hline 3,8 & 1,1 & 0,763 & 0,757 & 0,7 \\
\hline 5,0 & 1,12 & 0,909 & 0,839 & 0,76 \\
\hline 6,4 & 1,14 & 1,109 & 0,936 & 0,8 \\
\hline
\end{tabular}




\subsubsection{Modelo 1D del sistema de refrigeración original del motor}

Con la información experimental disponible se ensambló el modelo del sistema de refrigeración original del motor y se configuraron las características térmicas e hidráulicas requeridas por los submodelos seleccionados. En la Figura 5.4 se aprecia la representación en AMESim del sistema de refrigeración del motor. El enfriador de aceite se ha colocado para ajustar los caudales de refrigerante, pero no se ha establecido la magnitud exacta del calor intercambiado en él, por no tener la suficiente información. Así, todo el calor que se disipa en el modelo del motor proviene de la cámara de combustión y del enfriador de los gases de escape.

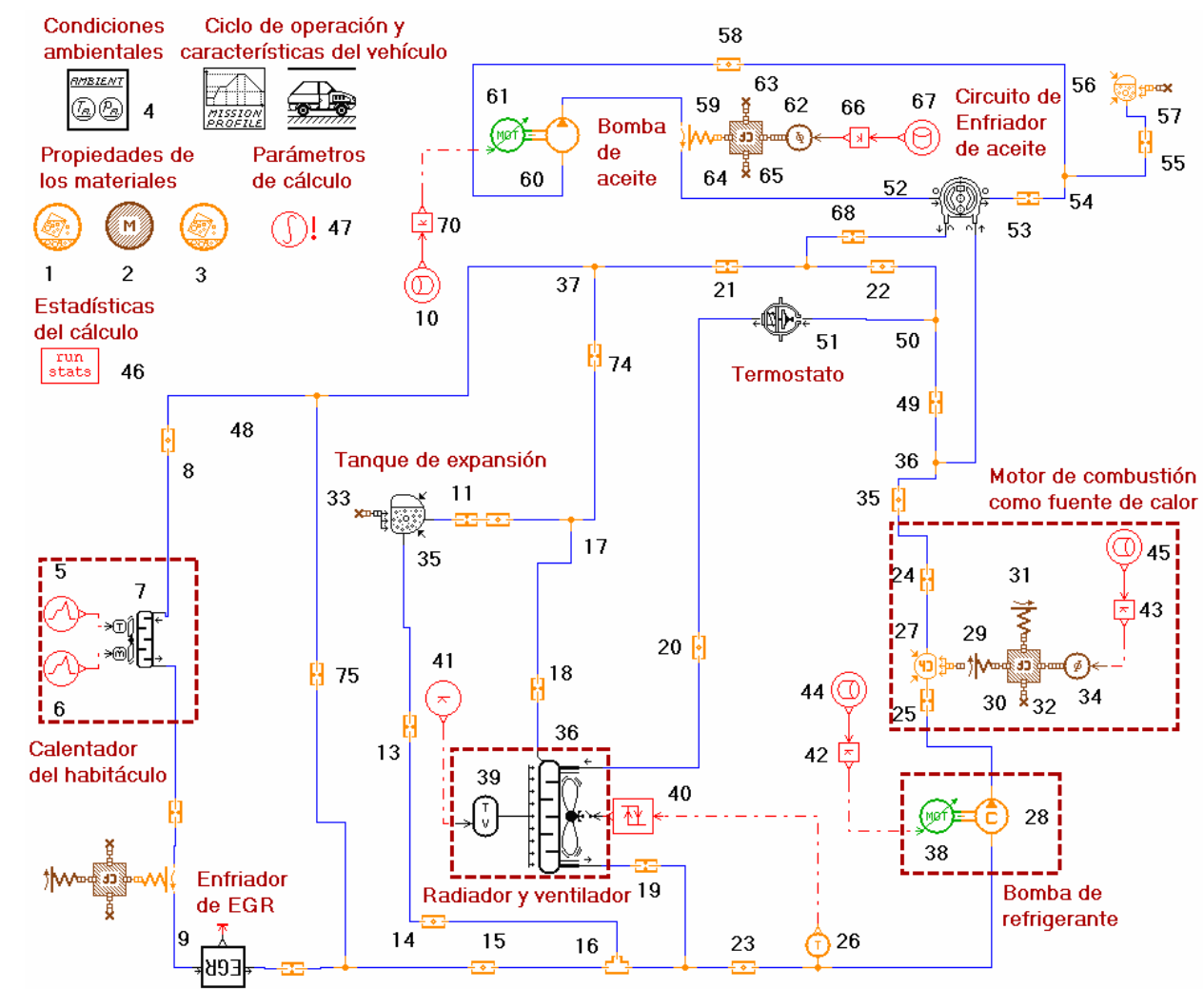

Figura 5.4. Modelo del sistema de refrigeración original del motor, ensamblado bajo el entorno del programa AMESim.

El modelo alimentado con las especificaciones técnicas reales, obtenidas después de las pruebas estacionarias, se simuló bajo la condición de carga y 
régimen constantes de $50 \mathrm{Nm}$ de par y $1500 \mathrm{rpm}$. La temperatura de referencia inicial para la estructura del motor fue de $20^{\circ} \mathrm{C}$.

En la Figura 5.5 se puede observar la comparación de los flujos volumétricos modelados y experimentales de refrigerante por los ramales del circuito, bajo las condiciones de operación constante. Las discrepancias durante cerca de los primeros 250 segundos se deben mayormente a la ligeramente menor densidad del fluido a bajas temperaturas en el modelo. Los resultados desde el punto de vista hidráulico son adecuados, dado que los gastos de refrigerante a lo largo de los ramales del sistema se acercan a los valores reales con una desviación del orden del $5 \%$.

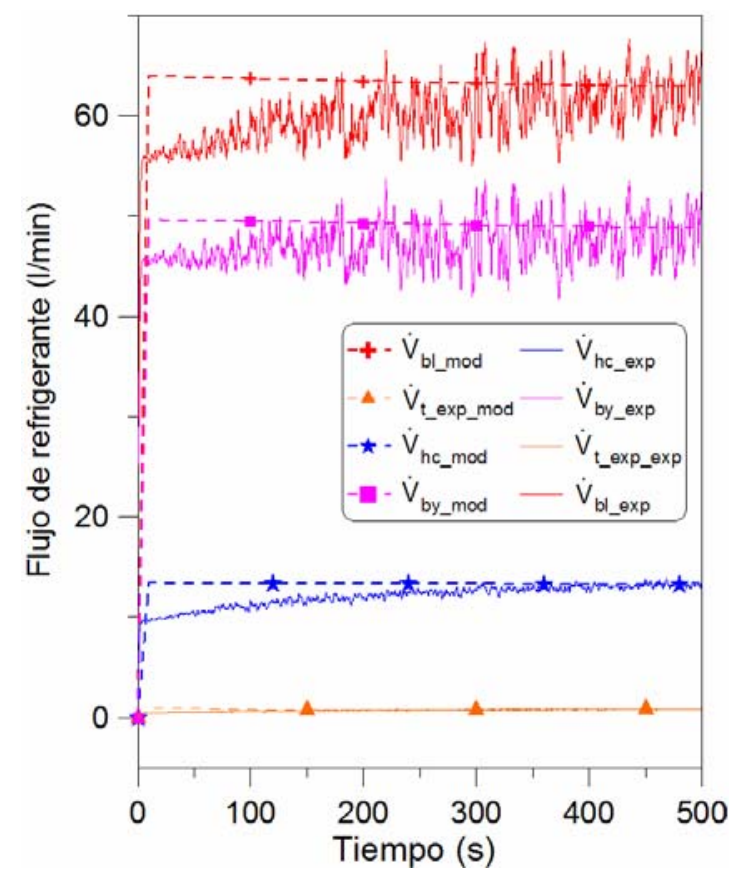

Figura 5.5. Gastos de refrigerante predichos $y$ experimentales a través de los ramales del sistema de refrigeración original.

Es de anotar que, para el modelado del sistema de refrigeración, las mangueras se han considerado rígidas y adiabáticas (por facilidad de convergencia rápida del programa). En el comportamiento de los flujos experimentales se presentan fluctuaciones inherentes al sistema real, por la fluctuación natural de las revoluciones del motor y la naturaleza elástica de las conexiones del sistema, lo que no se reproduce con el modelo. Los gastos 
másicos de más importancia son los que atraviesan el bypass y el ramal del calentador del habitáculo.

Tras haber ajustado la respuesta hidráulica, se emprendió el análisis térmico del modelo. En esta fase se examinó el perfil de respuesta de la temperatura del refrigerante considerando la masa sensible estimada y los flujos de calor al refrigerante. Aparte de los flujos de refrigerante por los diferentes ramales del circuito, como se anotó en el capítulo tercero, para un motor con fuentes y sumideros de calor definidos, la masa es el factor que más afecta la respuesta del sistema, como puede verse en la gráfica de la Figura 5.6, en la cual se ha simulado la evolución de la temperatura del refrigerante para cuatro masas diferentes del motor.

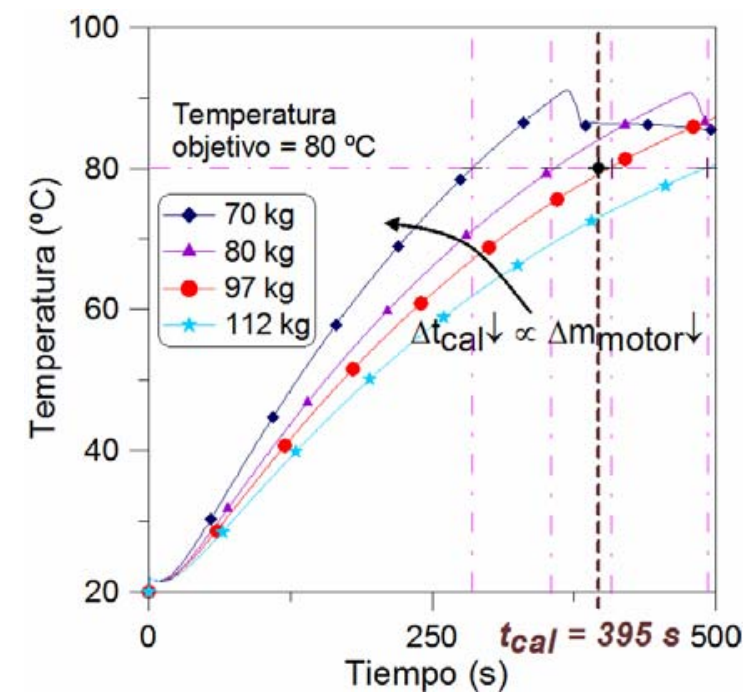

Figura 5.6. Ejemplo de sensibilidad de la respuesta la temperatura del refrigerante a la variación de la masa del motor .

El motor se modeló como una restricción de $185 \mathrm{~mm}^{2}$, ubicada a la salida de la bomba, en serie con un acumulador de 1,75 litros que transfiere calor a una masa metálica (45/55 aluminio/fundición) de $97 \mathrm{~kg}\left(5253 \mathrm{~kg} / \mathrm{m}^{3}\right.$ de densidad, calor específico de $C p=628 \mathrm{~J} /(\mathrm{kg} \cdot \mathrm{K})$ y conductividad térmica $k=102$ $\mathrm{W} /(\mathrm{m} \cdot \mathrm{K})$, a través de un área de transferencia de calor con el refrigerante de $130000 \mathrm{~mm}^{2}$, bajo un coeficiente de película de $10200 \mathrm{~W} /\left(\mathrm{m}^{2} \mathrm{~K}\right)$. En la Figura 5.7 se comparan las temperaturas simulada y experimental del refrigerante a la salida del motor. 
La respuesta del modelo es muy cercana a lo largo del calentamiento del motor. En la misma figura se ha graficado el error del modelo en la estimación de la temperatura del refrigerante en relación con los valores experimentales.

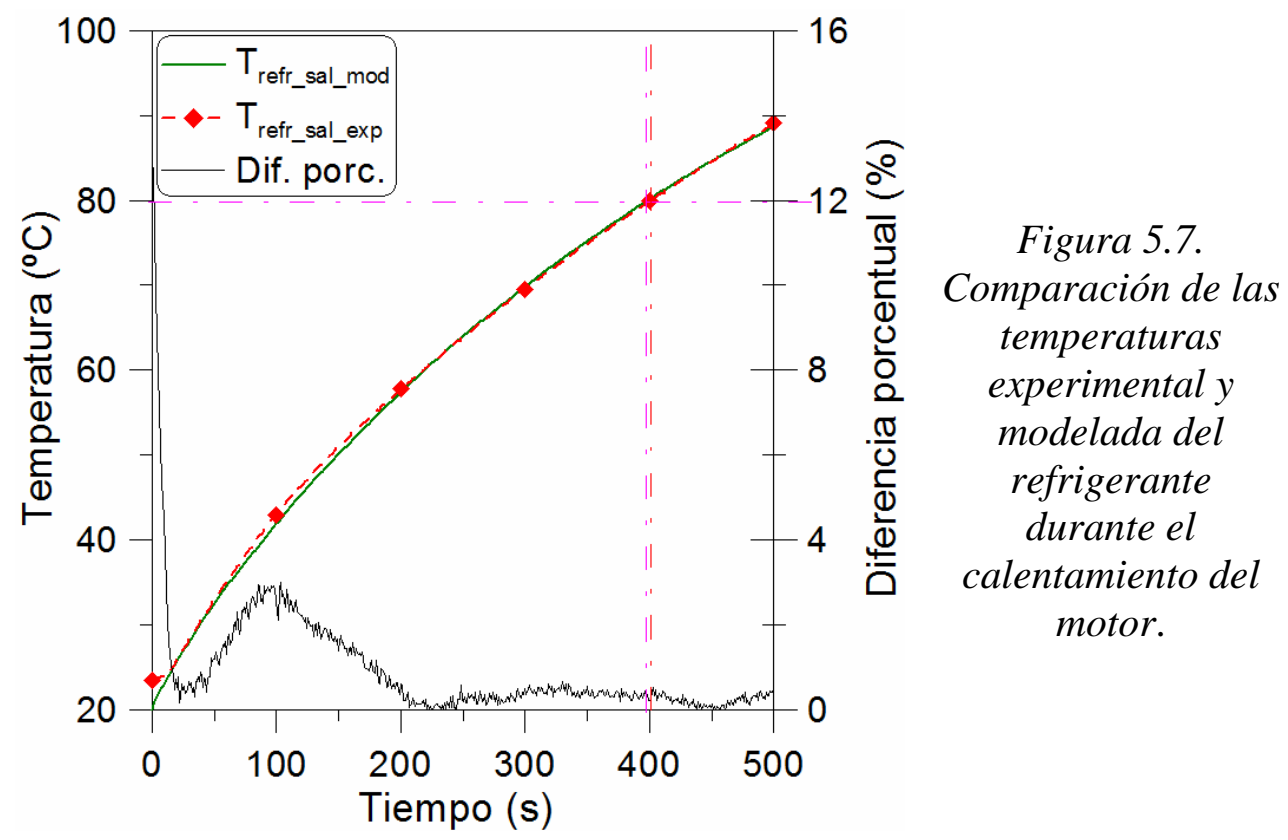

Como se puede ver en la Figura 5.7, el error del modelo comparando con la respuesta experimental durante el calentamiento bajo condiciones de operación constantes es menor del $4 \%$, excepto para los primeros 10 segundos, tiempo durante el cual el motor aún está operando a ralentí, en el ensayo experimental. El tiempo requerido para alcanzar los $80^{\circ} \mathrm{C}$ es de 400,5 segundos, mientras que el correspondiente tiempo experimental es de 395 segundos.

Una vez ajustado el modelo bajo condiciones de operación estabilizadas, y que la respuesta hidráulica y térmica del sistema bajo condiciones de carga constante se consideró satisfactoria, se cambió el modo de operación del modelo a condiciones de conducción bajo ciclo de operación NEDC. El calor suministrado durante el ciclo de conducción tiene el comportamiento ilustrado en la Figura 5.8. 


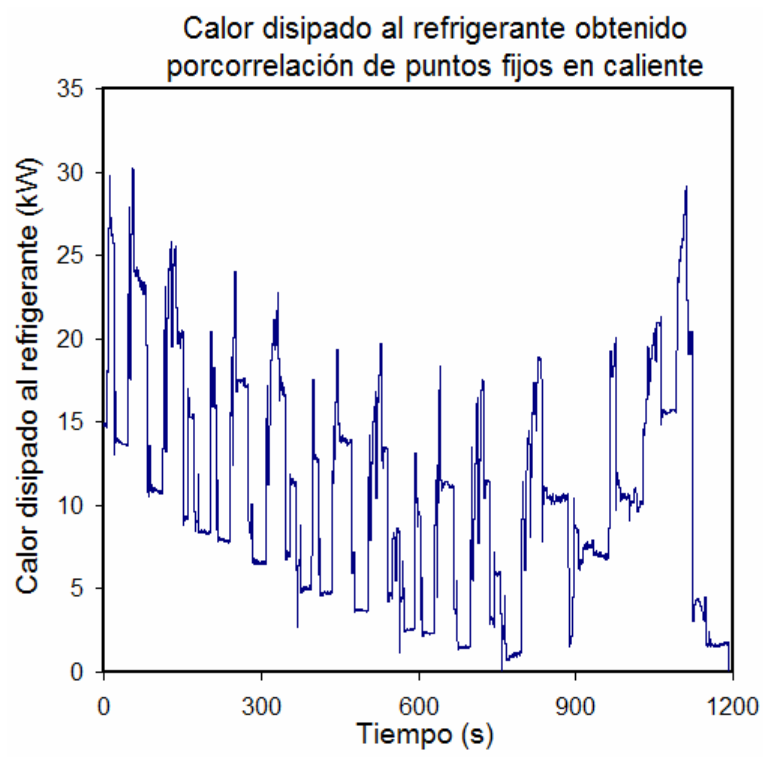

Figura 5.8. Flujo de calor disipado a las paredes del motor, calculado con la correlación de la expresión (3.29).

La comparación de las temperaturas experimental y modelada del sistema se ilustra en la gráfica de la Figura 5.9. La respuesta del modelo mantiene el perfil de la temperatura con algunas desviaciones menores, atribuibles a las diferencias entre el submodelo del motor y el motor real. Efectivamente, en el submodelo la parte exterior del bloque del motor no se ha modelado y tampoco se ha modelado la interacción del aceite del motor con otras partes diferentes al pistón y al cilindro. Además, los calores del mapa térmico introducidos corresponden a puntos de operación estacionarios, que no cubren la totalidad de los puntos de operación, y el modelo interpola entre los valores suministrados cuando pasa por puntos de operación que no se encuentran en el mapa. Las tablas de calores intercambiados del calentador del habitáculo y del enfriador de EGR también tienen una resolución muy gruesa y el programa realiza allí interpolaciones, con efectos directos sobre la curva de respuesta de temperatura del motor. No obstante lo dicho, el modelo mantiene el perfil de la respuesta experimental de la temperatura del refrigerante y además alcanza la temperatura objetivo en 721 segundos, muy cerca del tiempo experimental de 713 segundos. Cuando el refrigerante alcanza temperaturas ya cercanas para la apertura del termostato (cuando el termostato empieza a abrirse), la respuesta del modelo se separa mucho de la respuesta experimental, principalmente porque los modelos del termostato y del flujo de aire por el radiador no han sido refinados. La característica del ventilador del motor se ha introducido, pero lo importante en el modelado del 
aire es la dependencia del flujo del aire por el radiador en función de la velocidad del vehículo. Esto necesitaría de un modelado al menos unidimensional del lado del aire del sistema de refrigeración, lo cual queda fuera del alcance de la presente tesis. Recordando que el objetivo principal del modelo es la reproducción del comportamiento durante el calentamiento del motor, puede decirse que el modelo sirve a esos propósitos con suficiencia.

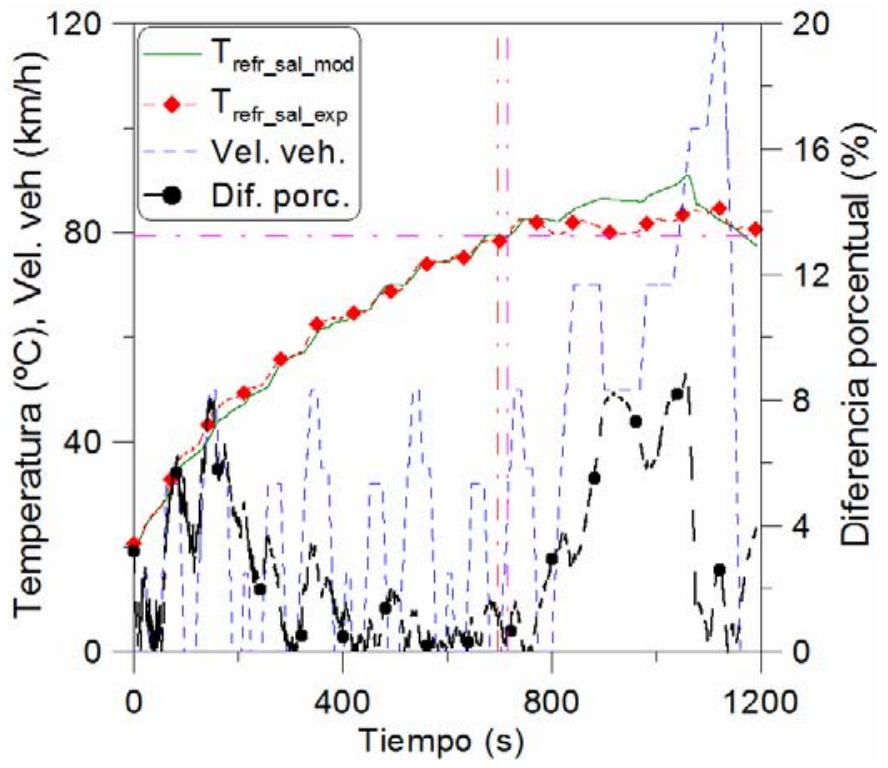

Figura 5.9.

Temperatura del refrigerante a la salida del motor durante la operación de éste bajo un ciclo de conducción NEDC.

Conformes con la capacidad del modelo para reproducir el comportamiento termohidráulico del sistema de refrigeración original del motor, se emprende el modelado y la validación de las modificaciones al sistema de refrigeración conducentes a lograr un menor tiempo de calentamiento.

\subsection{Modelado de las modificaciones al sistema de refrigeración del motor para reducir el tiempo de calentamiento}

En el capítulo tercero se analizó ya la influencia de la masa del motor y de la cantidad de refrigerante en el sistema sobre el tiempo de calentamiento. Todas las medidas conducentes a que la masa del motor reciba el mayor flujo 
posible de calor son medidas que reducen el tiempo de calentamiento: reducción de la masa del motor, reducción de la cantidad de refrigerante en el sistema y reducción del flujo de refrigerante a través de las camisas de refrigeración del motor.

El flujo reducido de refrigerante a través de las camisas del motor causaría una reducción del coeficiente de transferencia de calor y, en consecuencia, una reducción del calor disipado al refrigerante. Por otra parte, asumiendo que el motor disipa la misma cantidad de calor independientemente del gasto másico de refrigerante, puede concluirse que la temperatura del refrigerante se incrementaría proporcionalmente.

Si se pudiera cortar el flujo de refrigerante a través del circuito externo (retención de la masa de refrigerante atrapada en las camisas de refrigeración), la masa sensible del motor adquiriría más rápidamente su temperatura de operación y la diferencia entre las temperaturas de entrada y salida estaría limitada solamente por el retraso debido a la cantidad del refrigerante circulante. No obstante, la legislación requiere también que los tiempos de desempañamiento y descongelamiento de los vidrios del vehículo y de calentamiento del habitáculo en invierno sean pequeños, haciendo obligatoria la circulación de refrigerante a través del calentador del habitáculo desde el arranque del motor. Por tanto no es posible cortar la circulación de refrigerante a través del calentador del habitáculo, aunque es posible cortar la circulación por los otros ramales del sistema durante el calentamiento.

Basándonos en el análisis hecho, en este trabajo se ha propuesto estudiar la respuesta térmica del motor operando bajo diferentes topologías del sistema de refrigeración, resultantes de la incorporación de dos válvulas en el sistema original: una válvula controlada on-off ubicada en el ramal de bypass, para reducir el flujo de refrigerante, y una segunda válvula localizada en el ramal hacia el tanque de expansión, planeada para reducir el volumen total de refrigerante participante en la parte externa del circuito de refrigeración. Las válvulas están destinadas a operar totalmente abiertas o totalmente cerradas, exclusivamente. Las válvulas se proponen de una capacidad de paso consistente con los caudales originales que circulan por los ramales del bypass y el tanque de expansión. 
La ventaja de las modificaciones propuestas al sistema de refrigeración consiste en que no demandan la incorporación de estrategias sofisticadas de control y son fáciles de implementar sin demandar prácticamente cambios en el sistema original, excepto por la disponibilidad del espacio ocupado. La operación de instalación se reduce a desmontar las dos mangueras de los ramales actualmente existentes, cortarlas y cerrar de nuevo los ramales con las partes cortadas y las válvulas incorporadas. Un mando operado automáticamente podría controlar la posición de las dos válvulas durante el calentamiento del motor.

El modelo del sistema de refrigeración original desarrollado se empleó para simular las tres modificaciones resultantes de la introducción de las dos válvulas, con la asunción de que el motor disipa al refrigerante la misma cantidad de calor, independientemente de la topología del sistema de refrigeración.

Las válvulas sugeridas, denominadas $V_{b p} \mathrm{y} V_{\text {t.exp }}$, se instalan en los ramales del bypass y de descarga al tanque de expansión, respectivamente. Las configuraciones resultantes son las de la tabla 5.5. Con las dos válvulas abiertas se tiene la misma configuración del sistema original o configuración base (denominada M1); cortando el flujo a través de la válvula $V_{b p} \mathrm{y}$ manteniendo el flujo a través del ramal del tanque de expansión, $V_{t . \text { exp }}$, se ajusta la configuración M3; si, por el contrario, se abre la válvula $V_{b p}$ y se cierra la válvula $V_{\text {t.exp}}$, se tiene la configuración M4. La configuración M2 corresponde al caso cuando las dos válvulas introducidas están cerradas.

Tabla 5.5. Configuraciones nuevas del sistema de refrigeración.

\begin{tabular}{ccc}
\hline Configuración & Válvula bypass, $V_{b p}$ & Válvula tanque de expansión, $V_{t . e x p}$ \\
\hline M2 & Cerrada & Cerrada \\
M3 & Cerrada & Abierta \\
M4 & Abierta & Cerrada \\
\hline
\end{tabular}




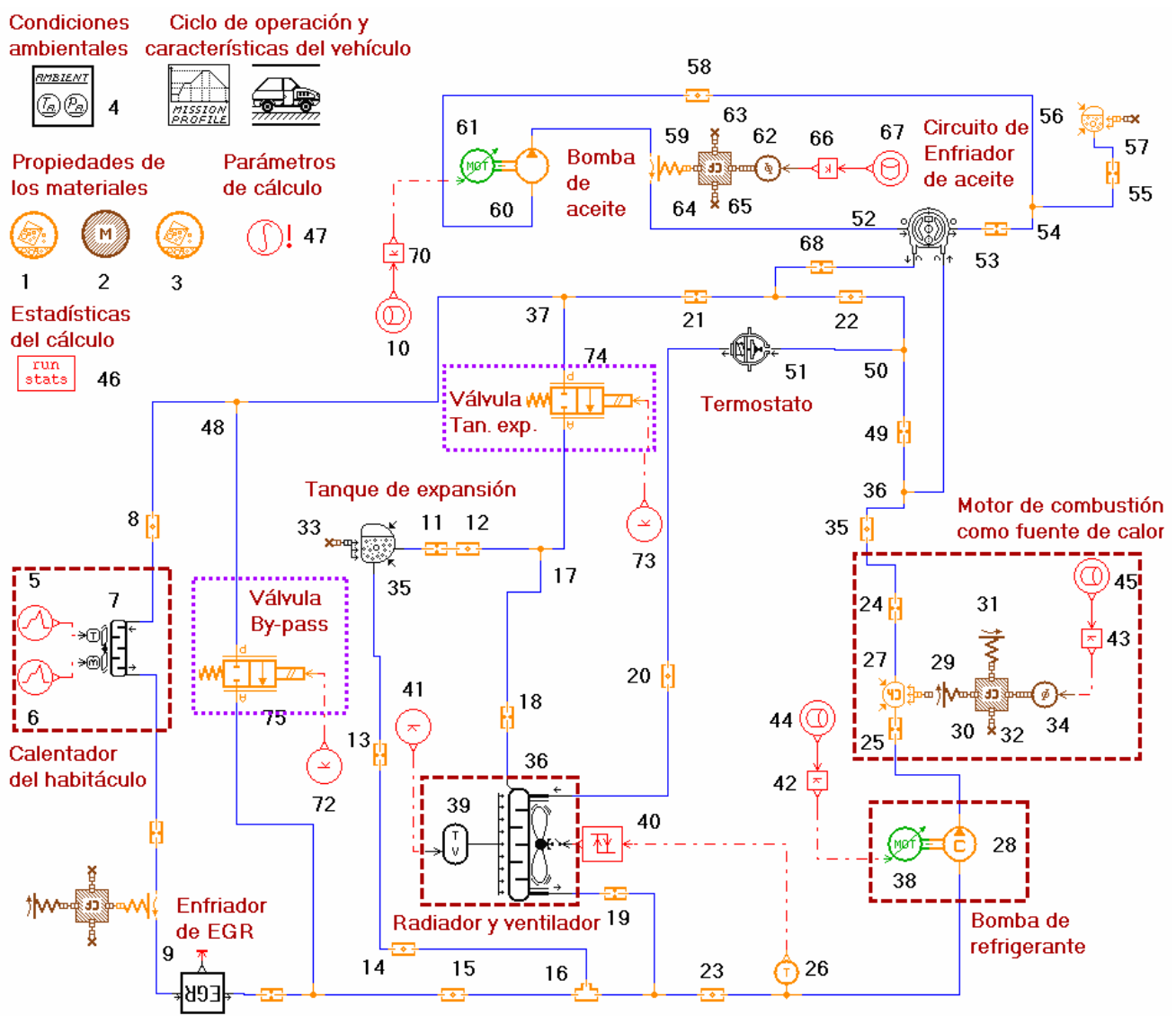

Figura 5.10. Configuración del modelo del sistema de refrigeración con la introducción de las dos válvulas propuestas. Actuando sobre los controladores 72 y 73 se abren o se cierran las válvulas en el modelo, con lo que se simulan los "cuatro posibles circuitos".

\section{Resultados de la simulación de las configuraciones propuestas}

Siendo rigurosos, el sistema de refrigeración no se modifica introduciendo las dos válvulas sugeridas y el modelo elaborado para el sistema original sólo tiene que modificarse introduciendo en los ramales del bypass y del tanque de expansión los submodelos para las válvulas nuevas. Seleccionando de la librería de componentes termohidráulicos un mismo submodelo para las dos válvulas, se realizó la simulación de las tres configuraciones nuevas, bajo el régimen de carga constante y bajo el ciclo de conducción NEDC. En los 
ramales a modificar se modelaron básicamente el paso o el cierre de ese ramal con un elemento tipo válvula de dos vías-dos posiciones, que tiene una resistencia tal que el caudal del circuito original no se altera cuando la restricción permite el paso de refrigerante. En consecuencia el esquema del modelo es prácticamente el mismo. Sí hay que anotar que, de quererlo, se podría montar válvulas con control continuo de la apertura, pero el interés en el estudio está limitado al mejoramiento del calentamiento, y la simulación con válvulas sencillas es suficiente. El esquema bajo el que se logra simular la operación de las alternativas al sistema de refrigeración es el representado en la Figura 5.10.

Bajo las condiciones constantes de operación simuladas en este capítulo (50 $\mathrm{N} \cdot \mathrm{m}$ de par y $1500 \mathrm{rpm}$ ) los gastos de refrigerante obtenidos fueron los mostrados en la tabla 5.6, para las cuatro configuraciones posibles.

Tabla 5.6. Flujo total de refrigerante para las cuatro configuraciones del motor, $\mathrm{l} / \mathrm{min}$.

\begin{tabular}{lllll}
\hline Configuración & M1 & M2 & M3 & M4 \\
Flujo de refrigerante & 53,8 & 18,3 & 19,7 & 52,7 \\
\hline
\end{tabular}

El gasto menor de refrigerante se logró con la configuración M2, contrario a la configuración M1 a la que le correspondió el mayor gasto, por proveer el mayor número de vías para el refrigerante $\mathrm{y}$, en consecuencia la menor impedancia hidráulica. Con las configuraciones M2 y M3, el gasto de refrigerante puede reducirse en cerca del $64 \%$, en comparación con la configuración M1.

Después de simular el sistema de refrigeración bajo las tres configuraciones nuevas, se efectuaron las simulaciones con el motor operando bajo un ciclo de conducción NEDC, con la obtención de las gráficas de temperaturas del refrigerante a lo largo del ciclo como se ilustran en la Figura 5.11.

Para las configuraciones M2 y M3 se observó la misma tendencia de cambio de la diferencia de temperaturas, pero el cambio relativo está en favor de la configuración M2. Para esta configuración el refrigerante que pasa por el bloque alcanza la temperatura de $80{ }^{\circ} \mathrm{C}$ después de 539 segundos. Aunque la 
configuración M3 es similar en las magnitudes de los flujos de refrigerante a la configuración M2, su mayor volumen asociado de refrigerante prevalece, haciendo que sea la más lenta en alcanzar la temperatura objetivo de $80^{\circ} \mathrm{C}$. El refrigerante se calienta más rápidamente para la configuración M4 que para la M1, por tener un menor volumen de refrigerante asociado, a pesar de que sus flujos de refrigerante son similares.

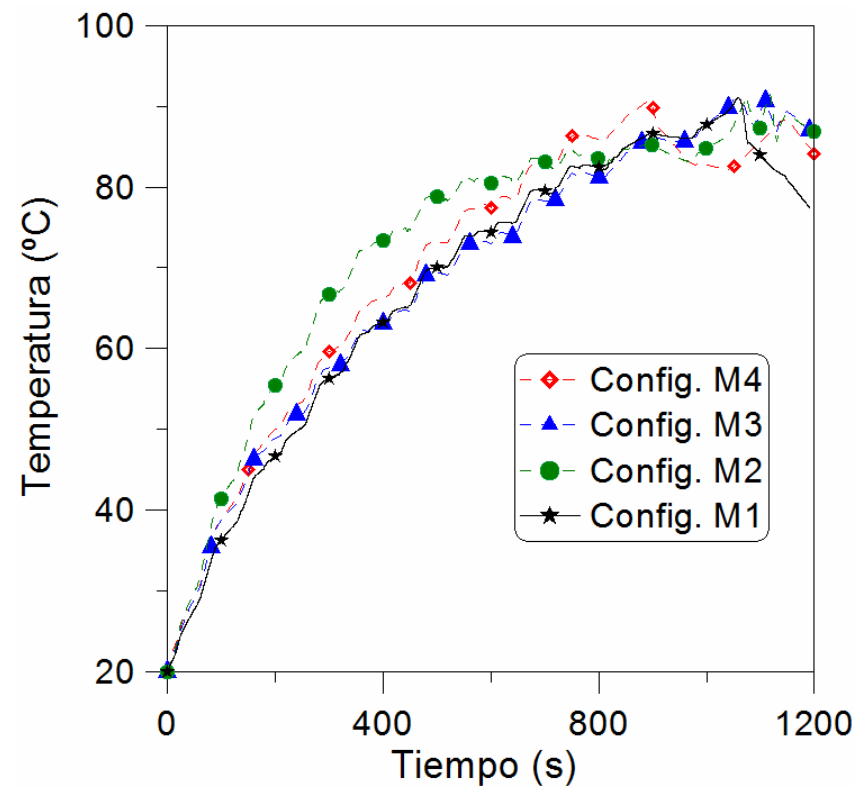

Figura 5.11.

Temperatura del refrigerante a la salida del motor para las cuatro configuraciones modeladas del sistema de refrigeración, bajo el ciclo de conducción NEDC.

\subsection{Validación experimental de las modificaciones propuestas y valoración de su influencia sobre las emisiones y el consumo de combustible}

Para validar los resultados de la simulación se emplearon las mismas instalaciones utilizadas durante las pruebas de caracterización del motor. Se instalaron dos válvulas mecánicas: una de $40 \mathrm{~mm}$ de diámetro de paso instalada entre la caja de agua y la entrada al motor, como se muestra en la Figura 5.12 y una segunda válvula de $6 \mathrm{~mm}$ de diámetro instalada entre la caja de agua y el tanque de expansión. 


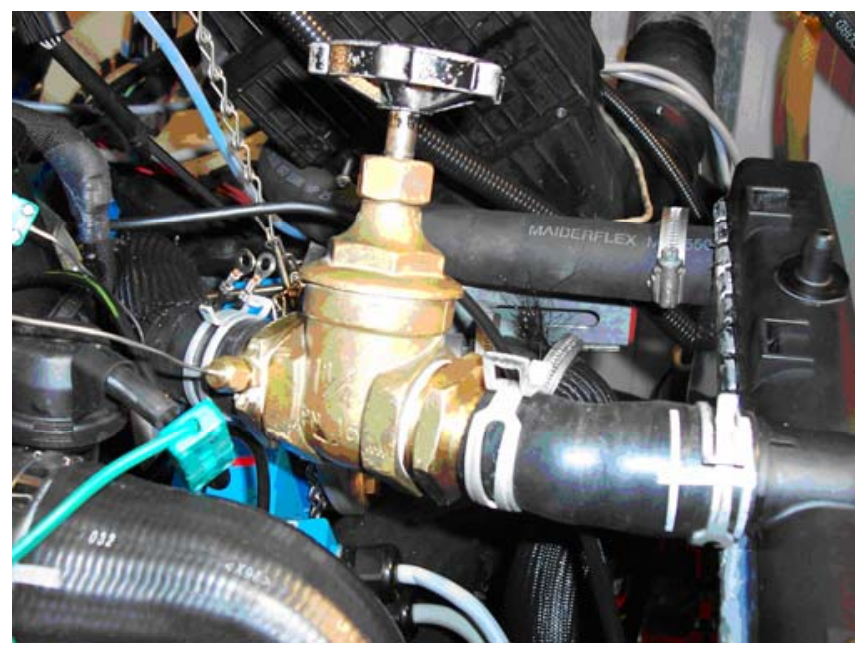

Figura 5.12. Instalación de la válvula mecánica en el ramal de bypass del sistema de refrigeración.

Para medir el posible impacto en el consumo de combustible y en las emisiones, asociado con las modificaciones del circuito, se instaló instrumentación adicional, constituida por un medidor gravimétrico de flujo de combustible y un colector integral de emisiones. Las mediciones de emisiones incluyeron el monoxido de carbono, (CO), los hidrocarburos no quemados, (HC), el material particulado (PM) y los óxidos de nitrógeno $\left(\mathrm{NO}_{\mathrm{x}}\right)$, tomados antes del catalizador.

Los valores de los contaminantes en $\mathrm{l} / \mathrm{km}$ se obtuvieron mediante un método estándar de postratamiento European Directive ECE-R-83/02.. Luego se extrajeron los valores acumulados en cada prueba y con cada configuración para los cuatro intervalos ECE y el intervalo EUDC. Finalmente, con estos valores se calcularon un valor medio y un intervalo de confianza para cada configuración.

\subsubsection{Flujos de refrigerante experimentales de todas las configuraciones}

La evolución obtenida de los flujos de refrigerante durante el ciclo NEDC para todas las configuraciones se ilustra en la Figura 5.13. Las relaciones de los flujos entre las configuraciones guardan correspondencia con las relaciones entre los flujo modelados bajo carga constante. Como se derivó de los resultados del modelo, los menores flujos de refrigerante corresponden a la configuración M2. 

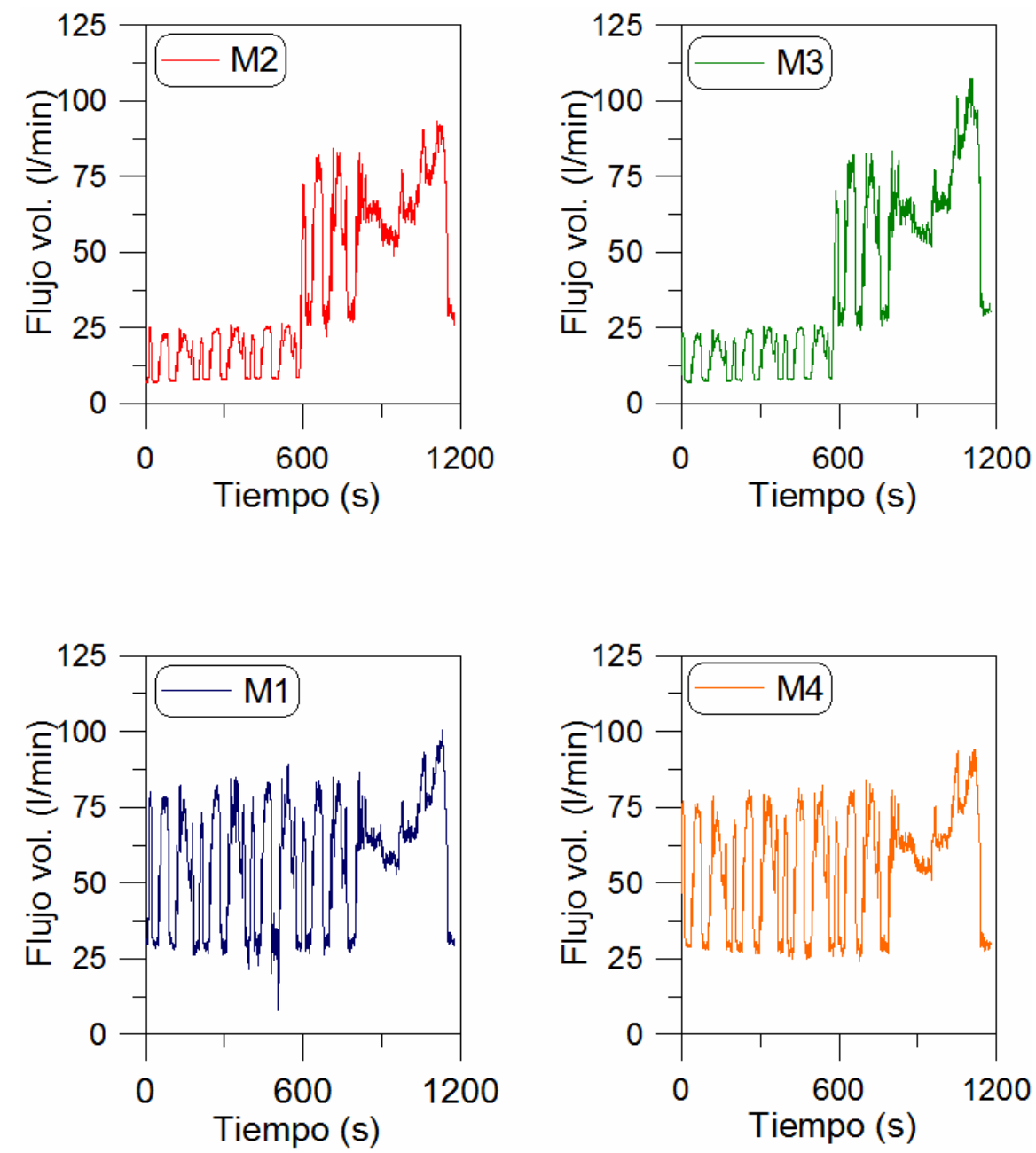

Figura 5.13. Evolución de los flujos de refrigerante durante el ciclo NEDC para todas las configuraciones.

\subsubsection{Respuesta de temperatura del refrigerante}

La Figura 5.14 muestra los perfiles de la temperatura del refrigerante que resultan de las pruebas experimentales con las cuatro configuraciones del sistema conduciendo el motor bajo el ciclo de operación NEDC. Como se observa en esta figura la temperatura experimental del refrigerante se comporta de una manera satisfactoria en relación con los modelos correspondientes. 


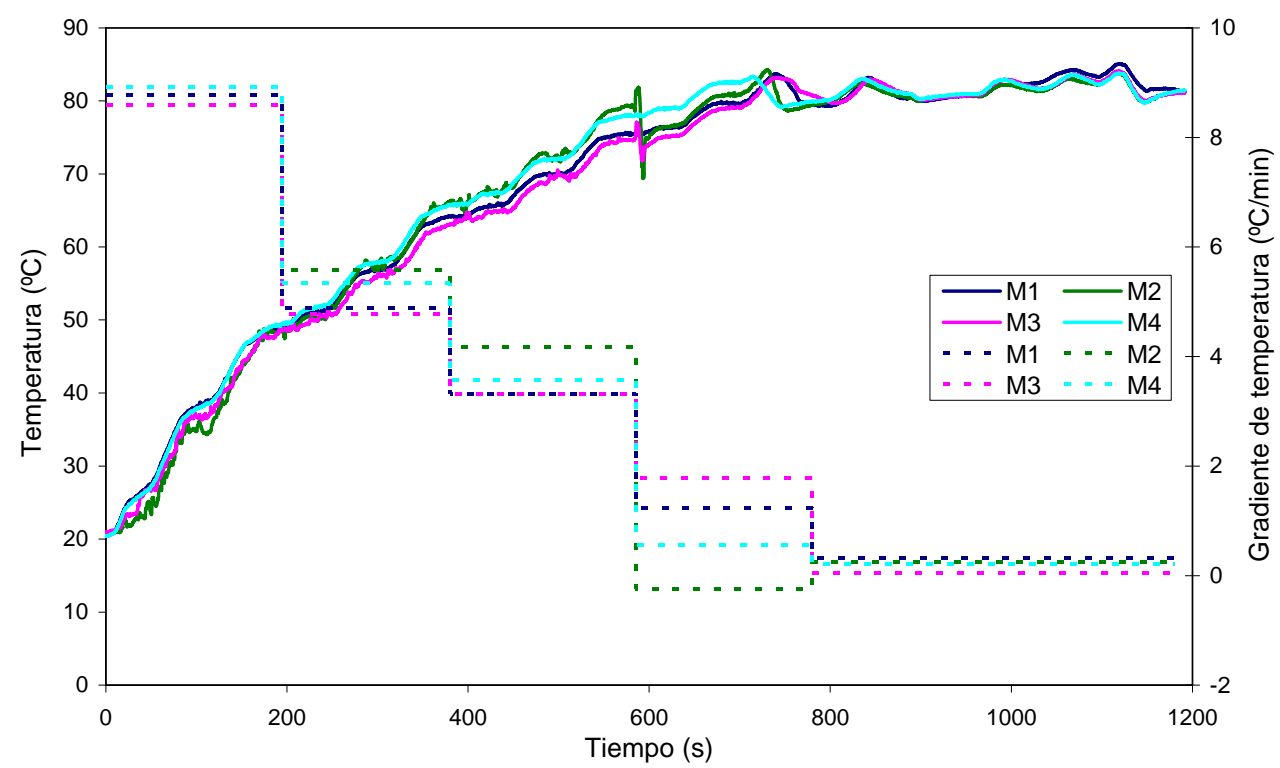

Figura 5.14. Temperaturas medidas del refrigerante durante la operación del motor bajo las cuatro configuraciones del sistema de refrigeración.

En la Figura 5.14, en líneas de trazos se presentan los gradientes de la temperatura del refrigerante para cada uno de los intervalos que conforman el ciclo. Al final del primer intervalo ECE, el gradiente de temperatura fue casi el mismo para las cuatro configuraciones. El gradiente global de temperatura se redujo para todas las configuraciones durante el segundo intervalo ECE, presentándose el mayor gradiente para la configuración M2, debido al menor flujo de refrigerante circulando por el motor y al volumen menor de refrigerante en el circuito. La influencia del volumen de refrigerante es significativa en la respuesta de la temperatura del sistema de refrigeración durante el último intervalo ECE del ciclo de conducción.

El menor tiempo para alcanzar los $80{ }^{\circ} \mathrm{C}$ correspondió a la configuración M2. La Figura 5.15 muestra los perfiles de respuesta experimental y modelada de la temperatura del refrigerante correspondientes a la configuración M2. Cualitativa y cuantitativamente las respuestas experimental y predicha fueron muy cercanas, hasta el momento en que el termostato se abrió. 


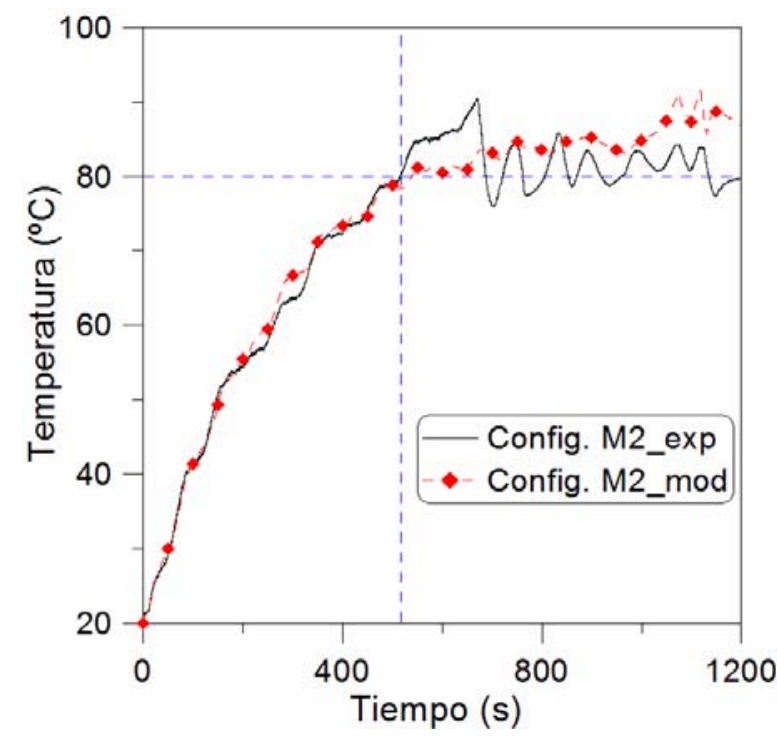

Figura 5.15. Perfiles modelado y experimental de la respuesta de la temperatura del refrigerante bajo la configuración M2, bajo el ciclo de conducción NEDC.

La tabla 5.7 resume los resultados de la comparación de las respuestas de las cuatro configuraciones, sobre la base del tiempo requerido por el refrigerante para alcanzar los $80^{\circ} \mathrm{C}$.

Tabla 5.7. Tiempo requerido para que el refrigerante alcance los $80^{\circ} \mathrm{C}$ en las configuraciones alternativas y su comparación con el tiempo del sistema original M1.

\begin{tabular}{cccc}
\hline Configuración & $\begin{array}{c}\text { Experimental, (s) } \\
\pm \text { CI 0.95(s) }\end{array}$ & Con respecto a M1 & Modelada \\
\hline M1 & $713,4 \pm 9,18$ & - & 721 \\
M2 & $554,5 \pm 9,03$ & $\mathbf{- 2 2 , 3} \%$ & 539 \\
M3 & $716,0 \pm 5,23$ & $\mathbf{0 , 4} \mathbf{\%} \sim \mathbf{0} \%$ & 730 \\
M4 & $641,8 \pm 5,41$ & $\mathbf{- 1 0 , 0} \%$ & 655 \\
\hline
\end{tabular}

M2 es la más rápida en el calentamiento; M4 es más rápida que M3. El efecto del volumen total de refrigerante (menor en M4) es más influyente que el del flujo de refrigerante (menor en M3). No hay mayores diferencias entre las configuraciones M1 y M3. El efecto del volumen total de refrigerante es muy importante. 


\subsubsection{Emisiones y consumo de combustible}

En las Figuras 5.16 - 5.21 se presentan representaciones en diagramas de barras de los resultados experimentales obtenidos para diferentes configuraciones del sistema de refrigeración en relación con las emisiones contaminantes y el consumo de combustible con discriminación para cada intervalo ECE y para el ciclo completo. Los resultados se presentan de una manera relativa tomando como base la configuración original M1 del motor. Para referencia, con líneas de trazos se representan las dispersiones correspondientes a la configuración M1 ( $\sigma /$ mean ).

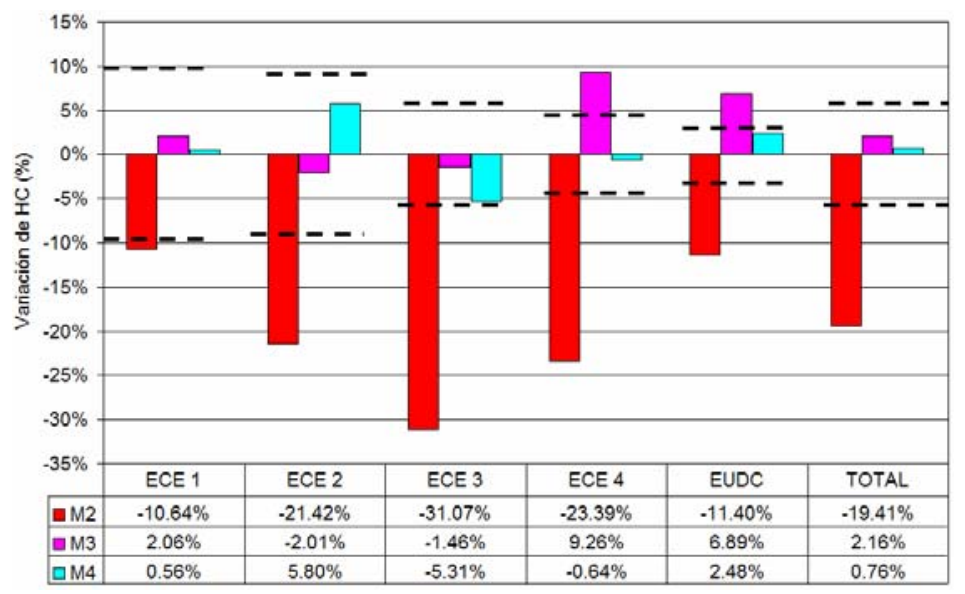

Figura 5.16. Emisiones de HC (g/km), comparación con M1.

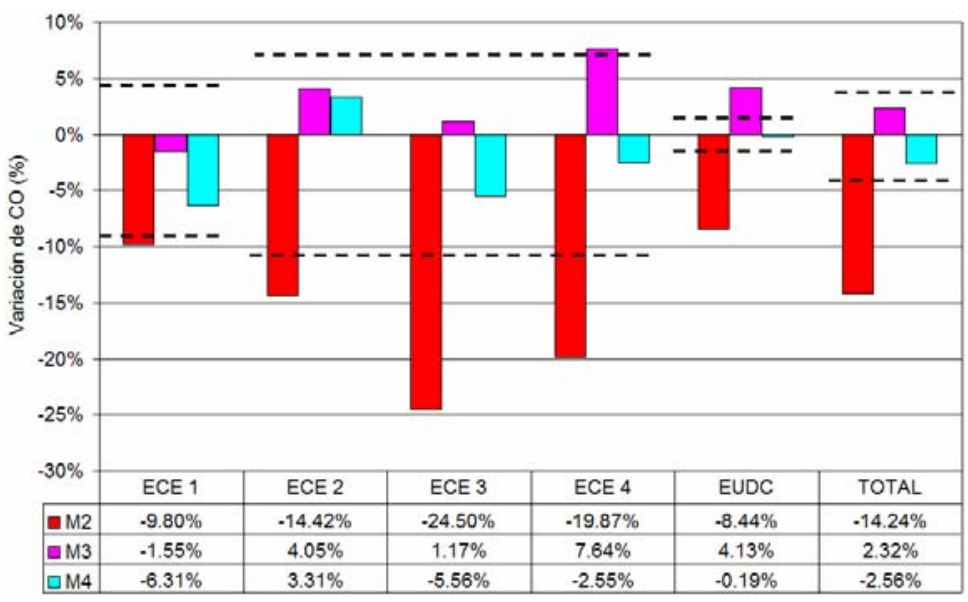

Figura 5.17. Emisiones de CO ( $\mathrm{g} / \mathrm{km})$, comparación con M1. 


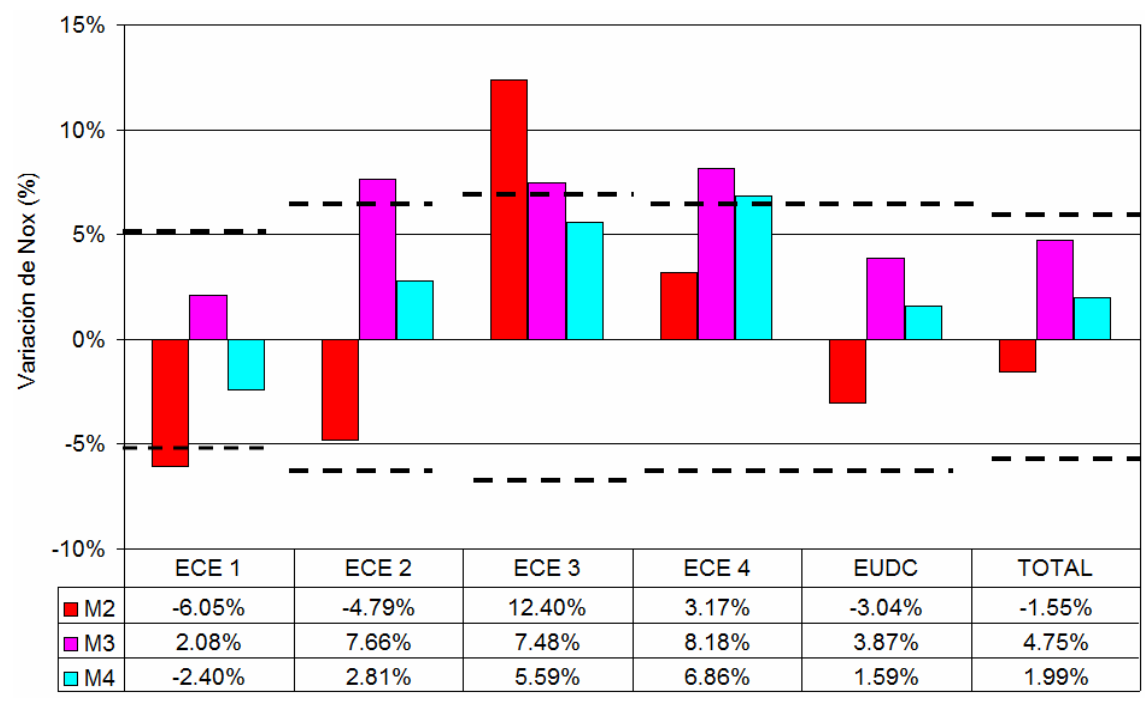

Figura 5.18. Emisiones de NOx ( $\mathrm{g} / \mathrm{km})$, comparación con M1.

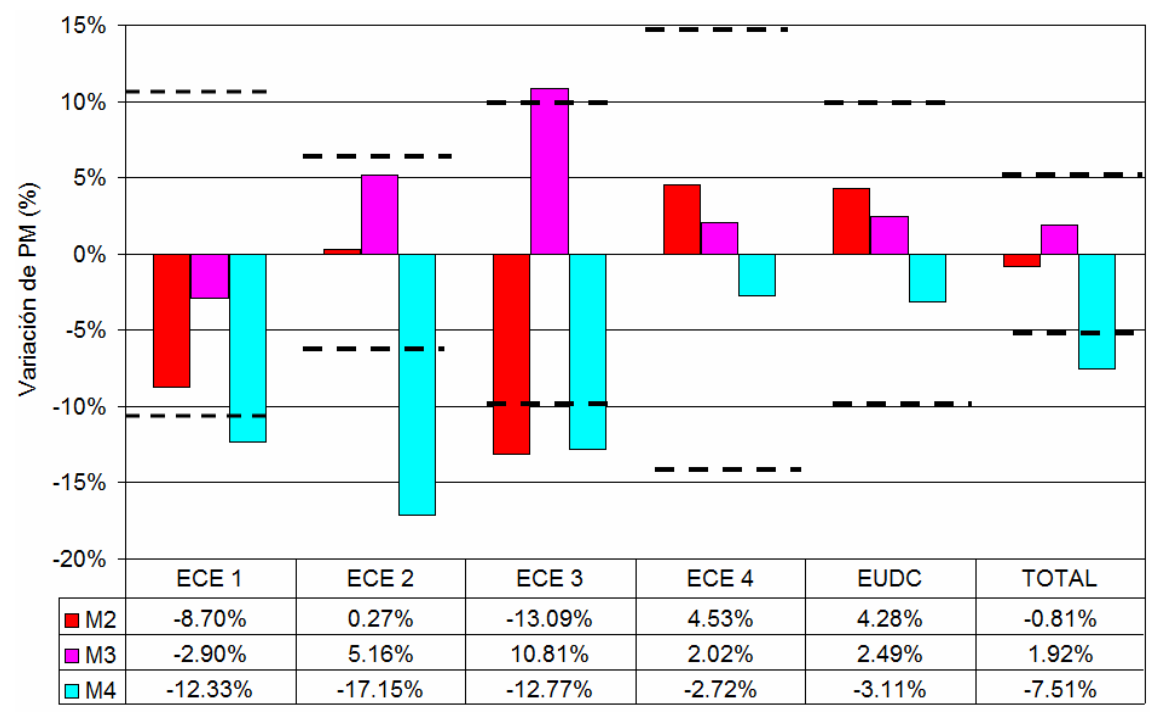

Figura 5.19. Emisiones de PM ( $\mathrm{g} / \mathrm{km})$, comparación con M1. 


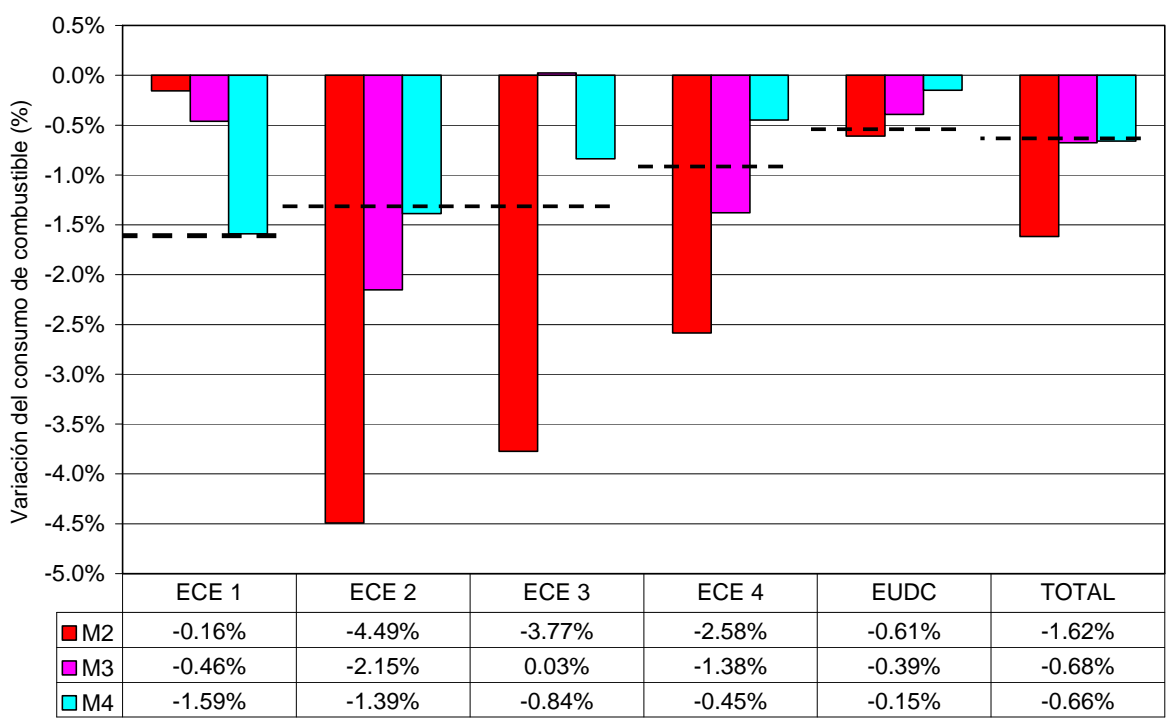

Figura 5.20. Consumo de combustible (l/100km), comparación con M1.

No se apreciaron efectos claros de las configuraciones M3 y M4 sobre las emisiones ni sobre el consumo de combustible. Con relación al esquema original M1, la configuración M2 exhibió una reducción significativa del consumo de combustible y de las emisiones de HC y CO del $2 \%$, 20 \% y 15 $\%$, respectivamente. Con relación a las emisiones de PM y NOx, ninguna de las configuraciones alternativas permitió mejorar de manera apreciable los valores correspondientes a la configuración original del sistema de refrigeración (M1).

Tabla 5.8. Resultados acumulados al finalizar los primeros tres intervalos ECE. Comparación porcentual en relación con M1.

\begin{tabular}{|c|ccccc|}
\hline Configuración & NOx & CO & HC & PM & $\begin{array}{c}\text { Consumo de } \\
\text { combustible }\end{array}$ \\
\hline M2 & $-1,28$ & $\mathbf{- 6 , 5 6}$ & $\mathbf{- 1 1 , 4 1}$ & $\mathbf{1 1 , 0 9}$ & $-\mathbf{2 , 9 1}$ \\
M3 & 4,97 & $\mathbf{0 , 8 1}$ & $-0,56$ & $\mathbf{1 , 2 1}$ & $-0,83$ \\
M4 & 1,08 & $-3,37$ & $\mathbf{0 , 4 4}$ & $\mathbf{- 1 3 , 4 1}$ & $\mathbf{- 1 , 3 1}$ \\
M1 & $\pm 5,85$ & $\pm 4,34$ & $\pm 6,68$ & $\pm 5,63$ & $\pm 1,07$ \\
\hline
\end{tabular}


-Los valores de la fila M1 indican la dispersión en los ensayos M1: $\sigma$ / mean .

En la tabla 5.8 se presenta una comparación del consumo de combustible y las emisiones del motor al final del tercer intervalo ECE del ciclo de conducción, en relación con los valores producidos con la configuración original M1.

\subsection{Conclusiones}

En este capítulo se ha presentado el estudio del calentamiento de un motor HSDI, cuyo balance térmico y características termohidráulicas fueron determinados en el capítulo tercero. Inicialmente se ha modelado el sistema de refrigeración con su configuración original y se ha simulado su respuesta bajo una carga y velocidad constantes del motor, con lo que se ha podido acercar el comportamiento hidráulico y térmico al del sistema real. Se ha explicado después cómo el sistema original puede modificarse de manera sencilla para que el motor caliente más rápido, demostrando con la simulación y luego con la experimentación esas posibilidades de mejora.

El modelado y la simulación se han realizado con la ayuda del programa computacional Amesim, los fundamentos de cuyos submodelos fueron brevemente descritos en el capítulo cuarto. En los modelos se han probado cuatro configuraciones considerando sólo aperturas y cierres totales de las válvulas, pero está claro que con, los modelos, las válvulas bien pueden modelarse para que sus secciones de paso se puedan variar de manera continua.

Con ayuda del modelo desarrollado se estudiaron las respuestas térmicas de las configuraciones propuestas para reducir el tiempo de calentamiento. Sin afectar el diseño original del sistema de refrigeración, sólo incorporando en el circuito original dos válvulas de bajo costo, sin necesidad de estrategias especiales y costosas de control, se ha concluido a partir de los resultados del modelo la posibilidad de reducir el tiempo de calentamiento del motor tanto bajo condiciones constantes de operación, como durante la conducción bajo un ciclo de conducción NEDC. Las modificaciones propuestas se implementaron físicamente en el motor real y se aprovechó para evaluar las 
mejoras o los cambios causados en el consumo de combustible y en las emisiones.

En general las predicciones del modelo fueron muy acordes con los resultados experimentales tanto en su evolución a lo largo del tiempo como en magnitud (con un error del $4 \%$ ), queriendo esto decir que el modelo es apropiado para estudiar el desempeño térmico del motor. Sí se presentan discrepancias luego que el motor ha calentado y que el termostato se ha abierto. Esta parte del ciclo, tras la apertura del termostato queda para ser ajustada en trabajos futuros y demanda de la caracterización experimental y con cálculos CFD del lado del aire.

El error durante el primer intervalo ECE puede deberse a inexactitudes en la estimación de la transferencia de calor a las paredes y también a la simplificación del modelo, que es un modelo 1D con sólo una masa de motor intercambiando calor con el refrigerante, el modelo es sencillo. También se puede mejorar el modelado de la transferencia de calor entre el aceite y las partes metálicas del motor.

Con relación a la influencia de la arquitectura del sistema de refrigeración sobre el desempeño del motor, se ha concluido que son muchas las mejoras que pueden lograrse si se implementan sistemas de refrigeración suficientemente flexibles para controlar el volumen y los gastos del refrigerante a través de los ramales del circuito de refrigeración como una estrategia para acelerar el calentamiento del motor, con lo que se reduce el consumo de combustible y se reducen las emisiones contaminantes.

Para el motor modelado se demostró que modificando ligeramente el sistema de refrigeración de una configuración original a una configuración M2 se logra una reducción significativa del tiempo de calentamiento del 22,3 \% en el tiempo necesario por el motor para alcanzar la temperatura objetivo de 80 ${ }^{\circ} \mathrm{C}$ a la salida del bloque del motor. La reducción mencionada en el tiempo de calentamiento de 713,4 \pm 9.18 segundos a 554,5 \pm 9.03 segundos se traduce en una reducción del consumo total de combustible del 1,62\%, y en reducciones de 1,55 \%, 14,24\%, 19,41 \% y 0,81 \%, en las emisiones de NOx, CO, HC y PM, respectivamente, al final del ciclo de conducción NEDC. 
Por último, se puede comentar que la creación de modelos y su simulación con programas computacionales permiten una reducción substancial en el número requerido de ensayos de motor. Esto es importante por razones económicas y también porque en las pruebas de homologación bajo el nuevo ciclo de conducción europeo (NEDC) el tiempo requerido para empezar una nueva prueba, esperando a que las temperaturas del material se estabilicen totalmente a $20^{\circ} \mathrm{C}$, es de cerca de siete horas.

\subsection{Bibliografía}

[5.1] Broatch, A., Luján, J. M., Ruiz, S., Olmeda, P. Measurement of hydrocarbon and carbon monoxide emissions during the starting of automotive DI Diesel engines. Int. J. Technology, vol.9, $\mathrm{n}^{\circ} 2$, 129140, 2008.

[5.2] Chen, C., Veshagh, A. A one-dimensional model for in-cylinder heat convection based on the boundary layer theory. SAE paper 921733.

[5.3] Mohan, K. V, Arici, O., Yang, S., Johnson, J. H. A computer simulation of the turbocharged Diesel engine as an enhancement of the vehicle engine cooling system simulation. SAE paper 971804.

[5.4] Campbell, N. A. F., Hawley, J. G., MacGregor, S. A. Incorporating nucleate boiling in a precision cooling strategy for combustion engines. SAE paper 971791.

[5.5] Campbell, N. A. F., Hawley, J. G., Robinson, K., Leathard, M. J. A review of predictive analysis applied to IC engine coolant heat transfer. J. Inst. Energy, vol. 73, pp. 78-86, 2000.

[5.6] Allen, D. J., Lasecki, M. P. Thermal management evolution and controlled coolant flow. SAE paper 2001-01-1732.

[5.7] Pang, H. H., Brace, C. J. Review of engine cooling technologies for modern engines. Proc. Instn. Mech. Engrs., part D: J. Automobile Engineering, vol. 11, pp.1209-1215, 2004.

[5.8] Cortona, E., Onder, C. H. Engine thermal management with electronic cooling pump. SAE paper 2000-01-0965.

[5.9] Cho, H., Jung, D., Assanis, D. N. Control strategy of electric coolant pumps for fuel economy improvement. Int. J. Automotive 
Technology, vol.6, $\mathrm{n}^{\circ}$ 3, pp. 269-275, 2005.

[5.10] Chanfreau, M., Gessier, B., Farkh, A., Geels, P.Y. The need for an electrical water valve in a thermal management intelligent system THEMISTM. SAE paper 2003-01-0274.

[5.11] Chalgren, R. D. Jr., Barron, L. Jr. Light duty Diesel advanced thermal management. SAE paper 2005-01-2020.

[5.12] Robinson, K., Campbell, N. A. F., Hawley, J. G., Tilley, D. G. A review of precision engine cooling. SAE paper 1999-01-0578.

[5.13] Robinson, K., Hawley, J. G., Hammond, G. P., Owen, N. J. Convective coolant heat transfer in internal combustion engines. Proc. Instn. Mech. Engrs., part D: J. Automobile Engineering, vol. 217, pp. 133-146, 2003.

[5.14] Porot, P. A., Ménégazzi, P., Ap, N. S. Understanding and improving evaporative engine cooling at high load, high speed by engine tests and 3D calculations. SAE paper 971792.

[5.15] Jaichandar, S., Tamilporai, P. Low heat rejection engines - An overview. SAE paper 2003-01-0405.

[5.16] Torregrosa, A. J., Olmeda, P., Martín, J., Degraeuwe, B. Experiments on the influence of inlet charge and coolant temperature on performance and emissions of a DI Diesel engine. Exp. Therm. Fluid Sci., vol. 30, issue 7, pp. 633-641, 2006.

[5.17] Ladommatos, N., Xiao, Z., Zhao, H. The effect of piston bowl temperature on diesel exhaust emissions. Proc. Instn. Mech. Engrs., part D: J. Automobile Engineering, vol. 219, pp. 371-388, 2005.

[5.18] Strupp, N., Lemke, N., Tegethoff, W., Köhler, J. MAC simulation using Modelica/Dymola. 7th IIR Gustav Lorentzen Conf. Natural Working Fluids, Trondheim, Norway, 2006.

[5.19] GT-Power User’s manual (GT-Cool). Versión 5.2. Diciembre 2001.

[5.20] FlowMaster user’s manual. Versión 7.2.

[5.21] AMESim user’s manual. Versión 7.1. Septiembre 2008.

[5.22] Sjöstedt, C. J. On the modular modelling for dynamical simulation with application to fluid systems. Licentiate thesis, Stockholm, 2005.

[5.23] Modelica homepage, www.modelica.org (consultada en agosto de 2008). 
[5.24] Kaplan, J. A., Heywood, J. B. Modelling the spark ignition engine warm-up process to predict component temperatures and hydrocarbon emissions. SAE paper 910302.

[5.25] Jarrier, L. Champoussin, J. C., Yu, R., Gentile, D. Warm-up of a D. I. Diesel engine: experiment and modeling. SAE paper 2000-010299.

[5.26] Torregrosa, A. J., Olmeda, P., Degraeuwe, B., Reyes, M. A concise wall temperature model for DI diesel engines. Appl. Therm. Eng., vol. 26, pp. 1320-1327, 2006.

[5.27] Lehner, C., Parker, G., Arici, O. Johnson, J. Design and development of a model based feedback controlled cooling system for heavy duty diesel truck applications using a vehicle engine cooling system simulation. SAE paper 2001-01-0336.

[5.28] Incropera, F. P., DeWitt, D. P. Introduction to heat transfer, John Wiley, New York, 1996.

[5.29] The Mathworks homepage, www.themathworks.com.

[5.30] Watanabe, N., Kubo, M., Yomoda, N. An 1D-3D integrating numerical simulation for engine cooling problem. SAE paper 200601-1603.

[5.31] Plazas, A. T., Modelado unidimensional de inyectores common rail Diesel. Tesis doctoral, Universidad Politécnica de Valencia, 2005.

[5.32] Kays, W. M., London, A. L. Compact Heat Exchangers. McGraw Hill, 1984.

[5.33] Torregrosa, A. J., Olmeda, P., Broatch, A., Romero, C. Assessment of the influence of different cooling system configurations on engine warm-up, emissions and fuel consumption. Int. J. Automotive Technology, vol. 9, Nº 4, pp. 447-458, 2008.

[5.34] European Directive ECE-R-83/02. 


\section{Capítulo 6}

\section{Diseño, modelado y simulación del sistema de refrigeración del motor de una locomotora}

Resumen

6.1. Introducción

6.2. Definición de las especificaciones del motor y diseño preliminar del sistema de refrigeración.

6.2.1. Especificaciones de diseño del sistema de refrigeración (tarea técnica).....

6.2.2. Cálculo preliminar de los componentes del sistema de refrigeración

6.2.3. Disposición de los componentes del sistema hidráulico y del sistema de ventilación

6.3. Modelado y simulación del sistema de refrigeración

6.3.1. Modelado y caracterización del sistema de ventilación 269

6.3.2. Caracterización de los componentes de los circuitos del refrigerante

6.3.3. Modelado en FlowMaster del sistema de refrigeración del motor de la locomotora 280

6.3.4. Alcance de resultados de los modelos. 287 
6.4. Validación experimental del sistema de refrigeración del motor de

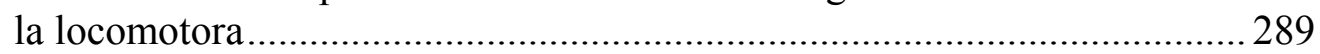

6.4.1. Instrumentación del sistema de refrigeración............................... 290

6.4.2. Resultados de las mediciones en el sistema de refrigeración operando el motor a plena carga ............................................................ 294

6.4.3. Resultados de las mediciones en el sistema de refrigeración operando el motor a plena carga ........................................................... 297

6.4.4. Comparación y análisis de las mediciones en el sistema de refrigeración operando el motor a plena carga con los resultados de la simulación 1D

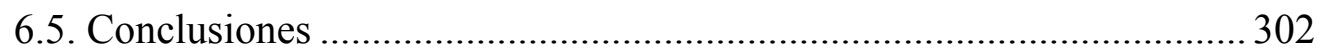

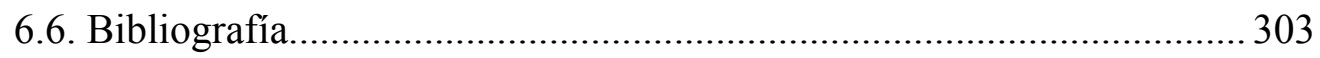




\section{Resumen}

En los capítulos anteriores se ha estudiado la transferencia de calor en los motores de automóviles y se ha hecho uso de la información experimental de operación del motor para predecir las temperaturas y los flujos de calor en la estructura del motor y en su circuito de refrigeración, empleando un modelo de predicción de temperaturas y luego un programa de modelado 1D. La parte conclusiva de esta tesis tiene que ver con el diseño asistido por herramientas de modelado y simulación de los sistemas de refrigeración de motores Diesel. A partir de las especificaciones del motor que propulsa una locomotora, la característica de la bomba y el punto de operación de ésta, se estima el calor que debe evacuarse, se seleccionan el ventilador y el radiador adecuados a esa capacidad térmica demandada y ajustados a las restricciones de espacio y ubicación dadas por la aplicación particular, se modela el sistema así ensamblado y se asegura el cumplimiento de las temperaturas del refrigerante y del aire para las condiciones de plena carga del motor. Después de esto, se ensambla el sistema de refrigeración real, se realizan las mediciones y se comparan los resultados experimentales con los reales. El sistema de refrigeración diseñado cumple con las especificaciones requeridas y la metodología de diseño se prueba como contrapropuesta a la práctica de ensamble de componentes basada totalmente en el ensayo, dejando en evidencia las ventajas del diseño integrado de las partes del refrigerante y del aire asistido por las herramientas computacionales.

\subsection{Introducción}

Actualmente sólo la industria automotriz empieza a introducir metodologías automatizadas para el diseño de sus sistemas refrigeración [6.1]-[6.5], siendo el diseño en aplicaciones para camiones y otros sistemas de transporte pesado una actividad aún no integrada o cerrada bajo una metodología única. Por otro, lado la tendencia en el diseño de los motores de combustión alternativos va haciendo que la casa matriz fabricante disponga la misma base estructural compuesta por bloque, cárter, culata(s), mecanismos manivela-deslizador y de distribución de gases y sistemas de alimentación de aire y combustible para un diverso mundo de aplicaciones y que sólo los sistemas de control, incluyendo el control de la temperatura del motor, dependan de la aplicación 
específica y sean diseñados por el usuario final, el ensamblador final de la aplicación.

Los objetivos de la industria del transporte mediante locomotoras son consonantes con los propios de los sistemas de transporte automotor en general: mejorar la economía de combustible y reducir las emisiones. Para cumplir con estos objetivos, la industria de las locomotoras ha enfocado sus investigaciones y programas de desarrollo a la creciente transferencia hacia la electrificación de la tracción, la utilización de motores múltiples en lugar de un solo motor, el estudio de unidades de potencia híbridas, el perfeccionamiento de los motores Diesel y las tecnologías de combustible, con una cada vez mayor preocupación por el control de emisiones, investigando la incorporación de catalizadores y sistemas de recirculación de gases de escape [6.2]. También ve oportunidades para mejorar la gestión térmica de los motores y del sistema de potencia en general, que directa o indirectamente conducen a la mejora del rendimiento del motor y a la reducción de las emisiones. No obstante esto, el desarrollo de los sistemas de gestión térmica en las locomotoras no va al compás del desarrollo de los motores dado que cada aplicación es individual, lo que hace difícil la implementación económica de programas de diseño generalizados; no es económicamente viable trabajar en la optimización de los sistemas de gestión térmica de las locomotoras cuando éstos son muy individualizados.

En las locomotoras modernas la potencia del motor, el sistema de refrigeración del aire fresco de admisión tras las etapas de compresión en los sobrealimentadores y su sistema de control de gases determinan la arquitectura de la parte hidráulica del sistema de refrigeración (topología del sistema de refrigeración con diferentes diseños para el enfriamiento de los gases de escape que se recirculan y el aire de admisión), mientras que el uso final de la locomotora determina en general la arquitectura de la parte de aire del sistema de refrigeración (sistema de ventilación). En la Figura 6.1 se muestran los principales esquemas de ventilación empleados en las locomotoras.

Lo dicho anteriormente sirve para situar el diseño de los sistemas de gestión térmica de las locomotoras. En este marco conceptual se inscribe el diseño o rediseño de los sistemas de refrigeración: dadas las características técnicas de un motor y su(s) bomba(s) de refrigeración, conocido su pasaporte funcional 
para los diferentes puntos de operación, se requiere diseñar y alojar un sistema de refrigeración compuesto por dos partes acopladas, una de circulación de refrigerante y otra de circulación de aire, de suerte que se observen las temperaturas del refrigerante a su paso por la estructura del motor y se cumpla con las exigencias de temperatura de otros fluidos que participan en la gestión térmica como el aire de admisión, el aceite del motor y de otros grupos hidráulicos, los gases de escape recirculados, etc.

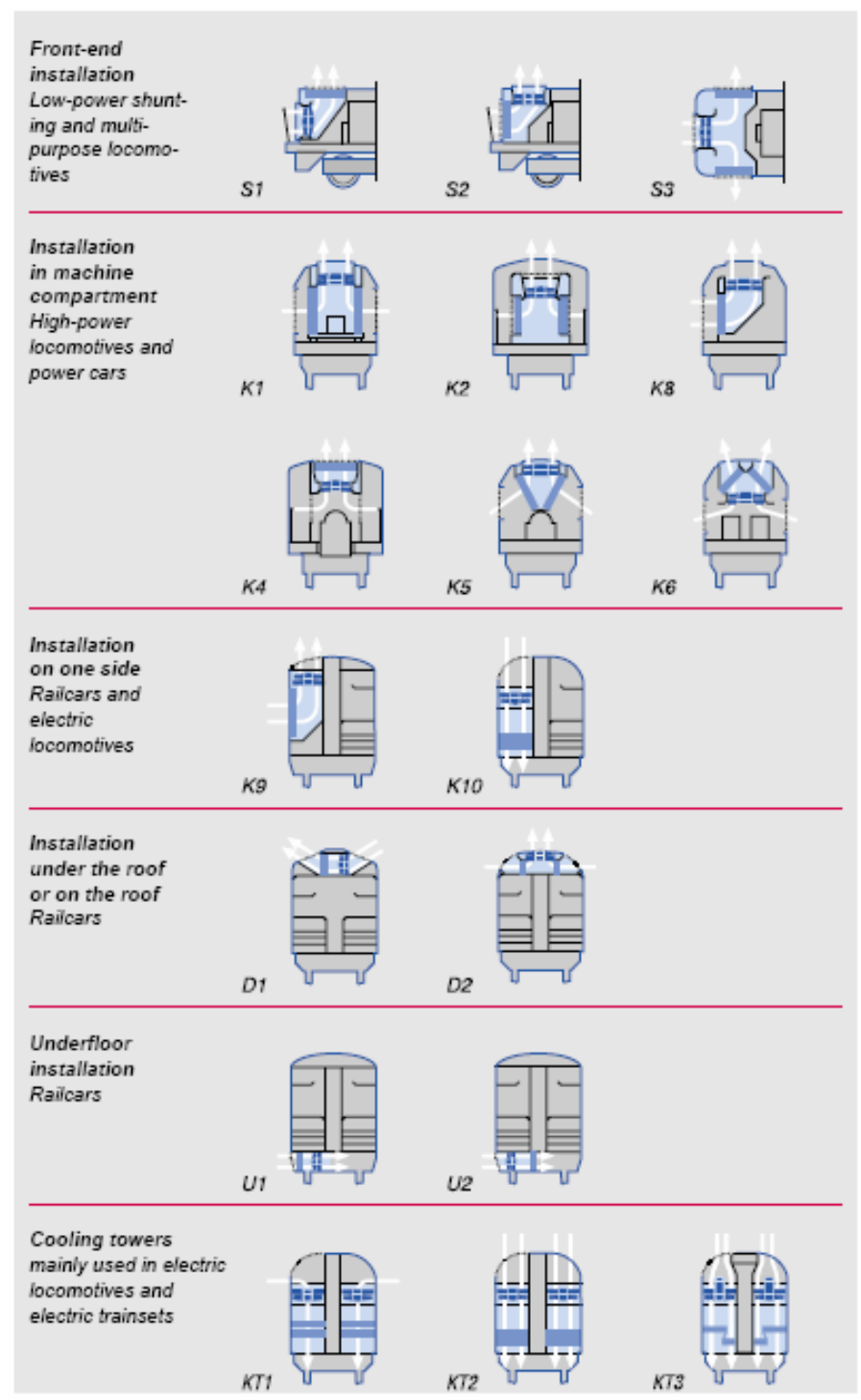

Figura 6.1.

Esquemas de ventilación empleados en los sistemas de refrigeración de las locomotoras [6.7]. 
En los siguientes parágrafos, inicialmente se formula el problema de diseño y se acotan las particularidades propias del sistema de refrigeración de la locomotora en estudio, con lo que se realiza el prediseño de los radiadores y el ventilador. Allí mismo se describe el proceso de selección de los ventiladores y se hace uso del programa de cálculo de radiadores, descrito en el capítulo cuarto, para seleccionar los radiadores del sistema. En el siguiente apartado se describe como, con la información obtenida y las ubicaciones relativas de los componentes se levantan los planos de los circuitos de refrigeración y se modelan los sistemas de ventilación para caracterizar las pérdidas de presión del lado del aire y proceder al modelado del sistema completo con el programa de modelado 1D. La tercera parte del capítulo se dedica a la descripción de los ensayos experimentales sobre el sistema ensamblado y operando a plena carga y se comparan los resultados obtenidos con los dados por el modelo. Se termina el capítulo con las conclusiones relativas a las ventajas del diseño asistido de los sistemas de refrigeración y los alcances de los resultados y la adecuabilidad de los modelos y su utilización para la simulación de cualesquiera condiciones de operación.

\subsection{Definición de las especificaciones del motor y diseño preliminar del sistema de refrigeración}

\subsubsection{Especificaciones de diseño del sistema de refrigeración (tarea técnica)}

Las especificaciones del motor cuyo sistema de refrigeración es objeto de diseño son las referenciadas en la Tabla 6.1. Se trata de un motor Diesel sobrealimentado con enfriador del aire de admisión, de $3170 \mathrm{~kW}$ de potencia efectiva a $900 \mathrm{rpm}, 15,5{ }^{\circ} \mathrm{C}, 97,73 \mathrm{kPa}$, con circuitos separados de refrigeración para el bloque del motor (en el que originalmente se incluye el enfriador del aceite y el compresor) y para el refrigerante del aire de admisión ("aftercooler").

Las características de las bombas del circuito del motor y del enfriador de aire son las ilustradas en la Figura 6.2 y la Figura 6.3, respectivamente. 
Tabla 6.1. Características técnicas iniciales del motor.

\begin{tabular}{ll}
\hline Motor & \\
\hline Tipo & Diesel 4T, 16 cilindros en V \\
Potencia, kW & 3170 a $900 \mathrm{rpm}$ \\
Revoluciones máximas, rpm & 950 \\
Diámetro/carrera del pistón, mm & $230,19 / 279,4$ \\
$\begin{array}{l}\text { Flujo estimado de refrigerante por el bloque } \\
\text { del motor (JW), 1/s }\end{array}$ & 63 \\
$\begin{array}{l}\text { Flujo estimado de refrigerante por el } \\
\text { enfriador del aire de admisión (AC), 1/s }\end{array}$ & 25,23 \\
Características del fluido & $50 / 50$ (agua/etilenglicol) \\
\hline
\end{tabular}

\section{Espacio disponible para radiadores}

Área disponible en cada costado del vagón, $\quad F_{f r_{\text {disp }}}=3,08 \mathrm{~m}^{2} .(2,8 \times 1,1$

$\mathrm{m}^{2}$ (L X B) $\mathrm{m}$ )

Disposición Vertical

Temperatura máxima del aire ambiente, ${ }^{\circ} \mathrm{C} \quad 35$

Temperatura de diseño de entrada del 85

refrigerante a los radiadores, ${ }^{\circ} \mathrm{C}$

\section{Enfriador de aceite}

Configuración

Disipación estimada de calor, $\mathrm{kW}$

Carcasa y tubos

Flujo estimado de refrigerante, $1 / \mathrm{s}$

439,21

57,72

\section{Tanque de expansión}

Capacidad, 1

Flujo estimado de refrigerante, $1 / \mathrm{s}$
150

57,72

\section{Compresor}

Disipación estimada de calor, $\mathrm{kW}$ 


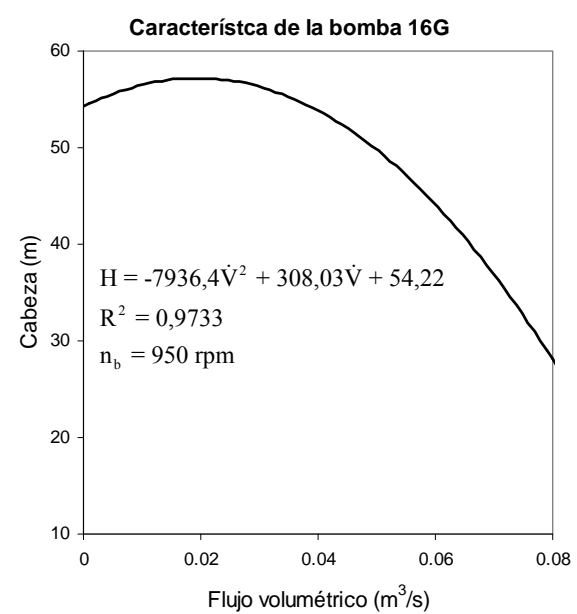

Figura 6.2. Característica de la bomba del circuito de refrigeración del motor.

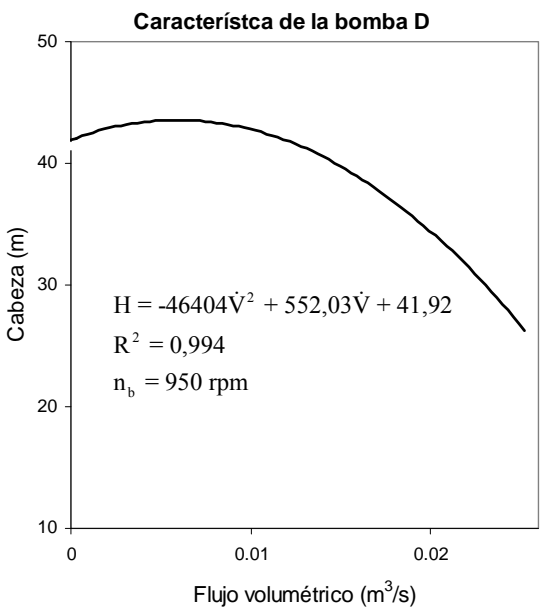

Figura 6.3. Característica de la bomba del circuito de refrigeración del enfriador de aire.

El proveedor del motor sugiere realizar la configuración del sistema de refrigeración según el plano de la Figura 6.4. Respetando la posición relativa de los componentes, el trazado del circuito queda definido, y en términos hidráulicos la atención se centra en limitar las pérdidas de presión que permitan que las bombas suministren el caudal correspondiente al punto de operación recomendado especificado en la tabla 6.1 . 


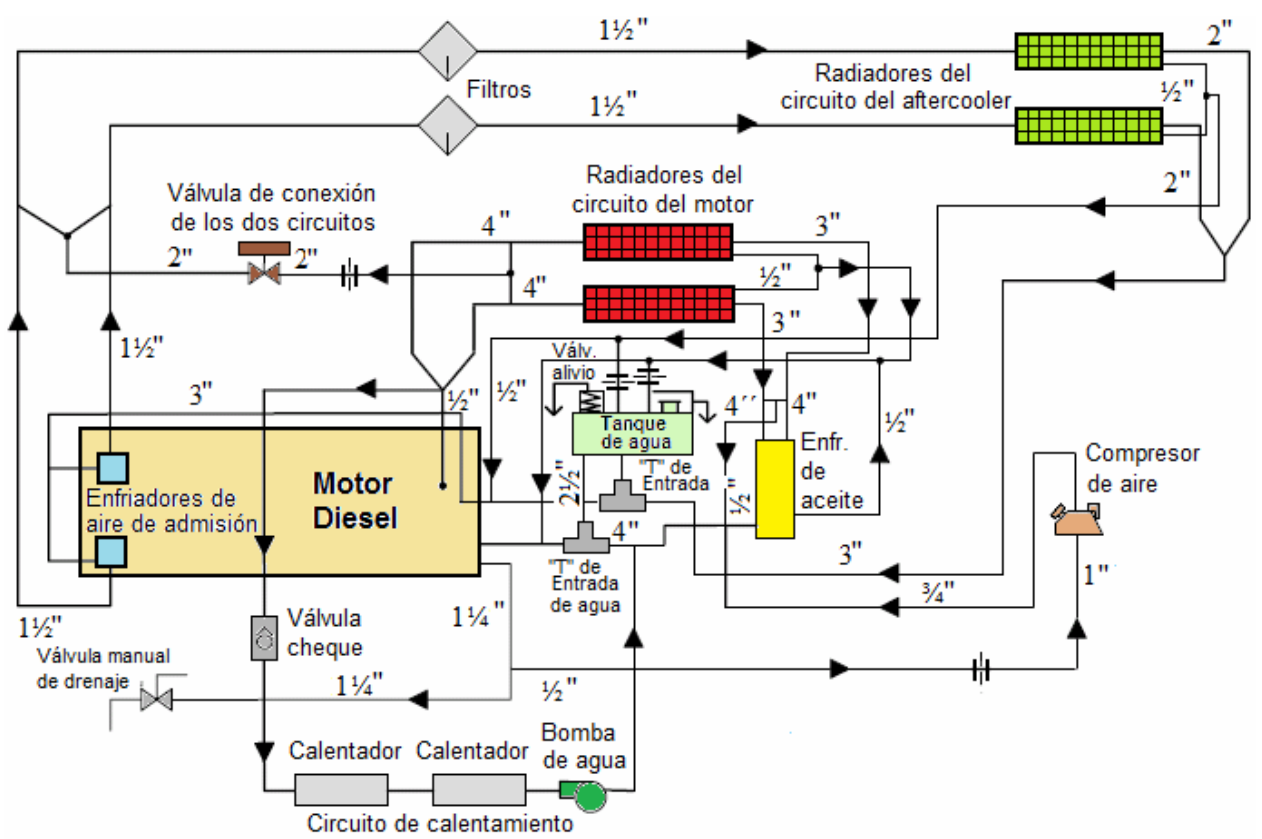

Figura 6.4. Plano esquemático propuesto del sistema de refrigeración del motor.

Descripción de la operación del circuito. El refrigerante es conducido a través de la camisa de refrigeración por la bomba centrífuga accionada por el motor (curva característica en la Figura 6.2). Un pequeño flujo paralelo de refrigerante se hace pasar por la camisa del compresor que alimenta todos los dispositivos neumáticos de la locomotora. A la salida del motor la corriente del refrigerante se bifurca para pasar por los dos radiadores ubicados en los costados, tras los cuales los flujos se reúnen de nuevo para retornar una parte por el enfriador de aceite y otra parte directo a la succión de la bomba. Una pequeña parte de refrigerante se envía al tanque de expansión. Los flujos que pasan por el compresor y el enfriador de aceite se unen a la salida de éste para sumarse al flujo que va directo desde los intercambiadores a la succión de la bomba.

El circuito de refrigeración del enfriador del aire de admisión ("aftercooler") tiene una configuración bastante sencilla: la segunda bomba independiente (curva característica en la Figura 6.3) también accionada por el motor hace circular el refrigerante por la camisa de los enfriadores y luego, por tuberías separadas, el refrigerante se lleva también a los radiadores pertinentes del 
circuito. A la salida de éstos la mayor parte del refrigerante regresa a la succión de la bomba. Una pequeña parte de refrigerante se envía al tanque de expansión.

Los dos circuitos de refrigeración tienen sus propios tanques de desaireación y, para algunas condiciones de operación, está previsto el mezclado de las corrientes de los circuitos a la salida de las camisas del motor y del "aftercooler" a través de una válvula de comunicación ("link valve"). La simulación que aquí se hace del sistema de refrigeración no contempla la operación de la válvula de comunicación, dado que importa predecir la capacidad de disipación máxima de los dos circuitos separados. Sin embargo, en el modelo construido sí está modelada la válvula.

El sistema de refrigeración previsto por el fabricante del motor no contempla válvulas termostáticas, realizándose el control de la temperatura se controlando el estado de los ventiladores y su frecuencia de giro.

\subsubsection{Cálculo preliminar de los componentes del sistema de refrigeración}

Como particularidad del sistema de refrigeración que aquí se diseña, y por recomendación del fabricante del motor como aparece en la tabla 1, los calores del motor y del aceite, por un lado, y el calor del enfriador de aire, por el otro lado, deberán disiparse a través de circuitos independientes para, entre otras razones, asegurar la facilidad de control de la temperatura del aire de admisión. Por la magnitud de la potencia térmica a disipar y por la arquitectura de las locomotoras similares a la de esta aplicación, el calor deberá disiparse a través de dos bancos de radiadores dispuestos a los costados del vagón de tracción, según una configuración parecida a la del esquema K5 de la Figura 6.1, con los radiadores del circuito del motor montados sobre los radiadores del circuito del aftercooler y utilizando dos ventiladores por consideraciones de control. Como el refrigerante será impulsado por las bombas propias del motor por los dos circuitos del sistema, durante el diseño preliminar se calcularán solamente los parámetros base para la selección de los ventiladores y los radiadores. Inicialmente se calcula el calor total evacuado al refrigerante por la correlación de Lahvic y luego se estima un reparto de ese calor entre los circuitos de refrigeración del motor (en el cual se incluye el enfriador de aceite y el compresor) y del enfriador del aire de admisión. Con los calores así obtenidos se determinan los parámetros 
base de los ventiladores y de los radiadores de ambos circuitos, asumiendo un reparto simétrico de los flujos de refrigerante, aire y calor entre los dos bancos de radiadores.

Estimación del calor disipado al refrigerante. El flujo de calor $Q_{r e f r}(\mathrm{~kJ} / \mathrm{s})$, a disipar a través de un sistema de refrigeración único calculado con la correlación empírica de Lahvic [6.23], expresión (4.1) es:

$\dot{Q}_{\text {refr }}[\mathrm{kW}]=\left(\begin{array}{l}8,66 V_{d} \cdot n+108,93 \cdot M_{T}+1119,74 \cdot N_{e} \\ -1010 \cdot V_{d}+2890\end{array}\right) / 3412,2=2264,84 \mathrm{~kW}$

Aceptando la magnitud del calor calculado como el valor ya corregido, $\dot{Q}_{\text {refr_c }}=\dot{Q}_{\text {refr }}$ y atendiendo las sugerencias del fabricante del motor, según el cual los calores evacuados por el circuito del motor y el circuito del enfriador corresponden al 72 y $28 \%$ del flujo de calor total disipado, respectivamente, se aproximan los calores a disipar en los dos circuitos del motor y del enfriador de aire: 1638,64 y 626,2 kW, en los dos grupos de radiadores correspondientes. Los parámetros básicos de selección de los radiadores y los ventiladores, calculados con las expresiones (4.5) - (4.17), previa consideración de las variables de cálculo descritas en la Tabla 6.2, se presentan en la Tabla 6.3.

Tabla 6.2. Variables y coeficientes de cálculo de los parámetros base

\begin{tabular}{|c|c|c|}
\hline Variable & Circ. motor & Circ. AC \\
\hline Variación de la temperatura del aire, $\Delta T_{\text {aire, }}{ }^{\circ} \mathrm{C}$ & 35 & 35 \\
\hline Variación de la temperatura del refrigerante, $\Delta T_{\text {refr }},{ }^{\circ} \mathrm{C}$ & 7 & 12 \\
\hline Temperatura de entrada del refrigerante, $T_{r e f r}^{e n}{ }^{r},{ }^{\circ} \mathrm{C}$ & 85 & 85 \\
\hline Temperatura de entrada del aire, $T_{\text {aire }}^{\text {en }}{ }^{\text {rad }},{ }^{\circ} \mathrm{C}$ & 35 & 35 \\
\hline Densidad del aire, $\rho_{\text {aire }}, \mathrm{kg} / \mathrm{m}^{3}$ & 1,015 & 1,015 \\
\hline Densidad del refrigerante, $\rho_{r e f r}, \mathrm{~kg} / \mathrm{m}^{3}$ & 1050 & 1050 \\
\hline Calor específico del aire, $C_{\text {paire }}, \mathrm{kJ} / \mathrm{kgK}$ & 1,18 & 1,18 \\
\hline
\end{tabular}


Calor específico del refrigerante, $C_{\text {prefr }}, \mathrm{kJ} / \mathrm{kgK}$

Coeficiente de convección entre el refrigerante y la pared de los tubos, $h_{\text {refr }}, \mathrm{W} / \mathrm{m}^{2} \mathrm{~K}$

Espesor de la pared de los tubos, $\delta, \mathrm{m}$

Conductividad el material de los tubos, $k, \mathrm{~W} / \mathrm{mK}$

Coeficiente de convección entre el aire y la pared de

los tubos, $h_{\text {aire }} \mathrm{W} / \mathrm{m}^{2} \mathrm{~K}$

$120 \quad 140$

Coeficiente de aristado, $\psi$

$120 \quad 170$

Velocidad del aire, $v_{\text {aire }}, \mathrm{m} / \mathrm{s}$

$6 \quad 6$

Velocidad de cálculo del aire por el área viva del

ventilador, $v_{\text {aire }}^{\prime}, \mathrm{m} / \mathrm{s}$

$27 \quad 27$

Coeficiente de compacidad volumétrica, $\varphi_{r}$

$0,7 \quad 0,7$

Presión generada por el ventilador, $p_{a i}, \mathrm{~Pa}$

$1000 \quad 1000$

Rendimiento del ventilador, $\eta_{v}$

$0,75 \quad 0,75$

Coeficiente del ventilador, $\psi_{a}$

Tabla 6.3. Parámetros base para la selección de los radiadores y el ventilador

Calor a disipar por el radiadr, $\dot{Q}_{r e f r}, \mathrm{~kJ} / \mathrm{s}$

Caudal de aire a través del radiador, $\mathrm{V}_{\text {aire }}, \mathrm{m}^{3} / \mathrm{s}$

$819,32 \quad 313,10$

Caudal de refrigerante a través del radiador, $\mathrm{V}_{\text {refr }}, 1 / \mathrm{s}$

$19,55 \quad 7,47$

Temperatura media del refrigerante, $T_{\text {refr }}^{m},{ }^{\circ} \mathrm{C}$

$31,40 \quad 7,00$

Temperatura media del aire, $T_{\text {aire }}^{m},{ }^{\circ} \mathrm{C}$

$81,50 \quad 79,00$

Coeficiente global de transferencia de calor, $U_{\text {refr, }}$,

$52,50 \quad 52,50$

$\mathrm{W} / \mathrm{m}^{2} \mathrm{~K}$

$99,23 \quad 131,19$

Área de superficie de refrigeración del radiador, $F_{\text {rad }}$, $\mathrm{m}^{2}$

Área frontal del radiador, $F_{f r}, \mathrm{~m}^{2}$ 
Profundidad del panel del radiador, $l_{\text {rad }}, \mathrm{mm}$

Velocidad tangencial del ventilador, $u, \mathrm{~m} / \mathrm{s}$

Diámetro externo del ventilador, $D_{v}, \mathrm{~m}(2 \mathrm{ud}$.)

Velocidad de rotación del ventilador, $n$, rpm

Potencia consumida por el ventilador, $N_{v}, \mathrm{~kW}$

Volumen del radiador, $V_{\text {rad }}$

Relación área de transferencia por volumen del

radiador, $\mathrm{m}^{2} / \mathrm{m}^{3}$

Relación $Q /\left(I T D \cdot F_{f r}\right)$

$\begin{array}{rr}222,34 & 193,65 \\ 78,47 & 78,47 \\ 1,46 & 0,97 \\ 1024 & 1549 \\ 45,6 & 19,9 \\ 0,62 & 0,21\end{array}$

$700,00 \quad 700,00$

$5,87 \quad 5,87$

La capacidad del sistema de refrigeración líquido, se estima en 600 litros, a partir de la potencia del motor $C_{r e f r}=0,2 \cdot N_{e}=600 l$ [4.35], repartidos en 450 litros para el circuito del motor y 150 litros para el circuito del enfriador de aire.

Los valores encontrados son orientativos para la selección de los radiadores y los ventiladores y deben conjugarse con las restricciones de espacio propias de la aplicación. Según las exigencias del diseño, a los costados del vagón de tracción se dispone de un espacio de hasta 2,8 por 1,1 metros a lo largo y alto, respectivamente, con lo que el área frontal del radiador puede ser de hasta $F_{f r}$ $=3,08 \mathrm{~m}^{2}$.

Selección de los radiadores y los ventiladores. Habiendo calculado los parámetros base para el cálculo de diseño detallado o para la selección de los radiadores y los ventiladores, y asumiendo dimensiones marco iguales para los dos tipos de radiadores (dimensiones máximas largo, alto y profundidad iguales por consideraciones de manufactura e instalación), se hace uso del programa de cálculo de radiadores y, comparando los valores de área frontal y relación área de transferencia/volumen con los correspondientes a las posibles geometrías conocidas de aplicaciones en otras locomotoras, se prueba con la geometría AXB10 (radiador de flujo cruzado) de dos pasos para el radiador del motor y de cuatro pasos para el radiador del enfriador de aire, con longitud de tubos $=2,71 \mathrm{~m}$, alto del panel del radiador $=1,045 \mathrm{~m} \mathrm{y}$ profundidad del panel del radiador de $299 \mathrm{~mm}$. El tipo de panel AXB10 tiene las características descritas en la Figura 6.5. 


\begin{tabular}{|c|c|c|c|}
\hline Superficie & \multicolumn{3}{|c|}{ AXB_10 } \\
\hline Tubo & \multicolumn{2}{|c|}{ Plano } & \\
\hline Aletas & \multicolumn{2}{|c|}{ Continuas } & \\
\hline Arrangement & \multicolumn{2}{|c|}{ En linea } & \\
\hline Pin pattern & \multicolumn{2}{|c|}{ Plano } & \\
\hline xt & \multicolumn{2}{|c|}{22.2250} & $\mathrm{~mm}$ \\
\hline$\underline{x l}$ & \multicolumn{2}{|c|}{15.875} & $\mathrm{~mm}$ \\
\hline$\phi$ tubo. Interior / Exterior & & & $\mathrm{mm}$ \\
\hline Aletas: Espesor / фexterior & 0.0762 & & $\mathrm{~mm}$ \\
\hline Densidad & 0.3937 & & aletas $/ \mathrm{mm}$ \\
\hline Lexterior & \multicolumn{2}{|c|}{17.907} & $\mathrm{~mm}$ \\
\hline фexterior & \multicolumn{2}{|c|}{3.759} & $\mathrm{~mm}$ \\
\hline Espesor & \multicolumn{2}{|c|}{0.4572} & $\mathrm{~mm}$ \\
\hline \multicolumn{4}{|c|}{ Cálculos } \\
\hline $\mathrm{pt}$ & \multicolumn{2}{|c|}{19.3779} & $\mathrm{~mm}$ \\
\hline$x d$ & \multicolumn{2}{|c|}{27.3124} & $\mathrm{~mm}$ \\
\hline$\sigma$ & 0.136 & 0.788 & - \\
\hline$\alpha$ & 108.1 & 754.8 & $\mathrm{~m}^{2} / \mathrm{m}^{3}$ \\
\hline$D_{h}$ & 5.02 & 4.18 & $\mathrm{~mm}$ \\
\hline$A_{\text {aletas }} / A_{\text {total }}$ & & 0.852 & $\mathrm{~m}^{2} / \mathrm{m}^{2}$ \\
\hline
\end{tabular}

Figura 6.5. Parámetros geométricos del tipo de de panel AXB10

En la Figura 6.6 y la Figura 6.7 se presentan la información de entrada y la información de salida del programa de cálculo de radiadores con el resultado final de la selección de uno de los radiadores para el circuito del motor.

\begin{tabular}{|c|c|c|c|c|c|}
\hline \multicolumn{5}{|c|}{ Unidades } & \multirow{2}{*}{\begin{tabular}{|l|} 
SI \\
--
\end{tabular}} \\
\hline Fluidos & \multicolumn{2}{|c|}{ Ethylen-Water 50} & \multicolumn{2}{|l|}{ Air } & \\
\hline Presión & \multicolumn{2}{|c|}{--} & \multicolumn{2}{|l|}{1} & bar \\
\hline Caudal (cálculos a Tmedio) & 31.4 & $\Rightarrow$ & 19545 & $\Rightarrow$ & $1 / \mathrm{s}$ \\
\hline $\mathrm{T}_{\text {entrada }}$ & 85 & $\Rightarrow$ & 35 & $\Rightarrow$ & ${ }^{\circ} \mathrm{C}$ \\
\hline Calor & \multicolumn{4}{|c|}{819.32} & kW \\
\hline Pasos: Counterflow/Crossflow & \multicolumn{2}{|l|}{2} & \multicolumn{2}{|l|}{0} & --- \\
\hline Máxima $\Delta \mathrm{P}$ & \multicolumn{2}{|l|}{1} & \multicolumn{2}{|l|}{0.2} & bar \\
\hline Superficie & \multicolumn{4}{|c|}{ AXB_10 } & --- \\
\hline Materiales & Aluminio pu & & Aluminio puro & & --- \\
\hline \multicolumn{6}{|c|}{ RESTRICCIONES GEOMÉTRICAS } \\
\hline Longitud tubos & 2710 & $\Rightarrow 1$ & L1 & & $\mathrm{mm}$ \\
\hline Espesor zona tubos & 299 & $\Rightarrow$ & L2 & & $\mathrm{mm}$ \\
\hline Altura & 1045 & $\Rightarrow$ & L3 & & $\mathrm{mm}$ \\
\hline
\end{tabular}

Figura 6.6. Cuadro de diálogo del programa de cálculo de radiadores con la información inicial para el cálculo de uno de los radiadores del motor. 


\begin{tabular}{|c|c|c|c|}
\hline \multicolumn{3}{|c|}{ Unidades } & SI \\
\hline Fluidos & Ethylen-Water 50 & Air & --- \\
\hline Presión & --- & 1 & bar \\
\hline Caudal (cálculos a Ti & 31.4 & 19545 & $1 / \mathrm{s}$ \\
\hline \begin{tabular}{l|l} 
Tentrada & \\
\end{tabular} & 85 & 35 & ${ }^{\circ} \mathrm{C}$ \\
\hline Calor & \multicolumn{2}{|c|}{819.32} & kW \\
\hline Pasos: Counterflow/l & 2 & 0 & --- \\
\hline Máxima $\Delta \mathrm{P}$ & 1 & 0.2 & bar \\
\hline Superficie & \multicolumn{2}{|c|}{ AXB_10 } & --- \\
\hline Materiales & Aluminio puro & Aluminio puro & --- \\
\hline \multicolumn{4}{|c|}{ RESTRICCIONES GEOMÉTRICAS } \\
\hline Longitud tubos & 2710 & $\mathrm{~L} 1$ & $\mathrm{~mm}$ \\
\hline Espesor zona tubos & 299 & L2 & $\mathrm{mm}$ \\
\hline Altura & 1045 & L3 & $\mathrm{mm}$ \\
\hline Tsalida & 77 & 77 & ${ }^{\circ} \mathrm{C}$ \\
\hline Calor & 883011 & & $\mathrm{~kW}$ \\
\hline$\Delta \mathrm{P}$ & 0.225 & 0.001 & bar \\
\hline
\end{tabular}

Figura 6.7. Cuadro de diálogo del programa de cálculo de radiadores con la información de salida de uno de los radiadores del motor.

El radiador seleccionado tiene una capacidad de disipación de calor bajo las temperaturas y flujos volumétricos entrados, de $883 \mathrm{~kW}$, frente a los $819 \mathrm{~kW}$ demandados. Las pérdidas de presión interna y externa son bien aceptables.

En la Figura 6.8 y la Figura 6.9 se presentan la información de entrada y la información de salida del programa de cálculo de radiadores con el resultado final de la selección de uno de los radiadores para el circuito del enfriador de aire de admisión.

\begin{tabular}{|c|c|c|c|c|c|}
\hline \multicolumn{5}{|c|}{ Unidades } & \multirow{2}{*}{\begin{tabular}{|c|} 
SI \\
--
\end{tabular}} \\
\hline Fluidos & Ethylen-Wate & 50 & Air & & \\
\hline Presión & $\ldots$ & & 1 & & bar \\
\hline Caudal (cálculos a Tmedio) & 7 & $\Rightarrow$ & 7469 & $\Rightarrow$ & V/s \\
\hline$T_{\text {entrada }}$ & 85 & $\Rightarrow$ & 35 & $\Rightarrow$ & ${ }^{\circ} \mathrm{C}$ \\
\hline Calor & \multicolumn{4}{|c|}{313.1} & kW \\
\hline Pasos: Counterflow/Crossflow & \multicolumn{2}{|l|}{4} & \multicolumn{2}{|l|}{0} & -- \\
\hline Máxima $\Delta \mathrm{P}$ & \multicolumn{2}{|l|}{1} & \multicolumn{2}{|l|}{0.2} & bar \\
\hline Superficie & \multicolumn{4}{|c|}{ AXB_10 } & -- \\
\hline Materiales & Aluminio pt & & Aluminio puro & & -- \\
\hline \multicolumn{6}{|c|}{ RESTRICCIONES GEOMÉTRICAS } \\
\hline Longitud tubos & 2710 & $\Rightarrow$ & L1 & & $\mathrm{mm}$ \\
\hline Espesor zona tubos & 299 & $\Rightarrow$ & L2 & & $\mathrm{mm}$ \\
\hline Altura & 1045 & $\Rightarrow 1$ & L3 & & $\mathrm{mm}$ \\
\hline
\end{tabular}

Figura 6.8. Cuadro de diálogo del programa de cálculo de radiadores con la información inicial para el cálculo de uno de los radiadores del enfriador de aire de admisión (AC). 


\begin{tabular}{|c|c|c|c|}
\hline \multicolumn{3}{|c|}{ Unidades } & SI \\
\hline Fluidos & Ethylen-Water 50 & Air & --- \\
\hline Presión & --- & 1 & bar \\
\hline Caudal (cálculos a Ti & 7 & 7469 & I/s \\
\hline \begin{tabular}{|l|l} 
Tentrada & \\
\end{tabular} & 85 & 35 & ${ }^{\circ} \mathrm{C}$ \\
\hline Calor & \multicolumn{2}{|c|}{313.1} & kW \\
\hline Pasos: Counterflow/d & 4 & 0 & --- \\
\hline Máxima $\Delta \mathrm{P}$ & 1 & 0.2 & bar \\
\hline Superficie & \multicolumn{2}{|c|}{ AXB_10 } & --- \\
\hline Materiales & Aluminio puro & Aluminio puro & -- \\
\hline \multicolumn{4}{|c|}{ RESTRICCIONES GEOMÉTRICAS } \\
\hline Longitud tubos & 2710 & $\mathrm{~L} 1$ & $\mathrm{~mm}$ \\
\hline Espesor zona tubos & 299 & L2 & $\mathrm{mm}$ \\
\hline Altura & 1045 & L3 & $\mathrm{mm}$ \\
\hline Tsalida & 72 & 76 & ${ }^{\circ} \mathrm{C}$ \\
\hline Calor & 333595 & & kW \\
\hline$\Delta \mathrm{P}$ & 0.111 & 0.000 & bar \\
\hline
\end{tabular}

Figura 6.9. Cuadro de diálogo del programa de cálculo de radiadores con la información de salida de uno de los radiadores del enfriador de aire de admisión (AC).

El radiador seleccionado tiene una capacidad de disipación de calor, bajo las temperaturas y flujos volumétricos entrados, de $333,6 \mathrm{~kW}$, frente a los 313 $\mathrm{kW}$ demandados. Las pérdidas de presión interna y externa son bien aceptables.

Durante la ejecución del programa de cálculo de los radiadores se han realizado algunos estudios paramétricos, estudiando la influencia de las variaciones de dimensiones y también de las fluctuaciones de las condiciones de operación. Luego de establecidos los paneles finales, se obtuvieron las características térmicas de los radiadores. En la Figura 6.10, a modo de ilustración se presenta la sensibilidad del calor transferido en el radiador del motor a la variación de la temperatura de entrada del refrigerante, lo que puede ocurrir durante el arranque del motor a una temperatura inicial de todo el sistema de $35^{\circ} \mathrm{C}$. Las tendencias coinciden con las reportadas por Oliet et al. [6.17].

Con la información entregada por el programa de cálculo de radiadores se han construido las características de disipación de calor, $Q(I T D \cdot A)=f\left(\dot{m}_{\text {refr }}, \dot{m}_{\text {aire }} / A_{f r}\right)$ de los radiadores de los subcircuitos del 
motor y el enfriador de aire, representadas en la Figura 6.11, necesarias para el modelado del sistema de refrigeración completo.

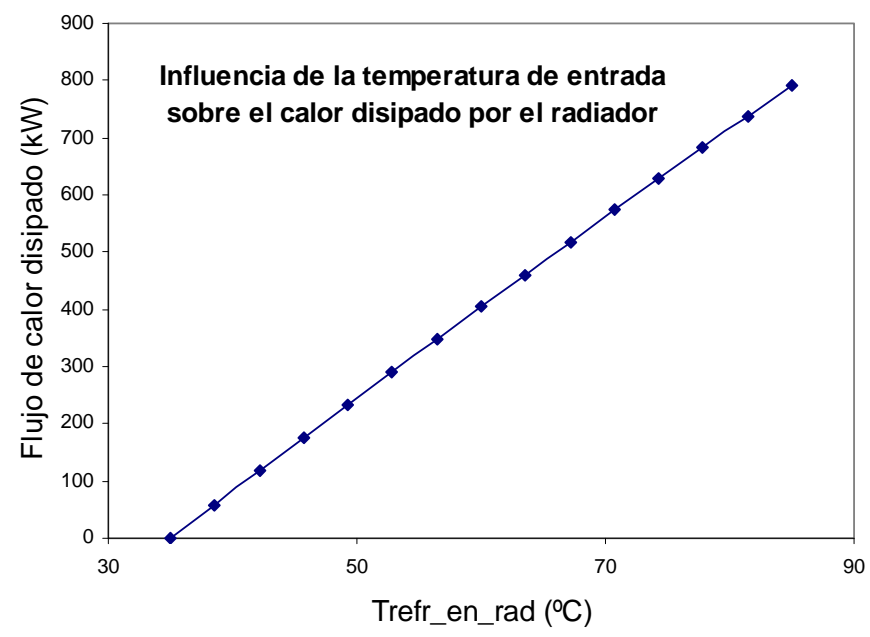

Figura 6.10. Variación del calor disipado por el radiador a medida que el motor calienta (la condición de arranque con plena carga es hipotética, pues el control del motor no permite su operación a plena carga estando aún frio).
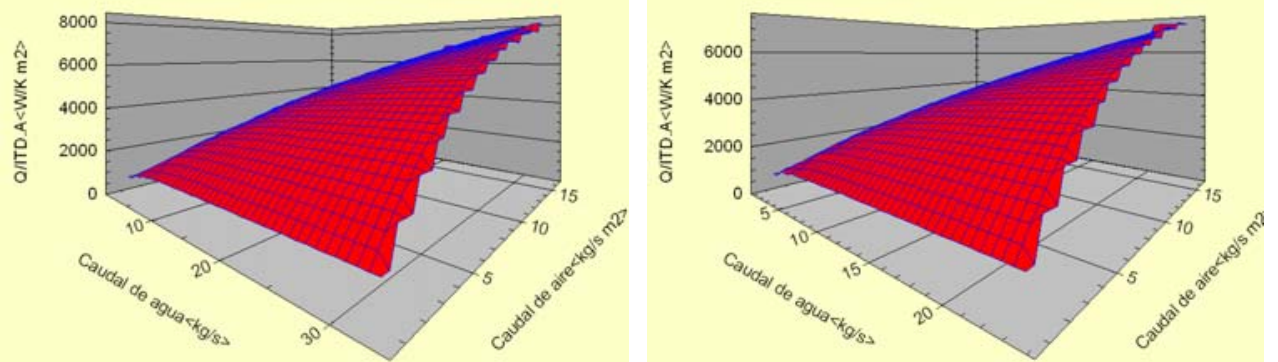

Figura 6.11. Características de los radiadores para los circuitos de refrigeración del motor (izquierda) y del enfriador de aire (derecha).

Con la información base del ventilador y por consideraciones de diseño y de control se seleccionan dos ventiladores iguales de 11 aspas con diámetro 
externo de $D_{v}=1372 \mathrm{~mm}$, para operar a $1800 \mathrm{rpm}$ y cuya característica es la ilustrada en la Figura 6.12. En el punto recomendado de operación el ventilador entrega un flujo volumétrico y una presión cercanos a $28 \mathrm{~m}^{3} / \mathrm{s}$ y $1380 \mathrm{~Pa}$, correspondientemente.

El ventilador puede ser accionado eléctricamente o hidráulicamente por medio de transmisiones hidrostáticas, y puede tener un control encendido/apagado o un control de velocidad, considerando señales de medidores de temperatura del refrigerante y del aire de carga del motor. Es una tendencia racional la utilización de varios ventiladores; los ventiladores más pequeños girando a mayores revoluciones consumen menos potencia y pueden apagarse completamente [6.27]. El estudio del control del sistema está fuera del alcance del trabajo aquí presentado.

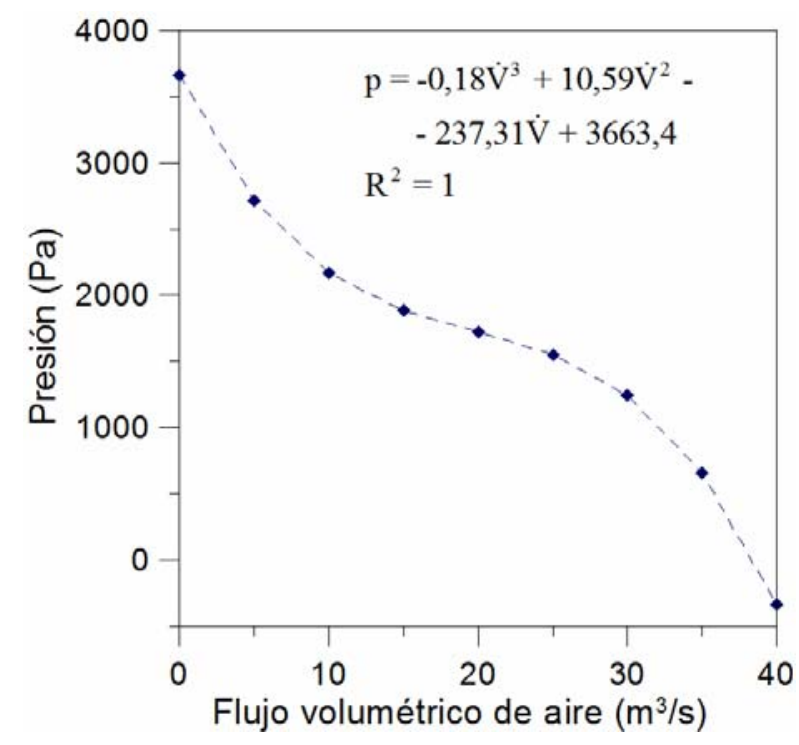

Figura 6.12.

Característica del

ventilador

seleccionado.

\subsubsection{Disposición de los componentes del sistema hidráulico y del sistema de ventilación}

En esta parte del proceso se define una configuración provisional del circuito con diámetros y longitudes tentativas de las tuberías y conexiones requeridas según la topología y las limitaciones impuestas por los componentes. 
Considerando los espacios disponibles en el vagón de tracción y observando la práctica de distribución de los sistemas en otras locomotoras, se ha decidido ubicar el tanque de expansión y el enfriador del aceite entre el espacio de radiadores y el espacio del motor. Se han seleccionado diámetros de tuberías y conexiones que se acomodan tanto a unas pérdidas razonables de presión, como al espacio permitido por la disposición de los elementos, los espacios entre éstos y la topología de la misma tubería. Se dispone la ubicación de los dos ventiladores en el techo del vagón, desde donde succionarán el aire desde los costados del vagón a través de rejillas protectoras, deflectores, pasándolo por los radiadores y el remanso a la salida de éstos. Los radiadores del motor van en la parte superior de cada módulo de radiadores, mientras que en la parte inferior van los radiadores para el circuito de refrigeración del aire de admisión. Los ventiladores generarán el tiro de succión del aire por la parte superior. En la Figura 6.13 se ilustra una distribución tipo de los bloques de componentes principales del sistema de refrigeración en una locomotora existente y que ha servido de referencia para disponer los componentes y trazar el recorrido de la tubería de los circuitos.

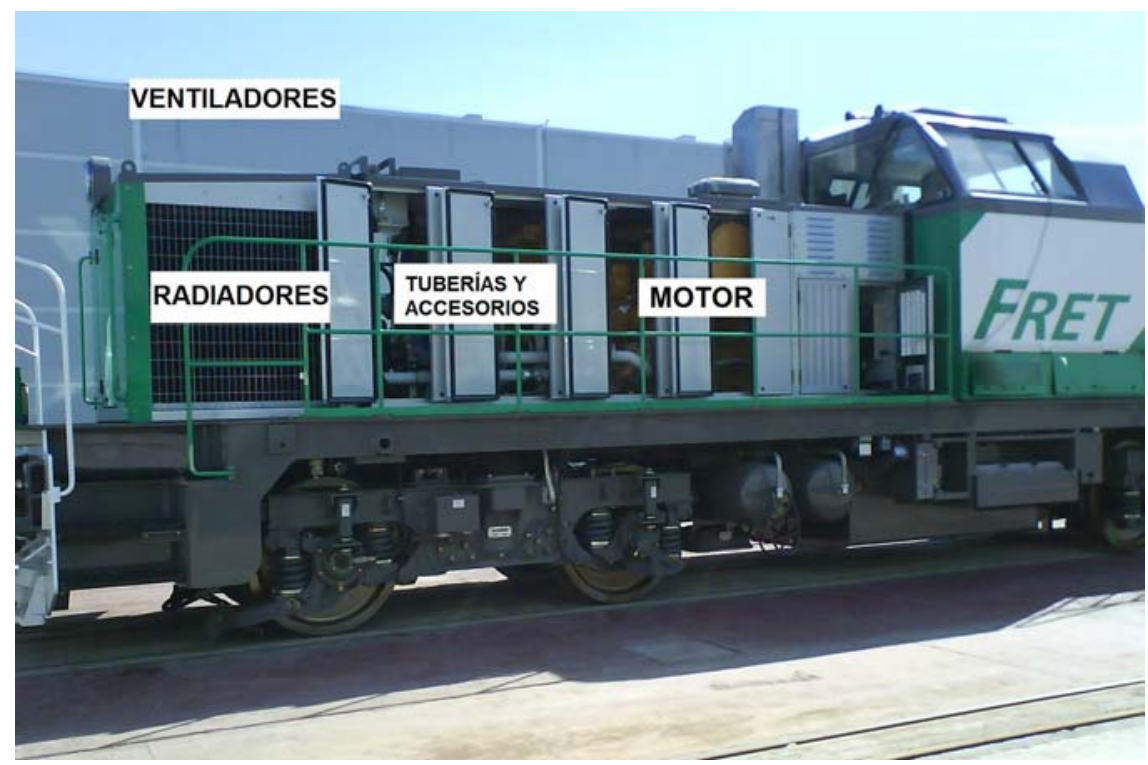

Figura 6.13. Disposición relativa de los grupos de componentes principales del sistema de refrigeración observada en una locomotora existente. 

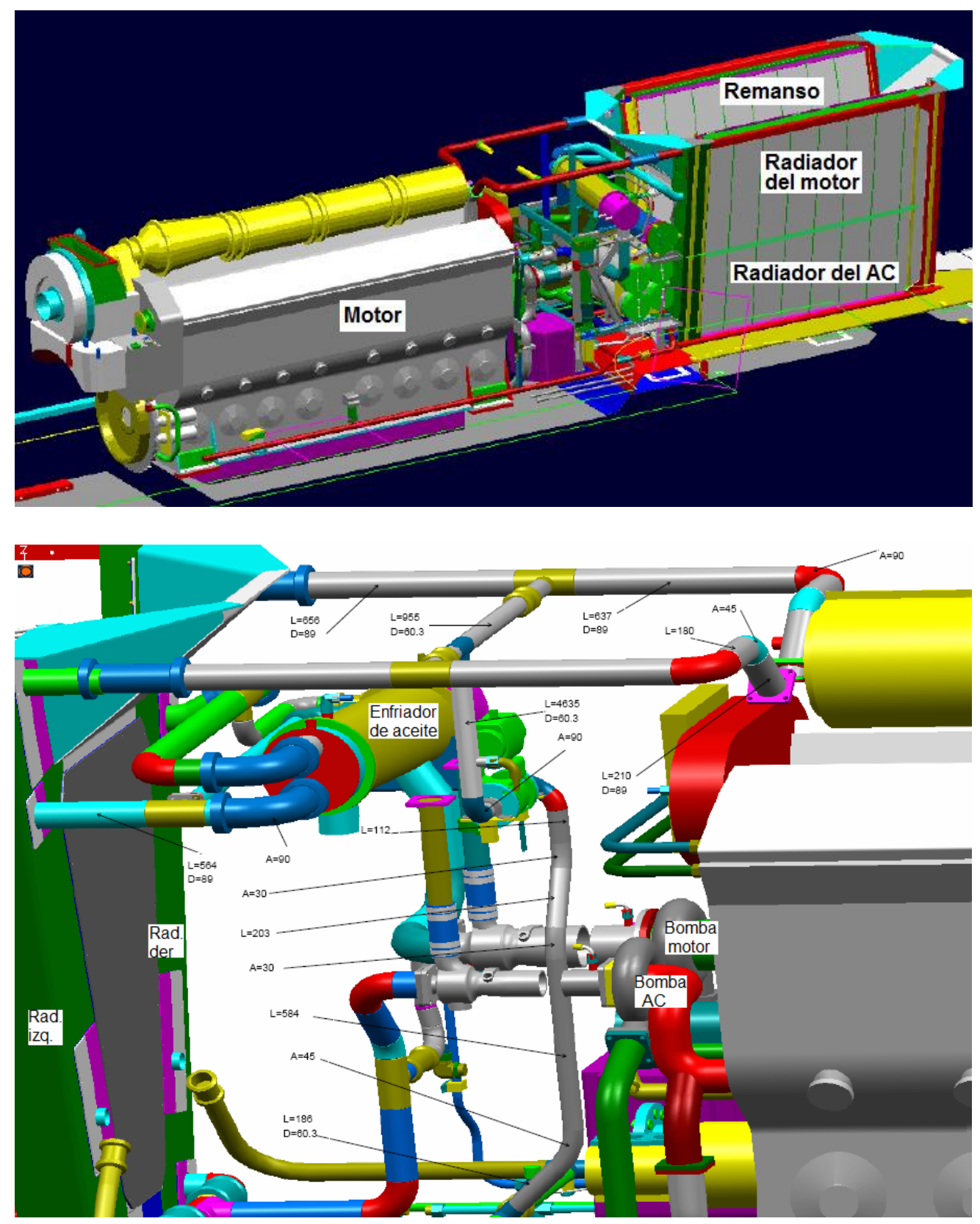

Figura 6.14. Vista de ensamble parcial de las conexiones entre el motor y los radiadores, en la que también se aprecian las bombas y el enfriador de aceite. 
En la Figura 6.14 se presenta una vista de conjunto del sistema de refrigeración diseñado. En la figura superior se puede ver la disposición relativa de los componentes (los ventiladores van justo sobre el remanso, en la parte superior), mientras que en la parte inferior se detallan las conexiones de las tuberías de los dos circuitos de refrigerante.

Habiendo seleccionado los componentes y realizado el trazado de la red hidráulica, el paso siguiente en el diseño del sistema de refrigeración es el modelado y la simulación de su funcionamiento, con el objetivo de analizar el comportamiento de los flujos y las temperaturas de refrigerante y de aire.

\subsection{Modelado y simulación del sistema de refrigeración}

El flujo a través de la rejilla, el enfocador, el radiador y la combinación del remanso y el ventilador es importante con relación al desempeño del sistema de refrigeración del motor. Todos los componentes mencionados influencian el perfil del flujo de aire a su paso por el radiador, y un buen análisis de la distribución de la velocidad permite realizar modificaciones para optimizar la calidad del flujo, mejorar la uniformidad de transfencia de calor de los radiadores, reducir las pérdidas de presión y, lo que es muy importante, reducir la potencia demandada por el ventilador, lo que a su vez se traduce en reducción del consumo de combustible.

\subsubsection{Modelado y caracterización del sistema de ventilación}

En esta tesis se ha utilizan los resultados del modelado del sistema de ventilación realizado con el programa PowerFlow ${ }^{\circledR}$. Este código está basado en volúmenes finitos con un mallado no estructurado. Para solucionar el campo de velocidades, el flujo turbulento se modela con un modelo k- $\varepsilon$ isotrópico, usando el modelo estándar $\mathrm{k}-\varepsilon$, junto con las funciones de la pared para representar las condiciones de contorno turbulentas en las capas contiguas a las paredes. Se consideran los tres mecanismos de transferencia de calor: convección, conducción y radiación. En el cálculo, se asume flujo incompresible turbulento (totalmente desarrollado) de aire seco, las paredes de contorno del sistema son consideradas adiabáticas. 
El modelo CFD del sistema de ventilación se ilustra en la Figura 6.15 y la Figura 6.16, donde se aprecia además la pérdida de presión tras el paso del aire por la rejilla.

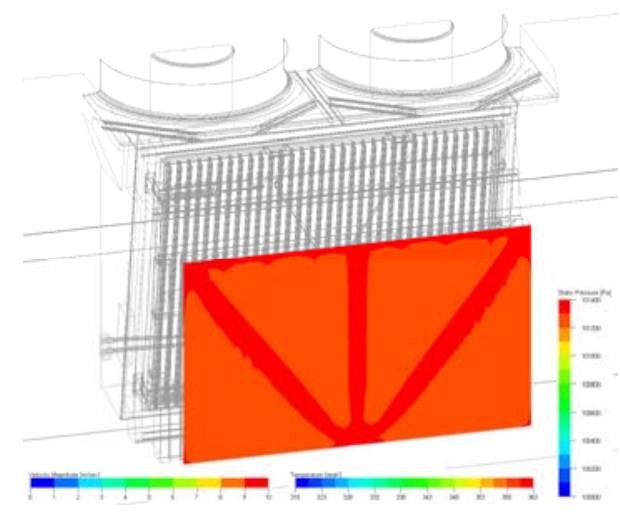

Figura 6.15. Modelo CFD del sistema de ventilación.

Distribución de las presiones antes de la rejilla.

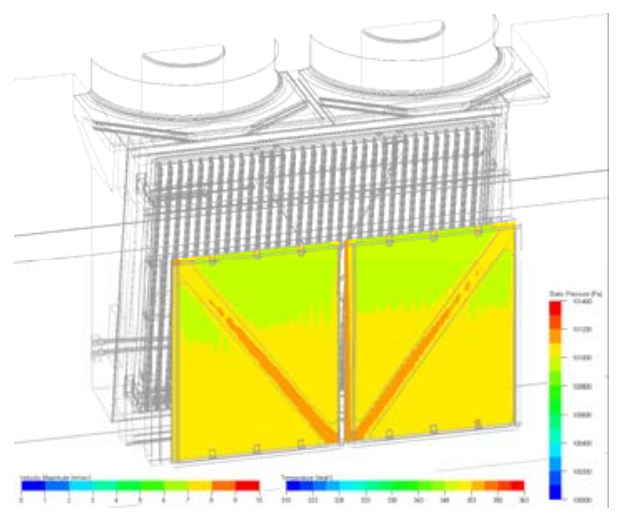

Figura 6.16. Modelo CFD del sistema de ventilación. Distribución de las presiones tras la rejilla.

En la solución del sistema de ventilación se consideran, como condiciones de entrada (no necesariamente las de operación, para caracterización hidráulica):

- $\quad$ Flujo másico de aire por radiador $=28,28 \mathrm{~kg} / \mathrm{s}$; temperatura de entrada del aire $=313,15 \mathrm{~K}$

- Salto de presión en los ventiladores, $\Delta p=1325 \mathrm{~Pa}$

Los radiadores se modelan como un medio poroso con área frontal $A_{f r}=2,71$ $\mathrm{m}^{2}$, con una disipación de calor de $747,33 \mathrm{~kW}$ para el radiador del motor $\mathrm{y}$ $325,30 \mathrm{~kW}$ para el radiador del "aftercooler".

En la Figura 6.17 y la Figura 6.18 se ilustran los campos de velocidades de paso del aire antes y después de la rejilla. 


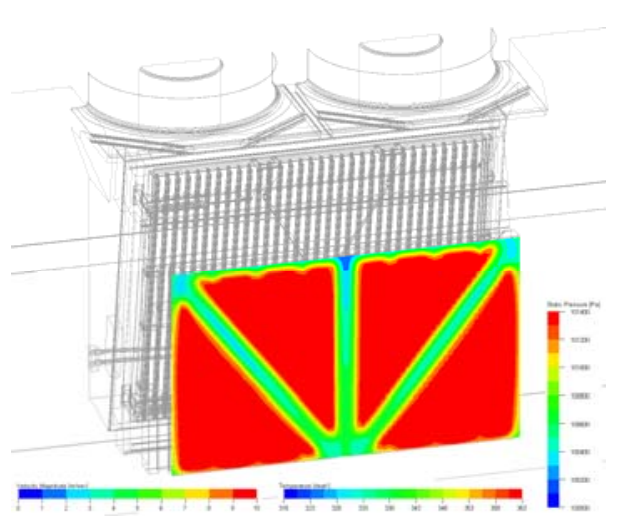

Figura 6.17. Campo de velocidades a la entrada al sistema, antes de la rejilla.

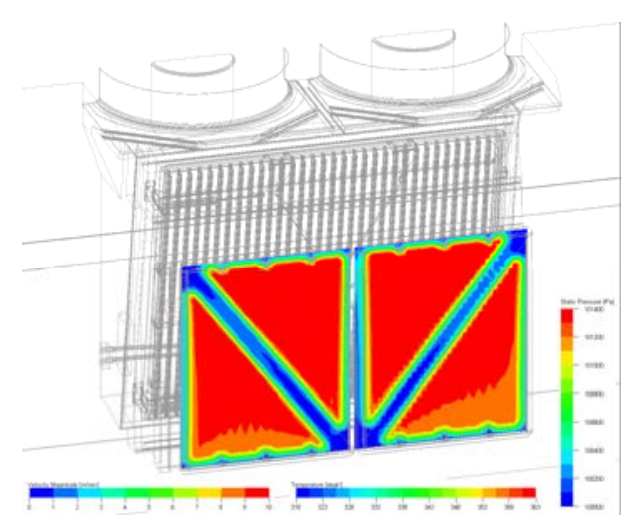

Figura 6.18. Campo de velocidades a la entrada al sistema, tras la rejilla.

La resistencia ofrecida por los radiadores al paso del aire produce la distribución de presiones mostrada en la Figura 6.19 y la Figura 6.20. La diferencia de los valores medios de las pérdidas de presion, junto con los caudales de paso, se utiliza para calcular el coeficiente de pérdidas de presión necesario posteriormente en el modelado termohidráulico del sistema.

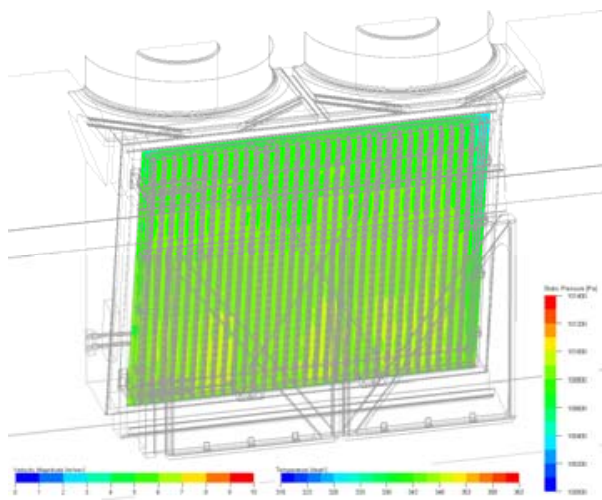

Figura 6.19. Variación de las presiones del aire antes de los radiadores.

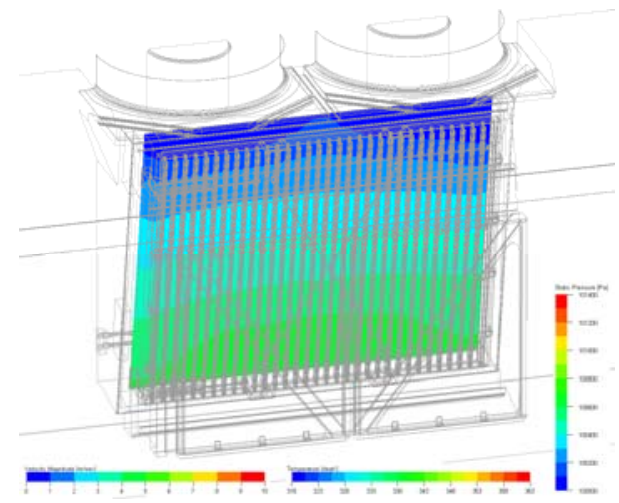

Figura 6.20. Variación de las presiones del aire después de los radiadores.

La diferencia de velocidades entre las caras de los radiadores puede seguirse en la Figura 6.21 y la Figura 6.22. Es evidente la no uniformidad a lo alto y 
ancho del radiador y también la mayor velocidad media para el radiador superior correspondiente al circuito del motor.

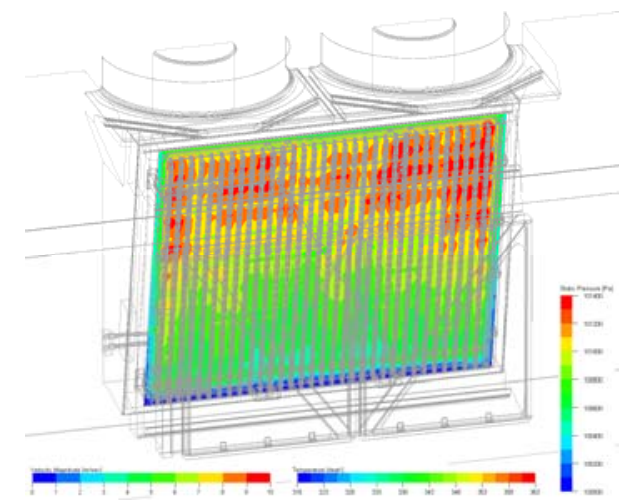

Figura 6.21.Campo de velocidades en la cara anterior de los radiadores.

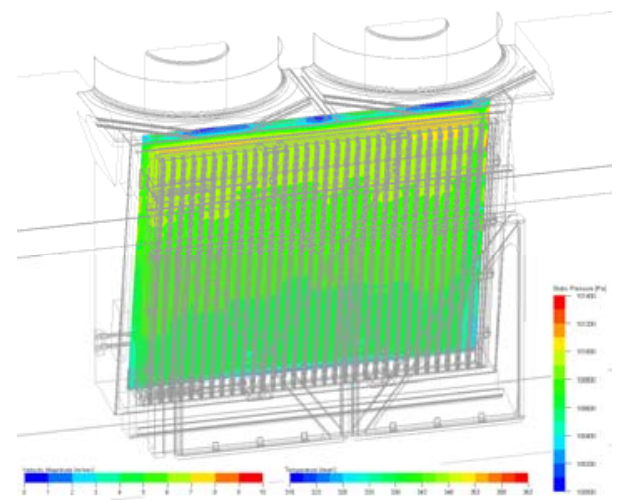

Figura 6.22. Campo de velocidades en la cara posterior de los radiadores.

La Figura 6.23 muestra claramente la no uniformidad de la temperatura a lo alto y lo ancho de los radiadores, mereciendo especial atención la diferencia de las temperaturas del aire a la salida del radiador del motor y del radiador del circuito del "aftercooler". En la Figura 6.24 se grafica la presión antes y después de los ventiladores. Asumiendo que los dos ventiladores trabajan igual, se ha representado en uno de ellos la presión antes de la salida y en el otro la presión después de la salida.

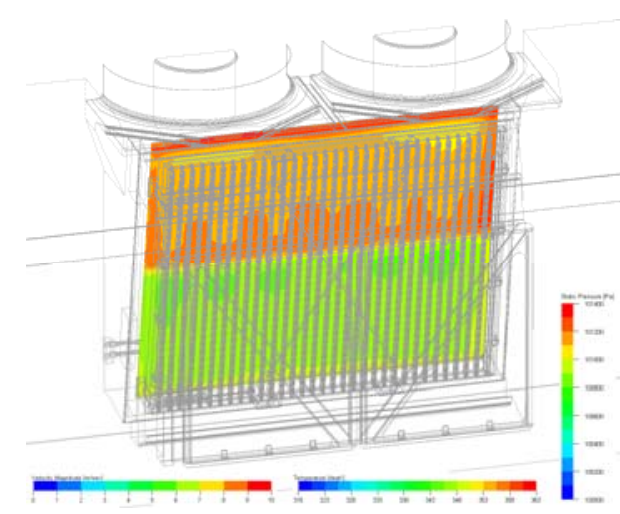

Figura 6.23. Distribución de la temperatura del aire en la cara posterior de los radiadores.

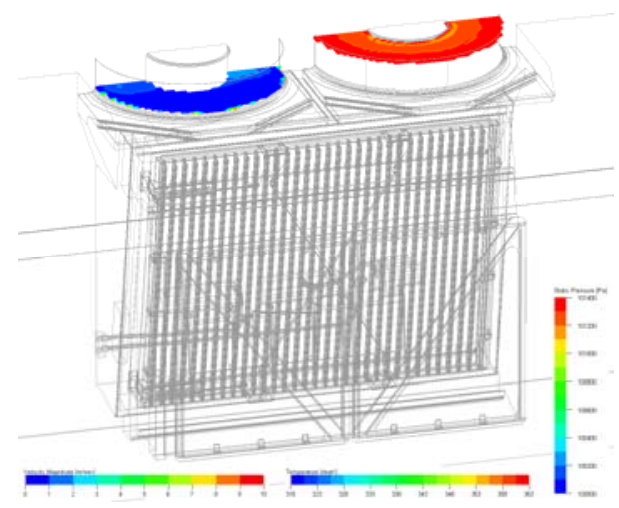

Figura 6.24. Distribución de la presión del aire antes y después de los ventiladores. 
Los valores medios de las pérdidas de presión del aire a su paso por el sistema de ventilación según el esquema del sistema mostrado en la Figura 6.25 se resumen en la Tabla 6.4.

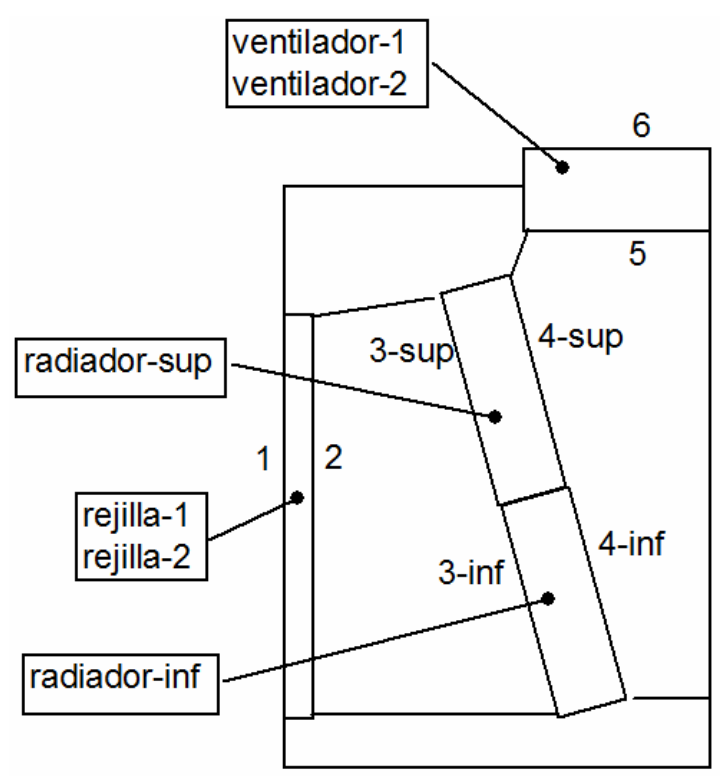

Figura 6.25. Distribución de la temperatura del aire en la cara posterior de los radiadores.

Tabla 6.4. Valores medios resultantes de la simulación del sistema de ventilación.

\begin{tabular}{lccc}
\hline \multicolumn{1}{c}{ Tramo } & $\Delta \mathbf{p}, \mathbf{P a}$ & $\Delta \mathrm{T},{ }^{\mathbf{0}} \mathbf{C}$ & Flujo de aire, $\mathbf{~ m}^{\mathbf{3}} \mathbf{s}$ \\
\hline 1-2 (rejilla1) & 288 & & \\
2-3 & 183,5 & & \\
3-4 (radiador motor) & 560 & 40,3 & 15,46 \\
3-4 (radiador AC) & 341 & 30,9 & 12,61 \\
4-5 (radiador motor) & 218,5 & & \\
4-5 (radiador AC) & 542,5 & & \\
5-6 (ventilador1) & 1307 & & \\
5-6 (ventilador2) & 1325 & & \\
\hline
\end{tabular}


Los valores de las pérdidas de presión sirven para calcular los coeficientes de pérdidas de presión, $K$ (considerando la expresión $\Delta p=K \dot{V}^{2}$ ), que deberán introducirse en las características del sistema de ventilación en el modelado 1D. Pueden calcularse los coeficientes individuales de los diferentes tramos, discriminando las pérdidas de presión por el radiador, o calcular un solo coeficiente integral de pérdidas y atribuírselo al radiador. Esto hace más liviano el cálculo y el error será admisible porque las pérdidas provienen en rigor de los cálculos CFD.

Los coeficientes de pérdidas permiten obtener las curvas de pérdidas de presión del sistema de ventilación que han de introducirse al programa de modelado 1D y que se ilustran en la Figura 6.26.

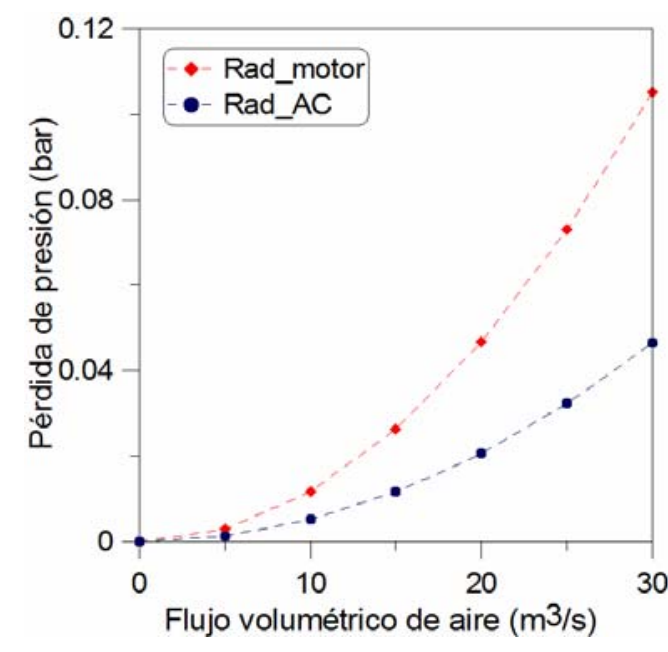

Figura 6.26. Pérdidas de presión al paso del aire por los radiadores del motor y del enfriador de aire.

En la caracterización de la parte de ventilación se requiere introducir la característica del ventilador, Figura 6.12. Al simular la operación de los ventiladores en conjunto con el conducto de ventilación, el punto de operación se establece según las magnitudes de las resistencias y los caudales correspondientes a esas resistencias predichas por PowerFlow, anotadas en la

Tabla $6.4: 15,46 \mathrm{~kg} / \mathrm{s}$ por cada radiador del motor y 12,61 por cada radiador del enfriador de aire, para un gasto másico total por banco de $28,07 \mathrm{~kg} / \mathrm{s}$. Siendo simétrico el sistema, el gasto másico total de aire será de $2 \cdot 28,07=$ $56,14 \mathrm{~kg} / \mathrm{s}$. 
La solución gráfica del sistema de aire tras conocer sus características de pérdidas de presión y la característica de los ventiladores, considerando simétrico el sistema de aire, se muestra en la Figura 6.27.

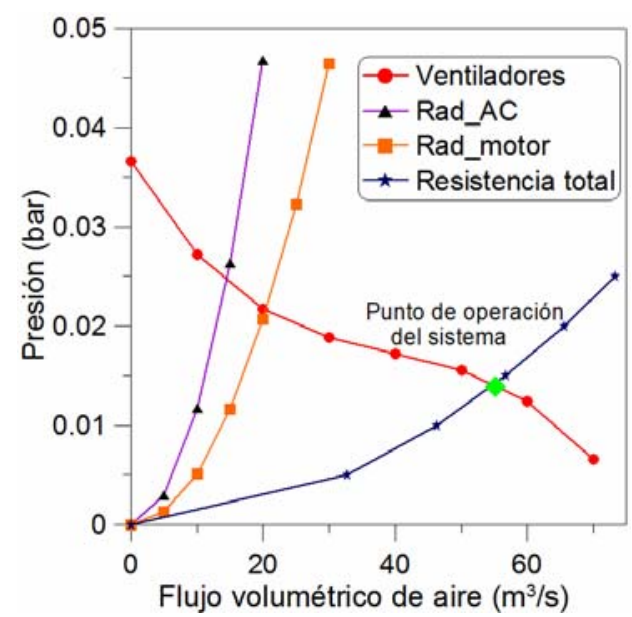

Figura 6.27. Equilibrio entre el tiro generado por los ventiladores y la resistencia al paso del aire para el punto de operación del sistema que satisface el balance térmico del motor y el enfriador de aire $(A C)$.

\subsubsection{Caracterización de los componentes de los circuitos del refrigerante}

En la tarea de caracterización se reúne toda la información mínima requerida por los submodelos del programa $1 \mathrm{D}$ en el cual se realiza el modelado y la simulación termohidráulica, y se caracteriza cada componente en la manera en que se requiere para alimentar el programa de modelado. Sin embargo, a efectos del modelado del circuito hidráulico y para acelerar la ejecución del programa de simulación, el circuito que contiene los componentes principales completos y con la identificación adecuada, simplifica previendo conservar las impedancias hidráulicas con elementos equivalentes pero en menor número, es decir, se limita el número de conexiones.

\section{Caracterización de las bombas}

Las bombas de los circuitos del motor (bomba 16G) y del enfriador de aire (bomba D) se caracterizan con sus curvas presión - caudal, según la información dada por el fabricante. Graficando en las características de las bombas los caudales nominales de operación para los circuitos $\left(0,0631 \mathrm{~m}^{3} / \mathrm{s} \mathrm{y}\right.$ $0,02524 \mathrm{~m}^{3} / \mathrm{s}$ para las bombas $16 \mathrm{G} \mathrm{y} \mathrm{D}$, respectivamente), se obtienen las 
presiones de las bombas para el régimen de motor de $950 \mathrm{rpm}$. Luego, utilizando las leyes de semejanza, se determinan los valores de las presiones (disponibilidad de presiones) y los caudales para el punto de máxima potencia del motor $(n=904 \mathrm{rpm})$. Para un punto de operación estable del sistema, la resistencia hidráulica (pérdidas de presión) debe igualarse a esa disponibilidad de presión del sistema de bombeo y con esta información se determinan las características equivalentes globales de pérdidas de presión por la expresión (4.37(b)) $\Delta p=K \cdot \dot{V}^{2}$. Las características de las bombas $16 \mathrm{G}$ y D son las representadas en la Figura 6.2 y la Figura 6.3, respectivamente.

La definición de las características equivalentes globales (a partir de la disponibilidad de las bombas) fija la máxima resistencia que puede imponerse en los circuitos, resistencia que debe repartirse entre los diferentes componentes concentrados y en las tuberías. Con estas consideraciones la característica de las pérdidas de presión permisibles para el circuito de refrigeración del motor queda definida por la expresión: $\Delta p_{\text {equiv_mot }}=1274 \cdot \dot{V}^{2}$. La característica de las pérdidas de presión permisibles para el circuito de refrigeración del enfriador de aire queda definida por la expresión $\Delta p_{\text {equiv_aft }}=8465,6 \cdot \dot{V}^{2}$.

\section{Caracterización térmica e hidráulica del motor, el enfriador de aire y los otros intercambiadores de calor}

El motor Diesel puede caracterizarse con la variación instantánea del calor disipado al refrigerante calculado con un modelo de predicción de temperaturas y flujos de calor (modelo térmico explicado en el capítulo tercero), pero para esto se necesitaría información de las variables instantáneas; lo mismo puede comentarse en relación al enfriador de aire. Ya en el apartado 6.2.2 se hallaron los calores aproximados evacuados por el motor y el enfriador de aire (1638,64 y 626,2 kW, respectivamente). El calor transferido por el motor comprende los calores intercambiados en el enfriador de aceite y el compresor, definidos en las especificaciones de la tarea como 439,21 y 105,41 kW, respectivamente. Por comparación con otros circuitos de refrigeración de locomotoras con motores de potencias similares se han tomado los valores para los coeficientes de resistencia de estos componentes. Cabe también comentar que de la exploración realizada de los valores de las pérdidas de presión externa permitidas en los motores comerciales similares, 
el margen dado para pérdidas externas en tuberías (las generadas por disposiciones de los radiadores según la aplicación) es del orden de $70 \mathrm{kPa}$ para los circuitos de refrigeración del bloque del motor y $35-40 \mathrm{kPa}$ para los circuitos de enfriador de aire.

El coeficiente de pérdidas de presión correspondiente a la camisa de refrigeración del motor se ha asumido como $0,6 \mathrm{mbar} \cdot \mathrm{h}^{2} / \mathrm{m}^{6}\left(7776 \mathrm{bar} \cdot \mathrm{s}^{2} / \mathrm{m}^{6}\right)$ y el área de paso se ha estimado en $0,002 \mathrm{~m}^{2}$. Para el enfriador de aire los valores correspondientes son $3,9 \mathrm{mbar} \cdot \mathrm{h}^{2} / \mathrm{m}^{6}\left(50544 \mathrm{bar} \cdot \mathrm{s}^{2} / \mathrm{m}^{6}\right)$ y $0,002 \mathrm{~m}^{2}$. En ausencia de información del proveedor del motor, estos valores se han tomado por comparación con los valores utilizados por [6.25].

Los valores de los coeficientes de pérdidas de presión y área del enfriador de aceite y del compresor se ajustan razonablemente también con información conocida en la literatura de elementos de este mismo tipo [6.25],[6.26].

Caracterización de los radiadores. Las pérdidas de presión del lado del refrigerante en los radiadores se obtuvieron durante el barrido conducente a la definición de las características térmicas. En la Figura 6.28 se ilustran las características obtenidas.

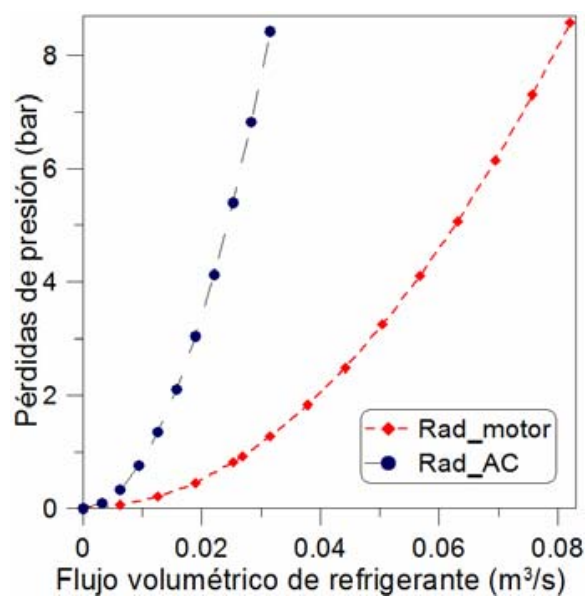

Figura 6.28. Pérdidas de presión al paso del refrigerante por los radiadores del motor y del enfriador de aire.

Para ambos radiadores el área representativa en el paso del refrigerante es igual al área de la boca de entrada, $A_{1}=0,00456 \mathrm{~m}^{2}$; el área representativa en el paso del aire es igual al área efectiva del panel del intercambiador, $A_{2}=$ 
2,6879 $\mathrm{m}^{2}$ y el diámetro hidráulico se toma igual al diámetro de la tubería, $d_{h}$ $=0,0762 \mathrm{~m}$.

\section{Caracterización de las tuberías y los accesorios}

Si el modelado que se va a hacer reproduce al detalle todos los accesorios y tuberías, lo importante está en caracterizar los componentes principales (motor, enfriador de aire, radiadores, intercambiadores adicionales y bombas), ya que el programa de modelado $1 \mathrm{D}$ dispone de una rica base de datos con la que se pueden caracterizar todos los accesorios (codos, uniones "T", tubos, mangueras, etc.). Como se comentó anteriormente, el propósito en la solución del problema que aquí se trata es modelar un sistema de refrigeración equivalente térmica e hidráulicamente, pero que pueda tener un reducido número de componentes. Esto hace que se deba resumir aquí sobre qué fundamentos se ha hecho esta simplificación.

Observando la topología de los circuitos del sistema, se ha realizado una reducción del sistema a un esquema con los mismos componentes principales, pero con un número reducido de conexiones. Se puntualizan las características de carga significativas en los ramales y subramales del circuito determinando las resistencias hidráulicas equivalentes de los tramos en serie y en paralelo y se validan los valores de los coeficientes de carga determinados por la ecuación de Colebrook. Para ilustración en la Figura 6.29 se muestra el esquema del subcircuito correspondiente al motor.

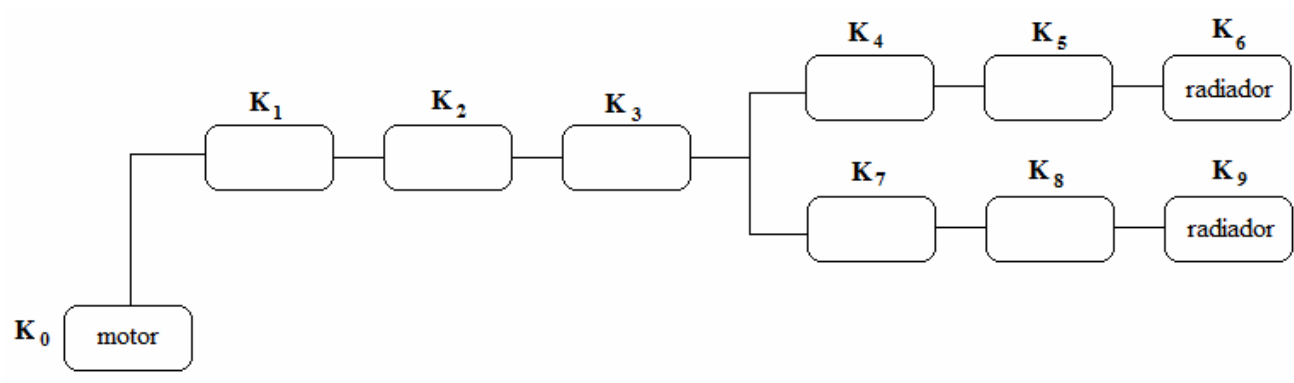

Figura 6.29. Simplificación del subcircuito del lado del motor.

Las pérdidas de presión por los radiadores del motor, caracterizadas en la Figura 6.28, quedan definidas por los coeficientes $K 6=K 9=1274 \mathrm{bar} \cdot \mathrm{s}^{2} / \mathrm{m}^{6}$. 
Las pérdidas de presión en las tuberías, según su longitud $L_{t}$ y diámetro $D_{t}$, se determinan tras resolver para cada una de ellas el coeficiente de Colebrook $\lambda$ [6.28]:

$$
\frac{1}{\sqrt{\lambda}}=-2 \log \left[\frac{\varepsilon}{3,7}+\frac{2,51}{\operatorname{Re} \sqrt{\lambda}}\right]
$$

donde $R e$ es el número de Reynolds, hallado como $\operatorname{Re}=v D_{t} / v, v$ es la velocidad del flujo, $v=\dot{V}_{\text {refr }} / A_{t}, \varepsilon$ es la rugosidad relativa. El coeficiente de Colebrook permite hallar las pérdidas de presión: $\Delta p=\rho \lambda L_{t} v^{2} / 2 D_{t} \mathrm{y}$, finalmente puede encontrarse el coeficiente de pérdidas de presión $K=\Delta p / \dot{V}_{\text {refr }}{ }^{2}$ para los elementos equivalentes $1-8$ en la Figura 6.29. El coeficiente $\lambda$ para todos los tubos tiene un valor de $0,018 \cong 0,02$, en la ecuación de Colebrook.

Para la unión en "Y", el coeficiente de pérdidas de presión K3 se halla como una pérdida singular. Luego de conocer los valores de todos los coeficientes de los componentes, se busca el coeficiente equivalente del sistema tras sucesivas sumas de resistencias en serie y en paralelo como se explica en el esquema de la Figura 6.30.

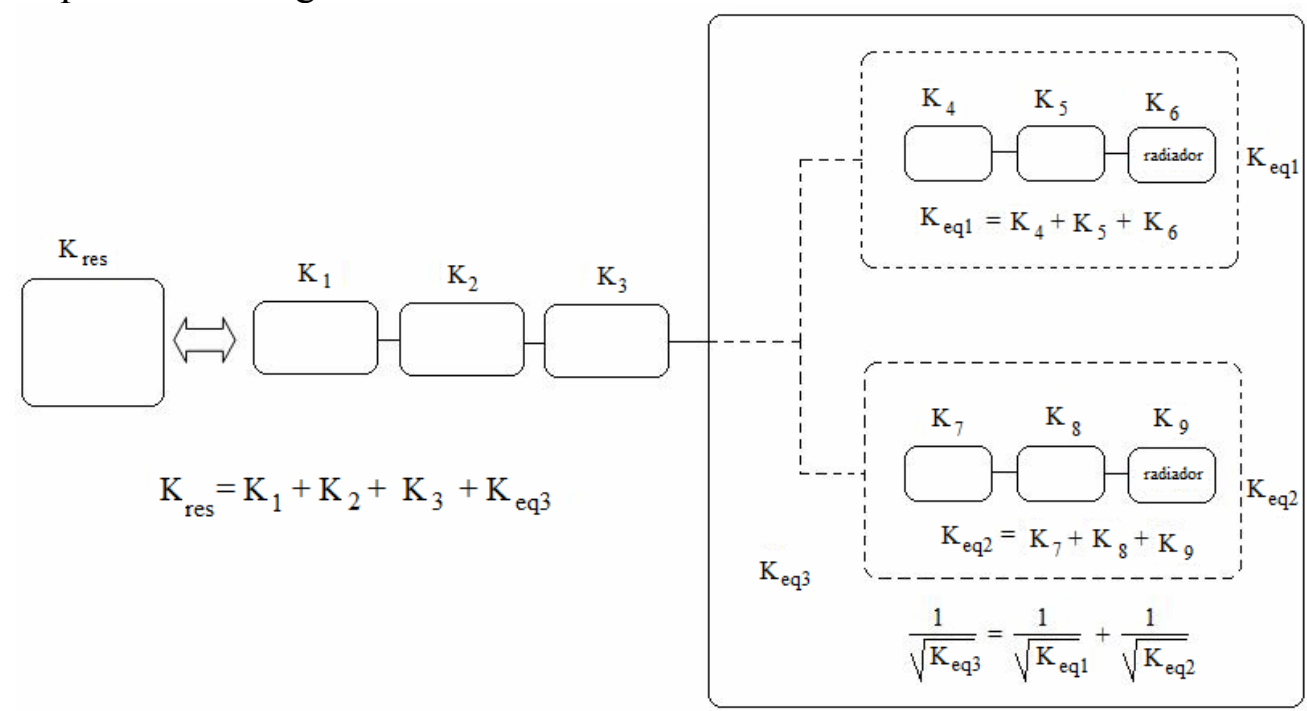

Figura 6.30. Reducción de las características de carga del subcircuito de refrigeración del motor. 
Los coeficientes de carga obtenidos son: $K_{\text {eq1 }}=1309 \mathrm{bar} \cdot \mathrm{s}^{2} / \mathrm{m}^{6}, K_{\text {eq2 }}=1309$ bar $\cdot \mathrm{s}^{2} / \mathrm{m}^{6}, K_{\text {eq } 3}=327 \mathrm{bar} \cdot \mathrm{s}^{2} / \mathrm{m}^{6}$ y $K_{\text {res }}=1334 \mathrm{bar} \cdot \mathrm{s}^{2} / \mathrm{m}^{6}$. En realidad estos coeficientes no se necesitan sino para hallar un esquema equivalente más sencillo del circuito y en el programa de modelado 1D no se introducen, a menos que en lugar de poner tuberías en el circuito reducido se pusieran pérdidas discretas (en tal caso los coeficientes hallados deberían dividirse por la densidad y el inverso del cuadrado del área: $\left.K_{D L}=K /\left(\rho / A^{2}\right)\right)$.

Para asegurar la correspondencia del modelo reducido y de los componentes equivalentes que lo integran, antes de componer el modelo en el programa 1D se han procesado las características de carga del sistema y de presión de las bombas en un proceso manual. En la Figura 6.31 se ven dichas características para los subcircuitos del motor y del enfriador de aire.
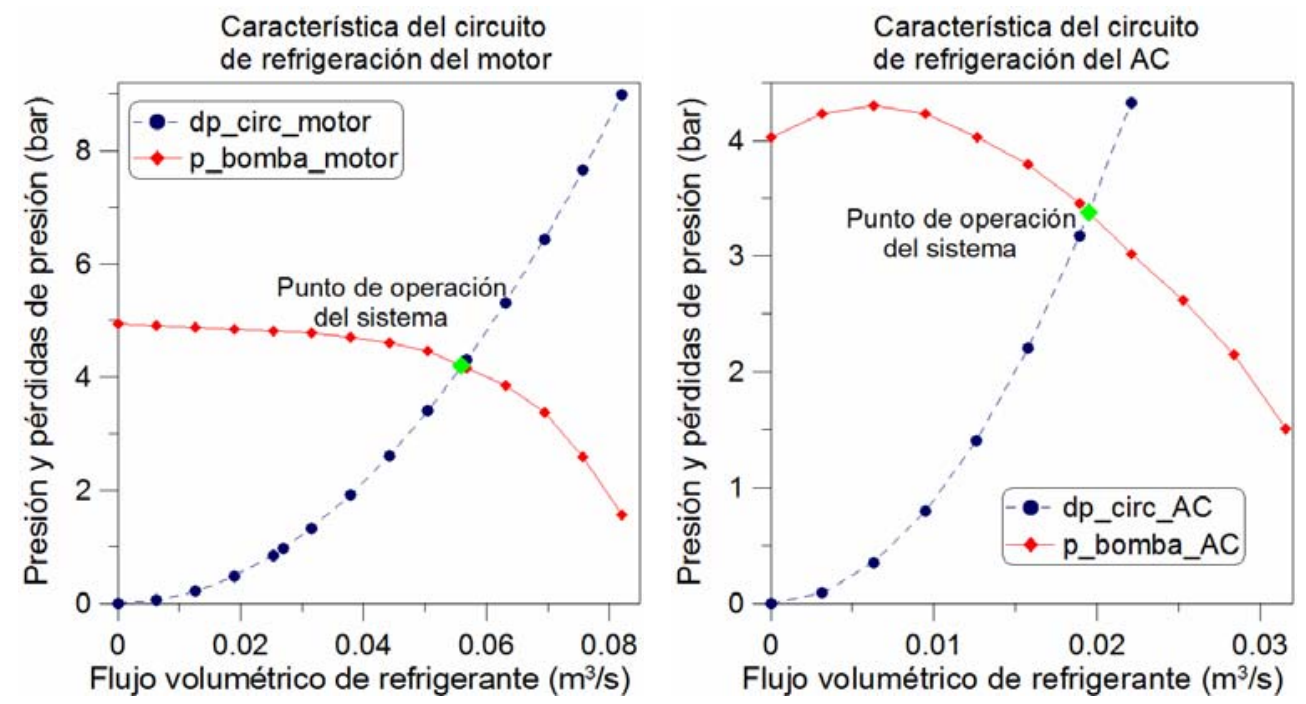

Figura 6.31. Solución gráfica de los subcircuitos del sistema de refrigeración.

\subsubsection{Modelado en FlowMaster del sistema de refrigeración del motor de la locomotora}

Teniendo en cuenta las consideraciones anteriores, el sistema de refrigeración obtenido del motor de la locomotora toma la presentación mostrada en la Figura 6.32. 


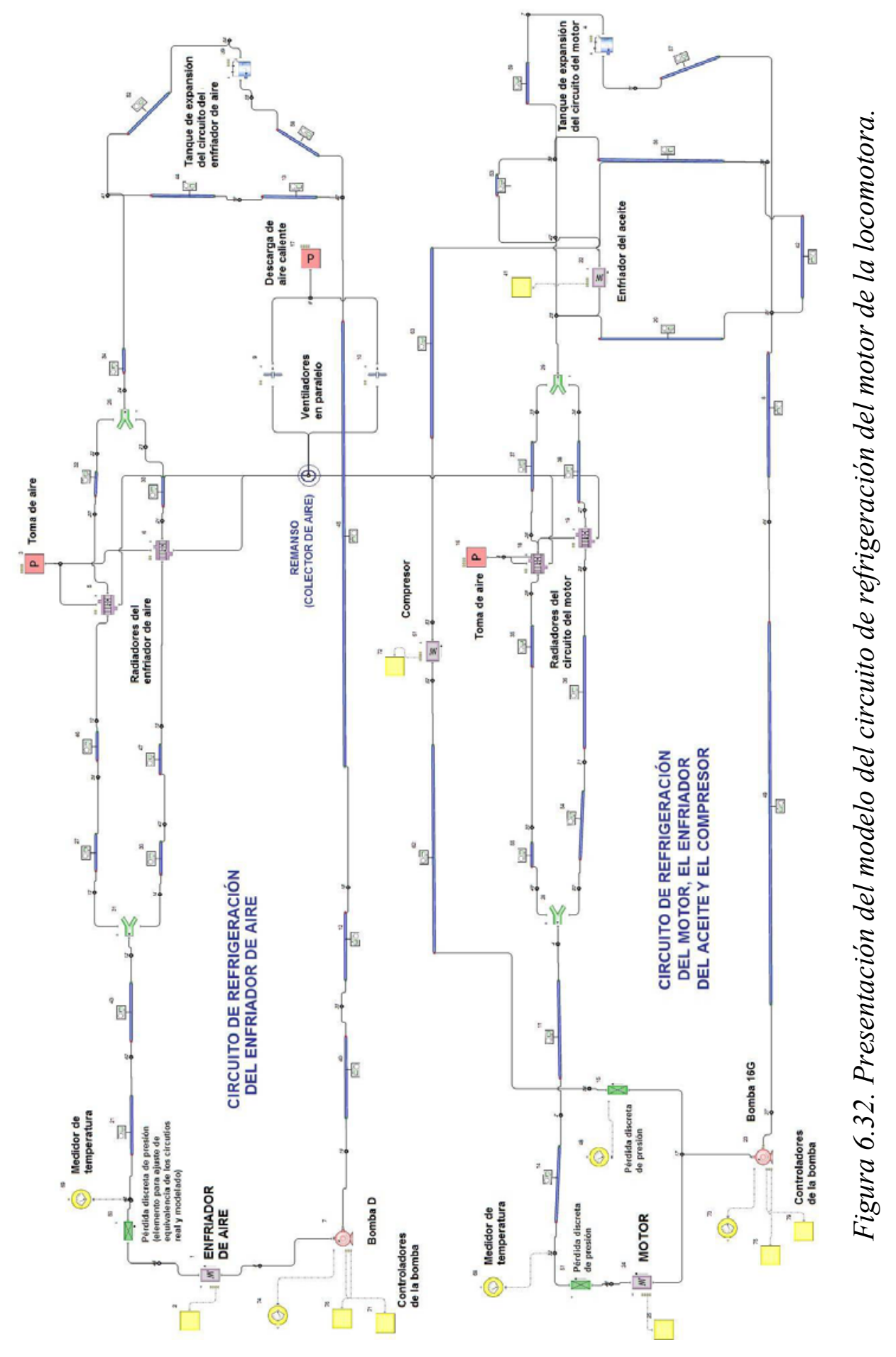


Los ramales hacia los radiadores tanto del motor como del enfriador de aire tienen prácticamente la misma configuración, excepto por las conexiones hacia el compresor de aire y hacia el enfriador de aceite. A la salida de los radiadores los flujos tanto de refrigerante como de aire confluyen y se dirigen hacia la succión de las bombas y de los ventiladores, respectivamente.

En el modelo, en la manera como se obtienen las pérdidas de presión, se logra que los caudales que fluyen sean los adecuados y no hay mucha preocupación en relación a la repartición concentrada de las pérdidas de presión, pues el circuito se modela con un circuito equivalente de menor número de accesorios. Entre los elementos equivalentes se han introducido pérdidas discretas de presión, que llevan la misma información hidráulica que un bloque de motor o un intercambiador de calor. Es decir, en una conexión de resistencias en serie en la que importa de forma neta la repartición de caudales, el método es válido. No lo es, si se requiere rigor en el análisis de las presiones en todos los componentes.

En el modelo simplificado se han conservado las tuberías de mayor longitud. Las más cortas y algunos elementos de unión y codos se han reemplazado por elementos de pérdidas concentradas como pérdidas discretas. Las pérdidas discretas de presión se configuran con un área de sección de paso y los coeficientes de pérdidas de presión bajo flujo directo y reverso. Es común la utilización de estos elementos en los circuitos hidráulicos a manera de "potenciómetros", para ajustar los caudales a los requeridos. También pueden usarse para ajustar las presiones en los sistemas y determinar las características de componentes ausentes que deban incorporarse al sistema físico.

Las uniones en "Y" se configuran a partir de la geometría de los elementos reales. Se les configuran los diámetros de los terminales, el ángulo entre los ramales menores y los coeficientes de pérdidas de presión para las direcciones posibles y en función del tipo de combinación en la que la unión opera (dividiendo o combinando flujos, etc.). Para definir los coeficientes de pérdidas se utilizan las funciones de pérdidas de presión de Miller [6.24].

Las tuberías del sistema son tuberías metálicas de acero laminado, con rugosidad del orden de $0,025 \mathrm{~mm}$. Al configurar su submodelo en el programa, se entran el diámetro y su longitud, la opción de cálculo de la 
fricción (Colebrook-White), la rugosidad interna del tubo, el coeficiente de fricción de Hazen-Williams, el factor de fricción y el número de nodos internos, requeridos para los cálculos hidráulicos, así como el tipo de material, el espesor de la pared, la temperatura externa y el coeficiente de transferencia de calor externo, requeridos para los cálculos térmicos.

El motor, el enfriador de aire, el enfriador de aceite y el compresor se modelan mediante intercambiadores genéricos, caracterizados por un área de paso, un coeficiente de pérdidas y un calor intercambiado fijo, suministrado por un elemento controlador. A través del controlador se puede entregar un perfil transitorio de calor disipado, si éste tuviera una variación temporal. Es la manera apropiada para mostrar la cantidad de calor intercambiado. Para propósitos de este trabajo los calores se entregaron mediante una tabla. Se ha asumido una entrega secuencial de los calores al refrigerante conducente a un calentamiento, desde un valor cero hasta el valor nominal del componente en el tiempo de 200 segundos. Se aclara que esto se hace para ilustración, pero que también sirve para explicar que si se tiene la variación en el tiempo del calor entregado al refrigerante, una forma de describir esto en el programa es mediante este tipo de controlador.

Las características de las bombas pueden introducirse como curvas sencillas de bomba (las dos características, la de presión contra caudal y la de par contra caudal), o también valiéndose de las características disponibles en FlowMaster en su base de datos. También se ingresan los valores de referencia de la presión y el caudal, correspondientes al punto nominal de operación de la bomba, así como los valores de inercia del rotor y del motor.

El procedimiento para introducir la información de los ventiladores es similar al utilizado para introducir las características de pérdidas de cualquier componente. Se ingresa la característica de crecimiento de la presión (bar) del aire con relación al flujo volumétrico $\left(\mathrm{m}^{3} / \mathrm{s}\right)$. Se asigna el diámetro externo de las aspas, el área de barrido o área neta de succión, la velocidad, y se especifica el punto de operación para el cual la característica seleccionada es la especificada

Los radiadores se caracterizan suministrando la superficie característica de la capacidad de transferencia de calor por diferencia de temperatura de entrada nominal referida al área del núcleo del radiador $\left(Q /\left(I T D \cdot A_{f a}\right)\right.$ en función de 
los flujos másicos de refrigerante y de aire por unidad de área frontal (figuras 5.28). La característica que se introduce corresponde a condiciones estándar, ya el programa automáticamente se acopla a la variación de las condiciones según evolucionen durante la simulación.

Otros elementos presentes en el modelo de la Figura 6.32 son los controladores y los medidores, a través de los cuales se le pasa información a los elementos principales y que se pueden configurar por programación mediante código. Mediante controladores en el modelo se fijan los valores de los calores a intercambiar en las fuentes térmicas y el estado y las revoluciones de las bombas y los ventiladores. Se han colocado medidores para conocer las temperaturas (variable de interés) a la salida del enfriador de aire, del motor y de la bomba.

Para propósitos de ilustración en la Figura 6.33 se destaca la conexión de los dos ventiladores en paralelo en el sistema, el componente 17 simula la atmósfera arriba de la locomotora; a este componente sólo se le define el tipo de fluido con el que opera, la presión y la temperatura. En la Figura 6.34 se muestra el cuadro de diálogo de los parámetros que se ingresan del ventilador.

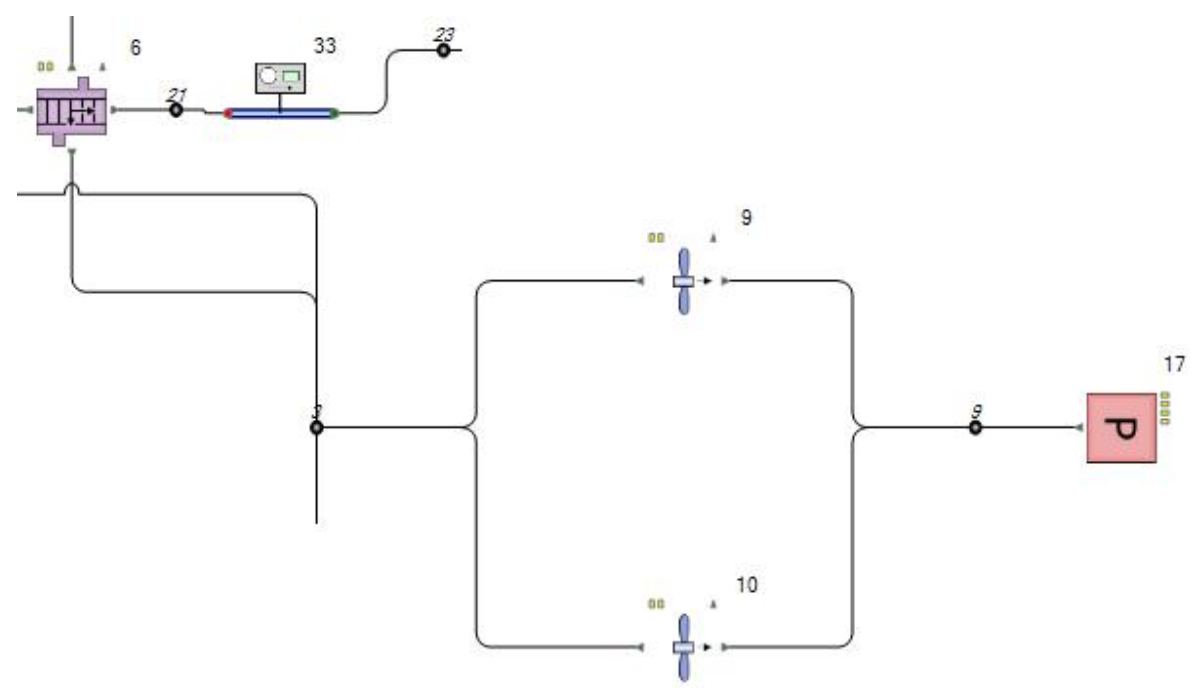

Figura 6.33. Detalle de los dos ventiladores en paralelo. 

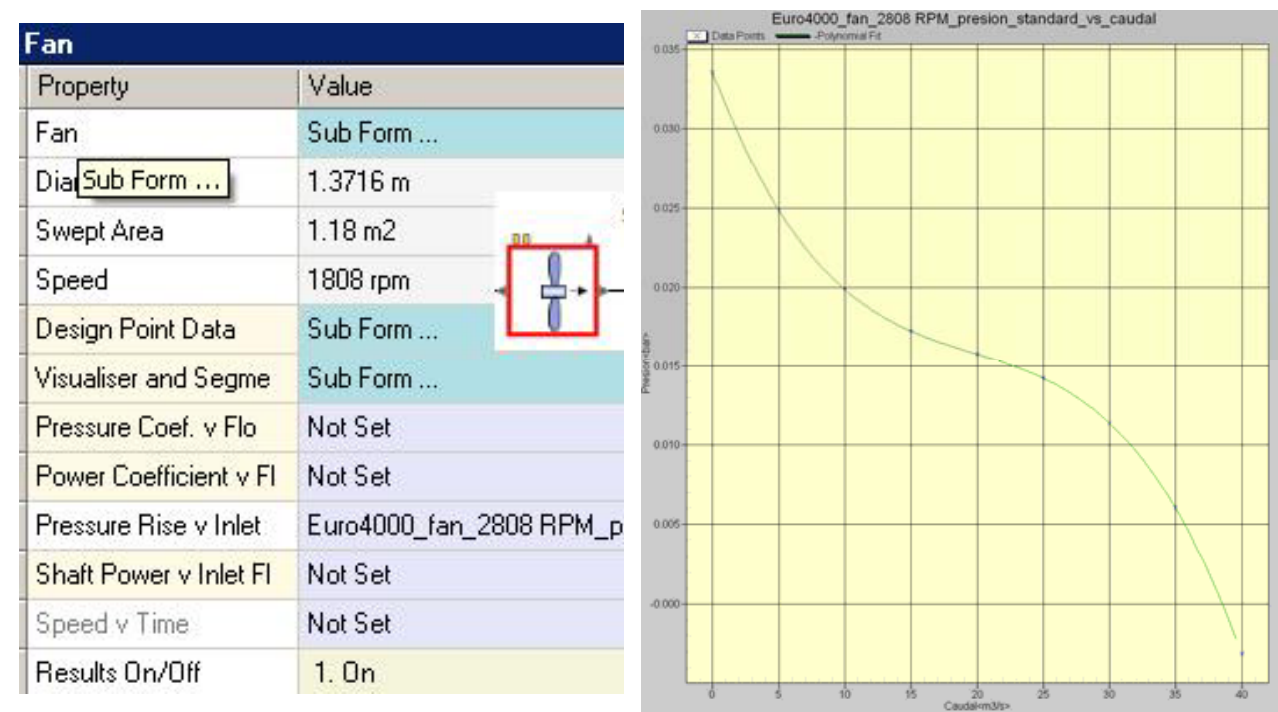

Figura 6.34. Caracterización del ventilador empleado en el sistema de refrigeración.

Se introducen los parámetros diámetro externo de las aspas, área de succión, las revoluciones y la característica de presión contra flujo (caudal volumétrico).

\section{Resultados de la simulación}

Construido el modelo y caracterizados los componentes, el primer paso de la simulación consiste en verificar la operación hidráulica del sistema, comprobando la obtención de los flujos de refrigerante y aire requeridos. Luego se realiza la simulación del proceso transitorio térmico. Como resultado de la simulación es posible conocer los caudales que pasan por cada componente y la energía transferida. De especial interés para el modelo aquí presentado es el análisis del comportamiento de la temperatura del aire y el refrigerante. Como la simulación se ha hecho para un punto constante de operación del sistema, bien podría prescindirse del despliegue gráfico de los resultados, pero para fines ilustrativos, a continuación se presentan las gráficas de resultados más relevantes según los propósitos del trabajo aquí presentado.

Los caudales de refrigerante (2) y de aire (4) obtenidos en los ramales del radiador del motor y del enfriador de aire se visualizan en la Figura 6.35. 


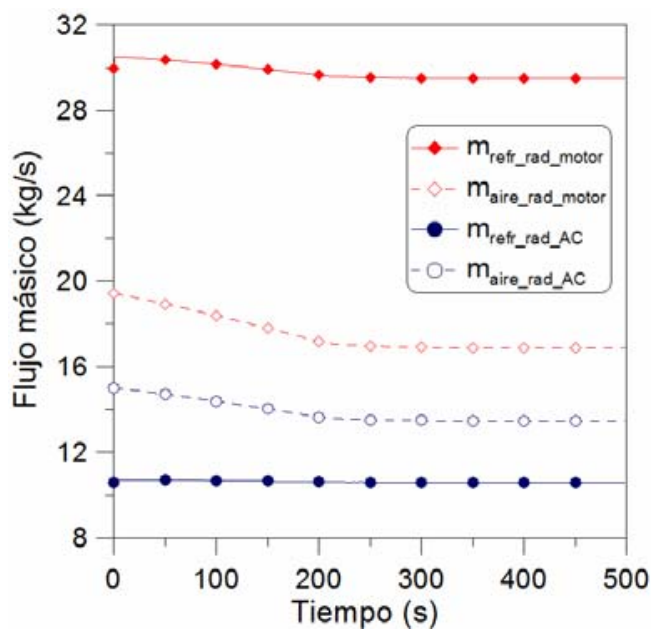

Figura 6.35. Flujos de aire y refrigerante a través de los radiadores del motor y del "aftercooler".

Los calores entregados al sistema de refrigeración se reflejan en la gráfica de la Figura 6.36. Estos calores fueron los aportados por el fabricante. En la misma figura se han ubicado las gráficas de los calores disipados por cada uno de los radiadores del motor y del enfriador de aire.
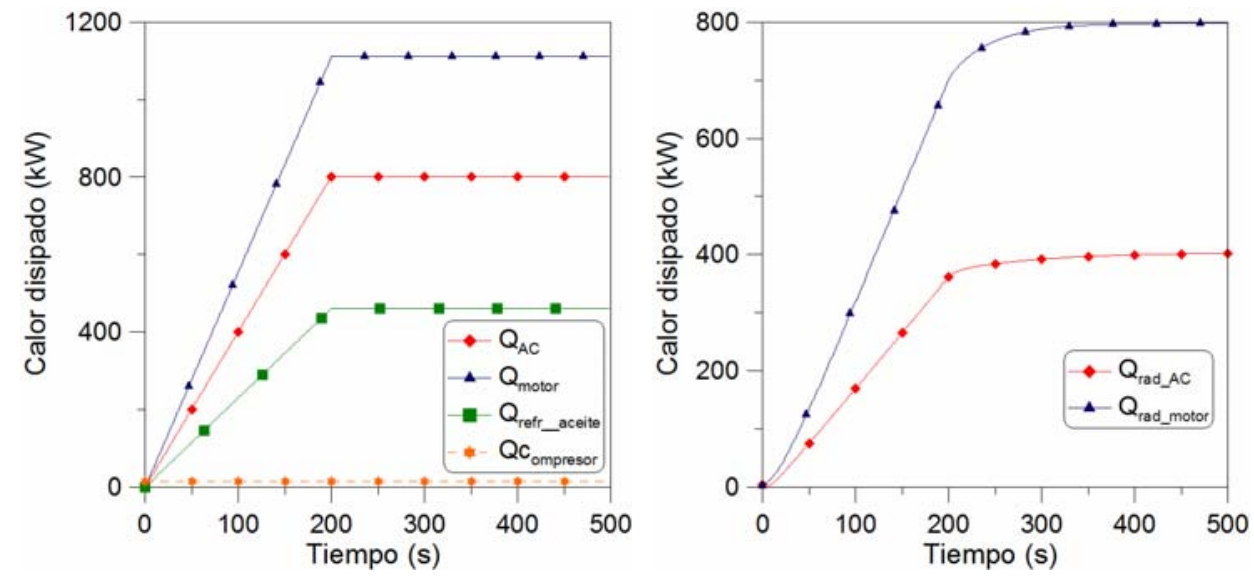

Figura 6.36. Calor aportado por el motor, el aftercooler, el compresor y el refrigerador de aceite al sistema de enfriamiento. A la derecha se ven las gráficas de los calores disipados por los radiadores.

En la Figura 6.37 se observan los resultados relativos a las temperaturas de entrada y salida de los fluidos al paso por uno de los radiadores del motor. 
Las gráficas correspondientes para uno de los radiadores del enfriador de aire se presentan en la Figura 6.38. Por los resultados del modelo se puede ver que las temperaturas de los fluidos se mantienen en valores aceptables, según las condiciones establecidas en la tarea técnica.

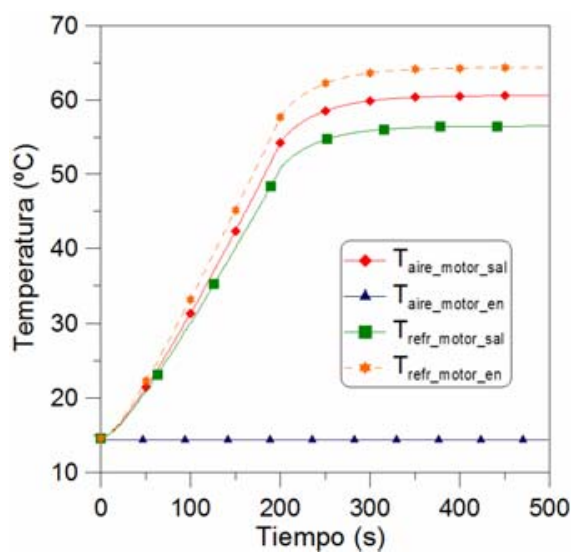

Figura 6.37. Temperaturas en los ramales de aire y refrigerante en uno de los radiadores del motor.

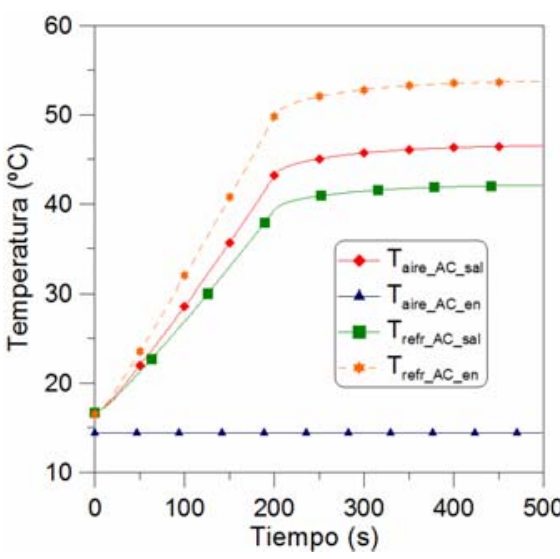

Figura 6.38. Temperaturas en los ramales de aire y refrigerante en uno de los radiadores del enfriador

\subsubsection{Alcance de resultados de los modelos}

Si bien el modelado de un sistema real puede tener sus ventajas, es importante ser consciente de las incertidumbres que la comparación de los resultados de la simulación con los resultados experimentales puede generar. Las comparaciones entre resultados experimentales y resultados de simulación pueden aceptarse con desviaciones de $+/-10 \%$. Esta desviación es prudente, dado que son muchas las variables que intervienen en el modelo y en todas ellas hay un rango de incertidumbre. Por ejemplo, si se considera la ecuación básica de disipación de calor en un intercambiador de calor, el cual es función de las propiedades del fluido, el flujo másico y las temperaturas de entrada y salida:

$$
\dot{Q}=\dot{m} C_{p}\left(T_{2}-T_{1}\right)=\dot{m} C_{p} \Delta T,
$$

se puede determinar la desviación o error posible, tras descomponer en la serie de Taylor y dejar sólo los términos de primer orden, como:

$$
\varepsilon \dot{Q}_{\Sigma}=(\partial \dot{Q} / \partial \dot{m}) \varepsilon \dot{m}+\left(\partial \dot{Q} / \partial C_{p}\right) \varepsilon C_{p}+(\partial \dot{Q} / \partial \Delta T) \varepsilon T
$$


Habiendo reemplazado los valores de las derivadas parciales en la expresión se obtiene:

$$
\varepsilon \dot{Q}_{\Sigma}=\left(C_{p} d T\right) \varepsilon \dot{m}+(\dot{m} d T) \varepsilon C_{p}+\left(m C_{p}\right) \varepsilon T
$$

Dividiendo ambos medios de esta expresión por $\dot{Q}=m C p d T$ pueden determinarse las desviaciones relativas:

$$
\varepsilon Q / Q=\varepsilon \dot{m} / \dot{m}+\varepsilon C_{p} / C_{p}+\varepsilon T / T
$$

Si se reemplazan las desviaciones de flujo, calor y temperaturas del modelo por las tolerancias de las variables en la última ecuación, se obtendría la contribución discreta de las desviaciones de las variables a la desviación total del flujo de calor predicho, no considerando las interacciones. Lo correcto es pensar que el modelo trabaja con unas tolerancias. Si se asumen las desviaciones, por ejemplo desviaciones para las variables: $\varepsilon \dot{m}=3 \%$; $\varepsilon C_{p}=0,012 ; \varepsilon T=1,5^{\circ} C$, entonces la desviación del flujo de calor predicho puede alcanzar el valor de $\varepsilon \dot{Q}_{\Sigma}=214,45 \mathrm{~kW}$.

Para apoyar un poco más este valor de desviación, podemos comparar con otras simulaciones realizadas con el mismo programa [6.23]. Se reproduce aquí una gráfica de barras, Figura 6.39, que compara temperaturas predichas con temperaturas experimentales en un sistema de Caterpillar (no tiene nada que ver con un motor de locomotora, pero sirve sólo para apoyar la admisibilidad de las desviaciones de temperaturas).

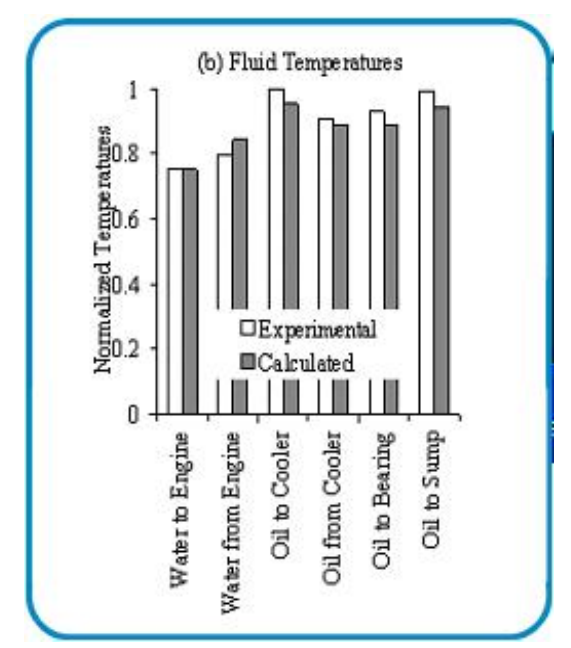

Figura 6.39. Comparación entre las temperaturas medidas $y$ predichas en un motor Caterpillar [6.23]. 
En dicho trabajo, se encontró que más del $95 \%$ de las predicciones de temperatura del modelo estaban dentro de un margen de error del $10 \%$ con relación a las mediciones experimentales del aire, el refrigerante, el lubricante y las temperaturas de superficie, validándose así la metodología analítica.

\subsection{Validación experimental del sistema de refrigeración del motor de la locomotora}

Diseñado, modelado y simulado el sistema de refrigeración, el paso siguiente es su validación experimental, la cual puede realizarse ó realizando mediciones en la vía, lo cual es costoso y complicado, o bien realizando las pruebas en una instalación apropiada para el efecto. En la empresa fabricante de las locomotoras se dispone de un banco de carga resistivo, al cual puede acoplarse la salida del generador de la locomotora para someterla a las posibles cargas y ciclos de prueba propias de su operación normal. También, de manera alternativa, el sistema de control de tracción de la locomotora permite un modo de operación denominado de autocarga con la unidad EM200, al cual puede recurrirse para la prueba. En el caso en estudio, el vagón de tracción de la locomotora con todos los sistemas del motor, generador eléctrico y motores conductores se dispuso en la sala de pruebas como lo ilustra la Figura 6.40.

El ensayo de la locomotora tiene por fin evaluar la capacidad y desempeño del sistema de refrigeración, valorado por la capacidad disipativa de los radiadores a plena carga y máxima velocidad de operación del motor. En la prueba se determina la disipación de calor del motor y del enfriador de aire, las temperaturas de operación del refrigerante, y la capacidad de disipación de calor del enfriador del aceite. Adicionalmente, la prueba sirve para comparar $\mathrm{y}$ validar los resultados de la simulación y para realimentar los procedimientos de la metodología de modelado y simulación aplicada en este capítulo.

La prueba de la locomotora se realizó en las condiciones climáticas naturales y por tanto no se reproducen las condiciones más exigentes. Tras validar el modelo, éste servirá para reproducir esas condiciones. 

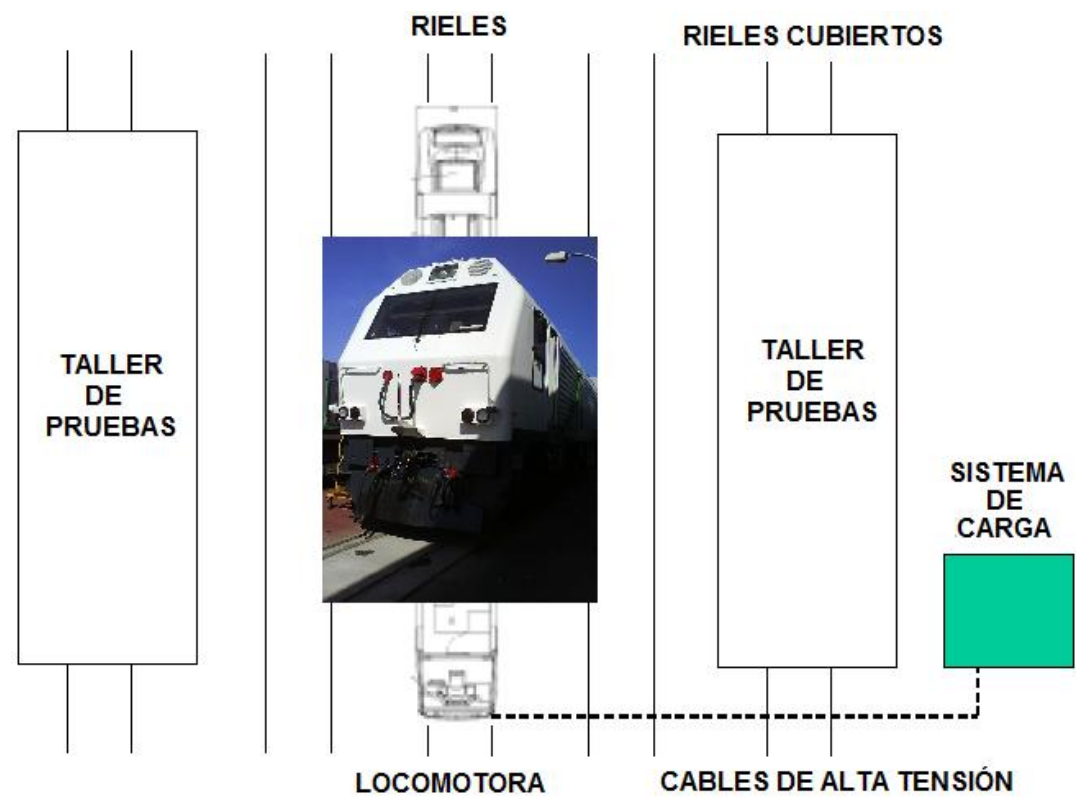

Figura 6.40. Disposición esquemática de la locomotora EURO4000 en la sala de pruebas.

\subsubsection{Instrumentación del sistema de refrigeración}

A lo largo de los ramales de los circuitos del sistema de refrigeración se instalaron medidores de presión y caudal para calcular la energía transportada y los calores transferidos en los componentes. También en el sistema de ventilación se instalaron medidores de velocidad de aire para medir el flujo de aire al paso por los radiadores y termopares para la medición de la temperatura.

En la Figura 6.41 se presenta el plano general de diseño del sistema de refrigeración con indicaciones de las posiciones de medida de temperaturas $(\mathrm{T})$, presiones $(\mathrm{P})$ y caudales $(\mathrm{F})$ de refrigerante. En las caras anterior y posterior del banco izquierdo de radiadores se posicionaron termopares como muestra la Figura 6.42, para la medición de la temperatura del aire. Las señales de los termopares fueron grabadas mediante un sistema de adquisición de datos, un osciloscopio y un ordenador portátil. En la Tabla 6.5 se resume la instrumentación utilizada en la prueba. 

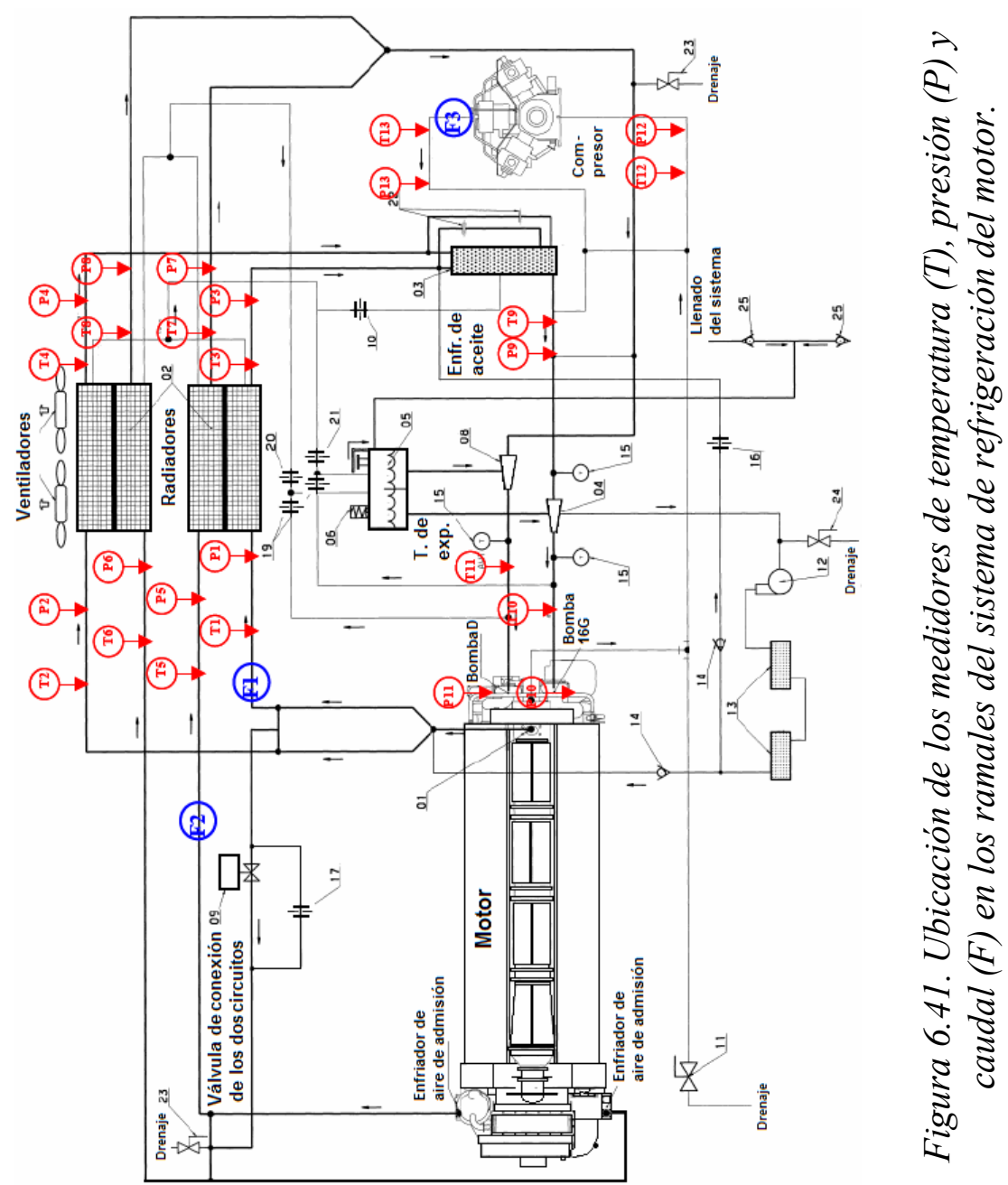
Tabla 6.5. Equipos e instrumentación utilizados en la prueba de la locomotora.

\begin{tabular}{|c|c|c|c|}
\hline Unid. & Elemento & Tipo & Función \\
\hline 2 & $\begin{array}{l}\text { Medidores de } \\
\text { caudal }\end{array}$ & $\begin{array}{l}\text { Siemens } \\
\text { MAG } 1100 \\
\text { DN80 }\end{array}$ & $\begin{array}{l}\text { Flujo refrigerante por } \\
\text { radiadores izquierdos de } \\
\text { motor y "aftercooler" }\end{array}$ \\
\hline 1 & $\begin{array}{l}\text { Medidor de } \\
\text { caudal }\end{array}$ & $\begin{array}{l}\text { Siemens } \\
\text { MAG } 1100 \\
\text { DN25 }\end{array}$ & $\begin{array}{l}\text { Flujo refrigerante por } \\
\text { compresor }\end{array}$ \\
\hline 13 & $\begin{array}{l}\text { Transductores } \\
\text { de presión } \\
\text { piezo-resistivos }\end{array}$ & $\begin{array}{l}\text { Danfoss y } \\
\text { Cerobar. } \\
\text { rango de } 0- \\
10 \text { bar }\end{array}$ & $\begin{array}{l}\text { Medición presión del } \\
\text { refrigerante. }\end{array}$ \\
\hline 13 & $\begin{array}{l}\text { Termopares } \\
\text { apantallados }\end{array}$ & Tipo K & $\begin{array}{l}\text { Medición de temperatura } \\
\text { del refrigerante en } \\
\text { diferentes puntos del } \\
\text { sistema. }\end{array}$ \\
\hline 21 & $\begin{array}{l}\text { Termopares } \\
\text { apantallados }\end{array}$ & Tipo K & $\begin{array}{l}\text { Medición de temperatura } \\
\text { de aire en los radiadores, } \\
\text { ducto de ventilación y } \\
\text { ambiente. }\end{array}$ \\
\hline 1 & $\begin{array}{l}\text { Sistema de } \\
\text { adquisición de } \\
\text { datos de } 30 \\
\text { canales }\end{array}$ & $\begin{array}{l}\text { IO Tech } \\
\text { Personal } \\
\text { Daq/55 }\end{array}$ & $\begin{array}{l}\text { Registro de variables } \\
\text { medidas. }\end{array}$ \\
\hline 1 & $\begin{array}{l}\text { Registrador de } \\
\text { información y } \\
\text { osciloscopio de } \\
16 \text { canales }\end{array}$ & Sefram 8420 & $\begin{array}{l}\text { Registro de variables } \\
\text { medidas. }\end{array}$ \\
\hline 1 & $\begin{array}{l}\text { Fuente de } \\
\text { alimentación }\end{array}$ & $\begin{array}{l}220 \mathrm{VAC}- \\
24 \mathrm{VDC}\end{array}$ & $\begin{array}{l}\text { Alimentación } \\
\text { instrumentación }\end{array}$ \\
\hline \multirow[t]{2}{*}{2} & $\begin{array}{l}\text { Columnas de } \\
\text { agua }\end{array}$ & & $\begin{array}{l}\text { Medición de caída de } \\
\text { presión del aire. }\end{array}$ \\
\hline & Anemómetro & $\begin{array}{l}\text { PROVA } \\
\text { AVM-03 }\end{array}$ & $\begin{array}{l}\text { Medición de velocidad } \\
\text { del aire. }\end{array}$ \\
\hline
\end{tabular}



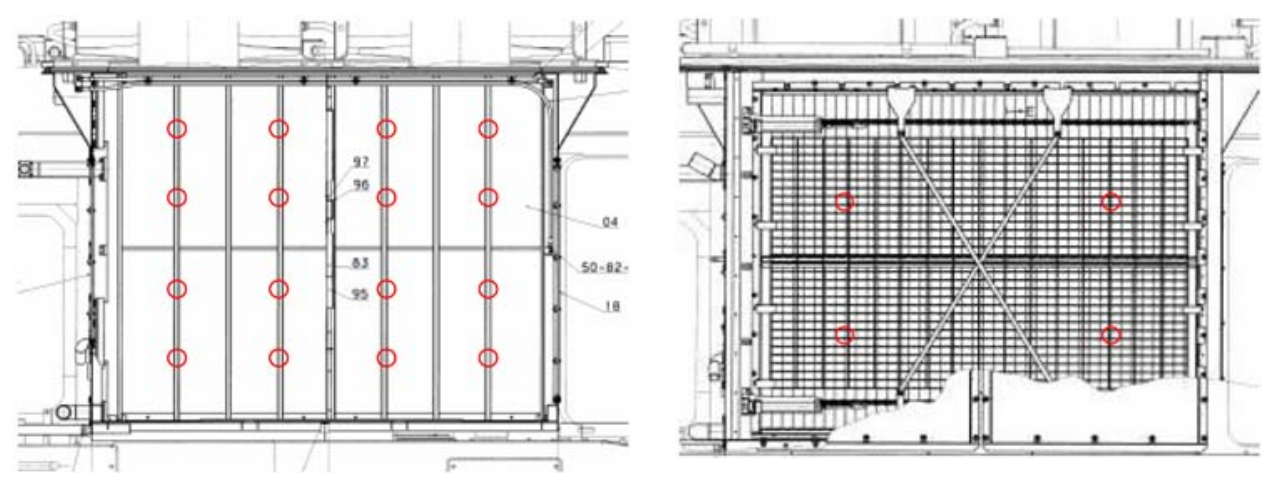

Figura 6.42. Posicionamiento de los termopares en las caras anterior y posterior de uno de los bandos de radiadores. En la parte superior se ubica el radiador del circuito del motor y en la parte inferior el correspondiente del circuito del enfriador de aire.

Algunos detalles de presentación de la instrumentación empleada se ilustran en las fotografías de la Figura 6.43.
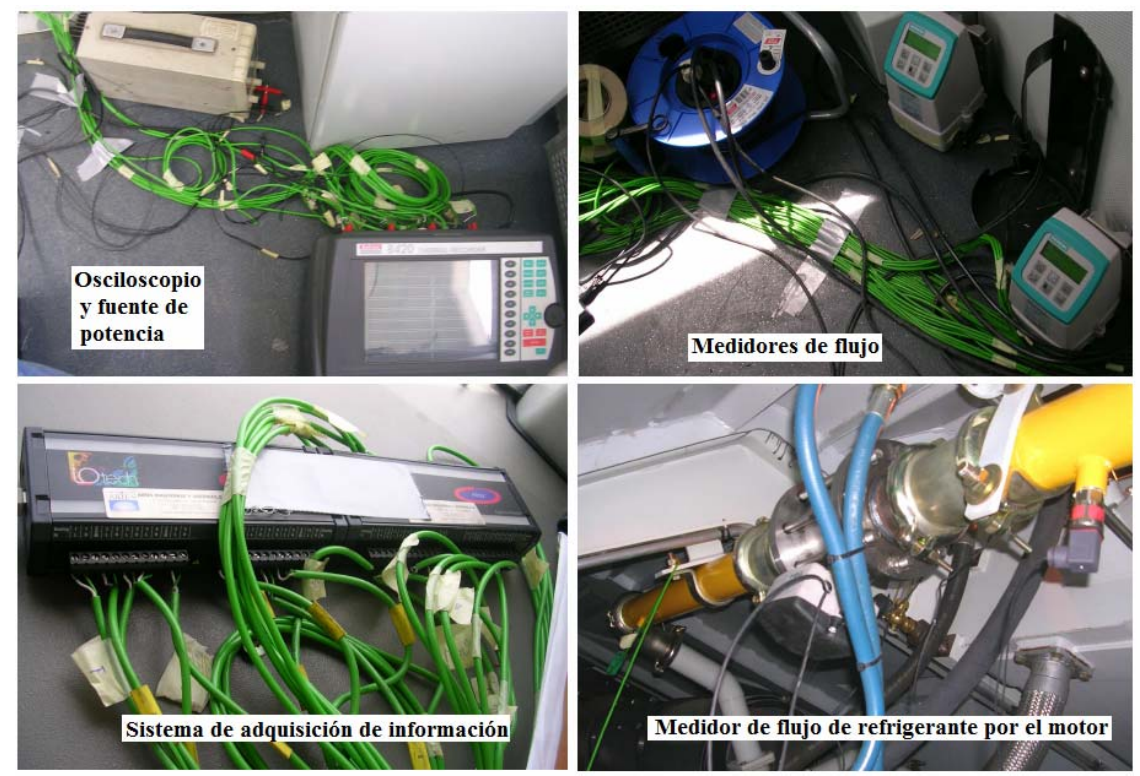

Figura 6.43. Instrumentación empleada en la medición de las variables del sistema de refrigeración. 
En el remanso del sistema de ventilación se posicionaron medidores de presión y velocidad del flujo de aire. En la Figura 6.44 se presenta una vista transversal del sistema de ventilación, en la que se marcan las posiciones de medida. A la derecha puede verse la utilización de un medidor de columna de agua para tomar una medición promediada de la presión en los puntos de medida.
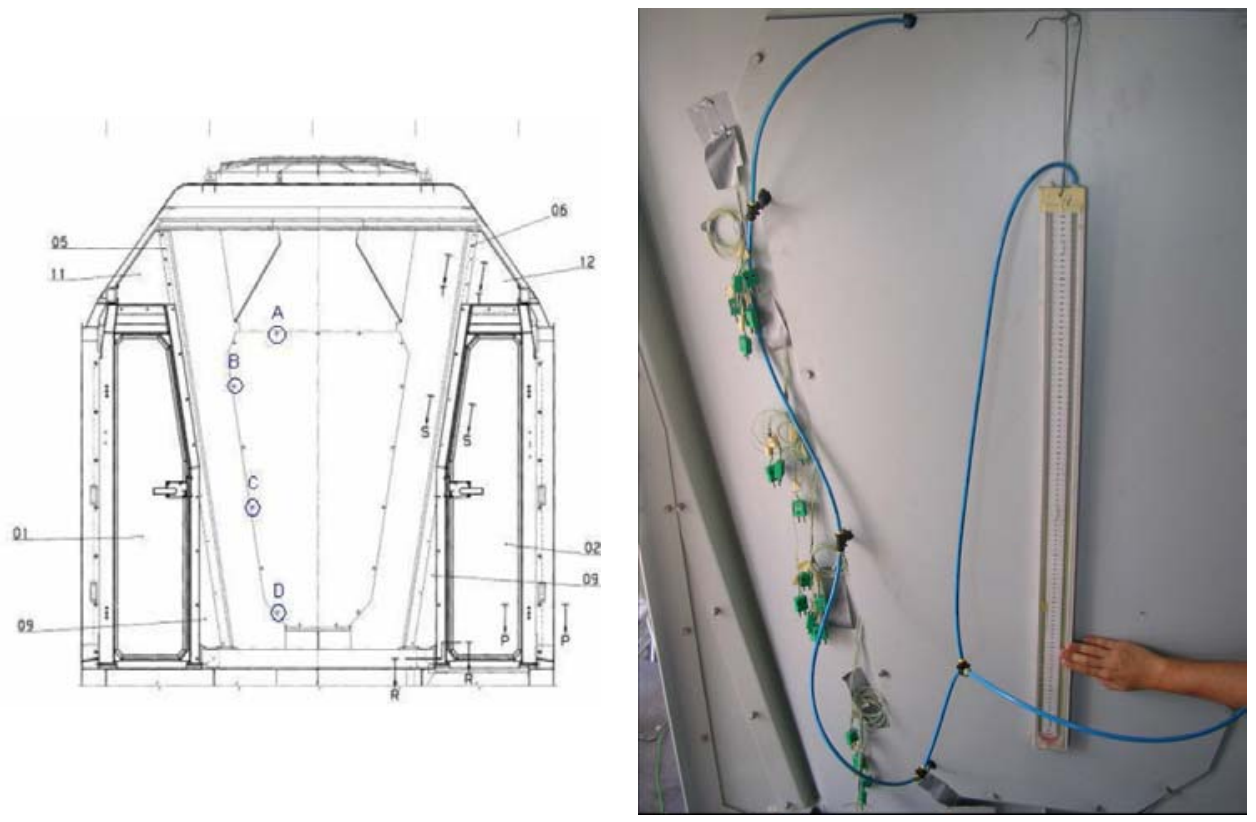

Figura 6.44. Distribución de los medidores de presión en el remanso de los radiadores.

\subsubsection{Resultados de las mediciones en el sistema de refrigeración operando el motor a plena carga}

Instalado el vagón de tracción de la locomotora en la sala de pruebas, bajo las condiciones detalladas en la Tabla 6.6, se estabilizaron las temperaturas y los flujos de refrigerante y aire como se reporta en la Tabla 6.7. 
Tabla 6.6. Condiciones iniciales de la prueba a plena carga.

\begin{tabular}{|l|lr|}
\hline \multicolumn{2}{|l|}{ Propiedades del fluido (bajo condiciones de prueba) } \\
\hline \multirow{4}{*}{ Refrigerante } & Tipo & agua/etilenglicol \\
& Mezcla & $50 / 50 \%$ \\
& Densidad & $-0,5506 \cdot T+1089,6 \mathrm{~kg} / \mathrm{m}^{3}$ \\
& Calor específico, $\mathrm{kJ} / \mathrm{kgK}$ & $0,0037 \cdot T+3,1879$ \\
\hline Aire (estimadas a & Densidad, $\mathrm{kg} / \mathrm{m}^{3}$ & 1,15 \\
$\left.T_{\text {aire }}^{m}=40^{\circ} \mathrm{C}\right)$ & Calor específico, $\mathrm{kJ} / \mathrm{kgK}$ & 1,01 \\
\hline Temperatura ambiente, ${ }^{\circ} \mathrm{C}$ & 15 \\
Presión ambiente, $\mathrm{kPa}$ & 101,3 \\
\hline
\end{tabular}

Tabla 6.7. Valores medios estabilizados de las temperaturas y los flujos de aire y refrigerante medidos durante la prueba a plena carga.

\begin{tabular}{lr}
\hline Radiador izquierdo del motor & \\
\hline Temperatura de entrada del refrigerante, ${ }^{\circ} \mathrm{C}$ & 61,9 \\
Temperatura de salida del refrigerante, ${ }^{\circ} \mathrm{C}$ & 54,8 \\
Flujo másico de refrigerante, $\mathrm{kg} / \mathrm{s}$ & 28,46 \\
Temperatura entrada del aire, ${ }^{\circ} \mathrm{C}$ & 14,43 \\
Temperatura de salida del aire, ${ }^{\circ} \mathrm{C}$ & 56,78 \\
Flujo másico medio medido de aire, $\mathrm{kg} / \mathrm{s}$ & 20,93 \\
\hline Radiador izquierdo del enfriador del aire & 52,8 \\
\hline Temperatura de entrada del refrigerante, ${ }^{\circ} \mathrm{C}$ & 41,4 \\
Temperatura de salida del refrigerante, ${ }^{\circ} \mathrm{C}$ & 9,62 \\
Flujo másico de refrigerante, $\mathrm{kg} / \mathrm{s}$ & 16,9 \\
Temperatura entrada del aire, ${ }^{\circ} \mathrm{C}$ & 50 \\
Temperatura de salida del aire, ${ }^{\circ} \mathrm{C}$ & 17,3 \\
\hline Flujo másico medio medido de aire, $\mathrm{kg} / \mathrm{s}$ & 56,4 \\
\hline Compresor & 56,6 \\
\hline Temperatura de entrada del refrigerante, ${ }^{\circ} \mathrm{C}$ & 1,856 \\
\hline Temperatura de salida del refrigerante, ${ }^{\circ} \mathrm{C}$ & 55,6 \\
Flujo másico de agua, $\mathrm{kg} / \mathrm{s}$ & 57,9 \\
\hline Enfriador de aceite & 56,92 \\
\hline Temperatura de entrada del refrigerante, ${ }^{\circ} \mathrm{C}$ \\
Temperatura de salida del refrigerante, ${ }^{\circ} \mathrm{C}$ & \\
Flujo másico de refrigerante, $\mathrm{kg} / \mathrm{s}$ &
\end{tabular}


Las temperaturas y las velocidades del aire a su paso por el radiador se distribuyeron en la superficie como se muestra en la Figura 6.45 y Figura 6.46 .

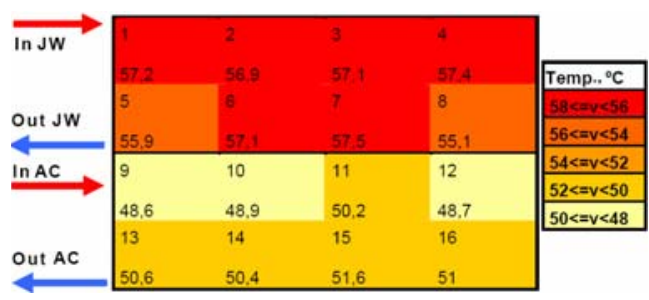

Figura 6.45. Distribución de la temperatura del aire a la salida del radiador izquierdo. $T_{\text {en_air }}=15^{\circ} \mathrm{C}$;

$$
\begin{aligned}
& T_{\text {en_refr_mot }}=61,9{ }^{\circ} \mathrm{C} ; \\
& T_{\text {en_refr_AC }}=52,8^{\circ} \mathrm{C} .
\end{aligned}
$$

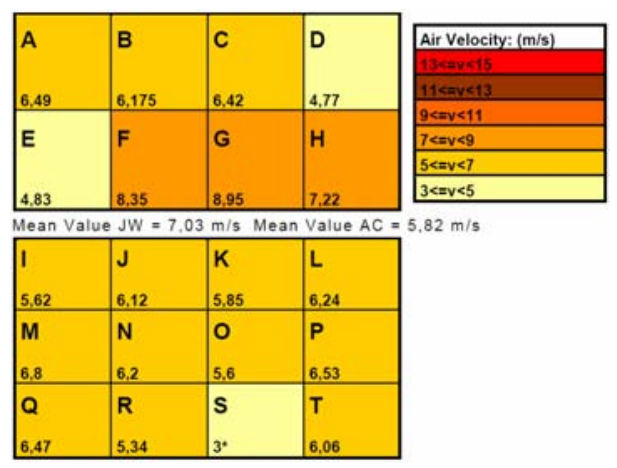

Figura 6.46. Velocidades de paso del aire por el banco de radiadores izquierdo.

Se observa en la Figura 6.45 la falta de uniformidad en la distribución de la temperatura del aire a lo alto y ancho de los radiadores, provocada a su vez por una falta de uniformidad en el flujo de aire, que se suma a la no uniformidad propia del enfriamiento del refrigerante al pasar por los tubos. Esta falta de uniformidad es influenciada por la geometría de todo el sistema de ventilación, el cual a su vez depende de la disponibilidad de espacio en el vagón y de la ubicación relativa de los componentes. La distribución experimental de las velocidades representada en la Figura 6.46 muestra, como se espera, una mayor velocidad de paso del aire por los radiadores del motor, ajustándose a una proporción cercana a 55/45 en favor de éstos. Los valores de las velocidades durante las mediciones presentan mucha fluctuación, sobre todo en algunas zonas en la sección de los radiadores. A pesar del promediado físico de las señales de los anemómetros, la medida experimental deja ciertas incertidumbres difíciles de cuantificar para el método de medición empleado y no permite una comparación concluyente en relación con los valores predichos por el modelo CFD. 


\subsubsection{Resultados de las mediciones en el sistema de refrigeración operando el motor a plena carga}

Calculando los calores intercambiados en los radiadores, el compresor y el enfriador de aceite por la expresión general $\dot{Q}=\dot{m} C_{p}\left(T_{2}-T_{1}\right)=\dot{m} C_{p} \Delta T$, y empleando los caudales y temperaturas del refrigerante, se obtienen los valores definidos en la Tabla 6.8.

Tabla 6.8. Cálculo de los calores transferidos en los intercambiadores, $\mathrm{kW}$

\begin{tabular}{lr}
\hline Calor transferido en el radiador del motor & 711,74 \\
Calor transferido en el radiador del aftercooler & 370 \\
Calor transferido en el enfriador del aceite & 461,13 \\
Calor transferido en el compresor & 3,27 \\
\hline
\end{tabular}

Los calores intercambiados en los radiadores se basan en los flujos de refrigerante y, teóricamente, deberían ser iguales a los valores calculados con los flujos y gradientes de temperatura del lado del aire. Sin embargo, hay discrepancias significativas como se reporta en la Tabla 6.9 en los valores de los flujos de aire obtenidos por balance térmico, en relación con los obtenidos experimentalmente. Los obtenidos por balance térmico son más cercanos a los obtenidos con la simulación CFD.

Tabla 6.9. Discrepancias entre los flujos másicos de aire obtenidos por balance térmico y los valores medios medidos y simulados con PowerFlow.

\begin{tabular}{lccc}
\hline \multicolumn{1}{c}{ Flujos másicos de aire, $\mathrm{kg} / \mathrm{s}$} & $\begin{array}{c}\text { Balance } \\
\text { térmico }\end{array}$ & Experimental & $\begin{array}{c}\text { Simulado } \\
\text { CFD }\end{array}$ \\
\hline $\begin{array}{l}\text { A través del radiador del motor } \\
\text { A través del radiador del }\end{array}$ & 16,55 & 20,93 & 15,46 \\
"aftercooler" & & 17,3 & 12,61 \\
\hline
\end{tabular}

Las diferencias entre los caudales de aire medidos y los obtenidos por balance térmico son bastante grandes, con lo que la evaluación de los resultados del modelo se hacen con los cálculos derivados del balance térmico. Aunque también puede argumentarse que el calor calculado con las temperaturas y 
calores del lado del refrigerante no está exento de errores, la misma naturaleza de los fluidos y las condiciones de turbulencia de éstos hacen que, comparativamente, los errores de medición sean mayores del lado del aire que del refrigerante. En estas últimas las mediciones son más estables. El mismo curso de las mediciones lo muestra así.

El desempeño del sistema de gestión térmica del motor se presenta en la Tabla 6.10, donde se resume el balance de los calores disipados y los generados. El remanente que cierra el balance térmico entre los calores calculados en las fuentes y los calculados en los disipadores, tras computar los calores disipados por los radiadores del motor y del aftercooler, es un calor cedido al ambiente.

Tabla 6.10. Balance térmico global del sistema de gestión térmica

\begin{tabular}{lll}
\hline Disipación de calor & $\mathbf{k W}$ & $\%$ \\
\hline Radiadores del motor & $-1423,4$ & 58,6 \\
Radiadores del aftercooler & -740 & 30,5 \\
Ambiente & -264 & 10,9 \\
Calor total disipado & 2427,4 & 100 \\
\hline Evacuación de calor & & \\
\hline Motor & +1161 & 47,8 \\
Enfriador de aire & +802 & 33,0 \\
Enfriador del aceite & $+461,13$ & 19,0 \\
Compresor & 3,27 & 0,1 \\
\hline
\end{tabular}

\subsubsection{Comparación y análisis de las mediciones en el sistema de refrigeración operando el motor a plena carga con los resultados de la simulación 1D}

Con la consideración hecha en el apartado anterior referente a los valores inconsistentes de los flujos de aire medidos, en la Tabla 6.11 y Tabla 6.12 se sintetizan las temperaturas, los flujos de refrigerante y aire y los calores transferidos en los radiadores obtenidos por vía experimental y por vía de la simulación. En las casillas de los valores de flujo de aire se han dejado los correspondientes a la simulación CFD. 
Tabla 6.11. Comparación de los valores medidos y los simulados en los radiadores del motor

\begin{tabular}{|l|c|c|c|c|c|}
\hline \multicolumn{2}{|c|}{ ÍTEM } & Simul. & Exper. & Diferencia & $\%$ Error \\
\hline \multirow{3}{*}{ Refrigerante } & Entrada T $\left({ }^{\circ} \mathrm{C}\right)$ & 64,26 & 61,9 & $\cong 2,36$ & 3,81 \\
& Salida T $\left({ }^{\circ} \mathrm{C}\right)$ & 56,37 & 54,8 & $\cong 1,57$ & 2,86 \\
& Enfriamiento $\left({ }^{\circ} \mathrm{C}\right)$ & 7,89 & 7,1 & $\cong 0,79$ & 11,13 \\
\hline \multirow{4}{*}{ Aire } & Entrada T $\left({ }^{\circ} \mathrm{C}\right)$ & 14,4 & 14,4 & $\cong 0$ & 0 \\
& Salida T $\left({ }^{\circ} \mathrm{C}\right)$ & 60,58 & 56,78 & $\cong 3,8$ & 5,63 \\
& Calentamiento $\left({ }^{\circ} \mathrm{C}\right)$ & 46,18 & 42,38 & $\cong 3,8$ & 8,97 \\
\hline \multicolumn{2}{|c|}{ Flujo de refrigerante $(\mathrm{kg} / \mathrm{s})$} & 29,49 & 28,46 & $\cong 1,03$ & 3,62 \\
\hline \multicolumn{2}{|c|}{ Flujo de aire $(\mathrm{kg} / \mathrm{s})$} & 16,89 & 16,55 & $\cong 0,34$ & 2,05 \\
\hline Calor disipado & \multirow{2}{*}{ Radiador1 } & 797 & 711,44 & $\cong 85,56$ & 12,04 \\
\hline
\end{tabular}

Hay un calor evacuado por tuberías y demás que el modelo puede no estar estimando bien. Aparte de esto están las desviaciones en las magnitudes medidas y simuladas (caudales y temperaturas).

Tabla 6.12. Comparación de los valores medidos y los simulados en los radiadores del "aftercooler"

\begin{tabular}{|c|c|c|c|c|c|}
\hline \multicolumn{2}{|c|}{ ÍTEM } & Simul. & Exper. & Diferencia & $\%$ Error \\
\hline \multirow{3}{*}{ Refrigerante } & Entrada T $\left({ }^{\circ} \mathrm{C}\right)$ & 53,69 & 52,8 & $\cong 0,9$ & 0,92 \\
& Salida T $\left({ }^{\circ} \mathrm{C}\right)$ & 41,42 & 41,4 & $\cong 0,02$ & 0,048 \\
& Enfriamiento $\left({ }^{\circ} \mathrm{C}\right)$ & 11,69 & 11,4 & $\cong 0,29$ & 2,54 \\
\hline \multirow{2}{*}{ Aire } & Entrada T $\left({ }^{\circ} \mathrm{C}\right)$ & 16,9 & 16,9 & $\cong 0$ & 0 \\
& Salida T $\left({ }^{\circ} \mathrm{C}\right)$ & 46,5 & 50 & $\cong 3,5$ & 7,0 \\
& Calentamiento $\left({ }^{\circ} \mathrm{C}\right)$ & 29,6 & 33,1 & $\cong 3,5$ & 3,02 \\
\hline \multicolumn{2}{|c|}{ Flujo de refrigerante $(\mathrm{kg} / \mathrm{s})$} & 10,5 & 9,62 & $\cong 0,88$ & 9,14 \\
\hline \multicolumn{2}{|c|}{ Flujo de aire $(\mathrm{kg} / \mathrm{s})$} & 13,44 & 11 & $\cong 2,44$ & 22,18 \\
\hline $\begin{array}{c}\text { Calor disipado } \\
(\mathrm{kW})\end{array}$ & Radiador1 & 408 & 370 & $\cong 38,16$ & 10,31 \\
\hline
\end{tabular}


Discusión. Las temperaturas del refrigerante al paso por las radiadores del motor, predichas por el modelo, son ligeramente superiores que las experimentales en términos absolutos $\left({ }^{\circ} \mathrm{C}\right)$, con un error porcentual de predicción de temperatura medio de entrada y salida, del 3,3\% y un error en el gradiente del refrigerante con respecto al experimental del $11 \%$ (el enfriamiento predicho por el modelo de diseño es mayor que el real obtenido). La temperatura predicha para el aire también es mayor, con un error del 3,8\%. Los flujos de refrigerante y de aire se predicen con una diferencia menor del 1\%. El calor disipado predicho es mayor en un $12 \%$ comparado con el experimental. Para la parte del enfriador de aire el análisis es similar, con menor error en la predicción de las temperaturas, pero mayor error en la predicción de los flujos de refrigerante y de aire. El modelo también predice un calor disipado superior en cerca de un $10 \%$.

Los flujos de refrigerante pueden ser predichos con mayor exactitud, completando el circuito con todos los componentes del sistema real. Se recuerda que la simplificación se ha hecho para hacer más ligero el modelo y facilitar la rapidez de los cálculos.

A pesar de que las temperaturas predichas por el modelo son superiores y los flujos son parecidos, se observa una mayor disipación de calor en el modelo, lo que quiere decir que el calor evacuado por el motor realmente es menor que el asumido o asignado al modelo. También deben considerarse los errores en las mediciones experimentales asociados a los instrumentos, al método de medición y al carácter turbulento de los flujos que se manejan. Con estas consideraciones $\mathrm{y}$, dado por un lado el cumplimiento general de las temperaturas y de los flujos y, por otro lado, la cercanía del calor disipado, con una diferencia respecto al valor experimental del $12 \%$, puede decirse que los radiadores del motor son apropiados dentro de los márgenes de tolerancias que se pueden aceptar en este tipo de cálculos [6.23] y que la manera en que se asumieron los calores ha sido al menos productiva.

Aparte de los resultados del caso ejemplo puntual que se ha resuelto, lo que importa para el presente trabajo es destacar la coherencia en la secuencia del proceso de diseño del sistema de refrigeración. Los calores previstos para el cálculo de los radiadores han sido muy cercanos a los reales y los métodos de cálculo de las resistencias, tanto del circuito hidráulico como de la parte de ventilación (con ayuda del programa CFD), han permitido calcular valores 
cercanos para los flujos del refrigerante y del aire. La secuencia del proceso se puede repetir las veces que sea necesario para optimizar la selección de los radiadores y el diseño del sistema de ventilación, pero antes debe pensarse en realizar una evaluación más detallada de las tolerancias dentro de las cuales se pueden realizar predicciones y mediciones en el sistema de refrigeración, pues son varias las fuentes de incertidumbre a pesar de estar en la dirección adecuada.

El principal objetivo de cualquier proceso de diseño de ingeniería debe ser el la definición de un producto con las características funcionales definidas bajo consideraciones claras de las incertidumbres y tolerancias involucradas. En el diseño del sistema de refrigeración se han realizado varias suposiciones desde el inicio (partiendo de la adopción de un valor de calor a disipar, que de entrada no se conoce con mucha aproximación). La acumulación de estas asunciones introduce variaciones en el diseño. Es por esto necesario entender dónde se concentran las inexactitudes y tratar de eliminar todas las fuentes de error posibles o, al menos, conocer con mayor certeza esas fuentes de error, escogiendo las configuraciones en las que estos errores sean menos probables. Para el caso concreto de este proyecto las principales incertidumbres, aparte de las debidas al diseño constructivo de los componentes, están asociadas a la distribución del flujo másico del aire a su paso por las secciones de los radiadores. En esto las técnicas experimentales empleadas deben refinarse más, pero además debe contarse con las especificaciones más detalladas de los componentes físicos. Como anotan Taylor et al. [6.29], la cuantificación de las incertidumbres en los problemas de ingeniería térmica se ve dificultada porque los proveedores de los componentes como bombas, ventiladores y accesorios, no proveen información relacionada con la incertidumbre de sus características, y porque también la información de ingeniería que reportan está basada generalmente en criterios de semejanza y leyes de escalado. En la gráfica de la figura 6.47 se ilustra la influencia que sólo las tolerancias en las características integrales del sistema de ventilación, asumiéndolas de $\pm 5 \%$ tanto en el flujo volumétrico como en la presión, podrían tener sobre el volumen de aire conducido a través de los radiadores.

Como se ve de la gráfica, el caudal de aire puede variar de 55 a $62 \mathrm{~m}^{3} / \mathrm{s}$, por la sola variación de las características constructivas de los componentes. 
Otras incertidumbres, más difíciles de estimar se asocian a la transferencia de calor entre las partes metálicas del sistema y el ambiente.

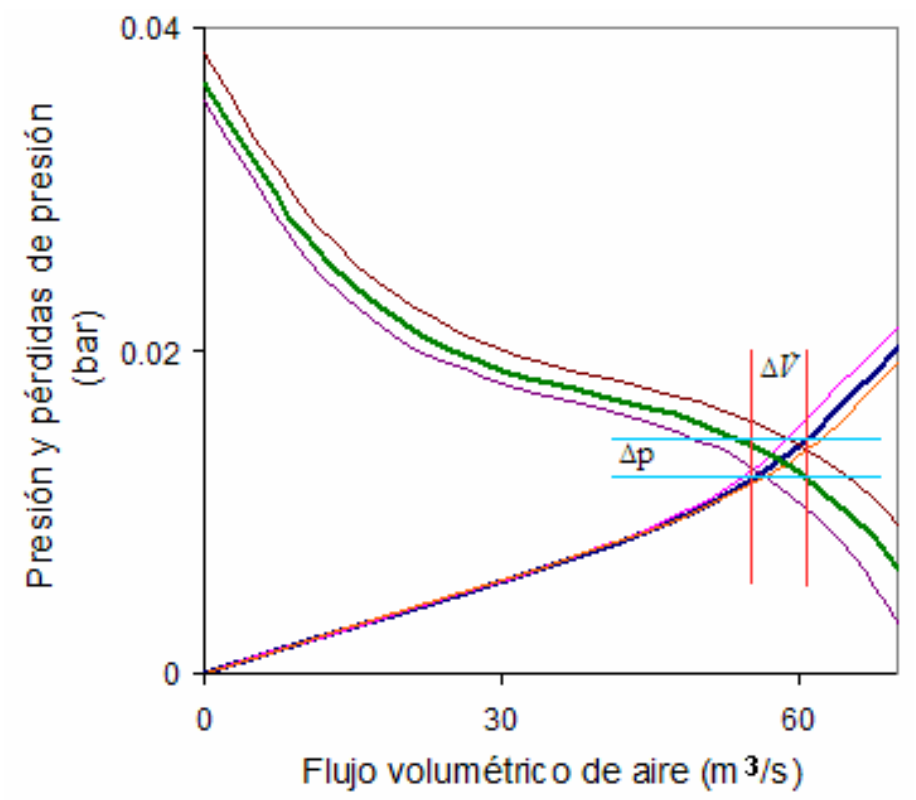

Figura 6.47. Sensibilidad del flujo de aire a través de los radiadores en función de unas tolerancias asumidas de $\pm 5 \%$ sobre las características integrales de pérdidas de presión en el sistema y de presión suministrada por los ventiladores

\subsection{Conclusiones}

En este capítulo se ha seguido un procedimiento de diseño o rediseño de sistemas de refrigeración de motores descrito en el capítulo cuarto. Conocidas las especificaciones básicas del motor y las particularidades de la aplicación, se ha estimado el calor a disipar, se han calculado los radiadores, se han seleccionado los ventiladores, se ha perfilado un trazado de los circuitos del sistema y se ha realizado su modelado para simular y comprobar que tanto el circuito hidráulico ensamblado como el de ventilación respondían a las necesidades presentadas en la tarea técnica. Se ha dedicado especial atención a ilustrar los resultados del modelado del flujo de aire a su paso por el recorrido del sistema de ventilación (sala de motor, radiadores, remanso, 
ventilador, atmósfera) y a su importante contribución en la determinación de las resistencias de todos los componentes que participan del circuito de aire.

Luego de presentar el diseño y comentar los principales detalles del modelado termohidráulico y la simulación, en las secciones posteriores del capítulo se trataron los aspectos relacionados con el montaje experimental, que por la naturaleza de la aplicación resulta ser la prueba del prototipo mismo. Se ha comentado la instrumentación empleada y se han desvelado las dificultades halladas en la medición del flujo de aire como variable. Con la información obtenida se han calculado los calores disipados por los radiadores y se ha estimado el calor disipado al ambiente.

Después de tener los resultados de la experimentación se han hecho comparaciones numéricas y cualitativas entre los resultados del modelo y los experimentales y se ha concluido que la metodología es adecuada, que es valiosa la ayuda de los programas de computación y que, conociendo las incertidumbres, el proceso seguido es acertado y acelera el proceso de diseño, acortando los tiempos entre la concepción del sistema y el ensayo del prototipo.

En la parte final se ha hecho énfasis en la necesidad de desarrollar actividades para conocer mejor las incertidumbres, tanto experimentales como de modelado, que desde el inicio del proceso se han tenido. Es de anotar aquí que los flujos de refrigerante pueden ser bien predichos por el modelo 1D si el sistema se modela con todos sus componentes, como se ha podido comprobar con el diseño y modelado de otro sistema de refrigeración, que por razones de espacio no se comentan aquí.

La metodología utilizada, aunque no automatizada, sirve a los propósitos planteados y agiliza el diseño actual de los sistemas de refrigeración para locomotoras.

\subsection{Bibliografía}

[6.1] Watanabe, N., Kubo, M. y Yomoda, N. An 1D-3D integrating numerical simulation for engine cooling problem. SAE paper 2006-01-1603. 
[6.2] Hill, N., Kollamthodi, S., Cross, S. Rail Diesel study, WP2 final report: Technical and operational measures to improve the emissions performance of Diesel rail, diciembre de 2005.

[6.3] Page, R. W., Hnatczuk, W. y Kozierowski, J. Thermal management for the 21 st century - improved thermal control and fuel economy in an army medium tactical vehicle. SAE paper 2005-01-2068.

[6.4] Sakai, T., Ishiguro, S., Sudoh, Y., Raab, G. y Hager, J. The optimum design of engine cooling system by computer simulation. SAE International Truck and Bus Meeting, Seattle, 1994.

[6.5] Puntigam, W., Hörmann, T., Bernasch, J., Schierl, K., Wiesler, B. y Hager J. Thermisches Management im Fahrzeug durch Kopplung unterschiedlicher Simulationsmodelle Wärmemangement des Kraftfahrzeuges IV, Essen, expert verlag, 2004 (consultado en http://www.vif.tugraz.at en diciembre de 2008).

[6.6] PowerFlow user's manual. Version 4.1.

[6.7] Kühlsysteme of the company Voith. http://www.voithturbo.com/kuehlsysteme-produkteuehlsysteme.htm (consultada en enero de 2008).

[6.8] Kays, W. M.; London, A. L. Compact heat exchangers. New York, 1984.

[6.9] Achachia, A., Cowell, T. A. Heat transfer and pressure drop characteristics of flat tube and louvered plate fin surfaces. Experimental Thermal and Fluid Science, vol. 1, pp. 147-157, 1988.

[6.10] Incropera, F.P. and DeWitt, D.P. Fundamentals of heat and mass transfer. New York, 1996.

[6.11] Namai, K., Muramoto, H., Mochizuki, S. Flow visualization in the louvered fin heat exchanger. SAE paper 980055.

[6.12] Tafti, D.K. and Cui, J. Computations of flow and heat transfer in a three dimensional multilouvered fin geometry. International Journal of Heat and Fluid Flow, 2002.

[6.13] Webb, R. L., Trauger, P. Flow Structure in the Louvered Fin Heat Exchanger Geometry. Experimental Thermal and Fluid Science, 
vol 4, pp. 205-214, 1991.

[6.14] Fuentes, D. y Gélvez, O. Cálculo de intercambiadores de calor por medio del método de los volúmenes finitos. $8^{\circ}$ Congreso Iberoamericano de Ingeniería Mecánica. Cusco, Octubre de 2007.

[5.15] Kakac S., Liu H.: Heat exchangers - selection, rating and thermal design, CRC Press LLC, 2002.

[6.16] Navarro, H. A. Y Cabezas-Gómez, L. A new approach for thermal performance calculation of cross-flow exchangers, Int. J. Heat Mass Transfer, vol. 48, pp. 3880-3888, 2005.

[6.17] Oliet, C., Oliva, A., Castro, J. y Pérez-Segarra, C.D. Parametric studies on automotive radiators. Appl. Therm. Eng., vol. 27, pp. 2033-2043, 2007.

[6.18] FlowMaster user's manual. Version 7.2.

[6.19] Wambsganss, M. W. Thermal Management in heavy vehicles: A review identifying issues and research requirements. Argone National Laboratory. Vehicle Thermal Management Systems 4 Conference. London, mayo 24-27, 1999.

[6.20] Kern, J., y Ambros, P. Concepts for a controlled optimized vehicle engine cooling system. Vehicle Thermal Management Systems Conference Proceedings, SAE P-314, pp. 357-362,1997.

[6.21] Huang, K. D., Tzeng, S. y Ma, W. Effects of anti-freeze concentration in the engine coolant on the cavitation temperature of a water pump. Applied Energy, vol. 79, issue 3, pp. 261-273, 2004.

[6.22] Flowmaster $N^{\circ}$ 11: Caterpillar and Argonne National Laboratory meet sound regulations with the help of Flowmaster, at a fraction of the cost and time (consultado en www.flowmaster.com en enero de 2008).

[6.23] Lahvic, T. R. Investigation of engine heat rejection, agosto 1986, Ford Motor Co.

[6.24] Miller, D. S. Internal flow systems, segunda edición, BHR Group Limited, 1996.

[6.25] GC-Flowmaster France. Alstom Transport locomotive cooling systems. Estudio del sistema de refrigeración de la locomotora Egipt, septiembre de 2002. 
[6.26] Caterpillar G3600 application and installation guide. Cooling Systems, enero de 2009.

[6.27] Staunton, N., Maughan, R., Jackson, K. Reduction of parasitic losses in heavy-duty Diesel engine cooling systems. Presentación en el Engine Expo 2008, Stuttgart Messe, mayo 6-8 de 2008.

[6.28] Lester, T. Solving for friction factor. ASHRAE Journal, Julio 2003.

[6.29] Taylor, R. P., Hodge, B. K., James, C. A. Estimating uncertainty in thermal systems analysis and design. Appl. Therm. Eng., vol. 19, pp. 51-73, 1999. 


\section{Capítulo 7}

\section{Conclusiones y trabajos futuros}

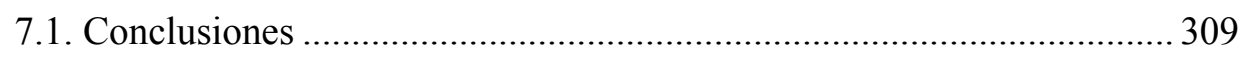

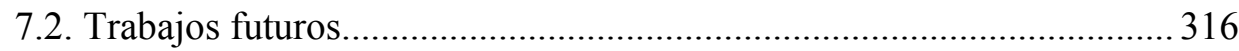


El trabajo llevado a cabo durante la realización de esta tesis ha estado encaminado al desarrollo de un estudio teórico-computacional-experimental de los fenómenos de transmisión de calor y del balance térmico en motores diesel, tanto dentro del cilindro como en el sistema de refrigeración, con el fin, por un lado, de contribuir a una mejor identificación y valoración de la distribución de las temperaturas y los flujos de calor en los componentes más próximos a la cámara de combustión durante la operación del motor en calentamiento y, por otro lado, con la intención de intervenir en el diseño de los sistemas de refrigeración para mejorar las prestaciones del motor durante el calentamiento. Adicionalmente, se ha querido presentar una propuesta metodológica de integración de herramientas de computación al diseño de los sistemas de refrigeración de motores de aplicación especial.

\subsection{Conclusiones}

Como fruto del trabajo realizado, se pueden extraer algunas conclusiones principales, derivadas directamente de las exposiciones hechas a lo largo de este escrito, y que se exponen a continuación, según el orden marcado por los diferentes capítulos. Relacionadas con el estudio del estado del arte del modelado y las mediciones experimentales, de manera amplia, puede afirmarse que:

- Para los estudios de la transferencia de calor en el motor de combustión interna se cuenta actualmente con programas computacionales CFD con enorme capacidad predictiva. Los códigos modernos integran submodelos cada vez mejores para modelar la rotura de las gotas del chorro de combustible, la distorsión y la resistencia al flujo del chorro, la impregnación con combustible de las paredes, con rebote de las gotas, la vaporización del combustible, la transferencia de calor transitoria y de flujo compresible, la inflamación y su asociada cinética multipasos, la combustión laminarturbulenta, la formación de NOx y de hollín, los flujos de masa por los intersticios de la cámara de combustión y la historia de la llama. Los modelos de turbulencia pueden predecir las estructuras de gran escala producidas por los flujos "squish" y provocadas por los chorros de combustible. Particularmente importantes son los modelos desarrollados bajo el entorno del código KIVA, el cual tiene la ventaja 
de ser un código abierto, al cual permanentemente se le han estado incorporando submodelos multidimensionales físicos y químicos. En las partes del aceite y el refrigerante, al igual que en los sistemas de intercambio de gases, el tratamiento multidimensional se ha ido incorporando progresivamente.

- Desde que Borman y Nishiwaki publicaron su revisión del estado del arte ha habido muchos avances en los medios o herramientas para evaluar la transferencia de calor en el motor. Las técnicas ópticas informatizadas cada vez se emplean con mayor propiedad para la determinación y valoración de la distribución de la temperatura del núcleo del gas en la cámara de combustión, la distribución de la temperatura de la capa límite térmica y el espesor de ésta, la distribución de la masa de hollín, el desarrollo del chorro de combustible y el comportamiento de la llama. A pesar de los logros en la evaluación de las propiedades de los depósitos, debe decirse que aún no parece disponerse de un modelo definido para la formación de los depósitos, debido a la complejidad de los factores involucrados. Esto representa un problema para las simulaciones CFD, por la necesidad de disponer de las temperaturas de las superficies y de las propiedades de los depósitos para predecir de manera exacta la transferencia de calor en la cámara de combustión. El problema se agrava por la multiplicidad de configuraciones de diseño de los motores y la naturaleza cambiante de los combustibles y los aceites. La evolución de la tecnología para la evaluación de los flujos de calor ha permitido interpretar mejor la variación de las temperaturas en la cámara de combustión y el fenómeno de la inversión del flujo de calor durante la carrera de expansión. Se ha adquirido una mejor conceptualización sobre los flujos bi- y tridimensionales del calor a través de las superficies de la cámara de combustión. Los experimentos conducentes a la identificación de los flujos del refrigerante y el aceite, las mediciones de las temperaturas y flujos de calor a través de las superficies de la camisa de refrigeración del motor promueven la mejor valoración de la transferencia de calor en el motor.

- Para valorar la transferencia de calor en los modelos multidimensionales, se emplean funciones de pared, estándar y modificadas, para determinar los esfuerzos cortantes y la transferencia 
de calor del fluido en las vecindades de la pared. Las inexactitudes derivadas de estos procedimientos han demandado la introducción de ajustes analíticos y empíricos para acercarse a los resultados experimentales. Al tiempo que las correlaciones empíricas y globales empleadas para valorar la transferencia de calor desde el gas a través de las paredes de la cámara de combustión proporcionan sólo estimativos del coeficiente de transferencia de calor y de la historia del flujo de calor, definidos en términos de la temperatura global del gas, el empleo de la función de pared estándar permite obtener resolución espacial, aunque subestima mucho los verdaderos niveles de flujo de calor y sus variaciones. Las mejoras hechas a la función de pared han permitido concluir sobre los modelos globales empíricos, que además de no proveer resolución espacial, también tienden a subestimar el calor transferido a las paredes durante la combustión. Se hacen actualmente desarrollos tratando con números de Reynolds bajos y modelos de acercamiento a la pared, complementados con condiciones de contorno de pared exactas. Para este propósito empieza a aplicarse el modelo $\zeta$-f. Los modelos teóricos multidimensionales se están usando para predecir el flujo y la transferencia de calor del lado del refrigerante, al igual que para la refrigeración del pistón.

- Son muchos los avances hechos en el modelado de la transferencia de calor radiativa. Varios grupos de investigación han desarrollado modelos de formación del hollín, el coeficiente de absorción, emisividad y deposición termoforética del hollín, requeridos para el modelado de la radiación. Los métodos numéricos para el cálculo de la ecuación de transferencia radiativa también se han perfeccionado, llegando a ser corriente el uso de métodos como el de Monte Carlo y el de ordenadas discretas.

- Los desarrolladores de códigos numéricos han ido respondiendo a las tareas complejas de modelado de los motores de combustión. El mallado dinámico durante el movimiento de válvulas y pistones, los chorros y la combustión, todo dentro de un ambiente totalmente turbulento está siendo superado progresivamente. Sin embargo, las simulaciones multidimensionales de los motores de combustión interna todavía requieren información experimental detallada como condiciones de contorno e información para su validación. 
Tras los trabajos de experimentación y modelado térmico del motor tratados en el tercer capítulo ha sido posible:

- Extender las capacidades del modelo térmico, para predecir los flujos de calor en el motor hacia los medios correspondientes a las condiciones de contorno, en particular hacia el refrigerante y el aceite, bajo condiciones de operación estacionarias y transitorias.

- Determinar el balance térmico del motor durante su calentamiento bajo carga constante y bajo un ciclo de conducción estándar. Las magnitudes de los calores intercambiados en los enfriadores de EGR y del calentador del habitáculo son similares, para las condiciones de los ensayos, por lo que el primero podría suministrar directamente su energía al segundo, si no tuviera que dedicarse para el tratamiento de las emisiones de NOx. Mirando los dos intercambiadores como recuperadores de calor, la capacidad media de recuperación de calor con sólo estos dos dispositivos, en las condiciones de operación ensayadas, es de aproximadamente un $20 \%$ de las pérdidas totales integradas en los gases de escape y el refrigerante. Las pérdidas de calor promedio totales (refrigerante y gases de escape), durante el calentamiento, constituyen una magnitud que podría ser suficiente para accionar un generador eléctrico y alimentar el sistema eléctrico del vehículo, si la realización tecnológica de esa energía fuera técnica y económicamente viable.

- Realizar la caracterización hidráulica y térmica del sistema de refrigeración. Las presiones en los componentes del sistema de refrigeración para diferentes caudales del refrigerante (varios puntos de operación estacionarios del motor) han sido medidas y con ellas se han determinado las características de pérdidas de presión vs. caudal, requeridas para el modelado $1 \mathrm{D}$ del sistema de refrigeración. La respuesta experimental de la temperatura del refrigerante durante el punto de operación de carga y régimen constantes ha servido para estimar la "masa sensible interna" del motor y del motor integrado al sistema de refrigeración.

- Predecir las temperaturas y los flujos de calor entre los nodos del modelo del motor, y obtener el mapa térmico de éste, junto con una correlación para cuantificar el calor disipado a través de las paredes de 
los cilindros, en función del régimen, la presión media efectiva y la temperatura del refrigerante.

- Concluir que las variables medias e instantáneas adquiridas durante el calentamiento del motor bajo carga constante y bajo ciclo de operación, a pesar de no ser adquiridas durante puntos de operación estabilizados, pueden emplearse en el modelo térmico y las temperaturas predichas son muy razonables, mostrándose así que una alternativa a la obtención de la información en caliente del motor y en condiciones estabilizadas para los puntos de operación representativos de un ciclo de calentamiento (actividad costosa y dispendiosa) es la adquisición de las variables durante el mismo ciclo de conducción.

En el capítulo cuarto se ha sugerido una metodología de diseño de sistemas de refrigeración que incorpora las consideraciones de diseño preliminares de los componentes del sistema a la sucesión (concebida como iterativa) de los trabajos de cálculo y modelado de los radiadores, el subsistema del refrigerante y el subsistema de ventilación. Los fundamentos teóricos sobre los que se basan, en general, los programas de cálculo de radiadores y de modelado $1 \mathrm{D}$ y 3D de los sistemas termohidráulicos han sido presentados también en este capítulo y, de manera particular, se ha comentado brevemente el modo de operación de los programas utilizados en esta tesis, resumiendo la información mínima demandada por los submodelos de los principales componentes del sistema de refrigeración. También en este mismo capítulo se han comentado las alternativas para el cálculo estimativo del calor disipado al refrigerante como información inicial requerida para el dimensionamiento del sistema de refrigeración, mostrando los amplios márgenes de los valores que puede tener ese calor para diferentes motores, y destacando las aproximaciones propuestas por Lahvic (empírica) y por Parish (conceptual) para el cálculo del mismo.

Con las características hidráulicas y térmicas del sistema de refrigeración original del motor evaluado en el capítulo tercero, y conociendo la respuesta de la temperatura del refrigerante durante el calentamiento bajo una carga constante, en el capítulo cuarto se ha construido el modelo 1D de ese sistema y se han ajustado los parámetros de los componentes para obtener en ellos las respuestas que mejor se acercan al sistema real. Los caudales de refrigerante por los ramales del circuito original tienen una desviación menor del $5 \%$. Se 
han introducido las tablas de los flujos de energía en los intercambiadores y en el motor para efectuar el modelado térmico. Habiendo asegurado que la respuesta del modelo se corresponde suficientemente bien con la respuesta del sistema de refrigeración real, con un error de temperatura a lo largo del período de calentamiento menor al $4 \%$, se ha simulado el desempeño del modelo en un transitorio de calentamiento de perfil definido por un ciclo de conducción europeo en ciudad (NEDC). Los resultados del modelo han sido satisfactorios en lo que tiene que ver con el seguimiento de la curva de calentamiento experimental y también con el tiempo al cual se alcanza la temperatura de consigna de $80{ }^{\circ} \mathrm{C}$. Como el tiempo de calentamiento puede disminuirse reduciendo el volumen que participa en el calentamiento del motor y también reduciendo el caudal, se modeló y simuló la operación del sistema modificado con dos válvulas dispuestas en las vías del by-pass y de la caja de agua al tanque de expansión, que podían trabajarse en posiciones totalmente abierta o totalmente cerrada. Como conclusión de la simulación se obtuvo una configuración alternativa al sistema original que proporcionaba menos tiempo de calentamiento. Las tres modificaciones modeladas, sencillas en su concepción, fueron validadas con ensayos experimentales, en los cuales se corroboraron los resultados del modelado. En las mismas pruebas experimentales se efectuaron mediciones de las emisiones contaminantes y del consumo de combustible. Los resultados han mostrado la ventaja de la modificación propuesta en una reducción del tiempo de calentamiento del motor del 22,3\% (el tiempo de calentamiento se baja de 713,4 segundos a 554,5 segundos), traduciéndose esto en una reducción del consumo de combustible en cerca del $3 \%$, y de las emisiones de CO y $\mathrm{HC}$ en 20 y $15 \%$, respectivamente. Además de esto, debe comentarse que la reducción del tiempo de calentamiento del motor mejora el confort de los ocupantes del habitáculo de pasajeros, al aumentar la energía disponible para su calentamiento.

En el penúltimo capítulo de la tesis se ha aplicado la metodología de diseño de sistemas de refrigeración al desarrollo del sistema de refrigeración de un motor para locomotora. La aplicación del motor es especial, muy diferente a la tradicionalmente encontrada en los vehículos, con dos sistemas de refrigeración, uno para el motor y otro para el enfriador del aire de admisión, y con consideraciones de distribución de espacio también muy particulares. A partir de las características de potencia y revoluciones del motor, de las características de las bombas, y de las condiciones de ubicación, se ha 
calculado el calor disipado al refrigerante utilizando la fórmula de Lahvic, y se han determinado los parámetros de los radiadores y los ventiladores. La simulación de los conductos de aire con los ventiladores y los radiadores seleccionados permite obtener las pérdidas de presión y las temperaturas del aire a su paso por el sistema. Estas características se han tenido en cuenta en el modelado y la simulación 1D de todo el sistema. Después de estar satisfechos con los resultados del modelo obtenidos con las características encontradas para los componentes, se ha efectuado el montaje real del sistema de refrigeración y se han registrado los valores de los caudales y las temperaturas requeridos para realizar el cálculo de los calores intercambiados en las fuentes (motor y enfriador de aire) y en los radiadores, encontrando que el sistema responde bien a las expectativas.

La utilización de los programas de modelado, objeto de los capítulos quinto y sexto, ha mostrado la gran ayuda que representan en la concepción y mejora de nuevos sistemas, pero también ha quedado claro que un factor a tener en cuenta a la hora de ponderar sus resultados es la consideración de los errores de medida, las tolerancias previstas en los modelos y la exactitud de los sistemas de medición. La naturaleza de las pruebas realizadas en los motores en este trabajo no ha hecho posible un estudio de los errores, pero se han hecho comentarios generales de las fuentes de discrepancias que podrían encontrarse entre los resultados experimentales y los resultados de los modelos. La consideración de los errores es importante no sólo en la valoración del modelo final, sino en todos los momentos del proceso de modelado, incluyendo los trabajos de caracterización.

El proceso de diseño de los sistemas de refrigeración se sugiere que es un proceso iterativo, en el cual deben sopesarse varias alternativas en la selección de los componentes y con esas alternativas debe haber un planteamiento sistémico de diseño, en el que aparte de la capacidad térmica del sistema se evalúen otras exigencias. La metodología así lo prevé, pero en lo que respecta al diseño especial del sistema de refrigeración que se ha presentado aquí, debe decirse que sólo se ha tenido en cuenta la capacidad del sistema en términos de disipación de calor. 


\subsection{Trabajos futuros}

Existen varios puntos en el trabajo aquí presentado, cuyo estudio puede ser profundizado en otros proyectos. Esos trabajos pueden agruparse en:

1. Trabajos experimentales a realizar en transferencia de calor en el motor.

2. Trabajos a realizar sobre el modelo térmico.

3. Trabajos a realizar con los programas de modelado

Con relación a los trabajos experimentales, dado que la selección de las condiciones de contorno es responsable de la exactitud de los cálculos de las temperaturas del motor, para obtener las distribuciones de flujo de calor se requiere mediciones de mayor resolución y más distribuidas. Es importante investigar sobre la formación de depósitos y sus propiedades. Como la exactitud de las predicciones de los modelos CFD depende de la exactitud de los submodelos que los soportan, éstos deben continuarse mejorando, con mucho acento en los modelos de la combustión y los chorros, las mezclas de aire y combustible en contacto con la superficie, la impregnación del chorro, los modelos de las gotas y las películas de combustible, y muy particularmente, con atención a los trabajos de determinación de las temperaturas instantáneas de superficie en la cámara de combustión y de estudio detallado del flujo de los gases en las vecindades de la pared que actualmente se están realizando. Para el lado del refrigerante, sería importante construir una maqueta para el estudio de la transferencia de calor del lado del refrigerante y simular condiciones de ebullición nucleada.

Relacionados con el modelo térmico, aunque éste no calcula los flujos de calor transportados por el refrigerante durante el calentamiento, sí permite predecir los flujos de calor transmitidos a las paredes de la cámara de combustión. Una actividad por realizar en el modelo es la extensión del modelo hasta la superficie externa del motor. Para mejorar la capacidad predictiva del modelo puede ser importante que éste:

- incorpore el modelo de fricción de manera separada;

- valide con mayor resolución las temperaturas del pistón; 
- considere en detalle los pares térmicos pistón-película de aceitesegmentos-película de aceite-cilindro;

- discrimine la topología del recorrido del refrigerante a través de la camisa de refrigeración;

- realimente las temperaturas predichas dentro de un ciclo de modelado, de suerte que sólo se deban introducir al modelo las temperaturas iniciales del aceite y el refrigerante. Así, para dentro de cada ciclo completo, el modelo podría ejecutarse para predecir las temperaturas de los nodos en el ciclo siguiente;

- se integre definitivamente e interactúe con los programas de predicción y diagnóstico de la combustión;

- Se complemente con una rutina de predicción de la dinámica de tracción del vehículo bajo un ciclo de conducción estándar, para realizar el cálculo de las temperaturas de pared y los flujos de calor en el motor bajo estas condiciones.

Para terminar, dada la enorme capacidad de los programas de modelado como Amesim, Flowmaster y Fluent, es importante desarrollar metodologías de cosimulación en las que se integren los modelos de conductor, dinámica del vehículo, combustión, control de emisiones, refrigeración y ventilación. 


\section{Apéndice A}

\section{A.1. Acerca de AMESim}

AMESim (sigla de "Advanced Modeling Environment for performing Simulations") es un programa multipuerto, en el que se intercambia información de flujo de energía entre componentes a través de los nodos de conexión. AMESim posee unas librerías primarias o estándar y unas librerías especializadas, como se puede apreciar en el cuadro de presentación de Amesim de la Figura A.1. Se tiene la posibilidad de crear modelos propios y supercomponentes.

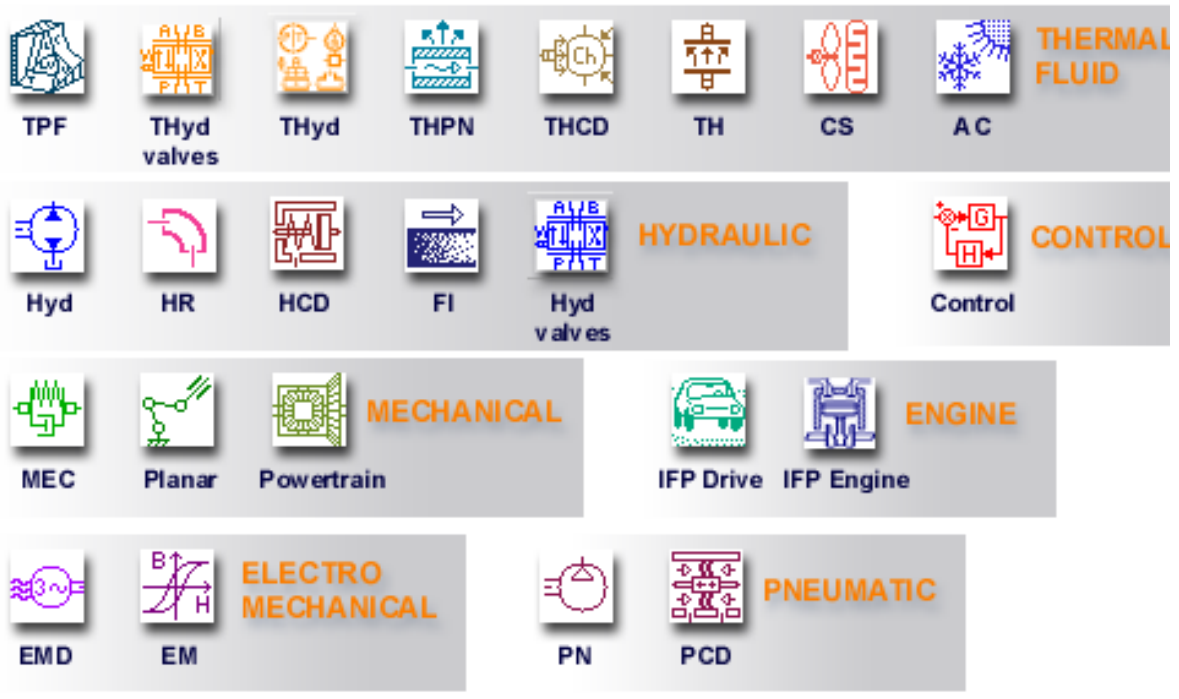

Figura A.1. Librerías disponibles en el programa AMESim para el modelado de sistemas [A.1]. 
Externamente AMESim puede conectarse a Matlab, Simulink, Matrixx y ADAMS, con la posibilidad de realizar co-simulación, cuando el modelo incluye también componentes modelados en entornos diferentes. Recientemente se le agregó una cantidad de herramientas de análisis lineal como diagramas de Bode, Nichols y Nyquist, lo mismo que análisis de lugar de las raíces. AMESim se complementa con programas adicionales como AMESet, AMECustom y AMERun que aportan flexibilidad y alternativas de modelado al usuario [A.1].

Utilizando AMESim los modelos se crean conectando íconos similares a los símbolos de los componentes hidráulicos. Posteriormente, basado en modelos matemáticos para los componentes que se encuentran en la base de datos, el programa construye automáticamente el modelo matemático del circuito completo y simula las características dinámicas del circuito. El modelado con AMESim fundamentalmente se reduce a los siguientes cinco pasos:

- Construcción del esquema del sistema, utilizando las diferentes librerías disponibles por el programa, bajo una representación cercana al plano constructivo disponible del sistema.

- Selección de los modelos matemáticos de los componentes, como el tipo de bomba a utilizar, tipo de tubería, tipo de conexión, etc. Los submodelos para los componentes del sistema pueden representar con mayor o menor aproximación la naturaleza física de los fenómenos que en él tienen lugar. Es claro que en la selección del modelo matemático, aparte de acudir a expresiones similares a las dadas por (4.37) - (4.52), es frecuente la utilización de tablas (mapas) característicos de los componentes.

- Definición de los parámetros de los modelos seleccionados, donde se especifican dimensiones, tolerancias, condiciones iniciales de simulación, etc. La caracterización experimental supone una parte importante en esta etapa del modelado.

- Ejecución de la simulación, donde se define el tiempo inicial y final de la simulación, los modos de integración, estabilización, modo de ejecución y la tolerancia para la convergencia. Se pueden analizar los escenarios de simulación, realizar análisis de sensibilidad, etc. En los programas comerciales 1D el usuario no se ve involucrado en la selección del método de solución de los sistemas, eso lo decide el programa, pero si tiene acceso al control de la simulación 
- Análisis de los resultados. En esta fase el usuario puede hacer análisis de los resultados mediante el procesamiento mediante análisis de respuesta dinámica, transformada rápida de Fourier, análisis del lugar de las raíces, posibilidad de realizar diseño de experimentos y optimización del modelo, entre otras posibilidades. Como característica importante vale la pena citar la facilidad de realizar operaciones con los resultados y de editar los resultados de esas operaciones.

Del grupo de librerías “Thermal Fluid” y “Cooling System” de AMESim, los submodelos sobre los que se fundamenta el ensamble de un sistema de refrigeración convencional moderno son los mostrados en la Figura A.2.

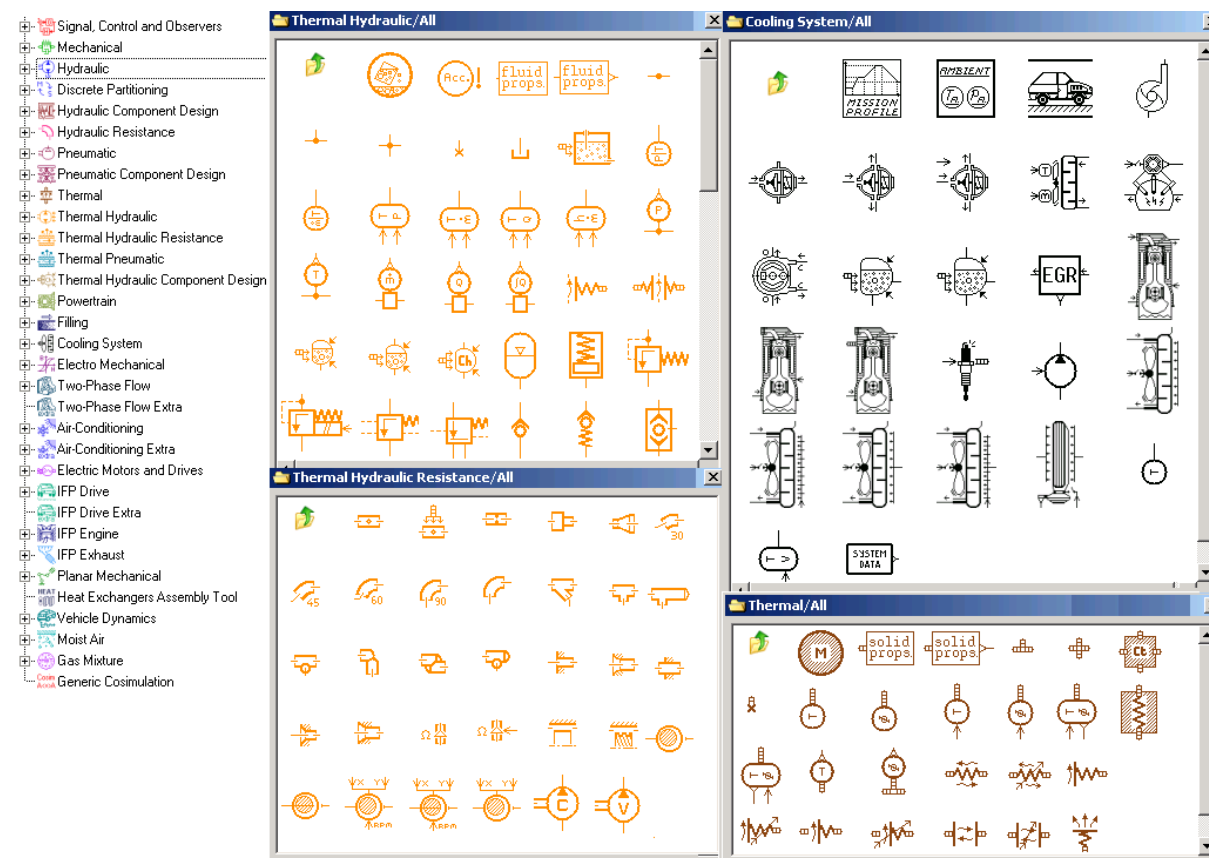

Figura A.2. Submodelos de AMESim ${ }^{\circledR}$ empleados en el ensamble del modelo de un sistema de refrigeración.

Una descripción detallada de los métodos empleados en el programa AMESim para la solución de los modelos 1D puede seguirse en el trabajo de Plazas [A.1]. Sin el ánimo de reproducir los componentes que componen el modelo y que se encuentran en la documentación técnica del programa AMESim, y sólo con propósitos ilustrativos, en la tabla 4.2 se han copiado 
algunos de los submodelos que pueden utilizarse para componer un modelo de sistema de refrigeración.

Tabla A. 1. Extracto de submodelos del programa AMESim, utilizados en la composición de modelos de sistemas de refrigeración.

\begin{tabular}{|c|c|}
\hline Icono definido por AMESim & Parámetros a definir \\
\hline $\begin{array}{l}\text { CSES1. Simulación de vehículo. } \\
\text { Calcula las revoluciones del motor } \\
\text { como función de la velocidad del } \\
\text { vehículo y las relaciones de la caja de } \\
\text { velocidades. } \\
\text { Los parámetros a definir pueden } \\
\text { depender del submodelo escogido. }\end{array}$ & $\begin{array}{l}\text { Archivo con velocidad de } \\
\text { referencia del motor y } \\
\text { velocidad del vehículo en } \\
\text { función de la relación de } \\
\text { transmisión } \\
\text { - Archivo con la posición del } \\
\text { pedal de aceleración en } \\
\text { función del tiempo } \\
\text { - Archivo con la información } \\
\text { del par del motor en función } \\
\text { de la posición del pedal y de } \\
\text { las revoluciones del motor. }\end{array}$ \\
\hline $\begin{array}{l}\text { CSMP2. Velocidad del vehículo, } \\
\text { Relaciones de la caja de velocidades y } \\
\text { perfiles de inclinación de la calzada. }\end{array}$ & 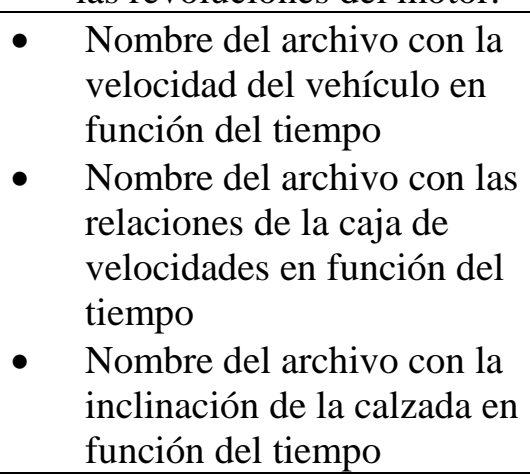 \\
\hline $\begin{array}{l}\text { CSEN00. Motor para vehículo, } \\
\text { elemento termohidráulico. }\end{array}$ & $\begin{array}{ll}\text { - } & \text { Área equivalente } \\
\text { - } & \text { Volumen de refrigerante } \\
\text { involucrado } \\
\text { - } & \text { Índice del fluido } \\
\text { - } & \text { Archivo con el calor } \\
\text { intercambiado entre el motor } \\
\text { y el refrigerante, en función } \\
\text { de la potencia del motor y } \\
\text { las revoluciones }\end{array}$ \\
\hline
\end{tabular}




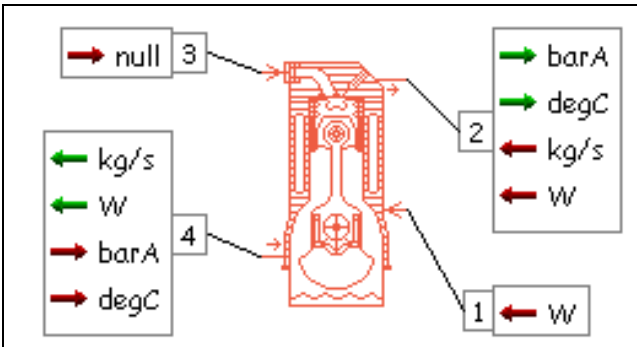

El flujo volumétrico en este componente se calcula a partir de las presiones en los puertos 2 y 4 , el área equivalente, el máximo coeficiente de flujo y el número de Reynolds. El flujo volumétrico se calcula por la ecuación de Bernoulli modificada

$Q_{v}=A_{e q} \sqrt{2 \Delta p / \rho}$

CSEN00. Motor para vehículo, elemento termohidráulico con capacitancia térmica.

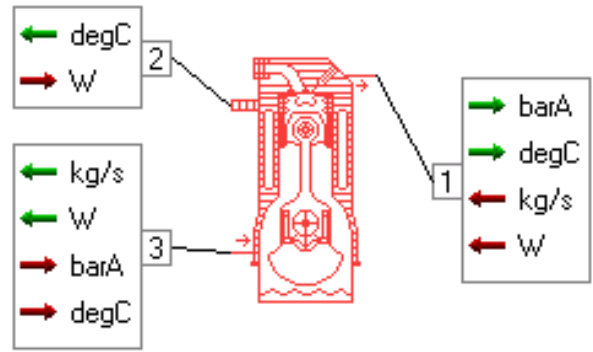

Con este submodelo el calor se entrega por el puerto2. Aparte de ésto y la posibilidad de atribuirle una masa, el submodelo se comporta como el anterior.

CSP1. Bomba centrífuga. Es el submodelo para una bomba centrífuga con característica de operación tabulada $d p=f\left(Q, N_{\text {pump }}\right)$.
- Archivo con el calor suministrado por la EGR (si la versión del submodelo lo requiere).

Otra información adicional puede requerirse dependiendo del submodelo empleado para definir la información del vehículo.
- Volumen de refrigerante

- Masa del material (masa térmicamente sensible)

- Área equivalente

- Área de transferencia de calor de la camisa del motor

- Coeficiente de transferencia de calor

- Índice del fluido

- Índice del material del motor
- Relación de transmisión entre la bomba y el motor

- Volumen de refrigerante asociado con la bomba

- Eficiencia total de la bomba 


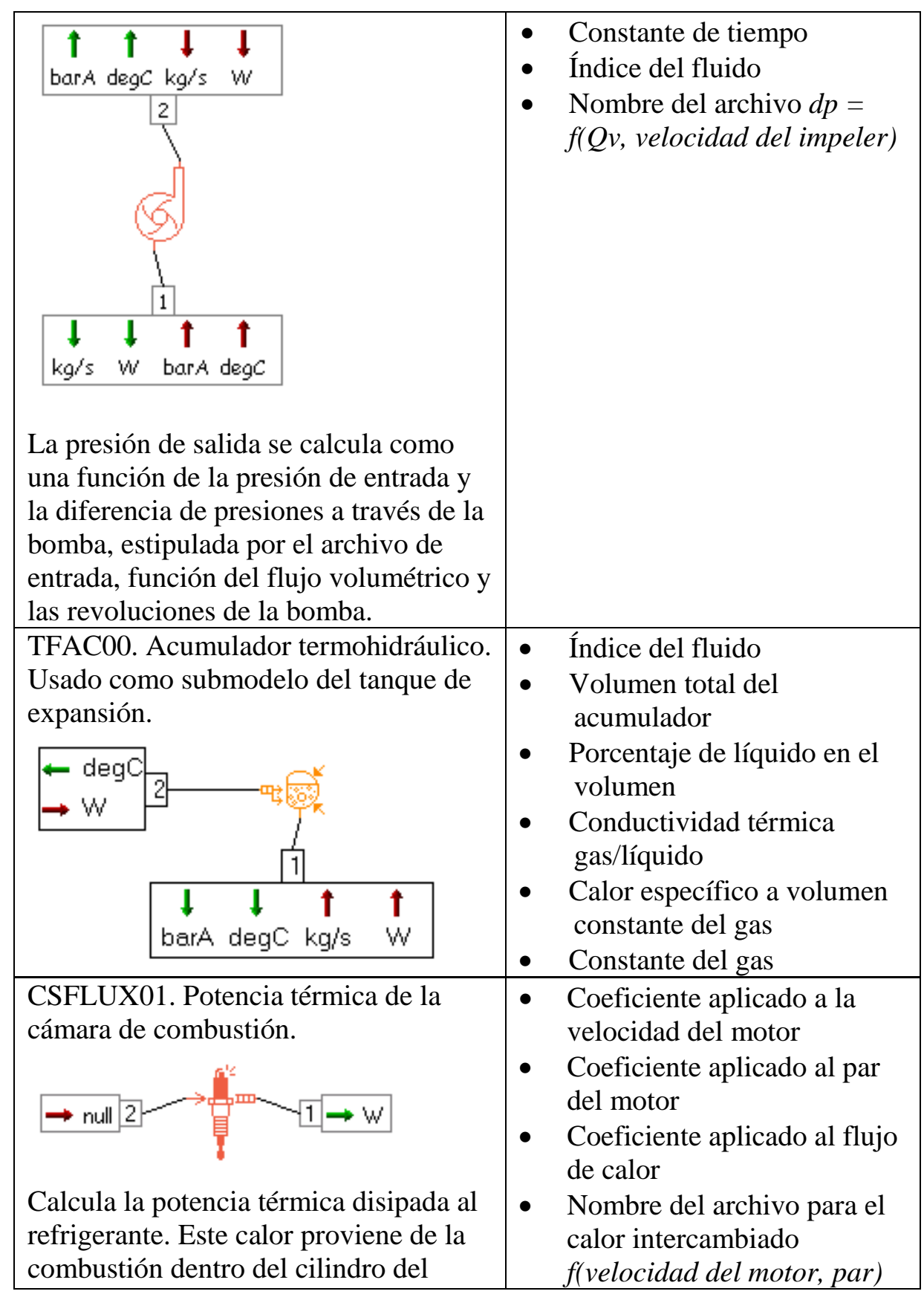




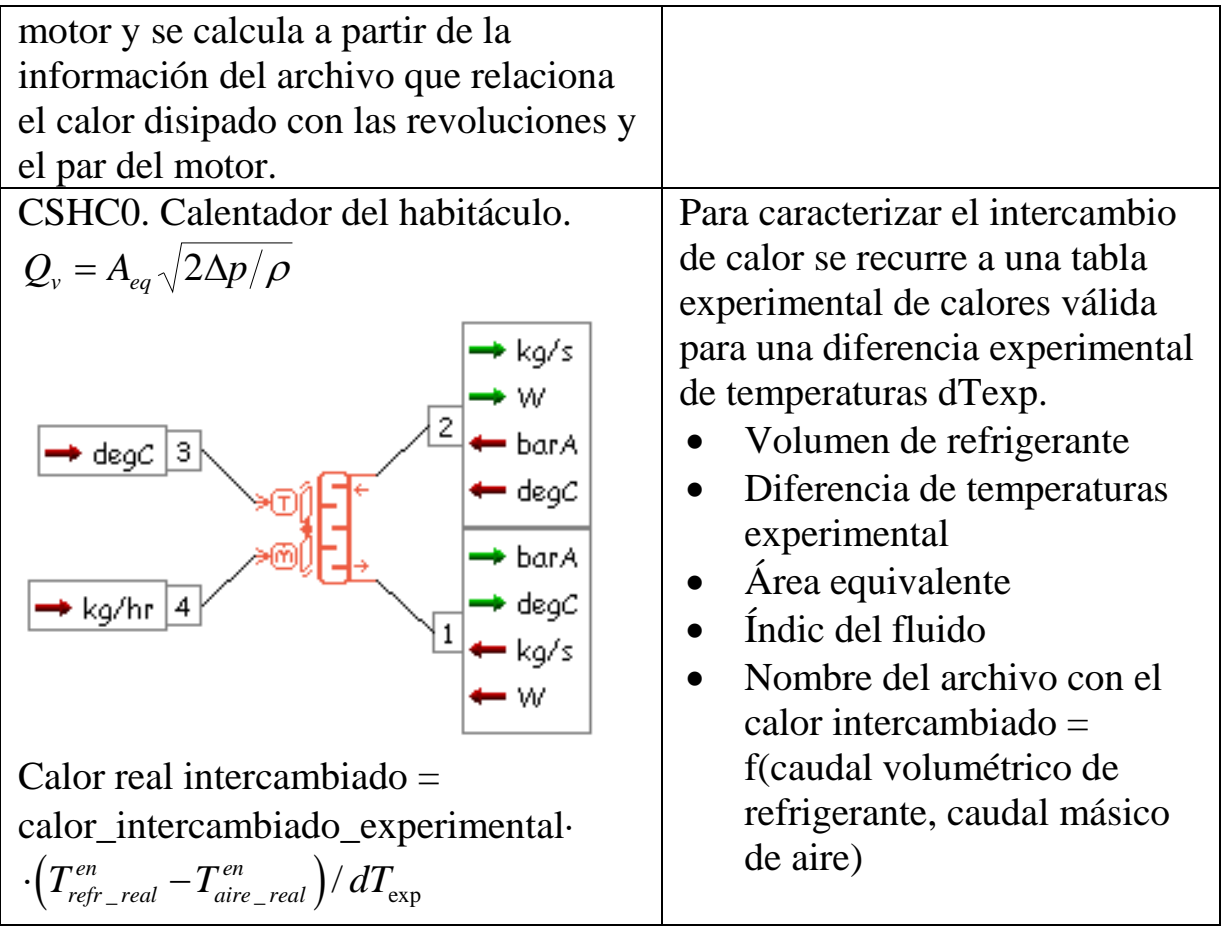

\section{A.2. Acerca de FlowMaster y PowerFlow}

Como se mencionó anteriormente, a diferencia de Amesim, cuyos métodos de solución se basan en las técnicas bond graph y multipuerto, FlowMaster, también multipuerto, hace uso del método de las características para resolver las ecuaciones diferenciales ordinarias (ODES) y las ecuaciones algebraicas diferenciales (DAES) a las cuales se reducen las ecuaciones diferenciales parciales (PDES) que constituyen el modelo matemático del sistema. Para el modelado y la simulación del sistema de refrigeración que se presentará en el capítulo sexto se ha escogido el programa computacional FlowMaster.

Flowmaster originalmente fue concebido para simular los efectos dinámicos causados por los cambios bruscos de presión en circuitos complejos, la sistematización de la información de los componentes y los circuitos lo hace muy versátil y potente para ser compartido por múltiples usuarios en diferentes puestos de trabajo. En comparación con Amesim la información 
que se suministra de los componentes tiene una presentación más física y matemática, pues hace uso de las características de diseño de ingeniería de los componentes, a diferencia de Amesim, en el que se introducen con frecuencia tablas de valores (tal es el caso de la información suministrada para las bombas y para los radiadores). No obstante lo dicho, Amesim da la posibilidad para que el usuario pueda ingresar expresiones de funciones para los componentes.

En la Figura A.3 se ilustra una pantalla de diálogo del programa FlowMaster, no hay una presentación de submodelos como en Amesim y el usuario accede a carpetas con bases de datos de submodelos genéricos (plantillas). Aparte de esta diferencia de organización de la información, el modelado con FlowMaster sigue los mismos cinco pasos descritos para Amesim.

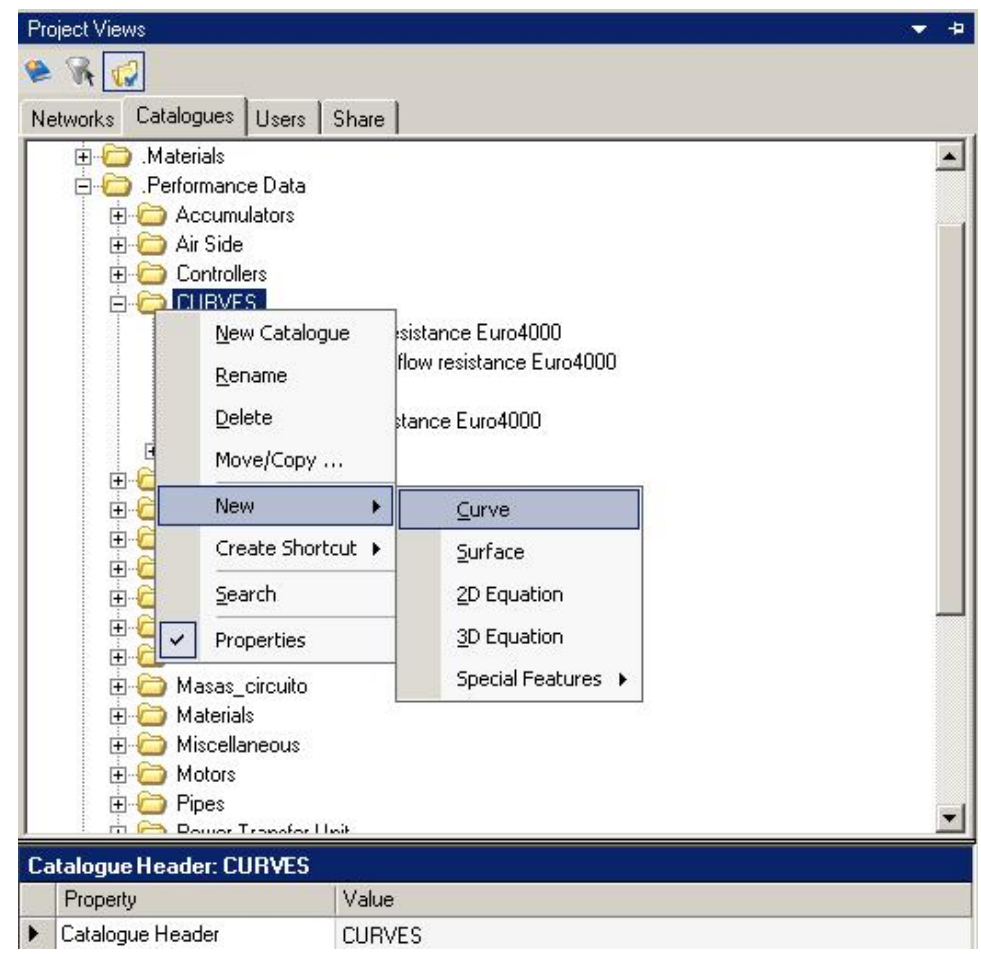

Figura A.3. Presentación de la interfaz de usuario del programa FlowMaster. Secuencia seguida para crear la curva característica de un componente en FlowMaster. 
En la Figura A.4 se ilustra la creación de la característica de una bomba centrífuga. Ingresados los valores de presión y caudal conocidos FlowMaster crea la función de ajuste de la característica. FlowMaster presenta excelentes facilidades para editar, modificar, escalar, ajustar y renombrar las funciones introducidas de los componentes. También es muy flexible la visualización de los resultados y la operación sobre gráficas. Como característica especial se anota además la posibilidad de editar un reporte muy completo del sistema modelado.

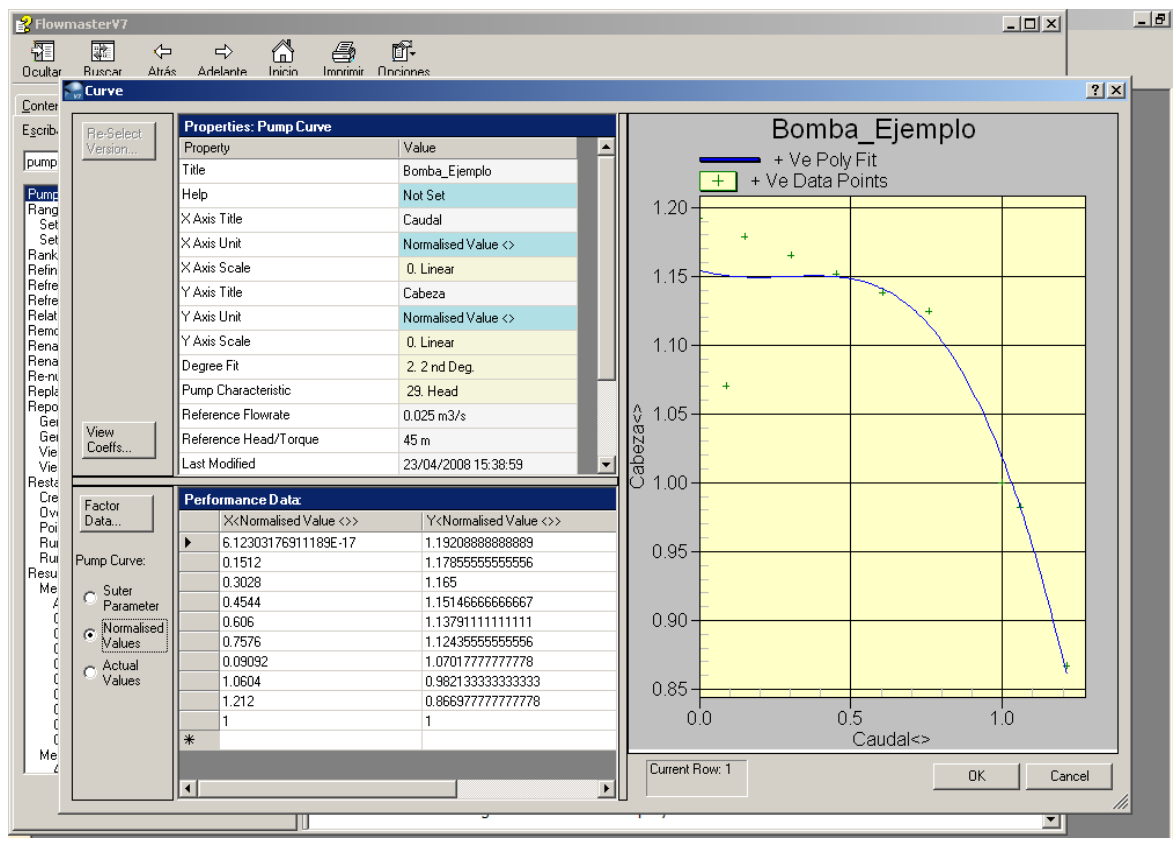

Figura A.4. Cuadros de diálogo y de datos y curva normalizada de la bomba.

FlowMaster incluye una extensa base de información para describir los principales componentes de los sistemas de refrigeración, con la opción de que esta base pueda ampliarse por parte del usuario; una mayor información sobre la operación y fundamentos de FlowMaster puede encontrarse en la referencia [A.4].

El empleo de un programa de modelado 3D en el diseño de la parte de ventilación sirve para asegurar un buen diseño en lo referente a la distribución uniforme del flujo en cualquier sección de paso y en particular en las 
secciones de paso adyacentes a los radiadores y a los ventiladores. Los resultados de las distribuciones de presiones y velocidades pueden usarse para caracterizar en los programas de modelado $1 \mathrm{D}$ los conductos y los componentes y también pueden utilizarse para realizar segmentación de las secciones que aseguren en los modelos 1D una mejor reproducción de las distribuciones de flujo, especialmente en los radiadores. Para el modelado 3D es corriente el empleo de códigos comerciales como Star-CD ${ }^{\circledR}$, Fluent ${ }^{\circledR}$, PowerFlow $^{\circledR}$. En el capítulo sexto se utilizan los resultados del modelado del sistema de ventilación realizado con el programa PowerFlow ${ }^{\circledR}$. Este código es basado en volúmenes finitos con un mallado no estructurado. Para solucionar el campo de velocidades, el flujo turbulento se modela con un modelo k- $\varepsilon$ isotrópico, usando el modelo estándar k- $\varepsilon$ de Launder y Spalding [A.5], junto con las funciones de la pared para representar las condiciones de contorno turbulentas en las capas contiguas a las paredes. Se consideran los tres mecanismos de transferencia de calor: convección, conducción y radiación. El núcleo es modelado como un medio poroso. La pérdida de momento a través del medio poroso se calcula con la ley de Darcy, donde la pérdida de presión se calcula a partir del coeficiente de resistencia inercial y viscosa. El calor disipado por el núcleo se modela usando un modelo basado en una macro de intercambiador de calor en PowerFlow [A.7], el cual usa el NTU del intercambiador de calor para predecir el calor transferido al aire. Empleando la mezcla agua/glicol como refrigerante, se modela el NTU del radiador. La succión de los ventiladores se modela con saltos de presión. Algunas asunciones hechas por el modelo son: el flujo es turbulento estable, el aire es incompresible, el aire es seco, las paredes del sistema son consideradas adiabáticas.

\section{A.3. Bibliografía}

[A.1] AMESim user’s manual. Versión 7.1. Septiembre 2008.

[A.2] Sjöstedt, C. J. On the modular modelling for dynamical simulation with application to fluid systems. Licentiate thesis, Stockholm, 2005.

[A.3] Plazas, A. T., Modelado unidimensional de inyectores common rail Diesel. Tesis doctoral, Universidad Politécnica de Valencia. España, 2005. 
[A.4] FlowMaster user's manual. Versión 7.2.

[A.5] Launder, B., Spalding, D. The numerical computation of turbulent flows. Comp Methods in App. Mech and Eng., vol. 3, , pp. 269289, 1974.

[A.6] Miller, D. S. Internal flow systems, segunda edición, BHR Group Limited, 1996.

[A.7] PowerFlow user's manual. Version 4.1. 